

Assinatura:

\section{Luca Meacci}

\section{A two-component model of the red blood cell membrane and other mathematical models in medicine}

Thesis submitted to the Instituto de Ciências Matemáticas e de Computação - ICMC-USP - in accordance with the requirements of the Computer and Mathematical Sciences Graduate Program, for the degree of Doctor in Science. FINAL VERSION

Concentration Area: Computer Science and Computational Mathematics

Advisor: Prof. Dr. Gustavo Carlos Buscaglia 
Ficha catalográfica elaborada pela Biblioteca Prof. Achille Bassi e Seção Técnica de Informática, ICMC/USP, com os dados inseridos pelo(a) autor(a)

Meacci, Luca

M479t A two-component model of the red blood cell membrane and other mathematical models in medicine / Luca Meacci; orientador Gustavo Carlos Buscaglia. -- São Carlos, 2021. $268 \mathrm{p}$.

Tese (Doutorado - Programa de Pós-Graduação em Ciências de Computação e Matemática Computacional) Instituto de Ciências Matemáticas e de Computação, Universidade de São Paulo, 2021.

1. Red blood cell. 2. Mathematical modeling. 3. Biological fluid mechanics. 4. Lipid membrane. 5. Cytoskeleton. I. Buscaglia, Gustavo Carlos, orient. II. Título. 


\section{Luca Meacci}

\section{Um modelo de dois componentes da membrana dos glóbulos vermelhos e outros modelos matemáticos na medicina}

Tese apresentada ao Instituto de Ciências Matemáticas e de Computação - ICMC-USP, como parte dos requisitos para obtenção do título de Doutor em Ciências - Ciências de Computação e Matemática Computacional. VERSÃO REVISADA

Área de Concentração: Ciências de Computação e Matemática Computacional

Orientador: Prof. Dr. Gustavo Carlos Buscaglia 

To the people who have accompanied me and to those I have not yet met. 

I would like first to express my gratitude to my supervisor, Professor Gustavo Buscaglia, whose guidance, patience, and support have been fundamental in completing my journey. I would also to thank the other members of his team, Professor Roberto Federico Ausas and Professor Fernando Mut.

I would like to acknowledge Professor Mario Primicerio, Professor Angiolo Farina, Dr. Vincenzo di Bari, Professor David Alistair Gray, Professor Michiel Bertsch, Professor Bruno Franchi, Professor Maria Carla Tesi, and Professor Gujji Murali Mohan Reddy.

In addition, I would like to thank my colleagues Franciane Fracalossi Rocha and Débora de Oliveira Medeiros.

All of them have contributed in some form to the realization of this work.

The work was supported by grants from the INCT-MACC (Instituto Nacional de Ciência e Tecnologia - Medicina Assistida por Computação Científica), approved from CNPq (Conselho Nacional de Desenvolvimento Científico e Tecnológico) of Brazil. The research was developed making use of the computational resources (Euler cluster) of the CeMEAI (Center for Mathematical Sciences Applied to Industry) financed by FAPESP (Fundação de Amparo à Pesquisa do Estado de São Paulo) of Brazil (Grant 2013/07375-0). The author acknowledges the Foundation CAPES (Coordenação de Aperfeiçoamento de Pessoal de Nível Superior) of the Ministry of Education of Federal Republic of Brazil for economic support (Grant PROEX-9740044/D).

Sâo Carlos, 15 March 2021 

"Better Medicine Through Mathematics?"

(David C. Dale · Michael C. Mackey) 



\section{RESUMO}

MEACCI, L. Um modelo de dois componentes da membrana dos glóbulos vermelhos e outros modelos matemáticos na medicina. 2021. 265 p. Tese (Doutorado em Ciências Ciências de Computação e Matemática Computacional) - Instituto de Ciências Matemáticas e de Computação, Universidade de São Paulo, São Carlos - SP, 2021.

Esta tese consiste na apresentação de uma série de artigos para a definição de um modelo matemático original de dois componentes para a membrana dos glóbulos vermelhos. O modelo, inspirado nos melhores modelos disponíveis atualmente, considera o citoesqueleto como uma estrutura elástica não linear discreta. A novidade do trabalho proposto é acoplar esse esqueleto com modelos contínuos ao invés dos modelos discretos mais comuns (dinâmica molecular, métodos de partículas) da bicamada lipídica. A interação do citoesqueleto sólido com a bicamada, que é um fluido bidimensional, é feita por meio de forças de adesão adaptando algoritmos de adesão sólido-sólido eficientes. $\mathrm{O}$ tratamento contínuo das partes do fluido é bem justificado por argumentos de escala e leva a problemas numéricos muito mais estáveis e precisos quando, como é o caso, o tamanho das moléculas $(0.3 \mathrm{~nm})$ é muito menor do que o tamanho geral ( $\simeq 8000 \mathrm{~nm}$ ). A versão mais avançada do modelo também leva em consideração a contribuição do citosol, modelado como um fluido interno obedecendo a um comportamento Newtoniano.

A formulação computacional do modelo pode ser usada para estudar o comportamento normal e patológico dos glóbulos vermelhos em processos transitórios lentos. Nos artigos, muitas simulações e aplicações são mostradas. Uma certa atenção foi dada para apresentar a boa postura matemática do modelo.

O trabalho de tese foi realizado no âmbito de atividade de pesquisa de apoio ao Instituto Nacional de Ciência e Tecnologia em Medicina Assistida por Computação Científica (INCT - MACC) do Brasil. O documento contém artigos adicionais publicados contendo novas propostas de modelos matemáticos com aplicações relevantes na medicina. Esses tópicos adicionais lidam com a manipulação e controle ótimo de partículas em micro-fluidos, a modelagem de progresso e tratamento de doenças como câncer com a presença de células-tronco cancerosas e doença de Alzheimer, e um modelo epidemiológico sobre a propagação do COVID-19 que simula a reação social devido à fadiga pandêmica.

Palavras-chave: Glóbulo vermelho, Modelagem matemática, Mecânica biológica dos fluidos, Membrana lipídica, Citoesqueleto, Interações fluido-sólido, Simulações numéricas, Controle ótimo, Manipulação de partículas, Células-tronco cancerosas, Doença de Alzheimer, COVID-19, Fadiga pandêmica. 



\section{ABSTRACT}

MEACCI, L. A two-component model of the red blood cell membrane and other mathematical models in medicine. 2021. 265 p. Tese (Doutorado em Ciências - Ciências de Computação e Matemática Computacional) - Instituto de Ciências Matemáticas e de Computação, Universidade de São Paulo, São Carlos - SP, 2021.

This thesis consists in the presentation of a series of papers for the definition of an original two-component mathematical model for the red blood cell membrane. The model, inspired by the best models currently available, considers the cytoskeleton as a discrete non-linear elastic structure. The novelty of the proposed work is to couple this skeleton with continuum models instead of the more common discrete models (molecular dynamics, particle methods) of the lipid bilayer. The interaction of the solid cytoskeleton with the bilayer, which is a two-dimensional fluid, is done through adhesion forces adapting efficient solid-solid adhesion algorithms. The continuous treatment of the fluid parts is well justified by scale arguments and leads to much more stable and precise numerical problems when, as is the case, the size of the molecules $(0.3 \mathrm{~nm})$ is much smaller than the overall size $(\simeq 8000 \mathrm{~nm})$. The most advanced version of the model also takes into account the contribution of the cytosol, modeled as an internal fluid obeying a Newtonian behaviour.

The computational formulation of the model can be used to study normal and pathological behavior of red blood cells in slow transient processes. In the papers, many simulations and applications are shown. A certain attention was done to present the mathematical well-posedness of the model.

The thesis work was conducted within research activity supporting the National Institute of Science and Technology in Medicine Assisted by Scientific Computing (INCT - MACC) of Brazil. The document contains additional published papers containing new proposals of mathematical models with relevant applications in medicine. These additional topics concern the manipulation and optimal control of particles in micro-fluids, the modeling of progress and treatment of diseases such as cancer with the presence of cancer stem cells and Alzheimer's disease, and an epidemiological model on the COVID-19 spread that mimics the social reaction due to pandemic fatigue.

Keywords: Red blood cell, Mathematical modeling, Biological fluid mechanics, Lipid membrane, Cytoskeleton, Fluid-solid interactions, Numerical simulations, Optimal control, Particles manipulation, Cancer stem cells, Alzheimer's disease, COVID-19, Pandemic fatigue. 


\section{Statement:}

The present thesis is a collection of seven published articles:

MEACCI, L.; DI BARI, V.; AUSAS, R. F.; MUT, F.; GRAY, D. A.; BUSCAGLIA, G. C. A mathematical model of a single seed oleosome. Results in Applied Mathematics, v. 9, p. 100128, (Elsevier), 2021.

DOI: https://doi.org/10.1016/j.rinam.2020.100128

MEACCI, L.; PRIMICERIO, M.; BUSCAGLIA, G. C. Growth of tumours with stem cells: The effect of crowding and ageing of cells. Physica A: Statistical Mechanics and its Applications, 2021, p. 125841, (Elsevier), 2021.

DOI: https://doi.org/10.1016/j.physa.2021.125841

MEACCI, L.; BUSCAGLIA, G. C.; MUT, F.; AUSAS, R. F.; PRIMICERIO, M. A new twocomponent approach in modeling red blood cells. Communications in Applied and Industrial Mathematics, v. 11, p. 55-71, 2020.

DOI: https://doi.org/10.2478/caim-2020-0004 Site: https://content.sciendo.com/view/journals/caim/11/1/articlep55.xml

BERTSCH, M.; FRANCHI, B.; MEACCI, L.; PRIMICERIO, M.; TESI, M. C. The amyloid cascade hypothesis and Alzheimer's disease: A mathematical model. European Journal of Applied Mathematics, v. 01, p. 1-20, 2020.

DOI: https://doi.org/10.1017/S0956792520000339

MEACCI, L.; BUSCAGLIA, G. C. AUSAS; MUT, F. A Red Blood Cell Cyto-Bilayer Interaction Model. XXXIX Congresso Nacional de Matemática Aplicada e Computacional - CNMAC 2019, Uberlândia (MG), September 16-20th, 2019. Proceeding Series of the Brazilian Society of Computational and Applied Mathematics, 7(1), 2020.

DOI: https://doi.org/10.5540/03.2020.007.01.0344

MEACCI, L.; PRIMICERIO, M. Mathematical models for tumours with cancer stem cells. Computational and Applied Mathematics, vol. 37, no 5, p. 6544-6559, (Springer) 2018.

DOI: https://doi.org/10.1007/s40314-018-0707-2

MEACCI, L.; FRACALOSSI ROCHA, F.; SILVA ALVES, A.; PRAMIU, P. V.; BUSCAGLIA, G. C. A Trajectory Planning Model for the Manipulation of Particles in Microfluidics. TEMA. Trends in Computational and Applied Mathematics, v. 19, p. 509, 2018.

DOI: http://dx.doi.org/10.5540/tema.2018.019.03.509

with other four papers in the submission or revision phase, and it is completed by an introduction and conclusions. 
INTRODUCTION . . . . . . . . . . . . . . . . . . . 19

$1.1 \quad$ Two-component model of red blood cell membrane . . . . . . . . 20

1.2 Miscellaneous models of microfluidic control, disease progression, and epidemiology . . . . . . . . . . . . . . . . . 25

1.2.1 Manipulation and trajectory planning of particles in microfluidics . 25

1.2.2 Mathematical modeling of tumours with cancer stem cells . . . . 25

1.2.3 Mathematical model for Alzheimer's disease progression . . . . . . . 26

1.2.4 COVID-19 spread mathematical model with pandemic fatigue impact 27

2 A NEW TWO-COMPONENT APPROACH IN MODELING RED BLOOD CELLS . . . . . . . . . . . . . . . . . . 29

$2.1 \quad$ Mathematical formulation . . . . . . . . . . . . 31

$2.1 .1 \quad$ Lipid bilayer model . . . . . . . . . . . . . . . . . . . 32

2.1.2 Cytoskeleton model . . . . . . . . . . . . . . . . . . . 34

2.1.3 Interaction Model . . . . . . . . . . . . . . . . . 35

$2.1 .4 \quad$ Model summary . . . . . . . . . . . . . . . . 36

$2.2 \quad$ Numerical simulations . . . . . . . . . . . . . 36

$2.3 \quad$ Achievements and future opportunities . . . . . . . . . 43

3 MODELING A RED BLOOD CELL CYTOSKELETON: INSIGHTS, TIPS, AND CODE . . . . . . . . . . . . . . . . 47

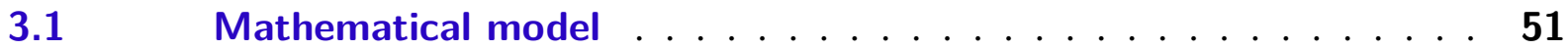

3.1.1 A case-study example: one spectrin . . . . . . . . . . . 53

3.1.2 Derivation of the complete system . . . . . . . . . . . . . . . 54

3.1.3 Cytoskeleton coarse-grained scaling . . . . . . . . . . . 55

3.1.4 Dynamical cytoskeleton and symmetry defects characterizations . . 58

3.1.5 Some in-depth observations: the virtual optical tweezers experiment 61

$3.2 \quad$ Achievements and future opportunities . . . . . . . . . . 65

4 A RED BLOOD CELL CYTO-BILAYER INTERACTION MODEL . 67

4.1 The cytoskeleton-bilayer interaction model . . . . . . . 68

$4.2 \quad$ Achievements and future opportunities . . . . . . . . . . 71 
$5.1 \quad$ Mathematical formulation . . . . . . . . . . . . 74

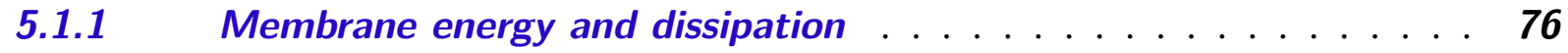

5.1.2 Lipid layer model . . . . . . . . . . . . . . . . . . 78

$5.2 \quad$ Results and Simulations . . . . . . . . . . . . . . 79

5.2.1 Numerical methods . . . . . . . . . . . . . . . . . . . . . . 79

5.2.2 Hypothesis, equilibrium, and lipid layer stress . . . . . . . . . 79

5.2.3 Senescence: a virtual experiment . . . . . . . . . . . . . 81

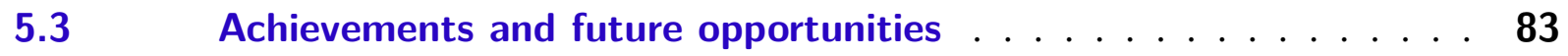

6 A MULTI-COMPONENT RED BLOOD CELL COMPUTATIONAL MODEL: A NEW MATHEMATICAL FORMULATION . . . . . 85

$6.1 \quad$ Methods and Models . . . . . . . . . . . . . . 85

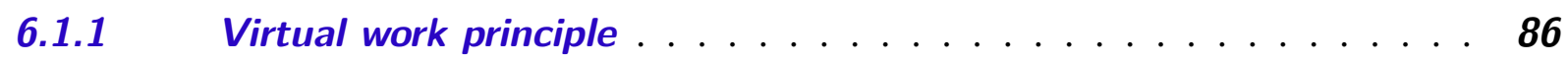

6.1.2 Dissipation and Boussinesq-Scriven law . . . . . . . . 87

6.1.3 Laplace-Beltrami identity and Canham-Helfrich energy . . . . . . 88

6.1.4 Inextensible membrane and osmotic equilibrium constrains . . . . 89

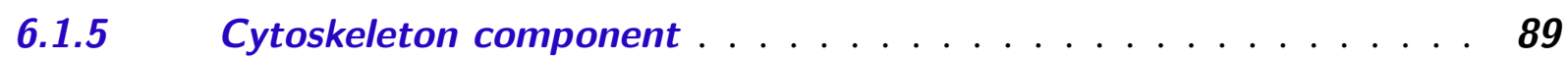

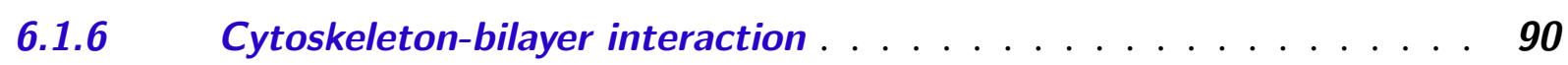

$6.1 .7 \quad$ Model summary . . . . . . . . . . . . . . . . . . . 91

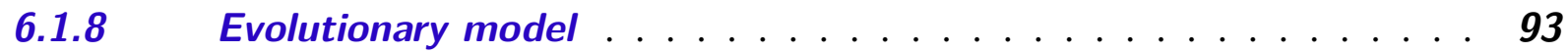

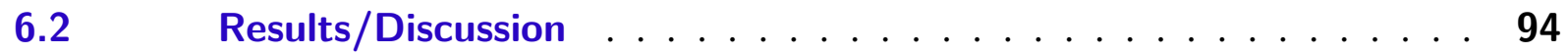

6.2.1 Discretization and numerical approach . . . . . . . . . . . 94

6.2.2 Equilibrium and resting shape . . . . . . . . . . . . 97

6.2.3 Stretching by optical tweezers . . . . . . . . . . . . . . 101

6.2.4 Validation through experimental measurements . . . . . . . . 102

$6.3 \quad$ Achievements and future opportunities . . . . . . . . . 106

7 A TRAJECTORY PLANNING MODEL FOR THE MANIPULATION OF PARTICLES IN MICROFLUIDICS . . . . . . . . . . 111

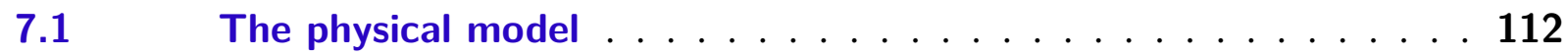

7.1.1 Particle manipulation along a predefined path . . . . . . . . . 114

7.1.2 Determining the path via arrival time optimization . . . . . . . 115

$7.2 \quad$ Calculation of $V(\vec{x}, \breve{d}) \ldots \ldots \ldots \ldots \ldots \ldots \ldots$

$7.3 \quad$ Optimizing trajectories by Bellman's principle . . . . . . . . . 119

$7.4 \quad$ Results . . . . . . . . . . . . . . . . . . 121

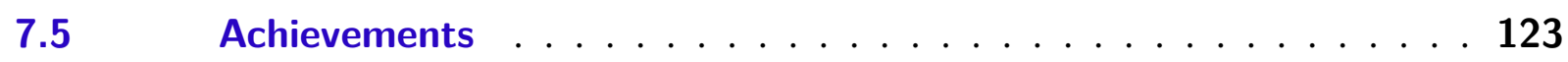

8 MATHEMATICAL MODELS FOR TUMOURS WITH CANCER

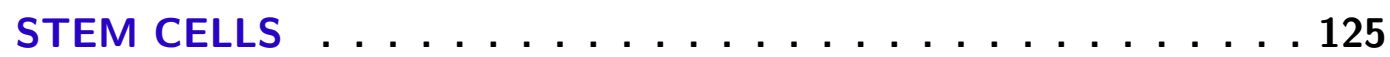

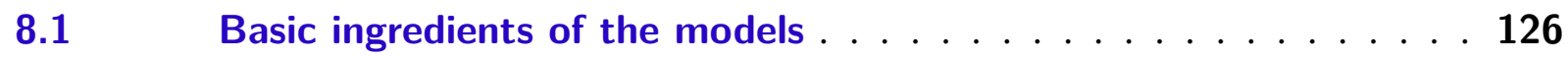


$8.2 \quad$ Agent-based models . . . . . . . . . . . . . . . . . 127

$8.3 \quad$ Mean field approximation $\ldots \ldots \ldots \ldots \ldots \ldots$

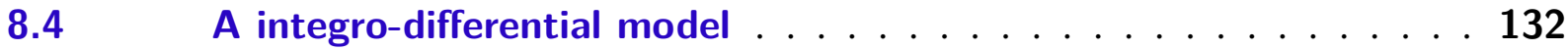

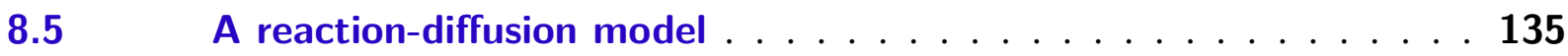

$8.6 \quad$ Achievements and future opportunities $\ldots \ldots \ldots \ldots$

9 GROWTH OF TUMOURS WITH STEM CELLS: THE EFFECT OF CROWDING AND AGEING OF CELLS . . . . . . . . . . 141

$9.1 \quad$ Cellular automata . . . . . . . . . . . . . . . . . . 144

$9.2 \quad$ Compartmental models . . . . . . . . . . . . . . . 146

9.3 Continuous age-structure for the non-stem cells . . . . . . . . 152

9.4 Achievements and future opportunities . . . . . . . . . . 158

10 THE AMYLOID CASCADE HYPOTHESIS AND ALZHEIMER'S DISEASE: A MATHEMATICAL MODEL . . . . . . . . . . . 159

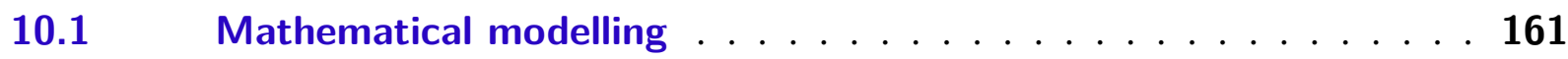

10.2 The model . . . . . . . . . . . . . . . . . 163

$10.3 \quad$ A specific example . . . . . . . . . . . . . . . 166

10.3.1 Dynamics of AD: local effect . . . . . . . . . . . . . . 167

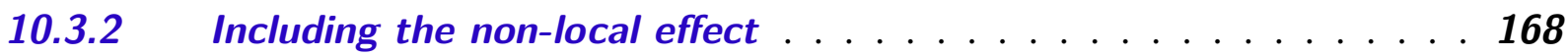

10.3.3 Global existence of solutions of system (10.10) . . . . . . . . 171

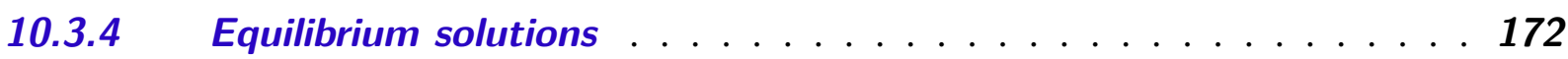

10.3.5 Parameter dependence . . . . . . . . . . . . . . . . . . . 175

10.4 Achievements and future opportunities . . . . . . . . . 176

11 PANDEMIC FATIGUE IMPACT INTO COVID-19 SPREAD: A MATHEMATICAL MODELING ANSWER TO THE ITALIAN SCENARIO 177

$11.1 \quad$ Model . . . . . . . . . . . . . . . . . . . . . . . 180

11.2 The case of Italy: numerical results . . . . . . . . . . . . . 184

$11.3 \quad$ Achievements and future opportunities . . . . . . . . . 188

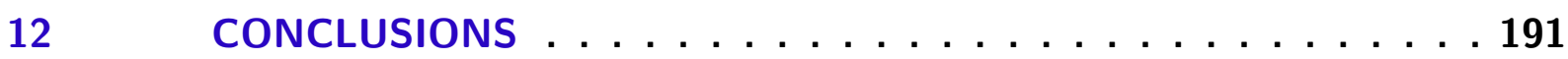

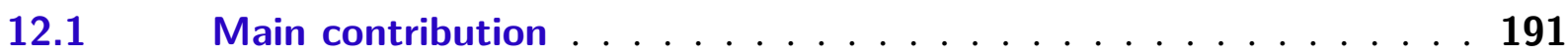

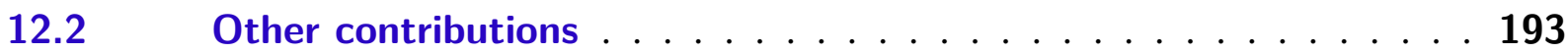

12.3 Ongoing and future works $\ldots \ldots \ldots \ldots \ldots \ldots$

BIBLIOGRAPHY . . . . . . . . . . . . . . . . . . . . . . . . . . 197

APPENDIX A CODE FOR MODELING A RED BLOOD CELL CYTOSKELETON . . . . . . . . . . 229 
B.1 A little study with Mathematica . . . . . . . . . . . 241

B.1.1 A hybrid potential . . . . . . . . . . . 245

APPENDIX C INTERACTION BETWEEN CROWDING AND GROWTH IN TUMOURS WITH STEM CELLS: CONCEPTUAL

MATHEMATICAL MODELLING . . . . . . . . . . 247

C.1 Cellular automata modelling . . . . . . . . . . . . 249

C.1.1 Cellular automata simulations . . . . . . . . . . . . . . . 251

C.2 Modelling based on differential equations . . . . . . . . . . 254

C.2.1 Numerical simulations . . . . . . . . . . . . . . . 257

C.3 Well-posedness of the differential equations based model . . . . 259

C.4 Concluding comments . . . . . . . . . . . . 262

ANNEX A LIST OF PUBLICATIONS AND CONFERENCE PARTIC-

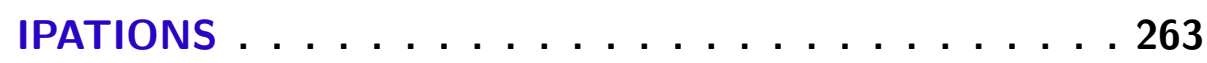


CHAPTER

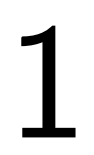

INTRODUCTION

The key role of mathematics in the development of science and technology is an argument old as the beginning of modern science, with Galileo Galilei who proposed this idea and Isaac Newton proved it (VÁZQUEZ, 2001). But it is in recent years, also due to the development of new technologies, that a growing and flourishing interest has specifically focused on the use of mathematical and computational modeling in life sciences (STEWART, 2011) and medicine (HOPPENSTEADT; PESKIN, 2013; TAKEUCHI; IWASA; SATO, 2007). These efforts have accelerated the growth of a new domain of science, with areas of concern that cover all scales of human biology and pathology, including epidemiology. New challenges arise constantly to provide rational and mechanistic explanations, as well as solutions in biology and medicine through the mathematical approach (COVENEY, 2020). On the other hand, the current prospects encourage to look to the future with confidence with respect to successfully addressing new problems (BAO et al., 2014) and to understand a "large number of mutually interdependent complex physiologic networks in the human body and to apply that understanding to maintaining the functions for which nature designed them". (WEST, 2014). In this sense, Cohen (2004) said the now well-known expression the "mathematics is biology's next microscope, only better". This context may lead us to ask the legitimate question proposed by Dale and Mackey (2015): "Better medicine through mathematics?"

This thesis does not claim to give a comprehensive answer to this question. It proposes specific solutions to real problems in medicine with new and original contributions largely already recognized by the scientific community and published in international journals. This research activity supports the National Institute of Science and Technology in Medicine Assisted by Scientific Computing (INCT - MACC), with the headquarter in InCor-HCFM USP (the largest Latin American Center for Cardiovascular Medicine). Since its foundation on November 2008, the INCT - MACC has aimed to to transfer for the Health System innovative medical applications, within the Brazilian Program of National Institutes of Science and Technology. In particular, the contents of this thesis, adhere to INCT - MACC mission to conduct and promote 
research and development in the frontier of knowledge in scientific computing and its applications in medicine, especially the computer modeling and simulation of physiological systems that comprise the human body. In this line the work of this thesis has the INCT-MACC common objective to connect, in a consistent manner, areas (medicine+computer science+modeling) that a priori progress separately and, consequently, to contribute to produce a new area of research in Brazil.

We addressed problems and phenomena taken from various fields of biology and medicine, from cell membranes mechanics to COVID-19 spreading, passing from cancer with stem cells and Alzheimer's disease treatments. Such results are achieved in the last four years through the development and strengthening of a network of international collaborations. The INCT - MACC essence is to foster a lively and fertile environment with internationally renowned researchers, which can also provide guidance and scientific advice to ensure the project is kept at the state of the art in terms of scientific computing applied to medicine. For this reason, besides the main content of this thesis on a new model for the red blood cell membrane, we present the concretization of other significant works. The author working inside the INCT MACC associated Applied Mathematics and Scientific Computing Department (Laboratório de Matemática Aplicada e Computação Científica) of the Institute of Mathematics and Computer Sciences (São Carlos Campus, SP) of the University of São Paulo (USP), led by Professor Gustavo Carlos Buscaglia, has promoted and benefited from the direct cooperation (among all) of the following persons and institutions: Professor Roberto Federico Ausas (Institute of Mathematics and Computer Sciences, University of São Paulo, Brazil), Professor Fernando Mut (Bioengineering Department, George Mason University, USA), Professor Mario Primicerio (Department of Mathematics “U. Dini”, University of Florence, Italy), Professor David Alistair Gray and Dr. Vincenzo di Bari (School of Biosciences, University of Nottingham, United Kingdom), Professor Bruno Franchi and Professor Maria Carla Tesi (Department of Mathematics, University of Bologna, Italy), and Professor Michiel Bertsch (Department of Mathematics, University of Rome "Tor Vergata", Italy). To consult the complete list of the author's activities in the last four years, the reader is referred to Annex A. We introduce in the following sections the chapters contents.

\subsection{Two-component model of red blood cell membrane}

In recent years, a multi-component approach in red blood cell (RBC) mathematical/computational modeling has been taking hold as new formulations and techniques have been introduced. The biological motivation hinges on the same RBC composite architecture. During its 120 days average life cycle, the RBC is subjected to strong stresses and deformations, as it traverses the human circulatory system in capillaries of the order of magnitude of its own size. Its ability to deform and assume the typical biconcave shape again is closely connected with its physiological function of transporting oxygen to tissues and organs. Hence the need to consider 
the contribution of each component of the erythrocyte structure to simulate the mechanical behavior exhaustively.

The human RBC physiognomy is rich and complex (LUX, 2016), but could be simplified for our modeling objectives, as shown in Figure 1. With an average width of $7.5 \mu \mathrm{m}$ and height of $2 \mu \mathrm{m}$, the RBC is surrounded by a double layer made of phospholipid molecules (in yellow in Figure 1), called lipid bilayer of $5 \mathrm{~nm}$ of thickness. This cellular membrane is complemented by a cytoskeleton, which contributes to the flexibility and stability of the cell. Such a network consists of long twisted strands of spectrin and actin filaments (in Figure 1 colored in purple). Transmenbranic proteins allow the cytoskeleton to attach (and detach) to the bilayer, contributing to an interactional adhesion between the two components. Finally, the cytoplasm (in green in Figure 1) is the part of the cell which is contained within the entire cell membrane. It is essentially a fluid composed of the cytosol and organelles and rich in hemoglobin, an ironcontaining biomolecule that can bind oxygen. In the RBC case, the nucleus is absent so that it can contain more haemoglobin to increase oxygen carry capacity.

Figure 1 - Red blood cell (RBC) main components structure.

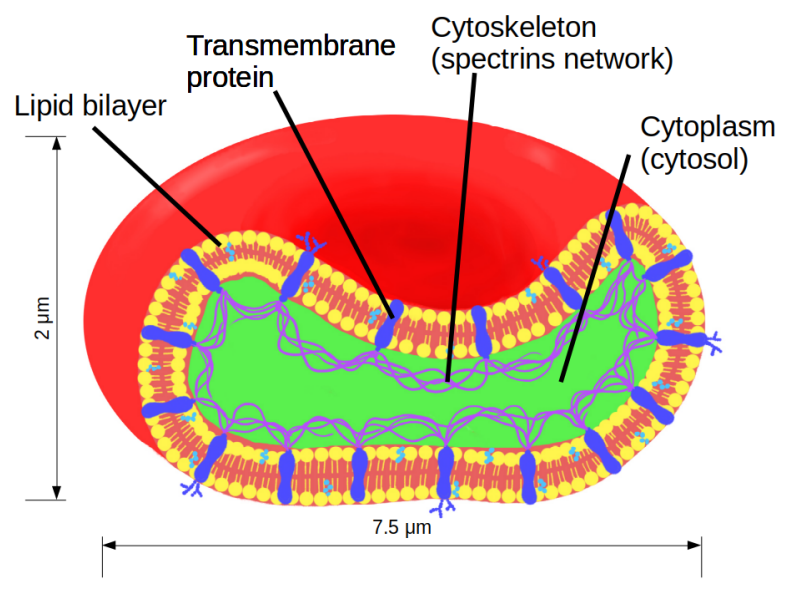

It is a difficult task to succeed in synthesizing the multiple approaches adopted to try to mimic mechanical behavior. All of them have strengths and elements of weakness (LI; LU; PENG, 2020). Some reviews are been presented illustrating a general discussion of numerical simulation challenges of flowing blood cells (FREUND, 2014; JU et al., 2015). However, we can opt to divide them into two large classes: continuum-based and particle-based models. We propose in the Figure 2 a schematic overview and summary of the contributions that in some way represent the best practices that have contributed to growth in the field of mathematical/computational RBC modeling, and from which other works have followed. Continuum-based models arise naturally when one aspires to describe fluid-structure interactions between the RBCs and the surrounding flow. They have the advantage to benefit of the experience of the well established continuum mechanics community of robust and accurate numerical methods (BARTHES-BIESEL, 2016). Some of them are represented by the implementation of boundary integral method (BIM) (RAMANUJAN; POZRIKIDIS, 1998; VEERAPANENI et al., 2011) and immersed boundary 
method (IBM) (YAZDANI; BAGCHI, 2011; HEINTZ, 2015). In the case of simple shear flows, they can be successfully adopted. On the other hand, particle-based models, which treat both the fluid and the cell membrane as particulate materials, can easier take into account the nonhomogeneous RBC structure, introducing elements like cytoskeleton spectrins, proteins, defects, and so on (LI et al., 2018). Nonetheless, although coarse-grained methods are eligible, they usually are computationally very expensive for large-scale applications. This class represents a very extensive field to which multi-particle collision dynamics (MPCD) methods (NOGUCHI; GOMPPER, 2005; MCWHIRTER; NOGUCHI; GOMPPER, 2009), coarse-grained molecular dynamics (CGMD) techniques (LI; LYKOTRAFITIS, 2012) and dissipative particle dynamics (DPD) methods (PIVKIN; KARNIADAKIS, 2008; FEDOSOV; CASWELL; KARNIADAKIS, 2010; PENG et al., 2013; LI; LYKOTRAFITIS, 2014) belong. They also reflect the choice of some most recent and complete works for the whole cell study, consisting in OpenRBC code (TANG et al., 2017) and LAMMS molecular dynamics simulator (FU et al., 2017). A special mention deserves the work of Peng et al. (2010, 2011) (PENG; ASARO; ZHU, 2010; PENG; ASARO; ZHU, 2011), for having well focused the idea that to model a RBC an holistic vision on several levels is necessary. In addition, the cytoskeleton is a component that has acquired space with dedicated studies. A common choice in this direction is to adopt non-linear elastic models of the worm-like chain type. Finally, we aim to highlight approaches that use the curvature concept according to the Canham-Helfrich model (ARROYO; DESIMONE, 2009; BARRETT; GARCKE; NÜRNBERG, 2015; RODRIGUES et al., 2015a). This assumption allows a continuous description of the lipid bilayer and consequently lends itself to the use of efficient numerical methods such as finite elements method (FEM). From this idea, very recently our group of authors presented a two-component model that mimics the behavior of the lipid bilayer, the cytoskeleton and the interaction dynamics between the two parts.

In Chapter 2, we present a computational modelling approach to study normal and pathological behavior of red blood cells in slow transient processes that can not be accompanied by pure particle methods (which require very small time steps). The basic model, inspired by the best models currently available, considers the cytoskeleton as a discrete non-linear elastic structure. The novelty of the proposed work is to couple this skeleton with continuum models instead of the more common discrete models (molecular dynamics, particle methods) of the lipid bilayer. The interaction of the solid cytoskeleton with the bilayer, which is a two-dimensional fluid, will be done through adhesion forces adapting efficient solid-solid adhesion algorithms. The continuous treatment of the fluid parts is well justified by scale arguments and leads to much more stable and precise numerical problems when, as is the case, the size of the molecules $(0.3 \mathrm{~nm})$ is much smaller than the overall size $(\simeq 8000 \mathrm{~nm})$. In this chapter we display some numerical simulations that show how our approach can describe the interaction of an RBC with and exogenous body as well as the relaxation of the shape of an RBC toward its equilibrium configuration in the absence of external forces.

In Chapter 3, we provide a mathematical model for a red blood cell (RBC) cytoskeleton. 
Figure 2 - Comparative summary review of RBC component models (best practices).

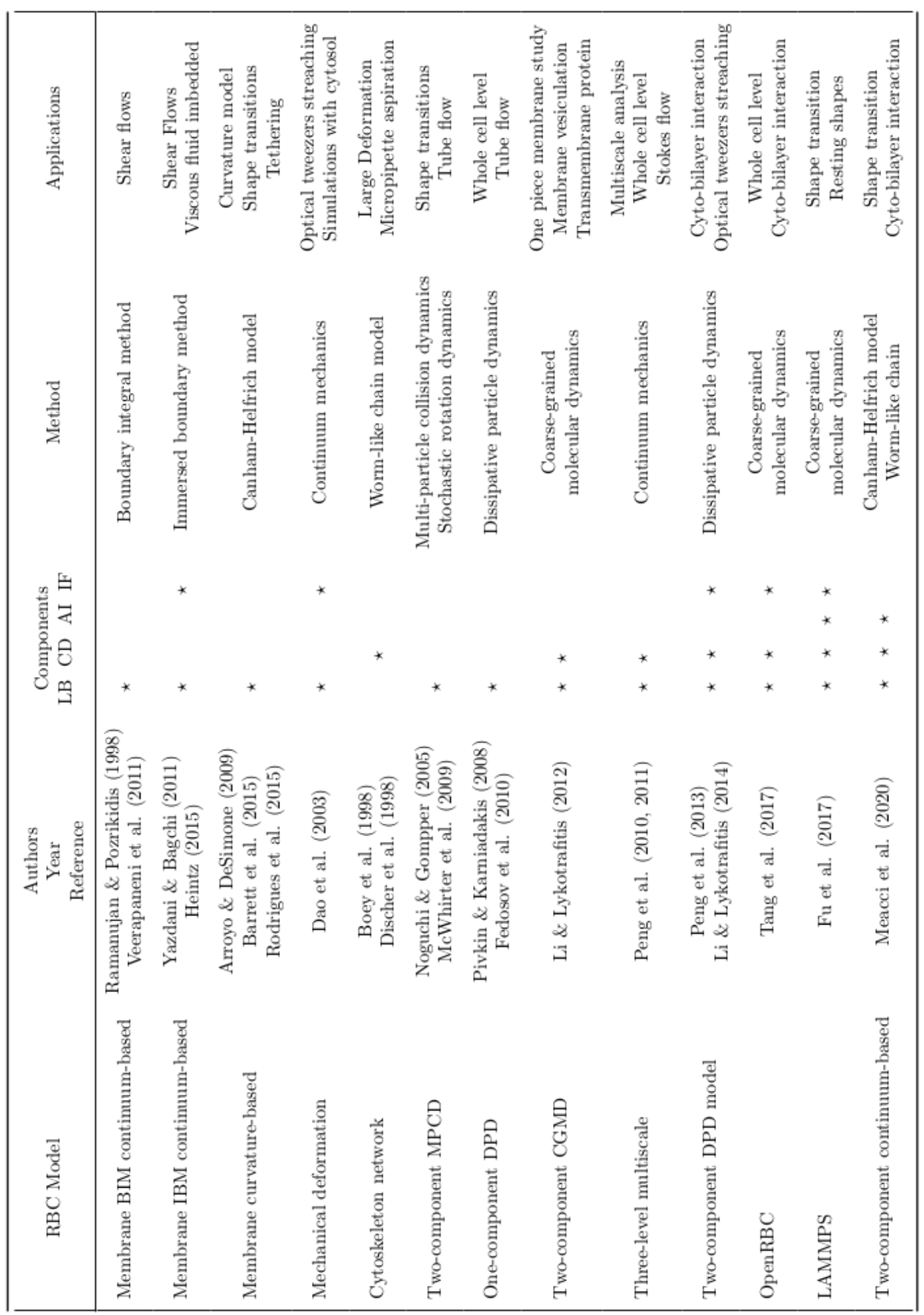

The motivation for this work lies in further elucidating the role that the cytoskeleton assumes in determining the remarkable RBC mechanical properties and in the need to consider the cytoskeleton component in the latest and most advanced single-cell mathematical models. In this process, a worm-like chain force was adopted, as it is already used in biological applications, and 
the virtual work principle was applied. We tested the model in some simple virtual experiments gaining insights over the system behaviours, including single spectrin equilibrium, cytoskeletal symmetry defects impact, and stress transmission and distribution within the network. In addition, we suggest modelling tips as well as coarse-grained scaling techniques and virtual optical tweezers experiments. In the Appendix A we provide also the implementation of the model into an Octave code, as ready-to-use tool to implement the model.

In order to develop a single-whole red blood cell model, it is necessary to model the interaction between the lipid bilayer and the cytoskeleton. To mimic these interactions is a current and open problem with important applications in medicine. In Chapter 4, we provide a mathematical formulation of a soft-body adhesion model in presence of a continuous lipidic membrane. In particular, we hypothesize to apply the Lennard-Jones potential in order to provide a mathematical formulation of a soft-body adhesion model between the above-mentioned components of an erythrocyte. The Mathematica use is effective to simulate and quickly visualize the interaction energy and force by changing parameters. In the Appendix B we report the Wolfram code to visualize a dynamical application for the cytoskeleton-membrane interaction.

The study of the red blood cell membrane has allowed us to acquire useful skills in many interdisciplinary fields. In Chapter 5, we report for the first time a mathematical approach to model the behaviour of a single oleosome (oil body) within a seed cellular environment. This work has been followed up through visits and active collaboration with the School of Biosciences of the University of Nottingham, in Sutton Bonington Campus (United Kingdom). To describe the behaviour of the oleosome membrane, we adopted a dynamical continuum model based on the principle of virtual work where the intrinsic energy of the lipid membrane is assumed to obey the Canham-Helfrich model with the rheology of the viscous interface governed by the Boussinesq-Scriven law. To show the suitability of this approach to study the mechanical behaviour of oleosomes, we present some numerical simulations of a single oleosome deformation occurring under in vivo and ex vivo conditions. This work aims to show how the mathematical and computational modelling allows studying the impact of otherwise hard-to-measure physical quantities in this field of biological applications.

Finally in Chapter 6, we present a new mathematical/computational formulation for a multi-component red blood cell model. The merit of this work is to take into account (i) the lipid bilayer behavior, (ii) the cytoskeleton dynamics, (iii) the interaction activity between them, and (iv) the internal cytoplasm flow. Following the line of the previous works we use the advantage to adopt the continuum approach for the membrane modeling. The well-posedness of the multi-component model is illustrated; presenting first the stationary model problem, then the evolutionary model problem, and finally the discrete evolutionary model problem. The availability of the model is tested with virtual experiments such as equilibrium resting shape process and optical tweezers stretching. 


\subsection{Miscellaneous models of microfluidic control, disease progression, and epidemiology}

\subsubsection{Manipulation and trajectory planning of particles in microflu- idics}

Many important microfluidic applications, also in medicine, require the control and transport of particles immersed in a fluid. In Chapter 7, we propose a model for automatically planning good trajectories from an arbitrary point to a target in the presence of obstacles. It can be used for the manipulation of particles using actuators of mechanical or electrical type. We present the mathematical formulation of the model and a numerical method based on the optimization of travel time through the Bellman's principle. The implementation is focused on square grids such as those built from pixelated images. Numerical simulations show that the trajectory tree produced by the algorithm successfully avoids obstacles and stagnant regions of the fluid domain. To control the trajectory of the particles, we use system actuators to induce fields of velocities appropriate to the desired path. A detailed explanation of the numerical resolution of these problems, according to MAC (Marker and Cell) discretization, is exposed in our previous work (MEACCI et al., 2017).

\subsubsection{Mathematical modeling of tumours with cancer stem cells}

Mathematical models for the growth of tumours in the presence of stem cells (CSCs) and differentiated tumour cells (CCs) are presented and discussed in Chapter 8. The CSCs are assumed to be immortal and multipotent, i.e. capable of generating several possible lineages of CCs that may undergo ageing and apoptosis. We present a synthetic review of some models that have been proposed in the literature and we report the development of new original models, to open a complete multi-approach window on modellization of the problem. Numerical simulations are displayed that exhibit the so-called "tumour paradox" that consists in the fact that tumours with a larger death rate for the $\mathrm{CC}$ may grow faster than tumours with a lower death rate for the same class of cells. The occurrence of this paradox has important consequences also on the strategy of treatment of tumours.

In Chapter 9, we focus on the effect of crowding and ageing of cells. Each $\mathrm{CC}$ is characterised by two indexes, related to the differentiation lineage and the class of age, respectively. The effect of crowding is taken into account, assuming that mitosis can be hindered by the presence of cells near the would-be mother cell. Two families of models are proposed. First, models based on cellular automata are considered, whose evolution is governed by stochastic rules. Then, by averaging over the cells with the same pair of indexes, we obtain a deterministic model that consists of a system of Ordinary Differential Equations (ODEs) whose unknown functions are the fractions of the cells in each lineage and the class of age. The system presents a novelty 
with respect to the other compartmental models proposed in the literature as it cannot be solved hierarchically because of the presence of the crowding effect. Numerical simulations based on the two families of models give the same qualitative results. The final section of the paper is devoted to the case in which the age distribution of the CCs is continuous and not discrete. In this case, an interesting mathematical problem is obtained that consists of one ODE for the fraction of CSCs and $m$ first-order Partial Differential Equations (PDEs); one for each lineage of CCs.

In Appendix C, we propose and discuss a conceptual modellization of the growth of tumors in the presence of immortal multipotent CSCs and of several lineages of differentiated CCs. It is assumed that the replication of CSCs can be symmetric or asymmetric with a prescribed mean ratio. For the CCs aging, mitosis and apoptosis are taken into account. We assume that replication can be hindered by the local crowding of the cells in the vicinity of the mother cell. The model is implemented in the framework of 3D cellular automata whose dynamics is governed by stochastic rules. Some simulations are displayed showing the growth of a tumor and of the fractions of different lineages and age classes of CCs. Then, we present an approach in which the same dynamics of aging, replication and apoptosis are considered, but the aim is to study the time evolution of the fractions of the different families of cells averaged over the total volume. The dynamics is governed by a system of ODEs, thus by deterministic rules. Nevertheless, numerical simulations of the solution of this system show qualitative similarity with the results of the CA, although in this case the crowding effect is no longer a local effect but is also averaged over the total volume. Moreover, we prove the mathematical well-posedness of this model in a general framework.

\subsubsection{Mathematical model for Alzheimer's disease progression}

In Chapter 10, we present a conceptual mathematical model for Alzheimer's disease (AD). This work was conducted thanks the collaboration of Mario Primicerio and Michiel Bertsch (as well as Professors in their respective universities) as Affiliated of the Institute for Calculus Applications "Mauro Picone" of the National Research Council of Italy and Members of the Accademia dei Lincei (founded in 1603; it is the oldest scientific academy in the world).

According to the so-called amyloid cascade hypothesis, we assume that the progression of $\mathrm{AD}$ is associated with the presence of soluble toxic oligomers of beta-amyloid. Monomers of this protein are produced normally throughout life, but a change in the metabolism may increase their total production and, through aggregation, ultimately results in a large quantity of highly toxic polymers. The evolution from monomeric amyloid produced by neurons to senile plaques (long and insoluble polymeric amyloid chains) is modelled by a system of ordinary differential equations (ODEs), in the spirit of the Smoluchovski equation.

The basic assumptions of the model is that, at the scale of suitably small representative volumes (REVs) of the brain, the production of monomers depends on the average degradation of the neurons and in turn, at a much slower time-scale, the degradation is caused by the number 
of toxic oligomers. To mimic the prion-like diffusion of the disease in the brain, we introduce an interaction among adjacent REVs, that can be assumed to be isotropic or to follow given preferential patterns. We display the results of numerical simulations which are obtained under some simplifying assumptions. For instance, the amyloid cascade is modelled by just three ODEs, and the simulations refer to abstract 2D domains, simplifications which can be easily avoided at the price of some additional computational costs. Since the model is suitably flexible to incorporate other mechanisms and geometries, we believe that it can be generalised to describe more realistic situations.

\subsubsection{COVID-19 spread mathematical model with pandemic fatigue impact}

The COVID-19 outbreak has generated psychological repercussions for the populations affected by the pandemic. These consequences of the epidemic also at the same time can be causes of a non-controlled spread of the virus. This occurs when peoples stress assumes to be a "pandemic fatigue", leading people to feel demotivated about following recommended behaviours to protect themselves and others. In Chapter 11, we present a new generalized SIQRD (susceptible-infected-quarantined-recovered-dead) model performed by Ordinary Differential Equations (ODEs), where we have implemented the pandemic fatigue impact effect. The mathematical well-posedness of the model is proven. We show the numerical results to be consistent with the transmission dynamics data characterizing epidemic of COVID-19 outbreak in Italy in 2020 and we provide a measure of the possible pandemic fatigue impact. The model can be used to evaluate public health interventions and to prevent with specific actions the possible damages resulting from the possible social phenomenon of relaxation with respect to the observance of the preventive rules imposed. 

CHAPTER

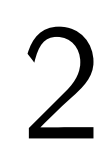

\section{A NEW TWO-COMPONENT APPROACH IN MODELING RED BLOOD CELLS}

Red blood cells (RBC) occupy between 35 and $50 \%$ of the total blood volume in the human circulatory system. Their mechanical characteristics are highly relevant for the biological functions of blood, not only because they have direct consequences in hemodynamics (POPEL; JOHNSON, 2005; MCHEDLISHVILI; MAEDA, 2001) but also because the way RBC respond (e.g., to shear stress) may impact the synthesis of nitric oxide and thus the regulation of vascular tonus (ULKER et al., 2009).

In human blood, a RBC (or erythrocyte) has the shape of a biconcave disk, flattened and depressed in the centre, with a cross-section of dumbbell shape (LI-GUO et al., 2010). In its undeformed state, the discoid has a diameter of $6-8 \mu \mathrm{m}$ and its average thickness is $2 \mu \mathrm{m}$. Typical erythrocytes have a volume of $90 \mu \mathrm{m}^{3}$ and a surface area of $136 \mu \mathrm{m}^{2}$ (see e.g. (JU et al., 2015)). Therefore, while in blood vessels with a diameter larger than $200 \mu \mathrm{m}$ (like arteries) the flow of the blood can be modeled in terms of a homogeneous non-newtonian fluid, when we consider the circulation in vessels whose internal diameter is comparable with the size of RBCs (arterioles, venules, and capillaries) it becomes essential to consider the blood as a suspension of erythrocytes and to take into account their morphological evolution.

Indeed, blood can flow in capillaries with diameter smaller than that of RBCs, because RBCs change their shape from the original biconcave one to that of a bullet or of a parachute and then recover their initial state. Therefore modeling erythrocytes is essential for an appropriate description of the blood flow and its functions in microcirculation (FASANO; SEQUEIRA, 2017). Analogous situations are frequent: as long as it is possible to apply a continuum approach by averaging the relevant quantities over a small volume the available methods are relatively simple and well established (SVAHN; BERG, 2007); however, sometimes intrinsic heterogeneities become fundamental because different components react differently to the same external stimulus (BAO et al., 2014). In those cases even a single-cell approach can be instrumental for modeling 
the system or for developing therapeutic strategies (CARLO; LEE, 2006). The present paper aims to contribute under this regard.

A human RBC is a nucleus-free cell whose basic mechanical structure consists of a cytoskeleton formed by a spectrin network and of a fluid-like lipid bilayer. In what concerns Mechanics, lipid bilayers are two-molecule-thick sheets of phospholipid molecules which are known to function as osmotic barriers with viscous tangential behavior and bending resistance. The spectrin network is like an internal reinforcement thought to play a role in the stability of the RBC's shape (RODRÍGUEZ-GARCÍA et al., 2015). The spectrin network attaches, through a junction complex (JC), to proteins that are "floating" in the bilayer among the (much smaller) lipid particles. The JCs can be associated to nodes of a network of fibers with elastic or viscoelastic behavior. The attachment of the JCs to the bilayer is strong enough for them to remain attached in most physiological conditions, but under extreme conditions it is known that detachment occurs before the bilayer itself breaks (PENG et al., 2013).

In (LI et al., 2014; CHANG; LI; KARNIADAKIS, 2017) a computational scheme was presented in which both the bilayer and the cytoskeleton are modeled as a discrete system of interacting particles. This requires particles describing the behavior of the lipid molecules, of the spectrin fibers, of the JCs, and also possibly of the inner and outer three-dimensional liquids. More precisely, the cytoskeleton is described as a set of JCs (nodes) connected by nonlinear springs obeying a worm-like-chain law (other laws have also been considered (FEDOSOV; CASWELL; KARNIADAKIS, 2010)). The typical length of the spectrin filaments is about 70 nanometers, which corresponds to a number of about 30,000 nodes for the cytoskeleton. This number is small enough for the numerical simulation of the complete cytoskeleton to be feasible. For certain studies that require low-cost solvers, some coarse graining rules are available that allow to reduce the number of nodes by a factor of 10 or 100 without significant loss in physical accuracy (FEDOSOV; CASWELL; KARNIADAKIS, 2010).

The use of particles to describe the lipid bilayer, on the other hand, is more questionable. The number of molecules in the bilayer is about 700 million, so that each of the numerical particles must model thousands or tens of thousands of molecules for the computing cost to be affordable.

Taking these facts into account, we adopt the following strategy: maintaining the discrete description of the cytoskeleton as in (LI et al., 2014; CHANG; LI; KARNIADAKIS, 2017), we model the lipid bilayer in terms of a continuous surface fluid. In particular, we adopt the viscous liquid shell model with Canham-Helfrich bending energy described by Arroyo et al. (ARROYO; DESIMONE; HELTAI, 2010), with a discretization based on the method of Rodrigues et al. (2015a) that has sufficient generality to accomplish this task. The two sub-systems are coupled by adhesion forces that bind the cytoskeleton and the bilayer, inspired by the computational contact formulations for soft body adhesion of Sauer (SAUER, 2012).

In this paper we describe the model and display some numerical simulations in specific 
situations that show how our approach is promising in view of future applications. In particular, we consider a case of interaction with an exogenous body, and the relaxation of an RBC from a deformed configuration towards its equilibrium state, in absence of external forces.

\subsection{Mathematical formulation}

We consider a red blood cell as a mechanical system whose configuration is described by the state $\mathscr{X}$ of the cytoskeleton and the state $\mathscr{Y}$ of the lipid bilayer. In Figure 3 we show a scheme of a RBC depicting the two components of the model. The configuration $\mathscr{X}$ represents a state of the cytoskeleton, which in our model is a set of $N_{X}$ balls of radius $R$ representing the junctional complexes of the cytoskeleton. In this way, appropriate coordinates for $\mathscr{X}$ are the positions of the nodes of the cytoskeleton model, which are the centers of the aforementioned balls. These coordinates will be denoted by $\left\{X^{j}\right\}_{j=1}^{N_{X}}$. Consequently, the instantaneous motion of the cytoskeleton nodes are described by $U^{j}=\frac{d X^{j}}{d t}$.

Figure 3 - Sketch of the problem.

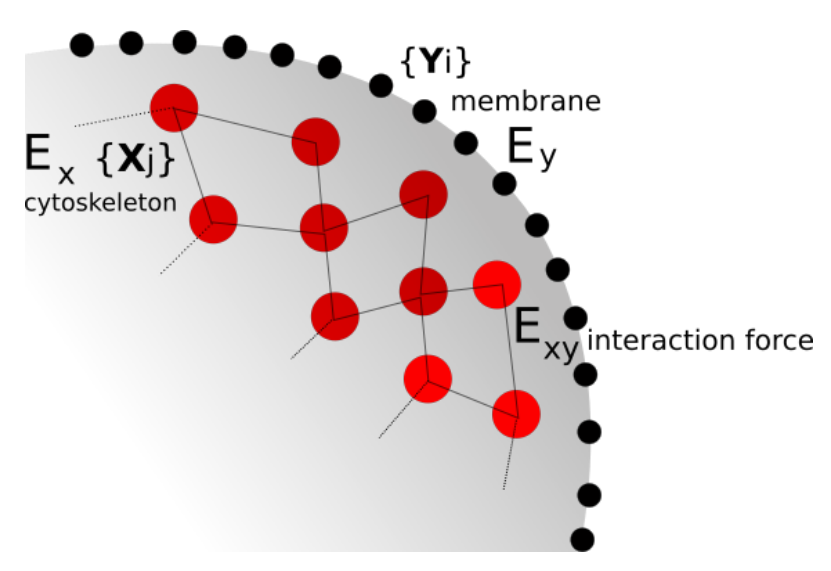

Similarly, $\mathscr{Y}$ is a configuration of the lipid bilayer, which in the exact problem is an element of an infinite-dimensional manifold of possible membrane shapes of fixed volume and surface area. For numerical purposes, however, we parameterize the configuration $\mathscr{Y}$ by the positions of $N_{Y}$ points, the nodes of the bilayer model. The coordinates of any configuration $\mathscr{Y}$ are thus $\left\{Y^{i}\right\}_{i=1}^{N_{Y}}$. From these coordinates the geometrical position of the bilayer,

$$
\Gamma(\mathscr{Y})=\left\{y \in \mathbb{R}^{3} \mid y \text { belongs to the bilayer surface }\right\},
$$

can be reconstructed. The instantaneous motion of the bilayer particles is characterized by the rates of change of $\mathscr{Y}$,

$$
\mathscr{W}=\frac{d \mathscr{Y}}{d t} .
$$

This means that the tracking of the surface is Lagrangian. The discrete velocity $w$, evaluated at the $i$-th bilayer node, coincides with that of the lipid particle at $Y^{i}(t)$ (i.e., $w^{i}=\frac{d Y^{i}}{d t}$ ). In this latter sentence "lipid particle" is to be understood not as a lipid molecule but as a small macroscopic chunk of lipid material, in the spirit of Continuum Mechanics. 
The energy $\mathscr{E}$ of the proposed two-component system (that clearly depends on $\mathscr{X}$ and $\mathscr{Y})$ is decomposed into the sum

$$
\mathscr{E}(\mathscr{X}, \mathscr{Y})=\mathscr{E}_{X}(\mathscr{X})+\mathscr{E}_{Y}(\mathscr{Y})+\mathscr{E}_{X Y}(\mathscr{X}, \mathscr{Y})
$$

where $\mathscr{E}_{X}$ is the intrinsic skeleton energy, $\mathscr{E}_{Y}$ the intrinsic bilayer energy, and $\mathscr{E}_{X Y}$ the interaction energy between the cytoskeleton and the bilayer.

We consider as starting point for the mathematical formulation of the problem the principle of virtual work demanding that the virtual change in energy of the system for an admissible virtual variation of the configuration variables plus the work done by the dissipative forces equals the work done by the external forces (LANCZOS, 1970). The corresponding expression can be formally written as

$d_{X} \mathscr{E}(\mathscr{X}, \mathscr{Y}) \bullet \delta \mathscr{X}+d_{Y} \mathscr{E}(\mathscr{X}, \mathscr{Y}) \bullet \delta \mathscr{Y}+\mathscr{D}(\mathscr{X}, \mathscr{Y}, \mathscr{U}, \mathscr{W}) \bullet(\delta \mathscr{X}, \boldsymbol{\delta} \mathscr{Y})=\mathscr{F}_{X} \bullet \delta \mathscr{X}+\mathscr{F}_{Y} \bullet \delta \mathscr{Y}$,

where

- $d_{X} \mathscr{E}(\mathscr{X}, \mathscr{Y}) \bullet \delta \mathscr{X}$ is the infinitesimal change $\delta \mathscr{E}$, when the state of the system is perturbed from $(\mathscr{X}, \mathscr{Y})$ to $(\mathscr{X}+\delta \mathscr{X}, \mathscr{Y})$,

- the bullet $\bullet$ is an appropriate duality product which will take on meaning based on the single component model as presented below,

- $d_{Y} \mathscr{E}(\mathscr{X}, \mathscr{Y}) \bullet \delta \mathscr{Y}$ is the infinitesimal change $\delta \mathscr{E}$, when the state of the system is perturbed from $(\mathscr{X}, \mathscr{Y})$ to $(\mathscr{X}, \mathscr{Y}+\delta \mathscr{Y})$,

- $\mathscr{D}(\mathscr{X}, \mathscr{Y}, \mathscr{U}, \mathscr{W}) \bullet(\delta \mathscr{X}, \boldsymbol{\delta} \mathscr{Y})$ is the dissipation of the system (i.e., the work of its internal dissipative forces), when the system is perturbed by $(\boldsymbol{\delta} \mathscr{X}, \boldsymbol{\delta} \mathscr{Y})$,

- the right-hand side is the virtual work of external forces.

Explicit expressions for the terms in (5.7) are derived from the mathematical modeling of each of the two components (bilayer, cytoskeleton) of the system, and of their interaction, as briefly introduced below.

\subsubsection{Lipid bilayer model}

Being $\Gamma$ the average surface of the bilayer at time $t$, let $H$ be the average curvature of $\Gamma$, $\check{n}$ the normal vector and $\kappa$ the mean curvature vector, defined as $\kappa=H \check{n}$. We also need the tangential projector $\mathbb{P}=\mathbb{I}-\check{n} \otimes \check{n}$. The tangential gradient $\nabla_{\Gamma}$ is the operator defined as $\nabla_{\Gamma} f=\mathbb{P} \nabla \widehat{f}$, where $f: \Gamma \rightarrow \mathbb{R}$ is any function and $\widehat{f}$ an arbitrary extension of $f$ to an open neighborhood of $\Gamma \subset \mathbb{R}^{3}$. The constraints of constant volume and constant surface are imposed 
by means of Lagrange multipliers, namely a uniform pressure difference $p$ and a space-dependent surface tension field $\sigma$, respectively.

Together with these definitions, we assume linear viscous dynamics of the lipid bilayer (SCRIVEN, 1960; ARROYO; DESIMONE; HELTAI, 2010) and the Canham-Helfrich bilayer energy given by (CANHAM, 1970; HELFRICH, 1973; SEGUIN; FRIED, 2014)

$$
\mathscr{E}_{Y}=\frac{C_{c h}}{2} \int_{\Gamma}\|\kappa\|^{2}
$$

where $C_{c h}$ is a constant. Then, we can compute the part $\mathscr{D}_{Y}$ of the virtual dissipation that is intrinsic to the bilayer $(\delta \mathscr{X}=0, \delta \mathscr{Y}=v$ ) as

$$
\mathscr{D}_{Y} \bullet v=\int_{\Gamma} 2 \mu D_{\Gamma} w: D_{\Gamma} v
$$

where $\mu$ is the surface viscosity, and the virtual change in energy as

$$
d_{Y} \mathscr{E}_{Y} \bullet v=C_{c h} \int_{\Gamma}\left[(\mathbb{I}-2 \mathbb{P}) \nabla_{\Gamma} \kappa: \nabla_{\Gamma} v+\frac{1}{2}\left(\nabla_{\Gamma} \cdot \kappa\right)\left(\nabla_{\Gamma} \cdot v\right)\right]
$$

We need a few more definitions to completely define the mathematical model of the bilayer. The first two of them are the experimentally observed constraints of isochoricity (constant volume, enforced by osmotic pressure) and inextensibility (constant area of each bilayer parcel). These constraints are enforced through the Lagrange multipliers $p$ (internal pressure) and $\sigma$ (surface tension, a scalar field on $\Gamma$ ). Notice that, as any Lagrange multiplier, $p$ and $\sigma$ are unknowns of the problem and thus depend on the configuration of the bilayer and on the force applied on it. The last needed definition is that of the interaction force field, $f^{\Gamma}$, that represents in an $L^{2}$ sense all the interaction terms in (2.4), i.e.,

$$
d_{Y} \mathscr{E}_{X Y} \bullet v+\mathscr{D}_{X Y} \bullet v=-\int_{\Gamma} f^{\Gamma} \cdot v
$$

In this way, the bilayer mathematical problem, assumed the interaction force $f^{\Gamma}$ known for the moment, reads: Find the unique fields $w, \sigma, \kappa$ and the only $p \in \mathbb{R}$ such that

$$
\begin{aligned}
\int_{\Gamma} 2 \mu D_{\Gamma} w: D_{\Gamma} v-p \int_{\Gamma} v \cdot \check{n}+\int_{\Gamma} \sigma \nabla_{\Gamma} \cdot v+ & \\
+C_{c h} \int_{\Gamma}\left[(\mathbb{I}-2 \mathbb{P}) \nabla_{\Gamma} \kappa: \nabla_{\Gamma} v+\frac{1}{2}\left(\nabla_{\Gamma} \cdot \kappa\right)\left(\nabla_{\Gamma} \cdot v\right)\right] & =\int_{\Gamma} f^{\Gamma} \cdot v, \\
\int_{\Gamma} \xi \nabla_{\Gamma} \cdot w & =0, \\
\int_{\Gamma} \kappa \cdot \zeta & =\int_{\Gamma} \mathbb{P}: \nabla_{\Gamma} \zeta, \\
\int_{\Gamma} w \cdot \check{n} & =0,
\end{aligned}
$$


$\forall(v, \xi, \zeta) \in V \times Q \times K$, where $V$ and $K$ are essentially $\left(H^{1}(\Gamma)\right)^{3}$ and $Q=L^{2}(\Gamma)$. The equation (2.11) corresponds the weak form of the Laplace-Beltrami identity, while (2.10) and (2.12) are the constraints of fixed surface area and volume, respectively.

The methodology to solve this part of the bi-component model arises from the discretization of the above variational problem in space and in time. This has already been developed and published (RODRIGUES et al., 2015b). It incorporates automatic adjustment of the time step and surface remeshing (LÖHNER, 1996). The theoretical framework comes from the works by Dziuk, Elliot, and others (BONITO; NOCHETTO; PAULETTI, 2010; DZIUK; ELLIOTT, 2007; DZIUK; ELLIOTT, 2013; RUSU, 2005).

In this contribution we incorporate the interaction with the cytoskeleton, as shown in Section 2.1.3 below. For that purpose, we first introduce in Section 2.1.2 the model for the internal skeletal component of the RBC.

\subsubsection{Cytoskeleton model}

The cytoskeleton will be treated by following the nature of its components, basically a spectrin fiber network with special joints (GRATZER, 1981). The model considers a mesh made of a set of nodes (junctions) joined by molecular chains represented by worm-like chains (HANSEN et al., 1996; FEDOSOV; CASWELL; KARNIADAKIS, 2010; FEDOSOV et al., 2011). The number of the elements of the skeleton is large $\left(\sim 10^{5}\right)$ but numerically tractable. Also, coarse-grained models are available in the literature to save computing effort (FEDOSOV; CASWELL; KARNIADAKIS, 2009).

According to these premises, the elastic spectrin mesh energy $\mathscr{E}_{X}$ is defined as follows

$$
\mathscr{E}_{X}=\sum_{j}\left[\frac{k_{B} T \ell_{m}\left(3 x_{j}^{2}-2 x_{j}^{3}\right)}{4 \ell_{p}\left(1-x_{j}\right)}+\frac{k_{p}}{(n-1) \ell_{j}^{n-1}}\right],
$$

where $\ell_{j}$ is the filament length $j, \ell_{m}$ is the maximum extension of these filaments, $x_{j}=\ell_{j} / \ell_{m}, \ell_{p}$ is the persistence length, $k_{B} T$ is the unit of energy and $n$ and $k_{p}$ are parameters. A homogenized version of this model corresponds to an elastic shell of shear modulus (FEDOSOV et al., 2011)

$$
G_{0}=\frac{\sqrt{3} k_{B} T}{4 \ell_{p} \ell_{m} x_{0}}\left(\frac{x_{0}}{2\left(1-x_{0}\right)^{3}}-\frac{1}{4\left(1-x_{0}\right)^{2}}+\frac{1}{4}\right)+\frac{\sqrt{3} k_{p}(n+1)}{4 \ell_{0}^{n+1}}
$$

with $\ell_{0}$ the equilibrium spacing and $x_{0}=\ell_{0} / \ell_{m}$, but, as said, our formulation keeps the wormlike chains without homogenizing them. The virtual change in energy is readily obtained by differentiating (2.13) with respect the vector of nodal coordinates. In this way, if no intrinsic dissipation is attributed to the cytoskeleton, if the interaction force on the $j$-th node is denoted by $F^{j}$ and if $m$ denotes the effective mass of each node, the equations governing the cytoskeleton movement are

$$
m \frac{d^{2} X^{j}}{d t^{2}}+\frac{\partial \mathscr{E}_{X}}{\partial X^{j}}=F^{j}
$$


The inertia is very small at the scale of the junction complexes, so that in this contribution we adopt $m=0$. These equations are numerically integrated with an implicit Newmark scheme, which is well established in solid mechanics.

\subsubsection{Interaction Model}

The interaction between the bilayer and the cytoskeleton is modeled as adhesion of soft bodies, adapting the formulation of Sauer (SAUER, 2012) based on the models and available data (FREUND; LIN, 2004; KUUSELA; ALT, 2009; PAJIC-LIJAKOVIC; MILIVOJEVIC, 2014; PENG et al., 2013). Denoting by $\Upsilon$ the adherent surface of the cytoskeleton, the contact energy, here denoted with $\mathscr{E}_{X Y}$, takes the general form

$$
\mathscr{E}_{X Y}=\int_{\Gamma} \int_{\Upsilon} \beta_{\Gamma} \beta_{\Upsilon} \phi\left(\left\|x^{\Gamma}-x^{\Upsilon}\right\|\right) d x^{\Gamma} d x^{\Upsilon}
$$

where $\phi$ is the potential of interaction (in Joule $/ \mathrm{m}^{4}$ ) and $\beta_{\Gamma}, \beta_{\Upsilon}$ are dimensionless scalars. One popular choice for $\phi$ is the Lennard-Jones (LJ) potential (ISRAELACHVILI, 2011), i.e.,

$$
\phi(d)=\varepsilon\left(\frac{r_{0}}{d}\right)^{k}-2 \varepsilon\left(\frac{r_{0}}{d}\right)^{k / 2}
$$

where $d$ is the distance between two particles and $r_{0}$ and $\varepsilon$ are length and energy scales, respectively. This potential has a minimum of value $-\varepsilon$ at $d=r_{0}$, it is repulsive for $d<r_{0}$ and attractive if $d>r_{0}$.

The force on the bilayer resulting from this energy, to be substituted in the right-hand side of (2.9), is

$$
f^{\text {con, },}\left(x^{\Gamma}\right)=-\beta_{\Gamma} \int_{\Upsilon} \beta_{\Upsilon} \phi^{\prime}\left(\left\|x^{\Gamma}-x^{\Upsilon}\right\|\right) \frac{x^{\Gamma}-x^{\Upsilon}}{\left\|x^{\Gamma}-x^{\Upsilon}\right\|} d x^{\Upsilon}
$$

Attributing a spherical shape of radius $R$ and center $c$ to $\Upsilon$, this integral can be computed analytically as an explicit function $\mathscr{G}\left(\left\|x^{\Gamma}-c\right\|-R, r_{0}, \varepsilon\right)$ times the unit vector along $x^{\Gamma}-c$. The function $\mathscr{G}$ diverges when $d=\left\|x^{\Gamma}-c\right\|-R$ tends to 0 , since $d<0$ implies interpenetration. Such singularity is too difficult to deal with numerically. Instead, we regularize the model replacing it by

$$
\widetilde{\mathscr{G}}\left(d, r_{0}, \varepsilon\right)= \begin{cases}\mathscr{G}\left(d, r_{0}, \varepsilon\right) & \text { if } d \geq z \\ \mathscr{G}\left(z, r_{0}, \varepsilon\right)+\frac{\partial \mathscr{G}}{\partial d}\left(z, r_{0}, \varepsilon\right)(d-z) & \text { otherwise }\end{cases}
$$

The function $\widetilde{\mathscr{G}}$ is defined for all $d \in \mathbb{R}$, thus allowing a softer contact with some interpenetration. The parameter $z$ is taken as $\alpha r_{0}$, with $0<\alpha<1$. A typical value is $\alpha=0.9$.

Similarly, the force exerted on a point $x^{\Upsilon}$ of the adherent surface of the cytoskeleton by the bilayer $\Gamma$ is given by

$$
f^{\mathrm{con}, \Upsilon}\left(x^{\Upsilon}\right)=\beta_{\Upsilon} \int_{\Gamma} \beta_{\Gamma} \phi^{\prime}\left(\left\|x^{\Gamma}-x^{\Upsilon}\right\|\right) \frac{x^{\Gamma}-x^{\Upsilon}}{\left\|x^{\Gamma}-x^{\Upsilon}\right\|} d x^{\Gamma} .
$$


It is immediate to see that $\int_{\Gamma} f^{\text {con, } \Gamma}+\int_{\Upsilon} f^{\text {con, }, ~}=0$, as expected from the action-reaction principle (see (MEACCI et al., 2020a) for more details). The contact force only acts along the surface normal, it is complemented by a drag force that models the tangential attachment of the citoskeleton nodes to the bilayer anchoring proteins as

$$
f^{d, \Gamma}\left(x^{\Gamma}\right)=-\int_{\Upsilon} \eta\left(w\left(x^{\Gamma}\right)-\frac{d x^{\Upsilon}}{d t}\right) d x^{\Upsilon}, \quad f^{d, \Upsilon}\left(x^{\Upsilon}\right)=\int_{\Gamma} \eta\left(w\left(x^{\Gamma}\right)-\frac{d x^{\Upsilon}}{d t}\right) d x^{\Gamma},
$$

where $\eta$ is a drag coefficient that depends on $\left\|x^{\Gamma}-x^{\Upsilon}\right\|$. We remark that, in the case of the cytoskeleton, the set $\left\{X^{i}\right\}_{i=1}^{N_{X}}$ refers to the coordinates of the centers of the nodes, while $\Upsilon$ consists of spheres $\Upsilon^{j}$ of a given radius around $X^{j}$, i.e., the surfaces of the junctions. The net force per unit area acting on the bilayer is thus $f^{\Gamma}=f^{\operatorname{con}, \Gamma}+f^{d, \Gamma}$, while the net force on node $j$, to be equilibrated by the worm-like chains (and possibly external forces) is

$$
F^{j}=\int_{\Upsilon^{j}}\left(f^{\text {con, }, \Upsilon}\left(x^{\Upsilon}\right)+f^{d, \Upsilon}\left(x^{\Upsilon}\right)\right) d x^{\Upsilon} .
$$

In Figure 4 we plot the contact part of $F$ (i.e., taking $\eta=0$ ) and of $\mathscr{E}_{X Y}$ as a function of $d$ for a node of radius $R=0.07$ in the vicinity of a typical biconcave surface (fixed). The maximum (positive) value of the force corresponds to the maximum adhesion force of the node to the surface. Notice that it takes place at $d \simeq r_{0}$. Similarly, the minimum value of the interaction energy corresponds to the adhesion energy and its position to the equilibrium distance. The regularization is evident in the repulsion forces, which depend approximately linearly with $d$ for $d$ much smaller than $r_{0}$.

\subsubsection{Model summary}

The main unknowns of the proposed two-component RBC model (2C-RBC-M) are the bilayer configurations $\Gamma(t)$ and the junctions' positions $X(t)$, which evolve according to the instantaneous velocity field $w(t)$ and nodal-velocity vector $U(t)$, respectively. At each instant $t$, the system (2.9)-(2.12) coupled to (2.15) determine $w$ and $U$ (remember that we are assuming $m=0$ for simplicity). The coupling arises from the interaction terms $f^{\Gamma}$ and $F^{j}$. Because the effects of bilayer bending, cytoskeleton stretching and surface-to-surface adhesion are derived from energy functions $\left(\mathscr{E}_{Y}, \mathscr{E}_{X}\right.$ and $\mathscr{E}_{X Y}$, respectively) and the additional forces are dissipative, we automatically have a bound for the total energy

$$
\frac{d}{d t}\left(\mathscr{E}_{X}+\mathscr{E}_{Y}+\mathscr{E}_{X Y}\right) \leq 0
$$

The model equations are certainly too involved to attempt analytical treatment, so that they are discussed through numerical simulations in what follows.

\subsection{Numerical simulations}

While the model for the cytoskeleton is discrete by definition, the model for the bilayer is continuous and needs to be discretized. We consider triangulated surfaces in 3D space as 
Figure 4 - Interaction force $F$ and energy $\mathscr{E}_{X Y}$ induced by $\widetilde{\mathscr{G}}\left(d, r_{0}, \varepsilon\right)$, with $\alpha=0.89$ and $k=6$.
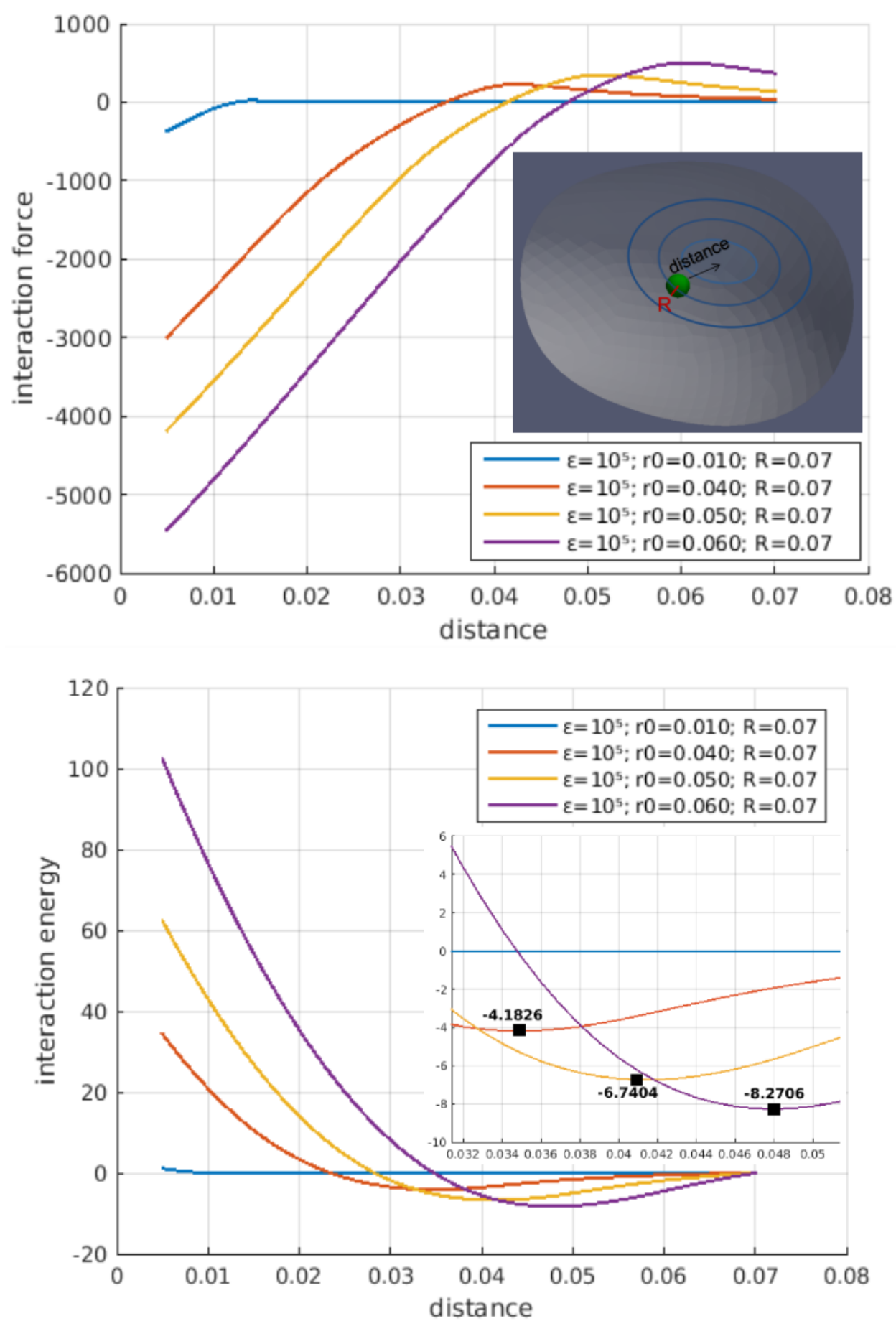

approximations for $\Gamma(t)$, which for a fixed mesh connectivity are uniquely described by the vector $Y$ of vertex positions. Time is discretized so that a sequence of triangulated surfaces $\Gamma^{0}, \Gamma^{1}, \ldots, \Gamma^{n}, \ldots$ are computed, corresponding to vertex positions $Y^{0}, Y^{0}, \ldots, Y^{n}, \ldots$. Following Rodrigues et al. (2015a), on each $\Gamma^{n}$ we define the piecewise-affine finite element space $P_{1}^{n}=$ $\left\{f \in C^{0}\left(\Gamma^{n}\right):\left.f\right|_{K}\right.$ is affine, $\forall K$ triangle in $\left.\Gamma^{n}\right\}$ and the approximation spaces for velocity, surface and curvature $W_{h}^{n}=\left(P_{1}^{n}\right)^{3}, Q_{h}^{n}=P_{1}^{n}, K_{h}^{n}=\left(P_{1}^{n}\right)^{3}$. We define $\Delta t=t_{n+1}-t_{n}$ and we update the 
nodal positions in a Lagrangian way, specifically,

$$
X^{j, n+1}=X^{j, n}+U^{j, n+1} \delta t, \quad Y^{j, n+1}=Y^{j, n}+w^{j, n+1} \delta t .
$$

With these choices, a fully discrete semi-implicit formulation of the bilayer can be built (RODRIGUES et al., 2015a) and coupled to a fully implicit time discretization of (2.15). At each time step, a nonlinear system of equations is thus built with unknowns $\left(w_{h}^{n+1}, \sigma_{h}^{n+1}, \kappa_{h}^{n+1}, p^{n+1}, U^{n+1}\right) \in$ $W_{h}^{n} \times Q_{h}^{n} \times K_{h}^{n} \times \mathbb{R} \times \mathbb{R}^{3 N_{X}}$ and solved by Newton's iterative procedure. Notice that the formulation is semi-implicit in that the bilayer mesh $\Gamma^{n}$ is kept frozen throughout the iterations.

The first simulation we present here to provide insight into the behavior of the $2 \mathrm{C}-\mathrm{RBC}$ $\mathrm{M}$ consists of the interaction of the bilayer surface with a single cytoskeleton sphere. If the contact energy is weak ( $\varepsilon$ small) the sphere simply adheres to the bilayer without further effects. However, as $\varepsilon$ is increased and becomes comparable to $\varepsilon_{1}=\frac{C_{c h}}{R^{2} r_{0}^{2}}$, the adhesion forces become strong enough to locally deform the bilayer towards wrapping around the sphere. To illustrate this we have considered a single sphere of radius $R=0.8$, with a contact length scale $r_{0}=0.1$. These values imply $\varepsilon_{1} \simeq 3000$. The bilayer initially has a biconcave shape with dimensions 7.8 , 7.8 and 2.2 along the $x, y$ and $z$ directions, respectively. We situate the sphere inside the bilayer at a distance $\approx r_{0}$ and simulate the relaxation of the system for several values of $\varepsilon$ between 1000 and 9000 . The complete setup and some quantitative results of the attained equilibrium are listed in the Table 1. In Figure 5 we display the different equilibrium shapes of the bilayer colored according to the mean curvature. For $\varepsilon=1000$ (and smaller) the perturbation in the bilayer shape caused by the sphere is very small. For $\varepsilon=3000$ and larger the bilayer tends to wrap around the sphere, with its global shape deforming so as to attain an equilibrium under these conditions.

Figure 5 - Screenshots of the simulation of one ball $(R=0.8)$ for different values of $\varepsilon$ of the RBC in the relaxation process at the equilibrium.

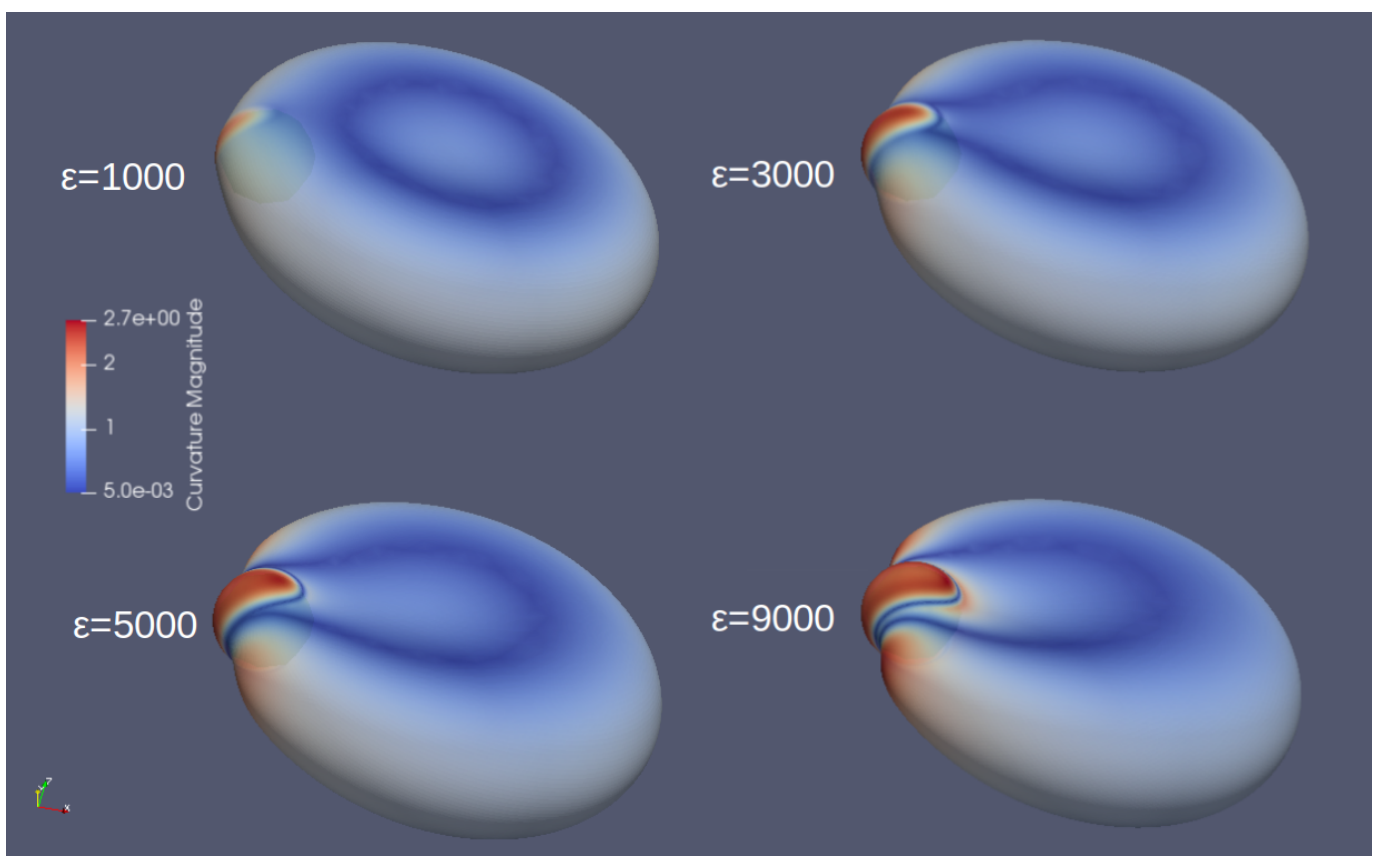


Table 1 - Parameters setup and numerical solutions at the equilibrium of the virtual experiment of adhesion between the bilayer and one internal spherical node.

\begin{tabular}{c|c|c|c|c|c|c|c|c}
$\varepsilon$ & $k$ & $R$ & $r_{0}$ & $C_{c h}$ & $\mathscr{E}_{\mathscr{Y}}$ & $\mathscr{E}_{\mathscr{X} \mathscr{Y}}^{\infty}$ & $p$ & $\sigma$ \\
\hline 1000 & 6 & 0.8 & 0.1 & 20 & $9.08 \times 10^{2}$ & $-2.20 \times 10^{2}$ & -10.6 & $-1.40 \times 10^{3}$ \\
3000 & 6 & 0.8 & 0.1 & 20 & $9.51 \times 10^{2}$ & $-1.16 \times 10^{3}$ & -9.5 & $-1.50 \times 10^{3}$ \\
5000 & 6 & 0.8 & 0.1 & 20 & $1.01 \times 10^{3}$ & $-2.60 \times 10^{3}$ & -8.5 & $-1.60 \times 10^{3}$ \\
7000 & 6 & 0.8 & 0.1 & 20 & $1.07 \times 10^{3}$ & $-3.90 \times 10^{3}$ & -7.5 & $-1.70 \times 10^{3}$ \\
9000 & 6 & 0.8 & 0.1 & 20 & $1.13 \times 10^{3}$ & $-5.50 \times 10^{3}$ & -6.0 & $-1.80 \times 10^{3}$ \\
\hline
\end{tabular}

Such studies are interesting in relation to the bilayer's reaction to exogenous bodies. A possible application concerns the modeling of the process of invasion of merozoites in the malaria disease, as shown by recent works (HILLRINGHAUS et al., 2019) and (HILLRINGHAUS et $a l ., 2020)$. Figure 6 shows how the appropriate use of the interaction force between the lipid bilayer and an external body can be suitable in such applications. On the top-left of the Figure we show microscopy images during the infection process, taken from the work of Riglar et al. (RIGLAR et al., 2011), while below we show selected snapshots of simulations performed with the 2C-RBC-M. In such applications the transient solution takes on particular interest and the proposed model allows to study the evolution of otherwise inaccessible physical quantities, as shown on the right panel of the Figure 6.

Figure 6 - Simplified simulation of the invasion of merozoite in malaria disease performed with the proposed model. Microscopy images taken from (RIGLAR et al., 2011).
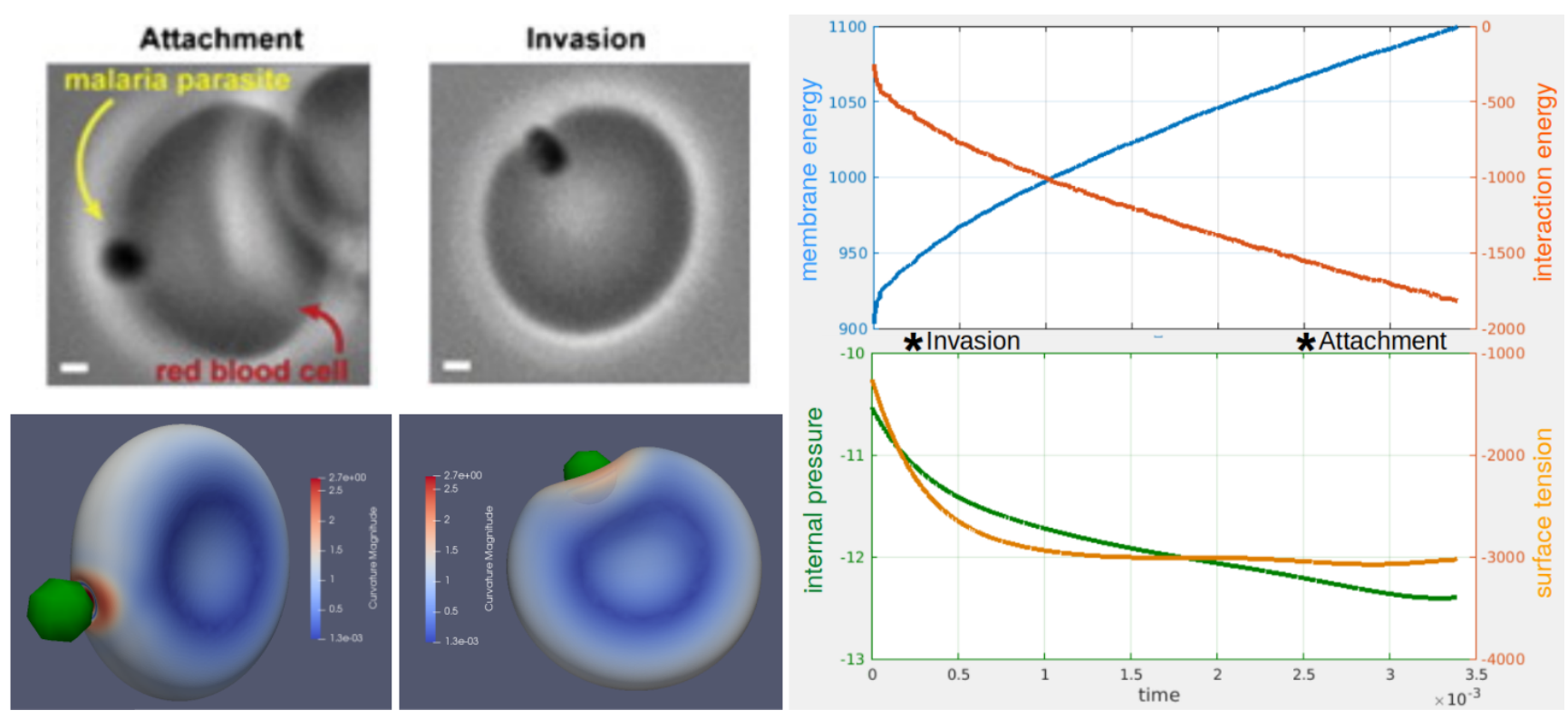

We now turn to study configurations more akin to the RBC membrane, involving the interaction of the bilayer with many connected solid bodies so as to mimic the cytoskeleton component of the RBC. The bilayer is energetically unable to locally deform so as to wrap around the nanometric cytoskeleton nodes. However, since the nodes are elastically connected, their combined action can indeed affect the global behavior of the bilayer. We assess the ability of the 
2C-RBC-M to relax towards equilibrium from an initial arbitrary shape maintaining the bilayer and cytoskeleton components attached. It is known that RBCs are able to return undamaged to their original shape after substantial deformations, such as those undergone inside the capillaries (FISCHER, 2004). There is no doubt that a close relationship exists between the shape of the bio-membranes and their curvature and line tension (BAUMGART; HESS; WEBB, 2003), as well the minimum energy of bending can be considered a possible explanation of the biconcave shape of the human RBC (CANHAM, 1970). Moreover, the equilibrium configurations of the bilayer (alone) depend on its reduced volume, defined as $v_{r}=\frac{6 \sqrt{\pi} V}{A^{3 / 2}}$, being $A$ and $V$ the surface area and internal volume, respectively (SEIFERT, 1997). The typical RBC has $v_{r} \approx 0.65$, so that we select as initial condition an oblate ellipsoid with the same $v_{r}$.

Following previous coarse-graining studies (PIVKIN; KARNIADAKIS, 2008; FEDOSOV; CASWELL; KARNIADAKIS, 2010), we set a simplified cytoskeleton with 350 nodes and discretized the bilayer with a triangulation with $10^{4}$ nodes. For the time discretization we adopted a variable time step $\Delta t$ in the range $\left[10^{-7}, 10^{-5}\right]$. Notice that the time unit is $\mu L^{2} /\left(k_{B} T\right)$, which with $L=1 \mu \mathrm{m}$ and $\mu \approx 10^{-8} \mathrm{~Pa} \cdot s \cdot \mathrm{m}$ can be estimated as $\approx 2$ seconds. Unless otherwise specified, the setup of parameters of the following simulations is reported in Table 2. Concerning the interaction parameters, it is possible to estimate the adhesion energy of $N$ spheres that do not deform the lipid bilayer locally as $\approx 2 \pi^{2} \varepsilon r_{0}^{3} R N$. The value of $\varepsilon=5000$ was chosen such that the adhesion energy is $\approx 400$ and thus comparable to the bilayer bending energy. This makes the adhesion to be strong enough for the cytoskeleton to be able to deform the bilayer by pulling from it without detaching. Other necessary data are the persistence length $\ell_{p}$ and the constant $k_{p}$, which we adjust so that the relaxed length of each cytoskeleton fiber is equal to its length in the initial configuration.

Table 2 - Setup of parameters of the relaxation process simulations.

\begin{tabular}{rclc} 
Value & Symbol & Description & Model \\
\hline 1.0 & $k_{B} T$ & Energy unit & (WLC) \\
2 & $n$ & POW exponent & (WLC) \\
1.0 & $\beta_{\Gamma}$ & Bilayer interaction constant & (INT) \\
1.0 & $\beta_{\Upsilon}$ & Cyto interaction constant & (INT) \\
6 & $k$ & Coefficient Lennard-Jones potential & (INT) \\
5000 & $\varepsilon$ & Interaction strength constant & (INT) \\
0.05 & $r_{0}$ & Equilibirum cyto-membrane surface distance & (INT) \\
0.1 & $R$ & Radius of cyto-sphere & (INT) \\
1.0 & $\mu$ & Viscosity & (BIL) \\
20.0 & $C_{c h}$ & Canham constant & (BIL) \\
117.5 & $A_{0}$ & Initial RBC surface area & (BIL) \\
79.6 & $V_{0}$ & Initial RBC volume & (BIL)
\end{tabular}

The initial bilayer shape is thus taken as an ellipsoid with lengths 8,8 , and 2.4 along the $x, y$, and $z$ axis, respectively. The initial positions of the cytoskeleton nodes lie at a distance $\approx r_{0}$ from it. In Figure 7 we show the evolution of the RBC model with and without the cytoskeleton. 
Also plotted is the total energy as a function of time, which in the case with cytoskeleton contains the contributions of $\mathscr{E}_{X}$ and $\mathscr{E}_{X Y}$. In both cases the model relaxes to a quite realistic biconcave shape at $t \approx 10^{-3}$, with a monotonous decay of the total energy. The full model has a sudden decrease of energy at the first instants, corresponding to the adjustment of the distances between the junctions and the bilayer. The relaxed shapes are shown in the figure colored with the local mean curvature. They are very similar, but in the one corresponding to the full model the small local effect of each cytoskeleton node on the adjacent bilayer is clearly visible. Notice that in this case $\varepsilon=5000 \ll \varepsilon_{1} \approx 8 \times 10^{5}$, so that it is not expected that the bilayer will significantly tend to wrap around the cytoskeleton nodes.

Table 3 - Quantities of numerical solutions at the equilibrium of the relaxation experiment of onecomponent (without cytoskeleton) and full RBC two-component model.

\begin{tabular}{c|c|c|c|c|c|c} 
Case & $\mathscr{E}^{\infty}$ & $\mathscr{E}_{\mathscr{Y}}^{\infty}$ & $\mathscr{E}_{\mathscr{X}}^{\infty}$ & $\mathscr{E}_{\mathscr{X} \mathscr{Y}}^{\infty}$ & $p$ & $\bar{\sigma}$ \\
\hline One-component & $9.0 \times 10^{2}$ & $9.0 \times 10^{2}$ & - & - & -10.6 & $-12.8 \times 10^{2}$ \\
Two-component & $1.9 \times 10^{4}$ & $9.1 \times 10^{2}$ & $2.1 \times 10^{4}$ & $-3.0 \times 10^{3}$ & -10.1 & $-38.2 \times 10^{2}$ \\
\hline
\end{tabular}

Some relevant global quantities at the equilibrium are reported in Table 3. The introduction of the cytoskeleton component does not affect the bending energy significantly, while a considerable decrease in average surface tension and some increase of the internal pressure can be noted. These observations are consistent with the cytoskeleton exerting an average inwards pull on the bilayer at equilibrium. In order to better understand the impact of the presence of the cytoskeleton in the evolution of the whole system we present below a series of virtual experiments that take this equilibrium as initial condition. We perturb this configuration by changing the equilibrium length $\ell_{0}$ of each spectrin fiber by a constant factor. For example, if we set $\ell_{e q}=0.9 \ell_{0}$, it means that each cytoskeleton fiber will tend to contract towards $90 \%$ of its initial length. The execution of this study has highlighted a deformation interval in which, under our hypotheses, the cytoskeleton interacts with the lipid membrane while maintaining its configuration and attachment to the bilayer.

Table 4 - Global physical quantities corresponding to the steady states reached after different perturbations of the equilibrium lengths of the cytoskeleton fibers.

\begin{tabular}{c|c|c|c|c|c|c}
$\ell_{e q}$ & $\mathscr{E}^{\infty}$ & $\mathscr{E}_{\mathscr{Y}}^{\infty}$ & $\mathscr{E}_{\mathscr{X}}^{\infty}$ & $\mathscr{E}_{\mathscr{X} \mathscr{Y}}^{\infty}$ & $p$ & $\bar{\sigma}$ \\
\hline $0.8 \ell_{0}$ & $21.26 \times 10^{3}$ & $9.07 \times 10^{2}$ & $23.29 \times 10^{3}$ & $-29.35 \times 10^{2}$ & -9.0 & $-12.5 \times 10^{3}$ \\
$0.9 \ell_{0}$ & $19.88 \times 10^{3}$ & $9.05 \times 10^{2}$ & $21.96 \times 10^{3}$ & $-29.88 \times 10^{2}$ & -9.7 & $-7.2 \times 10^{3}$ \\
$1.0 \ell_{0}$ & $19.30 \times 10^{3}$ & $9.1 \times 10^{2}$ & $21.62 \times 10^{3}$ & $-30.02 \times 10^{2}$ & -10.1 & $-3.8 \times 10^{3}$ \\
$1.1 \ell_{0}$ & $19.77 \times 10^{3}$ & $9.09 \times 10^{2}$ & $21.85 \times 10^{3}$ & $-29.98 \times 10^{2}$ & -10.9 & $-1.4 \times 10^{2}$ \\
$1.2 \ell_{0}$ & $20.34 \times 10^{3}$ & $9.11 \times 10^{2}$ & $22.43 \times 10^{3}$ & $-29.92 \times 10^{2}$ & -11.0 & $5.5 \times 10^{1}$ \\
\hline
\end{tabular}

In Table 4 we list the results of some main quantities corresponding to contraction or extension factors between $80 \%$ to $120 \%$. As evident from the cases shown in Figure 8, the average surface tension of the bilayer increases as the cytoskeleton tends to expand. This increase eventually leads to positive surface tension values which could reveal a mechanical-biological 
reality. As we have previously seen, the one-component model with a fixed reduced volume of the order $v_{r} \approx 0.65$ leads to a biconcave-shaped membrane with a negative surface tension (see also (VEERAPANENI et al., 2009)). Nevertheless there is experimental evidence that the effective surface tension of a RBC is positive and of the order of $10^{-7} \mathrm{~J} / \mathrm{m}^{2}$ (GOV; ZILMAN; SAFRAN, 2003; BETZ et al., 2009). In the case $\ell_{e q}=1.2 \ell_{0}$ the execution of our model leads to an average surface tension of comparable value (the scale for $\sigma$ is $k_{B} T / L^{2} \simeq 4 \times 10^{-9} \mathrm{~J} / \mathrm{m}^{2}$ ). This outcome suggests that the cytoskeletal network exerting an expansive force on the bilayer may reconcile the difference in the sign of the surface tension between theory and experiments. From the screenshots in Figure 8, we can appreciate the local influence of the nodes of the cytoskeleton. Probably this type of influence is excessive and is due to an exaggeration in the weight given to the interaction force ( $\varepsilon$ can be chosen lower), but it is interesting to validate the model and draw conclusions on the interdependence of the components of the erythrocyte.

We conclude the study by analyzing the extreme cases. If the relaxed length of the spectrin fibers is reduced further, some cytoskeleton nodes detach from the bilayer. In fact, this phenomenon occurs whenever $\ell_{e q}<0.8 \ell_{0}$. In Figure 9 we show the time evolution of a RBC with $\ell_{e q}=0.7 \ell_{0}$, for which detachment of the cytoskeleton starts at $t \approx 0.002$ put into evidence by a sudden increase of the bending energy. In this time interval we can observe the counterpart downward jump of the energy of the cytoskeleton (see graph on top-center). The specific nodes that first detach are shown in the image (on top-right), which also show that the detachment induces a positive local surface tension (see screenshot on top-left). This event is followed by a contraction of the entire network of spectrins which, remaining attached to the a part of the bilayer, contribute to giving the peculiar RBC shape shown in the last screenshot.

The opposite extreme case is when the spectrin network has its equilibrium lengths increased beyond $1.2 \ell_{0}$. We examine in Fig. 10 the numerical results of the simulation imposing $\ell_{e q}=1.3 \ell_{0}$. The compression along the individual spectrins grows while they try to find space for expansion subject to the constraint imposed by the surrounding bilayer. As expected, the spectrin network eventually buckles and locally entangles, as shown in the figure's insert. This event occurs when a series of spectrins of a junctional complex are an over-tension condition compared to the others ones (see the detail on below-left). Notably, this buckling takes place without the nodes detaching from the bilayer. This folding phenomenon leads to a complex evolution of the bilayer energy and results in a perturbation of the biconcave shape of the red blood cell, as shown in the screenshot. This behavior corresponds to an unpredictable and fluctuating trend of the internal pressure (as shown by the graph in the low-center). A final image (on the right) shows the local effect of nodes folding in terms of local surface tension. 


\subsection{Achievements and future opportunities}

In this work we have presented a new approach for modeling a single RBC. According to this, we have motivated the need of a new two-component model for a single RBC. We have coupled the established worm-like-chain approach for the cytoskeleton to a continuum model for the lipid bilayer based on a viscous liquid-shell model with Canham-Helfrich bending energy. Another original contribution is represented by the introduction of the adhesion forces modelling the attachment of the cytoskeleton nodes to the bilayer integral proteins. We tested the two-component RBC approach, firstly investigating the interaction of the bilayer surface with a single internal and external body. Then we provided the implementation of the $2 \mathrm{C}-\mathrm{RBC}-\mathrm{M}$ concerning the relaxation of the cell, and comparing it with the one-component model. We have also reported a short study by stressing the bilayer through the perturbation of the cytoskeleton equilibrium state. These virtual experiments not only showed the consistency of the mathematicalcomputational model but also have suggested physical considerations about the RBC membrane system.

Understanding better the behaviour of a red blood cell means to give a strong contribution to the comprehension of life. Thanks to modern technology, the accuracy of mathematical models can be proven by experimental laboratory tests. Conversely, mathematical modeling can help to identify the reasons behind what is physically observed. Our next goal is to adapt the code in order to simulate the fundamental experiments of micropipette aspiration and optical tweezing. Our deliverable is a software for the "in silico" (or virtual) simulation of RBCs, that extends the range of spatial and temporal scales of current simulators as the OpenRBC code (TANG et al., 2017) and the implementation in LAMMPS of Fu and co-workers (FU et al., 2017). 
Figure 7 - Screenshots of the simulation of the evolution of the RBC in the relaxation process $(t=0.15)$.
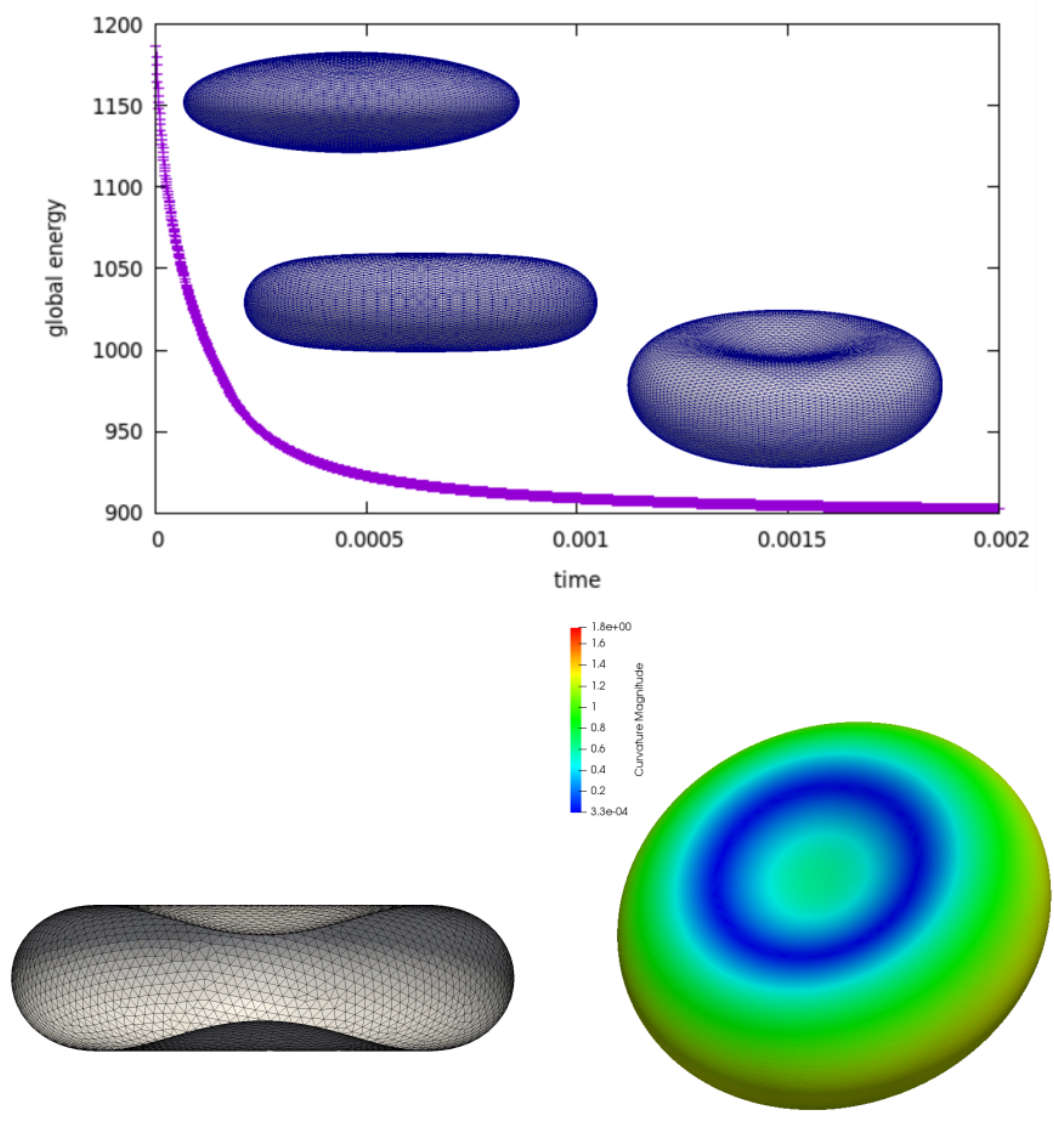

(a) Lipid bilayer neglecting cytoskeleton and interaction forces.
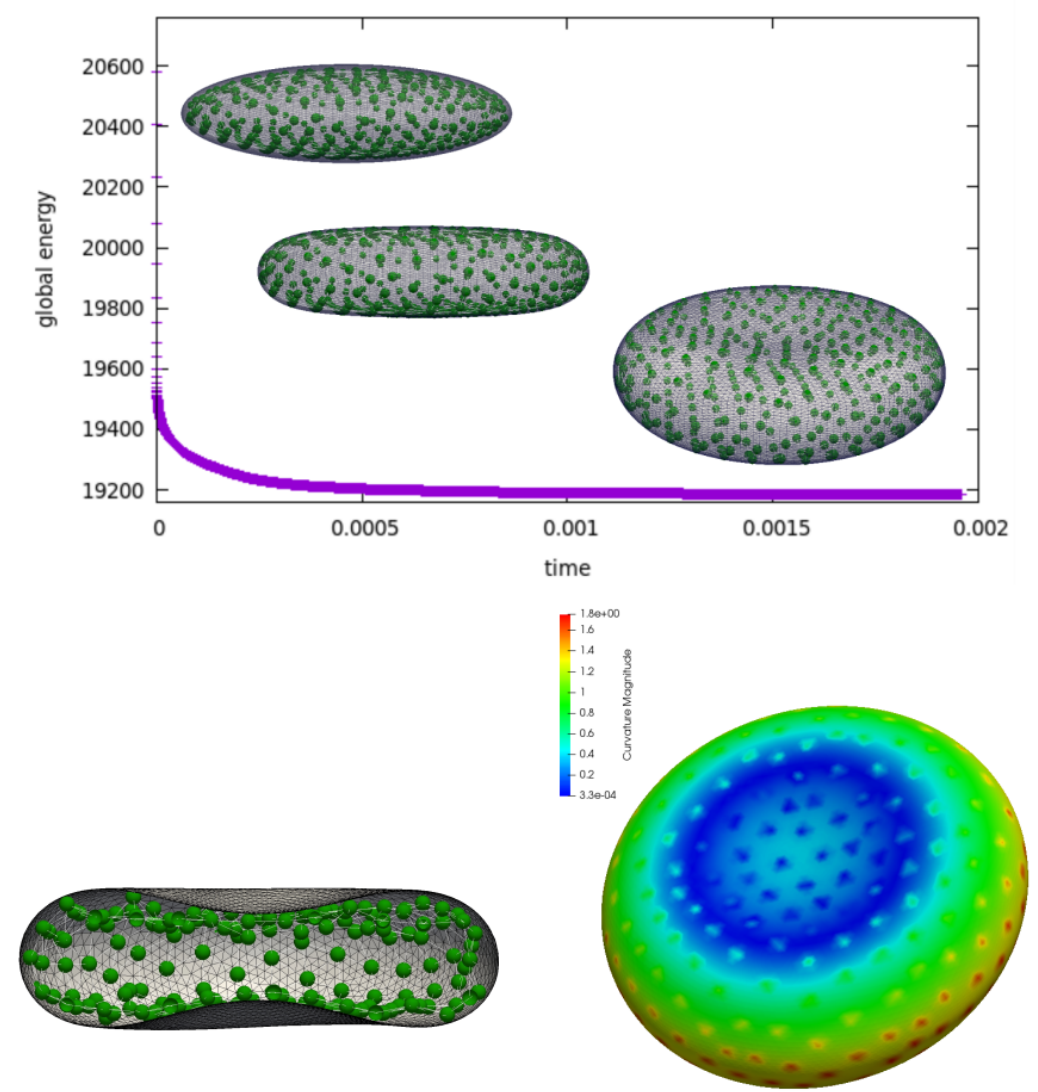

(b) Lipid bilayer considering cytoskeleton and interaction forces. 
Figure 8 - Configuration of the RBC at equilibrium, by contracting and extending the cytoskeleton. The surface tension is displayed with the colors.

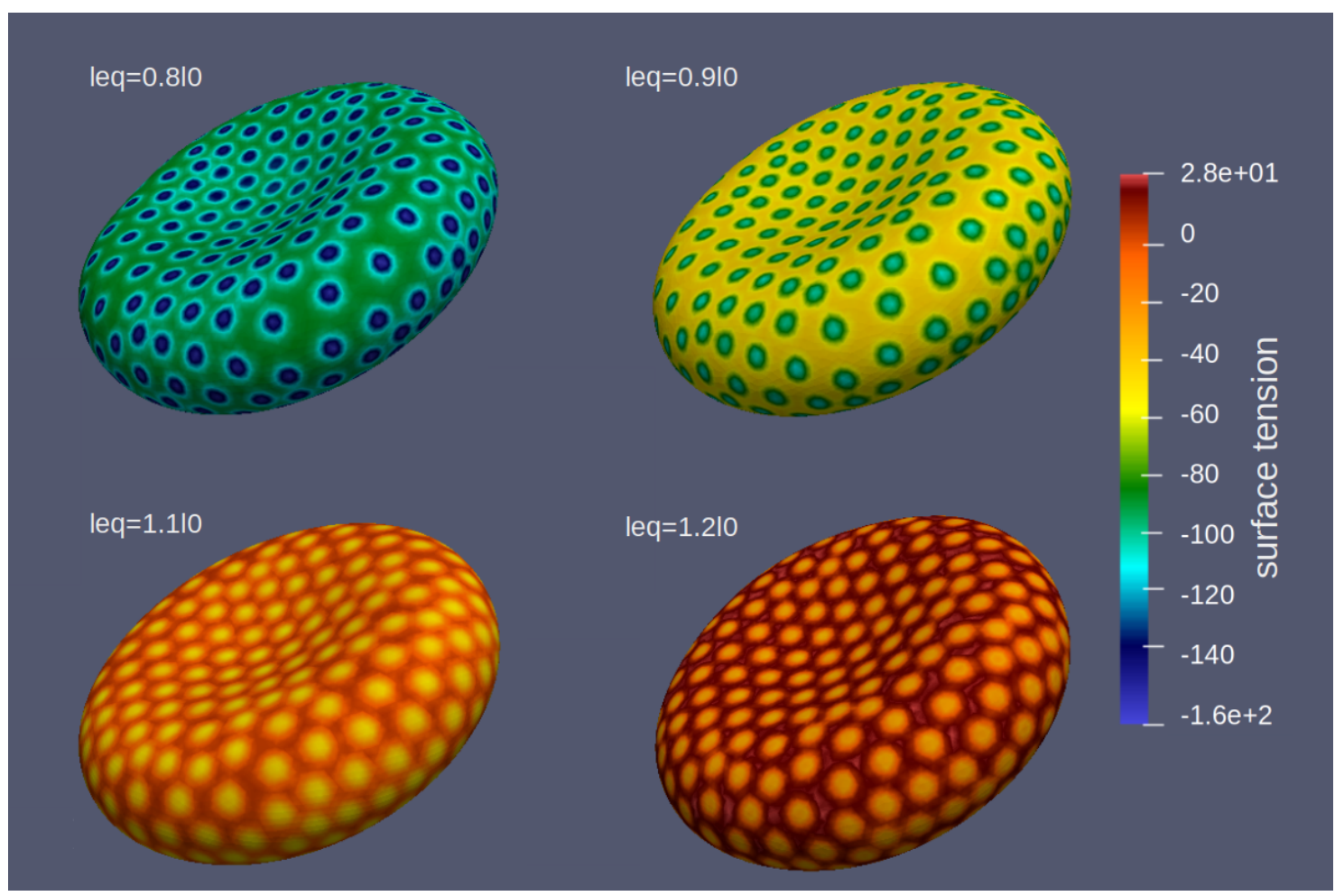

Figure 9 - Time evolution of a RBC a $\ell_{e q}=0.7 \ell_{0}$ is imposed, with "detaching" phenomenon of cytoskeleton network.

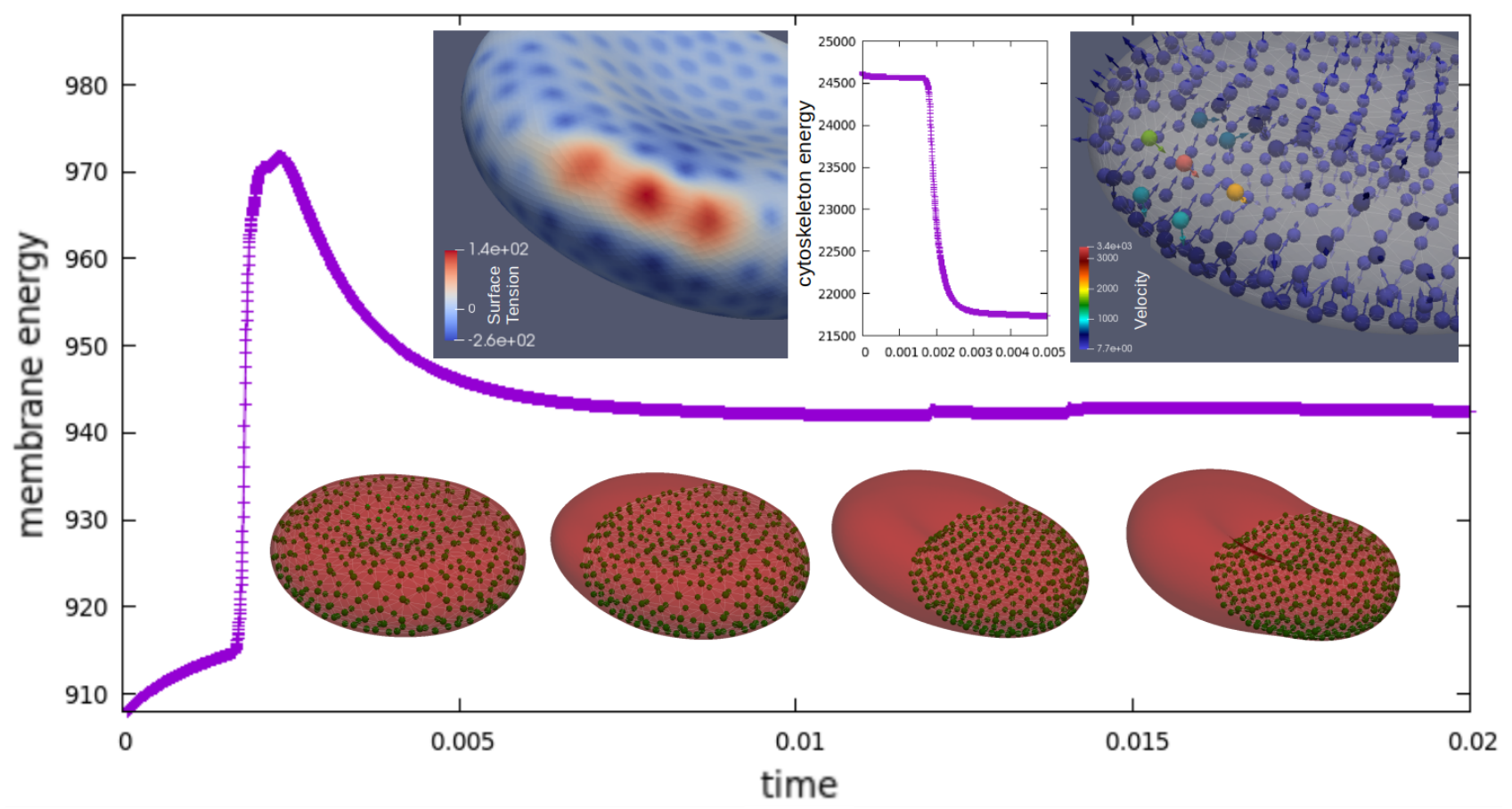


Figure 10 - Time evolution of a RBC a $\ell_{e q}=1.3 \ell_{0}$ is applied, with "folding" effect of cytoskeleton network.

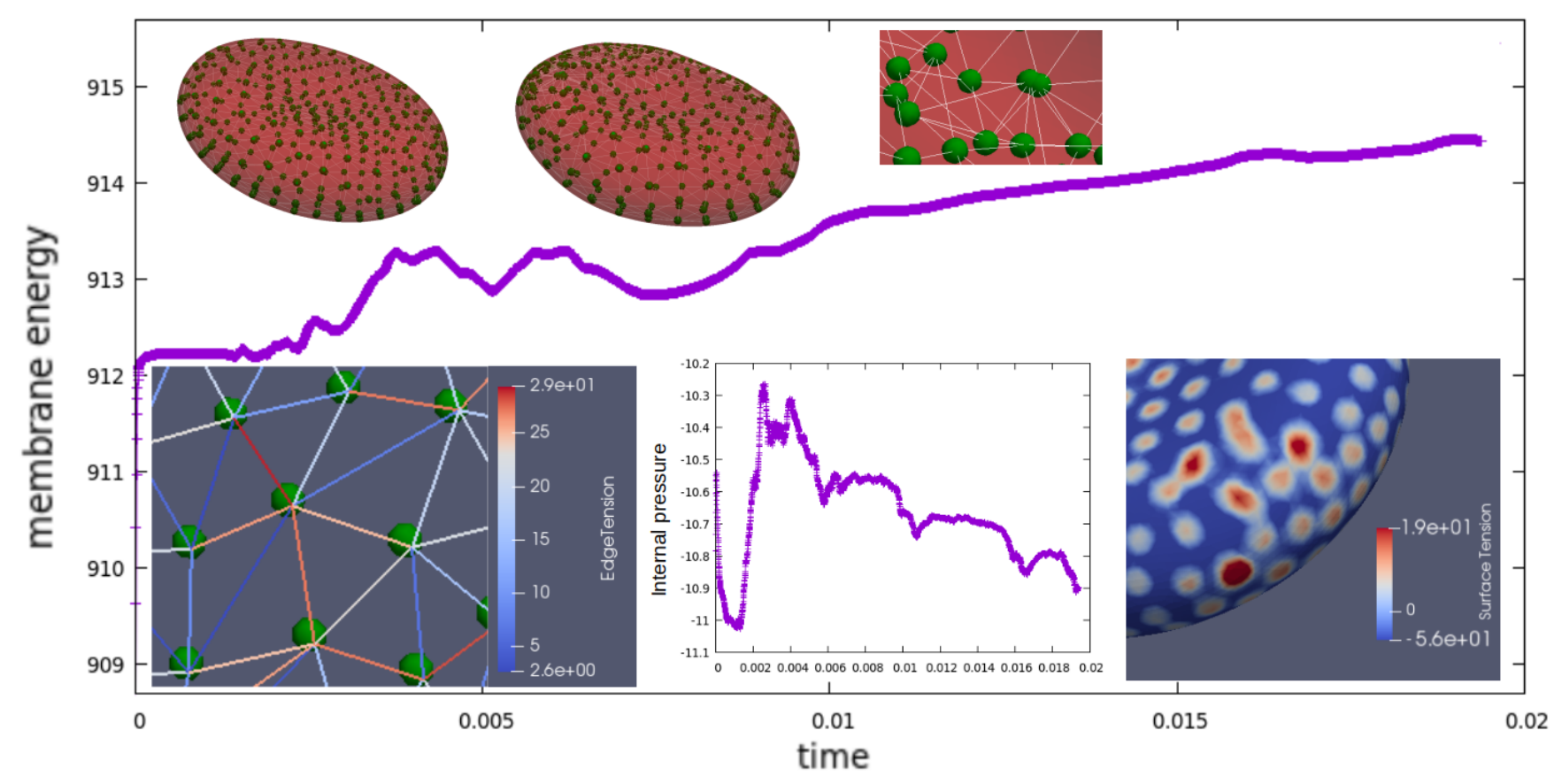


CHAPTER

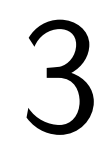

\section{MODELING A RED BLOOD CELL CYTOSKELETON: INSIGHTS, TIPS, AND CODE}

Mathematical modeling of living cells has been experiencing a continuous fruitful interest for decades now (BAO; SURESH, 2003; LIM; ZHOU; QUEK, 2006), also benefiting from the development of micro- and nano-scale cell technologies (UNAL et al., 2014). The important interactions between the mechanical responses and the physiological functions of living cells makes this inter-disciplinary field of research one of the most challenging and promising with significant implications in biological sciences and medicine (BAO et al., 2014). Single-cell studies (ANDERSON; REJNIAK, 2007; CARLO; LEE, 2006) and, in particular, on red blood cells have gained attention (LI; VLAHOVSKA; KARNIADAKIS, 2013) thanks to the increasing advance of computational techniques (TANG et al., 2017; FU et al., 2017). The red blood cell (RBC) is an excellent candidate for this type of investigations because despite being one of the simplest cells (for example, mature erythrocytes are non-nucleated), it possesses peculiar membrane mechanical characteristics, which ensure exceptional versatility and stability.

It is estimated that during its 120-days life-span, a single RBC circulates around the human body about a million times (LI et al., 2007). This life cycle is not without obstacles. The erythrocyte demonstrates a remarkable flexibility and resilience by withstanding a considerable amount of shear stresses and severe deformation during the micro-circulation even in capillaries thinner than its own size (average diameter of $7.8 \mu \mathrm{m}$ ) or in the spleen filtration, and continuously recovering its biconcave shape on stress release. These extraordinary mechanical properties are attributed to the RBC unique architecture (DUMITRU et al., 2018). The structural component enabling mechanical flexibility in an healthy human RBC is composed of three interconnected elements: (i) a fluid-like phospholipid-cholesterol bilayer that contributes to bending resistance; (ii) a two-dimensional spectrin network, that is, an underlying network of filamentous cytoskeletal 
proteins; (iii) trans-membrane proteins anchoring together the lipid bilayer and spectrin domains. Several works have gradually unrevealed answers to the many questions on the exceptional capacity of the erythrocyte membrane to maintain its physical properties and consequently physiological functions (for example, to capillary transport of hemoglobin to deliver oxygen to the cells) studying the the complex structural organization of the erythrocyte membrane (PICAS et al., 2013) (BORGHI; BROCHARD-WYART, 2007). There is common agreement in literature that the mechanical stability and flexibility necessary to withstand the deformations experienced during the RBC life can be largely attributed to its cytoskeleton (MOHANDAS; GALLAGHER, 2008; DISCHER, 2000).

The structural characterization of the RBC cytoskeleton has already been identified with sufficient clarity from the 70s and 80s (HAINFELD; STECK, 1977; LUX, 1979; GRATZER, 1981). Spectrins are the most abundant cytoskeletal proteins which are organized by forming long and flexible heterodimers and integrating multi-protein complexes, usually called junctional complexes (JCs). This complex organization is reported to be two-dimensional and quite regular, revealing an approximate hexagonal symmetry (LIU; DERICK; PALEK, 1987). The spectrin network envelopes the entire surface of the RBC membrane (BENNETT; BAINES, 2001) and it is linked via band 4.1 proteins or ankyrins to integral proteins embedded in the lipid bilayer (LUX, 2016). The typical length of a spectrin filament is about $70 \mathrm{~nm}$, leading to about $10^{5}$ edges in a human RBC cytoskeleton. An electron microscopy image of this peculiar network structure is shown in the left-hand panel in Figure 11. As shown in the right-hand panel of Figure 11, a recent study published by Nans, Mohandas and Stokes (NANS; MOHANDAS; STOKES, 2011) has enabled to produce the first three-dimensional representation of the RBC cytoskeleton utilizing cryo-electron tomography.

Figure 11 - Electron microscope image of a RBC cytoskeleton structure (left-hand panel) (LUX, 2016) and cryo-electron tomography (right-hand panel) (NANS; MOHANDAS; STOKES, 2011) showing details of the cytoskeleton.
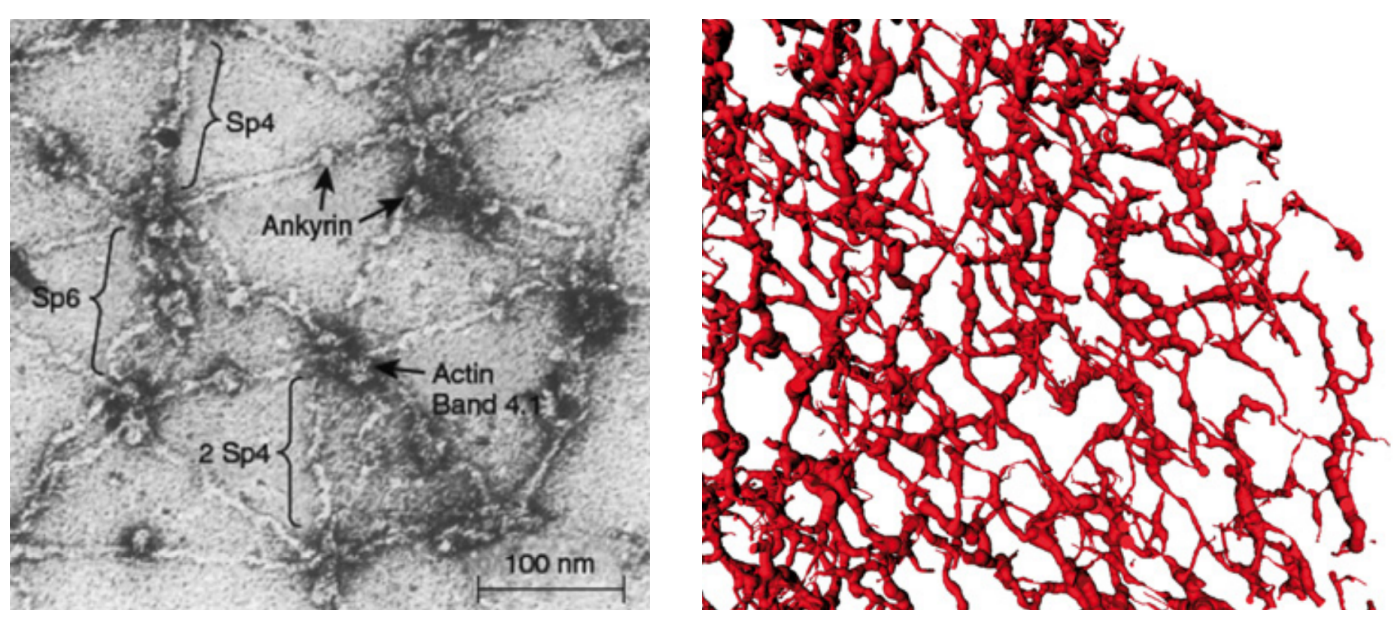

As schematically shown in Figure 12, the network architecture can easily stretch and contract again upon stress removal. Based on comparisons between rest (A) and expanded (B) 
states, we hypothesise that the spectrin filaments substantially reorganize as the membrane is stretched during cell deformation. This peculiar behaviour is due to the flexible and elastic characteristics of the spectrin domains that assume a triple helical structure (insert $\mathrm{C}$ in Figure 12).

Figure 12 - Scheme of the transition between the (A) intact and (B) expanded forms of the erythrocyte skeleton and $(\mathrm{C})$ of the triple helical spectrin structure. Image modified from (NANS; MOHANDAS; STOKES, 2011)

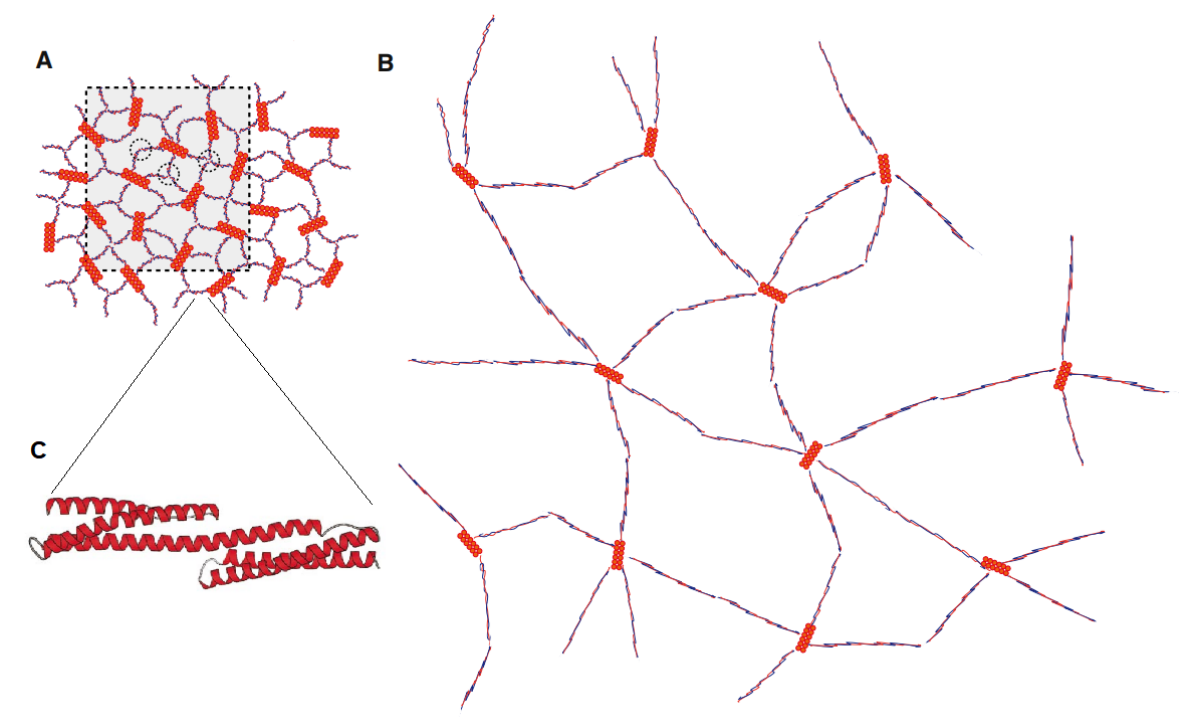

It is biologically clear that the mechanical properties associated with bilayer-cytoskeletal cooperative role strongly influence biorheology, erythrocyte function, and the onset and progression of red blood cell diseases (SVETINA et al., 2004) (SURESH, 2006; PENG et al., 2013; DISCHER; BOAL; BOEY, 1998). A very recent work of Gómez et al. provides evidence that the $\mathrm{RBC}$ surface tension and bending modulus are very responsive to the interaction between the lipid bilayer and the cortical cytoskeleton (GÓMEZ et al., 2020). A computational modelling approach that separately considers each component of the erythrocyte has recently proved useful in the analysis of healthy (PENG; ASARO; ZHU, 2010; LI; LYKOTRAFITIS, 2012; LI et al., 2014) and diseased (CHANG et al., 2016; CHANG; LI; KARNIADAKIS, 2017) erythrocytes. In these works, the common choice is to model the lipid bilayer with a bending surface energy, elastic and viscous interactions, and thermal fluctuations using particle-based techniques. A positive feature of this approach is to easily account for the detailed membrane structure and the critical biophysical processes at cellular and sub-cellular scales, such as membrane fluctuations and defective structure. A weak point of this approach is however represented by the fact that the computational costs can increase considerably to become very expensive with the reduction of the modeling scale. Alternative modeling approaches consist in the adoption of a continuum-based models, according to which the RBC membrane layer is usually treated as a two-dimensional viscous liquid-shell homogeneous material (FENG; KLUG, 2006) (ARROYO; DESIMONE; HELTAI, 2010; RODRIGUES et al., 2015a; BARTHES-BIESEL, 2016). The continuum framework provides consistent approximations of large-scale molecular systems with less adjustable 
parameters with respect to particle-based approaches, and are generally numerically more stable and less computationally costly (LI; LU; PENG, 2020). In both approaches, in order to develop a two-component model, an explicit mathematical description of the spectrin network is needed.

The cytoskeleton is a complex structure with non-trivial dynamics. In this study we aim to answer the following questions: Is it possible to capture the basic characteristics of the cytoskeleton, while at the same time obtaining a model capable of simulating its dynamics with a simple code able to be run even by a common computer? Can those computational tools, such as coarse-grained scaling techniques, be adopted by using not so sophisticated and articulated methods?

In this paper we aimed to provide a set of tools to facilitate RBC cytoskeleton mathematical modeling, giving tips over coarse grained scaling techniques, parameters choice to set an equilibrium spectrin length or computational approach to simulate a virtual optical tweezers experiment. Indeed, by delivering a cytoskeletal-only component-based model, a complementary goal of this work was to further elucidate the role of the cytoskeleton on determining the overall RBC mechanical behaviour. We draw some insights on different mechanical aspects such as cytoskeletal symmetry defects impact and stress transmission and distribution within the network, emphasizing findings while highlighting the limitations of using a cytoskeletal-only componentbased model for mimicking the mechanics of the whole RBC membrane. The computation model here presented may be useful for completing single RBC models.

With such purposes in mind, in Section 3.1 we describe the model abstraction process by discussing the main spectrin hallmarks and by introducing the worm-like chain force model to simulate the network behaviour. In Sub-section 3.1.1 a case-study of a single spectrin domain is presented. By adopting the principle of virtual work, in Sub-section 3.1.2 the complete model for the whole cytoskeletal system is shown and used in a relaxation test, i.e., when no external force is applied to the cytoskeleton and it relaxes to equilibrium. In order to provide a step-by-step guide for the use and implementation of such model, the Sub-section 3.1.3 is dedicated to coarsegrained scaling methods. This approach is particularly helpful to reduce the computational cost while preserving the network mechanical properties. We also show two examples of how this model can be used to study RBC cytoskeleton. Firstly, in the Sub-section 3.1.4 we focus on the implications of symmetry defects, which occur also taking into account the dynamic nature of the cytoskeletal network. Then, in Sub-section 3.1.5 we present a virtual implementation of an optical tweezers simulation to assess the potential of the model to describe the behaviour of the cytoskeleton in transient processes.

This chapter significantly expands the work presented at the VI ERMAC - Regional Meeting of Applied and Computational Mathematics at Unesp in Bauru/SP (Brazil) and published in the Proceedings of the Congress (MEACCI et al., 2019). In order to give more solidity to the results of this work, we provide in Appendix A the implementation of the mathematical model in Octave. GNU Octave is software featuring a high-level programming language, mostly 
compatible with Matlab and free under the terms of the GNU General Public License. We also list some brief comments to the commands of the main routine and the functions used to accompany the reader in understanding the code. Furthermore, to help the reader to test and easily use the code, we dispense with the corresponding source Octave codes in GitHub repository (at the URL: $<$ https://github.com/LucaMeacci/RBC_cytoskeleton_mathmodel>) with the archives concerning the initial geometry conditions to simulate the numerical tests presented in the text.

\subsection{Mathematical model}

The starting point of the model we propose refers to the single "brick" of the cytoskeleton: the spectrin. In the work of Marko and Siggia (MARKO; SIGGIA, 1995), an interpolating model was applied with an ideal chain behaviour at very small extension and with a divergent-tension behaviour at large extension (DISCHER; BOAL; BOEY, 1998). This approach is known as the worm-like chain (WLC) model in polymer physics, or sometimes as the Kratky-Porod WLC model, and is used to describe the behavior of semi-flexible polymers. The WLC model provides a description for an isotropic rod that is continuously flexible. The WLC model is particularly suited for describing stiffer polymers, with successive segments exhibiting cooperative interaction, all of which point in approximately the same direction. Several biologically important polymers can be effectively modeled as WLC strands, including double-stranded DNA, unstructured RNA, and unstructured polypeptides (proteins) (BAO, 2002; VASQUEZ et al., 2016). We opted for this model to describe the non-linear force-displacement behavior of each individual spectrin.

Chan, Haverkamp and Hill (CHAN; HAVERKAMP; HILL, 2010) recently proposed a force extension formula for the WLC model using the variational principle. The authors showed that Marko and Siggia's approach yields up to 6\% error compared to the exact solution in the intermediate force regime. Despite this error, Marko and Siggia's formula is currently the most commonly used one by biologists and chemists to adjust their experimental data. Therefore we will adopt it. The WLC force $f_{W L C}$ as a function of instantaneous length is modeled as:

$$
f_{W L C}=-\frac{k_{B} T}{p}\left\{\frac{1}{4(1-x)^{2}}-\frac{1}{4}+x\right\}, \quad x=\frac{L}{L_{\max }} \in[0,1),
$$

where $L_{\max }$ is the maximum length of the filament, $L$ is the instantaneous length of the filament ( $x$ is the dimensionless ratio between $L$ and $L_{\max }$ ), $p$ is the persistence length, $k_{B}$ is the Boltzmann constant, and $T$ is the temperature. We remark that the persistence length $p$ is a basic mechanical property quantifying the stiffness of a polymer and, more specifically, the characteristic length scale over which the chain maintains a certain direction (CHEN et al., 2012). Figure 13 shows the graph of the WLC force according to the equation (3.1) where $k_{B}=1.3806 \times 10^{-23} \mathrm{~J} / \mathrm{K}$, $T=300 \mathrm{~K}$ and $p=39 \times 10^{-9} \mathrm{~m}$. This arbitrary configuration reflects the similar behavior of experimental data referring to a portion of a double stranded DNA (GROSS; REUSKEN, 2011). 
Figure 13 - Graphic of the WLC force $\left(k_{B}=1.3806 \times 10^{-23} \mathrm{~J} / \mathrm{K}, T=300 \mathrm{~K}\right.$ and $p=39 \times 10^{-9} \mathrm{~m}$.

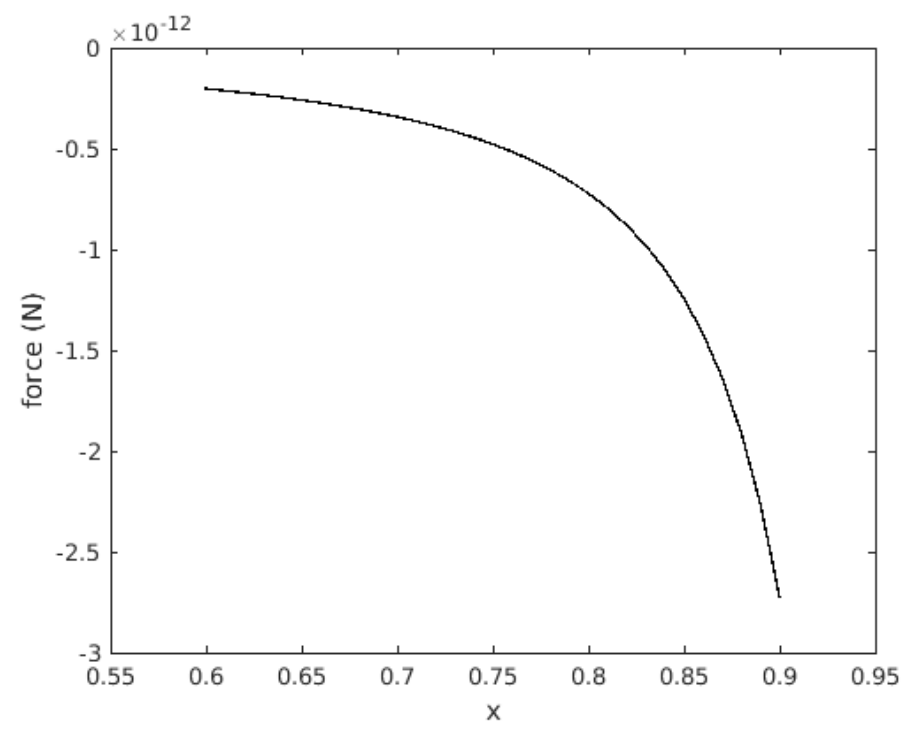

By integrating the equation (3.1) we obtain the corresponding potential, $\mathrm{V}(\mathrm{L})$, of a string of length $L$,

$$
V(L)=\int_{0}^{L} f_{W L C}(\xi) d \xi=\frac{k_{B} T L_{\max }}{4 p} \frac{3 x^{2}-2 x^{3}}{1-x} .
$$

The whole cytoskeleton will be treated following the nature of its components, basically a spectrin fiber mesh with special joints. The mesh is considered as a set of nodes joined together by molecular chains. The idea of using a finite network model to predict the macroscopic elastic shear modulus and the erythrocyte membrane skeletal area expansion module based on its microstructure has been used in the literature (HANSEN et al., 1996) and recently demonstrated to be accurate with nonlinear WLC forces (DISCHER, 2000) (FEDOSOV; CASWELL; KARNIADAKIS, 2010; PAN; CASWELL; KARNIADAKIS, 2010; JU et al., 2015). A mesh scheme representing the whole cytoskeleton is shown in Figure 14.

Figure 14 - Mesh scheme representing the cytoskeleton. The nodes are indicated in red while the edges in blue.

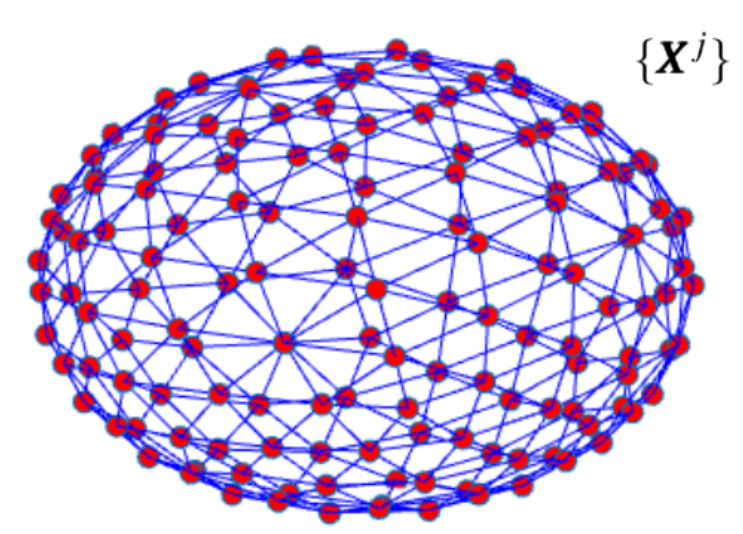

As shown in Figure 14, we can mathematically consider for the nodes to be a set of 
vertex points $\mathbb{X}=\left\{X^{j}\right\} \in \mathbb{R}^{3 \times N_{v}}$, being $N_{v}$ the number of vertexes. The vertices are connected by $N_{e}$ edges and form a two-dimensional network of triangles. For the matrix $\mathbb{X}$ corresponds to a length matrix $\hat{x}=\left\{x_{j}\right\} \in \mathbb{R}^{N_{e}}$. This way of building the network satisfies the hypothesis of the (average) hexagonal organization of the RBC cytoskeleton.

Having defined this geometry of the domain, the (whole) potential energy of the spectrin mesh can be evaluated taking into account all spectrins, leading to the following formulation:

$$
\mathscr{E}_{X}(\mathbb{X})=G(\hat{x})=\sum_{j}\left[\frac{k_{B} T \ell_{m}\left(3 x_{j}^{2}-2 x_{j}^{3}\right)}{4 p\left(1-x_{j}\right)}+\frac{k_{p}}{(n-1) \ell_{j}^{n-1}}\right],
$$

where $\ell_{j}$ is the filament length (spring) $j, \ell_{m}$ is the maximum length of these filaments, $x_{j}=\ell_{j} / \ell_{m}$, $p$ is the persistence length , $k_{B} T$ is the power unit, and $n$ and $k_{p}$ are parameters. The first term in the equation (3.3) is the attractive potential (WLC). We observe that in addition we include an second term to express the repulsive force (for $n>0$ ) between neighboring nodes, concerning the so-called power force (POW). We denote therefore the formulation (3.3) as WLC-POW model (FEDOSOV; CASWELL; KARNIADAKIS, 2010; XIAO et al., 2016; YUN; XIANG; WANG, 2018) and, a nonzero equilibrium spring length is defined by the balance of these two forces (ZHOU et al., 2016).

\subsubsection{A case-study example: one spectrin}

Considering a system consisting of only two nodes, $X^{1}$ and $X^{2}$, and only one spectrin, we refer to the (3.3) to evaluate the potential energy that results in

$$
\mathscr{E}_{X}\left(X^{1}, X^{2}\right)=G(x)=\frac{k_{B} T \ell_{m}\left(3 x^{2}-2 x^{3}\right)}{4 p(1-x)}+\frac{k_{p}}{\ell_{m} x},
$$

where we assume that $n=2$ and $\ell_{0}$ is the edge equilibrium length. Assuming $\ell_{m}=r \ell_{0}$, equation (3.4) can be written as:

$$
\frac{4 p}{k_{B} T \ell_{0} r} G(x)=\frac{3 x^{2}-2 x^{3}}{1-x}+A \frac{1}{x}
$$

with $A=\frac{4 p k_{p}}{k_{B} T \ell_{o}^{2} r^{2}}$.

Let us assume now that we are interested in a priori setting the equilibrium length $\ell_{0}$. Taking into account (3.5) the problem is: Find the $A$ value that makes $G(x)$ minimum at $x=1 / r$, i.e., $\frac{d}{d x} G(x=1 / r)=0$. For example, in the simulations of Figures 15 and 16 we show the results when $r=2.2$ and $\ell_{0}=3$, respectively. We note that under this assumption we obtain $A=0.86$ and, as in the case $k_{B} T=1$ and $p=1$, this implies $k_{p}=9.4743$. Using this method, we show the results related to two simulations where in both cases the final position of the 2 nodes will be $[-1.5,0,0]$ and $[1.5,0,0]$. Depending on the different initial positions of the nodes, we are able to show the cases of compression and extension in Figures 15 and 16, respectively. In the panels concerning the cytoskeleton tensions, we refer to the sum over all spectrin molecules of the WLC and POW force components. As we can observe, at the final (relaxed) state the tensions are equal in the module but opposite. 
Figure 15 - The nodes move from the initial positions $X^{1}(t=0)=(-3,0,0)$ and $X^{2}(t=0)=(3,0,0)$ with a respective length to the equilibrium ones with the desired length $l_{0}=3$ (Compression case).
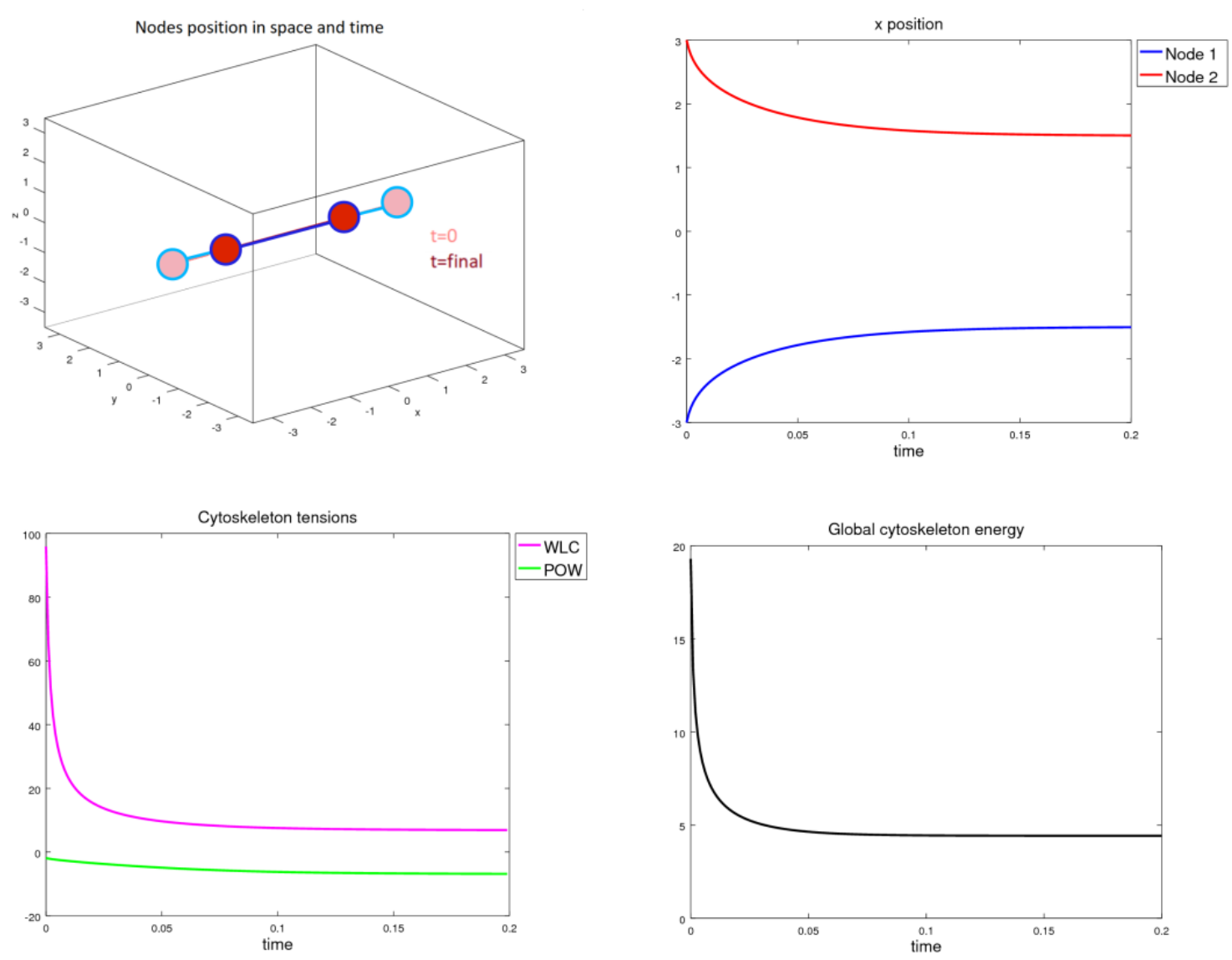

\subsubsection{Derivation of the complete system}

The principle of virtual work requires that the energy variation for an allowable virtual configuration variation to be equal to the virtual work performed by dissipative, inertial and system externalities (LANCZOS, 1970). From this principle the system of equations to describe the cytoskeleton dynamics can be derived:

$$
\frac{\partial \mathscr{E}_{X}}{\partial X_{j}}+\mathscr{D}_{X}^{j}+M^{j} \frac{d U^{j}}{d t}=F_{X}^{j} \quad \forall j=1 \ldots N_{Y},
$$

referring to the definition of $\mathscr{E}_{X}$ given by equation (3.3). Moreover, $\mathscr{D}_{X}^{j}$ denotes the contribution of the cytoskeleton dissipation with $F_{X}^{j}$ the external force and $M^{j} \frac{d U^{j}}{d t}$ the inertial contribution $\left(U^{j}=\frac{d X^{j}}{d t}\right)$. Concerning the dissipation of energy, a friction model is chosen, that is, $\mathscr{D}_{X}^{j}=$ $\beta_{\text {fric }} U^{j}$, where $\beta_{\text {fric }}$ a scalar. We remark that this hypothesis is a simplified one since the energy dissipation in the real system comes from the dissipation of the lipid layer and surrounding fluid, which are not considered here.

Through the (3.6) system it is possible to study the dynamics of the cytoskeleton with respect to time. Figure 17 presents a relaxation test, i.e., when no external force is applied to 
Figure 16 - The nodes move from the initial positions $X^{1}(t=0)=(-0.5,0.5,0)$ and $X^{2}(t=0)=(3,0,0)$ with a respective length to the equilibrium ones with the desired length $l_{0}=3$ (Extension case).
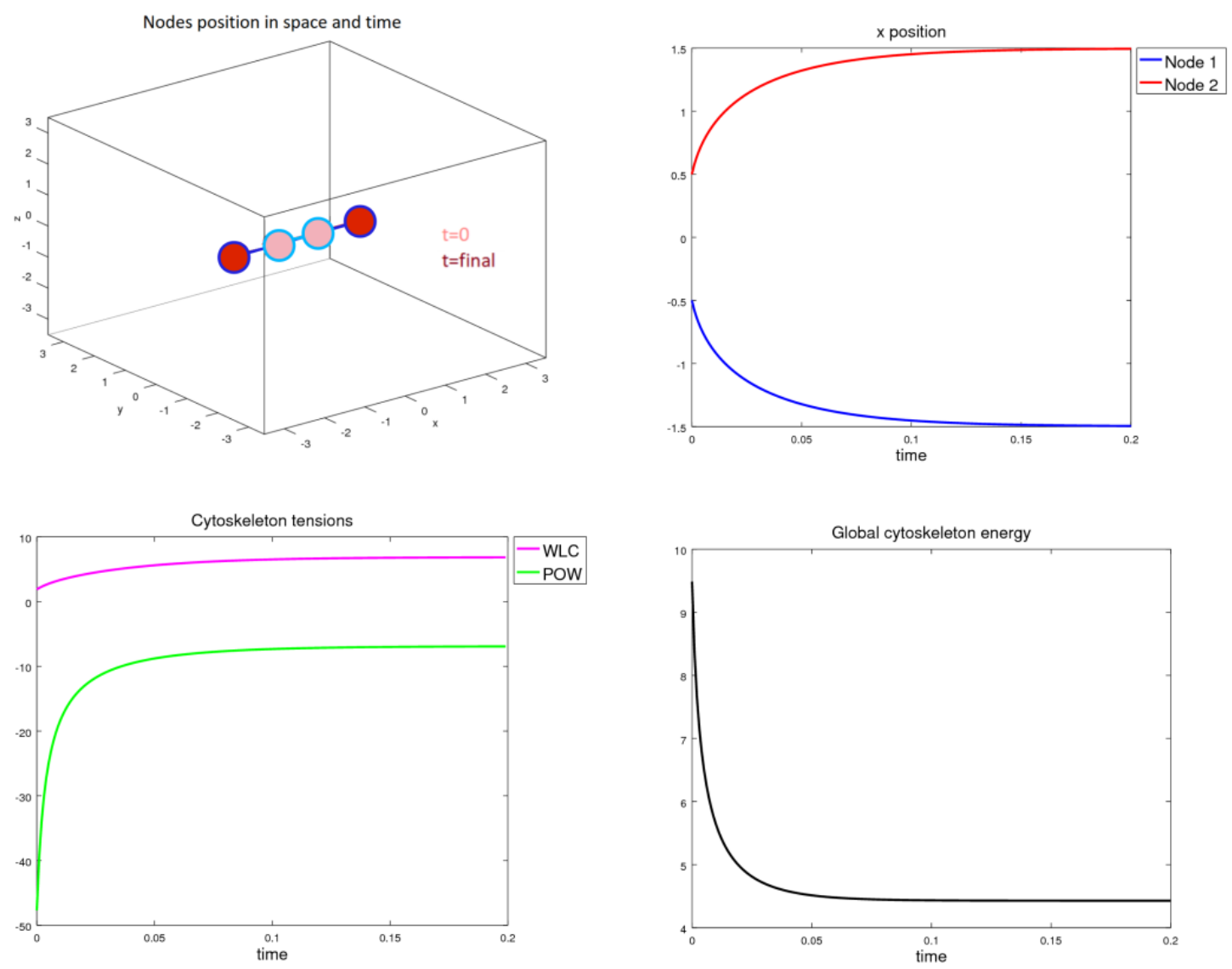

the cytoskeleton $\left(F_{X}^{j}=0, \forall j=1 \ldots N_{Y}\right)$. The initial cytoskeleton configuration was schematized to be of ellipsoidal shape, with 42 nodes and 80 edges. The presented model is numerically solved by the Newton-Raphson iterative method and with an arbitrary configuration as follows: $p=0.0375, K_{b}=1, T=1, k_{p}=1, \ell_{0}^{j} \approx 1, \ell_{m}=2.2$. The contraction process of a RBC during the relaxation time can be seen in the inserts of Figure 17 in conjunction with the profile of the cytoskeleton energy relaxation $\mathscr{E}_{X}$, according to equation (3.3). In the Appendix, we report the code to reproduce such a simulation. It should be stressed that this does not aim to reproduce real results but only to test the model. In order to facilitate the understanding of a computational implementation of the mathematical model, we will provide specific comments on the code for solving the system (3.6).

This model could be particularly useful to perform virtual experiments to help achieving the characterization of erythrocyte and cytoskeleton deformation.

\subsubsection{Cytoskeleton coarse-grained scaling}

The number of the junctional complexes (JCs) in a human RBC varies within the range of $27000-45000$, with each JC occupying a surface of $3000-5000 \mathrm{~nm}^{2}$ (SWIHART et al., 
Figure 17 - Simulation of the relaxation process of the cytoskeleton.

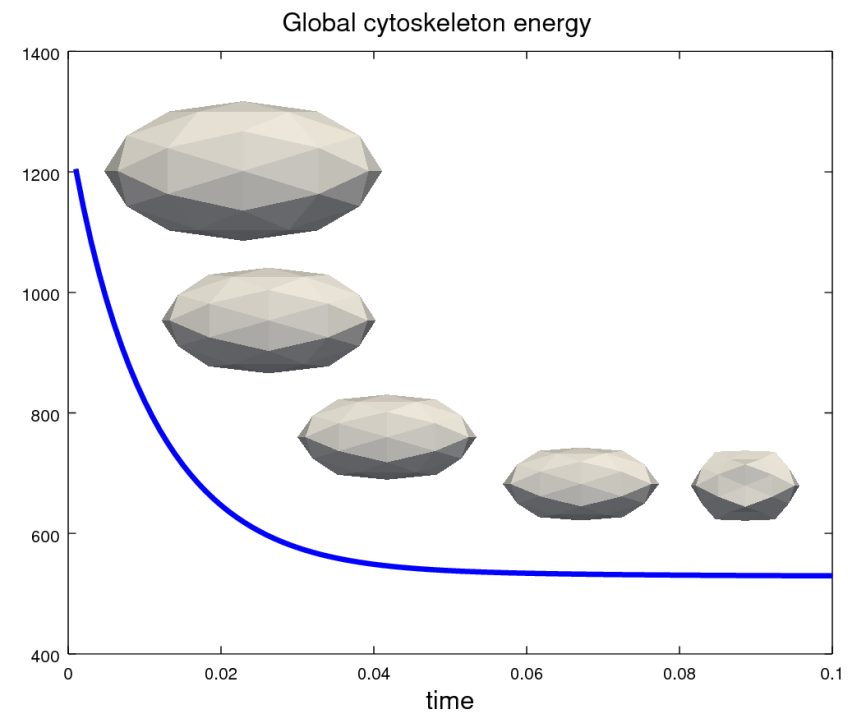

2001) and with $135 \mu \mathrm{m}^{2}$ (EVANS, 2018) being the average area of a whole RBC. These values correspond to an equilibrium spectrin length between $59-76 \mathrm{~nm}$. Considering these dimensions and fixing the number of vertices $N_{v} \approx 27000$, it is possible to estimate the parameters of the WLC-POW model of equation 3.3, yielding a persistent length value of $p=14.68 \mathrm{~nm}$ and a power $(n=2)$ force parameter $k_{p}=1.66 \times 10^{27} \mathrm{Nm}^{2}$ (at the room temperature of $T=300 \mathrm{~K}$ ) (FEDOSOV; CASWELL; KARNIADAKIS, 2010).

Although modern computer technology is capable of managing simulations with such numbers, it is useful a approach to reduce the size of the system while preserving its mechanical properties. This option to reduce computational cost is even more relevant if we consider that the model for the cytoskeleton could be accompanied by the one for the lipid bilayer in order to obtain a two-component RBC membrane model. One solution is to decrease the vertexes number $\left(N_{v}\right)$ compared with the real spectrin-level while at the same time maintaining the physical model response by appropriately scaling the parameters. We were inspired by this sort of calibration in the reduction of system cardinality, typical of the so-called coarse-graining methods (PIVKIN; KARNIADAKIS, 2008; NOGUCHI, 2009).

Let us use a smaller number of $N_{v}^{c}$ (the superscript $c$ is for "coarse-grained") with respect to the reference model number of $N_{v}$. We refer to the work by Fedosov et al. with respect to the re-scaling of a filament length (FEDOSOV; CASWELL; KARNIADAKIS, 2010). Taking into account geometrical considerations on the conservation of the area, it is possible to estimate the new coarse-grained spectrin equilibrium length

$$
\ell_{0}^{c}=\ell_{0} \sqrt{\frac{N_{v}-2}{N_{v}^{c}-2}},
$$

and similarly, estimate the new maximum coarse-grained spectrin length $\ell_{m}^{c}$. Using this as starting point, we provide a coarse-grained scaling method leading to the definition of the new coarse- 
grained persistent length $p^{c}$ and $k_{p}^{c}$ power force constant. Let first consider only one spectrin. By substituting the equation (3.7) into the (3.4), we can define again the persistent length and power force constant in order to equate the potential spectrin energy. However, the new coarse-grained system energy $\mathscr{E}_{X}(\mathbb{X})$, according to the equation (3.3), is given by the sum over all the edges, and the coarse-grained configuration has a lower filaments number than the previous one. We remark that a closed triangulated surface with hexagonal symmetry assumes a number of edges of $\frac{N_{v}-2}{N_{v}^{c}-2}$. Taking into account this observation, in order to maintain approximately unchanged the potential energy we provide the following parameters adjustment

$$
p^{c}=p\left(\frac{N_{v}-2}{N_{v}^{c}-2}\right)^{-1 / 2}, \quad k_{b}^{c}=k_{b}\left(\frac{N_{v}-2}{N_{v}^{c}-2}\right)^{n-1 / 2}
$$

We aim to test the efficacy our coarse-grained procedure by presenting a virtual experiment to evaluate the mechanical behavior of a reduced spectrin network. We set a reference arbitrary configuration of a spherical cytoskeleton of a radius of $\approx 1$, with $N_{v}=1429$, $0.1<\ell_{j}<0.24, p=0.0104$, and $k_{p}=1$. These values correspond to an equilibrium configuration, i.e., a configuration in which the mesh remains stationary if not subject to external forces. We proceed by dividing the number of nodes by at least a factor 2 each time, thus creating 3 new reduced cases. The detailed values are shown in Table 5. We can calculate the respective

Table 5 - Parameters setup and computed physical quantities concerning the coarse-grained test cases.

\begin{tabular}{cccccccc}
\hline \hline case & $N_{v}$ & $\ell_{0}$ & $p$ & $k_{p}$ & $f_{\text {ext }}$ & $\mathscr{E}_{\mathscr{X}}^{\infty}$ & $d_{\max }^{a}$ \\
\hline 0 & 1429 & $0.1<\ell_{j}<0.24$ & 0.0104 & 1 & 500 & $6.09 \times 10^{4}$ & 2.46 \\
1 & 619 & $0.16<\ell_{j}<0.34$ & 0.0068386 & 3.5173 & 500 & $6.10 \times 10^{4}$ & 2.44 \\
2 & 339 & $0.2<\ell_{j}<0.46$ & 0.0050540 & 8.7135 & 500 & $6.11 \times 10^{4}$ & 2.41 \\
3 & 145 & $0.34<\ell_{j}<0.62$ & 0.0032922 & 31.523 & 500 & $6.12 \times 10^{4}$ & 2.37 \\
\hline \hline
\end{tabular}

parameters with the new small-size grids using the equation (3.8). As expected, the new setups (shown in Table 5) essentially keep the coarse-systems in equilibrium balance. Let us now proceed with a experiment to test the new system mechanical response. In fact, we apply an assigned outgoing external force with a certain module $f_{\text {ext }}$ to the 6 extreme mesh nodes with respect to each axis, as schematized in the left-panel of Figure 18. The idea is to quantify the deformation of the cytoskeleton over time. To this purpose, in the right-hand panel of Figure 18 we display the graphs of the maximum average deformation with respect to the axes, $d_{\text {max }}^{a}$. As shown, the maintenance of the system behavior can be considered satisfactory. Even in the worst case (from case 0 to case 3 with $f_{\text {ext }}=500$ ), a reduction of the grid size of the $90 \%$ corresponds to a final variation of the maximum average diameter of $4 \%$. We can also appreciate a minimal variation of the network energy $\mathscr{E}_{\mathscr{X}}^{\infty}$ at the equilibrium. 
Figure 18 - Scheme of coarse grained test (on the left) and graph of the maximum average diameter $d_{\max }^{a}$ over time for the test cases (on the right).

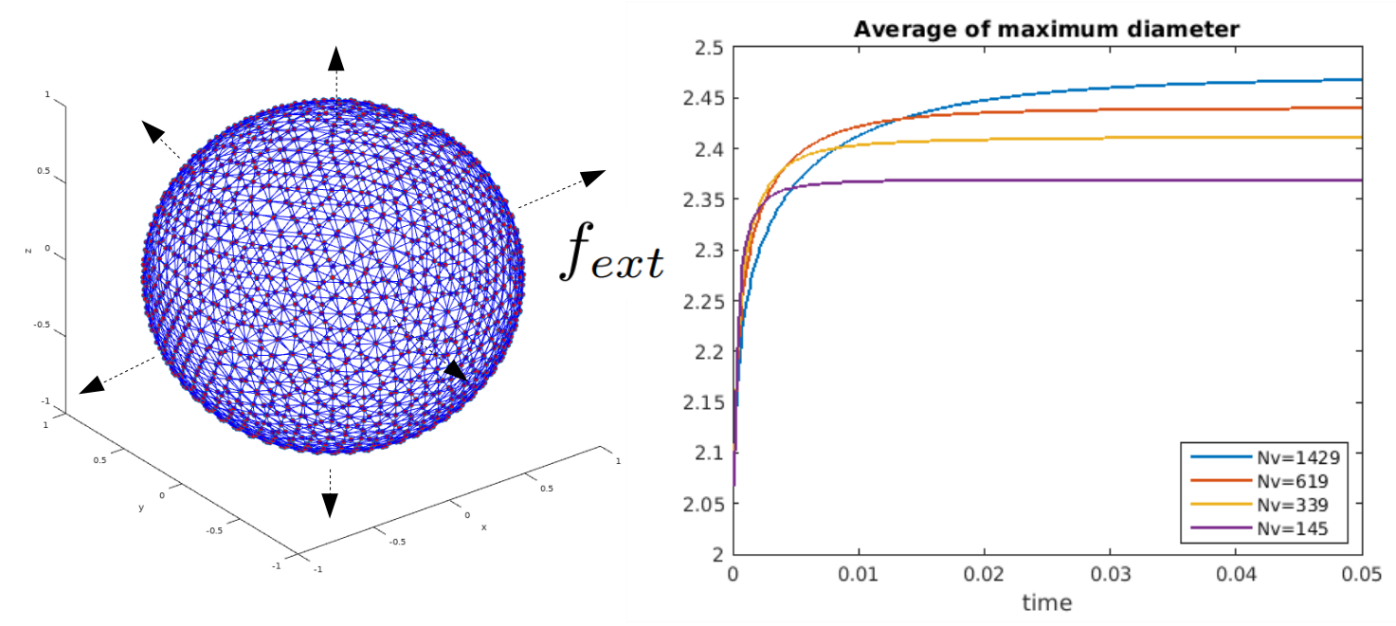

\subsubsection{Dynamical cytoskeleton and symmetry defects characteriza- tions}

As mentioned above, the spectrin network has a quite regular structure over the entire RBC surface. Nevertheless, its hexagonal symmetry is not perfect and contains many defects (BENNETT, 1989). Thanks to the atomic force microscopy visualisation technique we can observe these irregularities (TAKEUCHI et al., 1998; SWIHART et al., 2001). The cytoskeletal filaments can break and reconnect themselves as it occurs when a RBC experiences stresses passing through a small capillary. This phenomenon leads to a continual dynamic reformation of the spectrin links (ZHANG; BROWN, 2008). The transient dissociation process (GOV; SAFRAN, 2005) and active self-assembled network (GOV, 2007) significantly impact on the mechanical properties of the erythrocyte (LI et al., 2007).

It should be stressed that it is not the goal of this work to provide a simulator based on a dynamically evolving connectivity mesh, although this function may be complemented with some modifications (see (NOGUCHI, 2009)) of the code attached in the Appendix. Instead, we aim to provide insights and shed light on the impact of defects in the cytoskeletal geometry (at local level) using the implementation of a simple set of examples.

Let us consider a small portion of the spectrin network with a fixed number of 23 vertexes and the usual hexagonal symmetry, as shown in Figure 19. The aim of this test is to evaluate the response of the system as a result of the deformation generated by the application of an outward force $f_{\text {ext }}$ with parallel direction to the $x$ axis to the nodes at the left (labeled with 1,10,19) and right (labeled with 5, 14, 23) extremities. We refer to this kind of grid as the case 0 . For defined system values, as reported in Table 6, we can evaluate the final equilibrium energy $\mathscr{E}_{\mathscr{X}}^{\infty}$, and the maximum diameters of the mesh $d_{x}$ and $d_{y}$ along the $x$ and $y$, respectively. A visualization of this final system configuration is shown in the second panel of Figure 20, referring to the case 0 . 
Figure 19 - Reference cytoskeletal networking mesh.

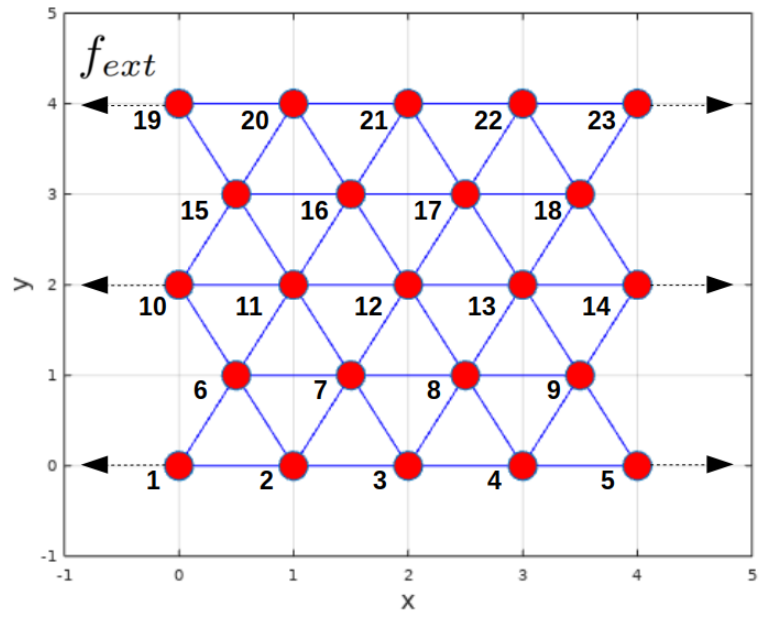

In Figure 20 the graphs of $\mathscr{E}_{\mathscr{X}}^{\infty}$ (third panel) and of the diameters $d_{x}$ and $d_{y}$ (forth panel), both resulting from a certain module of $f_{\text {ext }}$, are shown. The case 0 results reveal certain nonlinear trends of the calculated measures.

Table 6 - Parameters setup and computed physical quantities concerning the symmetry defects test cases.

\begin{tabular}{cccccccc}
\hline \hline case & $N_{e}$ & $p$ & $k_{p}$ & $f_{\text {ext }}$ & $\mathscr{E}_{\mathscr{X}}^{\infty}$ & $d_{x}$ & $d_{y}$ \\
\hline 0 & 50 & 0.0375 & 35 & 100 & 2502.8 & 6.30 & 3.38 \\
1 & 46 & 0.0375 & 35 & 100 & 2367.4 & 7.55 & 3.07 \\
2 & 42 & 0.0375 & 35 & 100 & 2183.9 & 7.91 & 3.18 \\
3 & 50 & 0.0375 & 35 & 100 & 2480.3 & 6.76 & 3.55 \\
\hline \hline
\end{tabular}

With the same criterion, we tested the response of other types of network. To build the network concerning the case 3 , we introduced defects to the reference grid of case 0 . We eliminated the edges between the nodes $7-8,11-12,12-14$, and $16-17$ so as to obtain the grid of case 1 and then removed also the links between the vertexes $6-7,8-9,15-16$, and $17-18$. We remark that, for each case it corresponds to a constant decrease of the total edges number $N_{e}$. However, this operation does not correspond to a linear increase in diameters $d_{x}$ and $d_{y}$. Specifically, the simulations reveal that for a unitary percentage reduction on the quantity of spectrins, a $d_{x}$ lengthening of the $0.9 \%$ and $1.3 \%$ occurs from case 0 to 1 and from case 1 to 2 , respectively. A first evidence is therefore that the mechanical response does not depend linearly on the number of spectrin links dissociation. We highlight this challenging aspect in the characterization of defects.

However, within the erythrocyte, not only the breakage of spectrin links occurs but also the re-connection of the cytoskeletal filaments. The occurrence of this process introduces another problem, i.e., the evaluation of non-symmetric geometries. For example, one spectrin end does not necessarily re-associate with the same JC. To illustrate this situation, let us discuss another simple test reported as case 3 . 
Figure 20 - Results of the symmetry defects test cases. For each case, we report (from left to right) initial and final network configuration and profiles of the final cytoskeletal equilibrium energy $\mathscr{E}_{\mathscr{X}}^{\infty}$ and of the diameters $d_{x}$ and $d_{y}$ over the external imposed force $f_{\text {ext }}$.
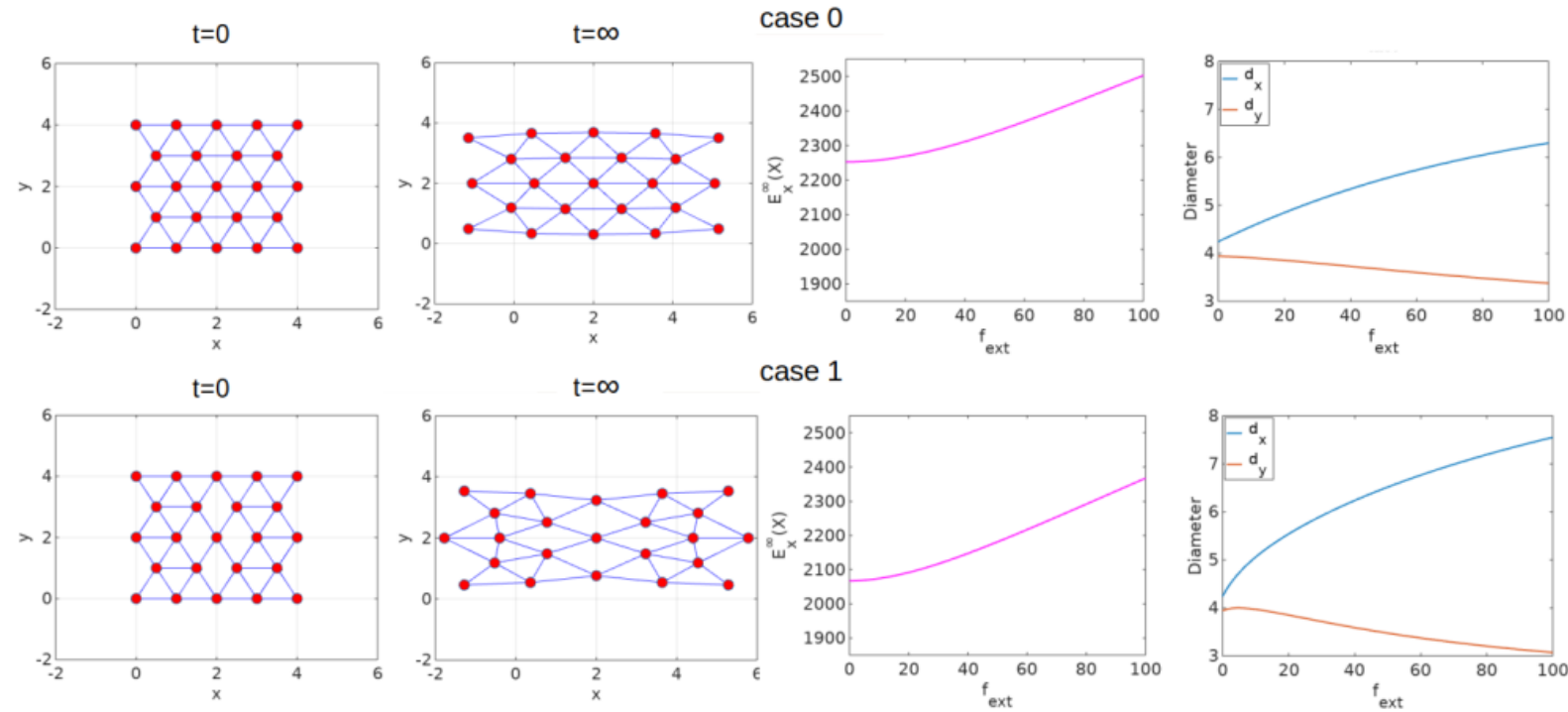

case 1
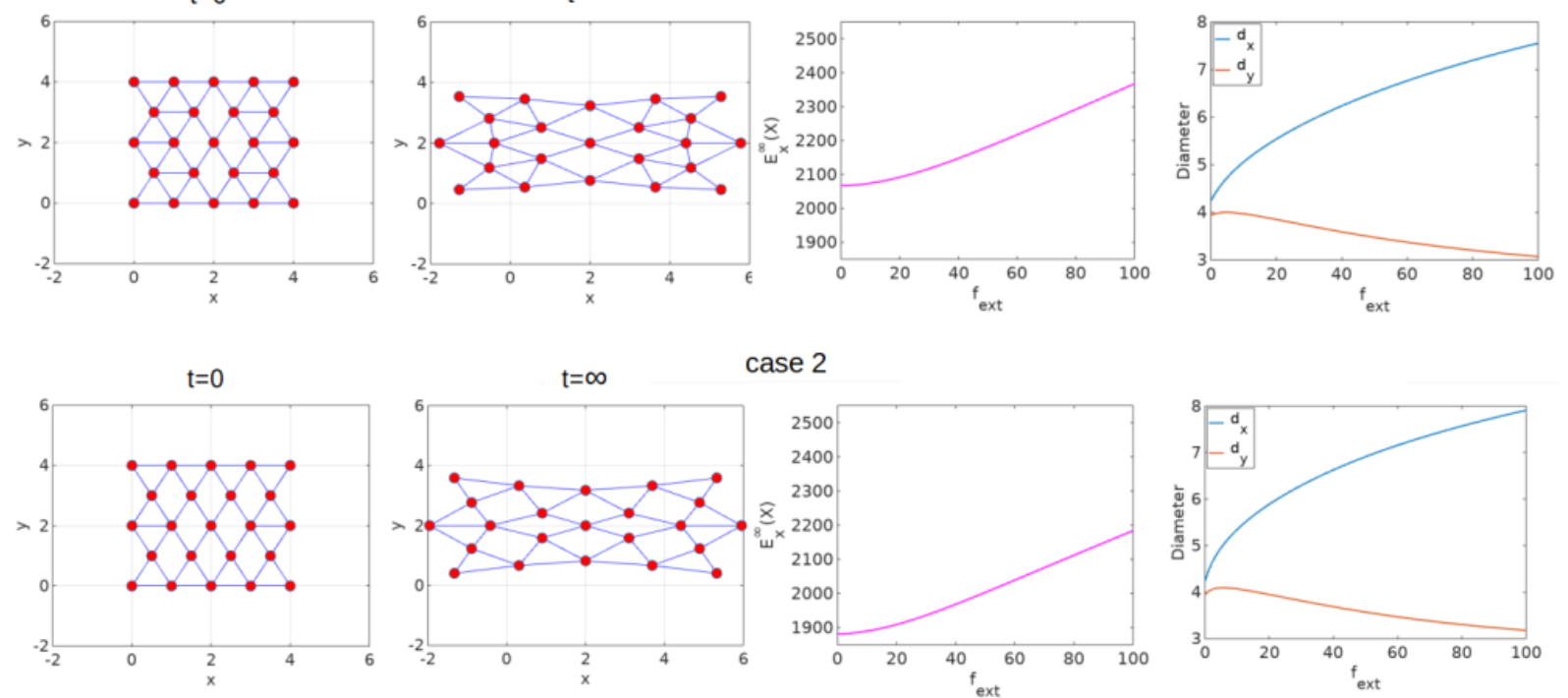

case 2
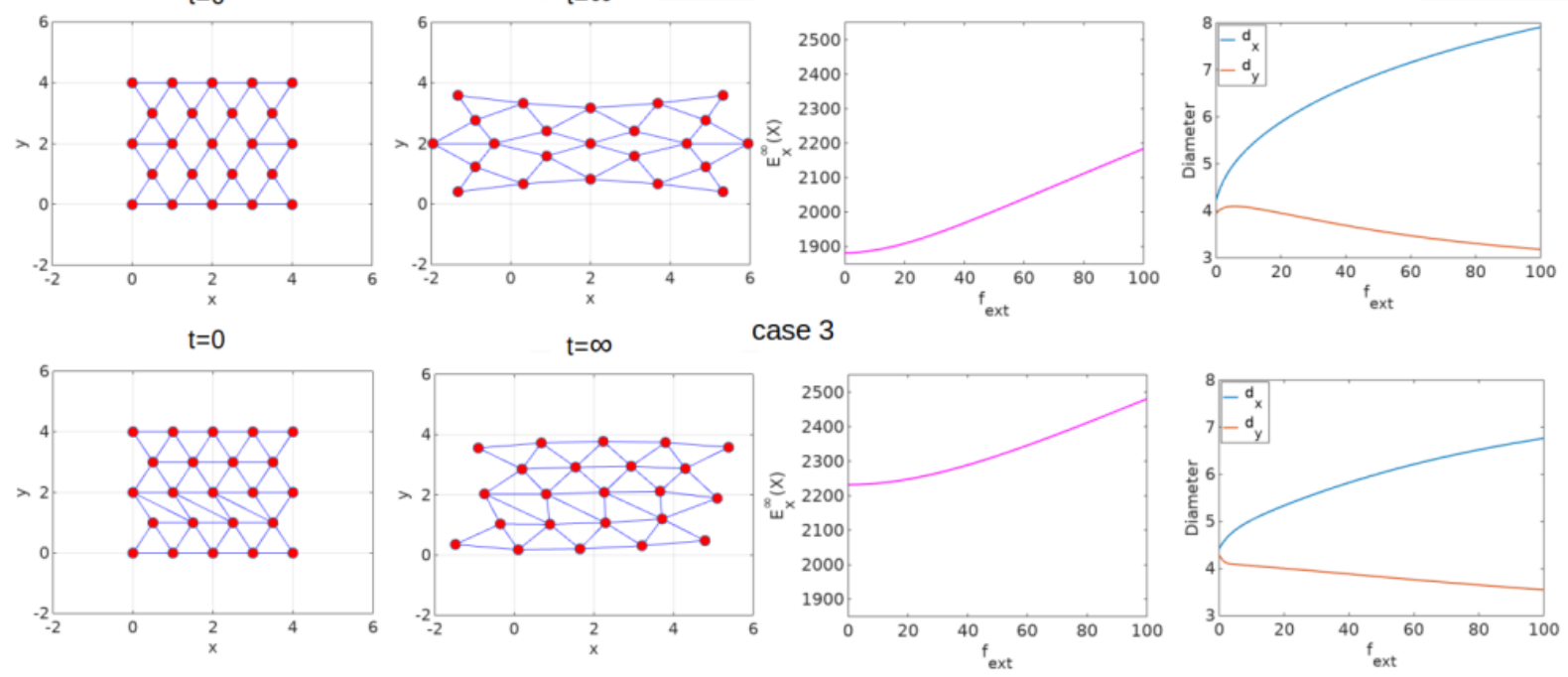

The actin filaments at the nodes seem to be limited to cross-link with no more than eight spectrin filaments. This behaviour controls the rearrangement, clearly favouring the most probable option of six connections but also limiting the types of defects (LIU; DERICK; PALEK, 1987). On building the case 3 we took into account the mentioned characteristics. We simulated the breaking of the edges $6-11,7-12$, and $8-13$ of the case 0 and connect the nodes pairs $7-10,8-11$, and $9-11$. We note that the edges number is the same between the two cases. Let us now compare the response to the same stretching virtual experiment of the case 3 with the case 0 . A relative $\mathscr{E}_{\mathscr{X}}^{\infty}$ variation of $0.9 \%$ does not correspond to a similar behavior of the system. We observe a significant $d_{y}$ increase of $7.3 \%$, also due to the rotation of the entire network during the stretching process. This case introduces a further element of complexity. We obtain similar energy but different deformation behavior. The impact of the connection geometry is another 
challenging aspect in the characterization of the cytoskeleton defects and represents an open field of research in the improvement of the current understanding of RBC mechanical proprieties.

\subsubsection{Some in-depth observations: the virtual optical tweezers ex- periment}

This last section emphasises the potential of the model and also its limitations, justifying the need for the lipid bilayer component integration and opening the opportunity for future developments. To this end, we employ a widely used technique (in the laboratory and computationally) to study RBC mechanical behavior: RBC stretching via optical tweezers. Through this method we can deform a cell in one or more directions by trapping points on the cell surface (HENON et al., 1999; DAO; LIM; SURESH, 2003).

The experiment we reproduced virtually is schematized in Figure 21. We performed RBC stretching using one common setup (MILLS et al., 2004) consisting in anchoring two micro-beads at diametrically opposite points of the RBC membrane. The left bead was attached to a glass immobile surface slide while the right bead was trapped by a laser beam applying an external force $f_{\text {ext }}$. In this process, we could measure the response of the RBC in terms of diameter in the direction of stretching, the axial diameter $D_{a}$, and the diameter orthogonal to the stretching direction named as transverse diameter $D_{t}$ (SIGÜENZA; MENDEZ; NICOUD, 2017).

Figure 21 - Scheme of a common optical tweezers technique to stretch a RBC.

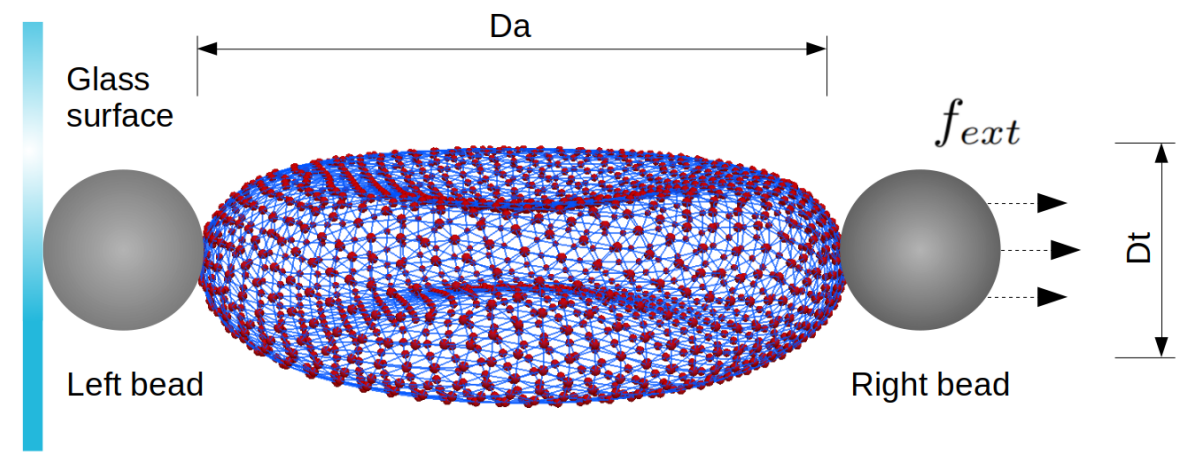

We started from an initial cytoskeletal network respecting the typical equilibrium shape expressed by the equation

$$
y=R \sqrt{1-\frac{x^{2}+z^{2}}{R}}\left[c_{0}+c_{1} \frac{x^{2}+z^{2}}{R^{2}}+c_{2} \frac{\left(x^{2}+z^{2}\right)^{2}}{R^{4}}\right],
$$

with a suitable parameters set-up, as for example $R=3.91(\mu \mathrm{m}), c_{0}=0.0135805, c_{1}=1.001279$, and $c_{2}=-0.561381$ (PIVKIN; KARNIADAKIS, 2008). The mesh dimensions values were $N_{v}=1458$ and $N_{e}=4368$.

Our experiment is unconventional, in fact it is virtual, because we aimed at stretching only the cytoskeleton, not counting the lipid bilayer. However, experimentally the bead is attached to 
the external lipid membrane and not directly to the cytoskeleton. To overcome this situation, we considered the presence of a (fictitious) surface fixed to the bead with an adhesion interaction potential of the Lennard-Jones type with the neighboring cytoskeletal nodes (SAUER, 2012). We also established that this adhesion has a limit bound in all tests: if this value is exceeded, the node is released from the bead. Then, to simulate the laser beam role and the bead movement (with consequent imposition of an external force $f_{\text {ext }}$ ), we applied a constant velocity to the bead.

We draw some considerations by proposing the results of simulations tuning the energy unit $k_{B} T$, given by the product between the Boltzmann constant $k_{B}$ and the temperature $T$. The persistence length $p$ and the power constant $k_{p}$ are set in order to obtain (for each case) the equilibrium with the initial spectrin configuration. For each choice of $k_{B} T$ we plot in Figure 22 the graphs of the energy $\mathscr{E}_{\mathscr{X}}$ and the the axial diameter $D_{a}$ over time. We also present, for a few specific time instants, the visualization of the network with nodes and edges.

Figure 22 - Results of the optical tweezers simulations varying $k_{B} T$. For each test, the graphs of the energy $\mathscr{E}_{\mathscr{X}}$ (on the top) and the the axial diameter $D_{a}$ (on the bottom) over time is shown. Some visualizations of the cytoskeletal network are reported at specific time points.
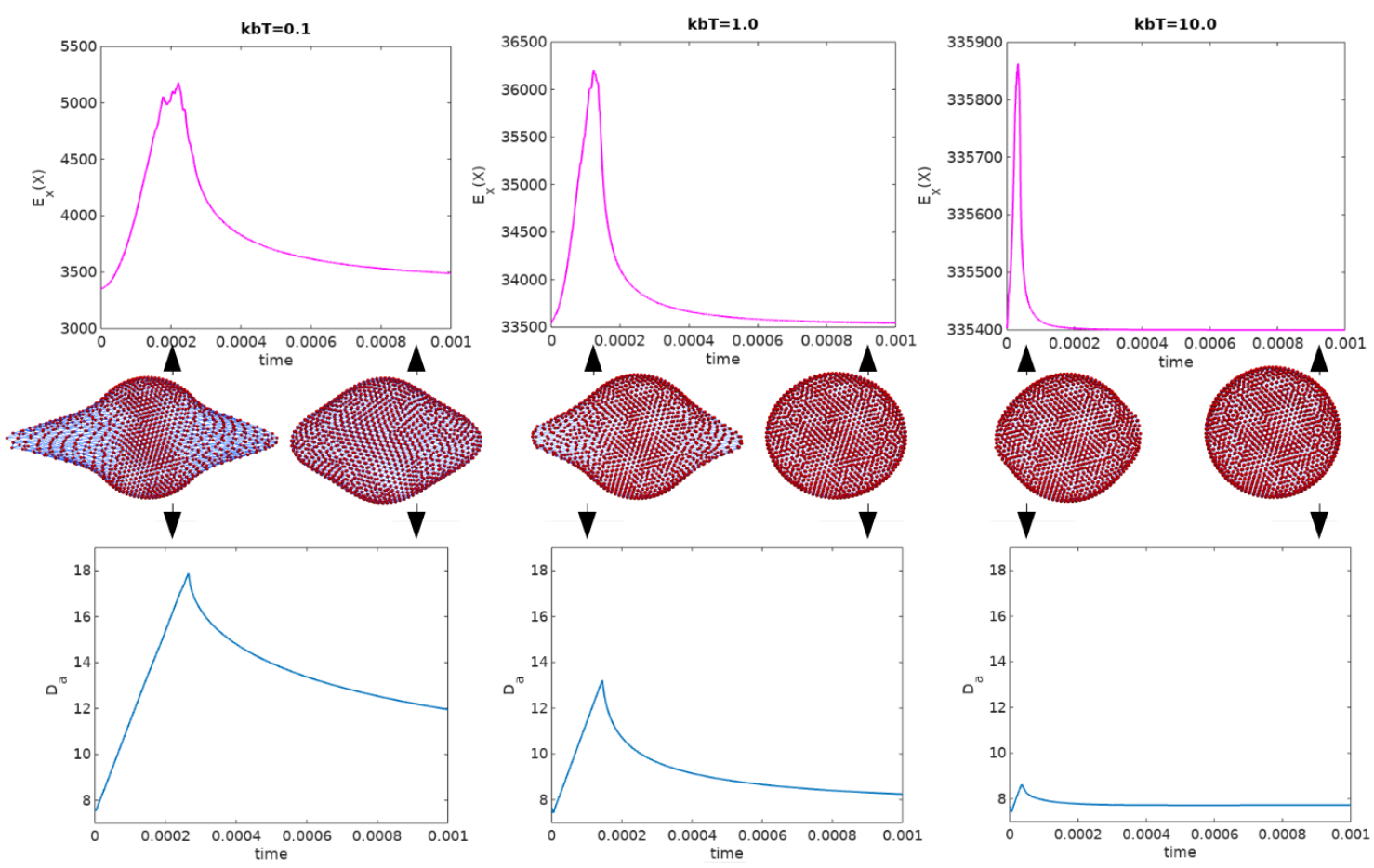

The orders of magnitude of the energies are different and respect the $k_{B} T$ imposed. However, all the behaviors reach a maximum. This point represents the moment when the RBC detaches itself from the bead. The larger is the $k_{B} T$ value, the greater is the force needed to deform the erythrocyte. Having set a constant attractive potential of the bead, it is reasonable that the higher is the value of $k_{B} T$, the sooner the RBC is detached. This implies that the elongation at the time of detaching is greater in the case with smaller $k_{B} T$, as we can see from the panels in Figure 22. After the moment of detaching, the relaxation phase occurs, described by the gradual downfall of the energy value. We note that the energy decreases faster for larger values 
of $k_{B} T$. This aspect agrees with the fact that the recovery shape time (or "shape relaxation time"), i.e., the time necessary for the erythrocyte to recover its initial shape, is considered inversely proportional to the membrane shear modulus and consequently to the $k_{B} T$ (HOCHMUTH; WORTHY; EVANS, 1979) (TAN; XU, 2019).

In the graphs of $D_{a}$ the trend of the diameter reaching the maximum value before decreasing as a result of contraction is shown. A noteworthy fact is that the process of stretching and relaxing does not seem to be fully reversible. This is highlighted by the fact that the diameter does not return to its original value. A similar inability (with due proportion) is also reflected with respect to the diameter $D_{t}$. This evidence allows to hypothesise that the ability to recover form is not an intrinsic property of the cytoskeleton. Therefore, the RBC shape recovering ability may result from the lipid layer contribution.

From the comparison of the simulations results with the experimental data, we can further shed light on the model consistency and infer some considerations. We introduce the quantities corresponding to the relative diameters increase (in the elongation phase) $D_{a}^{r}=\left(D_{a}-D_{a}^{0}\right) / D_{a}^{0}$ and $D_{t}^{r}=\left(D_{t}-D_{t}^{0}\right) / D_{t}^{0}$, with superscript 0 referring to the initial dimension. In Figure 23 we show on the left-panel the the results of our only-one component model of the cytoskeleton. Qualitatively, we can observe a positive correlation between $D_{a}^{r}$ and $D_{t}^{r}$, which agrees with physical intuition that larger stretching axial elongation causes more transverse contraction of the cells.

Figure 23 - Graphs of relative axial diameter $D_{a}^{r}$ with respect to the relative transverse $D_{t}^{r}$. Results of our model simulations (on the left) and experimental measurements (on the right) of RBC samples taken from (LI; LIU, 2008)
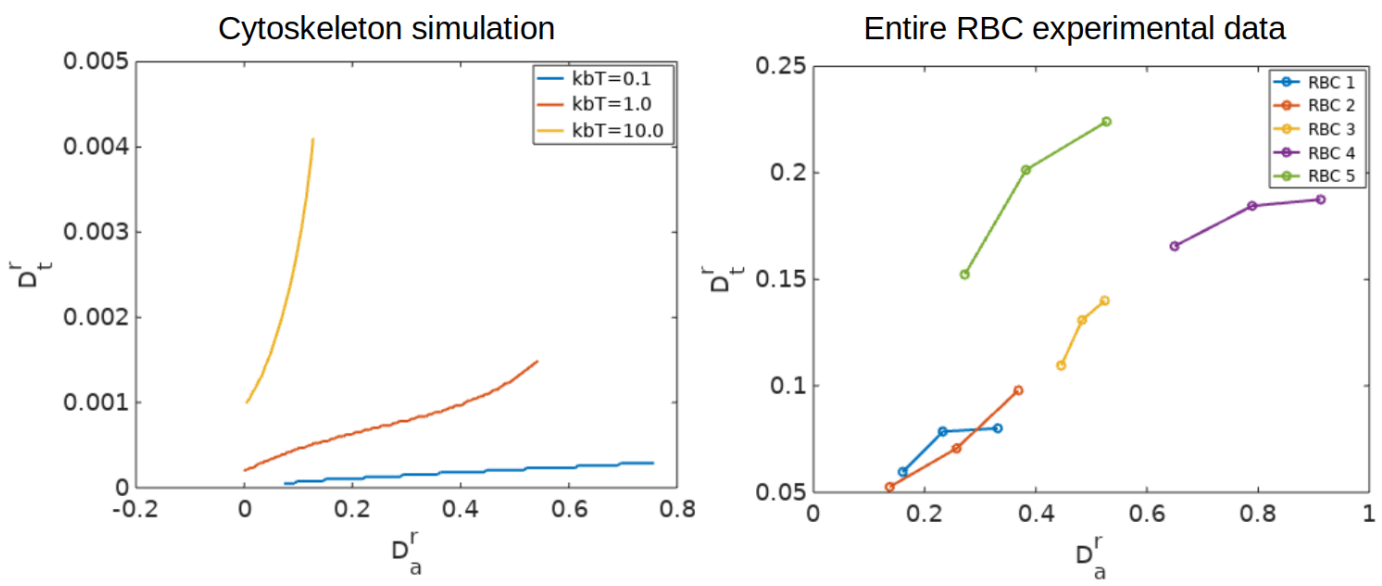

Nevertheless, such a correlation is insufficient comparing it to the laboratory results. On the left-panel of Figure 23 we report the same graphs concerning the entire RBC experimental data for a certain small sample of RBCs, as provided by Li et al.(LI; LIU, 2008) This divergence is another evidence of the mechanical role of the lipid bilayer. In fact, the bending energy induced by the lipid bilayer provides resistance to membrane wrinkle and distortion (POZRIKIDIS, 2003a; FEDOSOV; CASWELL; KARNIADAKIS, 2010). For this reason, to obtain a full RBC 
membrane mathematical model consistent with the experimental results, we need to add the component for the bending.

Although the only cytoskeletal component model cannot be used to mimic the whole RBC mechanical behavior, this quite simple tool (on its own or in conjunction with othercomponent models) has significant and considerable applications. A topic of great interest and impact also in the most recent scientific research is represented by the the study of the internal cell stress distribution as a result of external solicitations (LOPEZ; VALENTINE, 2015).

Figure 24 - Some time configuration of the spectrin network in the virtual optical tweezers experiment with $k_{B} T=1$, showing the stress transmission and distribution. The legend is in the center.

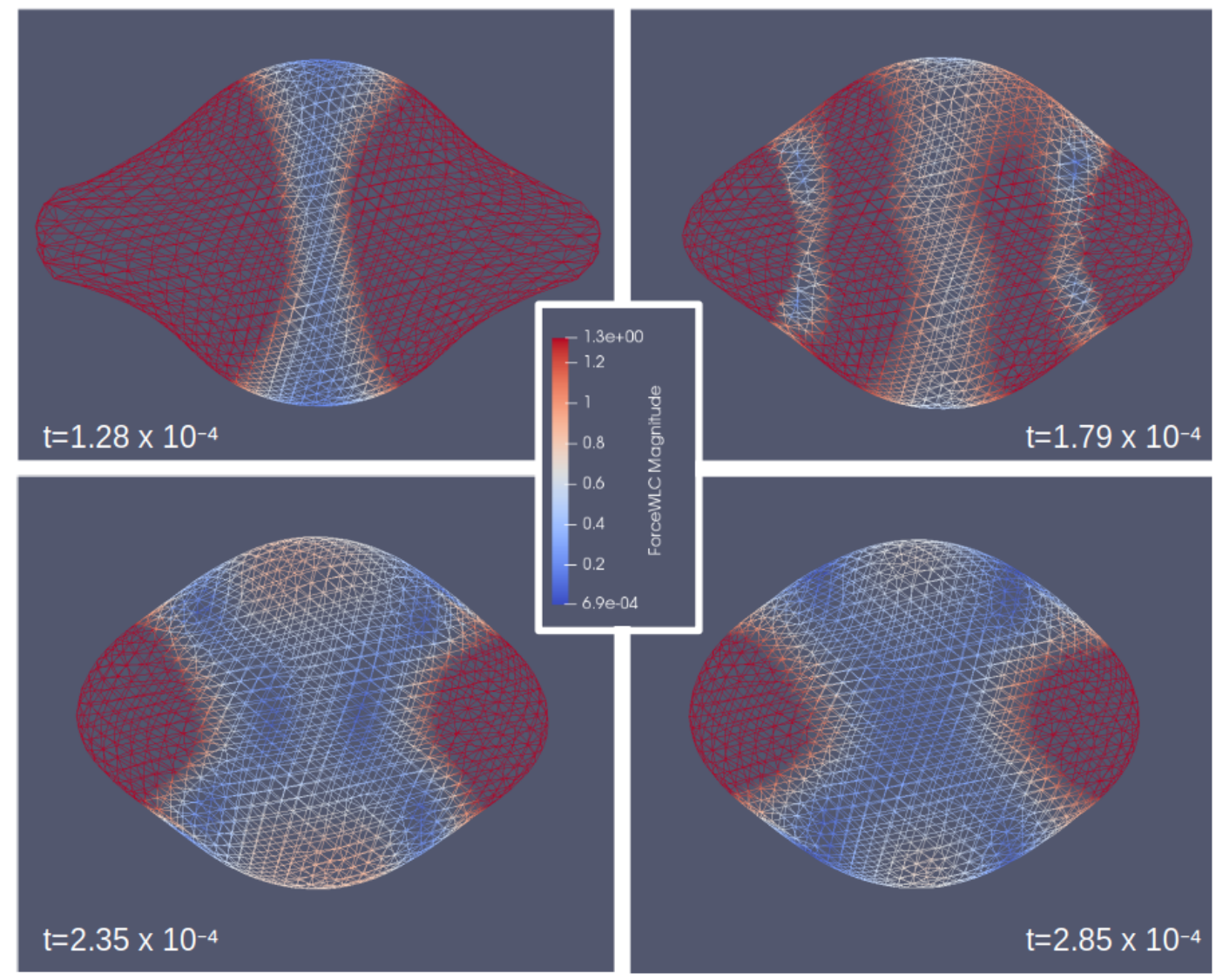

On the panels of Figure 24, we show the internal cytoskelton network of the previous streaching experiment with $k_{B} T=1$. In particular the filaments edges are colored according to the magnitude of force arising from the WLC model of equation (3.3). A non-trivial aspect is represented by the understanding of how spectrin rigidity and cross-linking allow forces (in this case highlighted in red color) to be transmitted within the RBC tissue. Let us comment and briefly analyze the simulation images. The top-left panel concerns the configuration of the network during the stretching process. We can observe as a stretch-induced stress front advances symmetrically from the two extremities towards the center and with a circular profile of a certain radius. The other three images instead concern the relaxation phase. We can note as they show not only the tendency to recover the shape but also how the forces distribution dynamically goes to the homogeneous condition. The resulting behaviors can reveal a certain degree of complexity, 
such as the presence of traveling waves or particular symmetries. All of these constitute an opportunity to advance our understanding by integrating the computational model, which we provided, with experimental results to further characterize the complex internal behaviors and stress transmission in the cytoskeletons of living cells.

\subsection{Achievements and future opportunities}

The study of the erythrocyte cytoskeleton is a well-established topic. Nonetheless, the mathematical and computational modeling of the cytoskeleton still presents several challenging aspects. A first objective of this work was to provide the basic tools necessary for computational study for those (mathematicians and not) who approach to study the cytoskeleton by highlighting useful tips. Indeed a question that motivated this work concerned the possibility of reproducing the main cytoskeleton properties with a simple model (consisting of a small number of code lines). Through tests chosen and built ad hoc, we opted to shed light on salient aspects so to discuss model potential and limitations, and provide insights on coarse-grained scaling procedures and symmetric geometry defects. Through the virtual experiment proposed on cytoskelton stretching by optical tweezers, we have determined that the ability to recover the shape is not an intrinsic property of the cytoskeleton. The comparison with the experimental data showed the need to consider the bending contribution due to the lipid bilayer in order to obtain a more realistic RBC membrane behavior. Broader developments can be integrated with the here described model and in particular with cytoskeleton-bilayer interaction (MEACCI et al., 2020a). In the Appendix, we enclose a (commented) numerical code in Octave free language as a tool ready-to-use.

The investigation of certain properties of the cytoskeletal mechanobiology continues to be essential (FAI et al., 2017) as well the improvement of the biological membrane modeling (RAJAGOPAL; HOLMES; LEE, 2018). The mechanics of spectrin networks are complex. However, we have seen how we can obtain evidences and clarifications with a simple simulation code. There are still many questions and open challenges to be addressed. The combination between experimental research and mathematical and computational modeling represents an open field in the progress of the understanding of erythrocyte functions and mechanics. Further research in this subject is of considerable scientific interest and we believe that through future multidisciplinary efforts, it will continue to provide new insights and positive impact on our daily life. 

CHAPTER

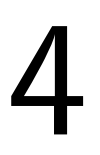

\section{A RED BLOOD CELL CYTO-BILAYER INTERACTION MODEL}

Experimental observations of human RBC behavior in flow mimicking the microcirculation reveal dramatic deformations and rich dynamics. This peculiar hallmark is due to the structure of healthy human RBC. It is a biconcave nucleus-free cell approximately $8 \mu \mathrm{m}$ in diameter and $2 \mu \mathrm{m}$ in thickness. It is primarily composed by a fluid-like lipid bilayer contributing to the bending resistance and an attached spectrin network, that is, a cytoskeleton maintaining cell shape and facilitating its motion. The lipid and spectrin domains are connected by transmembrane proteins. The alterations in cell geometry, membrane properties, as well as the interactions associated with the lipid bilayer and the cytoskeleton could lead to impaired functionality, as to deliver oxygen to the tissues (KIM; LEE; SHIN, 2015) and therefore strongly influence the biomechanics of RBCs (SURESH, 2006).

A computational modeling approach that separately accounts for each component has recently proved useful in the analysis of healthy and diseased RBCs (CHANG et al., 2016; CHANG; LI; KARNIADAKIS, 2017; LI et al., 2014). According to this line of research the cytoskeleton is modeled as junctions (nodes) that are joined by springs that obey a WormLike-Chain (WLC) law and the lipid bilayer is considered as a surface of Dissipative-ParticleDynamics (DPD) particles endowed with bending energy, elastic and viscous interactions. The cytoskeleton-bilayer interaction consists of a short-range force between the nodes of the network and the bilayer particles.

We remark that a particle-based simulation for the bilayer could require to agglomerate tens of thousands of lipid molecules into a single numerical particle, since the typical number of lipid molecules is about 700 million. This unavoidable fact brings to the consequence that "it is difficult to have a precise idea of the scales involved in DPD simulations", as Li et al. (2014) says. This scenario leads to consider a continuum approach for the lipid bilayer component as more natural. Indeed, continuous models provide consistent approximations of large scale 
molecular systems with fewer adjustable parameters than particle-based approaches, and usually are numerically more stable and cost-effective.

Recently, a research group of the ICMC of the USP developed a semi-implicit finite element method that was proved to be useful for studying the behavior of viscous lipid membranes. This computational model is presented in (RODRIGUES et al., 2015a), adopting the viscous liquid-shell model with Canham-Helfrich bending energy described by arroyo2010role. We opted to refer to the same discretization to achieve our aim i.e. to arrive at a hybrid two-component whole-RBC model, with a discrete WLC network for the cytoskeleton but with a continuous surface fluid model for the lipid bilayer.

This means to face the problem to couple the two components by the adhesion forces that mimic the attachment of the cytoskeleton nodes to the bilayer integral proteins. This study is not only necessary to close the model but also is a non-trivial central point. Although the biomechanics of the RBC have been object of extensive studies and work, Peng et al. (2013) affirm that "the mechanical properties of the interactions between the lipid bilayer and the cytoskeleton ... via the pinning connections of transmembrane proteins are still largely unknown" and also Li and Lykotrafitis (2014) recognize that "are not yet fully understood". Nevertheless, there exist biological evidences that bilayer-cytoskeletal interactions are responsible for significant changes in terms of biorheology, erythrocyte function and progress of RBC diseases (SURESH, 2006), as for instance in the case of sickle cell disease (LIU et al., 1991) or during the mechanical filtering process when the RBCs pass through the interendothelial slits in the spleen (SAFEUKUI et al., 2012).

This study is aimed to provide a mathematical formulation of the forces that model the interactions between the cytoskeleton, composed of a discrete set of nodes connected by WLC-type forces and the bi-lipid membrane modeled as a continuous surface that behaves as a two-dimensional fluid.

\subsection{The cytoskeleton-bilayer interaction model}

According to the macroscopic contact modeling, the behavior of two interacting bodies is governed by the principles of continuum mechanics and the unilateral constraint that the bodies may not penetrate each other. On the other hand, when the problem size decreases to the nanoscale, the emergence of atomic effects affects the contact mechanics. The quasi-continuum contact model introduced by sauer07ijnme combines features from both the continuum and the molecular approach.

Let us consider $\Omega_{10}$ and $\Omega_{20}$ the reference configurations of the two bodies, with a given atomic density $\beta_{10}$ and $\beta_{20}$ depending on the atomic structure, respectively. Then, let us consider $\varphi_{1}=x_{1}\left(X_{1}, t\right)$ and $\varphi_{2}=x_{2}\left(X_{2}, t\right)$ are the motions mapping the reference configurations $\Omega_{10}$ and $\Omega_{20}$ onto $\Omega_{1}$ and $\Omega_{2}$ and the atomic density $\beta_{10}$ and $\beta_{10}$ onto $\beta_{1}$ and $\beta_{2}$, respectively (see 
(SAUER, 2012)). Now, we consider two arbitrary points $x_{1} \in \Omega_{1}$ and $x_{2} \in \Omega_{2}$, whose distance is given by $r=\left|x_{1}-x_{2}\right|$. In the proposed model, we assume that two atoms (or molecules) located at $x_{1}$ and $x_{2}$ interact with each other via an interatomic potential $\phi(r)$. A popular choice is the so-called Lennard-Jones potential,

$$
\phi(r)=\varepsilon\left(\frac{r_{0}}{r}\right)^{k}-2 \varepsilon\left(\frac{r_{0}}{r}\right)^{k / 2},
$$

where $r_{0}$ and $\varepsilon$ are a length and an energy scale, respectively. The force $F(r)$ between the two atoms is given by the gradient

$$
F(r)=-\frac{\partial \phi}{\partial r}=\frac{k \varepsilon}{r_{0}}\left(\frac{r_{0}}{r}\right)^{k+1}-\left(\frac{r_{0}}{r}\right)^{(k / 2)+1} .
$$

It can be seen that $r_{0}$ is the equilibrium distance between the atoms, i.e. the distance where $F=0$, and that $\varepsilon$ is the energy of the well at $r=r_{0}$. We can see that when to atoms are close enough to each other, there is a repulsion between them. Otherwise, it occurs an attraction between the atoms with a maximum potential when $r=r_{0}$ and the force tends to zero as $r$ increases and goes to $r=\infty$.

We can define the contact energy as the total interaction energy between all particle pairs located at positions $x_{1} \in \Omega_{1}$ and $x_{2} \in \Omega_{2}$. Adopting this approach, we deduce the bilayercitoskeleton interaction model as soft-body adhesion. For this purpose let us denote by $\Upsilon$ the adhesive surface of the cytoskeleton. In the continuum limit we obtain the contact energy given by

$$
\mathscr{E}_{\text {con }}=\int_{\Gamma} \int_{\Upsilon} \beta_{\Gamma} \beta_{\Upsilon} \phi\left(\left\|x^{\Gamma}-x^{\Upsilon}\right\|\right) d x^{\Gamma} d x^{\Upsilon},
$$

where $\beta_{\Gamma}$ and $\beta_{\Upsilon}$ are non-dimensional scalars (or simply constants) that model the adhesive strength of each surface. The forces arising from this energy are obtained by considering variations $\delta x^{\Gamma}$ and $\delta x^{\Upsilon}$ in the positions of bilayer and cytoskeleton particles

$$
d_{\Upsilon} \mathscr{E}_{c o n} \cdot \delta \Upsilon+d_{\Gamma} \mathscr{E}_{c o n} \cdot \delta \Gamma=-\int_{\Gamma} f^{\mathrm{con}, \Gamma}\left(x^{\Gamma}\right) \cdot \delta x^{\Gamma} d x^{\Gamma}-\int_{\Upsilon} f^{\mathrm{con}, \Upsilon}\left(x^{\Upsilon}\right) \cdot \delta x^{\Upsilon} d x^{\Upsilon}
$$

where $f^{\mathrm{con}, \Gamma}\left(x^{\Gamma}\right)$ is the net force at $x^{\Gamma}$ produced by the contact interaction with the whole of $\Upsilon$,

$$
f^{\text {con }, \Gamma}\left(x^{\Gamma}\right)=-\beta_{\Gamma} \int_{\Upsilon} \beta_{\Upsilon} \phi^{\prime}\left(\left\|x^{\Gamma}-x^{\Upsilon}\right\|\right) \frac{x^{\Gamma}-x^{\Upsilon}}{\left\|x^{\Gamma}-x^{\Upsilon}\right\|} d x^{\Upsilon},
$$

and $f^{\text {con, }, \Upsilon}\left(x^{\Upsilon}\right)$ is the corresponding contact force at $x^{\Upsilon}$ with the whole bilayer surface $\Gamma$,

$$
f^{\text {con }, \Upsilon}\left(x^{\Upsilon}\right)=\beta_{\Upsilon} \int_{\Gamma} \beta_{\Gamma} \phi^{\prime}\left(\left\|x^{\Gamma}-x^{\Upsilon}\right\|\right) \frac{x^{\Gamma}-x^{\Upsilon}}{\left\|x^{\Gamma}-x^{\Upsilon}\right\|} d x^{\Upsilon} .
$$

Notice that, taking $\delta x^{\Gamma}=\delta x^{\Upsilon}$ a constant vector, that $\int_{\Gamma} f^{\text {con, } \Gamma}+\int_{\Upsilon} f^{\text {con, },}=0$, as expected from Newton's third principle.

Let us further elaborate the model so as to only perform integrals on $\Gamma$, taking advantage of our hypothesis that the adhesive surface $\Upsilon$ of the cytoskeleton consists of rigid spheres. Let 
$\Upsilon$ consist of one single sphere $S$, with center $X$ and radius $R$ as in Figure 25. The distance $D=\left\|x^{\Gamma}-X\right\|$ is assumed to be greater than $R$, so that the distance between $x^{\Gamma}$ and $S$ is $d=D-R$. In particular, we can define

$$
e\left(x^{\Gamma}, X\right)=\hat{e}\left(\left\|x^{\Gamma}-X\right\|-R\right)=\int_{S} \phi\left(\left\|x^{\Gamma}-x^{\Upsilon}\right\|\right) d x^{\Upsilon},
$$

where $S$ is the sphere with center $X$ and radius $R$. We can re-write the (4.7) in function of $d$ as

Figure 25 - Scheme with in red a node of the cytoskeleton and in grey a piece of bilayer.

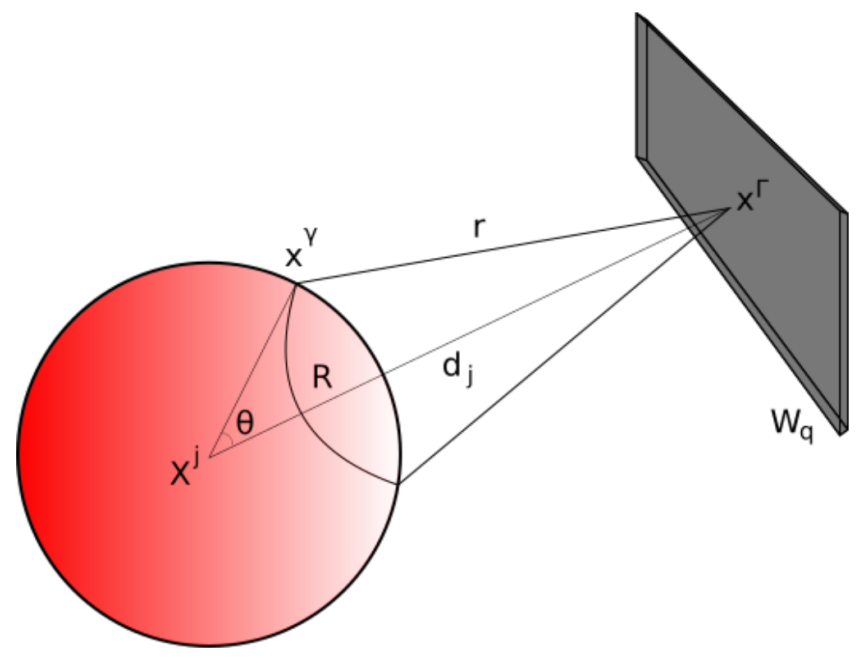

follows

$$
\hat{e}(d)=\int_{d}^{d+2 R} \phi(r) \frac{2 \pi r}{1+d / R} d r
$$

so that, adding up all nodes of the cytoskeleton,

$$
\mathscr{E}_{\text {con }}=\sum_{j} \beta_{j} \sum_{q} \beta_{\Gamma} \hat{e}\left(\left\|x^{\Gamma, q}-X^{j}\right\|-R\right) W_{q}
$$

where $j$ and $q$ are the indexes over the cytoskeleton nodes and the quadrature rule, respectively and $W_{q}$ is the quadrature weight. Therefore the (4.4) becomes

$$
d_{\Upsilon} \mathscr{E}_{c o n} \cdot \delta \Upsilon+d_{\Gamma} \mathscr{E}_{c o n} \cdot \delta \Gamma=\sum_{j} f^{\text {con }, j} \cdot \delta X^{j}+\sum_{q} f^{\mathrm{con}, \Gamma}\left(x^{\Gamma, q}\right) \cdot \delta x^{\Gamma, q} W_{q}
$$

The explicit expressions for the forces (4.5) and (4.6) are

$$
\begin{gathered}
f^{\mathrm{con}, j}\left(X^{j}\right)=\beta_{j} \sum_{q} \beta_{\Gamma} W_{q} \hat{e}^{\prime}\left(\left\|x^{\Gamma, q}-X^{j}\right\|-R\right) \frac{x^{\Gamma, q}-X^{j}}{\left\|x^{\Gamma, q}-X^{j}\right\|}, \\
f^{\mathrm{con}, \Gamma}\left(x^{\Gamma}\right)=-\beta_{\Gamma} \sum_{j} \beta_{j} \hat{e}^{\prime}\left(\left\|x^{\Gamma}-X^{j}\right\|-R\right) \frac{x^{\Gamma}-X^{j}}{\left\|x^{\Gamma}-X^{j}\right\|} .
\end{gathered}
$$

Now, using the (4.1) we can evaluate the (4.7), obtaining

$$
\hat{e}(d)=\frac{2 \pi \varepsilon}{1+d / R}[\mathscr{F}(d+2 R)-\mathscr{F}(d)]
$$


where the auxiliary function $\mathscr{F}(x)$ is as follows

$$
\mathscr{F}(x)=\frac{4}{k-4} \frac{r_{0}^{k / 2}}{x^{k / 2-2}}-\frac{1}{k-2} \frac{r_{0}^{k}}{x^{k-2}} .
$$

We can thus provide the explicit expressions

$$
\begin{aligned}
f^{\text {con }, j} & =\sum_{q} \beta_{\Gamma} W_{q} \beta_{j} 2 \pi \varepsilon R_{j}\left\{-\frac{1}{\left(d_{j}+R_{j}\right)^{2}}\left[\mathscr{F}\left(d_{j}+2 R_{j}\right)-\mathscr{F}\left(d_{j}\right)\right]+\right. \\
& \left.+\frac{1}{d_{j}+R_{j}}\left[\mathscr{F}^{\prime}\left(d_{j}+2 R_{j}\right)-\mathscr{F}^{\prime}\left(d_{j}\right)\right]\right\} \frac{x^{\Gamma}-X^{j}}{\left\|x^{\Gamma}-X^{j}\right\|}= \\
& =\beta_{j} \sum_{q} \frac{\beta_{\Gamma} W_{q}}{d_{j}+R_{j}}\left\{-\hat{e}\left(d_{j}\right)+2 \pi \varepsilon R_{j}\left[\mathscr{F}^{\prime}\left(d_{j}+2 R_{j}\right)-\mathscr{F}^{\prime}\left(d_{j}\right)\right]\right\} \frac{x^{\Gamma, q}-X^{j}}{\left\|x^{\Gamma, q}-X^{j}\right\|}
\end{aligned}
$$

and

$$
\begin{aligned}
f^{\mathrm{con}, \Gamma} & =-\sum_{j} \beta_{\Gamma} \beta_{j} 2 \pi \varepsilon R_{j}\left\{-\frac{1}{\left(d_{j}+R_{j}\right)^{2}}\left[\mathscr{F}\left(d_{j}+2 R_{j}\right)-\mathscr{F}\left(d_{j}\right)\right]+\right. \\
& \left.+\frac{1}{d_{j}+R_{j}}\left[\mathscr{F}^{\prime}\left(d_{j}+2 R_{j}\right)-\mathscr{F}^{\prime}\left(d_{j}\right)\right]\right\} \frac{x^{\Gamma}-X^{j}}{\left\|x^{\Gamma}-X^{j}\right\|}= \\
& =\beta_{\Gamma} \sum_{j} \frac{\beta_{j}}{d_{j}+R_{j}}\left\{\hat{e}\left(d_{j}\right)-2 \pi \varepsilon R_{j}\left[\mathscr{F}^{\prime}\left(d_{j}+2 R_{j}\right)-\mathscr{F}^{\prime}\left(d_{j}\right)\right]\right\} \frac{x^{\Gamma, q}-X^{j}}{\left\|x^{\Gamma, q}-X^{j}\right\|}
\end{aligned}
$$

where

$$
\mathscr{F}^{\prime}(x)=\frac{-2 r_{0}^{k / 2}}{x^{k / 2-1}}+\frac{r_{0}^{k}}{x^{k-1}}
$$

\subsection{Achievements and future opportunities}

Optical tweezers are instruments used to stretch a RBC directly in one or more directions by trapping beads that are strategically attached to the cell surface (DAO; LIM; SURESH, 2003). This equipment is well-suitable for studying the interaction effects between the cytoskeleton and the lipid membrane. In our case, a virtual optical tweezing experiment is a good test to verify the consistency of our model. The numerical implementation revealed the applicability of the formulation for the cyto-bilayer interaction model. The results also reveal a complex behavior which can be a subject of an in-depth study.

In Figure 26 is plotted the magnitude of the contact force of a single node (circled in yellow) over time. As we can see, during the stretching process with the typical folded RBC shape, the cyto-note goes through phases in which it attaches and detaches itself from the membrane.

This study with the corresponding mathematical formulation for the cyto-bilayer RBC interaction provides the tool for a complete new two-component continuum-discrete model for a single RBC. Indeed, it can contribute to shed light on this difficult and relevant issue that is aimed to understand the interactions between the lipid bilayer and the cytoskeleton using a soft-adhesion body approach. 
Figure 26 - Graph of the norm of the contact force a particular cyto-node (circled in yellow) and corresponding screenshots of the optical tweezing numerical simulation.

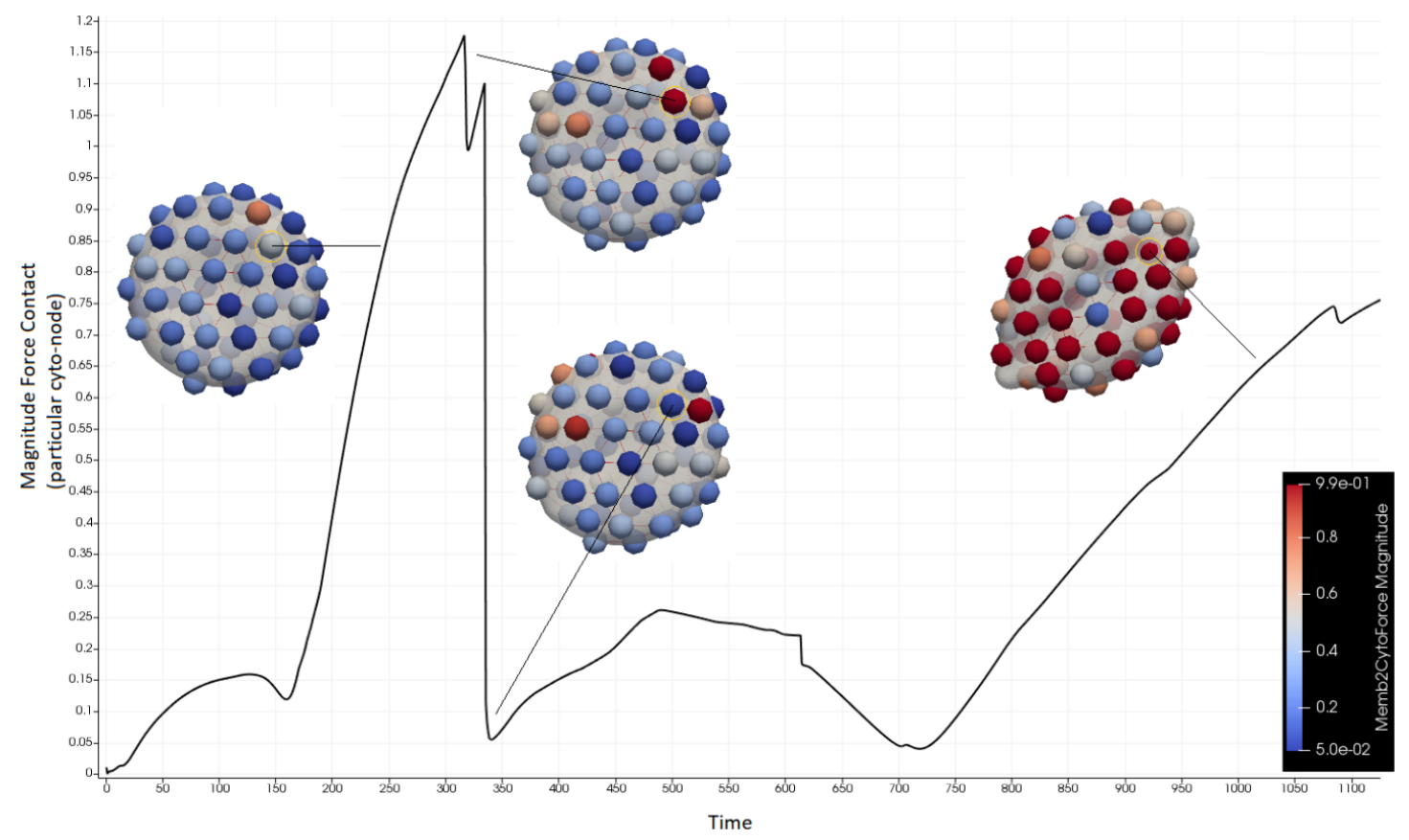


CHAPTER

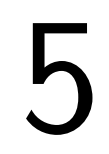

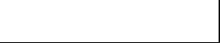

\section{A MATHEMATICAL MODEL OF A SINGLE SEED OLEOSOME}

In plant tissues energy is commonly stored in the form of carbohydrates and lipids. These energy resources support periods of active metabolism such seed germination and initial growth (MURPHY; VANCE, 1999). The most abundant lipid molecules in plants are triglycerydes (or triacylglycerols), which are stored in specialised subcellular organelles called oleosomes, also referred to as "oil bodies", "lipid bodies" or "spherosomes". The size and composition of these organelles are affected by type of plant and of tissue (seeds, fruit mesocarps, leaves, microalgae), nutritional status, and environmental factors (HUANG, 1992). In order to understand their biogenesis and functional role, oleosomes have been isolated from natural and genetically engineered plants and their structure and composition extensively investigated (BRASAEMLE; WOLINS, 2012; ZWEYTICK; ATHENSTAEDT; DAUM, 2000; NAPIER; STOBART; SHEWRY, 1996; BOURGEOIS et al., 2018; WANNER; FORMANEK; THEIMER, 1981; HUANG, 1992; HUANG, 1996; NIKIFORIDIS, 2019). The oleosomes recovered from seeds are recognised to have a size mostly ranging between 0.2 to $2.5 \mu \mathrm{m}$ (CHAPMAN; DYER; MULLEN, 2012). Oleosome structure consists of a neutral lipid rich core stabilised by a monolayer membrane of phospholipids (PLs) and unique integral proteins (TZEN, 2011). The neutral lipid core includes the triacylglycerol molecules (TAG, molecules composed of three fatty acyl chains esterified to a glycerol backbone) and sterol esters. The PLs are oriented to expose the acyl moieties to the TAGs and the polar head group towards the external cytosol environment. Among the interfacial proteins, oleosin is the most abundant accounting for $75-90 \%$ of the total proteins, together with two minor proteins, caleosins and steroleosins (TZEN, 2012). In the accepted structural model of oleosomes found in angiosperm seeds, oleosin is believed to provide coverage to the PL head groups and to be exposed to the cytosol (TZEN; HUANG, 1992).

In recent years, oleosomes have attracted growing attention as a sustainable natural 
ingredient for food and pharmaceutical applications. To fulfill this purpose, oleosomes are recovered using aqueous media from seeds (soybean, rapeseed, pumpkin, etc.) or nuts (pecan nut) and applied to manufacture dairy-free alternatives to conventional milk and yogurts (CHIRICO et al., 2018; ADAMS et al., 2012; ZHANG et al., 2017; NIKIFORIDIS; MATSAKIDOU; KIOSSEOGLOU, 2014) as well as to produce natural cosmetics and pharmaceutical systems (BETTINI et al., 2013). Despite its potential, molecular biology, food sciences, and chemistry have enabled much of the progress in this research area while mathematical studies are lacking. In recent years the mathematical and computational modelling of biological systems has significantly evolved proving to be a useful tool for the study of both biological membranes (STEIGMANN, 2017) and single cell phenomena (BAO; SURESH, 2003). In the case of living cells this kind of approach proved to be a suitable tool to understand and characterise the mechanical responses of cells subjected to both transient and dynamic loads (LIM; ZHOU; QUEK, 2006) and continues to be a field of research for active and enthusiastic interest underpinning innovations of technologies of the future (RAJAGOPAL; HOLMES; LEE, 2018).

The goal of this paper is to address new interdisciplinary research questions: Can a mathematical modelling approach help to understand the physico-chemical and mechanical behaviour of oleosomes? And if so, can the tools of applied mathematics directly tackle some of the challenges associated with the study of oleosomes in applied bio-soft matter science? Therefore, the aim of this work was to test the suitability of a mathematical approach to model the behaviour of a single oleosome within a seed cellular environment. The long term objective of this work is to develop mathematical predictive tools to improve our knowledge of the mechanical and physico-chemical behaviour of oleosomes in-vivo (i.e., within seed environment) and ex-vivo (i.e., in technological applications).

\subsection{Mathematical formulation}

From a mathematical point of view, we can consider an oleosome as a mechanical system that has a configuration described by the state $\mathscr{Y}$ of the monolayer membrane of phospholipids and proteins.

In Figure 27 we show a scheme of our mathematical abstraction of an oleosome. The configuration $\mathscr{Y}$ is a configuration of the lipid membrane, which in the exact problem is an element of an infinite-dimensional manifold of possible membrane shapes. For numerical purposes, however, it is modeled as a finite-dimensional manifold spanned by generalized coordinates. In our case we adopt parameterisations that are defined by the positions of $N_{Y}$ points, the nodes of the membrane model. The coordinates of any configuration $\mathscr{Y}$ are thus $\left\{Y^{i}\right\}_{i=1}^{N_{Y}}$. We remark that the nodes do not correspond to any specific membrane constituent. From these coordinates the geometrical position of the membrane, $\Gamma(\mathscr{Y})=\left\{y \in \mathbb{R}^{3} \mid y\right.$ belongs to the membrane surface $\}$, can be reconstructed and, in the discrete case, being $F^{K}(\xi)=\sum_{j=1}^{3} Y^{C(K, j)} N^{j}(\xi)$, admits the 
Figure 27 - Oleosome sketch with the triacylglycerol (TAG) matrix (in yellow) surrounded by a membrane (a portion of which in orange) of phospholipids and proteins. In the mathematical abstraction, the nodal points $\left\{Y^{i}\right\}$ (in grey) characterize the $\mathscr{Y}$ membrane configuration.

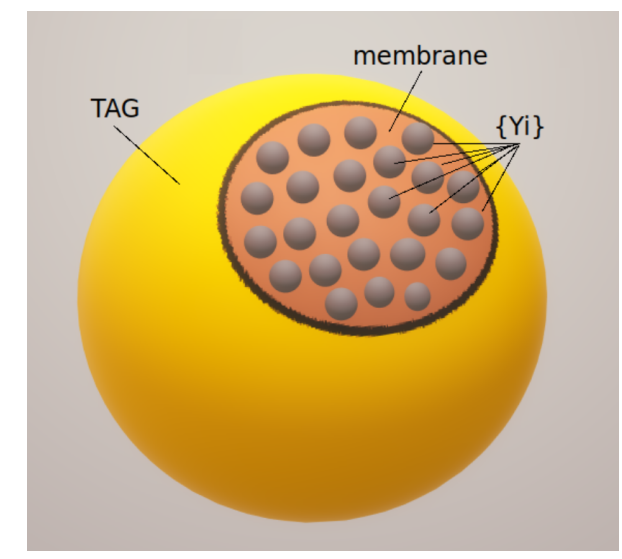

explicit form

$$
\Gamma(\mathscr{Y})=\left\{y \in \mathbb{R}^{3}: y=F^{K}(\xi), \xi \in \hat{K}, K=1, \ldots, M_{Y}\right\}
$$

where $C(\cdot, \cdot)$ is the connectivity matrix (that is, $C(K, j)$ is the index of the $j$-th node of triangular element number $K$ ), $\hat{K} \in \mathbb{R}^{2}$ is the master element and $N^{j}: \hat{K} \rightarrow \mathbb{R}$ are the shape (or basis) functions (see also (TASSO; BUSCAGLIA, 2013)). This discretisation of the surface leads to a faceted (triangulated) $\Gamma(\mathscr{Y})$, over which a normal field $n$ (constant by element) is defined.

The instantaneous motion of the membrane particles is characterised by the rates of change of $\mathscr{Y}$, as follows $\mathscr{W}=\frac{d \mathscr{Y}}{d t}$. This means that the tracking of the surface is Lagrangian and that, in the discrete case, the velocity of the $i$-th membrane node, $W^{i}=\frac{d Y^{i}}{d t}$, coincides with that of the lipid particle at $Y^{i}(t)$. In this latter sentence "lipid particle" is to be understood not as a single molecule but as a small but macroscopic portion of membrane, in the spirit of Continuum Mechanics. Other fields over $\Gamma(\mathscr{Y})$ will be discretised in the same way as the geometry. More specifically, the vector field $w$ that represents the motion given the nodal velocities $\left\{W^{i}\right\}$ is

$$
w\left(F^{K}(\xi)\right)=\sum_{j=1}^{3} W^{C(K, j)} N^{j}(\xi) .
$$

Another important field over $\Gamma(\mathscr{Y})$ is the mean curvature vector $\kappa$, equal to $H n$, with $H$ the mean curvature, with nodal values $\left\{K^{i}\right\}_{i=1}^{N_{Y}}$ when discretized, so that

$$
\kappa(y \in \Gamma(\mathscr{Y}))=\kappa\left(F^{K}(\xi)\right)=\sum_{j=1}^{3} K^{C(K, j)} \mathscr{N}^{j}(\xi) .
$$

The geometrical equation for $\kappa$ is the Laplace-Beltrami identity $-\Delta_{\Gamma} x=\kappa$ that in weak form reads

$$
\int_{\Gamma(\mathscr{Y})} \nabla_{\Gamma} x \cdot \nabla_{\Gamma} \zeta=\int_{\Gamma(\mathscr{Y})} \kappa \cdot \zeta,
$$


where $x$ is the identity mapping,

$$
x(y)=y, \quad x\left(F^{K}(\xi)\right)=\sum_{j=1}^{3} Y^{C(K, j)} \mathscr{N}^{j}(\xi),
$$

and $\nabla_{\Gamma} x$ is the tangential projector $\mathbb{P}$,

$$
\mathbb{P}=\nabla_{\Gamma} x(y)=\mathbb{I}-n(y) \otimes n(y) .
$$

The energy of the proposed system is the intrinsic membrane energy $\mathscr{E}_{Y}$ that clearly depends on $\mathscr{Y}$. To study the evolution of the system, we will refer to the "principle of virtual work", that, in our case, states that the work done by the energies yet defined for an admissible virtual variation of the configuration variables and the work done by the dissipative forces is equal to the work done by the external forces of the system (LANCZOS, 1970). The corresponding expression can therefore be formally written as

$$
d_{Y} \mathscr{E}(\mathscr{Y}) \bullet \delta \mathscr{Y}+\mathscr{D}(\mathscr{Y}, \mathscr{W}) \bullet \delta \mathscr{Y}=\mathscr{F}_{Y} \bullet \delta \mathscr{Y}
$$

where

- $d_{Y} \mathscr{E}(\mathscr{Y}) \bullet \delta \mathscr{Y}$ is the infinitesimal change of energy $\delta \mathscr{E}$, when the state of the system is perturbed from $\mathscr{Y}$ to $\mathscr{Y}+\delta \mathscr{Y}$,

- the bullet $\bullet$ is an appropriate duality product which will be detailed later (Sec. 5.1.1),

- $\mathscr{D}(\mathscr{Y}, \mathscr{W}) \bullet(\boldsymbol{\delta} \mathscr{Y})$ is the dissipation of the system (i.e., the work of its internal dissipative forces), when the system is perturbed by $\delta \mathscr{Y}$, and,

- $\mathscr{F}_{Y} \bullet \delta \mathscr{Y}$ is the virtual work of external forces.

An explicit expression of the lipid layer model is presented below, describing in detail its mathematical formulation arising from equation (5.7).

\subsubsection{Membrane energy and dissipation}

Let us now consider the term $d_{Y} \mathscr{E}(\mathscr{Y})$ of the Equation (5.7). The intrinsic energy $\mathscr{E}_{Y}$ of the layer is given adopting the Canham-Helfrich model (CANHAM, 1970; HELFRICH, 1973), i.e., considering the contribution of the curvature $\kappa$. The bending rigidity (or stiffness) is expressed by the parameter $c_{\mathrm{CH}}$. Moreover, we introduce the addition due to the surface tension $\sigma$ as follows

$$
\mathscr{E}_{Y}(\mathscr{Y})=\int_{\Gamma(\mathscr{Y})}\left(\frac{c_{\mathrm{CH}}}{2}\|\kappa\|^{2}+\sigma\right) d \Gamma
$$

Since the momentum equations are obtained from the virtual work principle, an expression for the variation of $\mathscr{E}_{Y}$ is needed. This expression describes the consequence of a perturbation of the 
layer shape along a vector field $z$ defined on $\Gamma(\mathscr{Y})$. The shape derivative of a functional $\mathscr{I}$ in the direction of a virtual vector field $z$ is defined as

$$
d \mathscr{I}(\mathscr{Y}) \bullet z=\lim _{\varepsilon \rightarrow 0} \frac{\mathscr{I}(\mathscr{Y}+\varepsilon z)-\mathscr{I}(\mathscr{Y})}{\varepsilon}
$$

where in synthetic form $\mathscr{Y}+\varepsilon z=\{Y+\varepsilon z, Y \in \mathscr{Y}\}$. According to (BONITO; NOCHETTO; PAULETTI, 2010) for the Canham-Helfrich energy we obtain

$$
d \mathscr{E}_{Y}(\mathscr{Y}) \cdot z_{n}=\int_{\Gamma(\mathscr{Y})}\left[c_{\mathrm{CH}}\left(-\Delta_{\Gamma} H-\frac{1}{2} H^{3}+2 K H\right)+\sigma H\right] z_{n} d \Gamma,
$$

with $z_{n}=z \cdot n$ being the normal component of $z$ and $n$ the exterior normal to $\mathscr{Y}, \Delta_{\Gamma}$ the LaplaceBeltrami operator, and $H$ the mean curvature. Note that tangential gradient operator $\nabla_{\Gamma}$ is defined by $\nabla_{\Gamma} f=\mathbb{P} \nabla \widehat{g}$, where $g: \Gamma \rightarrow \mathbb{R}$ is any function and $\widehat{g}$ an arbitrary extension of $g$ to an open neighborhood of $\Gamma \subset \mathbb{R}^{3}$. We remark that the curvature force occurs exclusively in the normal direction: tangential motions of $\mathscr{Y}$ do not change its shape. With some calculations (see (BONITO; NOCHETTO; PAULETTI, 2010)) the (5.10) leads to

$$
\begin{aligned}
d \mathscr{E}_{Y}(\mathscr{Y}) \cdot z & =\int_{\Gamma(\mathscr{Y})}\left\{c _ { \mathrm { CH } } \left[(\mathbb{I}-2 \mathbb{P}) \nabla_{\Gamma} \kappa: \nabla_{\Gamma} z+\right.\right. \\
& \left.\left.+\frac{1}{2}\left(\nabla_{\Gamma} \cdot \kappa\right)\left(\nabla_{\Gamma} \cdot z\right)\right]+\sigma \nabla_{\Gamma}\right\} \cdot z d \Gamma,
\end{aligned}
$$

where we remember $x$ is the identity mapping, $\kappa$ obeys to the (5.4), and $\mathbb{P}=\mathbb{I}-\check{n} \otimes \check{n}$ is the tangential projector.

The dissipation function of the layer $\mathscr{D}(\mathscr{Y}, w) \bullet z$ is also needed. This is defined similarly to the viscous dissipation in volume fluids,

$$
\mathscr{D}_{Y}(\mathscr{Y}, w) \bullet z=\int_{\Gamma(\mathscr{Y})} \Sigma: D_{\Gamma} z d \Gamma
$$

where $D_{\Gamma} z=\frac{1}{2} \mathbb{P}\left(\nabla_{\Gamma} z+\nabla_{\Gamma} z^{T}\right) \mathbb{P}$ represents the virtual surface strain rate of field $z$.

The rheology of a viscous interface $\Gamma$ is governed by the Boussinesq-Scriven law (BOUSSINESQ, 1913) (SCRIVEN, 1960) (GROSS; REUSKEN, 2011), which is the tangential analog to the Newtonian constitutive law, leading to the following viscous dissipative surface stress

$$
\Sigma_{v}=\lambda \nabla_{\Gamma} \cdot w \mathbb{P}+2 \mu D_{\Gamma} w
$$

where $\lambda$ and $\mu$ are surface viscosity coefficients. Consequently, we can write the dissipation term (5.12) as follows:

$$
\mathscr{D}_{Y}(\mathscr{Y}, w) \bullet z=\int_{\Gamma(\mathscr{Y})}\left[\lambda \nabla_{\Gamma} \cdot w \nabla_{\Gamma} \cdot z+2 \mu D_{\Gamma} w: D_{\Gamma} z\right] d \Gamma
$$




\subsubsection{Lipid layer model}

Over $\Gamma$ we define the space of kinematically admissible motions, enforced with restrictions of local volume preservation, which is given by

$$
\mathscr{V}(\Gamma(\mathscr{Y}))=\left\{w: \Gamma(\mathscr{Y}) \longrightarrow \mathbb{R}^{3} \text {, s.e., } \int_{\Gamma(\mathscr{Y})} w \cdot n d \Gamma=0\right\}
$$

Specifically, the condition concerning the volume the osmotic equilibrium $\int_{\Gamma} w \cdot n d \Gamma=0 \Rightarrow$ $\frac{d \mathscr{V}}{d t}=\int_{\Gamma} w \cdot n d \Gamma=0$. In discrete time, nevertheless, this condition is not satisfied exactly. This fact may lead to an accumulation of volume errors. The restriction can be therefore implemented as

$$
\int_{\Gamma} w \cdot n d \Gamma-\frac{\mathscr{V}^{*}-\mathscr{V}(t)}{\tau_{\mathscr{V}} \mathscr{V}(t)}=0
$$

Such equation forces $\mathscr{V}(t)$ to the value $\mathscr{V}^{*}$ with the characteristic time $\tau_{\mathscr{V}}$. The actual velocity field $w$ and any virtual ones $v$ must belong to $\mathscr{V}(\Gamma(\mathscr{Y}))$ for all configurations $\mathscr{Y}$ of the surface.

In this context, the virtual work principle for the layer, with $\delta \mathscr{Y}$ represented by the vector field $z$, reads

$$
d \mathscr{E}_{Y}(\mathscr{Y}) \cdot z+\mathscr{D}_{Y}(\mathscr{Y}, w) \cdot z=\int_{\Gamma(\mathscr{Y})} f \cdot z d \Gamma
$$

$\forall z \in \mathscr{V}(\Gamma(\mathscr{Y}))$

In the model adopted here we have greatly simplified the interaction with the surrounding fluid. In fact, the only force the surrounding fluid exerts on the layer comes from a uniform pressure difference, denoted by $p$, between the interior and exterior of $\Gamma$. We observe in fact that the (5.16) leads to an internal pressure $p$ uniform, which is expressed on the surface as a superficial force

$$
f_{p}=p n
$$

With these deductions, we arrive at the following algebraic-differential problem: Solve

$$
\frac{d Y}{d t}=w(Y, t)
$$

where $(w, \kappa, p) \in \mathscr{W} \times K \times \mathbb{R}$ such that

$$
\begin{array}{r}
\int_{\Gamma} \lambda \nabla_{\Gamma} \cdot w \nabla_{\Gamma} \cdot z+2 \mu D_{\Gamma} w: D_{\Gamma} z+ \\
+c_{\mathrm{CH}} \int_{\Gamma}\left[(\mathbb{I}-2 \mathbb{P}) \nabla_{\Gamma} \kappa: \nabla_{\Gamma} z+\frac{1}{2}\left(\nabla_{\Gamma} \cdot \kappa\right)\left(\nabla_{\Gamma} \cdot z\right)\right]= \\
=p \int_{\Gamma} z \cdot n+\int_{\Gamma} f^{\mathrm{ext}, \Gamma} \cdot z-\int_{\Gamma} \sigma \nabla_{\Gamma} \cdot z, \\
\int_{\Gamma} \kappa \cdot \zeta=\int_{\Gamma} \mathbb{P}: \nabla_{\Gamma} \zeta, \\
\int_{\Gamma} w \cdot n=\frac{\mathscr{V}^{*}-\mathscr{V}}{\tau_{\mathscr{V}}},
\end{array}
$$


$\forall(z, \zeta) \in \mathscr{W} \times K$

Above, $K$ is the mean-curvature-vector space, essentially equal to $\left(H^{1}(\Gamma)\right)^{3}$. The space $Q=L^{2}(\Gamma)$ corresponds to the surface tension field. The force field $f^{\text {ext }, \Gamma}: \Gamma \rightarrow \mathbb{R}^{3}$ encompasses all forces acting on the layer by external interventions.

\subsection{Results and Simulations}

\subsubsection{Numerical methods}

For the layer continuum one, we need to discretize it. We consider triangulation surfaces in 3D space, which for a fixed mesh connectivity are uniquely described by the vector $Y$ of vertex positions. Time is discretized so that a sequence of triangulation surfaces $\Gamma^{0}, \Gamma^{1}, \ldots, \Gamma^{n}, \ldots$ are computed, corresponding to vertex positions $Y^{0}, Y^{0}, \ldots, Y^{n}, \ldots$. Following the approach proposed by Rodrigues et al. (2015a), on each $\Gamma^{n}$ we define the piecewise-affine finite element space $P_{1}^{n}=\left\{f \in C^{0}\left(\Gamma^{n}\right):\left.f\right|_{K}\right.$ is affine, $\forall K$ triangle in $\left.\Gamma^{n}\right\}$ and the approximation spaces for velocity, surface and curvature $W_{h}^{n}=\left(P_{1}^{n}\right)^{3} / \mathbb{R}, Q_{h}^{n}=P_{1}^{n}, K_{h}^{n}=\left(P_{1}^{n}\right)^{3}$. We define $\Delta t=t_{n+1}-t_{n}$ and we update the nodal positions in a Lagrangian way, specifically, $Y^{j, n+1}=Y^{j, n}+W^{j, n+1} \Delta t$. With these definitions we can arrive at a fully discrete non-linear problem corresponding to the weak formulation of the system (5.20) - (5.22) only introducing one temporal stabilization term $-\int_{\Gamma^{n}} \tau_{k} \nabla_{\Gamma} w_{h}^{n+1}: \nabla_{\Gamma} \zeta_{h}$, suggested by Bänsch (B ̈̈NSCH, 2001) and described also in (RODRIGUES et al., 2015a), for which the usual choice is $\tau_{k}=\Delta t$. In Sections 5.2.2 and 5.2.3 we present some preliminary results of some numerical resolution with finite elements method (FEM) of this fully-discrete numerical model. We avoid a detailed description of the parameters setup to focus on the qualitative observation of the results. Evidences show the uncertainty of evaluating dynamical parameters as well material quantities such as the surface tension (WHITE, 1980). The simulations presented in the following sections aim at illustrating the possibilities and the potentiality of the model to shed light over the role and measuring of the physical quantities. This model can be used in combination with laboratory work.

\subsubsection{Hypothesis, equilibrium, and lipid layer stress}

We discretise the surface of the oleosome in a surface of triangles, i.e., in a mesh with 642 nodes 1280 edges. For the time discretisation we adopted a time step $\Delta t$ of the magnitude order of $10^{-3}$ time unit. We refer to an ellipsoidal initial form of the oleosome with an average radius of the order of 1 , corresponding to and initial volume and initial surface area of $V_{0}=4.15$ and $A_{0}=13.24$, respectively. Also we put an arbitrary Canham-Helfrich constant $c_{\mathrm{CH}}=1$ that corresponds to establish the energy unit to $1 k T$. We recall that according our model we assume condition (a) of constant volume, due to the fact that we consider the oil fluid inside the membrane incompressible, and the hypothesis (b) of variable surface area that guarantees the deformability of the lipid layer. 
With respect to the set-up of dynamical parameters, we take $\mu=1$ since any other value would only change the time scale but not the equilibrium configuration. Since it has not been experimentally proven otherwise, the ratio $\mu / \lambda$ is set to 1 , which gives similar significance to shear and dilatational dissipation actions. It should be kept in mind that here we are focusing on natural and/or laboratory studies (as hydration or drying out) which are slow processes. A reasonable choice of surface tension $\sigma$ corresponds to that such that the characteristic length $L$ (which is $R$ ) of the system is of the order of $\sqrt{\frac{c_{\mathrm{CH}}}{\sigma}}$. The value of $\sigma$ affects the internal pressure value at equilibrium, for instance in the case of a sphere of $p=\frac{2 \sigma}{R}$. We will focus on the possibility of evaluating the internal pressure value because in the laboratory process of oleosomes extraction, the pressure influences the probability of breaking them and therefore the quality of the final extract. Extracting intact oleosomes is of paramount importance to formulate stable natural oil-in-water emulsions for food and pharma applications. Another factor possibly responsible for the fragmentation of the lipid membranes during the extraction process is the development of regions with very large bending moment, defined as $M=-2 c_{\mathrm{CH}} H$ (GUCKENBERGER; GEKLE, 2017).

Let us first introduce the simulation with our model of a relaxation process, i.e., starting from a initial configuration and in absence of external forces, we allow the system to evolve towards the equilibrium state. In Figure 28 we display the time evolution of the interfacial layer and the magnitude of the mean curvature vector $\kappa$ in the case with $\sigma=1$. The top-left panel corresponds to the initial configuration. It appears clear that, although the oleosome starts from a stressed condition (assuming it has deformed by external forces as shown in the top left panel), it converges with time to its equilibrium condition which is spherical and therefore with constant average curvature (bottom right panel). From a physical and mathematical point of view this behaviour is due to the tendency of the system to go to a condition of minimal energy. In Figure 29 we can see as the energy decreases over time achieving a "plateau" level modelled using different values of $\sigma$. This configuration corresponds also to a minimum value of the pressure that the core liquid oil exerts from inside toward the membrane (pressure data shown in the center-panel of Figure 29). We note that these results are consistent with the previous discussion over the internal pressure.

As we can see from the previous results (Fig. 29), the surface tension influences the velocity of degrowth of energy but not its equilibrium value, a fact which is coherent because the organelle (without external perturbations) tends to a sphere. Let us now suppose to subject the lipid membrane to a compression stress between two rigid planes. In this case the evolution of the internal pressure is not of trivial calculation. In Figure 30 we report some snapshots of the evolution of the membrane shape and the corresponding solution for the internal pressure over time and for different values of the interfacial tensions. We are also interested in evaluating the bending moment during the process. In Figure 30 on the bottom panel we show the evolution of the maximum magnitude of the bending moment evaluated by the model (with $\sigma=1$, because 
Figure 28 - Panels of the evolution of the lipid layer during the relaxation process: at initial condition ( $t=0$, top left panel) and at time $t=0.26$ (top right), $t=0.73$ (bottom left) and $t=3.85$ (bottom right).
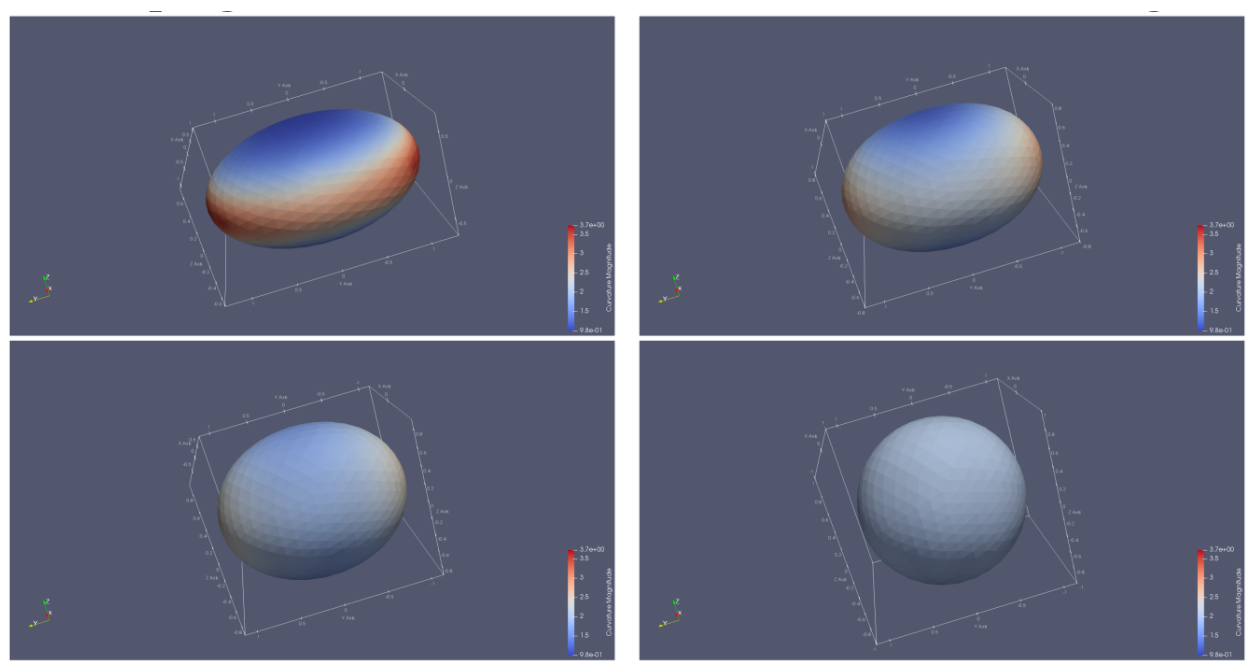

Figure 29 - Lipid layer energy and internal oleosome pressure (insert) over time. A qualitative detail about the velocity field is shown.

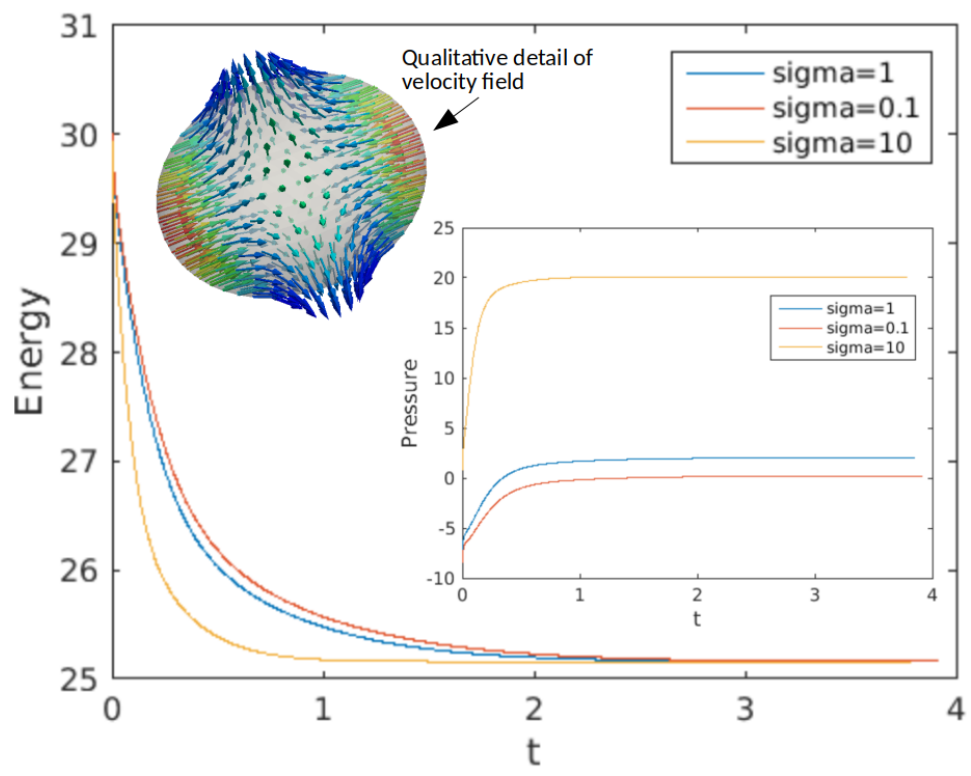

with the other values of $\sigma$ the graph is analogous). These simulations results allow to study the impact of otherwise hard-to-measure physical quantities as well as gaining information over the possible breaking limit of the interfacial layer under stress.

\subsubsection{Senescence: a virtual experiment}

Compression stress is not only a key element of laboratory (or industrial) processes but also of natural phenomena. In order to show the potentiality of this new approach we present here a virtual experiment of seeds senescence. As it can be seen from the microscopy picture of Peng and Tzen (PENG; TZEN, 1998) of a sesame seed (Fig. 31), it is observed experimentally that inside the seed evolving towards its the senescence phase (when water is lost) the oleosomes 
Figure 30 - Time evolution of the internal pressure (top) and the bending moment (below) exercising compression stress.
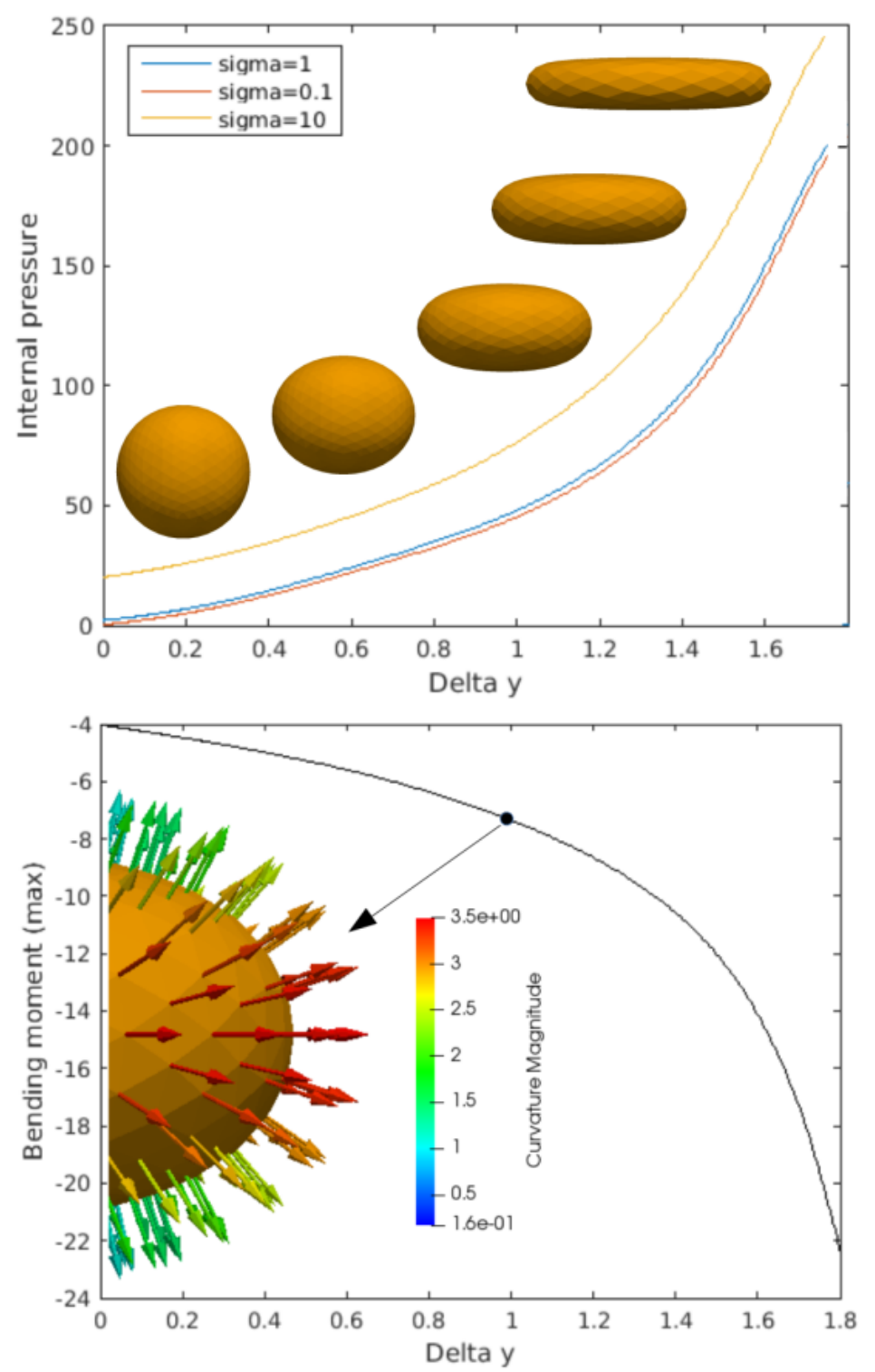

come into contact with each other and take polygonal shapes to allow a better packing within the available space.

We simulated the natural phenomenon while applying our model to a virtual senescence experiment to reproduce the events occurring in vivo. For sake of example, one oleosome is hypothesised to be surrounded by four other rigid organelles, increasingly approaching towards the centre and exerting a contact interaction. The insert plotted in Figure 31 shows as (from an initial spherical shape) the oleosome, due to influence of the interaction with the approaching organelles, assumes the peculiar shape in agreement with what is observed experimentally in seed tissues. The apparently polygonal shape reveals (as shown in a blue-scale line around the layer) a complex curvature distribution, with flat parts of the membrane, others with an almost circular curvature (where interaction with external organelles is negligible) and areas with maximum 
Figure 31 - Electron microscopy of a cell in a mature sesame seed (adopted and modified from (PENG; TZEN, 1998)). The typical polygonal shape assumed by oleosomes due to the interaction/contact with other organelles can be observed. The insert contains a digital reproduction of an oleosome displaying its polygonal shape.

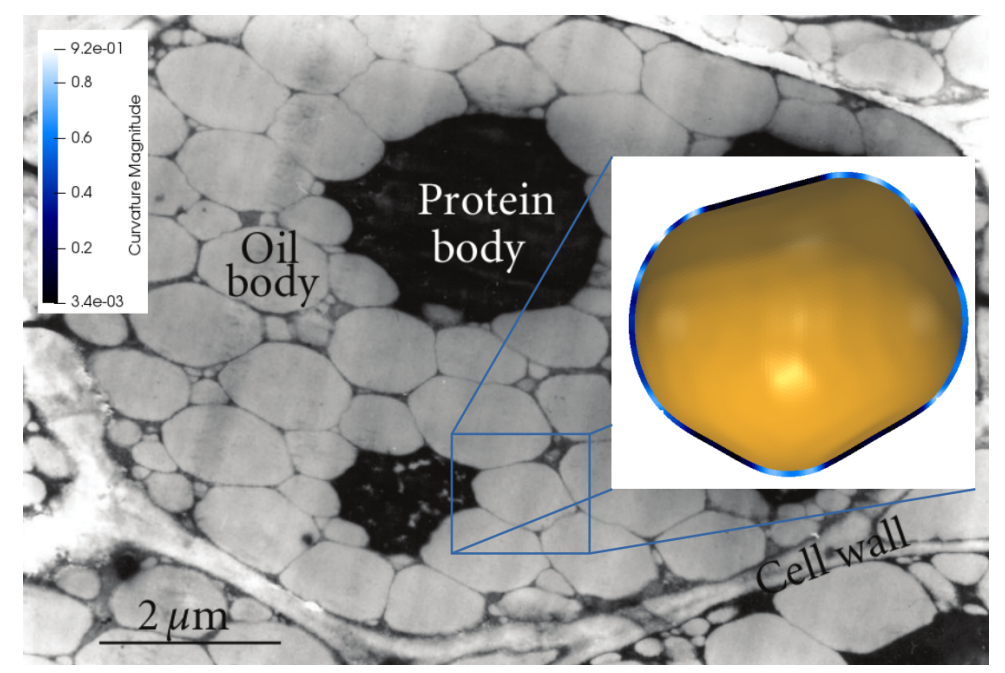

curvatures (and consequently, as we have seen, maximum bending moments).

\subsection{Achievements and future opportunities}

In this work we presented for the first time a model of a single oleosome, providing a detailed mathematical formulation. Although only qualitative, these results show the potential of such approach. Therefore, the main outcome of this interdisciplinary concept study is to offer a starting point for a new research line combining a mathematical modelling approach with laboratory experimental results for oleosome research.

Understanding and modelling the behaviour of oleosomes is relevant from both a fundamental and applied science point of view. These organelles display in-vivo the remarkable ability to preserve their structural integrity over a wide temperature and hydration range (FRANDSEN; MUNDY; TZEN, 2001), (BARRE et al., 2018), (TZEN, 2012), which triggers fundamental questions referring to the membrane fluid behaviour and mechanical properties under those environmental conditions. We believe mathematical modelling could provide new insights to understand oleosomes behaviour during seed maturation, storage and energy mobilisation. Understanding their ex-vivo (i.e., following extraction from plant seed tissues) functionalities is also pivotal for food and pharmaceutical applications; oleosomes could be used as natural alternative for emulsion formulations (i.e., ice cream, mayonnaise, and salad dressings). This approach would allow reducing synthetic emulsifiers usage, processing costs and environmental impacts providing support to tackle global sustainability challenges. 

CHAPTER

\section{6}

\section{A MULTI-COMPONENT RED BLOOD CELL COMPUTATIONAL MODEL: A NEW MATHEMATICAL FORMULATION}

The developments of various mathematical formulations and of complementary numerical techniques have led to continuous improvements and to an ever-increasing differentiation of the contributions of the various RBC components. The novelty of this work is consider a fullcomponent model, i.e., simulating (i) the lipid bilayer behavior, (ii) the cytoskeleton dynamics, (iii) the interaction activity between them, and (iv) the internal cytoplasm flow. In a previous work, the authors justified the choice and the advantage to adopt the continuum approach for the membrane modeling; this new multi-components mathematical formulation is presented and achieved based on the same line. The availability of the model is tested with virtual experiments such as resting shape process and optical tweezers stretching.

The remainder of this paper is organized as follows. The Section 6.1 concern the detailed mathematical abstraction of the model, considering the various components and pointing out the cytoplasm fluid introduction. In Section 6.2 we present the suitability of the model to simulate classical RBC experiments and we provide some considerations of discussion. Finally, in the Section 6.3, we summarize the article achievements and we suggest future applications of the model.

\subsection{Methods and Models}

We suppose that from the mathematical point of view, a RBC is a domain $\bar{\Omega} \subset \mathbb{R}^{3}$. The frontier $\partial \Omega$ models the lipid bilayer and we denote it with $\Gamma$. The inside of the domain $\Omega$ is filled with a fluid of constant viscosity $\mu_{f}$, to simulate the cytoplasm. The bilayer receives an interaction force from the cytoskeleton, this is in the real system through the possible anchoring 
Figure 32 - Domain.

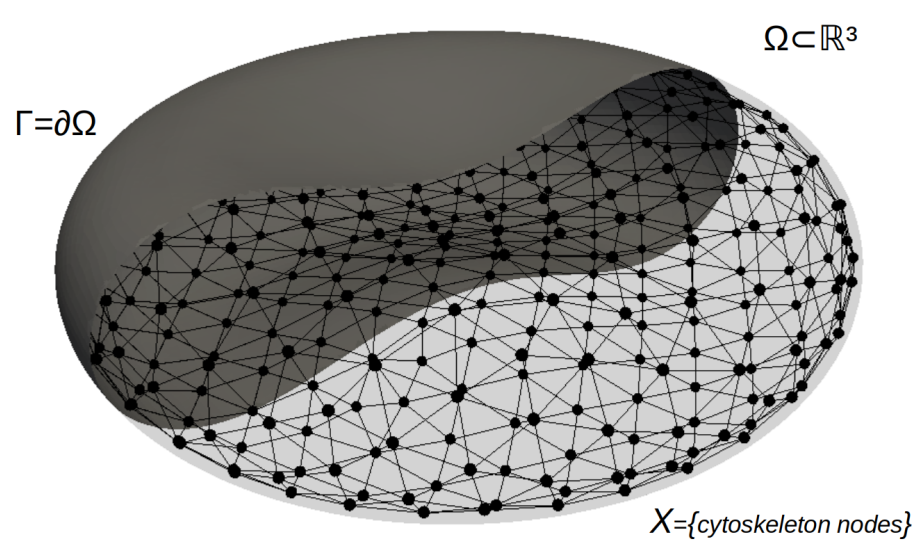

to the transmenbranic proteins. In our abstraction process, this adhesion form is mimicked with a potential defined ad hoc. Finally, the cytoskeleton is schematized as a set $X$ of $N_{X}$ nodes connected to each other by elastic links. We can see a representation of such a domain in Figure 32 .

\subsubsection{Virtual work principle}

We aim to describe the motion of $\bar{\Omega} \subset \mathbb{R}^{3}$ under the action of viscoelastic and external forces. We opt for the application of the virtual work principle (CAPECCHI, 2012), that in our case reads

$$
\int_{\bar{\Omega}} \Sigma: D z=-d \mathscr{E} \Gamma(z)+\int_{\bar{\Omega}} f \cdot z, \quad \forall z \in Z
$$

being $\Sigma$ the tangential stresses tensor, $\mathscr{E}_{\Gamma}$ the elastic membrane energy, $f$ in the net interaction force with the surroundings, $Z$ the space of virtual admissible velocities, and $D z$ the virtual strain rate. The left side of (6.1) is the dissipation term due to the motion/deformation of $\Gamma$, while $d \mathscr{E}_{\Gamma}(z)$ denotes the first variation of $\mathscr{E}_{\Gamma}$ along the virtual velocity field $Z$.

The mathematical formulation (6.1) is very concise and therefore efficient. We consider one unique domain $\bar{\Omega}$ to describe the cytoplasmatic internal fluid and the lipid bilayer, being it simply its border. This fact implies that we can use one only velocity field $W=\left\{w \mid w: \bar{\Gamma} \rightarrow \mathbb{R}^{3}\right\}$ to describe, for a given time instant, the configuration of both components. Although we have one only variable $w \in W$, however the equations are defined according to the domain zone, as it happens for example in the case of dissipation where we split the different contributions of the internal fluid and of the external membrane. Furthermore, the integral over $f$ contains not only completely external forces but also the contribution in the form of interaction of the cytoskeleton to the bilayer. This explicit description implicitly requires the formulation of the cytoskeleton dynamics. In the following sections, we provide a step-by-step complete mathematical model, introducing assumptions and detailing the components. 


\subsubsection{Dissipation and Boussinesq-Scriven law}

As previously mentioned, we divide the dissipation term into 2 parts

$$
\int_{\bar{\Omega}} \Sigma: D z=\int_{\Gamma} \Sigma_{\Gamma}: D_{\Gamma} z+\int_{\Omega} \Sigma: D z
$$

Let us focus firstly into the term concerning the lipid bilayer. It is preliminarily necessary to introduce some notations and surface differential operators. For further information, we suggest to consult the works of Buscaglia \& Ausas (BUSCAGLIA; AUSAS, 2011) and Biria et al. (BIRIA; MALEKI; FRIED, 2013). Nonetheless, in this paper, we refer to the analog of the common symmetric gradient $D z=\left(\nabla z+\nabla z^{T}\right) / 2$ to indicate the corresponding surface operator

$$
D_{\Gamma} z=\frac{1}{2} \mathbb{P}\left(\nabla_{\Gamma} z+\nabla_{\Gamma} z^{T}\right) \mathbb{P},
$$

where the tensor $\mathbb{P}$ is the tangential projector onto the surface $\Gamma$,

$$
\mathbb{P}=\mathbb{I}-\check{n} \otimes \check{n}
$$

$\check{n}$ is the outward normal vector to $\Gamma$, and $\nabla_{\Gamma}$ is the tangential or surface gradient operator, defined as

$$
\nabla_{\Gamma} g=\mathbb{P} \nabla \widehat{g}
$$

with $g: \Gamma \rightarrow \mathbb{R}$ is any function and $\widehat{g}$ an arbitrary extension of $g$ to an open neighborhood of $\Gamma \subset \mathbb{R}^{3}$. We remark that the corresponding surface Laplacian $\Delta_{\Gamma} g$ is given by $\nabla_{\Gamma} \cdot\left(\nabla_{\Gamma} g\right)$. Finally, the surface gradient of a vector, as in the case of $\nabla_{\Gamma} z$ in equation (6.3) is a matrix as follows

$$
\left\{\nabla_{\Gamma}\right\}_{i j}=\left\{\nabla_{\Gamma} z_{i}\right\}_{j}
$$

being $w_{i}$ the i-th $z$ component.

Once these geometrical and differential preliminaries are established, we introduce the costitutive law for the rheology of the viscous interface. According to Boussinesq-Scriven law (BOUSSINESQ, 1913; SCRIVEN, 1960; GROSS; REUSKEN, 2011), the stress tensor related to $\Gamma$ is given by

$$
\Sigma_{\Gamma}=\left(-p_{S}+\lambda \nabla_{\Gamma} \cdot w\right) \mathbb{P}+2 \mu D_{\Gamma} w
$$

where $\lambda$ and $\mu$ are surface viscosity coefficients, and $p_{S}$ is a surface thermodynamic pressure that requires a closure law. We assume also that the bilayer is an inextensible membrane, i.e. an area-preserving interface. Therefore, the condition of inextensibility holds,

$$
\nabla_{\Gamma} \cdot w=0
$$

Under the constraint (6.8), making $\lambda$ tend to infinity, we obtain the classical result that there exists a surface tension $\sigma$ such that $\lim _{\lambda \rightarrow+\infty}\left(-p_{S}+\lambda \nabla_{\Gamma} \cdot w\right)=\sigma$. Consequently, for an inextensible membrane, we have a closure law for $p_{S}$ and the (6.7) becomes

$$
\Sigma_{\Gamma}=\sigma \mathbb{P}+2 \mu D_{\Gamma} w
$$


That means that finally we can write the dissipation term for $\Gamma$ as follows

$$
\int_{\Gamma} \Sigma_{\Gamma}: D_{\Gamma} z=\int_{\Gamma} 2 \mu D_{\Gamma} w: D_{\Gamma} z+\int_{\Gamma} \sigma \nabla_{\Gamma} \cdot z
$$

Let us now consider the dissipation term of (6.2), concerning the internal bulk fluid of $\Omega$. In this case, the classical Newtonian law (BARRETT; GARCKE; NÜRNBERG, 2016) reads

$$
\Sigma=\left(\lambda_{f} \nabla_{\Gamma} \cdot w\right) \mathbb{I}+2 \mu_{f} D w
$$

where $\lambda_{f}$ and $\mu_{f}$ are fluid viscosity coefficients, and $\mathbb{I}$ is the identity tensor (identity matrix). Similarly to the previous case, we apply the incompressibility condition for the fluid i.e., $\nabla \cdot w=0$. By tending $\lambda_{f}$ to infinity we can introduce the variable internal fluid pressure $p$, obtaining the explicit description of the dissipative term for the fluid

$$
\int_{\Omega} \Sigma: D z=\int_{\Omega} 2 \mu_{f} D w: D z+\int_{\Omega} p \nabla \cdot z
$$

which is the weak form of the usual result for the Stokes equations $-\mu_{f} \Delta w+\nabla p=0$.

\subsubsection{Laplace-Beltrami identity and Canham-Helfrich energy}

We introduce one more variable, the mean curvature vector $\kappa$; it is equals to $H n$, with $H$ the mean curvature), which defines a field over $\Gamma$. The geometrical equation for $\kappa$ satisfies the Laplace-Beltrami identity $-\Delta_{\Gamma} \chi=\kappa$ that in weak form is

$$
\int_{\Gamma} \kappa \cdot \zeta=\int_{\Gamma} \nabla_{\Gamma} \chi \cdot \nabla_{\Gamma} \zeta=\int_{\Gamma} \mathbb{P}: \nabla_{\Gamma} \zeta, \quad \forall \zeta \in L^{2}(\Gamma)
$$

where $\chi$ is the identity mapping on $\Gamma$, i.e., $\chi(x)=x, \forall x \in \Gamma$, and $\nabla_{\Gamma} \chi=\mathbb{P}$.

The intrinsic bilayer energy $\mathscr{E}_{-}$is assumed to obey the Canham-Helfrich model (CANHAM, 1970; HELFRICH, 1973), according to

$$
\mathscr{E}_{\Gamma}=\frac{c_{\mathrm{CH}}}{2} \int_{\Gamma}\|\kappa\|^{2}
$$

We are interested in explicitly evaluating the term $d \mathscr{E} \Gamma(z)$ of equation (6.1), that is the $\mathscr{E}$ variation as a consequence of a perturbation of the bilayer shape along a vector field $z$ defined on $\Gamma$. We recall that the shape derivative of a functional $\mathscr{I}$ in the direction of a virtual vector field $z$ is defined as $d \mathscr{I}(z)(x)=\lim _{\varepsilon \rightarrow 0} \frac{\mathscr{I}(x+\varepsilon z)-\mathscr{I}(x)}{\varepsilon}$, where $x \in \Gamma$. When the (6.14) holds, with some calculations (see (BONITO; NOCHETTO; PAULETTI, 2010; RODRIGUES et al., 2015a)), we obtain

$$
d \mathscr{E}_{\Gamma}(z)=c_{\mathrm{CH}} \int_{\Gamma}\left[\nabla_{\Gamma} \kappa: \nabla_{\Gamma} z-\nabla_{\Gamma} z\left(\nabla_{\Gamma} \chi+\nabla_{\Gamma} \chi^{T}\right): \nabla_{\Gamma} \kappa+\frac{1}{2}\left(\nabla_{\Gamma} \cdot \kappa\right)\left(\nabla_{\Gamma} \cdot z\right)\right]
$$

or equivalently, with respect to the tangential projector, the (6.15) can be written as

$$
d \mathscr{E}_{\Gamma}(z)=c_{\mathrm{CH}} \int_{\Gamma}\left[(\mathbb{I}-2 \mathbb{P}) \nabla_{\Gamma} \kappa: \nabla_{\Gamma} z+\frac{1}{2}\left(\nabla_{\Gamma} \cdot \kappa\right)\left(\nabla_{\Gamma} \cdot z\right)\right],
$$

that finally we consider in the formulation of our model. 


\subsubsection{Inextensible membrane and osmotic equilibrium constrains}

Because of (6.8), the tangential motion may be area preserving, being $\frac{d \mathscr{A}}{d t}=\int_{\Gamma} \nabla_{\Gamma} \cdot w=$ 0 . In discrete time, nevertheless, this condition is not satisfied exactly; $\mathscr{A}$ can shift from the correct value $\mathscr{A}^{*}$. For numerical purposes, the area restriction can be therefore implemented as

$$
\int_{\Gamma} \nabla_{\Gamma} \cdot w=\frac{\mathscr{A}^{*}-\mathscr{A}(t)}{\tau_{\mathscr{A}} \mathscr{A}(t)}
$$

in order to force $\mathscr{A}(t)$ to the $\mathscr{A}^{*}$ value with the characteristic time $\tau_{\mathscr{A}}$.

Similarly for the volume, the osmotic equilibrium establishes that the enclosed volume may be fixed to a $\mathscr{V}^{*}$ value. Then, $\int_{\Gamma} w \cdot n d \Gamma=0 \Rightarrow \frac{d \mathscr{V}}{d t}=\int_{\Gamma} w \cdot n d \Gamma=0$. However similarly to the area case we set

$$
\int_{\Omega} w \cdot n=\frac{\mathscr{V}^{*}-\mathscr{V}(t)}{\tau_{\mathscr{V}} \mathscr{V}(t)}
$$

where $\tau_{\mathscr{V}}$ is the characteristic time to force $\mathscr{V}(t)$ to $\mathscr{V}^{*}$.

The Lagrange multipliers are the surface tension field and pressure field spaces (Boyle law), for the volume and area restrictions, respectively.

\subsubsection{Cytoskeleton component}

The remarkable RBC mechanical properties are largely attributed to its cytoskeleton (MOHANDAS; GALLAGHER, 2008). To subsequently quantify the cytoskeleton interaction with the lipid membrane, we briefly provide a model for such a component. We can treat the cytoskeleton by following the nature of its components, basically a spectrin fiber network with special joints (GRATZER, 1981; FEDOSOV; CASWELL; KARNIADAKIS, 2009). The typical length of a spectrin filament is $70 \mathrm{~nm}$, leading to about $10^{5}$ edges for a single RBC. This number, even large, is numerically treatable. In addition, coarse-grained models have been developed to save computing effort (PIVKIN; KARNIADAKIS, 2008). For a comprehensive review of RBC cytoskeleton modeling details, we refer the reader to (MEACCI et al., 2021). As yet displayed in the Figure 32, mathematically we can consider for the nodes a set of vertex points $X=\left\{X^{j} \mid X^{j} \in \mathbb{R}^{3} \subset \Omega\right.$, with $\left.j=1 \ldots N_{X}\right\}$ which forms a two-dimensional network of triangles. The model considers this mesh made of a set of nodes (junctions) joined by $N_{e}$ molecular chains (edges) represented by worm-like chains (HANSEN et al., 1996; FEDOSOV et al., 2011).

In accordance with these preamble considerations, the elastic cytoskeleton mesh energy $\mathscr{E}_{X}$ can be read as

$$
\mathscr{E}_{X}=\sum_{j}\left[\frac{k_{B} T \ell_{m}\left(3 x_{j}^{2}-2 x_{j}^{3}\right)}{4 \ell_{p}\left(1-x_{j}\right)}+\frac{k_{p}}{\ell_{j}}\right],
$$

where $\ell_{j}$ is the filament length $j, \ell_{m}$ is the maximum extension of these filaments, $x_{j}=\ell_{j} / \ell_{m}, \ell_{p}$ is the persistence length, $k_{B} T$ is the unit of energy and $k_{p}$ is a parameter. We remark that the first term in equation (6.19) is the pure worm-like chain (WLC) attractive potential, while the second 
term is related to a repulsive force, called power force (POW). This is the reason why some authors refer to this approach as WLC-POW model (FEDOSOV; CASWELL; KARNIADAKIS, 2010).

By differentiating (6.19) with respect to the vector of nodal coordinates we can obtain the cytoskeleton force $F^{j}$ on the $j$-th node. We remark that the real equations governing the cytoskeleton movement are $m \frac{d^{2} X^{j}}{d t^{2}}+\frac{\partial \mathscr{E} X}{\partial X^{j}}=F^{j}$, where $m$ denotes the effective mass of each node. However the inertia is very small at the scale of the junction complexes, so that for the mathematical formulation we adopt $m=0$, i.e.,

$$
F^{j}=\frac{\partial \mathscr{E}_{X}}{\partial X^{j}}
$$

From a numerical point of view, nevertheless the complete equations are normally numerically integrated with an implicit Newmark scheme, which is well established in solid mechanics.

\subsubsection{Cytoskeleton-bilayer interaction}

The interaction between the membrane and the skeleton mimics the adhesion between these two components and the corresponding mutual transmission of mechanical properties. It deserves particular attention and is the last element for the closure of the mathematical formulation of the model. In particular, since we hypothesize that there are no other external forces onto $\Omega$, we define the last term of the right-hand side of the (6.1). For this goal, we benefit from recent progress on the computational modeling of the adhesion of soft bodies, adapting the formulation of Sauer (SAUER, 2012) (see also (FREUND; LIN, 2004; KUUSELA; ALT, 2009; PAJIC-LIJAKOVIC; MILIVOJEVIC, 2014; PENG et al., 2013)).

Let us denote by $\Upsilon$ the adherent surface of the cytoskeleton. The general form for the contact energy $\mathscr{E}_{c o n}$ is given by

$$
\mathscr{E}_{\text {con }}=\int_{\Gamma} \int_{\Upsilon} \beta_{\Gamma} \beta_{\Upsilon} \phi\left(\left\|x^{\Gamma}-x^{\Upsilon}\right\|\right) d x^{\Gamma} d x^{\Upsilon}
$$

where $\phi$ is the interaction potential (in Joule $/ \mathrm{m}^{4}$ ) and $\beta_{\Gamma}, \beta_{\Upsilon}$ are dimensionless scalars. In many applications, mathematical modelers opt for the Lennard-Jones potential (ISRAELACHVILI, 2011), i.e., $\phi(d)=\varepsilon\left(\frac{r_{0}}{d}\right)^{k}-2 \varepsilon\left(\frac{r_{0}}{d}\right)^{k / 2}$, where $d$ is the distance between two particles and, $r_{0}$ and $\varepsilon$ are length and energy scales, respectively. This potential has a minimum of value $-\varepsilon$ at $d=r_{0}$, it is repulsive for $d<r_{0}$ and attractive if $d>r_{0}$.

The contact force on the bilayer resulting from this energy therefore consists in

$$
f^{\text {con, }, ~}\left(x^{\Gamma}\right)=-\beta_{\Gamma} \int_{\Upsilon} \beta_{\Upsilon} \phi^{\prime}\left(\left\|x^{\Gamma}-x^{\Upsilon}\right\|\right) \frac{x^{\Gamma}-x^{\Upsilon}}{\left\|x^{\Gamma}-x^{\Upsilon}\right\|} d x^{\Upsilon} .
$$

Attributing a spherical shape of radius $R$ and center $c$ to $\Upsilon$, this integral can be computed analytically as an explicit function $\mathscr{G}\left(\left\|x^{\Gamma}-c\right\|-R, r_{0}, \varepsilon\right)$ times the unit vector along $x^{\Gamma}-c$. The function $\mathscr{G}$ diverges when $d=\left\|x^{\Gamma}-c\right\|-R$ tends to 0 , since $d<0$ implies interpenetration. To 
improve the numerical resolution, the authors recently proposed a variation of the regularized potential (MEACCI et al., 2020b). In a complementary manner, the force exerted on a point $x^{\Upsilon}$ of the adherent surface of the cytoskeleton by the bilayer $\Gamma$ is given by

$$
f^{\mathrm{con}, \Upsilon}\left(x^{\Upsilon}\right)=\beta_{\Upsilon} \int_{\Gamma} \beta_{\Gamma} \phi^{\prime}\left(\left\|x^{\Gamma}-x^{\Upsilon}\right\|\right) \frac{x^{\Gamma}-x^{\Upsilon}}{\left\|x^{\Gamma}-x^{\Upsilon}\right\|} d x^{\Gamma}
$$

As we expected from the action-reaction principle, $\int_{\Gamma} f^{\mathrm{con}, \Gamma}+\int_{\Upsilon} f^{\mathrm{con}, \Upsilon}=0$. For a more detailed exposition of the formulation and calculations, the authors recently presented a paper (MEACCI et al., 2020a). The contact force only acts along the surface normal. For this reason, it is complemented by a drag force that models the tangential attachment of the citoskeleton nodes to the bilayer anchoring proteins as

$$
f^{d, \Gamma}\left(x^{\Gamma}\right)=-\int_{\Upsilon} \eta\left(w\left(x^{\Gamma}\right)-\frac{d x^{\Upsilon}}{d t}\right) d x^{\Upsilon}, \quad f^{d, \Upsilon}\left(x^{\Upsilon}\right)=\int_{\Gamma} \eta\left(w\left(x^{\Gamma}\right)-\frac{d x^{\Upsilon}}{d t}\right) d x^{\Gamma},
$$

where $\eta$ is a drag coefficient that depends on $\left\|x^{\Gamma}-x^{\Upsilon}\right\|$. We highlight that the set $\left\{X^{i}\right\}_{i=1}^{N_{X}}$ concerns the coordinates of the centers of the nodes, while $\Upsilon$ consists of spheres $\Upsilon^{j}$ of a given radius around $X^{j}$, i.e., the surfaces of the junctions. The net force per unit area acting on the bilayer is thus $f^{\Gamma}=f^{\operatorname{con}, \Gamma}+f^{d, \Gamma}$, while the net force on node $j$, to be equilibrated by the worm-like chains, according to the (6.20), is

$$
F^{j}=\int_{\Upsilon^{j}}\left(f^{\text {con, }, \Upsilon}\left(x^{\Upsilon}\right)+f^{d, \Upsilon}\left(x^{\Upsilon}\right)\right) d x^{\Upsilon}
$$

\subsubsection{Model summary}

We now are able to sum all components to obtain the mathematical model formulation for the RBC configuration at a given instant time. We obtain the following problem: 
Stationary full model problem. Find $(w, \sigma, \kappa, p) \in W \times Q \times K \times \mathscr{P}$ such that

$$
\begin{aligned}
& \int_{\Omega} 2 \mu_{f} D w: D z-\int_{\Omega} p \nabla \cdot z+\int_{\Gamma} 2 \mu D_{\Gamma} w: D_{\Gamma} z+\int_{\Gamma} \sigma \nabla_{\Gamma} \cdot z+ \\
& +c_{\mathrm{CH}} \int_{\Gamma}\left[(\mathbb{I}-2 \mathbb{P}) \nabla_{\Gamma} \kappa: \nabla_{\Gamma} z+\frac{1}{2}\left(\nabla_{\Gamma} \cdot \kappa\right)\left(\nabla_{\Gamma} \cdot z\right)\right]=\int_{\Gamma} f^{\mathrm{con}, \Gamma} \cdot z, \\
& \int_{\Gamma} \xi \nabla_{\Gamma} \cdot w=\frac{\mathscr{A}^{*}-\mathscr{A}}{\mathscr{A} \tau_{\mathscr{A}}} \int_{\Gamma} \xi \\
& \int_{\Gamma} \kappa \cdot \zeta=\int_{\Gamma} \mathbb{P}: \nabla_{\Gamma} \zeta \\
& \int_{\Omega} q \nabla \cdot w=\frac{\mathscr{V}^{*}-\mathscr{V}}{\mathscr{V} \tau_{\mathscr{V}}} \int_{\Omega} q \\
& \frac{\partial \mathscr{E}_{X}}{\partial X^{j}}=\int_{\Upsilon^{j}}\left(f^{\text {con, }, \Upsilon}\left(x^{\Upsilon}\right)+f^{d, \Upsilon}\left(x^{\Upsilon}\right)\right), \quad j=1, \ldots, N_{x} \\
& \text { with, } \\
& \mathscr{E}_{X}=\sum_{k=1}^{N_{e}}\left[\frac{k_{B} T \ell_{m}\left(3\left(x_{k}\right)^{2}-2\left(x_{k}\right)^{3}\right)}{4 \ell_{p}\left(1-x_{k}\right)}+\frac{k_{p}}{\ell_{k}}\right], \quad x_{k}=\ell_{k} / \ell_{m}, \\
& f^{\text {con }, \Gamma}\left(x^{\Gamma}\right)=-\beta_{\Gamma} \int_{\Upsilon} \beta_{\Upsilon} \phi^{\prime}\left(\left\|x^{\Gamma}-x^{\Upsilon}\right\|\right) \frac{x^{\Gamma}-x^{\Upsilon}}{\left\|x^{\Gamma}-x^{\Upsilon}\right\|}, \\
& f^{\operatorname{con}, \Upsilon}\left(x^{\Upsilon}\right)=\beta_{\Upsilon} \int_{\Gamma} \beta_{\Gamma} \phi^{\prime}\left(\left\|x^{\Gamma}-x^{\Upsilon}\right\|\right) \frac{x^{\Gamma}-x^{\Upsilon}}{\left\|x^{\Gamma}-x^{\Upsilon}\right\|}, \\
& f^{d, \Upsilon}\left(x^{\Upsilon}\right)=\int_{\Gamma} \eta\left(w\left(x^{\Gamma}\right)-\frac{d x^{\Upsilon}}{d t}\right),
\end{aligned}
$$

$\forall(z, \xi, \zeta, q) \in W \times Q \times K \times \mathscr{P}$.

Above $W$ is the space of kinematically admissible motions, i.e., essentially $W=\{z$ : $\Omega \rightarrow \mathbb{R}^{3}, z \in C^{k}$ with $k$ enough large $\}$ with opportune restrictions, such as inextensible and volume-preserving motions. Then $K$ is the mean-curvature-vector space that can be equal to $\left(H^{1}(\Gamma)\right)^{3}$, the space $Q=L^{2}(\Gamma)$ corresponds to the surface tension field, and $\mathscr{P}=L^{2}(\Omega)$ is the pressure field space.

Proposition 2.7.1 Assuming to have computed the cytoskeleton configurations governed by (6.30) - (6.31) and related cyto-bilayer contact forces (6.32)-(6.34), if

- $\Gamma$ is smooth enough,

- $W=\left\{z: \Omega \rightarrow \mathbb{R}^{3}, z \in C^{k}\right.$ with $k$ enough large $\}$ with opportune restrictions,

- $K=\left(H^{1}(\Gamma)\right)^{3}$,

- $Q=L^{2}(\Gamma)$

- $\mathscr{P}=L^{2}(\Omega)$,

then, the stationary full model problem is well-posed.

Proof. Given a certain configuration of the cytoskeleton (and of the lipid membrane), we suppose 
to solve the equations for the cytoskeleton and to calculate the relative contact membrane forces, as assumed in the hypothesis. In the following, we denote with "smoothness" the property to possess a sufficient number of continuous derivatives over the domain. The model is related to a composite problem, which can be broken down into parts to prove the well-posedness. Let us proceed in three steps:

(i) We can solve the Laplace-Beltrami equation (6.28) and find the smooth $k$ solution. It is possible integrating by part the right-hand side of (6.28) and because $\Gamma, \chi$ and $\mathbb{P}$ are smooth (KASHIWABARA et al., 2015; BONITO; DEMLOW; NOCHETTO, 2020).

(ii) We now neglect the constrains (6.27) and (6.29), set $\sigma=p=0$, and substitute the $k$ solution into (6.26). We obtain essentially 2 bilinear forms:

$$
\mathscr{B}_{1}(w, z)=\int_{\Omega} 2 \mu_{f} D w: D z, \quad \mathscr{B}_{2}(w, z)=\int_{\Gamma} 2 \mu D_{\Gamma} w: D_{\Gamma} z
$$

one resolving the Boussinesq-Scriven problem for the velocity $w$ over $\Gamma$, and the other one concerning the classical Stokes problem for the velocity $w$, here in the domain $(\Omega)$, respectively. In both cases it is possible to choose an appropriate space $W \subset H^{1}(\Omega)^{3}$ such that the bilinear forms are coercive and therefore $W$ uniquely defined (CLÉMENT; MARTIN, 2016). For example, a possibility is to choose $W$ as the $H^{1}(\Omega)^{3}$ quotiented with rigid movement space.

(iii) We finally focus on $\sigma$ and $p$. In (6.27) and (6.29) the Lagrange multipliers enforce the area and volume preservation. Under these conditions, the existence and uniqueness of these last unknown variables are proved satisfying inf-sup conditions, as yet shown in similar in (RODRIGUES et al., 2015a). It is possible to verify that such requirements are fulfilled by $Q=L^{2}(\Gamma)$ and $\mathscr{P}=L^{2}(\Omega)$.

\subsubsection{Evolutionary model}

In this section, we extend the previous model, which describes the system configuration at a certain fixed time, to the evolutionary dynamics over time. In fact, if the previous case allows to study the model mathematically, from the computation point of view and for simulation purposes, it is crucial to introduce the time dependence. In particular, we denote with $\Omega(t)$ the configuration of the domain $\Omega$ at time $t$ and with $X(t)=\left\{X^{j}(t) \mid X^{j}(t) \in \mathbb{R}^{3} \subset \Omega(t)\right.$, with $\left.j=1 \ldots N_{X}\right\}$ the set of positions of all cytoskeleton nodes at time $t$. Our evolutionary generalization is expressed with an infinitesimal increase in time, taking into account the families of velocity field over $\Omega(t)$ and of velocity field of the cytoskeleton nodes $Y(t)=\left\{Y^{j}(t) \mid Y^{j}(t)=d X^{j}(t) / d t\right.$, with $\left.j=1 \ldots N_{X}\right\}$. We lead to the following problem. 
Evolutionary model problem. In presence of known initial configurations $\Omega(0)$ and $X(0)$, compute the evolution of configuration $\Omega(t)$ and $X(t)$, convected by the velocity field arising by solving the stationary full model problem and by choosing the time increment $\delta t$ such that

$$
\begin{array}{r}
\forall t, \forall X^{j}(t) \in X(t), \operatorname{dist}\left(X^{j}(t)+Y^{j}(t) \delta t, X^{j}(t+\delta t)\right) \leq C_{1} \delta t^{2}, \\
\forall t, \forall x \in \Gamma(t), \operatorname{dist}(x+w(x, t) \delta t, \Gamma(t+\delta t)) \leq C_{2} \delta t^{2}
\end{array}
$$

for some $C_{1}$ and $C_{2}$ and where $\operatorname{dist}(\cdot, \cdot)$ stands for both point-point and point-surface distance, on a case by case basis.

\subsection{Results/Discussion}

In this section we present the suggested discretization of the model and methods we use to numerically solve the evolutionary problem. We then show some results.

\subsubsection{Discretization and numerical approach}

Following the (6.35) and (6.36) assumptions, we define a time increment $\delta t$ inducing a time discretization so that

$$
t_{n+1}=t_{n}+\delta t, \text { with } t_{0} \text { given, } n=0,1, \ldots
$$

Let us now proceed to discretize the space domain. The cytoskeleton component is discrete by definition; so we do not need to discretize it. Concerning $\bar{\Omega}$, we have to distinguish the case of the boundary surface $\Gamma$ which solves the problem of Laplace Beltrami and concerning the Boussinesq-Scriven law from the internal fluid where the velocity and pressure arise by solving a Stokes problem in the three-dimensional domain $\Omega$.

Therefore, concerning $\Gamma$ we consider triangulation surfaces, which for a fixed mesh connectivity are uniquely described by triangle vertex position set. The time sequence of triangulation surfaces $\Gamma^{0}, \Gamma^{1}, \ldots, \Gamma^{n}, \ldots$ are computed and we define the piecewise affine finite element space

$$
P_{1}^{n}=\left\{f \in C^{0}\left(\Gamma^{n}\right):\left.f\right|_{K} \text { is affine, } \forall K \text { triangle in } \Gamma^{n}\right\}
$$

and with the same $\Gamma^{n}$ we consider the approximation spaces for velocity, surface and curvature set as

$$
\begin{array}{r}
W_{h}^{n}=\left(P_{1}^{n}\right)^{3} / R, \\
Q_{h}^{n}=P_{1}^{n}, \\
K_{h}^{n}=\left(P_{1}^{n}\right)^{3},
\end{array}
$$


where, as suggested in the previous section, we can opt for $R$ as the rigid movements space, i.e., formally $R=\left\{r: \mathbb{R}^{3} \rightarrow \mathbb{R}^{3} \mid r(x)=\omega \wedge x+\beta, \omega, \beta \in \mathbb{R}^{3}\right\}$.

Concerning $\Omega$, we similarly discretize the volume with tetrahedra, in order to obtain a time sequence of tetraized volumes $\Omega^{0}, \Omega^{1}, \ldots, \Omega^{n}, \ldots$, where we define the equal order piecewise affine finite element space

$$
P V_{1}^{n}=\left\{f \in C^{0}\left(\Omega^{n}\right):\left.f\right|_{T} \text { is affine, } \forall T \text { tetrahedron in } \Omega^{n}\right\}
$$

and the corresponding approximation spaces for the velocity and pressure fields in the volume, i.e.,

$$
\begin{array}{r}
W_{h}^{n}=\left(P V_{1}^{n}\right)^{3} / R, \\
\mathscr{P}_{h}^{n}=P V_{1}^{n} .
\end{array}
$$

We note that we have used a little abuse of notation for the spaces approximating the velocity, but the meaning, the analogy, and the passage between the superficial and volumetric domain is easy to understand.

We therefore come to the following new discrete problem:

Discrete evolutionary model problem. Update the nodal positions $x^{i, n}$ and $X^{j, n}$ of the domain ( $i$-th vertex of triangle on $\Gamma$ or tetrahedron in $\Omega$ ) and the cytoskeleton (center of the $j$-th cytoskeleton network node) respectively, according to

$$
\begin{gathered}
x^{i, n+1}=x^{i, n}+w_{h}^{i, n+1}\left(x^{i, n}\right) \delta t, \\
X^{j, n+1}=X^{j, n}+Y^{j, n+1} \delta t,
\end{gathered}
$$

such that the velocities $w_{h}^{i, n+1}$ and $Y^{j, n+1}=\left(X^{j, t+1}-X^{j, t}\right) / \delta t$ solve the following fully discrete problem, that demands to find $\left(w_{h}^{n+1}, \sigma_{h}^{n+1}, \kappa_{h}^{n+1}, p_{h}^{n+1}\right) \in W_{h}^{n} \times Q_{h}^{n} \times K_{h}^{n} \times \mathscr{P}_{h}^{n}$ in such a way 
that

$$
\begin{aligned}
& \int_{\Omega^{n}} 2 \mu_{f} D w_{h}^{n+1}: D z-\int_{\Omega^{n}} p_{h}^{n+1} \nabla \cdot z+\int_{\Gamma^{n}} 2 \mu D_{\Gamma} w_{h}^{n+1}: D_{\Gamma} z+\int_{\Gamma^{n}} \sigma_{h}^{n+1} \nabla_{\Gamma} \cdot z+ \\
& +c_{\mathrm{CH}} \int_{\Gamma^{n}}\left[(\mathbb{I}-2 \mathbb{P}) \nabla_{\Gamma^{\prime}} \kappa_{h}^{n+1}: \nabla_{\Gamma} z+\frac{1}{2}\left(\nabla_{\Gamma} \cdot \kappa_{h}^{n+1}\right)\left(\nabla_{\Gamma} \cdot z\right)\right]=\int_{\Gamma^{n}} f^{\mathrm{con}, \Gamma, n+1} \cdot z, \\
& \int_{\Gamma^{n}} \xi \nabla_{\Gamma} \cdot w^{n+1}+\int_{\Gamma^{n}} \gamma_{h}\left(\nabla_{\Gamma} \sigma_{h}^{n+1}-g_{h}^{n}\right) \cdot \nabla_{\Gamma} \xi=\frac{\mathscr{A}^{*}-\mathscr{A}^{n}}{\mathscr{A}^{n} \tau_{\mathscr{A}}} \int_{\Gamma^{n}} \xi \\
& \int_{\Gamma^{n}} \kappa^{n+1} \cdot \zeta-\int_{\Gamma^{n}} \tau_{k} \nabla_{\Gamma} w_{h}^{n+1}: \nabla_{\Gamma} \zeta=\int_{\Gamma} \mathbb{P}: \nabla_{\Gamma} \zeta \\
& \int_{\Omega^{n}} q \nabla \cdot w_{h}^{n+1}+\int_{\Omega^{n}} \gamma_{h}^{v}\left(\nabla p_{h}^{n+1}-h_{h}^{n}\right) \cdot \nabla q=\frac{\mathscr{V}^{*}-\mathscr{V}^{n}}{\mathscr{V}^{n} \tau_{\mathscr{V}}} \int_{\Omega^{n}} q \\
& \frac{\partial_{X}^{\mathscr{E}}{ }_{X}+1}{\partial X^{j, n+1}}-\left(\beta_{f} Y^{j, n+1}-M^{j} \frac{Y^{j, n+1}-Y^{j, n}}{\delta t}\right)=\int_{\Upsilon^{j, n+1}}\left(f^{\text {con, },, n+1}\left(x^{\Upsilon, n+1}\right)+f^{d, \Upsilon, n+1}\left(x^{\Upsilon, n+1}\right)\right), \\
& \mathscr{E}_{X}^{n+1}=\sum_{k=1}^{N_{e}}\left[\frac{k_{B} T \ell_{m}\left(3\left(x_{k}^{n+1}\right)^{2}-2\left(x_{k}^{n+1}\right)^{3}\right)}{4 \ell_{p}\left(1-x_{k}^{n+1}\right)}+\frac{k_{p}}{\ell_{k}^{n+1}}\right], \quad x_{k}^{n+1}=\ell_{k}^{n+1} / \ell_{m}, \\
& f^{\mathrm{con}, \Gamma, n+1}\left(x^{\Gamma, n+1}\right)=-\beta_{\Gamma} \int_{\Upsilon^{n+1}} \beta_{\Upsilon} \phi^{\prime}\left(\left\|x^{\Gamma, n+1}-x^{\Upsilon, n+1}\right\|\right) \frac{x^{\Gamma, n+1}-x^{\Upsilon, n+1}}{\left\|x^{\Gamma, n+1}-x^{\Upsilon, n+1}\right\|}, \\
& f^{\text {con, }, \Upsilon, n+1}\left(x^{\Upsilon}\right)=\beta_{\Upsilon} \int_{\Gamma^{n+1}} \beta_{\Gamma} \phi^{\prime}\left(\left\|x^{\Gamma, n+1}-x^{\Upsilon, n+1}\right\|\right) \frac{x^{\Gamma, n+1}-x^{\Upsilon, n+1}}{\left\|x^{\Gamma, n+1}-x^{\Upsilon, n+1}\right\|}, \\
& f^{d, \Upsilon, n+1}\left(x^{\Upsilon, n+1}\right)=\int_{\Gamma^{n+1}} \eta\left(w_{h}^{n+1}\left(x^{\Gamma, n+1}\right)-\frac{x^{\Upsilon, n+1}-x^{\Upsilon, n}}{\delta t}\right),
\end{aligned}
$$

$\forall(z, \xi, \zeta, q) \in W_{h}^{n} \times Q_{h}^{n} \times K_{h}^{n} \times \mathscr{P}_{h}^{n}$

We need to provide some notes to comment the new stabilization terms introduced and some generalizing additions compared with the evolutionary continuum model. The stabilization terms of the (6.48) and (6.50)

$$
\begin{gathered}
\int_{\Gamma^{n}} \gamma_{h}^{\mathscr{A}}\left(\nabla_{\Gamma} \sigma_{h}^{n+1}-g_{h}^{n}\right) \cdot \nabla_{\Gamma} \xi, \\
\int_{\Omega^{n}} \gamma_{h}^{\mathscr{V}}\left(\nabla p_{h}^{n+1}-h_{h}^{n}\right) \cdot \nabla q,
\end{gathered}
$$

are similar; they aim to fix the checkerboard modes due to the equal order interpolation of $w_{h}, \sigma_{h}$, and $p_{h}$, benefiting from the same idea of the works (see (CODINA et al., 2001)), In the (6.56) and (6.57), $\gamma_{h}^{\mathscr{A}}$ and $\gamma_{h}^{\mathscr{V}}$ are parameters. Then $g_{h}^{n}$ and $h_{h}^{n}$ are the $L^{2}(\Gamma)$-projection of $\nabla_{\Gamma} \sigma_{h}^{n+1}$ onto $\left(Q_{h}^{n}\right)^{3}$ and $L^{2}(\Omega)$-projection of $\nabla p_{h}^{n+1}$ into $\left(\mathscr{P}_{h}^{n}\right)^{3}$ respectively, i.e.,

$$
\begin{aligned}
& \int_{\Gamma^{n}} g_{h}^{n} \cdot z=\int_{\Gamma^{n}} \nabla_{\Gamma} \sigma_{h}^{n+1} \cdot z, \quad \forall z \in\left(Q_{h}^{n}\right)^{3}, \\
& \int_{\Omega^{n}} h_{h}^{n} \cdot z=\int_{\Omega^{n}} \nabla p_{h}^{n+1} \cdot z, \quad \forall z \in\left(\mathscr{P}_{h}^{n}\right)^{3} .
\end{aligned}
$$

In the equations (6.48) and (6.50) is heuristically verified that the characteristic times $\tau_{\mathscr{A}}$ and $\tau_{\mathscr{V}}$ can be set equal to $10 \delta t$. 
The other stabilization term concerns the Laplace-Beltrami equation (6.49), given by

$$
-\int_{\Gamma^{n}} \tau_{k} \nabla_{\Gamma} w_{h}^{n+1}: \nabla_{\Gamma} \zeta=0
$$

as provided by Bänsch (2001) to increase the temporal stability. Rodrigues et al. (2015a) suggest to set $\tau_{k}=\delta t$.

Furthermore, the equation (6.51) concerning the cytoskeleton dynamics was integrated with the component

$$
\beta_{f} Y^{j, n+1}-M^{j} \frac{Y^{j, n+1}-Y^{j, n}}{\delta t}
$$

to allow the introduction of: (i) a friction term, being $\beta_{f}$ a constant parameter, and (ii) the inertial effect due to the $M^{j}$ masses of the cytoskeleton junctions. In the following results, we consider these masses negligible, but the complete model can be numerically integrated with an implicit Newmark scheme and solved by a Newton-Raphson iterative procedure. Let us proceed to validate the computational formulation of the model through numerical tests.

\subsubsection{Equilibrium and resting shape}

We opt to use a relaxing process in order to test our computational formulation. This virtual experiment consists in letting the system tend to a minimum of energy (condition of equilibrium) without applying any external force. This test has value both from a mathematical point of view to evaluate the numerical consistency of the model, but also from a physical / biological point of view. In the case of the red blood cell, in fact, the resting form of the cell, which in healthy conditions has the well-known biconcave physiognomy, is closely related to its biological functionality. However, various states and forms of disease can give different morphologies to the red blood cell, with mechanical and physiological implications of various kinds. An example of a sample of diseased red blood cells seen under a microscope is shown in Figure 33.

To compare the model with the results presented in the literature, let us consider a sphere of radius $r=3.27 \mu \mathrm{m}$ as the reference form, as in the works of Li et al. (2005), Peng, Asaro and Zhu (2010). In such case the sphere surface area and the volume are $A_{\text {sphere }}=134.3709 \mu \mathrm{m}^{2}$ and $V_{\text {sphere }}=146.4643 \mu \mathrm{m}^{3}$, respectively. We suppose to gradually reduce the volume to a target value $V_{\text {target }}$, defining a reduced volume parameter as

$$
v_{\text {reduced }}=\frac{V_{\text {target }}}{4 / 3 \pi r^{3}}
$$

According to the benchmarking values, a reduced volume $v_{\text {reduced }} \approx 0.60$ corresponds to the classical biconcave shape; but this depends also on the possible variation of the area. For detailed studies of the resulting equilibrium shapes we refer the reader to the articles of Seifert, Berndl and Lipowsky (1991), Geekiyanage et al. (2019). 
Figure 33 - Heterogeneous sample of RBCs affected by stomatocytosis and echinocytosis under the scanning electron microscope and reported on (GEEKIYANAGE et al., 2019).

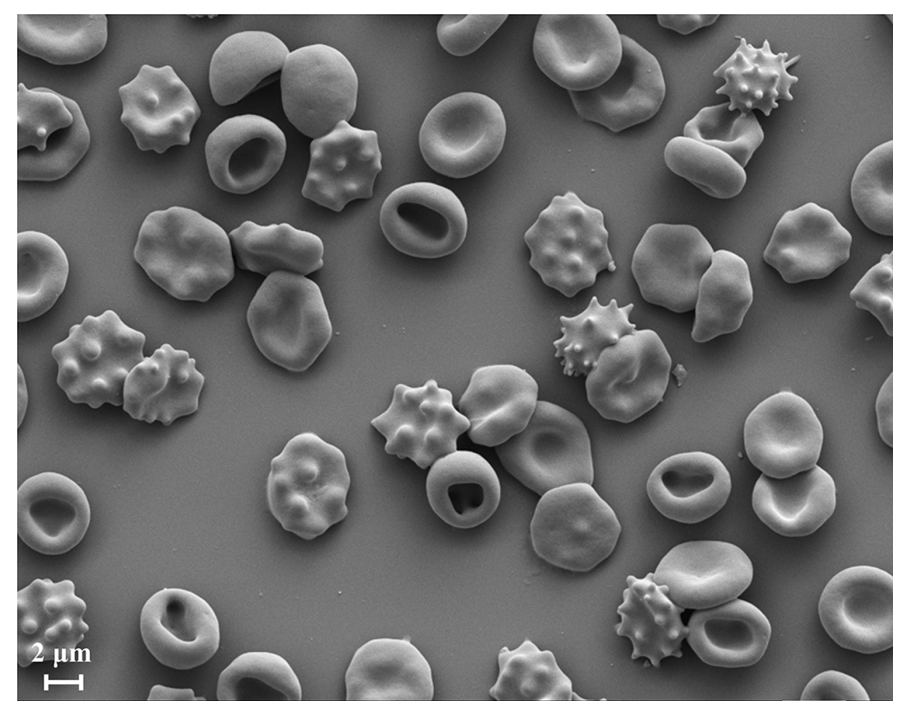

In our case, we refer to the parameters setup listed in Table 7 for all the model components, to solve our discrete model of equations (6.45) - (6.55). In the following test, we have introduced a cytoskeleton of 741 nodes of radius $R=0.05$ and a cytoplasmic internal fluid of viscosity $\mu_{f}=0.01$.

Table 7 - Setup of parameters of the relaxation process simulations.

\begin{tabular}{rclc} 
Value & Symbol & Description & Component \\
\hline 1.0 & $k_{B} T$ & Energy unit & (WLC) \\
2 & $n$ & POW exponent & (WLC) \\
1.0 & $\beta_{\Gamma}$ & Bilayer interaction constant & (INT) \\
1.0 & $\beta_{\Upsilon}$ & Cyto interaction constant & $($ INT) \\
6 & $k$ & LJ potential coefficient & $($ INT) \\
200 & $\varepsilon$ & Interaction strength constant & (INT) \\
0.05 & $r_{0}$ & Equilibrium cyto-membrane distance & (INT) \\
0.05 & $R$ & Radius of cyto-sphere & (INT) \\
1.0 & $\mu$ & Bilayer viscosity & (BIL) \\
20.0 & $C_{c h}$ & Canham constant & (BIL) \\
118.270 & $A_{\text {target }}$ & Target RBC surface area & (BIL) \\
67.747 & $V_{\text {target }}$ & Target RBC volume & (BIL) \\
0.01 & $\mu_{f}$ & Fluid viscosity & (CYTOSOL)
\end{tabular}

The safe red blood cell biconcave morphology is obtained. As we can see from Figure 34 , the biconcavity is particularly accentuated, due to a significant reduction in the target volume. The nodes of the cytoskeleton are visualized and the peculiar structure of the junction complexes can be seen, with the spectrins colored according to their tension. The internal fluid substantially reaches an uniform pressure, as expected. The results obtained are qualitatively consistent with those obtained with our two-component model presented in (MEACCI et al., 2020b). In 
particular, the resulting values of the main quantities are reported in Table 8 .

Figure 34 - Snapshot of the RBC system after the relaxing process test described in the sub-section 6.2.2. The lipid bilayer is visualized in red scale according to the curvature magnitude. A section of the internal fluid is displayed in sky-blue, according its pressure value. Indeed, the cytoskeleton network is plotted. The spectrin colors are referred to the edge tension.

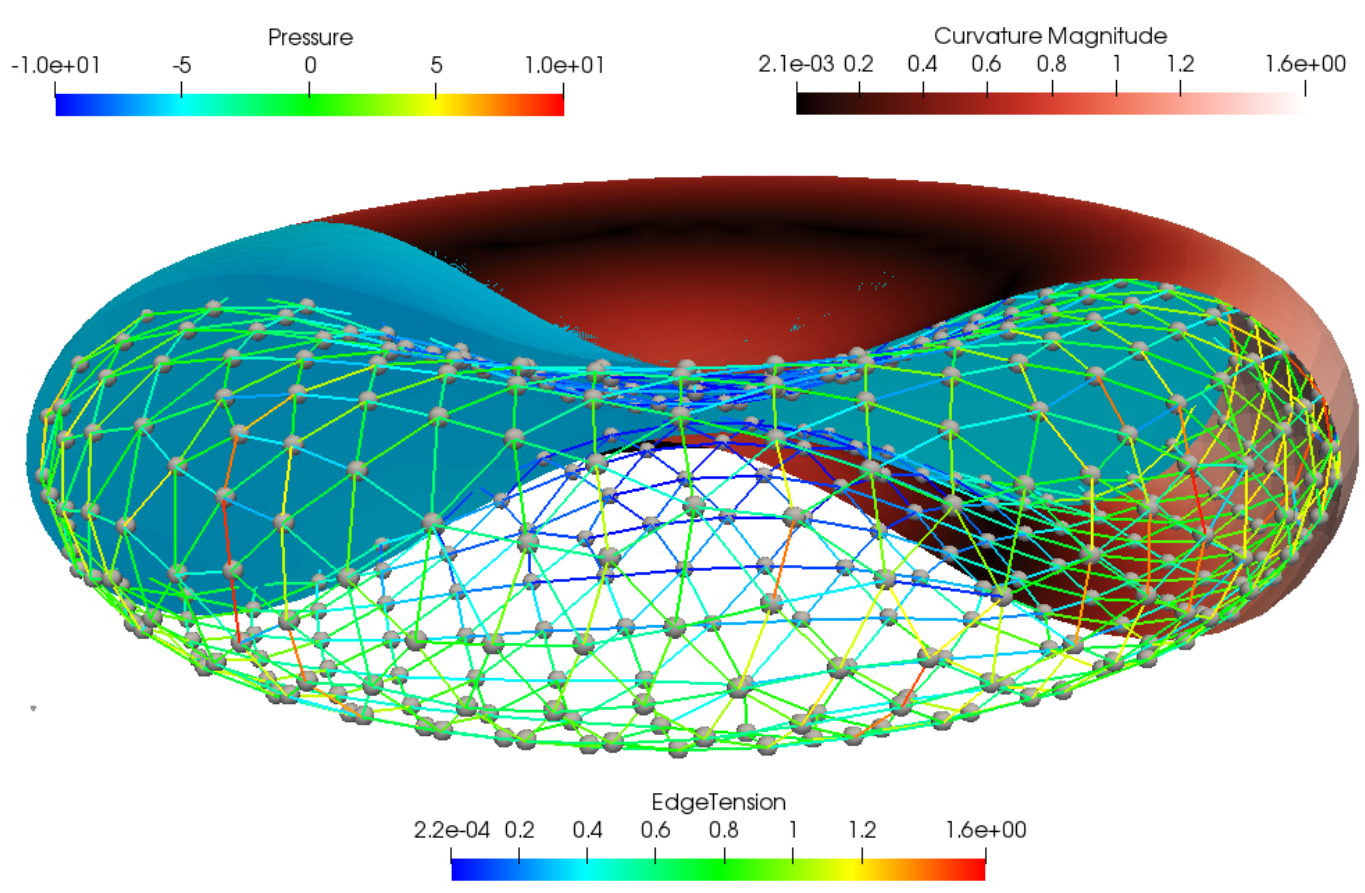

Table 8 - Quantities of numerical solutions the relaxation experiment of multi-component RBC model.

\begin{tabular}{c|c|c|c|c|} 
Case & $\mathscr{E} \Gamma$ & $\mathscr{E}_{X}$ & $\mathscr{E}_{\text {con }}$ & $p$ \\
\hline Biconcave relax & $10.38 \times 10^{2}$ & $1.4 \times 10^{4}$ & $-5.6 \times 10^{1}$ & -6.0
\end{tabular}

Following the same idea of the work of Peng, Asaro and Zhu (2010), we test the possibility to reach the prolate and stomatocyte shapes, imposing appropriate reduce volumes $v_{\text {reduced }}$ of 0.80 and 0.55 respectively. In Figure 35, we display with quantitative details the system configurations in both cases. We remark that, according our calculations, the internal cytoplasmatic pressure in the prolate case is positive. Such results need to be verified in terms of biological consistency.

The morphological RBCs change is a very interesting topic from a mechanical point of view. Is is known that, during the transition from the normal discoid shape to abnormal shapes, the membrane's shear, area, and bending moduli undergo significant variations (PARK et al., 2010). The range of morphological irregularities of red blood cells is very wide (FORD, 2013; RUEF; LINDERKAMP, 1999). Specific applications of the model on this type of variants can lead to evaluate measurements and interactions for an eventual characterization of the membrane irregularities. In Figure 36 we show some main cases obtained with our code. 
Figure 35 - Snapshots of the RBC system reproducing the prolate and stomatocyte shapes, occurring imposing a reduce volumes $v_{\text {reduced }}$ of 0.80 and 0.55 respectively.

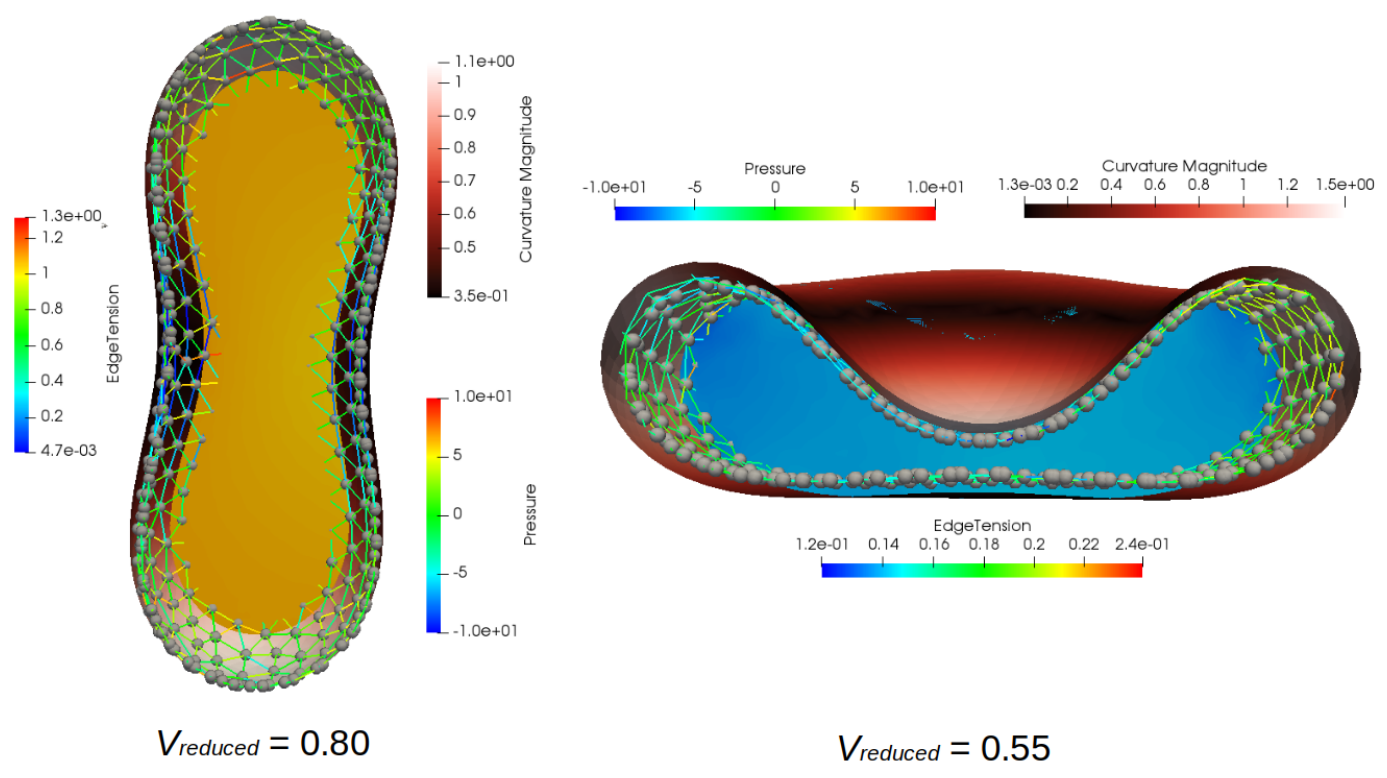

Figure 36 - Acanthocyte, elliptocyte, teardrop cell, and spherocyte example obtained with the code. The source of the photo under the microscope is (KEY, 2016)

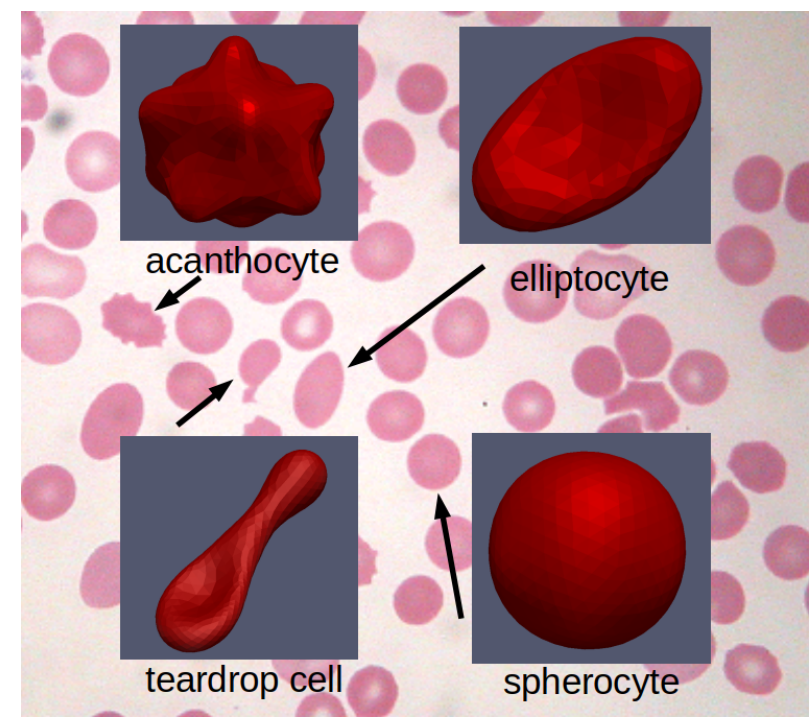

We conclude this section with a final remark. The relaxing process is important also because it is related to the ability of the RBC to recover its original shape. It is proven that the characteristic time for the resting shape is within the range of $0.1-0.3$ (HOCHMUTH; WORTHY; EVANS, 1979; CHANG et al., 2016; TANG et al., 2020).

If the virtual experiment of system relaxation can be considered useful for the verification of the computational formulation, on the other hand, we can better appreciate the characteristics of the multi-component model in simulations that foresee transient states. For this reason, in the following section we present a case of cell stretching with optical tweezers. 


\subsubsection{Stretching by optical tweezers}

We aim in this section to show the potential of the multi-component model, when used to study dynamic processes. The optical tweezers instrument provides for the possibility of stretching a RBC by two opposite beads attached to the bilayer. Being optical controlled, the beads can deform the RBC applying a certain force. In Figure 37 we can see how the RBC results in a certain time during this process, using our code to mimic the experiment. It is possible to study the RBC response with respect to the force applied. Our multi-component model, however allows to consider other details. Not only the external deformation and curvature distribution but the internal RBC behavior can be simulated. Figure 38 display the configuration of the internal cytoskeleton, that being attached to the lipid bilayer is affected and contributes to the global deformation. We can observe that junctional complexes closed to the regions attached by the beam present spectrins in overtension. This fact is due to the force that the cytoskeleton receives in the form of anchoring from the bilayer, through the interaction component of the model. Also, the cytoplasm dynamics are not trivial. Unlike the previous relaxation experiment, in this case the fluid is not in a rest state. As we can see in the plot of Figure 39, the cytoplasm pressure does not result to be homogeneous. In the volume most prone to stretching and in the zone subject to folding, a non-trivial pressure distribution is shown.

Figure 37 - Visualization of the lipid bilayer at a certain instant during the optical tweezers stretching. The surface is colored on red scale according to the curvature magnitude.

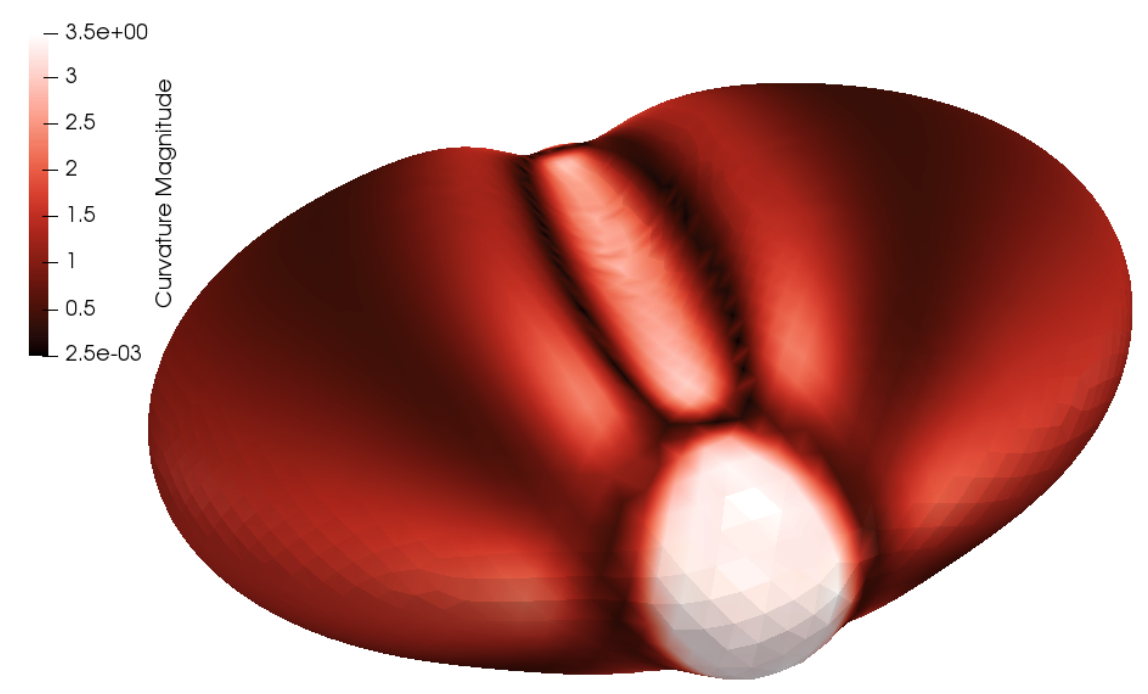

The model, considering each component as a distinct entity, allows to study the interaction phenomena between the parts, such as the detachment and reattachment of the cytoskeleton to the bilayer. Such effects are still to be fully understood and for this reason are of great interest; considered only in the most recent and advanced models available (CHANG et al., 2016).

In particular, the internal fluid viscosity is a sensitive parameter that affects the results. With the same longitudinal elongation in the graph in Figure 40 we show how the RBC transverse diameter $D_{t}$ evolves considering a fluid viscosity $\mu_{f}$ that varies from 0.01 to 10 . The panel of 
Figure 38 - Visualization of the cytoskeleton at a certain instant during the optical tweezers stretching. The edges are colored based on its tensions.

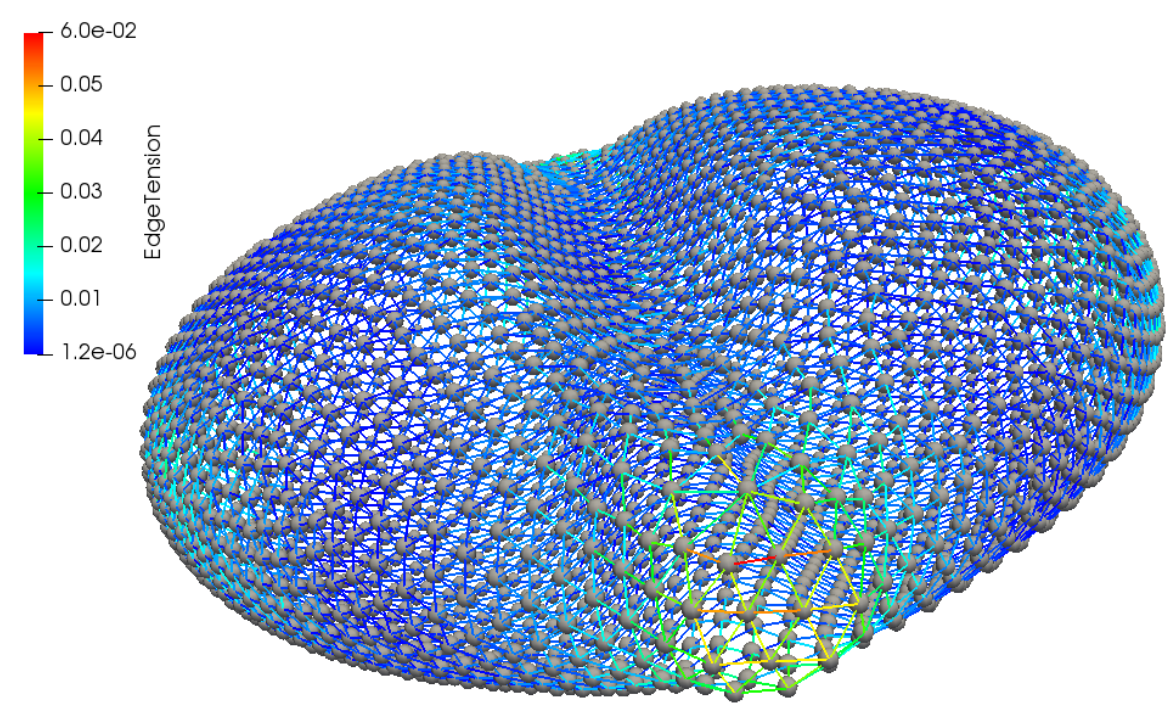

Figure 39 - Visualization of the internal RBC fluid at a certain instant during the optical tweezers stretching. The fluid is colored according its local pressure.

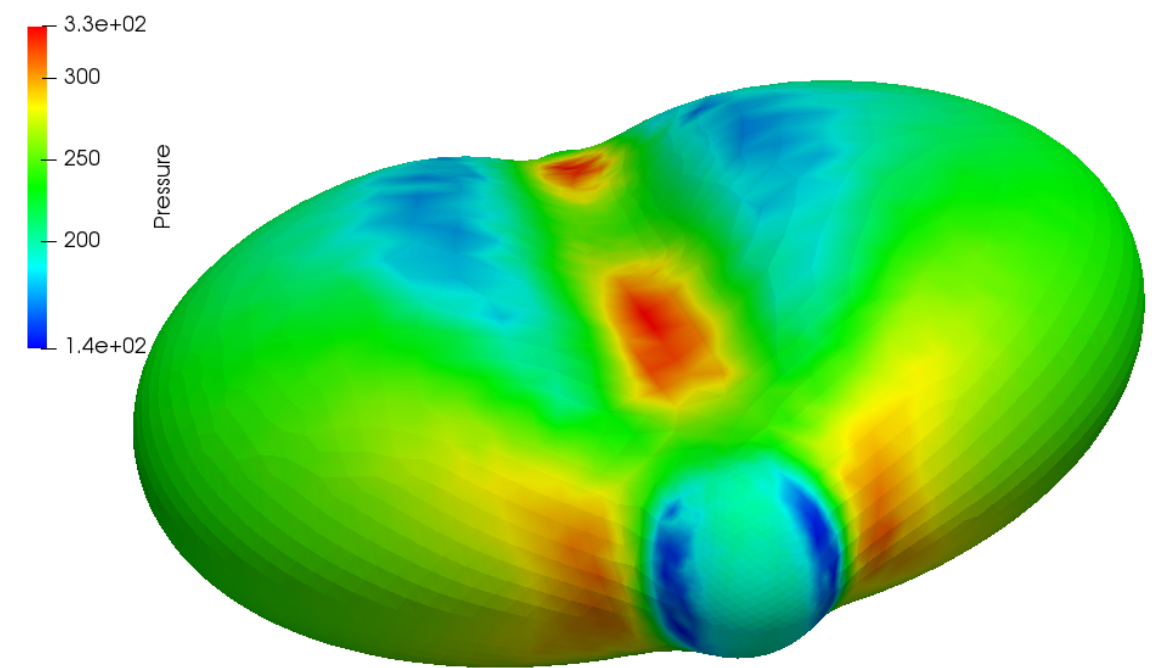

Figure 41 displays the extreme case highlight as during the deformation not only, as naturally expected, the internal pressure values are significantly different, but the RBC shape profile is notably dissimilar.

\subsubsection{Validation through experimental measurements}

Optical tweezers experiment is a "testbed" to evaluate the deformation characteristics of the red blood cell during the dynamic process. We aim to compare the transient characteristics of the virtual red blood cell modeled with our model with the experimental measurements in the laboratory and the best practices generated by the other mathematical models present in 
Figure 40 - Trasverse RBC diameter $D_{t}$ over the time, for a constant velocity of longitudinal elongation, under various cases of internal fluid viscosity $\mu_{f}$.

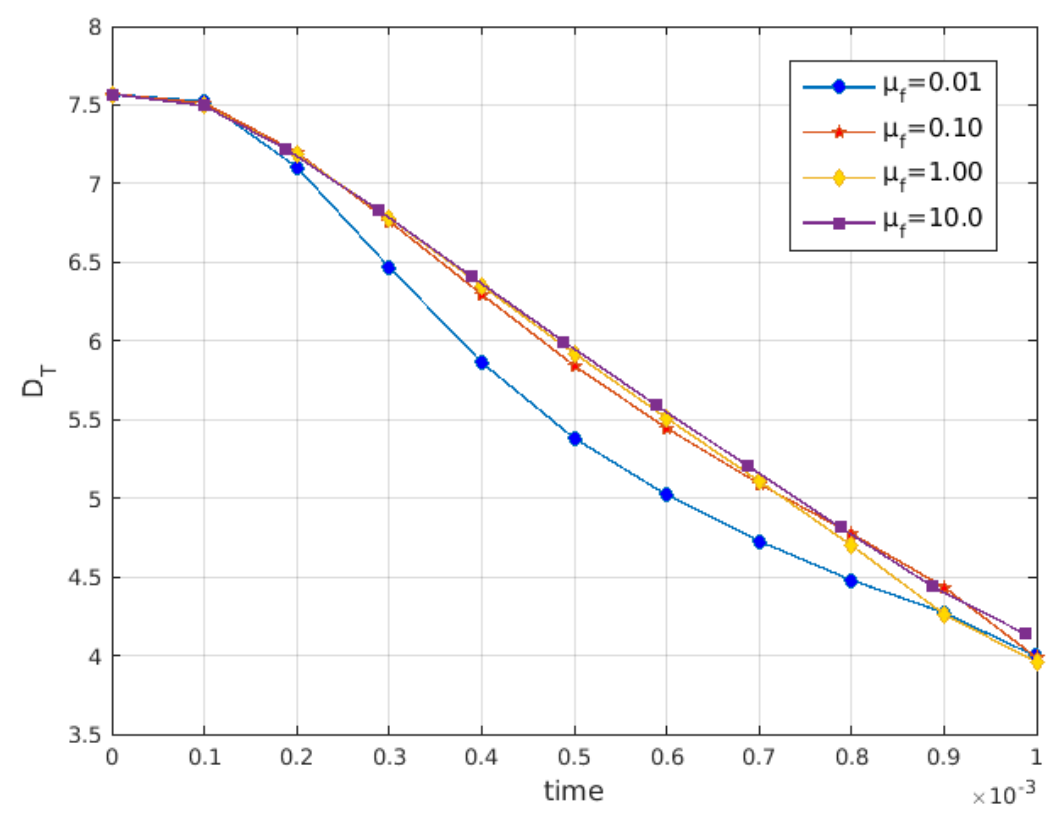

Figure 41 - Different views of an optical tweezers experiment in two assumptions of internal fluid viscosity $\mu_{f}=0.01$ on the left and $\mu_{f}=10.0$ on the right. We can observe the pressure field and the geometrical different resulting configuration.
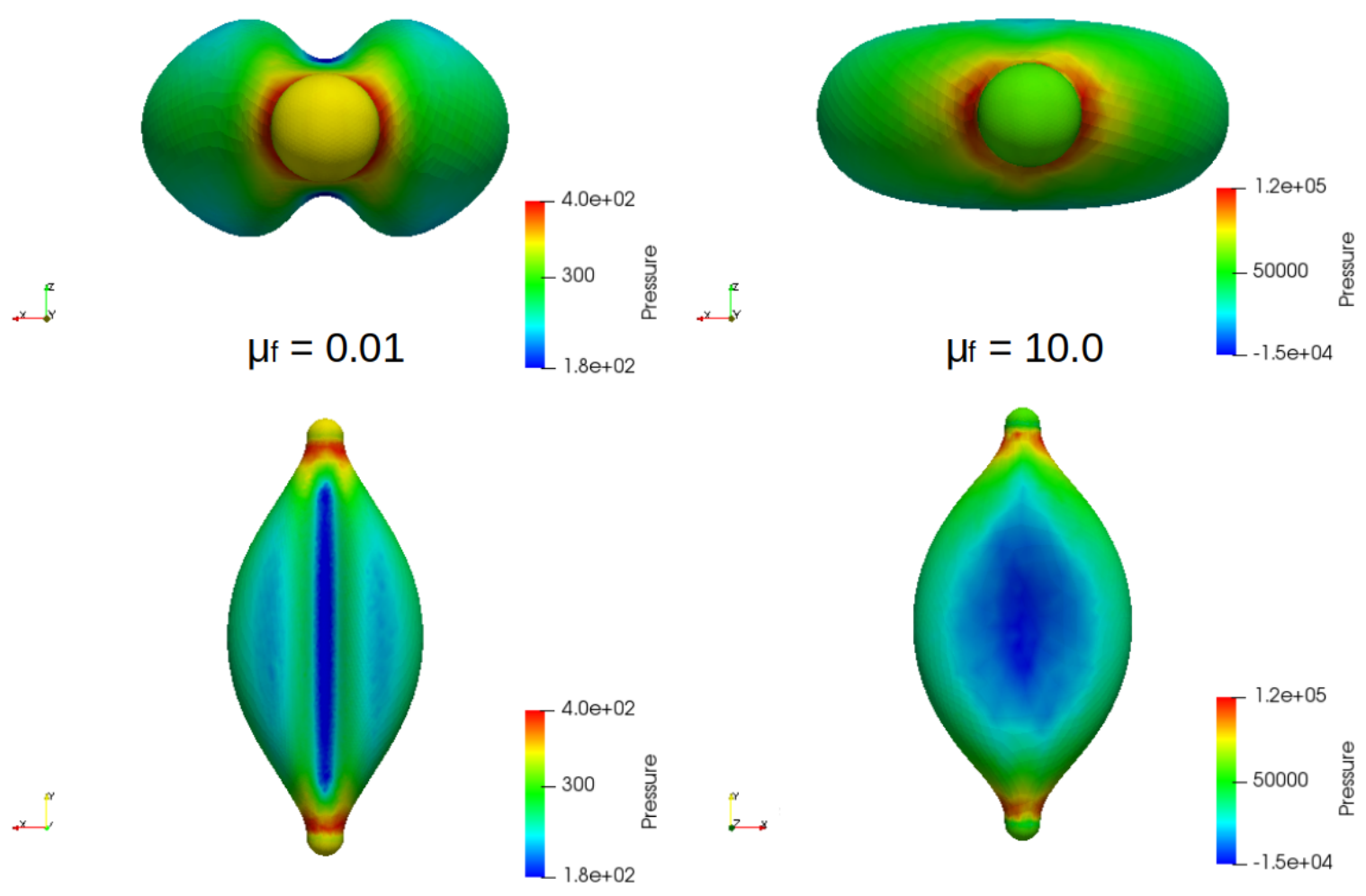

the literature. To this end, we introduce the quantities corresponding to the relative diameters increase (in the elongation phase). Let us consider to denote with $D_{a}$ and $D_{t}$ the longitudinal and 
transverse diameters with respect to the velocity direction of the optical tweezers, respectively. We define the longitudinal $D_{a}^{r}$ and transverse strain $D_{t}^{r}$ as follows

$$
D_{a}^{r}=\left(D_{a}-D_{a}^{0}\right) / D_{a}^{0}, \quad D_{t}^{r}=\left(D_{t}-D_{t}^{0}\right) / D_{t}^{0},
$$

with superscript 0 referring to the initial dimension.

Li and Liu (2008) provide an experimental nanomechanical characterization of red blood cells by applying stretching forces via optical tweezers. They have measured the cell deformation directly from microscopic digital images over a set of cell samples. They have considered five RBC samples with an original radius ranging from $2.87 \mu \mathrm{m}$ to $3.31 \mu \mathrm{m}$. In Figure 42 we plot the corresponding relative strains. For the reader's reference, we list the measurements in Table 9.

Other experimental/computational works concerning the stretching response of healthy and defective RBC can be considered to validate our model. In particular, we consider the results of the group of Suresh, Chang and Karniadakis (CHANG et al., 2016; SURESH et al., 2005), considering the average values of the relative strains of an healthy and infected RBC with the malaria parasite plasmodium falciparum. As we can see in Figure 42 and Table 9, an evident effect due to the paratisitization by P. flaciparum is a significant increase in the elastic stiffness. In the data, this fact is reflected in greater transverse stretching than a fixed longitudinal elongation.

Concerning our computational simulation scaling, we follow similar setup of the previous simulations. In particular, we consider an energy unit $k_{B} T=1$, a Canham constant $C_{c h}=20$, and an interaction strength constant $\varepsilon=5000$. The cytoskeleton consists of a network of around 750 nodes of radius 0.05 . We remark that the internal viscosity of a RBC is five times higher than plasma, i.e., $6 \times 10^{-3} \mathrm{~Pa} \cdot s$ (POZRIKIDIS, 2003b), so that considering our length scale of $1 \mu \mathrm{m}$ it leads to set $\mu_{f}=0.6$. During the virtual experiment, we consider an opposite velocity of each micro-bead of $200 \mu \mathrm{m}$ with respect to the unit time.

With the aim of evaluating the multi-component contribution of the model, we proceed to simulate the experiment by degree of completeness: (i) assuming only the lipid bilayer component, (ii) inserting the cytoskeleton, and finally (iii) also including the cytosol. For generality purpose, we also considered two cases of small (RBC1) and large (RBC2) RBCs of radius $2.85 \mu \mathrm{m}$ and $3.78 \mu \mathrm{m}$, respectively. In Table 9, we report our results and we compare them with the other data in Figure 42.

Comparing our simulation results with the other data, as we can observe on the left-panel of Figure 42, they reside within the variability window of the results described in literature. In general, the transverse deformation grows monotonically with respect to the longitudinal deformation with a certain slope and with a slight tendency to a convex growth. On the other hand, by the zoom shown on the right-panel we can highlight some different responses with respect to the physical components considered. Our results show that the presence of the cytoskeleton softens the growth of the curve. Such a behaviour is physically consistent being that the lipid bilayer, intrinsically fluid in character, requires stabilization by association with an underlying 
Figure 42 - Comparison between experimental data and our numerical results.
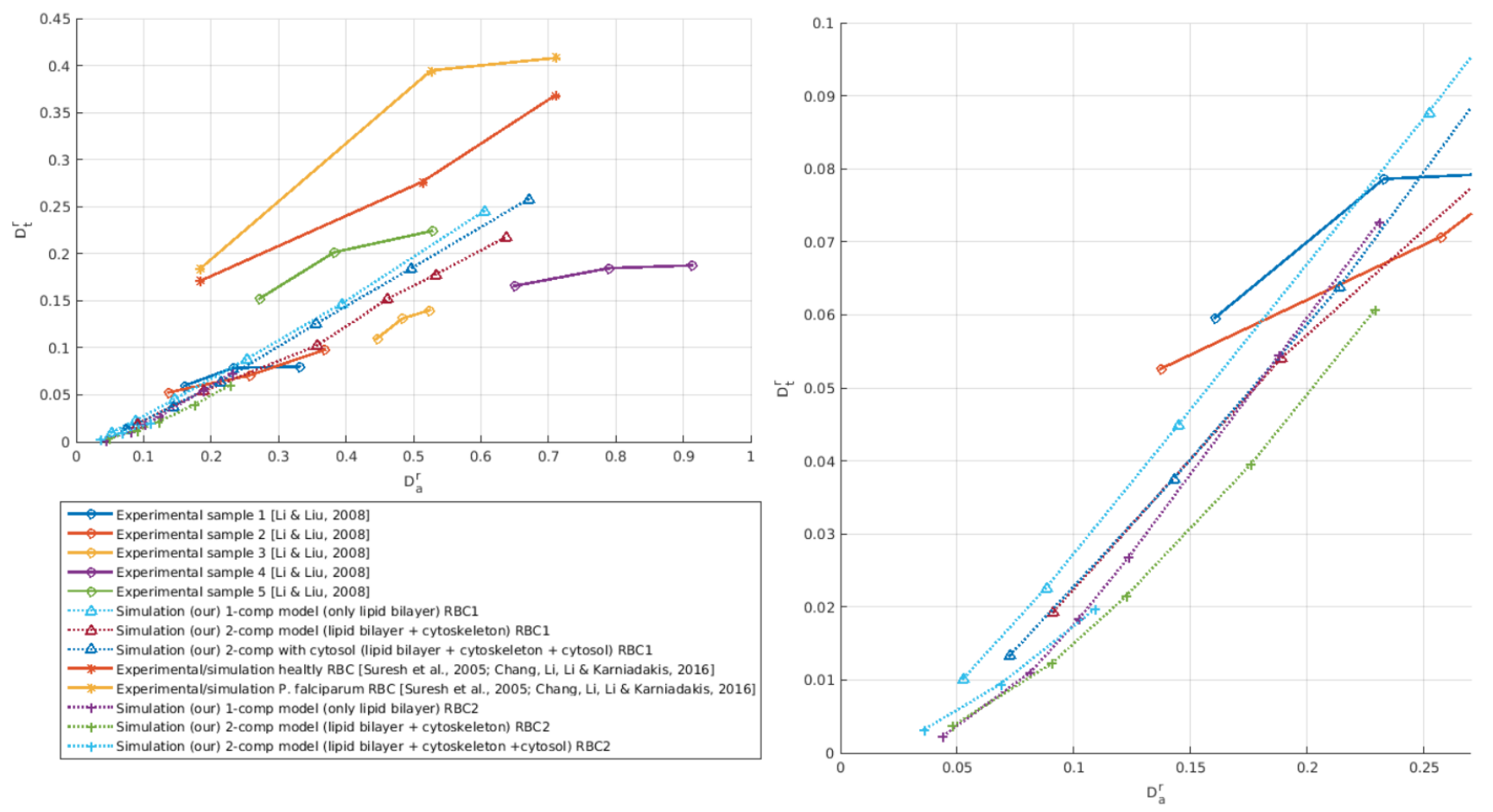

cytoskeleton (RAUCHER, 2008). Considering the introduction of the internal fluid, from the trend of the graphs, it seems that absence of the cytosol affects only relatively the RBC deformation. Moreover, comparing the 2-component model with and without internal fluid, the results indicate that for a fixed longitudinal elongation, the corresponding transverse diameter is slightly less in presence of the cytosol. This result, which may appear to be counter-intuitive, is explained by the behavior with respect to the axis perpendicular to the plane between the two diameters. In Figure 43, we display the transient configuration of the system, coloring the outer bilayer according to the magnitude of its curvature. The images suggest a visible difference between the two configurations. Without cytosol, the center of the biconcave disc digs less spherically, tending to make the fold of the membrane stretch towards the micro-beads. Such an effect is concomitantly reflected on the velocity field profile of the bilayer, which in the same central disk region in the case with cytosol it is on average lower than the case without cytosol. We remark that the internal pressure field in the cytosol case is not homogeneous and assumes a corresponding behavior. We can appreciate also the local influence on the pressure of the cytosol due to the presence of the nodes of the cytoskeleton.

The cytosol ability to avoid that the fold crosses for the entire longitudinal diameter appropriately illustrates how the cytosol serves to maintain the cell volume and precludes contact between different parts of the cell (DAO; LIM; SURESH, 2003).

We finally remark that we have found a certain discord in our simulations on the possibility that a cytoskeleton of an inactive nature (without the ability to rearrange itself) can remain 
Figure 43 - Visualization of our simulation configuration of the RBC1 at a fixed time during the stretching process, performed by our model with and without cytosol.

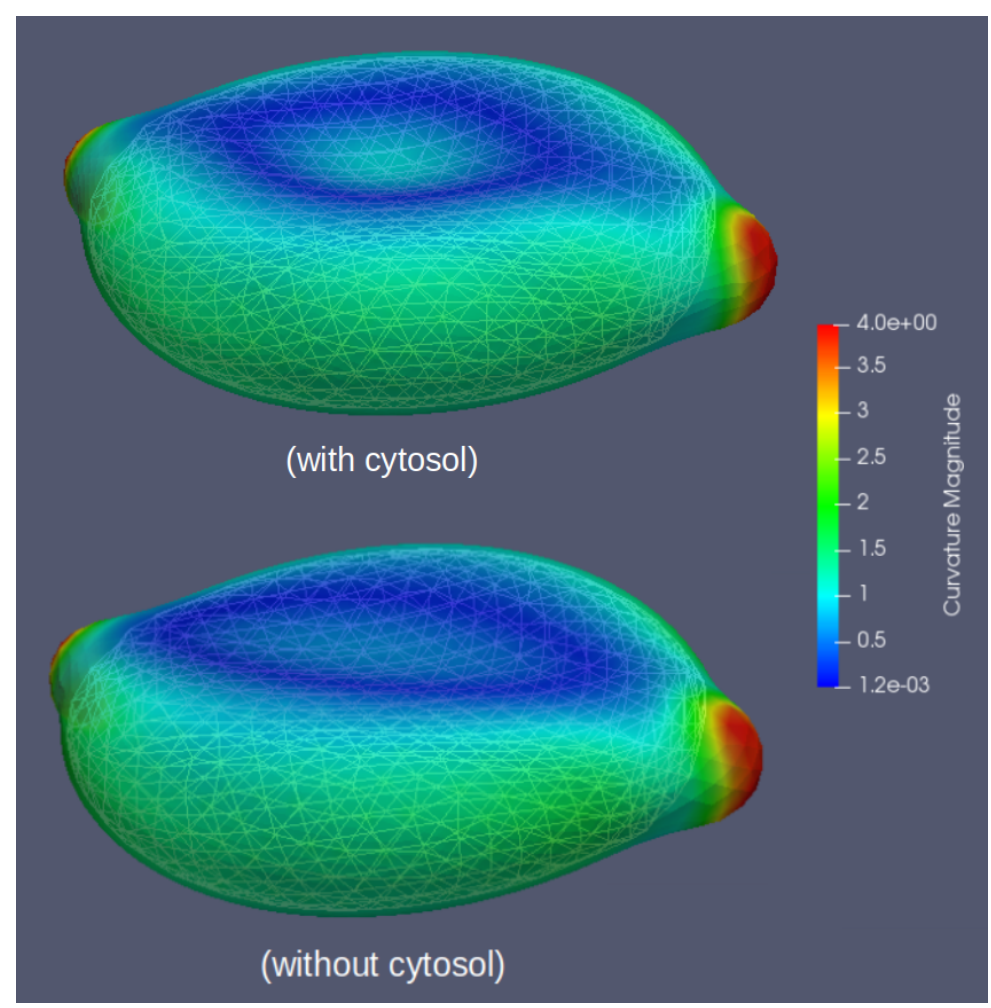

anchored to the bilayer for important efforts such as those present in the work of Chang et al. (2016). Nonetheless, we agree that spectrin detachment events are relatively infrequent and appear in extreme cases (CHANG et al., 2016; RODRÍGUEZ-GARCÍA et al., 2015). Furthermore, the formation of blebs (balloon-shaped membrane protrusions with no cytoskeleton content) turns out to be a possible phenomenon with physiological and mechanical implications (SPANGLER et al., 2011; PONTES et al., 2011). Our simulation results reinforce the idea that stress causes membrane rearrangements to adapt to the load and, in particular, that cytoskeleton is dynamic and can rapidly adapt to major changes in the cell shape (SHEETZ; SABLE; DÖBEREINER, 2006; LI et al., 2018; AVSIEVICH et al., 2020). An other speculative explanation is that the effective energy unit $k_{B} T$ should be reduced, resulting in a lower cytoskeleton stiffness (see (RODRÍGUEZ-GARCÍA et al., 2015)). In Figure 45 we show a zoom on the RBC of how the cytoskeleton can follow the membrane movement included in the region adjacent to the stretching due to the bead.

\subsection{Achievements and future opportunities}

In this Chapter, a new mathematical/computational formulation for a multi-component red blood cell model has been presented. The emphasis of the work is centered on the possibility of performing the mathematical modeling of the following RBC components: the lipid bilayer behavior, the cytoskeleton dynamics, the interaction activity between them, and the internal 
Figure 44 - Visualization of lipid bilayer velocity field and cytosol pressure field of the RBC1 at a fixed time during the stretching process, performed by our model with and without cytosol.

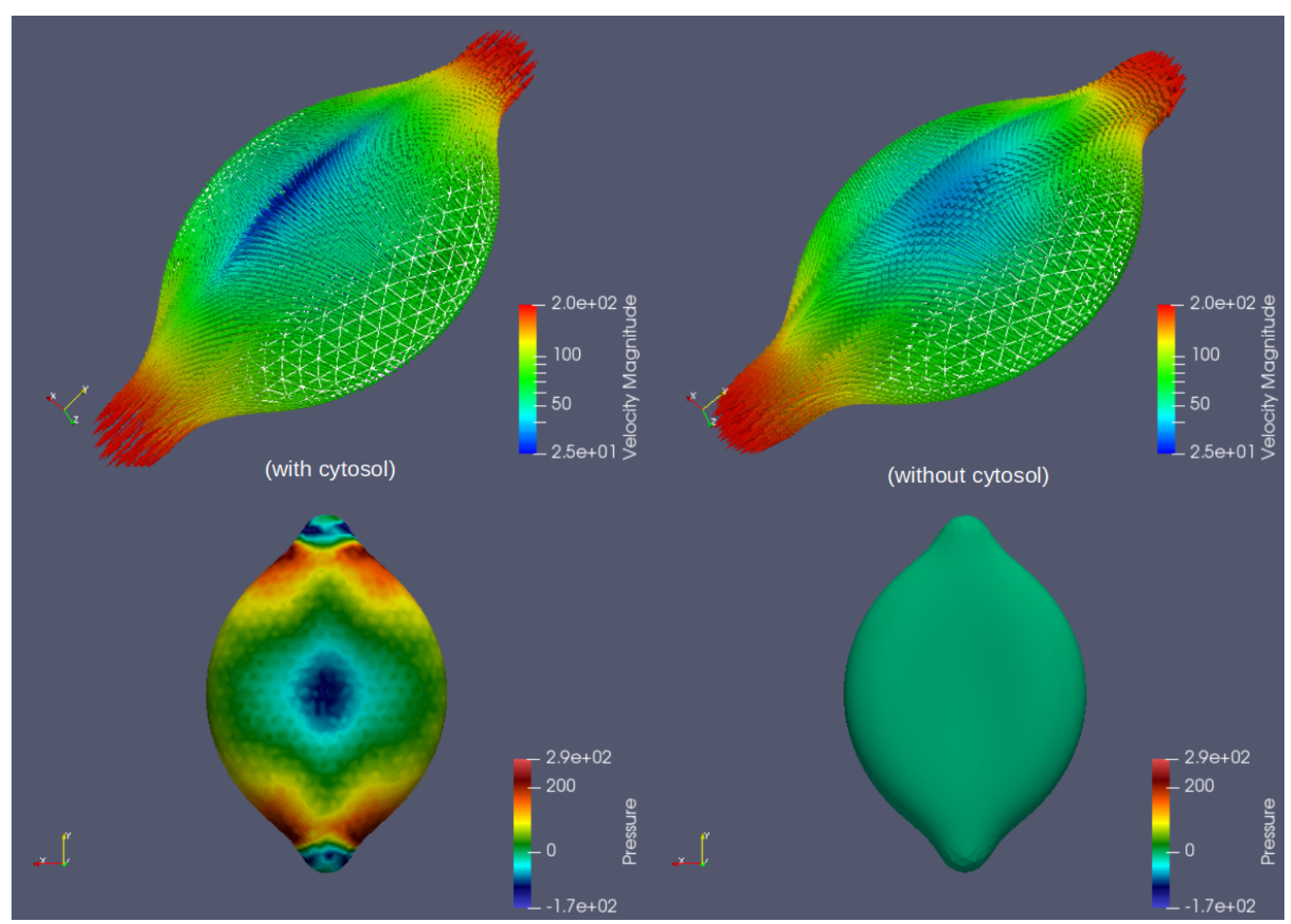

cytoplasm flow. Furthermore, the model considers a continuous definition of the lipid bilayer, allowing to use finite elements method. The text contains a detailed description of the construction of the model of the mathematical well-posedness, and it is tested with virtual experiments in order to show the potential and the advantages deriving from its implementation. Numerous studies and applications can be developed, implementing simulations on the basis of experimental observations. 
Figure 45 - Detail of the adhesive cyto-bilayer movement during optical streaching experiment with weakened cytoskeleton $\left(k_{B} T=10^{-3}\right)$.

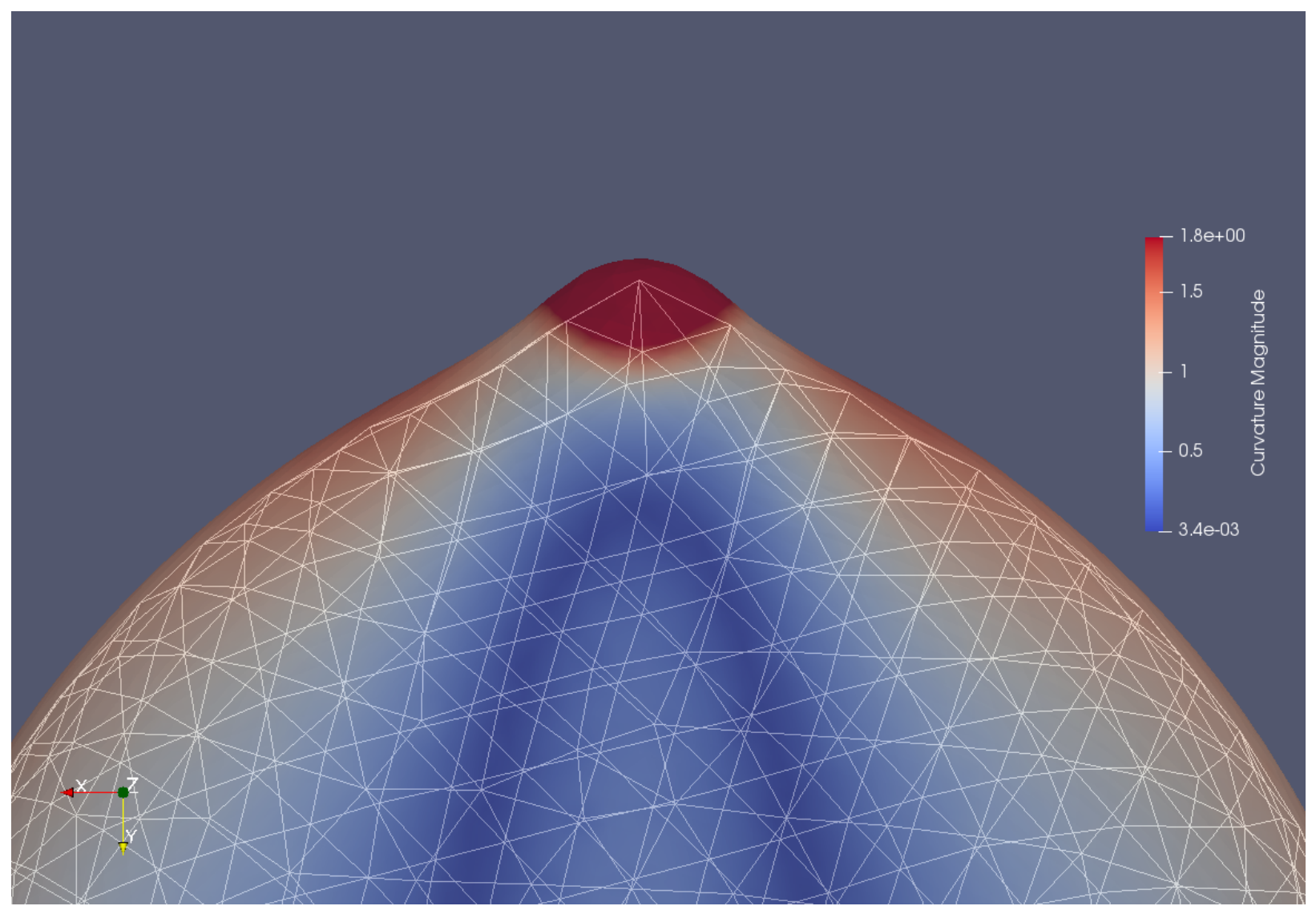


Table 9 - Experimental measurements and computational results on RBC deformation using optical tweezers. Our simulations are performed by 1-component (only lipid bilayer), 2-component (lipid bilayer and cytoskeleton) and 2-component with cytosol (lipid bilayer, cytoskeleton and internal fluid) models.

\begin{tabular}{|c|c|c|c|}
\hline Test (sample) & Original radius $(\mu \mathrm{m})$ & T. strain $D_{t}^{r}$ & L. strain $D_{a}^{r}$ \\
\hline \multirow[t]{3}{*}{ Li’s exp. 1} & 2.96 & 0.0596 & 0.1605 \\
\hline & & 0.0786 & 0.2327 \\
\hline & & 0.0801 & 0.3314 \\
\hline \multirow[t]{3}{*}{ Li's exp. 2} & 3.20 & 0.0526 & 0.1372 \\
\hline & & 0.0707 & 0.2574 \\
\hline & & 0.0979 & 0.3685 \\
\hline \multirow[t]{3}{*}{ Li’s exp. 3} & 2.87 & 0.1096 & 0.4456 \\
\hline & & 0.1309 & 0.4832 \\
\hline & & 0.1400 & 0.5240 \\
\hline \multirow[t]{3}{*}{ Li’s exp. 4} & 3.93 & 0.1656 & 0.6495 \\
\hline & & 0.1845 & 0.7891 \\
\hline & & 0.1875 & 0.9125 \\
\hline \multirow[t]{3}{*}{ Li's exp. 5} & 3.31 & 0.1523 & 0.2722 \\
\hline & & 0.2014 & 0.3822 \\
\hline & & 0.2241 & 0.5273 \\
\hline \multirow[t]{3}{*}{ Suresh \& Karnidakis's healthy } & 3.80 & 0.1711 & 0.1842 \\
\hline & & 0.2763 & 0.5132 \\
\hline & & 0.3684 & 0.7105 \\
\hline \multirow{3}{*}{ Suresh \& Karnidakis's P. falciparum } & 3.80 & 0.1842 & 0.1842 \\
\hline & & 0.3947 & 0.5263 \\
\hline & & 0.4079 & 0.7105 \\
\hline \multirow[t]{6}{*}{ Our sim. 1-comp RBC1 } & 2.85 & 0.0102 & 0.0526 \\
\hline & & 0.0226 & 0.0881 \\
\hline & & 0.0449 & 0.1447 \\
\hline & & 0.0877 & 0.2520 \\
\hline & & 0.1465 & 0.3927 \\
\hline & & 0.2449 & 0.6043 \\
\hline \multirow[t]{6}{*}{ Our sim. 2-comp RBC1 } & 2.85 & 0.0193 & 0.0912 \\
\hline & & 0.0540 & 0.1888 \\
\hline & & 0.1023 & 0.3570 \\
\hline & & 0.1516 & 0.4612 \\
\hline & & 0.1777 & 0.5321 \\
\hline & & 0.2175 & 0.6372 \\
\hline \multirow[t]{6}{*}{ Our sim. 2-comp with cytosol RBC1 } & 2.85 & 0.0133 & 0.0725 \\
\hline & & 0.0375 & 0.1428 \\
\hline & & 0.0639 & 0.2139 \\
\hline & & 0.1254 & 0.3553 \\
\hline & & 0.1842 & 0.4963 \\
\hline & & 0.2579 & 0.6702 \\
\hline \multirow[t]{6}{*}{ Our sim. 1-comp RBC2 } & 3.78 & 0.0022 & 0.0437 \\
\hline & & 0.0110 & 0.0811 \\
\hline & & 0.0184 & 0.1022 \\
\hline & & 0.0269 & 0.1235 \\
\hline & & 0.0545 & 0.1880 \\
\hline & & 0.0728 & 0.2308 \\
\hline \multirow[t]{5}{*}{ Our sim. 2-comp RBC2 } & 3.78 & 0.0037 & 0.0481 \\
\hline & & 0.0123 & 0.0907 \\
\hline & & 0.0215 & 0.1226 \\
\hline & & 0.0395 & 0.1758 \\
\hline & & 0.0607 & 0.2290 \\
\hline \multirow[t]{3}{*}{ Our sim. 2-comp with cytosol RBC2 } & 3.78 & 0.0032 & 0.0361 \\
\hline & & 0.0095 & 0.0686 \\
\hline & & 0.0197 & 0.1090 \\
\hline
\end{tabular}



CHAPTER

\section{7}

(1)

\section{A TRAJECTORY PLANNING MODEL FOR THE MANIPULATION OF PARTICLES IN MICROFLUIDICS}

Particle manipulation is an important topic in microfluidics, a science that studies the behavior of small-scale fluids. A growing interest exists in the steering of particles in microfluidic systems, especially in the biological and medical fields, to analize elements of very small size such as proteins or DNA. In particular, improving the ability to manipulate and to steer particles in microfluidics can allow to better examine, control, and treat biological materials. Some interesting examples are the microfluidic device presented in (ZHOU; WANG; LIN, 2010) or the use of optical tweezers as tools to move in a controlled way individual particles, as shown in (CURTIS; KOSS; GRIER, 2002).

The control of the trajectory of particles immersed in a fluid by boundary actuators is the topic presented in the articles by Chaudhary and Shapiro (CHAUDHARY; SHAPIRO, 2006) and Tuval et. al. (TUVAL et al., 2005). The microfluidic modeling in these works proposes the displacement of the particles from one point to another by a pre-established trajectory. The idea is to identify the location of the particle in real time so that a control algorithm calculates the demand on each actuator for the flow to carry the particle along the desired path. In Armani et. al., (ARMANI et al., 2005) and (ARMANI et al., 2012), some examples can be found of the use of control techniques. A generalization for the manipulation of particles in the three-dimensional case also exists in the study of Probst and Shapiro (PROBST; SHAPIRO, 2011).

In all published methods discussed above, a predefined trajectory is necessary as a datum, but no mention is given about how to build such a trajectory. In fact, in their examples the trajectory is introduced manually. The present work proposes a method to automatically define a "good" trajectory $\gamma^{*}$ that can then be used as input for those particle manipulation techniques. By "good" we intend that $\gamma^{*}$ not only avoids obstacles, but also avoids parts of the fluid domain where 
the actuators are ineffective at producing flow (stagnant regions, or regions of low controllability). These requirements are fulfilled by time-optimal trajectories, which are the ones proposed here. In fact, we propose an algorithm that works on a rectangular grid and could be implemented directly on a pixelated image of the domain. The trajectories are discrete in the sense that they are broken lines joining neighbor grid points. This is a very practical, though not smooth, parameterization of possible paths. The resulting trajectory $\gamma^{*}$ can eventually be smoothed out before passing it to the manipulation algorithm. The combination of the proposed method with the manipulation techniques of Shapiro and coworkers ((CHAUDHARY; SHAPIRO, 2006), (ARMANI et al., 2005), (ARMANI et al., 2012) and (PROBST; SHAPIRO, 2011)) would allow for an integrated system of detection, planned manipulation and control of particles towards an arbitrary target.

The presentation begins by describing the general physical model and recalling the techniques used for particle steering when the desired trajectory is known. This allows us to introduce several concepts needed for trajectory planning. Then the continuous problem of optimizing trajectories (Bellman's principle) with respect to arrival time is described, together with the proposed discretization. Finally, applications are reported in the section of numerical results. They show the good performance of the algorithm in finding suitable, sometimes counterintuitive, trajectories.

\subsection{The physical model}

We study the manipulation of a particle in a microfluidic device in the two-dimensional case, the extension to 3D being immediate. In fact, we can see the 2D case applicable to flows between flat plates parallel to the plane $x y$, in the case when the plates are sufficiently close.

Let us consider therefore a square domain $(0, L)^{2}=\Omega$, for the flow of a fluid without inertia with the presence of obstacles, walls and also of actuators. As we can see in the Figure 46 , the actuators act in some regions of the boundary of the domain $\Gamma_{N}, \Gamma_{S}, \Gamma_{E}$ and $\Gamma_{W}$, being placed on the respective north, south, east and west borders.

At each actuator we apply a value $X^{N}, X^{S}, X^{E}$ e $X^{W}$. Since the driving force of the flow is the difference between actuator values (pressure or electrical potential differences), we can fix $X^{E}$ to zero without losing generality and work only with the variables $\underline{X}=\left(X^{N}, X^{S}, X^{W}\right)^{T}$. Inertial effects are generally negligible in microfluidics. As a consequence, each $\underline{X} \in \mathscr{A}$ (where $\mathscr{A}$ is the set of admissible actuator values) leads to a velocity field according to the following linear relation

$$
\vec{v}(\vec{x}, \underline{X})=\underline{B}(\vec{x}) \underline{X}, \quad \vec{x} \in \Omega .
$$

The matrix $\underline{\underline{B}}(\vec{x})=\left(B^{N}(\vec{x}), B^{S}(\vec{x}), B^{W}(\vec{x})\right)^{T}$ contains as columns the components of the velocity field at the point $\vec{x}$ with $\underline{X}$ belonging to the canonical basis, i.e. $X^{N}=1, X^{S}=0$ and $X^{W}=0$ for the first column; $X^{N}=0, X^{S}=1$ and $X^{W}=0$ for the second one and $X^{N}=0, X^{S}=0$ 
Figure 46 - Sketch of the domain configuration.

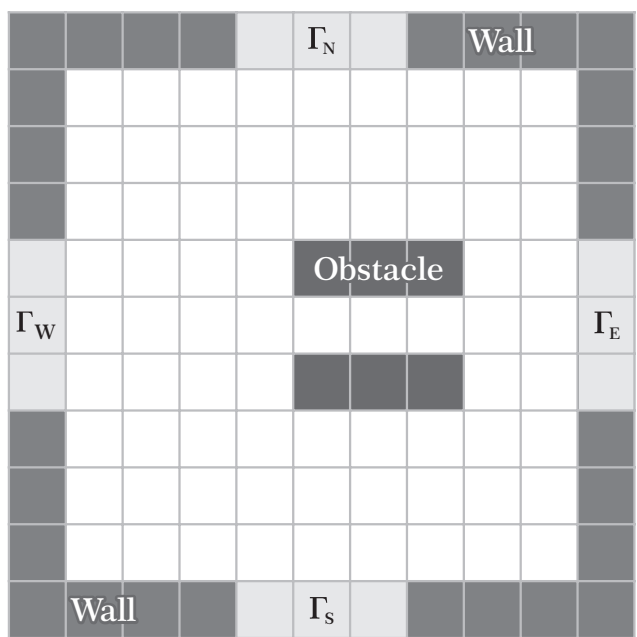

and $X^{W}=1$ for the third one. The Figure 47 shows an example of the velocity field for some points of the domain, generated by imposing the canonical basis.

Figure 47 - Velocity fields generated by imposing the canonical basis. All possible velocity fields are linear combinations of these three.
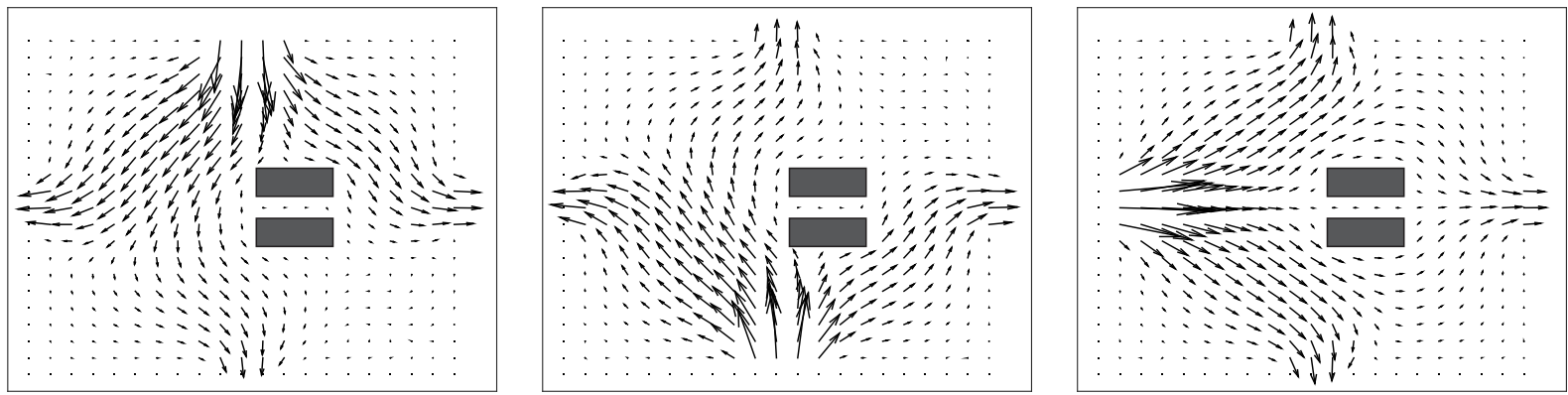

The admissible set of the controls $\mathscr{A}$ generates an admissible set of velocities $\mathbb{V}(\vec{x})$ on each point, defined by

$$
\mathbb{V}(\vec{x})=\{\vec{w} \in \Omega \mid \exists \underline{X} \in \mathscr{A}, \vec{w}=\underline{\underline{B}}(\vec{x}) \underline{X}\} .
$$

Let $P$ be a passive point particle whose initial position is $\vec{x}^{0}$. Each control function $\underline{X}(t):[0, T] \rightarrow \mathscr{A}$ moves $P$ along a trajectory $\vec{r}(t)$ that is the solution of the following problem

$$
\left\{\begin{array}{l}
\frac{d \vec{r}}{d t}=\vec{v}(\vec{r}(t), \underline{X}(t)), \\
\vec{r}(0)=\vec{x}_{0} .
\end{array}\right.
$$

The goal of this work is to answer the question of how to determine the control function $\underline{X}^{*}(t)$ that moves the particle $P$ from the position $\vec{x}^{0}$ to a target set $Z \subset \Omega$. The target set $Z$ may be a region of the domain where the particle is analyzed or subjected to some biochemical reaction, for example. In order to completely achieve this aim we have first to solve the problem of the 
particle manipulation through a predefined path. As explained in the following subsection, this approach introduces an optimization problem for maximizing the possible velocity. The solution of this preliminary problem allow us to have all the tools for achieving the main goal, which is to determine a "good" trajectory that consider the arrival time optimization.

\subsubsection{Particle manipulation along a predefined path}

Let us consider the determination of the control function $\underline{X}^{*}(t)$ when the path to move the particle $P$ from $\vec{x}^{0}$ to $Z$ is given. Let $\gamma$ be the chosen path that is a smooth curve in $\Omega$ joining $\vec{x}^{0}$ to $\vec{x}^{f} \in Z$. For every point $\vec{x} \in \gamma$, let $\check{\tau}(\vec{x})$ be the unit vector tangent to $\gamma$ at $\vec{x}$. In order to move $P$ along $\gamma$ it is necessary and sufficient that $\underline{\underline{B}}(\vec{r}(t)) \underline{X}(t)$ is parallel to $\check{\tau}(\vec{r}(t))$ for all $t$. Since the relation (7.1) does not depend explicitly on $t$, time becomes a parameter and will be omitted in what follows for conciseness. We have the following characterization:

Proposition 7.1 At a point $\vec{x}$ the control $\underline{X}$ generates a velocity vector $\vec{w}=\vec{v}(\vec{x}, X)$ parallel to an arbitrary direction $\check{d}$, if and only if

$$
\underline{\underline{M}}(\vec{x}, \breve{d}) \underline{X} \stackrel{\text { def }}{=}\left(\mathbb{I}-\underline{d}^{T} \underline{d}^{T} \underline{\underline{B}}(\vec{x}) \underline{X}=0,\right.
$$

where $\underline{d}$ is the (column) array representation of $\breve{d}$. Being $\underline{\underline{B}}(\vec{x})$ of full rank, and the number of controls equal to or greater than the number of spatial dimensions, the above equation has as its solution a non-trivial subspace $S(\vec{x}, \breve{d})$ of $\mathbb{R}^{n}$.

In particular, the maximum velocity that can be generated along direction $\check{d}$ is

$$
V(\vec{x}, \breve{d})=\max _{\underline{X} \in S(\vec{x}, \breve{d}) \cap \mathscr{A}} \underline{d}^{T} \underline{\underline{B}}(\vec{x}) \underline{X},
$$

and the maximizing argument $\underline{\hat{X}}(\vec{x}, \breve{d})$ is the control value that realizes the velocity $V(\vec{x}, \breve{d}) \check{d}$.

If the admissible set of controls is a box with minimum $\underline{X}^{-}$and maximum $\underline{X}^{+}$values for each control variable, the following classical linear optimization problem results:

Linear optimization problem: Maximize $\underline{c}^{T} \underline{X}$ over $\underline{X} \in \mathbb{R}^{n}$ (being $\underline{c}^{T}=\underline{d}^{T} \underline{\underline{B}}(\vec{x})$ ), with the restrictions

$$
\begin{aligned}
\underline{\underline{M}}(\vec{x}, \breve{d}) \underline{X} & =0, \\
\underline{X} & \geq \underline{X}^{-} \\
\underline{X} & \leq \underline{X}^{+}
\end{aligned}
$$

The maximum reached is the maximum velocity $V(\vec{x}, \check{d})$, and the maximizing argument is $\underline{\hat{X}}(\vec{x}, \breve{d})$.

With these elements, bringing back the time variable, the problem (7.2) becomes

$$
\left\{\begin{array}{l}
\frac{d \vec{r}}{d t}=V(\vec{r}(t), \check{\tau}(\vec{r}(t))) \check{\tau}(\vec{r}(t)), \\
\vec{r}(0)=\vec{x}_{0}
\end{array}\right.
$$


which will necessarily follow the curve $\gamma$ at maximum speed, and $\vec{r}(t)$ has been calculated, the control function arises from

$$
\underline{X}^{*}(t)=\underline{\hat{X}}(\vec{r}(t), \check{\tau}(\vec{r}(t))) .
$$

The control at every instant is uniquely determined by the position of the particle and the direction in which it is desired to move it. The problem of microfluidically controlling a particle to follow a predefined trajectory was addressed by Chaudhary and Shapiro (2006) and the work of Mathai et al. (2011), where the simplest constraints $\|\underline{X}\| \leq 1$, where $\|\cdot\|$ is the Euclidean norm are considered.

Remark 7.2. In (7.4) the vector $\underline{\hat{X}}$ can be multiplied by an arbitrary $\alpha(t) \in(0,1]$ if the desired speed of the particle is not the maximum attainable one at each point. For example, let

$$
V_{\min }(\gamma)=\min _{\vec{x} \in \gamma} V(\vec{x}, \check{\tau}(\vec{x})) .
$$

If $V_{\min }(\gamma)=0$, it means that it is impossible to reach the target $Z$ in finite time along the path $\gamma$. Otherwise $V_{\min }(\gamma)>0$, for any $V^{*} \leq V_{\min }(\gamma)$ the control function that moves $P$ along $\gamma$ at constant speed $V^{*}$ is obtained by first solving

$$
\left\{\begin{array}{l}
\frac{d \vec{r}}{d t}=V^{*} \check{\tau}(\vec{r}(t)), \\
\vec{r}(0)=\vec{x}_{0},
\end{array}\right.
$$

and then setting

$$
\underline{X}^{*}(t)=\frac{V_{\min }(\gamma)}{V(\vec{r}(t), \check{\tau}(\vec{r}(t)))} \underline{\hat{X}}(\vec{r}(t), \check{\tau}(\vec{r}(t)))
$$

\subsubsection{Determining the path via arrival time optimization}

When the path is not pre-defined, it can be chosen in several ways. Here we study the trajectory planning between $\vec{x}^{0}$ and the $Z$ target by minimizing the time of arrival. Other possibilities include minimizing path length, energy expenditure, and in general any cost function depending on the path and possibly on the associated control function.

For a point $\vec{x} \in \Omega$ (controllable, i.e., such that there exists at least one function $\underline{X}(t)$ which moves $P$ from $\vec{x}$ to $Z$ ) we can consider the function $T(\vec{x})$ defined as the minimum time required to take $P$ from $\vec{x}$ to $Z$. The path associated with $T(\vec{x})$ is an optimal trajectory because it moves $P$ to $Z$ in minimum time and defines, at each point, the optimal direction $\check{\tau}(\vec{x})$ (tangent to the optimal trajectory).

Bellman's principle states that if a point $\vec{y}$ is placed in the optimal path $\gamma^{*}$ of $\vec{x}$, then the optimal trajectory from $\vec{x}$ to $\vec{y}$ is the arc of $\gamma^{*}$ joining these two points, and the optimal trajectory of $\vec{y}$ to the target $Z$ is the final arc of $\gamma^{*}$. With this principle it is possible to obtain the Hamilton-Jacobi-Bellman equation for $T$, according to (CACACE; CRISTIANI; FALCONE, 2014):

$$
V(\vec{x}, \check{\tau}(\vec{x})) \check{\tau}(\vec{x}) \cdot \nabla T+1=0,
$$


where $\check{\tau}(\vec{x})$ is given by $\check{\tau}(\vec{x})=\arg \min _{\|\check{d}\|=1} V(\vec{x}, \check{d}) \check{d} \cdot \nabla T(\vec{x})$. We have that the associated Hamiltonian is $H(\vec{x}, \vec{p})=\min _{\|\check{d}\|=1} V(\vec{x}, \breve{d}) \check{d} \cdot \vec{p}$, which gives the equation in the usual form (see (SETHIAN; VLADIMIRSKY, 2001))

$$
H(\vec{x}, \nabla T(\vec{x}))+1=0 .
$$

The fundamental property we will use in the numerical treatment of this equation is the following (a direct consequence of Bellman's principle):

Proposition 7.3 Let $\vec{x} \in \Omega$, and $C$ a closed curve (surface in 3D) such that $\vec{x}$ is internal to $C$ and the target $Z$ is external to $C$. For each $\vec{y} \in C$, let $\zeta(\vec{y})$ be the minimum time required to carry a particle from $\vec{x}$ to $\vec{y}$. Then,

$$
T(\vec{x})=\min _{\vec{y} \in C}\{T(\vec{y})+\zeta(\vec{y})\} .
$$

Moreover, if $\vec{y}^{*}$ is a point of $C$ where the minimum is attained, $\vec{y}^{*}$ is the intersection of the optimal path $\gamma^{*}$ with the curve $C$.

If the curve $C$ is small enough (contained in a ball of ratio $\rho \ll 1$ ), we can consider just straight trajectories between $\vec{x}$ and $C$, which allows to approximate the equation (7.5), according to (CACACE; CRISTIANI; FALCONE, 2014), by

$$
T(\vec{x}) \approx \min _{\vec{y} \in C}\left\{T(\vec{y})+\frac{\|\vec{y}-\vec{x}\|}{V\left(\vec{x}, \frac{\vec{y}-\vec{x}}{\|\vec{y}-\vec{x}\|}\right)}\right\}
$$

and the optimal trajectory, locally, is given by the segment $\overline{\vec{x} \vec{y}^{*}}$.

\subsection{Calculation of $V(\vec{x}, \check{d})$}

To find the set of possible maximum velocities $V(\vec{x}, \breve{d})$ at a point $\vec{x}$, for each given direction $\breve{d}$, we have first to determine the admissible set of velocities $\mathbb{V}(\vec{x})$, according to the equation (7.1). We consider that the system actuators can be mechanical (imposing pressure) or electrical (imposing electrode voltage).

When the velocity field is generated by pressure sources, the governing equation for the flow is the Stokes equation, that in our setting defines the following boundary value problem

$$
\left\{\begin{aligned}
-\mu \nabla^{2} \vec{v}+\nabla p & =0 & & \text { in } \Omega, \\
\nabla \cdot \vec{v} & =0 & & \text { in } \Omega, \\
\sigma(p, \nabla \vec{v}) \cdot \vec{n} & =-X^{i} & & \text { on } \Gamma_{i}, \text { where } i \in\{N, S, E, W\}, \\
\vec{v} & =0 & & \text { on } \partial \Omega \backslash \cup_{i} \Gamma_{i},
\end{aligned}\right.
$$

where $\sigma(p, \nabla \vec{v})=-p \mathbb{I}+\mu\left(\nabla \vec{v}+\nabla \vec{v}^{T}\right)$ is the stress tensor and $\vec{n}$ the unit normal to the boundary. We remark that the boundary conditions for this problem are essentially that pressure is imposed on the actuators and null velocity is imposed on the obstacles and walls. 
When the system actuators are electrodes, the electric field is obtained from the electric potential $\Phi$ (or voltage) that solves the following problem

$$
\left\{\begin{aligned}
\nabla^{2} \Phi & =0 & & \text { in } \Omega, \\
\vec{E} & =-\nabla \Phi & & \text { in } \Omega, \\
\Phi & =X^{i} & & \text { on } \Gamma_{i}, \text { where } i \in\{N, S, E, W\} \\
\vec{E} \cdot \vec{n} & =0 & & \text { on } \partial \Omega \backslash \cup_{i} \Gamma_{i} .
\end{aligned}\right.
$$

The electric field induces a velocity field according to the relation

$$
\vec{v}=m_{e o} \vec{E}
$$

where $m_{e o}$ is the electrosmotic constant relative to the interaction between the fluid and the wall (KIRBY, 2010). In this case, Dirichlet boundary conditions for the voltage are imposed on the actuators, and Neumann homogeneous ones on the obstacles and walls.

A detailed explanation of the numerical resolution of these problems, according to MAC (Marker and Cell) discretization, is exposed in (MEACCI et al., 2017).

Remark 7.4 In fact, one could easily consider a combination of mechanical and electrical actuators. In this case, if a control variable $X_{i}$ corresponds to a mechanical actuator, the i-th column of matrix B in (7.1) would be the velocity field obtained by solving (7.7), while if it corresponds to an electrical actuator the equation to be solved would be (7.8). We have not considered problems with both kinds of actuators simultaneously to keep the exposition simpler.

The calculation of $V(\vec{x}, \breve{d})$ involves a resolution of the linear optimization problem of the Proposition 7.1 on all mesh nodes for a directions set $D$ defined by the user.

If v1, v2 and v3 are the three vectors (column) calculated with the hydrodynamic solver on the node $\vec{x}$, and d is a direction belonging to $D$, the following Octave code calculates $V(\vec{x}, \breve{d})$ :

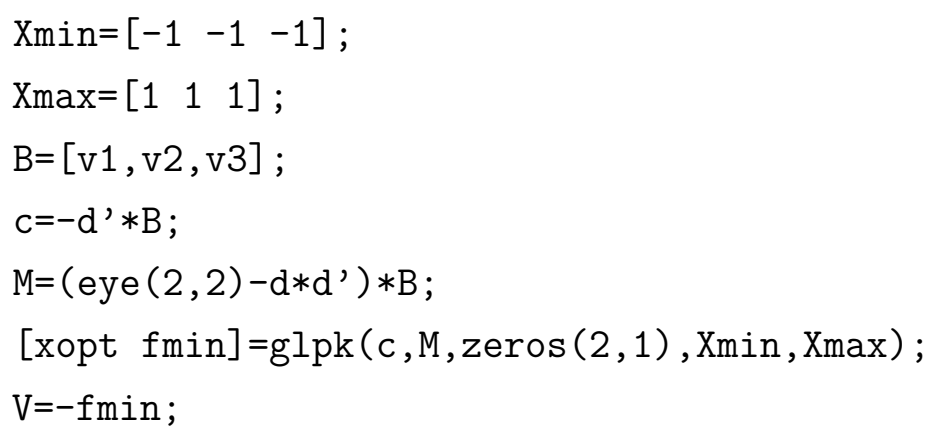

It is important to justify that the use of the negative sign in $\underline{c}=-\underline{d}^{T} \underline{\underline{B}}$ is because by pattern the function glpk minimizes instead of maximizing. We remark that the values $\underline{X}^{-}$and $\underline{X}^{+}$are in the vectors Xmin and Xmax. The value of the control to generate the velocity $\mathrm{V}$ in the direction $d$ is given by xopt. 
The Figure 48 (a) illustrates an example of the set $V(\vec{x}, \breve{d})$ obtained initially with the vectors

$\mathrm{v} 1=[1 ; 1.1] ; \quad \mathrm{v} 2=[-0.2 ; 0.6] ; \quad \mathrm{v} 3=[-0.21 ;-1.1] ;$

In this Figure 48 (a), the external polygon was generated using as minimum and maximum values the vectors

$X \min =[-1,-1,-1] ; \quad X \max =[1,1,1] ;$

When we change the vector of minimum values of the control variables to

$\mathrm{Xmin}=[-0.1,-0.1,-0.1]$;

we obtain a new set $V(\vec{x}, \breve{d})$, which corresponds to the internal polygon. Notice how the polygon that represents $V(\vec{x}, \breve{d})$ loses its symmetry about the origin when Xmin is different from -Xmax. We remark that the number of directions used to determine the region can influence the quality of the set of possible velocities generated. The Figure 48 (b) illustrates a set $V(\vec{x}, \breve{d})$ given by the values

$\mathrm{v} 1=[1 ; 1.1] ; \quad \mathrm{v} 2=[-0.2 ; 1.6] ; \quad \mathrm{v} 3=[-0.21 ;-1.1] ;$

where the internal polygon was generated using 8 directions and the external polygon was generated using 320 directions.

Figure 48 - Polygons representing the set $V(\vec{x}, \breve{d})$.

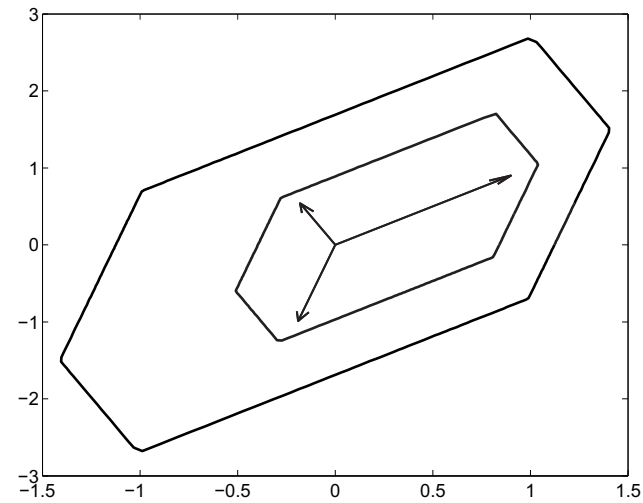

(a) With two different choices of minimum values of the control variables (see text).

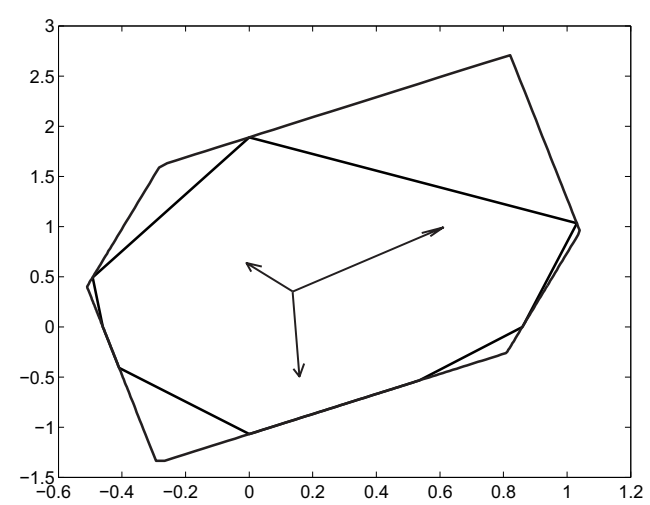

(b) With two different choices of the number of directions considered: 8 and 320 .

By performing the same procedure for all nodes of the mesh, we obtain the data necessary to calculate the optimal trajectory according to the Hamilton-Jacobi-Bellman equation. The Figure 49 shows the set $V(\vec{x}, \breve{d})$ for some points of the domain, where in the Figure 49 (a) the actuators are of the pressure type, and in the Figure 49 (b) the actuators are of the electrode type. 
We can see the variability of $V(\vec{x}, \breve{d})$, which is highly anisotropic and highly dependent on the geometric configuration as well as on the nature of the actuators. The regions where the polygons are smaller correspond to stagnant regions, i.e., regions where the velocity generated by the actuators hardly moves the particles at all. The regions where the polygons are bigger correspond to regions where the particles are easily transported. A "good" trajectory for manipulation avoids not just the obstacles but also these stagnant regions as much as possible.

Figure 49 - Representation of the set $V(\vec{x}, \breve{d})$ of the possible maximum velocities (in each of the 32 directions considered) in some points of the domain.

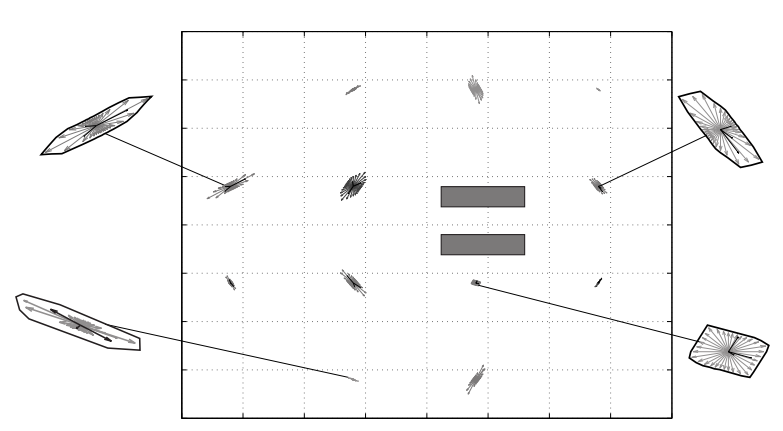

(a) Actuators of the pressure type.

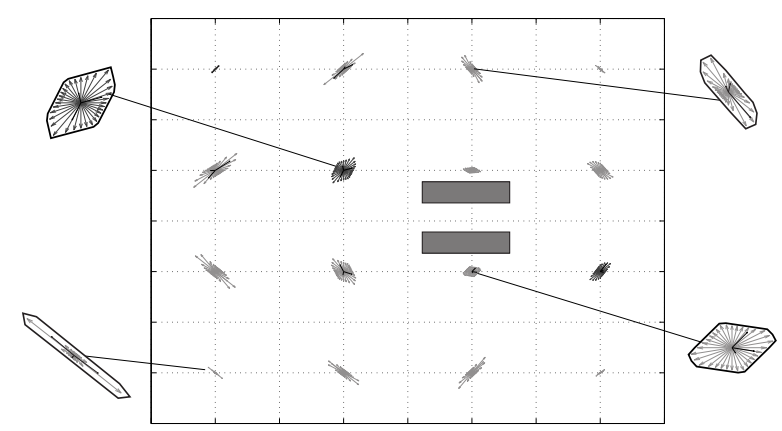

(b) Actuators of the electrod type.

\subsection{Optimizing trajectories by Bellman's principle}

The optimal trajectory, in terms of time, for moving a particle from a point $\vec{x}$ to a target point, solves the Hamilton-Jacobi-Bellman equation. A semi-Lagrangian discretization is considered for the function $T$. The equation (7.6) is approximated at each node $(i, j)$, taking the curve $C$ (denoted as $C_{i, j}$ ) as the boundary of the set of the four cells that share node $(i, j)$. The coordinates of node $(i, j)$ are $\vec{x}=\left(X_{i-1}, Y_{j-1}\right)$ (see Figure 50). For each direction $\breve{d}$, the trajectory from $\vec{x}$ to $C_{i, j}$ is approached by a straight line from $\vec{x}$ to $\vec{y}^{d} \in C_{i, j}$.

Figure 50 - Trajectory, approximated by a straight line, from $\vec{x}$ to $C_{i, j}$ for the direction $\check{d}$.

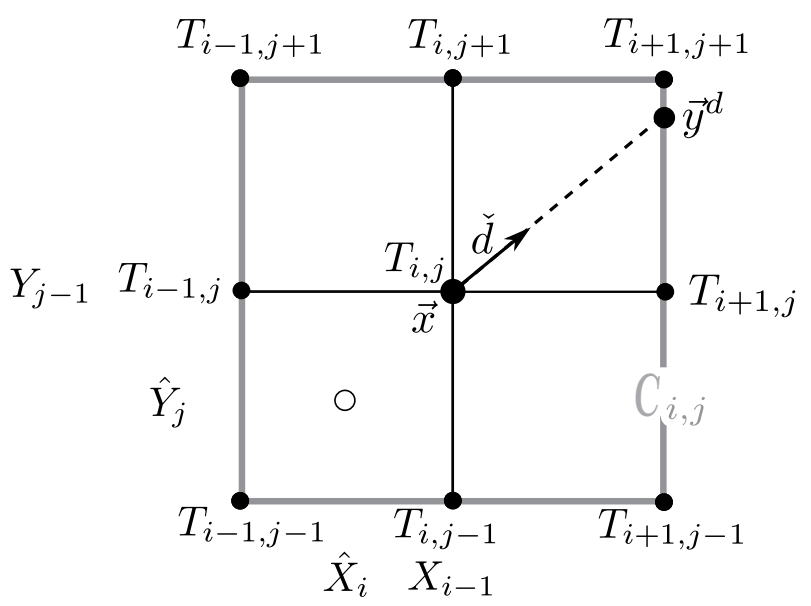


The values for the discrete approximation of $T_{i, j}$ are defined by

$$
T_{i, j}=\min _{\|\check{d}\|=1}\left\{T\left(\vec{y}^{d}\right)+\frac{\left\|\vec{x}-\vec{y}^{d}\right\|}{V(\vec{x}, \check{d})}\right\},
$$

where $T\left(\vec{y}^{d}\right)$ is an interpolation of the $T$ values at the nodes that are placed on $C_{i, j}$. In the case of the direction $\breve{d}$ illustrated in the Figure 50, for example, $T\left(\vec{y}^{d}\right)$ is calculated by linearly interpolating between $T_{i+1, j}$ e $T_{i+1, j+1}$. The direction of the optimal trajectory is that in which the minimum is attained, which is generally not parallel to $\nabla T$ because of the anisotropy of the problem.

To leave a completely discrete scheme we consider the following discretization of the set of directions $D$

$$
D=\left\{\breve{d}^{k}=\left(\cos \left(\theta_{k}\right), \sin \left(\theta_{k}\right)\right), \theta=\frac{(k-1) \pi}{m}, k=1, \ldots, m\right\} .
$$

The algorithm requires that $V(\vec{x}, \breve{d})$ has already been calculated at each vertex and has been stored as a table of values $V_{i, j}^{k}:=V\left(\vec{x}_{i, j}, \breve{d}^{k}\right)$, corresponding to each vertex $(i, j)$ and to each direction $\breve{d}^{k} . T_{i, j}$ is initialized to a large value $\left(10^{12}\right)$ on all nodes except those that belong to the target $Z$, which are initialized to zero. Then we iterate all the mesh nodes that do not belong to $Z$ updating $T$ according with the following equation

$$
T_{i, j} \longleftarrow \min _{\breve{d}=\breve{d}^{k} \in D}\left\{T\left(\vec{y}^{d}\right)+\frac{\left\|\vec{x}-\vec{y}^{d}\right\|}{V_{i, j}^{k}}\right\} .
$$

This algorithm is discussed in (CRISTIANI, 2013), including error estimators for the solution in the norm of $L^{\infty}(\Omega)$, which is important because the exact solution in general is not smooth and can not be understood in the classical sense.

Remark 7.5 (Complexity). Calculations necessary to start the previous algorithm are:

- Computing the matrix $\underline{B}$ according to the linear relation (7.1) for the $N$ nodes and $n$ actuators with cost $n \mathscr{O}\left(N^{a}\right)$, where $\mathscr{O}\left(N^{a}\right)$ is the cost of solving one hydrodynamic problem (tipically $a \approx$ from 1.3 to 1.5 );

- Computing $\left\{V_{i j}^{k}\right\}$ with cost $m N \mathscr{O}\left(n^{b}\right)$, obtained by the cost $\mathscr{O}\left(n^{b}\right)$ of solving one linear optimization problem (7.3) in dimension $n$ (tipically $b \approx 2$ ), for all $N$ nodes and all $m$ directions.

From (7.9) the cost of the HJB iteration is $\mathscr{O}(m N)$, and the number of required iterations is $\mathscr{O}\left(N^{1 / s}\right), s$ being the number of space dimensions. Summarizing, the total cost adds up to $\mathscr{O}\left(n N^{a}+m N n^{b}+m N^{1+1 / s}\right)$. Since $a \approx 1+1 / s$, in general the HJB iterations dominate the total cost, but if the number of actuators $n$ is large the cost of the linear optimization problems can also be significant. 


\subsection{Results}

In this section, we consider the set $D$ generated by 8 directions. The accuracy of the calculation depends on the number of the considered directions, and so does the computational cost. The choice of 8 directions has the advantage of not requiring the interpolation step, since the directions considered in (7.9), with $k=1, \ldots, 8$, are determined by the neighboring nodes. Therefore for each node $(i, j)$ the optimal trajectory is a line that connects it to the neighboring mesh node at which the minimum in (7.9) is attained. As a consequence, the set of numerical trajectories produced by our algorithm has a tree structure. For this reason, to plot the numerical solution we draw all the optimal trajectories that pass through the mesh nodes, which has the geometrical appearance of a tree rooted at the target $Z$.

In the next simulations, we consider a domain $\Omega=\left\{\left(x_{1}, x_{2}\right) \in \mathbb{R}^{2}, 0<x_{1}, x_{2}<10\right\}$ discretized in a grid of $40 \times 40$ nodes, except when indicated otherwise. When the flux is induced by an electrical potential we assume an electrosmotic costant $m_{e o}=1$ while when it is generated by a pressure source we consider viscosity $\mu=0.1$. The actuators' limit values are set to $\underline{X}^{-}=(-1,-1,-1)$ and $\underline{X}^{+}=(1,1,1)$.

Figure 51 shows the trees of optimal trajectories obtained for both electrical (subfigure (a)) and mechanical (subfigure (b)) actuators.

Figure 51 - Optimal trajectory configuration with range of actuators between -1 and 1 . The highlighted point is the arrival target.

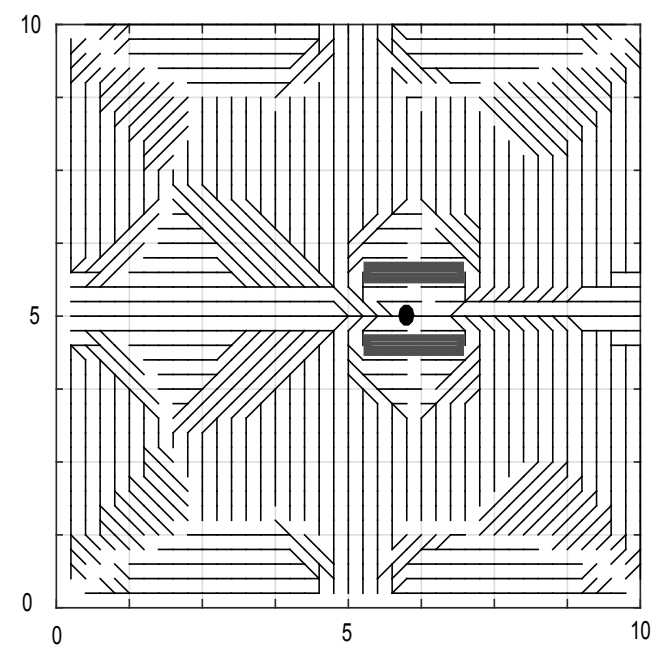

(a) Electrical potential.

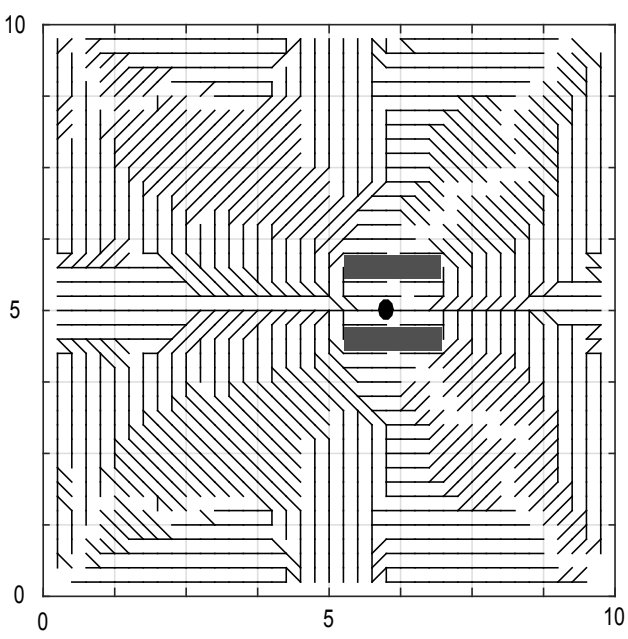

(b) Pressure.

One can notice that the trajectories between the two plates of the obstacle are essentially horizontal. This is because the actuators cannot generate significant vertical velocity there due to the presence of the plates. With this, it turns out that a particle that is between the plates but below or above $Z$ must first take a path to get out of the space between the plates and then 
walk back in towards the target point. We find worthy of note that the optimal trajectories tend to circumvent the obstacle so as to traverse regions (more distant to the obstacle) where the admissible velocities are higher. Notice also that the trajectory trees have similarities for both types of actuators, mainly differing at the obstacles' boundaries, where the mechanically-driven flow has zero velocity while the electrically-driven one only imposes the normal component of the velocity to be zero.

The model exhibits convergent behavior with respect to mesh refinement in all cases considered. As an example, in Figure 52 we compare the resulting trees for the electrically-driven model, with meshes $40 \times 40$ and $80 \times 80$.

Figure 52 - Effect of mesh refinement on the resulting trajectory trees, for the electrically-driven flow.

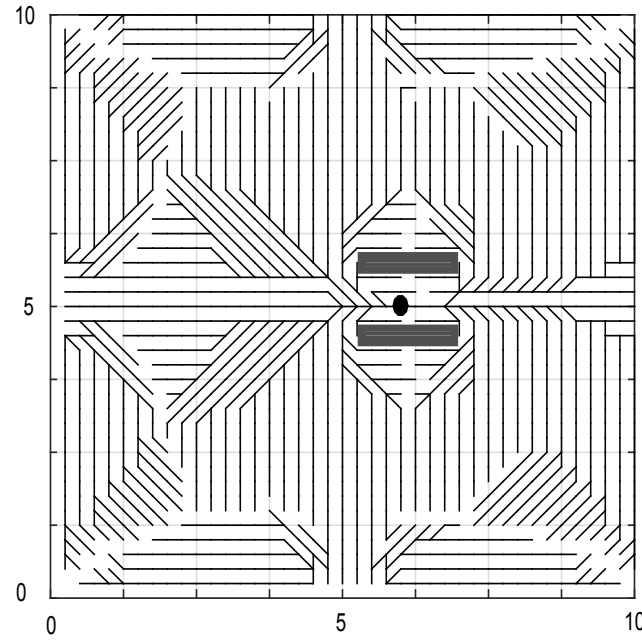

(a) Mesh size $40 \times 40$.

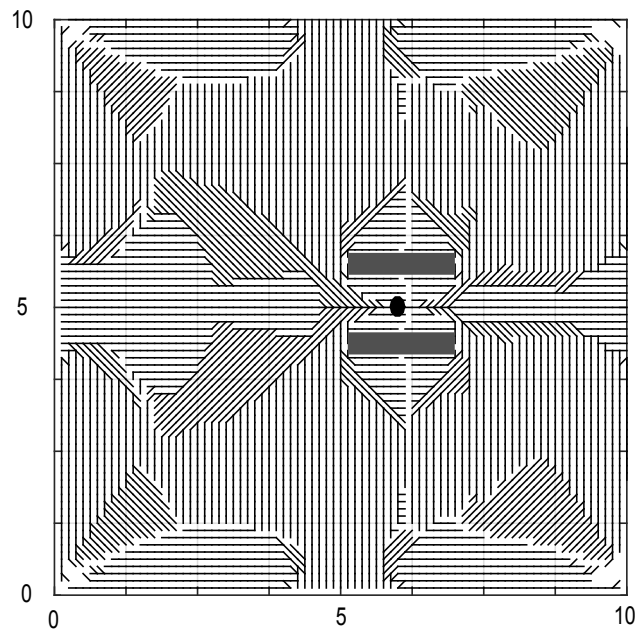

(b) Mesh size $80 \times 80$.

The optimal trajectories may be counterintuitive. Let us consider the flow generated by pressure actuators setting as target $Z$ a single point close to the obstacle as shown in Figure 53 . Then we consider two possible start points, labeled with the letters $A$ and $B$. In the case with $A$ as starting point, the model designs an optimal trajectory that is a straight segment. The travel time $t$ computed by integrating the field $V(\cdot, \breve{d})$ between $A$ and $Z$ along a straight line $\left(\check{\tau}_{1}=1, \check{\tau}_{2}=0\right)$ coincides with $t_{o p}=T(A)$, where $T$ is the solution of the discrete Hamilton-Jacobi-Bellman equation (7.9). On the other hand, when the starting point is $B$ the optimal trajectory proposed by the model is not straight. It assumes a form that is not of immediate intuition. The designed path, although it is longer than the straight path, results in a shorter travel time $t_{o p}=6.7$ than that obtained along the straight one $t=9.1$. This advantage is due to the greater efficiency of the actuators in the region traveled by the optimal trajectory. The values to be applied to the actuators to manipulate the particle along the proposed path are easily obtained from (7.4) and are shown in Figure 54 as functions of time for the case that has $B$ as starting point. As usual in time-optimal control, the actuator values exhibit a bang-bang-like behavior. The geometrical 
path is the most fundamental result since it avoids obstacles and stagnant regions. This path can then be traveled at non-optimal velocities, with smoother control functions, as discussed in Remark 7.2.

Figure 53 - Comparison of the optimal trajectories with straight ones.

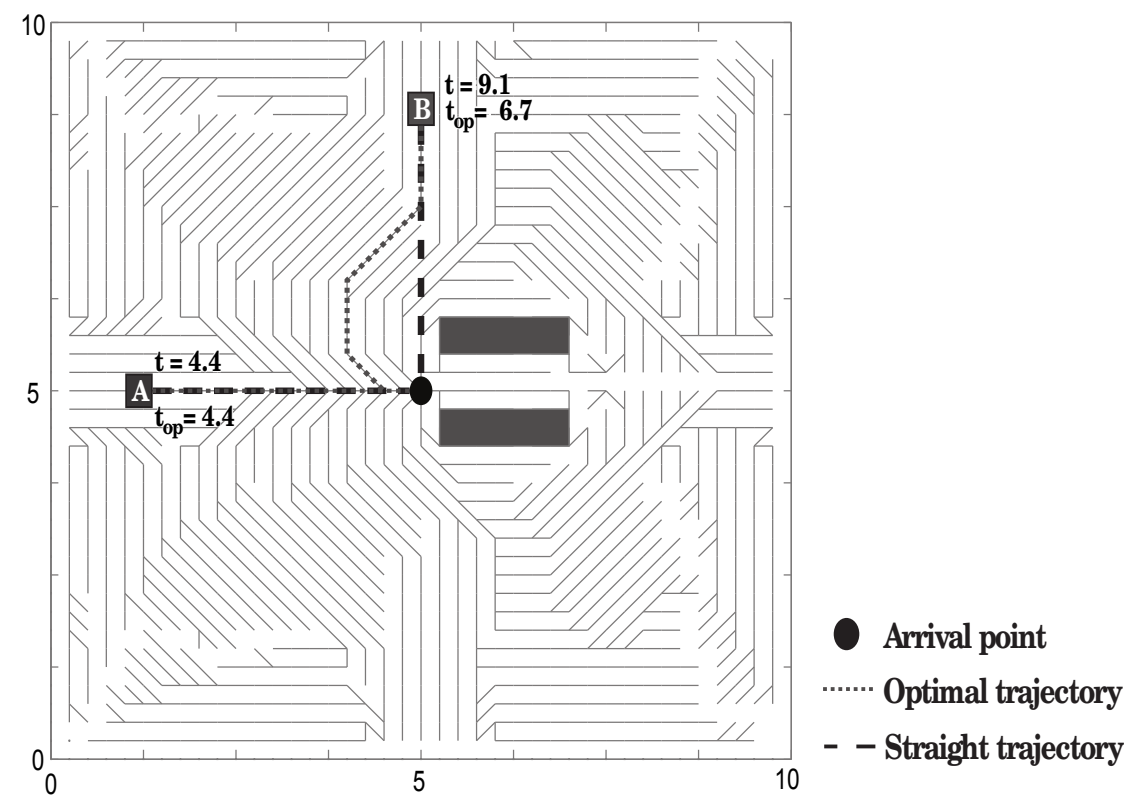

Figure 54 - Actuators values over time along the optimal path starting at $B$.

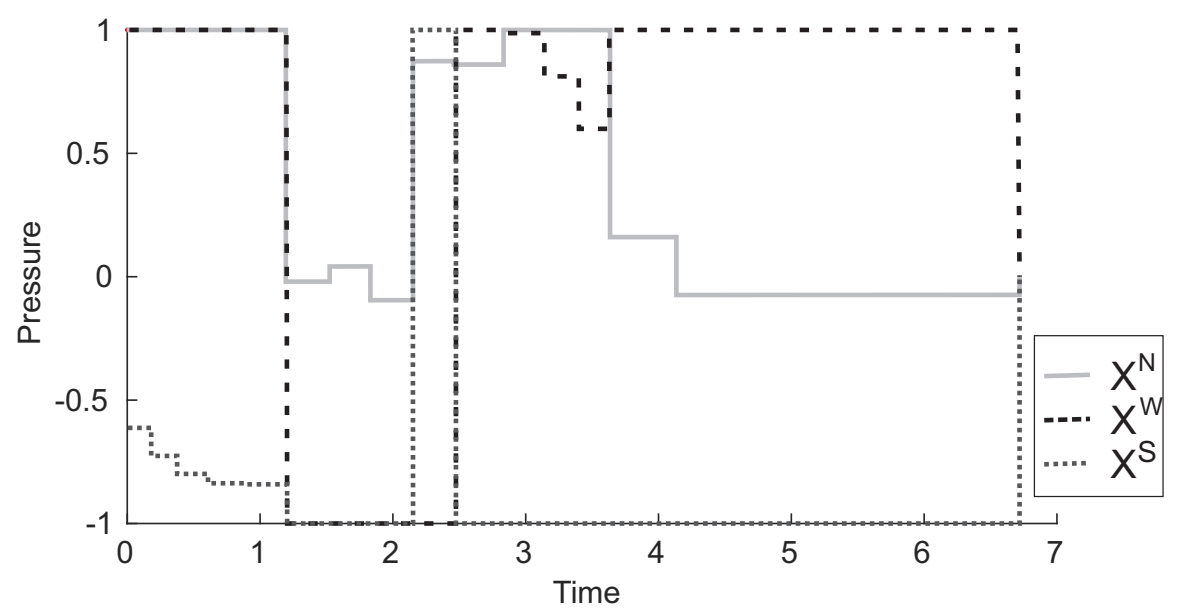

\subsection{Achievements}

We have presented an integrated model to identify convenient trajectories for the manipulation and control of particles in microfluidics. The achievement of the above-mentioned objective involved various mathematical problems:

- To find an admissible set of velocities $\mathbb{V}(\vec{x})$ on each point $\vec{x}$ generated by a set of admissible actuator values $\mathscr{A}$, solving 
- a Stokes problem for the velocity field induced by pressure sources;

- a Poisson problem for the electrical potential induced by a voltage imposed at electrodes, which in turn induces an electrosmotic velocity field;

- To determine the maximum admissible velocity $V(\vec{x}, \breve{d})$ at each point $\vec{x}$ and for each direction $\breve{d}$, through the resolution of a linear optimization problem;

- To provide the control functions $\underline{X}^{*}(t)$ arising from the resolution of an initial value problem for the trajectory ODE;

- To plan the trajectory by minimizing the arrival time through the numerical implementation of Bellman's principle.

The proposed method automatically indentifies a trajectory for a particle that we must manipulate from a departure point to the target in minimum time (and also provides the corresponding values to impose on the actuators). The numerical results show that the trajectories found not only avoid the obstacles, but also avoid the regions of the domain where the actuators are ineffective at producing flow. The numerical simulations show counterintuitive optimal trajectories in this kind of problem, justifying the use of mathematical models for designing automatic manipulation systems.

It should be noted that the proposed algorithm produces not just one optimal trajectory, but the whole tree of optimal paths, starting at any $x \in \Omega$. This apparent overkill is very important when applying the model under realistic conditions, in particular considering external perturbations (noise). In fact, if the sensor in the control loop detects that a perturbation has taken the particle to a position $\tilde{x}$ outside the original optimal path, the controller can immediately switch to the path in the optimal tree that passes throught $\tilde{x}$. In this way, and without any additional computation, the optimal tree can be used to correct perturbations so that the particle eventually reaches $Z$. 
CHAPTER

\section{8}

(1)

\section{MATHEMATICAL MODELS FOR TUMOURS WITH CANCER STEM CELLS}

In the last few years several papers have been devoted to the construction, discussion and analysis of mathematical models designed to describe of the growth of tumours in presence of cancer stem cells (CSC), see for instance (BETTERIDGE et al., 2006), (BORSI et al., 2015b), (ENDERLING et al., 2009), (FASANO; MANCINI; PRIMICERIO, 2016), (GANGULY; PURI, 2006), (HILLEN; ENDERLING; HAHNFELDT, 2013). The heterogeneity of tumour cells in breast cancers was put in evidence in (AL-HAJJ et al., 2003) where the term "tumorigenic cells" was introduced; this fact is now widely accepted (see e.g. (LATHIA et al., 2015)) and CSC have been identified in many cancers, as well in sarcomas and leukemia (see e.g. (DITTMAR; ZÄNKER, 2013)). The relevance of CSC in the progression of these and other tumours and the investigation of their role has been the object of many experimental papers as well as of more theoretically-oriented articles, see (MICHOR, 2008) and the bibliography cited therein as well as (DINGLI; MICHOR, 2006), (DITTMAR; ZÄNKER, 2013), (KAKARALA; WICHA, 2008), (LATHIA et al., 2015), (MADDALENA, 2014), (SOLÉ et al., 2008). One of the features of tumours with CSC is the so-called tumour-growth-paradox that consists in the fact that tumours with a larger death rate for ordinary (non-stem) cancer cells might grow bigger than tumours with a lower death rate for the same class of cells. Of course, since the death rate is influenced by medical treatment, understanding the reasons of this paradox can be relevant in the control of the tumours ((GUROVA; GUDKOV, 2003) and (WODARZ; KOMAROVA, 2007)). Concerning mathematical models, that are the object of this short review, we have to stress that they are not intended to give quantitative prediction of the progression of cancers, nor to include all possible factors and aspects that influence their growth. They are rather oriented to offer a conceptualization of the phenomenon and to isolate the mechanisms that seem to be crucial for the essential features of the evolution of tumours in presence of CSC. On the other hand, presenting and analyzing various mathematical modelling techniques and the corresponding numerical 
simulations outlines in particular the model-independence of the occurrence of the tumor growth paradox. In the models that we describe some drastic simplifications are introduced in order to provide a mathematical scheme such that the properties of its solutions can be investigated and simulated. On the other hand, it is important that the model does not contain a too high number of parameters, whose values would be hardly estimated form experimental data. In other words, as Albert Einstein said, "a model should be as simple as possible ... but not simpler". In this paper, we will present, analyze and dicuss some mathematical models that have been proposed in this context (see (BETTERIDGE et al., 2006), (BORSI et al., 2015b), (ENDERLING et al., 2009), (FASANO; MANCINI; PRIMICERIO, 2016), (HILLEN; ENDERLING; HAHNFELDT, 2013), (MADDALENA, 2014), (STIEHL; MARCINIAK-CZOCHRA, 2012)), avoiding technical details and displaying some numerical simulations. In addition, we will present the development of an original model based on cellular automata to provide a comprehensive overview on modelling of the phenomenon. We will see, in particular, in which sense and cases the models exhibit the paradoxical behaviour outlined above and we will mimic the response of tumours to different treatments. We will not describe mathematical models more based on stochastic arguments and/or dealing with the mutation of the normal cells to CSC ((GANGULY; PURI, 2006), (SUN; KOMAROVA, 2015)).

\subsection{Basic ingredients of the models}

The models that we will describe contain two basic simplifications. The first simplification consists in assuming that the population of tumour cells consists of just two sub-populations: cancer stem cells (CSC) and ordinary non-stem cells (CC) and that all the cells in each subpopulation have the same properties. This implies in particular that it is assumed (and this is the second drastic approximation) that cells have age-independent replicative potential and mortality. More specifically, for $\mathrm{CC}$ there is a fixed probability of generating new $\mathrm{CC}$ or of undergoing apoptosis, while CSC are assumed to be immortal and to be capable of generating new CSC (symmetrical mitosis) or CC (unsymmetrical mitosis), with a fixed ratio between the two probabilities. Of course, these are very strong semplifications because in practice multipotent cells generate various cell lineages and because cells are subject to senescence and their parameters (replication potential, mortality) vary accordingly. On the other hand, in the models consisting in systems of equations (ODE, integro diferential equations, reaction-diffusion equations) the unknown functions represent averaged fractions of CC and CSC and thus the characteristics of each cell (age among them) cannot be taken into account. This is the limit of this class of models that can represent just a conceptual idea of the complex phenomenon. Nevertheless, even with these simplifications, the models we discuss here are sufficiently rich and in particular they are able to describe the tumour paradox adequately. We may add that it could be possible to encompass age-dependence in the models, introducing compartmental models in which the unknown functions represent the fractions of cells belonging to a sequence of different classes of 
age, as in (WERNER et al., 2016), (MICHOR et al., 2005), (DINGLI; TRAULSEN; PACHECO, 2007) and (WERNER et al., 2011). The "cost" of this approach will be the introduction of a larger number of parameters that have to be fitted with experimental data.

An important effect that is included (although in a very idealized way) in all the models that will be described is the "crowding effect" ((BETTERIDGE et al., 2006), (BORSI et al., 2015b), (ENDERLING et al., 2009), (FASANO; MANCINI; PRIMICERIO, 2016), (HILLEN; ENDERLING; HAHNFELDT, 2013)). This means that mitosis is supposed to be inhibited when the density of cells in a neighborhood of the would-be mother cell exceeds a threshold value. Models based on the basic concepts of population dynamics but not taking into account the crowding effect have been proposed starting from (MICHOR et al., 2005) but were unable to explain the tumour growth paradox. In what follows we do not take into account explicitly the movement of cells induced by mutual pushing by effect of mitosis. In some sense, this mechanism is implicitly included in the mean field approximation (8.3) because the "crowding" term $1-u-v$ represents the availability of space in the whole region of interest ${ }^{1}$. According to some authors (e.g. (BETTERIDGE et al., 2006), (ENDERLING et al., 2009) and (HILLEN; ENDERLING; HAHNFELDT, 2013)) "pushing” effect can be included in integro-differential models (see Section 8.4) by adding diffusion and this is reflected, in reaction-diffusion models (see Section 8.5), by additive terms in the coefficients of $U_{x x}$ and $V_{x x}$. From the point of view of the mathematical well-posedness and of the qualitative aspects of the numerical simulations, this does not make any relevant difference (just the appearance of two more parameters). In the case of agent-based models one could possibly consider different probabilities of replication when space is available in a close neighborhood of the mother cell with respect to the case in which the space is available at some distance and some energy has to be spent to displace the neighboring cells.

\subsection{Agent-based models}

This approach is based on the so-called "cellular automata" (CA). As it is well-known, CA were introduced by John von Neumann (following the idea of Stanislaw Ulam) to study global properties from local processes (KARI, 2005) and are currently applied in several contexts $(\mathrm{CHOPARD} ; \mathrm{DROZ},)^{2}$. In the present case we imagine cells as living in a square (or cubic) lattice and we prescribe (probabilistic) rules for their motion, replication or death. When we run a simulation, the individual behaviour of each cell contributes to the evolution of the system that is visualized by the collective behaviour. Possibly, one may average over several simulations to get insight of the response of the model to different setup of parameters and conditions.

1 Possibly, this term could be modified by a different function of $u+v$ vanishing when the whole region is filled by tumour cells.

2 For an application to a social problem see (MEACCI; NUÑO; PRIMICERIO, 2012) and (MEACCI, 2015). 
According to the remark on the crowding effect, we assume that cells can proliferate only if in their neighborhood some lattice sites are vacant. It is also assumed that dying cells leave immediately blank the site they occupied. In the simplest version that we present here cells are supposed to be immobile, but also a random motion (e.g. diffusion) or drift can be incorporated in the model. Different definitions of "neighbourhood" are possible: for the 2-D lattice Von Neumann's or Moore's neighbourhood can be used (4 orthogonal neighbours - north, south, east, west - or 8 neighbours - adding northwest, northeast, southwest, southeast - respectively). But larger neighbourhood can also be used, such as squares of $5 \times 5$ lattice sites centered in the considered cell. In the simulation we present here, each site in the lattice grid can be in one of three different states:

- vacant sites (white),

- sites occupied by CSC (black),

- sites occupied by CC (red).

Starting from a given situation at time $t_{k}$ each CC (red) has a probability $\mu$ of undergoing apoptosis (becoming white). Then, the surviving cells have a probability $\rho$ of being potentially replicant. But, in this case, mitosis is only possible if there are vacant (white) sites in the chosen neighbourhood; if this situation occurs a daughter CC appears and one of these white sites becomes red. The situation with CSC is similar, but with two differences: (i) $\mu=0$, and (ii) if mitosis occurs a daughter CSC appears with probability $\delta$, while the unsymmetrical proliferation (i.e. the appearance of new born $\mathrm{CC}$ ) has probability $1-\delta$. The replication probability $\rho$ can be different for the two classes of cells ( $\rho_{u}$ for CSC, $\rho_{v}$ for CC) and could be assumed to be agedependent, i.e. decreasing for each mitosis undergone. We will neglect this latter fact and assume that the replication potential is age-independent. We also neglect the possibility of diffusion and movement of the cells, as considered e.g. in (BETTERIDGE et al., 2006), (ENDERLING et al., 2009) and (HILLEN; ENDERLING; HAHNFELDT, 2013). When the simulation stops before the growing tumour reaches the boundary of the grid, as in the simulations displayed in the sequel, it is not necessary to specify boundary conditions. In other cases the boundary of the grid (not necessarily rectangular) can be identified with a physical boundary beyond which no tumour cell can appear; this means that a form of no-flux boundary conditions are applied and this modifies the form of neighbourhood for the cells close to the boundary. Finally, to mimic an unbounded medium, it can be convenient to impose periodicity, i.e. the boundary "wraps" on itself so that the cells of each side of the square interact with cells of the opposite side. Here, we display some simulations. In Figure 55 the fraction of the lattice sites that are occupied by tumour cells (both CC and CSC) is displayed as a function of time for different mortalities of the CC. In particular, the two cases considered are with $\mu_{1}=0.005$ (blue line) and $\mu_{2}=0.01$ (red line). The parameters of this simulation are $\delta=0.2, \rho_{u}=0.01$ and $\rho_{v}=0.005$.

In Figures 56 and 57 we show the screenshots correspondent to the two simulations. 
Figure 55 - Fraction of the total number of tumour cells (CC and CSC) as function of time.

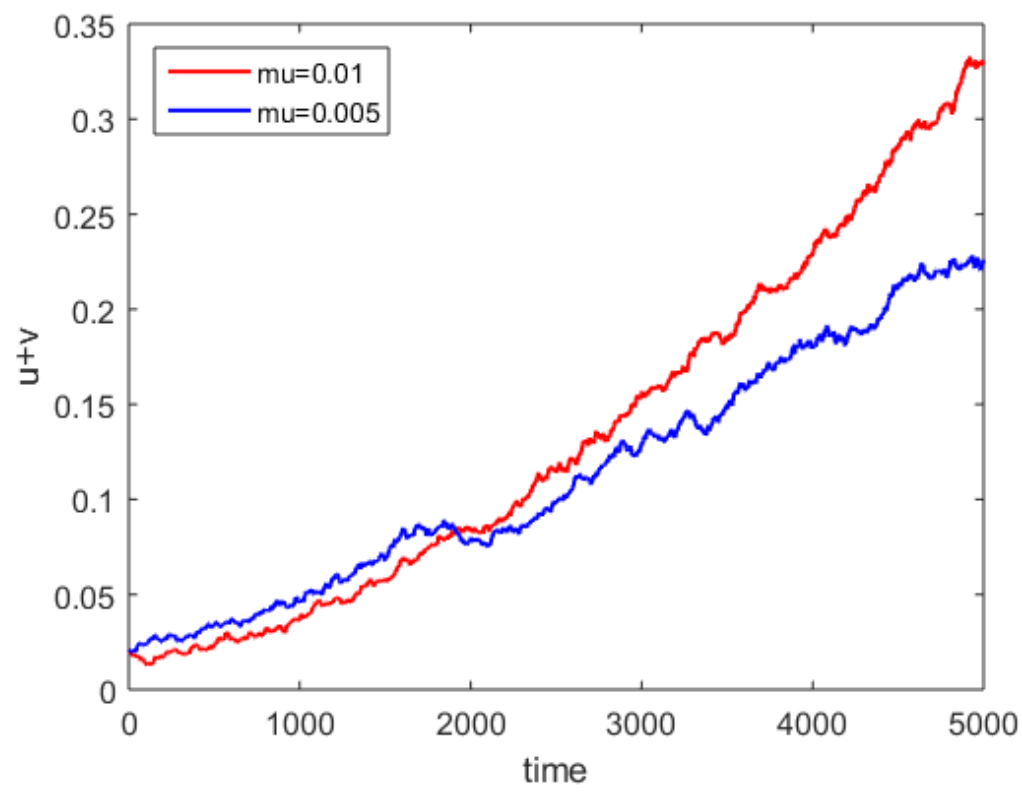

Figure 56 - Distribution of tumour cells CSC (black) and CC (red) for a mortality $\mu_{1}=0.005$ at certain times $(t=0, t=500, t=2000$ and $t=5000)$.
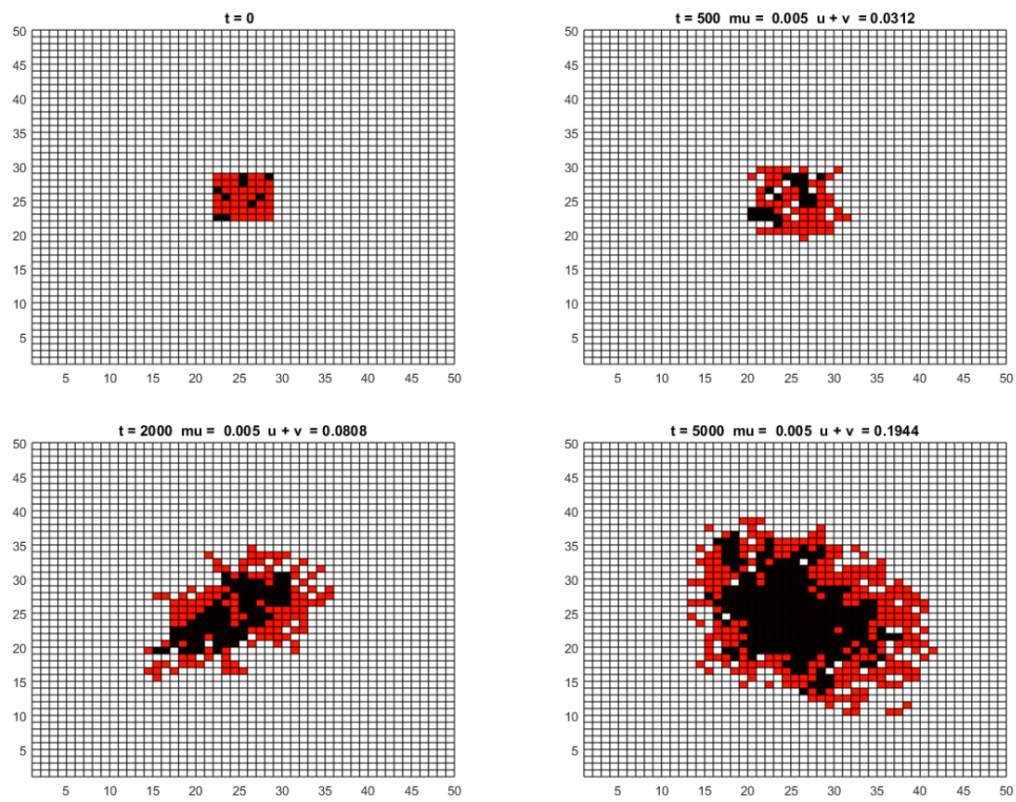
Figure 57 - Distribution of tumour cells CSC (black) and CC (red) for a mortality $\mu_{2}=0.01$ at certain times $(t=0, t=500, t=2000$ and $t=5000)$.
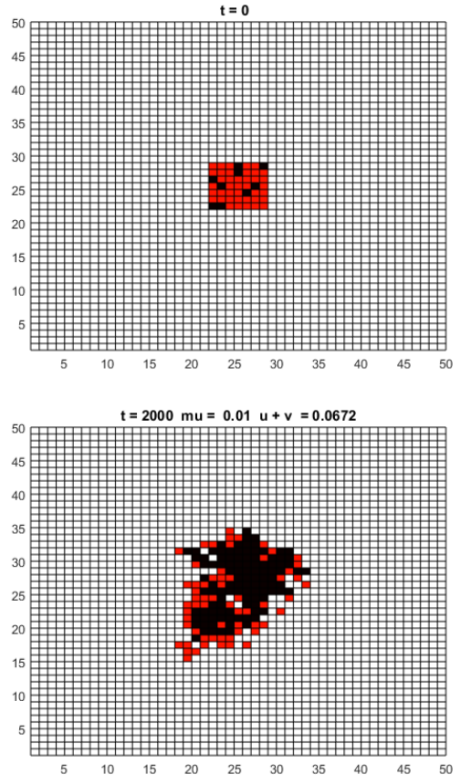
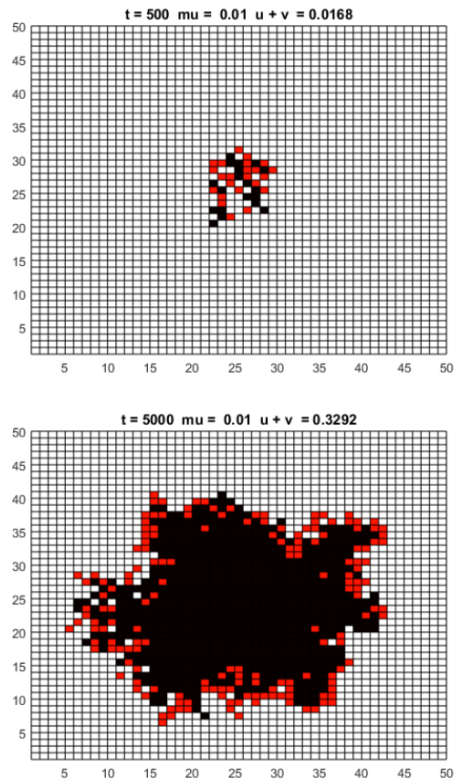

The results of these simulations show clearly that the model exhibits the tumour paradox. More precisely, a higher number of CC (case $\mu=0.005$ ) inhibits the growth of CSC and thus of the whole tumour. To conclude this section we consider the case in which, at a prescribed time, a treatment takes place such that all the $\mathrm{CC}$ are destroyed. Displaying the fraction of total number of tumour cells as function of time in the case with treatment and without treatment we see that the treatment produces instantly a reduction of the volume of the tumour but in the long run it does not cause a lower growth of the tumour (see Figure 58).

We conclude this Section with the following remark. If the initial situation in the CA simulation only consists of a set of red sites, black sites will never appear since CSC can only be generated by stem cells. Thus, this case would formally describe tumours in which stem cells are not present. But in this case the intrinsic approximations introduced in the modelling (in particular age-independence) lead to an oversimplified description in which the final steady state will consist either in a configuration with all white sites (if $\mu>\rho$ ) or in a fixed fraction $(<100 \%)$ of red sites and the tumour paradox does not appear. Therefore, our models only make sense if the initial fraction of CSC is positive.

\subsection{Mean field approximation}

Consider the situation described in the previous section with no flux boundary conditions and define $u\left(t_{k}\right)$ the fraction of the CSC, i.e. the number of the lattice sites occupied by CSC cells at time $t_{k}$, divided by the total number of sites. Similarly, $v\left(t_{k}\right)$ will represent the fraction of CC at time $t_{k}$. To obtain a mean field approximation, one formally considers that the neighbourhood of each cell is the entire grid, so that the fraction, at time $t_{k}$, of the vacant sites where a new cell 
Figure 58 - Fraction of total number of tumour cells (averaged over 20 simulations) as function of time in the case with treatment (blue line) and without treatment (red line). The treatment has the effect of the destruction of the CC cells at the time $t_{d}=100$. Here the setup of parameters is $\mu_{1}=0.005, \delta=0.2, \rho_{u}=0.01$ and $\rho_{v}=0.005$.

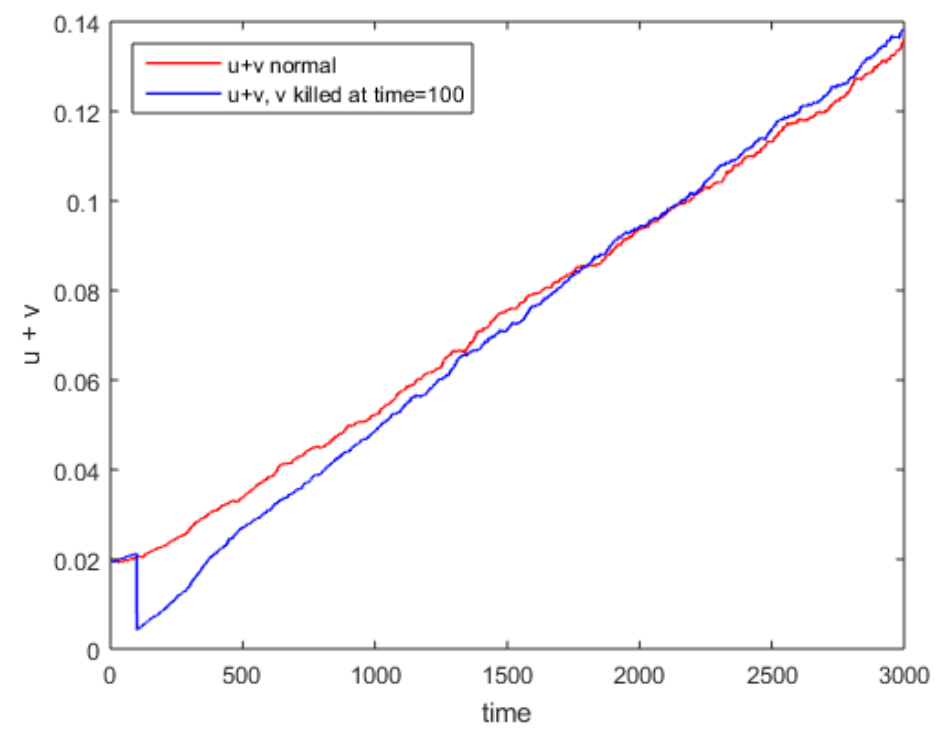

can appear is $1-u\left(t_{k}\right)-v\left(t_{k}\right)$. Therefore, if we look for a continuous evolution system, we are brought to the following pair of ordinary differential equations:

$$
\begin{gathered}
\frac{d u}{d t}=\rho_{u} \delta u(1-u-v), \\
\frac{d v}{d t}=\rho_{u}(1-\delta) u(1-u-v)+\rho_{v} v(1-u-v)-\mu v,
\end{gathered}
$$

According to the remark at the end of Section 8.2, our discussion is confined to tumours with cancer stem cells and thus we assume that $u(0)>0$.

We note that under this assumption the system (8.1)-(8.2) has an invariant set $\Sigma=\{(u, v)$ : $u>0, v \geq 0, u+v \leq 1\}$ and has an unique steady state $u=1$ and $v=0$ that is the only global attractor (see (BORSI et al., 2015b)) in the invariant set $\Sigma$. This corresponds to a situation in which, independently of the proliferation and death rate of the two subpopulations, the tumour invades the whole available space and consists of stem cells only. Of course, normalizing time, we have a system with three parameters $\delta, \rho=\rho_{v} / \rho_{u}$ and $\mu^{*}=\mu / \rho_{u}$. To simplify notation we will write $\mu$ instead of $\mu^{*}$ :

$$
\left\{\begin{array}{l}
\dot{u}=\delta u(1-u-v), \\
\dot{v}=(1-\delta) u(1-u-v)+\rho v(1-u-v)-\mu v .
\end{array}\right.
$$

Figure 59 shows that the model still exhibits the tumour paradox although this fact is less evident than in the examples displayed in Section 8.2. But this fact can be easily explained: the "crowding" effect is the key factor producing this paradox and in the CA models this effect is taken into account locally, whereas in the mean field approximation the term $(1-u-v)$ just 
represents the fraction of "empty spaces" respectively from their location or distance from the mother cell. To introduce a qualitative correction to this fact the term $1-p(p \equiv u+v)$ has been replaced by $1-p^{n}$ or $e^{-n p}-e^{-n}, n>1$ (see (BORSI et al., 2015b)). Also in this mean field approximation we can simulate a treatment that "kills" the $\mathrm{CC}$ at given time. Figure 60 shows an effect that is similar to the one for the CA model presented in Figure 58 and according to which a sudden decrease of the tumour cells does not provide long-term substantial benefits.

Figure 59 - Fraction of tumour cells $(u+v, u$ and $v$ separately) as function of time. The setup of parameter is $u_{0}=0.016$ and $v_{0}=0.0036$ (initial conditions, as for CA simulations), $\delta=0.2, \rho=0.5$ for the two cases $\mu_{1}=1.5$ and $\mu_{2}=0.5$.
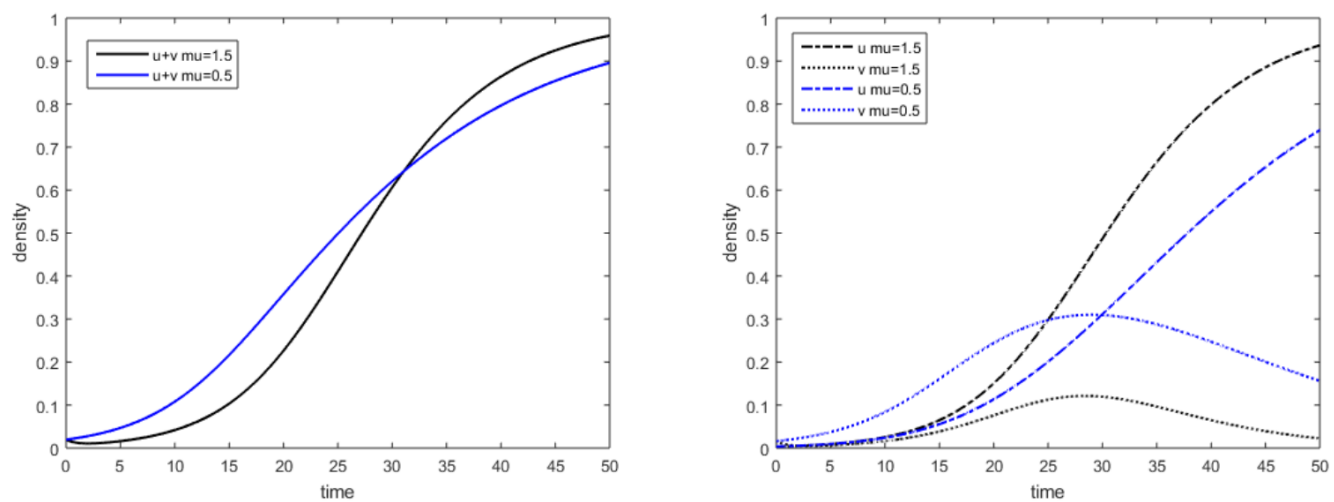

Figure 60 - Fraction of total number of tumour cells as function of time in the case with treatment (black line) and without treatment (blue line). The treatment has the effect of the destruction of the CC cells at the time $t_{d}=15$. Here the setup of parameters is $\rho=0.5, \mu=0.5$ and $\delta=0.2$.

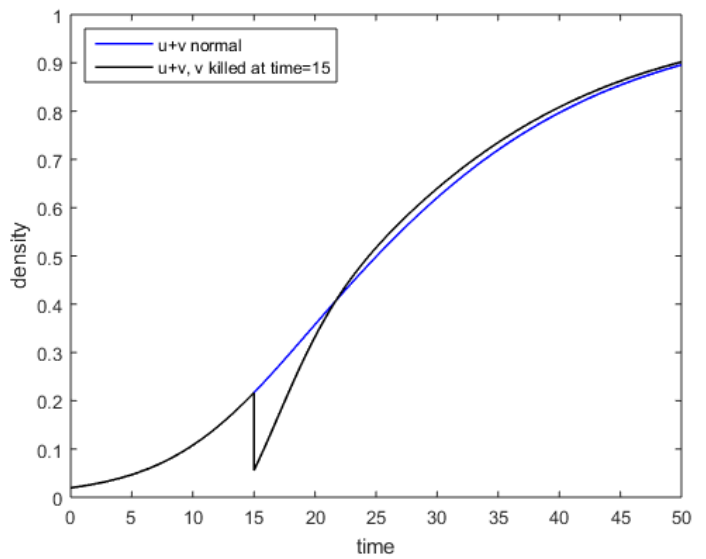

\subsection{A integro-differential model}

Of course, the mean-field approach only contains global information on the evolution of the tumour but does not take spatial variation into account. In the sequel we present a continuous time model that includes crowding as a local effect, in the sense that a daughter cell can be generated only if there is space available in the neighbourhood of its progenitor. The radius of 
this neighborhood is also depending on the possibility that cells are displaced to allow a mitosis to occur. Thus, the birth rate of CC's at location $\underline{x}$ originating from $\mathrm{CC}$ mother cells can be expressed as

$$
\left.\rho_{v} \int_{\Omega} K(|\underline{x}-\underline{y}|) F(u(\underline{x}, t)+v(\underline{x}, t)) v(\underline{y}, t)\right) d y,
$$

where $\Omega$ is the domain where the phenomenon takes place ( $\mathbb{R}^{3}$ for simplicity), $K$ is a positive decreasing function whose integral over $\mathbb{R}^{3}$ equals 1 and $F$ is a positive decreasing function such that $F(0)=1, F(1)=0$. In other words, $F$ takes into account the crowding and $K$ is a kernel indicating "how far" the proliferation capability of a cell can have an effect. Therefore, the system that has to be considered is the following:

$$
\begin{aligned}
\frac{\partial u(\underline{x}, t)}{\partial t} & =\rho_{u} \delta \int_{\Omega} K_{u}(|\underline{x}-\underline{y}|) F(p(\underline{x}, t)) u(\underline{y}, t) d y, \\
\frac{\partial v(\underline{x}, t)}{\partial t}= & \rho_{u}(1-\delta) \int_{\Omega} K_{u}(|\underline{x}-\underline{y}|) F(p(\underline{x}, t)) u(\underline{y}, t) d y \\
& +\rho_{v} \int_{\Omega} K_{v}(|\underline{x}-\underline{y}|) F(p(\underline{x}, t)) v(\underline{y}, t) d y \\
& -\mu v(\underline{x}, t),
\end{aligned}
$$

where we set

$$
p(\underline{x}, t)=u(\underline{x}, t)+v(\underline{x}, t)
$$

and we assumed, for the sake of generality, that the kernels could be different for CSC and CC. Again, we can assume

$$
F((p(\underline{x}, t))=1-p(\underline{x}, t)
$$

and typically

$$
K_{u}(|\underline{x}-\underline{y}|)=\frac{1}{\sqrt{\pi} \sigma_{u}} \exp \left(-\frac{(x-y)^{2}}{\sigma_{u}^{2}}\right) .
$$

In (BORSI et al., 2015b) it is shown (see also (MADDALENA, 2014)) that the model is mathematically well-posed (existence, uniqueness, continuous dependence on the data, boundedness of the solutions) and some numerical simulations are shown that provide evidence of the following basic facts: (i) if the mortality rate of $\mathrm{CC}$ is higher, then the tumour is more aggressive and (ii) incomplete treatment of cancers can enhance their growth. Indeed, CSC are less (or not) sensitive to treatments and hence the stress applied to the tumour acts in favour of a selection of stem cells, thus enhancing the fitness of tumour and its growth. For simplicity, numerical simulations are confined to one-dimensional situation. The choice of parameters is the following

$$
\rho_{u}=1, \quad \rho_{v}=0.5, \quad \delta=0.2
$$

and the kernels are taken as gaussian curves with

$$
\sigma_{u}=0.5, \quad \sigma_{v}=0.1
$$


The initial conditions are the following

$$
u(x, 0)=e^{-10 x^{2}}, \quad v(x, 0)=0 .
$$

The plots of Figures 61 and 62 correspond to two different values of the mortality of the CC. In Figure $61 \mu=0.2$ and the invasion of the tumour is clearly dominated by CC while CSC are in the centre of the cancer. In Figure $62(\mu=2)$ we see that the progression of the tumour is dominated by CSC. Finally, Figure 63 displays for two values of $\mu$ the time evolution of the total tumour mass

$$
\Pi(t)=\int_{-\infty}^{\infty} p(x, t) d x
$$

and the progression of the level line $p=0.8$. Indeed, the latter gives an idea of the motion of the boundary of the tumour and we can take its slope as a measure of the invasion speed of the tumour. Figure 63 (left) shows that tumours with $\mu=2$ have a total mass that, after some time, exceeds the mass of tumours with $\mu=0.2$. The slope of the curves in Figure 63 (right) shows that tumours with higher mortality of $\mathrm{CC}$ have an higher invasion speed. The tumour paradox is evident.

The model can also be used to visualize the effect of treatment. Take $\mu=0.2$ and assume that at $t=50$ we apply a radiation treatment that destroys a fraction $\phi$ of the $\mathrm{CC}$ and has no effect on CSC. Figure 64 displays $p$ at time $t=50$ (just after the treatment) and $t=100$ and the comparison with the case of no treatment. In Figure 65 the total tumour mass and the rate of invasion in the two cases are compared.

Figure $61-$ Plot of $u, v, p=u+v$ at selected time instants, with initial conditions 8.12 and parameters from 8.10 and 8.11. Case $\mu=0.2$.
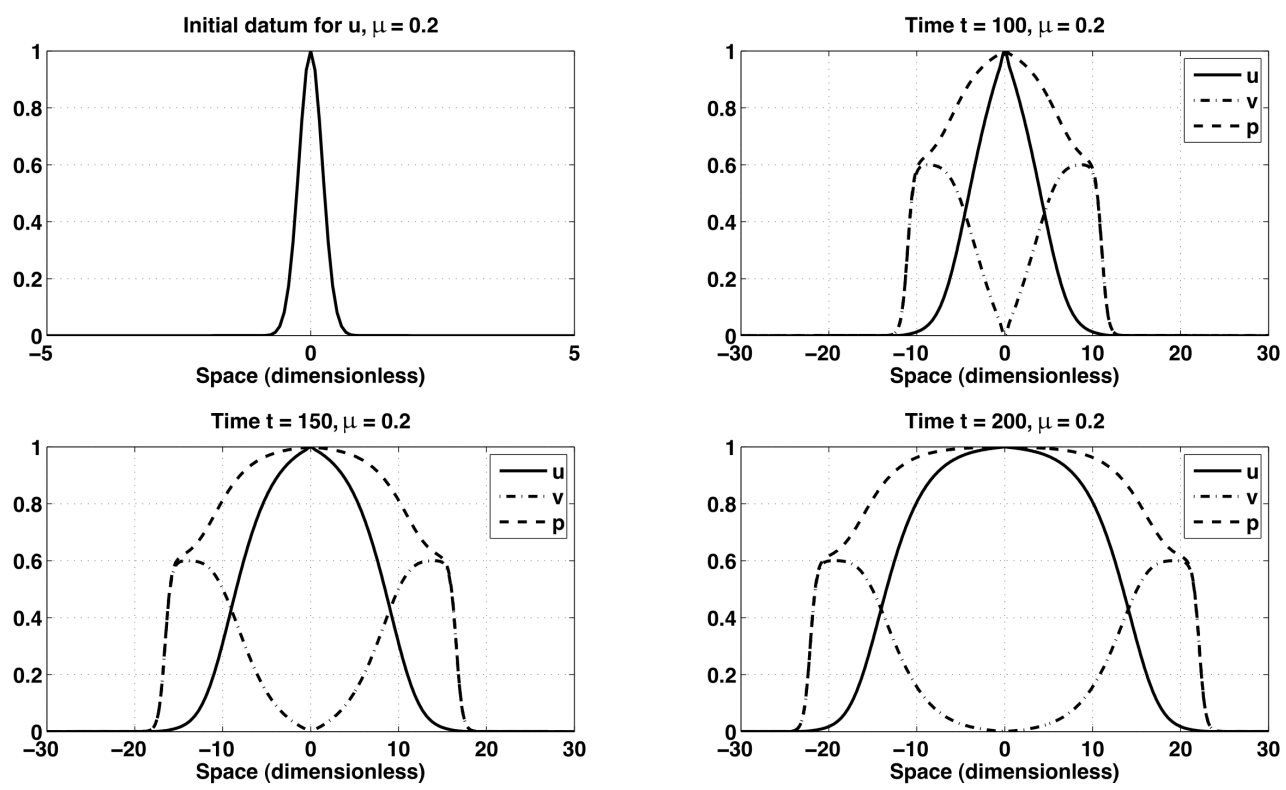
Figure $62-$ Plot of $u, v, p=u+v$ at selected time instants, with initial conditions 8.12 and parameters from 8.10 and 8.11. Case $\mu=2$.
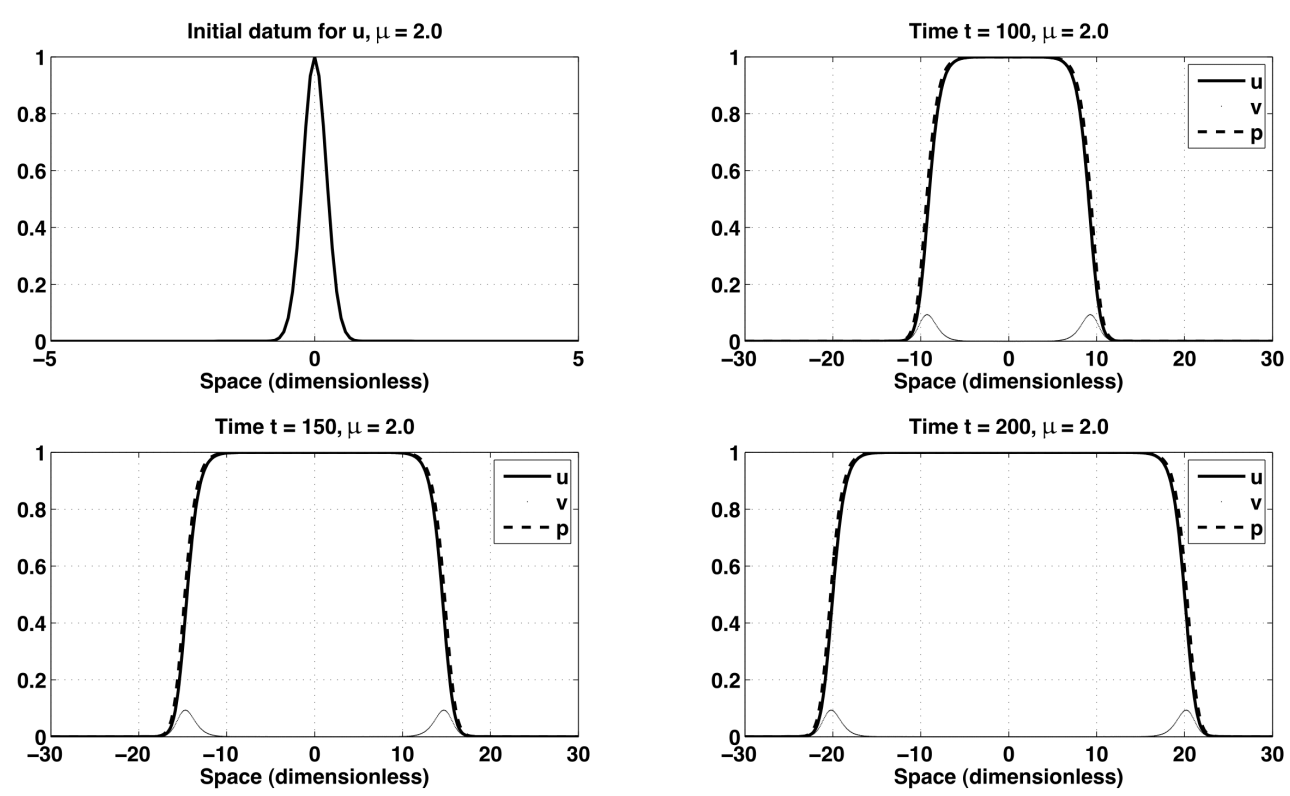

Figure 63 - Left: Time evolution of total population $\Pi(t)$, for $\mu=0.2$ (dotted line) and $\mu=2.0$ (solid line). Right: Time evolution of the level line $p=0.8$, for $\mu=0.2$ and $\mu=2.0$.
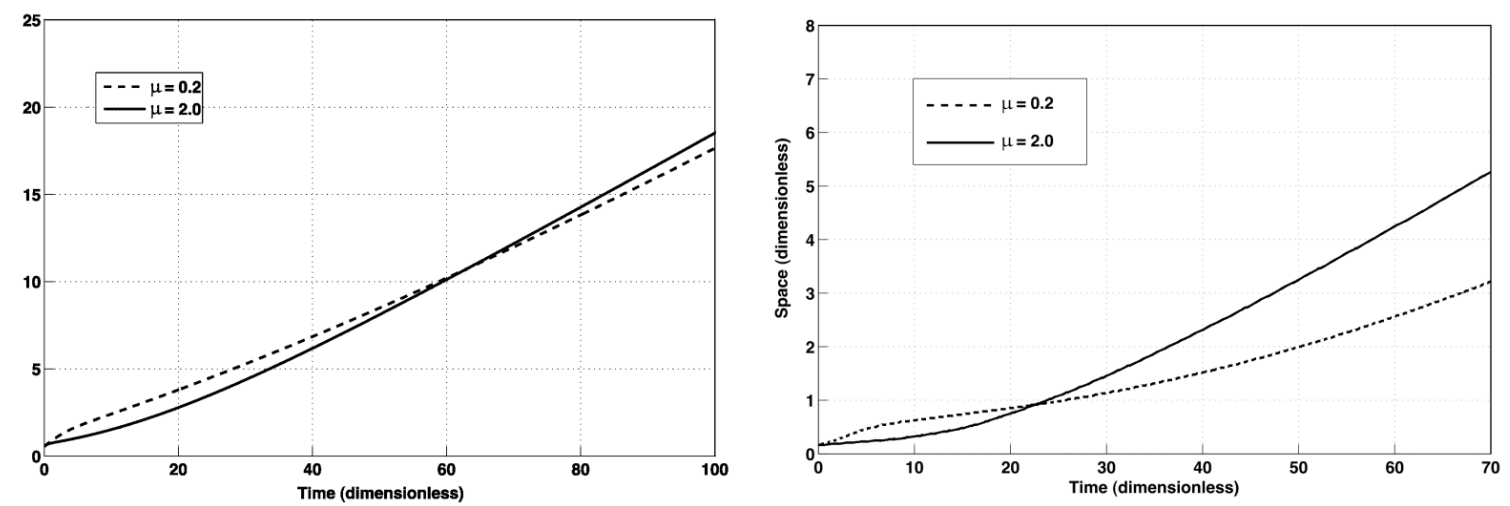

\subsection{A reaction-diffusion model}

Consider the integral appearing in the equation 8.5 in the one-dimensional case $(\Omega \equiv$ $(-\infty,+\infty))$ and approximate $u(y, t)$ till the second order

$$
\begin{aligned}
& \rho_{v} \int_{-\infty}^{+\infty} K(|x-y|) F((p(x, t)) v(y, t) d y \simeq \\
& \simeq \rho_{v} F\left((p(x, t)) \int_{-\infty}^{+\infty} K(|x-y|)\left[v(x, t)+(y-x) v_{x}(x, t)+(y-x)^{2} \frac{v_{x x}(x, t)}{2}\right] d y\right. \\
& \simeq \rho_{v} F\left((p(x, t))\left[A v(x, t)+B v_{x x}(x, t)\right],\right.
\end{aligned}
$$


Figure $64-$ Plot of $\mathrm{p}$ at selected time instants in case of radiation treatment at $t=50$. Case $\mu=0.2$.
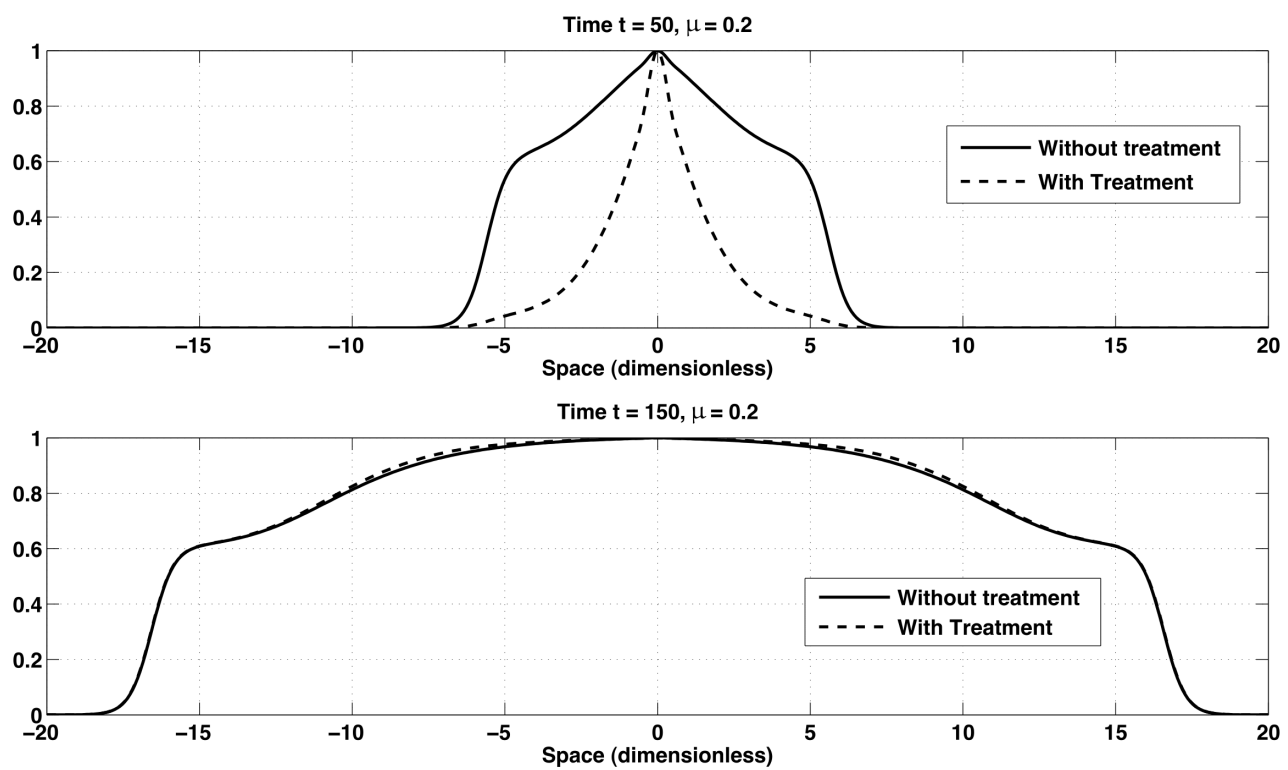

Figure 65 - Left: Time evolution of total population $\Pi(t)$, for $\phi=0.95$ and $\mu=0.2$. Right: Time evolution of the level line $p=0.8$, for $\phi=0.95$ and $\mu=0.2$
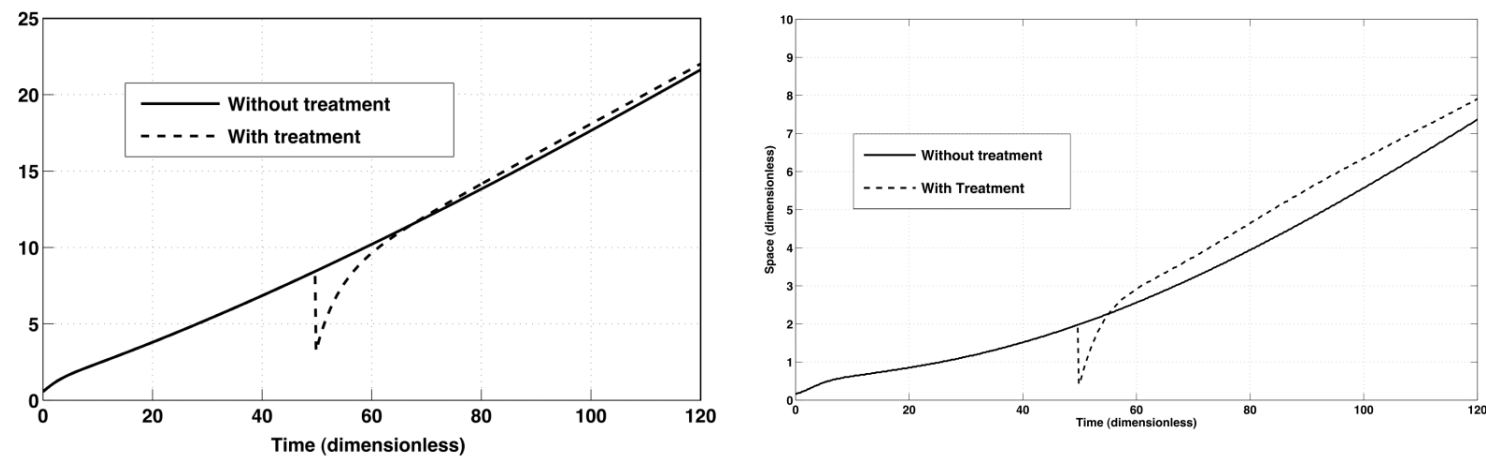

where $A$ is the integral of $K$ and $B$ is half of its second order momentum (the first order momentum vanishes since $K$ is symmetric). In particular, if $K$ is a Gaussian curve

$$
A=1, \quad B=\frac{\sigma_{v}^{2}}{4}
$$

By this approximation (see (CAPASSO; SERIO, 1978)), we transform system (8.5) - (8.6) in the following

$$
\begin{gathered}
\frac{\partial u}{\partial t}=\rho_{u} \delta F(p)\left[u+\frac{\sigma_{u}^{2}}{4} u_{x x}\right] \\
\frac{\partial v}{\partial t}=\rho_{u}(1-\delta) F(p)\left[u+\frac{\sigma_{u}^{2}}{4} u_{x x}\right]+\rho_{v} F(p)\left[v+\frac{\sigma_{v}^{2}}{4} v_{x x}\right]-\mu v .
\end{gathered}
$$

System (8.16) - (8.17) is a classical reaction-diffusion model (by the way an additional diffusion effect can be taken into account if it is present in the original setting of the problem). In 
(FASANO; MANCINI; PRIMICERIO, 2016) a proof is given of the local existence for problem (8.16) - (8.17) supplemented with smooth initial data and usual vanishing conditions at $\pm \infty$. Numerical simulations give results quite similar to the ones displayed in the previous section. Using the same setting of parameters and performing numerical simulations with $\mu=0.25$ and $\mu=2.50$ we have the following plots for $u$, for $v$ and for $p=u+v$ at the time $t=20$ (Fig. 66) and $t=40$ (Fig. 67). Only the part of the plot for $x>0$ is displayed. Figure 68 shows the time evolution of $\Pi$.

Figure 66 - Plots for $u$, for $v$ and for $p=u+v$ at the time $t=20$.
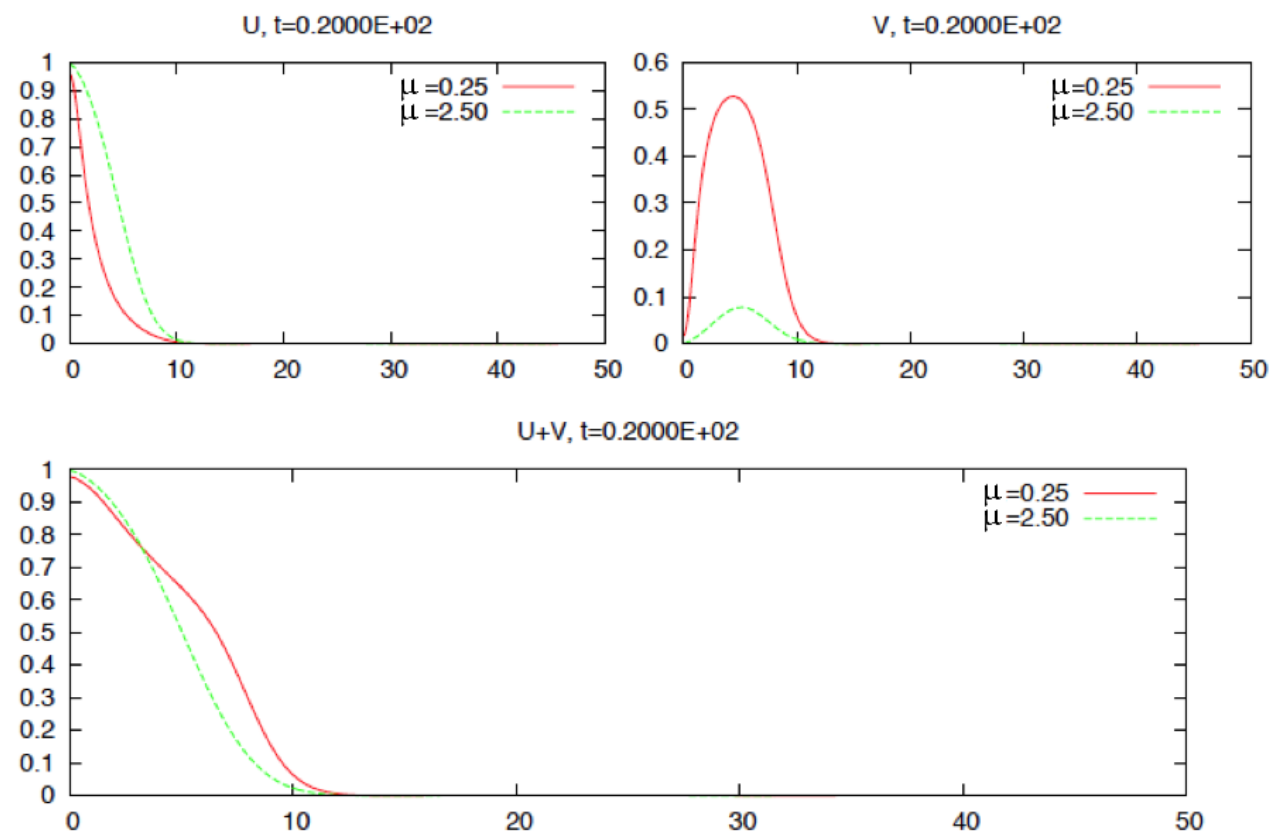

Figure $67-$ Plots for $u$, for $v$ and for $p=u+v$ at the time $t=40$.

$\mathrm{U}, \mathrm{t}=0.4000 \mathrm{E}+02$

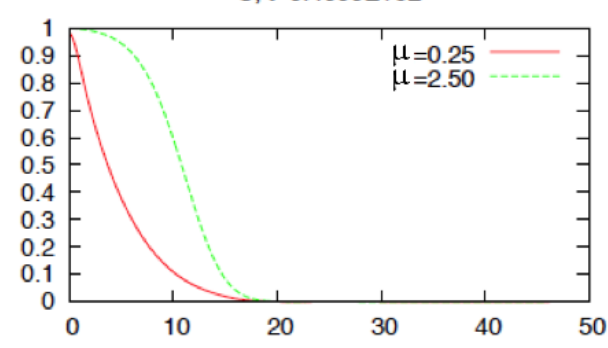

$\mathrm{U}+\mathrm{V}, \mathrm{t}=0.4000 \mathrm{E}+02$

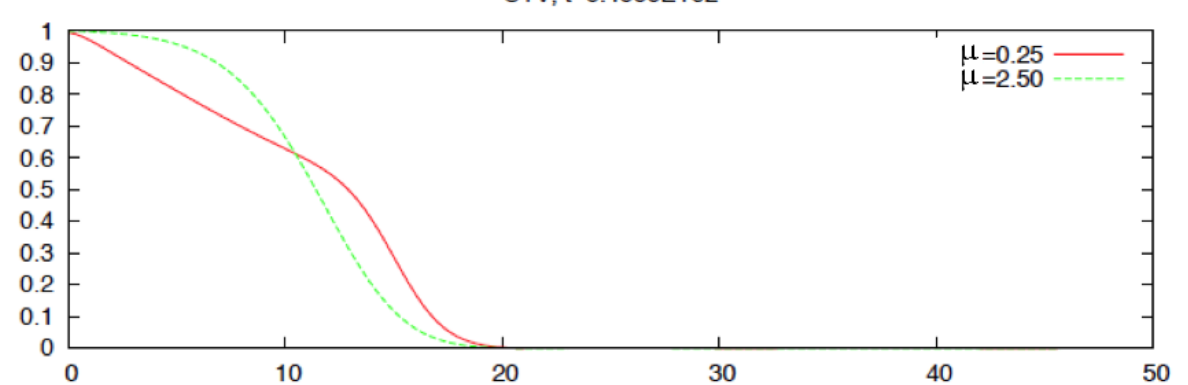


Figure 68 - Time evolution of $\Pi(t)$.

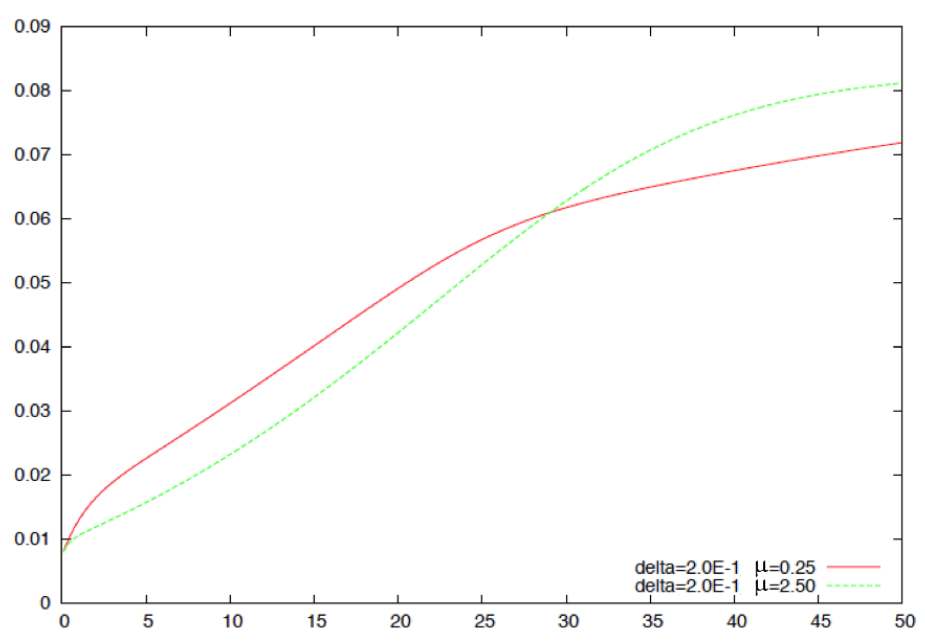

\subsection{Achievements and future opportunities}

We have presented four classes of mathematical models to describe the growth of a tumour characterized by the presence of cancer stem cells (CSC). The simpler model averages over the space variables and represents a sort of mean field approximation to the phenomenon thus resulting in a system of ordinary differential equations for the time evolution of the total number of CSC and of ordinary cancer cells (CC). Notwithstanding this (and other) drastic simplifications, our numerical simulations provide evidence of the basic features that characterize tumours with CSC, in particular the so-called "tumour paradox": if the mortality of the CC is increased the growth of the tumour is accelerated. If space dependence is taken into account, the mathematical aspects of the problem appear to be more complicated. We presented a model based on integro-differential equations and subsequently we have shown how this model (under suitable assumptions) can be transformed in a system of parabolic PDE with reaction diffusion terms. In these cases, numerical simulations have been performed in a one-dimensional setting and show how the radius of the tumour grows with time. Also in this case the effect of "tumour paradox" is present. Simulations with cellular automata have also been performed. In a two-dimensional grid three categories of sites are presents: sites where no cells are present, sites occupied by CSC and sites occupied by CC. The simulations show how the tumour grows, without any prescribed symmetry, and how the two families of cells proliferate. As in the cases described above, the "crowding" effect of the cells appear to be responsible for the occurrence of the tumour paradox. As a consequence, a chemical or radioactive treatment that destroys CC may have the effect of increasing, instead of controlling, the speed at which tumoral cells invade the surrounding space.

As we said, the models do not provide quantitative results but a conceptual framework to interpret experimental data. Simulations with parameters estimated by "in vitro" experiments could just give hints on the strategy of treatment (frequency and intensity of applications) and suggestions on situations to be avoided. Summing up, this paper shows that the tumour paradox can be explained on the basis of a few basic assumptions on the mechanisms of cell proliferation 
and that the phenomenon could be put in evidence in different classes of mathematical models, considering therefore a complete multi-approach point of view. 

CHAPTER

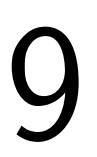

\section{GROWTH OF TUMOURS WITH STEM CELLS: THE EFFECT OF CROWDING AND AGEING OF CELLS}

The heterogeneity of cancer cells is currently considered to be a key factor in determining the evolution of many tumours. The basic idea in explaining the genesis of the inhomogeneities consists, in its simplest version, of assuming that the entire tumour is generated by a small number of cancer stem cells (CSCs) that are immortal and multipotent, in the sense that they are able to proliferate indefinitely and to produce either new-born CSCs or differentiated cancer cells (CCs). The differentiation can occur following distinct lineages of the CCs with their usual cycle of ageing, mitosis, and apoptosis (TOMASETTI; LI; VOGELSTEIN, 2017; TOMASETTI; VOGELSTEIN; PARMIGIANI, 2013). CSCs (originally called tumorigenic cells) were first isolated and described in the hematopoietic system (LAPIDOT et al., 1994), and the importance of their role in tumour growth has been described (REYA et al., 2001; BENÍTEZ; BARBERIS; CONDAT, 2019). In the early 2000's, the existence of CSCs was shown for solid tumours, such as breast and brain cancers (AL-HAJJ et al., 2003). Since then, the number of papers that present theoretical and experimental results in this area has been steadily increasing, and we will limit ourselves to quoting some review papers that contain extensive reference lists (BELLOMO; LI; MAINI, 2008; HUNTLY; GILLILAND, 2005; MICHOR, 2008; VISVADER; LINDEMAN, 2008) and, more recently, (BATLLE; CLEVERS, 2017; CLEVERS, 2011; KAKARALA; WICHA, 2008; LATHIA et al., 2015). Furthermore, we refer the reader to the webpage (Cancer Stem Cell News, 2018) containing a weekly updated list of published papers on CSCs.

In the past decade, many papers have also been devoted to mathematical modelling of the growth of tumours in the presence of CSCs, applying the methods of population dynamics. We can quote, e.g. (BELLOMO; DELITALA, 2008; GANGULY; PURI, 2006; WEEKES et al., 2014; GENTRY; JACKSON, 2013; WERNER et al., 2016; MICHOR et al., 2005; MARCINIAK- 
CZOCHRA; STIEHL; WAGNER, 2009; WERNER et al., 2011; KAVEH; KOHANDEL; SIVALOGANATHAN, 2016), as well as (WEISS; KOMAROVA; RODRIGUEZ-BRENES, 2017) that has an extensive bibliography in this area, updated to 2017.

Several papers use compartmental models: the unknown functions represent abundances of each type of cells $\left(v_{0}, v_{1}, \ldots, v_{N}\right)$ where $v_{0}$ is the fraction of CSCs and $v_{1}, \ldots, v_{N}$ is the fraction of the $N$ classes of successively differentiated cells. In the simplest case (differentiation in cascade), these functions obey $N+1$ ordinary differential equations of the type

$$
\left\{\begin{aligned}
\frac{d v_{0}}{d t} & =r_{0} v_{0}-a_{0} v_{0}-m_{0} v_{0}, \\
& \vdots \\
\frac{d v_{i}}{d t} & =a_{i-1} v_{i-1}-a_{i} v_{i}-m_{i} v_{i}+r_{i} v_{i}, \quad i=1,2, \ldots, N-1, \\
& \vdots \\
\frac{d v_{N}}{d t} & =a_{N-1} v_{N-1}-m_{N} v_{N}+r_{N} v_{N},
\end{aligned}\right.
$$

supplemented with initial conditions. Here $a_{i}(i=0,1, \ldots, N-1)$ represents the differentiation rate of the $i$-th class of cells, and $m_{i}$ and $r_{i}(i=0,1, \ldots, N-1)$ are the mortality and the reproduction rate without differentiation. Models of this class are called hierarchical models since the equations can be solved recursively, starting from the first one.

In other cases, agent-based computer models are used to find mechanisms that drive tumour development and progression; see, e.g. (ANDERSON et al., 2006; ENDERLING et al., 2009), as well the more recent works (MEACCI; PRIMICERIO, 2018; MONTEAGUDO; SANTOS, 2014; MONTEAGUDO; SANTOS, 2015; ROCHA et al., 2018; POLESZCZUK; ENDERLING, 2014; POLESZCZUK; HAHNFELDT; ENDERLING, 2015).

The stem cell assumption is also crucial for describing the tumour growth paradox that consists of an accelerated tumour growth that can be found with an increased cell death that, for example, can result from the immune response or from cytotoxic treatments (WODARZ; KOMAROVA, 2007; GUROVA; GUDKOV, 2003; ENDERLING et al., 2009).

Of course, this fact could have a crucial relevance in connection with the strategy of treatment, to avoid the fact that the latter produces a faster progression of the tumour (DINGLI; MICHOR, 2006; GUROVA; GUDKOV, 2003; WODARZ; KOMAROVA, 2007).

The occurrence of the paradox has been associated with a crowding effect (BETTERIDGE et al., 2006; HILLEN; ENDERLING; HAHNFELDT, 2013; BORSI et al., 2015a; FASANO; MANCINI; PRIMICERIO, 2016; MEACCI; PRIMICERIO, 2018), assuming that mitosis can be inhibited when the density of the cells in the vicinity of the would-be mother cells exceeds a threshold value. ${ }^{1}$ Indeed, numerical simulations based on different models

1 In (RODRIGUEZ-BRENES; KOMAROVA; WODARZ, 2011) general mechanisms of negative feedback are assumed in computational simulations. 
incorporating the crowding effect evidentiate this paradoxical behaviour.

The papers quoted in this context introduce two basic approximations: (i) they neglect the age-dependence of the replication potential and of the mortality of the non-stem cells, and (ii) they group all the non-stem cancer cells in a single population.

The present paper releases these assumptions and considers the simultaneous effects of crowding, multiple differentiation, and ageing.

Throughout this paper, we will assume that CSCs have zero mortality and a constant replicative potential, and that they are capable of generating new CSCs or ordinary non-stem cancer cells (CCs). In the first case, we speak of symmetrical mitosis, while the second case is denoted as asymmetrical mitosis ${ }^{2}$. The fraction of asymmetrical mitosis will be denoted by $d$ (usually larger than 90\%).

We will consider two families of models. In the first family, we present agent-based models consisting of the application of cellular automata whose evolution is governed by stochastic rules, in the sense that given probabilities of replication and apoptosis are prescribed for each class of age and for each differentiation lineage ${ }^{3}$. The models in the second family are deterministic, since the behaviour of the cells is averaged (in each class of age and in each differentiation lineage) as in typical mean field approximations. The mathematical structure of these models, presented in Section. 9.2, consists of a mean field approximation and results in a system of Ordinary Differential Equations (ODEs) in which the unknown functions are fractions of the age classes in each lineage of CCs, irrespective of their position in the region under consideration. Thus, the population of CCs is composed of $m$ sub-populations of differentiated cells with $n$ classes of age (from new-born to old). All of them can only generate new CCs and may undergo apoptosis. The replicative potential and the mortality rate of class $k$-th $(k=1,2, \ldots, m)$ and age $i(i=1,2, \ldots, n)$ will be denoted by $\rho_{k i}$ and $\mu_{k i}$, respectively. We are still in the class of compartmental models but, because of the presence of the crowding term depending on the total number of CCs, the system is much more complex and qualitatively different from the hierarchical models quoted above.

In Sections 9.1 and 9.2, we display some numerical simulations that show that both families of models give the same qualitative results and that both evidentiate the occurrence of the tumour paradox, i.e. an accelerated tumour growth when the mortality of the CCs is increased. It should be noted that the numerical values of the parameters used in the simulations are only speculative, but the role of this kind of conceptual model consists of giving the correct qualitative information regarding the complex mechanisms involved in the phenomenon.

2 As far as the mathematical models are concerned, it is immaterial whether different classes of differentiated CCs are generated in cascade or in parallel.

3 In the example that will be displayed in Section 9.1, all the probabilities are supposed to be spaceindependent and constant-in-time. Moreover, the ageing process is assumed to be deterministic. However, generalisations in these directions could be incorporated with some additional computational work. 
Finally, in Section 9.3, we consider a generalisation of the family of deterministic models, assuming that a continuous age distribution, instead of a structure in age classes, is given. The mathematical aspects of the problem are substantially different. Here, we have a system of one ODE for the evolution of the fraction of CSCs and $m$ first-order Partial Differential Equations (PDEs), one for each lineage of CCs.

\subsection{Cellular automata}

For the sake of simplicity, we will confine ourselves to the case of a single lineage of CCs (i.e., we take $m=1)$ and three classes of age $(n=3)$ : new-born cells $(i=1)$, adult cells $(i=2)$, and old cells $(i=3)$. More general cases give essentially similar results. We imagine the cells to be living in a square lattice ${ }^{4}$ of $50 \times 50$ sites, and we prescribe probabilistic rules for their replication or death. At each time step, each site in the lattice can be in one of the following states:

- vacant sites (white);

- sites occupied by a CSC (black);

- sites occupied by a young CC (red);

- sites occupied by an adult CC (orange);

- sites occupied by an old CC (yellow).

Starting from a given situation at time $t_{k}$, each CC (red, orange, or yellow) has a probability $\mu_{1}, \mu_{2}, \mu_{3}$ of undergoing apoptosis (becoming white) in the time interval corresponding to the time step of the Cellular Automata (CA). Then, the surviving cells have probabilities $\rho_{1}, \rho_{2}, \rho_{3}$ of being potentially replicant. However, in this case, mitosis is only possible if there are vacant (white) sites in the chosen neighbourhood; if this situation occurs a daughter CC appears and one of these white sites (randomly chosen) becomes red, as well as the site of the mother cell. The situation with CSCs is similar but with two differences: (i) $\mu=0$, and (ii) if mitosis occurs a daughter CSC appears with probability $1-d$, while the asymmetrical proliferation (i.e. the appearance of a new-born CC) has probability $d$. The replication probability is $\rho_{0}$.

Remark 9.1 Of course, the phenomenon might be much more complex with respect to this simplified scheme. For instance, possible dormancy of some CSCs should be considered, so that the replicative probability is not the same for all cells but a (possibly variable) stochastic distribution should be introduced. Similarly, distribution for ageing and mortality of the CCs could be taken into consideration. We remark that the model is sufficiently flexible to deal

4 A tri-dimensional lattice could easily be considered with a little computational work (see (MEACCI $e t$ al., 2019)). 
with these generalisations. We choose to keep the number of parameters as small as possible to illustrate some fundamental aspects of this complex phenomenon.

It is natural to assume that $\rho_{0}>\rho_{1}>\rho_{2}>\rho_{3}$ and that $\mu_{1}<\mu_{2}<\mu_{3}$.

In what follows we assume that dying cells leave the site they occupied immediately blank, but we can also simulate cases in which apoptosis gives rise to necrotic material that occupies the site for some time (grey sites), and, possibly, this material influences the surrounding cells (intoxicating effect).

According to the crowding effect, we have assumed that cells can proliferate only if in their neighbourhood ${ }^{5}$. Some lattice sites are vacant; more specifically, the replicative potential is scaled in the function of the sites that are vacant in the neighbourhood.

On the other hand, it is possible that the mother cell pushes the adjacent cells if all of the close neighbourhood is occupied. To take this effect into account, we consider a hierarchy of neighbourhoods: the 1st order is formed by the 8 sites of the Moore's neighbourhood, the second order is formed by the 24 cells of the $5 \times 5$ neighbourhood and so on. If the first-order neighbourhood is occupied, the algorithm counts the vacant sites in the 2nd order neighbourhood, and so on. However, we postulate that some energy is spent in the case of displacement, thus decreasing the replicative potential accordingly.

Finally, we remark that in our simulations, cells will be supposed to be immobile, but it is evident that random motion (e.g. diffusion) or drift can be incorporated into the model as well. In the sequel, we display results in the basic case (no intoxication nor pushing).

As the initial condition for the simulations displayed below, we took a single CSC situated in the centre of the grid. The setup of the parameters is

$$
d=0.9, \quad \rho_{0}=1, \quad \rho_{i}=\rho_{1}(1-i \varphi), \quad \mu_{i}=\mu_{1}(1+i \theta), \quad i=2,3 .
$$

Parameters $\theta$ and $\varphi$ were fixed to be 0.1 , while $\rho_{1}$ and $\mu_{1}$ were chosen in each experiment.

The choice of the value of the parameters is only speculative because the model is aimed at reproducing the characteristics of the evolution of tumour growth and not at giving an exact prediction of the dimension of the tumour. In fact, the role of conceptual modelling is to mimic the relevant features of the phenomenon. Nevertheless, even in this oversimplified model, it is, in principle, possible to experimentally determine realistic values for the parameters. We remark that

(i) choosing $\rho_{0}=1$ is nothing other than choosing the time unit equal to the average length

5 Different definitions of neighbourhood are possible: for the $2-D$ lattice, a Von Neumann or Moore neighbourhood can be used (4 orthogonal neighbours - north, south, east, west - or 8 neighbours - adding northwest, northeast, southwest, southeast - respectively). We use Moore neighbourhoods unless otherwise specified. 
of the reproduction cycle of CSCs under consideration;

(ii) the average fraction $d$ of the asymmetric reproduction can be determined by in vitro experiments;

(iii) in the simulations we have assumed that the permanence of the CCs in each age class is, on average, the same as the lifecycle of the CSCs, i.e. we chose the amplitude of the age classes accordingly, and this can be easily changed to correspond with the experimental conditions;

(iv) plausible values for $\theta$ and $\varphi$ could easily be found, once the amplitude of the age classes has been chosen.

We assume that at each time step the CCs that do not replicate or die change their state (from new-born to adult, to old). Thus, to take $\rho_{0}=1$ corresponds to assuming that the interval between the two replications of a CSC is of the same order as the time of permanence of a CC in an age class.

In Figure 69 we show some screenshots (at different times) of a simulation corresponding to $\rho_{1}=0.5$ and $\mu_{1}=0.25$.

It is a common characteristic of agent-based models that the individual behaviour of each cell contributes to the evolution of the system and that the latter can be visualised as a collective behaviour, possibly averaging over several simulations in the same conditions to evidentiate the response of the model to different setups of parameters and conditions. This is what is shown in Figure 70, where the averages are taken over 20 simulations. The curves show the evolution of the total number of cancer cells (CSCs and the three classes of CCs) as a function of time. Next, we compare the behaviour for two different values of the mortality rate. In Figure 71, the blue curve corresponds to $\mu_{1}=0.25$, while the magenta curve corresponds to $\mu_{1}=0.35$. The tumour paradox is clearly evident: a higher mortality of the CCs produces a faster progression of the tumour.

The same algorithm can be used to simulate the effect of a treatment that, for some prescribed time, destroys a fraction $g$ of the CCs. For instance, it assumes that in the time interval $t \in[200,350] \mu_{1}$ is switched from 0.25 to 1 . As can be seen in Figure 72 , the reduction of the dimensions of the tumour is only temporary. Comparing the situation with this aggressive treatment with the one with constant $\mu_{1}=0.25$ for all time, the paradox is evident.

\subsection{Compartmental models}

A general compartmental model for a system of CSCs and $m$ lineages of differentiated cells and $n$ classes of age can generally be written in the following form 
Figure 69 - Screenshots of the simulation of the evolution of the tumour growth according to the setup (9.2) and $\rho_{1}=0.5$ and $\mu_{1}=0.25$. In white, we display the vacant sites; in black we show the sites occupied by a CSC, while red, orange, and yellow show the sites occupied by a young, adult, and old CC, respectively.
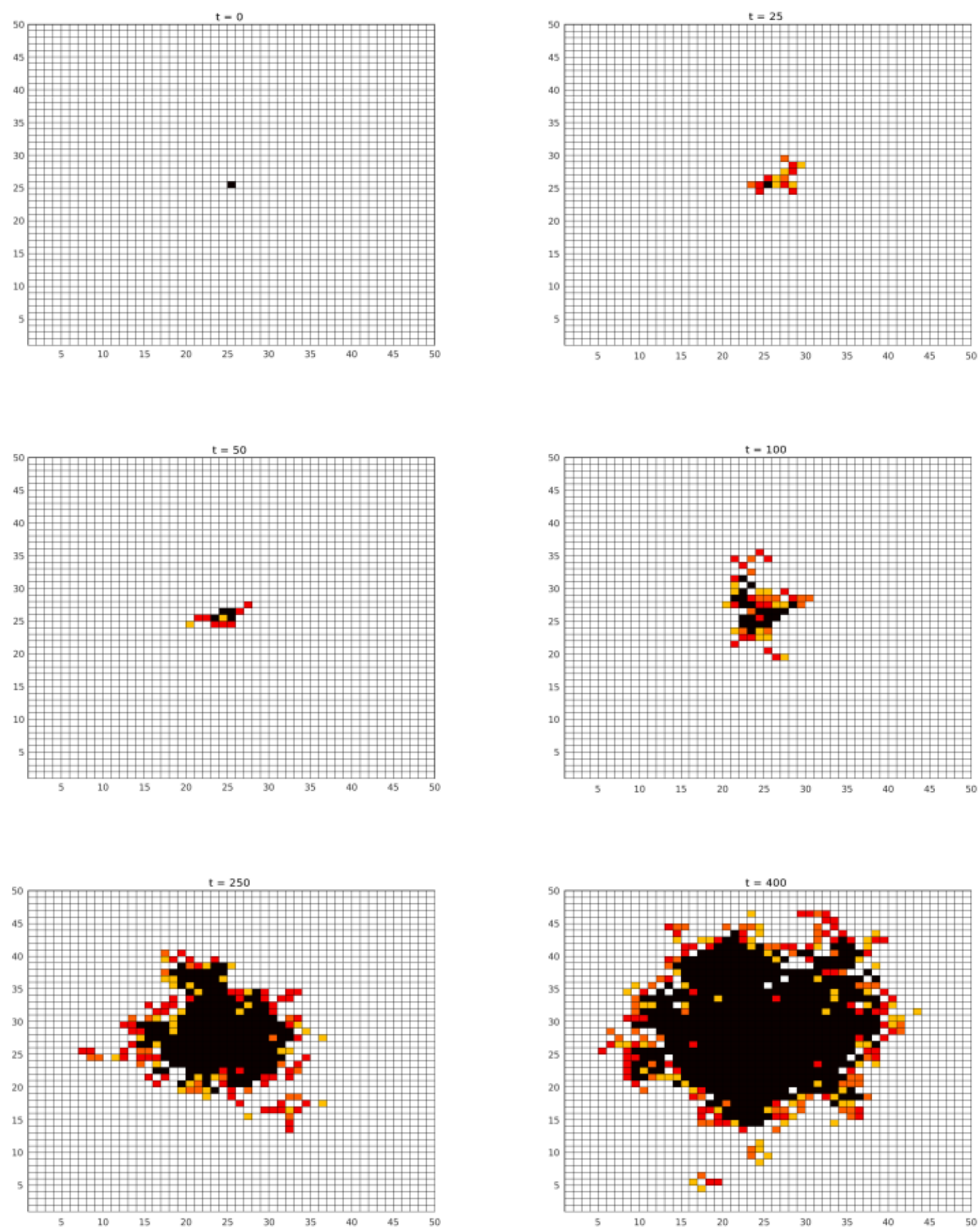
Figure 70 - Evolution of the fraction of cancer cells (CSCs on the top and the three classes of CCs on the bottom) as a function of time.
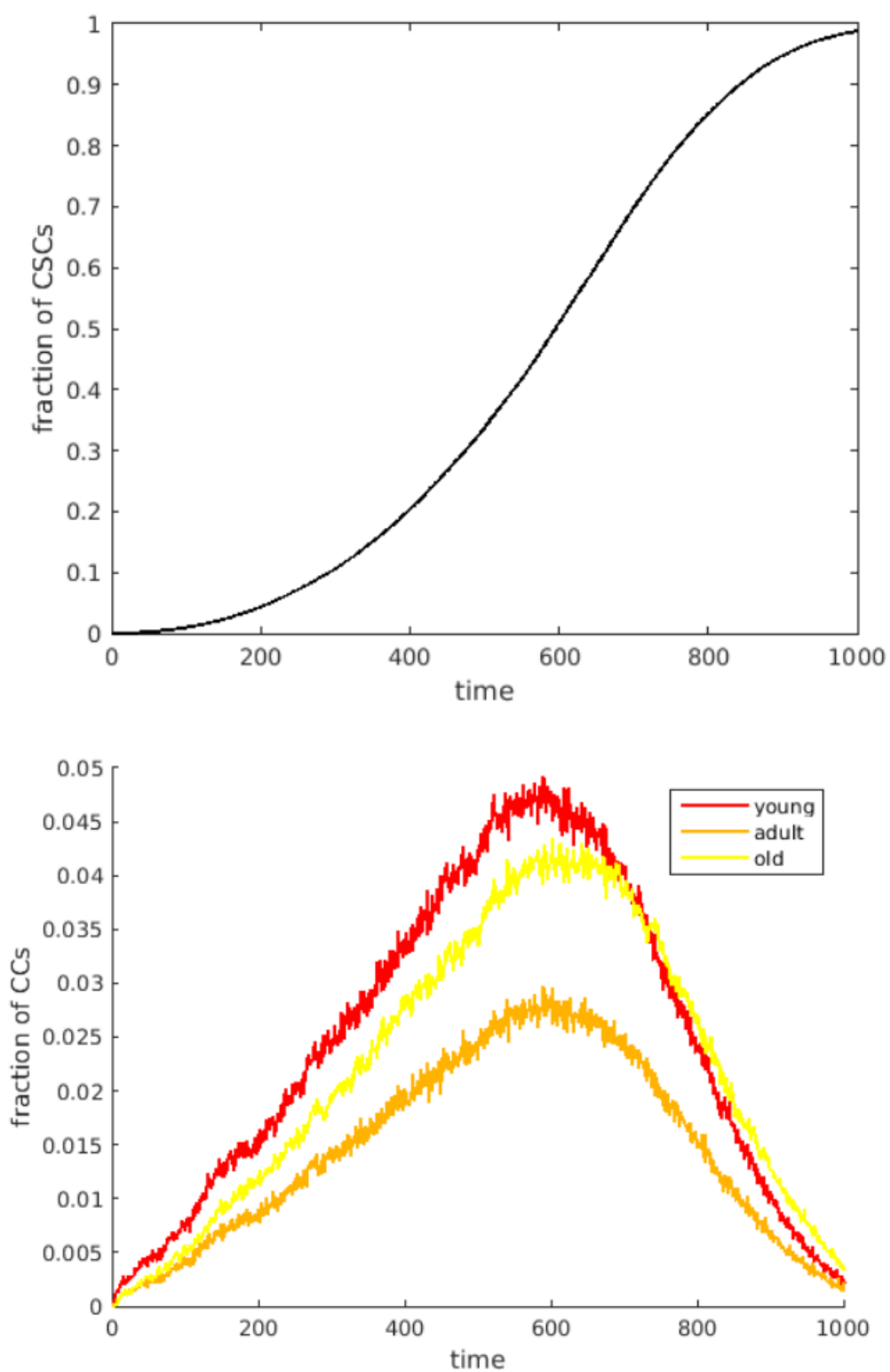

$$
\left\{\begin{aligned}
\frac{d u}{d t} & =\rho(1-d) u, \\
\frac{d v_{1 i}}{d t} & =\rho d_{i} u+2 \sum_{j=1}^{n} \sum_{k=1}^{m} \rho_{j k \neq 1 i} \rho_{j k \rightarrow i} v_{j k}-\sum_{k=1}^{m} \rho_{1 i \rightarrow k} v_{1 i} \\
& -\left(\mu_{1 i}+\psi_{i}\right) v_{1 i}+\rho_{1 i \rightarrow i} v_{i}, \\
& \vdots \\
\frac{d v_{k i}}{d t} & =\psi_{i} v_{k-1, i}-\psi_{i} v_{k i}-\mu_{k i} v_{k i}-\sum_{j=1}^{n} \rho_{k i \rightarrow j} v_{k i}, \\
& \vdots \\
\frac{d v_{n i}}{d t} & =\psi_{i} v_{n-1, i}-\mu_{n i} v_{n i}-\sum_{j=1}^{n} \rho_{n i \rightarrow j} v_{n i} .
\end{aligned}\right.
$$


Figure 71 - Evolution of the fraction of all cancer cells (CSCs and CCs) applying $\mu_{1}=0.25$ (blue curve) and $\mu_{1}=0.35$ (magenta curve).

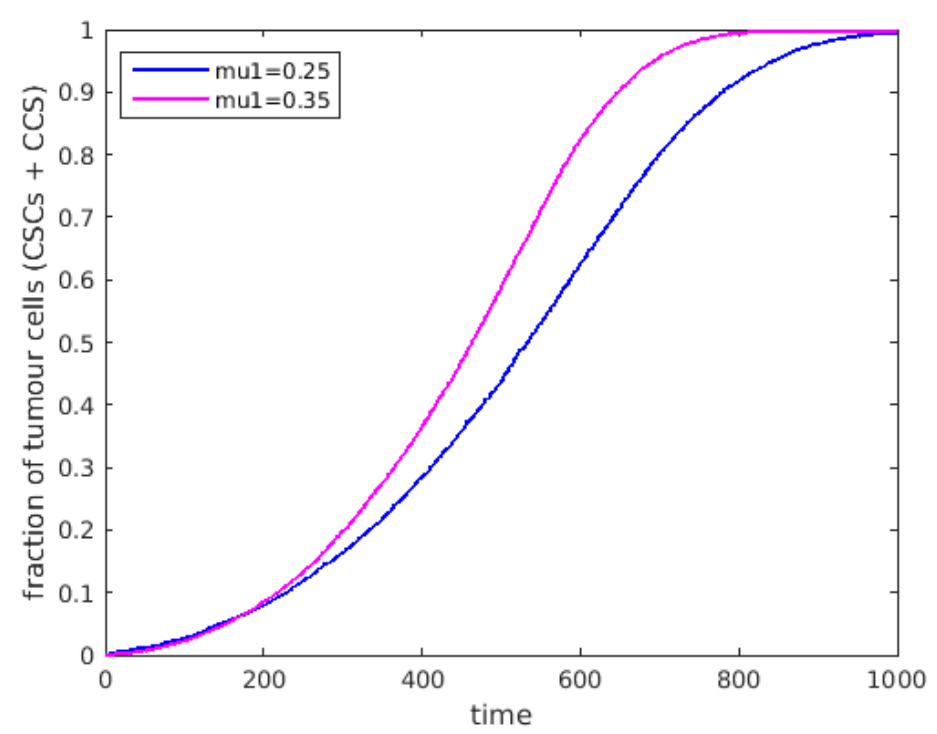

Figure 72 - Evolution of the fraction of all cancer cells (CSCs and CCs) applying $\mu_{1}=0.25$ (blue curve) and $\mu_{1}=1$ when $t \in[200,350]$ and $\mu_{1}=0.25$ elsewhere (green curve).

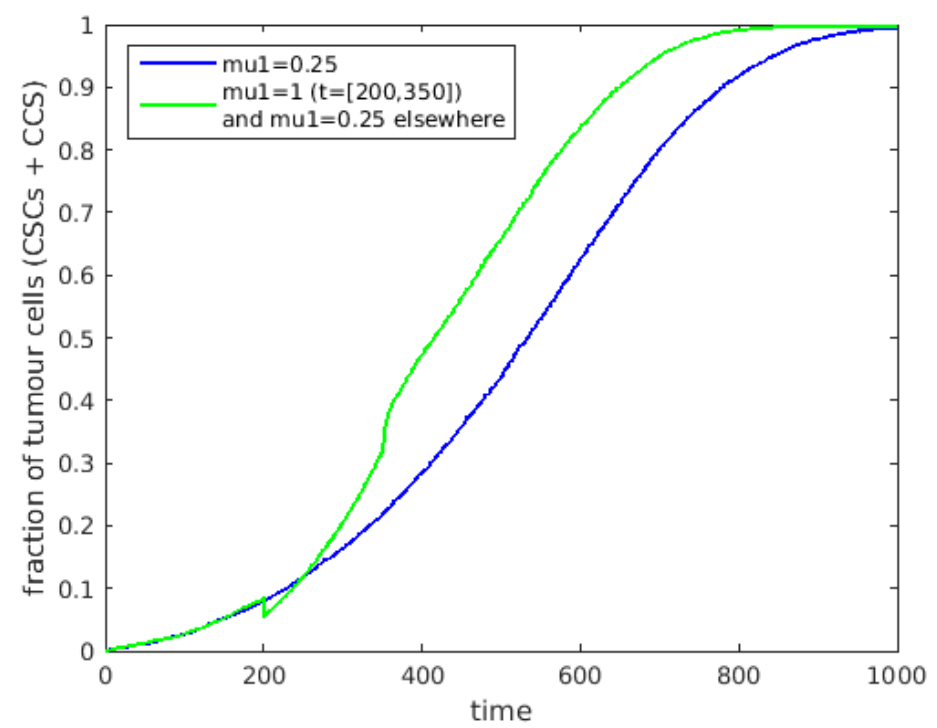

Here $v_{k i}$ is the fraction of cells of lineage $i$ and the class age $k, \rho$ is the replication potential of the CSCs; $d=\sum_{i=1}^{m} d_{i}$ is the ratio of asymmetrical mitosis ${ }^{6}, \mu_{k i}$ is the mortality of the cells in the compartment $(k i), \rho_{k i \rightarrow j}$ is the replication potential of the cells in compartment (ki) to produce cells in the lineage $j$, and $\psi_{i}$ represents the ageing for lineage $i$.

The system (9.3) is composed of $n \times m+1$ first-order ordinary differential equations that have to be solved starting from an equal number of initial conditions. It is clear that the

6 To save notation we have assumed that a mitosis with differentiation produces two cells (in the age class 1) of the same lineage. 
system represents a mean field approximation of the tumour, because the dependence of the cell fractions on the position is not taken into account. Of course, some of the parameters appearing in (9.3) may vanish. For instance, if each lineage of CC can only be generated by a CSC and evolves with age, independently of the others, then the only replication terms appearing in the equations are $\rho_{k i \rightarrow i}$.

So far, no crowding effect has been taken into account. In the spirit of the mean field approximation, we can introduce this effect by multiplying each replicative potential in (9.3) by a function $F(p)$ of the total fraction $p$ of all the cells present in the domain. Quantity $p$ is given by

$$
p=u+\sum_{j=1}^{n} \sum_{k=1}^{m} v_{j k}
$$

and the function $F(p)$ will be a decreasing function of $p$ such that $F(0)=1$ and $F(1)=0$.

It is clear at this point that the system is no longer hierarchical. Even in the simplest cases, the term $F(p)$ introduces in each equation the full set of the unknown functions. This means that is it not possible to solve the system recursively, and, of course, this fact introduces a relevant difference and complexity with respect to the other compartmental models quoted in the introduction of this Chapter 9.

To be specific, to avoid dealing with cumbersome notation and increasing the number of parameters to be chosen, from now on, we will consider the case $m=1$ (one lineage of non-stem cells) and three classes of age (new-born, adult and old cells) as we did in the simulations with cellular automata. Simultaneously, we will include the crowding factor $F(p)$ in the differential equations. We point out that all the simulations we will show can be duplicated in more complex cases with some additional computational work.

Thus we will study in detail the following system of ODE

$$
\left\{\begin{aligned}
\frac{d u}{d t} & =\rho_{0}(1-d) F(p(t)) u(t), \\
\frac{d v_{1}}{d t} & =\rho_{0} d F(p(t)) u(t)+\rho_{1} v_{1} F(p)+2 \rho_{2} v_{2} F(p(t))+2 \rho_{3} v_{3} F(p(t))-\mu_{1} v_{1}-\psi v_{1}, \\
\frac{d v_{2}}{d t} & =\psi v_{1}-\rho_{2} v_{2} F(p(t))-\mu_{2} v_{2}-\psi v_{2}, \\
\frac{d v_{3}}{d t} & =\psi v_{2}-\rho_{3} v_{3} F(p(t))-\mu_{3} v_{3},
\end{aligned}\right.
$$

$$
u(0)=u_{0} \geq 0, v_{1}(0)=v_{10} \geq 0, v_{2}(0)=v_{20} \geq 0, v_{3}(0)=v_{30} \geq 0,
$$


where

$$
p(t)=u(t)+v_{1}(t)+v_{2}(t)+v_{3}(t)
$$

and it is assumed that

$$
p(0)=u_{0}+v_{10}+v_{20}+v_{30} \leq 1 .
$$

The case $u_{0}=0$ would correspond to a model without CSCs and is simpler but outside our interest.

We will assume that $F(p)$ is a Lipschitz continuous decreasing function such that $F(0)=1$ and $F(1)=0$. For technical reasons, we will extend $F(p)$ so that

$$
F(p)=1 \text { for } p \leq 0, \quad F(p)=0 \text { for } p \geq 1
$$

The ageing $\psi$ represents the inverse of the time of permanence of the cells in each age class. Of course, the conclusions we will reach in this section also apply to cases in which there is a different value of $\psi$ for each class.

By standard techniques, it can be easily proved that the set $u \in(0,1), v_{i} \in(0,1)$ and $p \in(0,1)$ is invariant for system $(9.5)$.

As a consequence, $u=1, v_{i}=0, i=1,2,3$ is the unique equilibrium point for (9.5) (9.8). It is globally attractive and the solution of

$$
\frac{d u}{d t}=\rho_{0}(1-d) F(u) u
$$

is a super-solution for (9.5). For the case of $F(p)=1-p$, the solution of (9.10) is a logistic.

In the following simulations, we assume, as in section 9.1

$$
d=0.9, \quad \rho_{0}=1, \quad \rho_{1}=0.5, \quad \rho_{i}=\rho_{1}(1-i \varphi), \quad \mu_{i}=\mu_{1}(1+i \theta), \quad i=2,3 .
$$

We take $\theta=\varphi=0.1$ and $F(p)=1-p$, and the initial conditions are chosen in accordance with the simulations that we carried out with $\mathrm{CA}$

$$
u(0)=1 / 2500, \quad v_{1}(0)=v_{2}(0)=v_{3}(0)=0 .
$$

Since time has been normalised by choosing $\rho_{0}=1$, we set $\psi$ accordingly as the ratio of the average time between two replications (for CSCs without space constraints) and the average time spent in each age class. In what follows, we set $\psi=1$ according to the CA simulations. 
We can numerically solve the system (9.5) with initial conditions (9.6) with standard solvers for ordinary differential equations as the Matlab function ode45 that implements a Runge-Kutta method with a variable time step for efficient computation.

Figure 73 - Evolution of the normalised volume $p(t)$ of the tumour cells (CSCs and CCs) applying $\mu_{1}=0.25$ (blue curve) and $\mu_{1}=0.35$ (magenta curve).

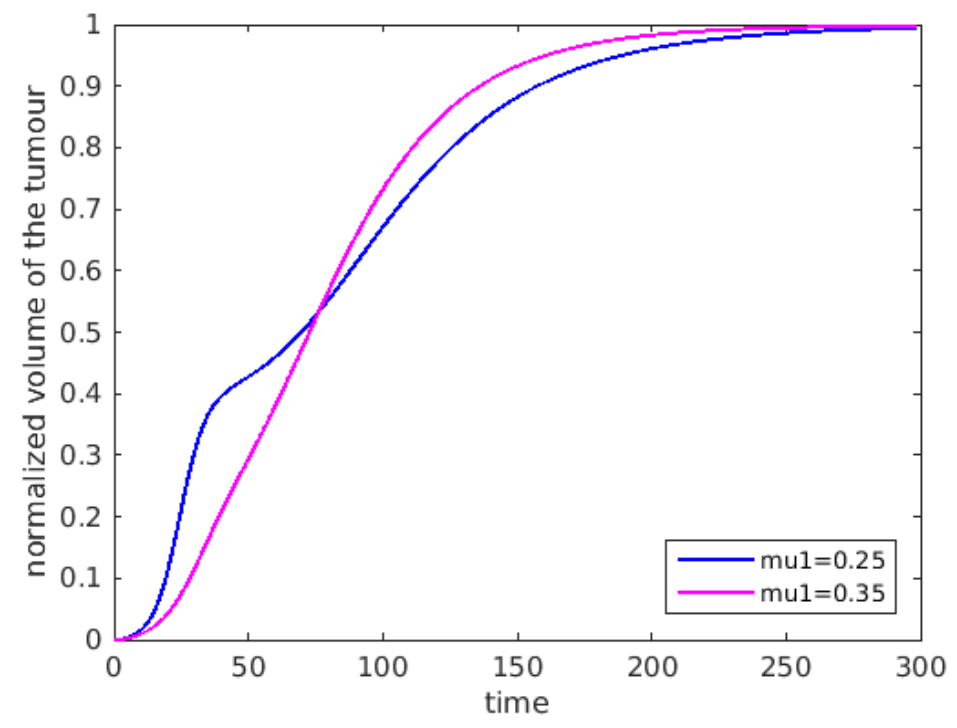

In Figure 73, we show the quantity $p(t)$ (representing the normalised volume of the tumour) and we compare the cases of $\mu_{1}=0.25$ and $\mu_{1}=0.35$. The curves exhibit the occurrence of the tumour paradox, although this fact is less evident than in the curves of Figure 71 that correspond to the CA approach. This fact can be easily explained in terms of the different choice of the crowding term: in the case of simulations with CA, crowding was introduced as a local effect, counting the empty sites in the vicinity of the mother cell. In the mean field approximation of this section we chose to mimic crowding by a term $F(p)$ and, thus, depending on the fraction of the total space available. This is also the reason why the speed of growth is larger in this case.

\subsection{Continuous age-structure for the non-stem cells}

In this section, we will model the evolution of the tumour assuming that there is a single lineage of CCs but considering the case of a continuous age structure.

As in the usual models of population dynamics, the age structure of the population of non-stem cells can be represented by introducing a function $v(a, t)$ such that, for any non-negative $t, a_{1}$ and $a_{2}\left(a_{1}<a_{2}\right)$, the integral

$$
\int_{a_{1}}^{a_{2}} v(a, t) d a
$$

represents the fraction of CCs that, at time $t$, have an age between $a_{1}$ and $a_{2}$. 
Assuming that the biological age coincides with the chronological age $\mathrm{e}^{7}$, the equation regulating the evolution of the age distribution in the population of the $\mathrm{CCs}$ will be:

$$
\frac{\partial v(a, t)}{\partial a}+\frac{\partial v(a, t)}{\partial t}=-\bar{\mu}(a) v(a, t)-\bar{\rho}(a) v(a, t) F(p(t)), \quad t>0, \quad a>0
$$

while the fraction $u(t)$ will still obey

$$
\frac{d u}{d t}=\rho_{0}(1-d) u(t) F(p(t)), \quad t>0
$$

where

$$
p(t)=u(t)+\int_{0}^{+\infty} v(a, t) d t, \quad t>0 .
$$

In (9.14), we denoted the mortality and replication potential of the CCs as functions of their age by $\bar{\mu}(a)$ and $\bar{\rho}(a)$.

The system has to be supplemented by initial conditions

$$
u(0)=u_{0}, \quad v(a, t)=v_{0}(a), \quad a>0,
$$

and by a boundary condition expressing the number of new-born CCs $(a=0)$ generated by the replication of the whole population, i.e.

$$
v(0, t)=v_{0}(0)+\rho_{0} d \int_{0}^{t} F(p(\tau)) u(\tau) d \tau+2 \int_{0}^{t} F(p(\tau)) \int_{0}^{+\infty} \bar{\rho}(a) v(a, \tau) d a d \tau
$$

We assume

H1 $\quad u_{0}>0, \quad \rho>0, \quad d \in(0,1)$,

H2 $0<\underline{b} \leq v_{0}(a) \leq \bar{b}<1$ is continuous in $[0, \alpha]$ and vanishes for $a>\alpha$,

H3 $0<\underline{C} \leq \int_{0}^{\alpha} v_{0}(a) d a+u_{0} \leq \bar{C}<1$.

We have the following immediate result

Proposition 9.2 For any solution (9.14) - (9.18) with data satisfying (H1) - (H3) and for any $T>0$, there exists $A>0$ such that:

$$
v(a, t)=0, \quad \text { for } a>A, \quad t \in(0, T) .
$$

\footnotetext{
7 Otherwise a constant factor would appear in the first term of the equation (9.14).
} 
Proof The characteristic lines for (9.14) are the straight lines $t=a+c$. Therefore (9.19) is satisfied for any $A \geq \alpha+T$. Q.E.D.

We define $S$ to be the support of $v$, and we will look for a pair $(u, v), u \in C^{1}[0, T]$, $v \in C(\bar{S}) \cap C^{1}(S \cap(0, T)), v \equiv 0$ in $(0, A) \times(0, T) \backslash S$ satisfying (9.17) and such that (9.14) holds in $(0, A) \times(0, T),(9.15)$ holds in $(0, T)$, and (9.18) is satisfied where the upper limit in the integrals is $A$ instead of $+\infty$.

We will assume:

H4 $\bar{\rho}(a)$ and $\bar{\mu}(a)$ are positive and continuous in $[0, A]$,

H5 $F(p)$ is a non-increasing Lipschitz-continuous function in $(-\infty,+\infty)$, with $F(p)=1$, $p \leq 0$ and $F(p)=0, p \geq 1$.

Let us consider the following auxiliary problem:

$$
\left\{\begin{array}{llll}
\frac{\partial w}{\partial a}+\frac{\partial w}{\partial t} & =g(a, t), & & 0<a \leq A, \quad 0<t<T, \\
w(a, 0) & =w_{0}(a), & & 0 \leq a \leq \alpha, \quad w_{0}(a)=0, \quad a \in[\alpha, A] \\
w(0, t) & =\Gamma(t ; w(a, t)), & & 0<t<T,
\end{array}\right.
$$

where

$$
g(a, t) \leq 0, \quad-b_{1} \leq w_{0}(a) \leq-b_{2}<0
$$

and $\Gamma(t ; w)$ is a functional such that for any bounded $w$ and suitably small $T$ it is:

$$
\begin{gathered}
-B_{1} \leq \Gamma(t ; w) \leq-B_{2}<0, \\
\left|\Gamma\left(t ; w_{1}\right)-\Gamma\left(t ; w_{2}\right)\right| \leq c(t)\left\|w_{1}-w_{2}\right\|,
\end{gathered}
$$

where $c(t)=O(T)$ and \|\| is the uniform norm.

We choose $\hat{w}(a, t) \in C([0, A] \times[0, T])$, and define $G(t)=\Gamma(t ; \hat{w}(a, t))$. Then we solve (9.20) where the third condition is replaced by $w(0, t)=G(t)$. 
We have immediately

$$
w(a, t)= \begin{cases}w_{0}(a-t)+\int_{0}^{t} g(z+a-t, z) d z, & t<a<A, 0<t<T, \\ G(t-a)+\int_{0}^{a} g(z, z+t-a) d z, & 0<a<t, 0<t<T .\end{cases}
$$

We denote the set of continuous functions $[0, A] \times[0, T]$ by $\Sigma$ such that $-C_{1} \leq w \leq$ $-C_{2}<0$ where $C_{1}>\max \left(B_{1}, b_{1}\right)$ and $C_{2}=\min \left(B_{2}, b_{2}\right)$. The procedure just described defines a mapping of $\Sigma$ into itself for suitable small $T$ because of (9.21), (9.22), (9.24).

Moreover, (9.23) guarantees that the mapping is contractive (reducing $T$ if necessary). Using Banach's fixed point theorem we conclude the proof of

Proposition 9.3 If (9.21) - (9.23) are satisfied, problem (9.20) has one unique solution in a suitable time interval $(0, T)$.

We are now in a position to prove the following

Theorem 9.4 Under the assumptions (H1) - (H5), the problem (9.14) - (9.18) has one unique solution for a suitable $T$.

Proof We again use a fixed point argument. We prescribe a continuous function $\tilde{p}(t)$ such that

$$
0<\underline{K} \leq \tilde{p}(t) \leq \bar{K}<1
$$

when $\bar{K}>C$ and $\underline{K}<\underline{C}$ of assumption (H3). Let $\tilde{F}(t)=F(\tilde{p}(t))$ and consider the problem

$$
\left\{\begin{array}{lll}
\frac{d u}{d t} & =\rho(1-d) \tilde{F}(t) u, & t \in(0, T), \\
\frac{\partial v}{\partial a}+\frac{\partial v}{\partial t}=-(\bar{\mu}(a)+\bar{\rho}(a) \tilde{F}(t)) v, & a \in(0, A), t \in(0, T),
\end{array}\right.
$$

with conditions

$$
u(0)=u_{0}, \quad v(a, 0)=v_{0}(a),
$$

$$
v(0, t)=v_{0}(0)+\rho d \int_{0}^{t} \tilde{F}(\tau) u(\tau) d \tau+2 \int_{0}^{t} \tilde{F}(\tau) \int_{0}^{+\infty} \bar{\rho}(a) v(a, \tau) d a d \tau .
$$


Hence

$$
u(t)=u_{0} \exp \left[\rho(1-d) \int_{0}^{t} \tilde{F}(\tau) d \tau\right]
$$

and the function

$$
w(a, t)=\ln v(a, t)
$$

solves a problem of type (9.20) where

$$
\begin{gathered}
g(a, t)=-\bar{\mu}(a)-\bar{\rho}(a) \tilde{F}(t) \\
w_{0}(a)=\ln v_{0}(a), \\
\Gamma(t ; w(a, t))=\ln \left[v_{0}+\rho d \int_{0}^{t} \tilde{F}(\tau) u(\tau) d \tau+2 \int_{0}^{t} \tilde{F}(\tau) \int_{0}^{A} \bar{\rho}(a) \exp [w(a, t)] d a d \tau\right] .
\end{gathered}
$$

Of course (9.29) and (9.30) guarantee that (9.21) is fulfilled since (H2), (H4), and (H5) are supposed to hold. Moreover, (H1) and (9.27) ensure that, for any bounded $w$, the functional $\Gamma$ defined by (9.33) is the logarithm of $v_{0}$ plus a positive function of $O(t)$ so that, since $v_{0}$ satisfies (H2), there exist two constants $B_{1}$ and $B_{2}$ such that (9.22) holds for a suitable $T>0$.

By reducing, if necessary, $T$ we have that (9.23) holds as well. Therefore, problem (9.26) - (9.28) is uniquely solvable by Proposition 9.2.

This means that, for any $\tilde{p}(t) \in C[0, T], 0<\underline{C} \leq \tilde{p} \leq \bar{C}<1$ we have found two functions $u(t)$ given by (9.29) and

$$
v(a, t)=\exp (w(a, t)),
$$

with

$$
v(a, t)=\left\{\begin{array}{lc}
v_{0}(a-t)+O(t), & t<a<A, \\
v_{0}(t-a)+O(t) & 0<a<t .
\end{array}\right.
$$

Therefore

$$
\int_{0}^{A} v(a, t) d a=\int_{0}^{A} v_{0}(a) d a+O(t) .
$$


But it is also

$$
u(t)=u_{0}+O(t)
$$

so that

$$
p(t)=u(t)+\int_{0}^{A} v(a, t) d a+O(t)
$$

and reducing $T$ if necessary we conclude

$$
\underline{K} \leq p(t) \leq \bar{K}
$$

This means that we have defined a mapping

$$
p(t)=\mathscr{T} \tilde{p}(t)
$$

that maps the set of continuous functions satisfying (9.34) into itself.

To prove that $T$ can be chosen so that $\mathscr{T}$ is a contraction is immediate: take $\tilde{p}_{1}$ and $\tilde{p}_{2}$ and define $\Delta \tilde{p}=\left\|\hat{p}_{1}-\hat{p}_{2}\right\|$ and $\Delta \tilde{F}=\left\|F\left(\tilde{p}_{1}\right)-F\left(\tilde{p}_{2}\right)\right\|$. Assume (H5) yields $\Delta \tilde{F} \leq D \Delta \tilde{p}$.

Now let $u_{i}(t), v_{i}(a, t)$ be the solutions of (9.26) - (9.28) with $\tilde{F}$ replaced by $F\left(p_{i}(t)\right), i=$ 1,2. We have

$$
\begin{aligned}
& \Delta u=\left|u_{1}-u_{2}\right|=O(t) \Delta \tilde{p}, \\
& \Delta v=\left|v_{1}-v_{2}\right|=O(t) \Delta \tilde{p} .
\end{aligned}
$$

Consequently

$$
\Delta p=\Delta u+\int_{0}^{A} \Delta v d a=O(t) \Delta \tilde{p}
$$

and $T$ can be chosen so that $O(t)<1$, thus proving the contractivity of the mapping.

The application of Banach's fixed point theorem concludes the proof. Q.E.D.

We conclude this section by remarking that the proof of existence and uniqueness can be extended as long as, for $t=T$, assumptions (H2) and (H3) are fulfilled for some constants $\underline{b}, \bar{b}$, $\underline{C}$ and $\bar{C}$. 


\subsection{Achievements and future opportunities}

We have presented two families of models for the growth of tumours in the presence CSCs and of CC's. We have considered the simultaneous effect of crowding, differentiation, and ageing. First, we have considered agent-based models based on the application of cellular automata. Then, a non-hierarchical compartmental model has been presented in which the crowding effect is not included as a local effect but is averaged over the whole domain. Numerical results of simulations for the two families of models have been displayed, showing the same qualitative behaviour. In particular, we have shown in both cases the occurrence of the tumour paradox: tumours in which the death rate of the CCs is higher may have faster growth. This fact is, of course, relevant in connection with the treatment strategies and, indeed, an example has been displayed in which the killing of tumour cells may not induce, in the long run, the slowing down of the growth of the cancer.

While in the models just described, the ageing has been modelled dividing the differentiated cells in age classes, in a final section, we have considered the more general case in which there is a continuous distribution in the age of the population of the differentiated cells. The resulting mathematical model is non-standard but its well-posedness has been proved. 
CHAPTER

10

\title{
THE AMYLOID CASCADE HYPOTHESIS AND ALZHEIMER'S DISEASE: A MATHEMATICAL MODEL
}

\begin{abstract}
Alzheimer's Disease (AD), a neurodegenerative disease with a huge social and economic impact, is the prevalent form of late life dementia (HURD et al., 2013). The biomedical knowledge about the AD-pathology is rapidly growing, as witnessed by a vast literature, but the etiology, the mechanisms and the progression of the AD-pathology, as well as their relationship to clinical and cognitive manifestations, are still far from understood. The trajectories of AD-progression in the patient's brain are highly stereotyped but vary individually. The lack of full understanding of the pathophysiology of AD-progression, its insidious onset and the clinical heterogeneity and variability in speed and pattern progression, severely complicate the rigorous characterisation and prognosis of the disease, and hampers informed, data-driven clinical intervention.
\end{abstract}

In this context, there is a pressing need to find and validate biomarkers to track and predict disease progression. Several biomarkers are currently under investigation: genetic analysis (APOE status, Presenilin, APP), cerebrospinal fluid homogenates (CSF tau protein, amyloid $\mathrm{A} \beta_{42}$ protein), imaging (atrophy, glucose PET for hypometabolism, AV45-PET for amyloid, T807- or AV1451-PET for tau) and cognitive tests (ADAS-cog, AVLT, MMSE).

In this paper we use the in silico approach, based on mathematical modelling and computer simulations, and supplementary to the huge amount of in vivo and in vitro research (see (GOOD; MURPHY, 1996; CRUZ et al., 1997; URBANC et al., 1999; MURPHY; PALLITTO, 2000; EDELSTEIN-KESHET; SPIROSS, 2002; ACHDOU et al., 2013; HELAL et al., 2013; FRIEDMAN; HAO, 2016; BERTSCH et al., 2017; BERTSCH et al., 2017; CARBONELL; ITURRIA-MEDINA; EVANS, 2018); we refer in particular to (CARBONELL; ITURRIAMEDINA; EVANS, 2018) for a comprehensive updated overview of mathematical approaches in 
the literature.

The general idea is to develop a highly flexible model of the interplay between the excess of $\mathrm{A} \beta_{42}$ protein in the Central Nervous System (CNS) and the progression of the disease, focussing on the early stage of the disease where there is still hope to intervene and stop the further development of the disease. Flexibility of the model is essential: it means that one can test several modelling hypotheses based on medical input and easily adapt the model to new medical insight.

Roughly speaking, the $\mathrm{A} \beta$ protein is normally produced during life by neurons in the CNS through intramembranous proteolysis of APP (amyloid precursor protein), a large transmembrane protein involved in signal transduction pathways (O'BRIEN; WONG, 2011). For unknown and partially genetic reasons, some neurons - referred to as malfunctioning neurons present an unbalance between produced and cleared $\mathrm{A} \beta$. This ultimately leads to the presence of highly toxic oligomers of, among other isoforms, $A \beta_{40}$ and $A \beta_{42}$. For the sake of simplicity, from now on we shall write $\mathrm{A} \beta$.

More precisely, $\mathrm{A} \beta$ oligomers are subject to two different phenomena:

- agglomeration, leading eventually to the formation of long, insoluble amyloid fibrils, which accumulate in spherical microscopic deposits known as senile plaques;

- diffusion through the microscopic tortuosity of the brain tissue.

In addition, recently it has been proposed that neuronal damage spreads in the neuronal net through a neuron-to-neuron prion-like propagation mechanism (BRAAK; TREDICI, 2011; RAJ; KUCEYESKI; WEINER, 2012).

Agglomeration can be articulated in several steps (CHIMON et al., 2007; ONO; CONDRON; TEPLOW, 2009): initial seeds, soluble small oligomers, protofibrils and insoluble polymers, and amyloid fibrils with a $\beta$-sheet conformation. However, this level of description is beyond the scope of our model, as will be explained in detail in the next section. The connection between $\mathrm{A} \beta$ and $\mathrm{AD}$ relies on the fact that, as is largely accepted, $\mathrm{A} \beta$ amyloid plays an important role in the process of the cerebral damage (the so-called amyloid cascade hypothesis (KARRAN; MERCKEN; STROOPER, 2011)). In particular some soluble A $\beta_{42}$ oligomers have been suggested as the principal cause of neuronal death and eventually dementia (WALSH; SELKOE, 2007).

Soluble $\mathrm{A} \beta$ shows a multiple neurotoxic effect: it induces a general inflammation that activates the microglia which in turn secretes proinflammatory innate cytokines (GRIFFIN et al., 1998) and, at the same time, increases intracellular calcium levels (GOOD; MURPHY, 1996), which ultimately leads to apoptosis and neuronal death.

Although senile plaques are associated with $\mathrm{AD}$, their presence is not strictly related to 
the severity of the disease. High levels of soluble $\mathrm{A} \beta$ correlate better with the presence and degree of cognitive deficits than plaque statistics. Indeed, diffuse amyloid plaques are commonly present in the brains of cognitively intact elderly people. Some authors (see, for instance (HAASS; SELKOE, 2007)) overturn the traditional perspective, and claim that large aggregates of $A \beta$ can actually be inert or even protective to healthy neurons. Analogously, $\mathrm{A} \beta$ monomers have been shown to lack neurotoxicity (SHANKAR et al., 2008) and have in fact been suggested to be neuroprotective (ZOU; GONG; YANAGISAWA K.AND MICHIKAWA, 2002; GIUFFRIDA et al., 2009).

In the present paper we introduce a model to describe the toxic effect of beta-amyloid on neurons. Proceeding in the spirit of mean field approximation, we divide a given cerebral region in a number of smaller subregions. In each of them we write a system of ordinary differential equations to describe the evolution of the number of beta-amyloid monomers, oligomers and plaques. These equations depend on the average health state of the neurons in the subregion under consideration, which is updated at given times (say every half a year, a relatively "long" period which reflects the slow evolution of the disease). The updating depends on both the amount of toxic oligomers which are present in the subregion itself and the average health state in neighbouring subregions. We present some numerical simulations to illustrate the behaviour of solutions and their parameter dependence.

We stress again that the present model only takes into account the evolution of the $\mathrm{A} \beta$, and ignores the role played by the microglia in neuronal death and in the formation of senile plaques. For these aspects, we refer for instance to (MEYER-LUEHMANN et al., 2008) and (EDELSTEIN-KESHET; SPIROSS, 2002). In the same spirit we ignore also the progressive degeneration of the brain due to aging.

The paper is organised as follows. In Section 10.1 we briefly review some of the mathematical models of Alzheimer's disease and in Section 10.2 we present the basic model. In Section 10.3 we discuss a specific example of the model and present numerical simulations. We also provide the corresponding source MATLAB codes, which are available in GitHub repository at the URL: <https://github.com/LucaMeacci/Alzheimer_MathModel_Ejam>. Finally, in the Appendix, existence, positivity and asymptotic behaviour of the example introduced in Section 10.3 are discussed.

\subsection{Mathematical modelling}

The mathematical model of aggregation and diffusion of $\mathrm{A} \beta$ analyzed in the present paper is based on the so-called Smoluchowski coagulation equations, originally introduced by Smoluchowski (SMOLUCHOWSKI, 1917) in 1917 to describe the binary coagulation of colloidal particles moving according to Brownian motion. Subsequently, these equations were used to model a variety of phenomena such as the evolution of a system of solid or liquid particles 
suspended in a gas (in aerosol science), polymerisation (in chemistry), aggregation of colloidal particles (in physics), formation of stars and planets (in astrophysics), red blood cell aggregation (in hematology), behaviour of fuel mixtures in engines (in engineering), etc. Moreover, several additional physical processes have been subsequently incorporated into the model (diffusion, fragmentation, condensation, influence of external fields, see, e.g. (DEACONU; TANRÉ, 2000; LAURENÇOT; MISCHLER, 2002; WRZOSEK, 1997)). We refer also to (DRAKE, 1972), (DUBOVSKII, 1994), and (ACHDOU et al., 2013; BERTSCH et al., 2017; BERTSCH et al., 2017; FRANCHI; LORENZANI, 2016; HERRERO; RODRIGO, 2005) for a more exhaustive account of the literature on the Smoluchowski system.

In spite of the large literature on applications of Smoluchowski equations in many branches of science, in the field of biomedical research their use seems to be rather limited. Here we only consider the applications of coagulation equations to mathematical models related to AD. For different approaches, in particular to prion-like diffusion and role of the tau-protein, we refer the reader to (CARBONELL; ITURRIA-MEDINA; EVANS, 2018).

As far as we know, Murphy and Pallitto (MURPHY; PALLITTO, 2000; PALLITTO; MURPHY, 2001) were the first ones who used Smoluchowski equations to describe $A \beta$ agglomeration, starting from an in vitro approach. More recently, a systematic approach to the modelling of $\mathrm{A} \beta$-agglomeration and the formation of senile plaques was carried on in a series of papers (ACHDOU et al., 2013; FRANCHI; TESI, 2012; BERTSCH et al., 2017; BERTSCH et al., 2018; BERTSCH et al., 2017; FRANCHI; LORENZANI, 2016; FRANCHI; LORENZANI, 2017; FRANCHI; HEIDA; LORENZANI, 2019; CHEN; TSENG; WARD, 2019; CRAFT; WEIN; SELKOE, 2002). In (ACHDOU et al., 2013; FRANCHI; TESI, 2012; CHEN; TSENG; WARD, 2019) the authors consider a model at microscopic scale. They use suitable Smoluchowski type equations to describe the diffusion and agglomeration of soluble $\mathrm{A} \beta$-oligomers of different lengths in small portion of the cerebral parenchyma, of the size of the soma of a single neuron (from 4 to $100 \mu \mathrm{m}$ ), and the formation of plaques, identified with insoluble assemblies of very long polymers. Some other phenomena were also included in the model, such as fragmentation of long polymers (FRANCHI; TESI, 2012) and clearance of A $\beta$ from the CSF (CHEN; TSENG; WARD, 2019).

A macroscopic model was proposed in (BERTSCH et al., 2017; BERTSCH et al., 2018). The authors couple the set of truncated Smoluchowski equations already used in (ACHDOU et $a l ., 2013)$ to a kinetic-type transport equation that models the spreading of neuronal damage, including the possibility of spreading through neuron-to-neuron prion-like transmission. The model takes into account both the microscopic phenomena of diffusion and aggregation of the $\mathrm{A} \beta$ peptide, characterised by a short time scale (of a few days, the time needed for the formation of the senile plaques: see (MEYER-LUEHMANN et al., 2008)) and the macroscopic spreading of the disease and the associated cerebral atrophy in large parts of the brain, with a long time scale (of several years, the time needed for the development of the disease). Remarkably, the model 
involves mathematical quantities (concentration of plaques, neuronal damage) that have a precise counterpart in terms of clinically observed parameters through PIB-PET (Pittsburgh compoundB, to detect senile plaques: see e.g. (COHEN et al., 2012)) and FDG-PET (fluorodeoxyglucose PET, to evaluate the brain metabolism of both glucose and oxygen: see e.g. (MOSCONI et al., 2010)).

Finally, the papers (BERTSCH et al., 2017; FRANCHI; LORENZANI, 2016; FRANCHI; LORENZANI, 2017; FRANCHI; HEIDA; LORENZANI, 2019) are dedicated to the transition from the microscopic scale to the macroscopic scale, through different mathematical procedures: (BERTSCH et al., 2017) adapts arguments from modern Boltzmann-type kinetic theory for multi-agent systems (PARESCHI; TOSCANI, 2013), while (FRANCHI; LORENZANI, 2016; FRANCHI; LORENZANI, 2017; FRANCHI; HEIDA; LORENZANI, 2019) rely on homogenisation procedures (in (FRANCHI; LORENZANI, 2016; FRANCHI; LORENZANI, 2017) neurons are assumed to be periodically distributed, whereas (FRANCHI; HEIDA; LORENZANI, 2019) introduces randomness of the distributions of neurons and the onset of the disease).

Recently, the production of the $\mathrm{A} \beta$ and then the onset of $\mathrm{AD}$ has been associated with the tau protein, a prion-like intra-neuronal protein (see e.g. (KAMETANI; HASEGAWA, 2013); we refer to (TATARNIKOVA; ORLOV; N.V, 2015) for a careful overview of the subject). Therefore also the mathematical modelling of prion-like proteins could be relevant to $\mathrm{A} \beta$ mdelling. We refer for instance to (GREER; PUJO-MENJOUET; WEBB, 2006; HELAL et al., 2013; PRüSS et al., 2006), but we refer once more to (CARBONELL; ITURRIA-MEDINA; EVANS, 2018) for an exhaustive panorama of the literature.

In most of the previous papers the diffusion of the amyloid as well as the (possible) prion-type infection is assumed to be uniform, whereas it has been observed that, if we are looking to a real macroscopic model, the disease diffuses between different regions of the brain according to the anatomical connection strength between them. To describe this kind of diffusion, a network model has been introduced in (MATTHÄUS, 2006; RAJ; KUCEYESKI; WEINER, 2012; RAJ et al., 2015; FORNARI et al., 2019; SCHÄFER; WEICKENMEIER; KUHL, 2019; WEICKENMEIER et al., 2019; FORNARI et al., 2020).

\subsection{The model}

As we explained in the Introduction, our basic assumption is that the progression of AD is related to the evolution of the amount of $\mathrm{A} \beta$ in the cerebro-spinal fluid (CSF). Amyloids are produced as monomers by neurons, diffuse in the CSF (with a diffusivity that decreases with their size), and may aggregate and polymerise, producing longer polymeric chains. In polymerisation (DEACONU; TANRÉ, 2000; LAURENÇOT; MISCHLER, 2002; WRZOSEK, 1997) the use of the Smoluchowski equation can be described as follows. For $k \in \mathbb{N}$, let $P_{k}$ denote a polymer of length $k$, formed by $k$ identical units (monomers). If the polymers are sufficiently close, they 
can merge into a single polymer whose length equals the sum of the length's of the two (only binary reactions are considered). The merge of a polymer of size $k$ with a polymer of size $j$ can be written as

$$
P_{k}+P_{j} \longrightarrow P_{k+j}
$$

We postulate that there exists a number $n$ such that polymers of length $<n$ are soluble and those of length $\geq n$ are immobile. In the spirit of the dynamics of continuous media, we adopt a strategy of mean field approximation and divide the cerebral region under consideration in a sufficiently large number of elements (representative elementary volumes, REV). A more precise discussion on the dimension of the REV will be presented when we will introduce the concept of "cerebral degradation". We denote by $w_{i}(t)$ the number of soluble amyloid polymers of length $i(i<n)$ contained in the given REV at time $t$, while $W_{n}(t)$ will denote the total number of immobile particles in the REV.

For the sake of simplicity we assume that there exists a constant $K>0$ which does not depend on $i$ and $j$ such that the aggregation rate $R_{i, j}$ of two soluble polymers of length $i$ and $j$ is given by

$$
R_{i, j}= \begin{cases}K w_{i} w_{j} & \text { if } i, j<n, i \neq j, \\ \frac{1}{2} K w_{i}\left(w_{i}-1\right) \approx \frac{1}{2} K w_{i}^{2} & \text { if } i, j<n, i=j .\end{cases}
$$

Similarly, assuming that two immobile polymers do not merge, we postulate that the aggregation rate for a soluble and an immobile polymer is given by

$$
R_{i, n}=R_{n, i}=K^{*} w_{i} W_{n} \quad \text { if } i<n
$$

Since the probability of merging is smaller if one of the two polymers is immobile, we assume that $K^{*}<K$.

The evolution of the number of monomers in a given REV is described by the differential equation

$$
\frac{d w_{1}}{d t}=-K w_{1} \sum_{j=1}^{n-1} w_{j}-K^{*} w_{1} W_{n}+\Lambda-M_{1} w_{1},
$$

where $\Lambda>0$ is the production rate of monomers and $M_{1}>0$ their mortality rate, i.e. the clearance due to both the phagocytic activity of the microglia and the possible reabsorption by blood vessels (see (PLOG; NEDERGAARD, 2018; BACYINSKI et al., 2017)). The evolution of the number of oligomers, $w_{s}(t)$ with $1<s<n$, is described by

$$
\frac{d w_{s}}{d t}=\frac{K}{2} \sum_{i+j=s} w_{i} w_{j}-K w_{s} \sum_{j=1}^{n-1} w_{j}-K^{*} w_{s} W_{n}-M_{s} w_{s}
$$

Finally, the differential equation for the number of immobile aggregates is

$$
\frac{d W_{n}}{d t}=\frac{K}{2} \sum_{i+j \geq n ; i, j<n} w_{i} w_{j}-M_{n} W_{n} .
$$


The next step is to model the progression of $\mathrm{AD}$. We associate the average degree of malfunctioning of the neurons in each REV with a parameter $a$ ranging from 0 to $1 ; a=0$ means that this portion of the brain is healthy, while $a=1$ corresponds to complete degeneration. At this point we discuss the dimension of the REV. It should be large enough to contain a sufficiently large number of neurons so that it makes sense to define the quantity $a$ as the average degree of malfunctioning of the neurons contained in the REV. On the other hand, the dimensions of the REV are to be taken small enough so that the variation of $a$ over the macroscopic scale of the cerebral region are identifiable. Ideally, the experimental resolution of the FDG-PET, extensively discussed in (MOSES, 2011), should suggest how to choose the size of the REV, which could very well depend on the part of the brain under consideration. In reality, the size of the REV is also determined by computational costs, an issue which becomes of critical importance in the case of 3D simulations. For each REV we assume that $a$ is a nondecreasing function of time, and its variation is essentially due to two different effects:

- a local effect, due to the level of toxicity of the amyloids in the REV itself;

- a non-local effect, induced by degradation of the adjacent REV's.

Concerning the local effect, the current opinion is that, while monomers are innocuous, neurons degenerate if the concentration of soluble toxic oligomers exceeds a given threshold. The relative influence $\gamma_{i}>0$ of each oligomer is still under investigation, but we can define the quantity

$$
D=\sum_{i=2}^{n-1} \gamma_{i} w_{i},
$$

and assume that degradation occurs when $D$ exceeds a threshold value $D^{*}>0$.

Since the typical timescale of degradation is much slower than that of aggregation, it is reasonable to assume that there exists $T>0$ such that $a$ is constant in each of the time intervals $(0, T),(T, 2 T),(2 T, 3 T), \ldots$, and update the value of $a$ at the times $T, 2 T, 3 T$ etc. Typically, $(0, T)$ can be thought of as a period of half a year.

Based on these considerations we postulate for the local effect the relation

$$
a(t)=a(m T)+\theta\left[D(m T)-D^{*}\right]_{+} \quad \text { for } m T<t \leq(m+1) T,
$$

where $[\cdot]_{+}$means the positive part and $\theta$ is a given positive constant.

To model the non-local effect, we assume that the REV's in which we divide the cerebral region under investigation are cubes of equal size stacked in such a way that each of them has faces in common with its neighbors. We define the neighborhood of a REV (located in the interior of the region) as formed by the 26 cubes that have at least one vertex in common with it. If we consider the region embedded in a "virtual frame" formed by cubes where $a$ is constantly equal to zero, the definition applies to every REV to be considered. At this point we update the value of $a$ in the following way

$$
a(t)=a(m T)+\sigma \sum\left[a_{k}(m T)-a(m T)\right]_{+} \quad \text { for } m T<t \leq(m+1) T,
$$


where the sum ranges over the 26 cubes in the neighborhood of the given REV, and $\sigma$ is a given positive constant. Condition (10.8) can be easily generalised. For example, we could assume that the influence of the neighbors is different in different directions, i.e. substituting the sum on the RHS by $\sum \sigma_{k}\left[a_{k}(m T)-a(m T)\right]_{+}$.

Combining (10.7) and (10.8), we obtain the law by which we update the value of $a$ in each REV of the region at times $T, 2 T, 3 T$ etc.

The final step in the modelling consists in specifying how the level of degeneration influences the dynamics of the amyloids. The influence is twofold: on one side the degeneration leads to the reduction of the number of "active" neurons, but on the other side it also causes an increased production of monomers by each neuron. Combining the two effects we can postulate that the number of monomers produced in the REV per unit time is given by

$$
\Lambda=A(1-a)(1+\beta a)
$$

where $A$ is the number of monomers produced in a healthy REV and $\beta>1$ is a prescribed constant. It is known that, during the desease, the maximum production of amyloid can be of 4-6 times larger than in a healthy brain (see e.g. (EDELSTEIN-KESHET; SPIROSS, 2002)). Accordingly, we choose $\beta=15$.

\subsection{A specific example}

To test the model, we consider the following simplified situation: we divide the total population of amyloids in just 3 subpopulations: monomers, toxic oligomers, and immobile aggregates (a larger number of subpopulations just requires a more cumbersome notation and only leads to a slight increase in computing time and a less transparent visualisation of the results).

We rescale $w_{i}(i=1,2,3)$ by a number $N$ to be chosen and define

$$
X(t)=\frac{w_{1}(t)}{N}, \quad Y(t)=\frac{w_{2}(t)}{N}, \quad Z(t)=\frac{w_{3}(t)}{N} .
$$

According to (NAG et al., 2011) the mass of a monomer is about $4.5 \mathrm{kDa}$, i.e. about $8 \times 10^{-12}$ nanograms. We choose $N=10^{11}$, corresponding to the order of magnitude of the number of monomers in a nanogram, so that $X$ represents, in order of magnitude, the mass of monomers in nanograms in the REV. For the evolution of the amyloids we choose one day as the unit time.

Setting $k=K / N, k^{*}=K^{*} / N$ and $\lambda=\Lambda / N$, the differential equations (10.3-10.5) take 
the form

$$
\left\{\begin{array}{l}
X^{\prime}=-k X^{2}-k X Y-k^{*} X Z-M_{1} X+\lambda, \\
Y^{\prime}=\frac{1}{2} k X^{2}-k X Y-k Y^{2}-k^{*} Y Z-M_{2} Y, \\
Z^{\prime}=\frac{1}{2} k Y^{2}+k X Y-M_{3} Z .
\end{array}\right.
$$

The production rate $\Lambda$ is given by (10.9) where the constant $A$ represents the production rate in a healthy tissue. Rescaling yields

$$
\lambda=\lambda_{0}(1-a)(1+\beta a) \quad \text { with } \lambda_{0}=A / N
$$

We observe that the production rate $\lambda_{0}$ depends on the total number of REV's used to describe the brain. Here we assume that we have about 500 REV's of equal size. Choosing $\lambda_{0}=2$ corresponds to a daily monomer production of 2 nanograms in each REV and 1000 nanograms in a healthy brain. Quantification of the monomer production in the brain is rather controversial in the literature. For example, Karran et al. (KARRAN; MERCKEN; STROOPER, 2011) mention an estimate of 4000 nanograms per day, where Bateman et al. (BATEMAN et al., 2006) mention only 60 nanograms. In this context our choice of $\lambda_{0}$ seems reasonable.

We choose the remaining constants in (10.10):

$M_{i}$ : Assuming that the daily clearance amounts to $1 \%$, we take $M_{i}=10^{-2}$ for $i=1,2,3$.

$k, k^{*}:$ Although in the literature there are various attempts to give explicit formula's for $k$ and $k^{*}$ (MURPHY; PALLITTO, 2000; PALLITTO; MURPHY, 2001; GILLAM; MACPHEE, 2013), we believe that their choice remains rather arbitrary. In the simulations below, we choose $k=10^{-4}$ and $k^{*}=5 \times 10^{-6}$. In this context we stress that our example is rather conceptual, and mainly aimed to illustrate the potentiality of the model.

Solving the system with initial data $X(0)=Y(0)=Z(0)=0$ we find the solution when $a=0$, as displayed in Figure 74.

The graphs suggest that there exist equilibrium values reached within a few months even when the initial data are far from equilibrium. Referring to the Appendix for some additional comments on system (10.10), we just note that the system can be scaled: if the volume of the REV is divided by a factor $m$, then dividing $\lambda$ by $m$ and multiplying $k$ and $k^{*}$ by $m$, the asymptotic values of $X, Y$ and $Z$, are also divided by $m$.

\subsubsection{Dynamics of AD: local effect}

Now we simulate the dynamics of AD. Assume that the threshold $D^{*}$ (that in this particular case is a value $Y^{*}$ ) for the normalised number of toxic oligomers in a single REV is $Y^{*}=22$. 
Figure $74-$ Solution of system (10.10) with $a=0$.

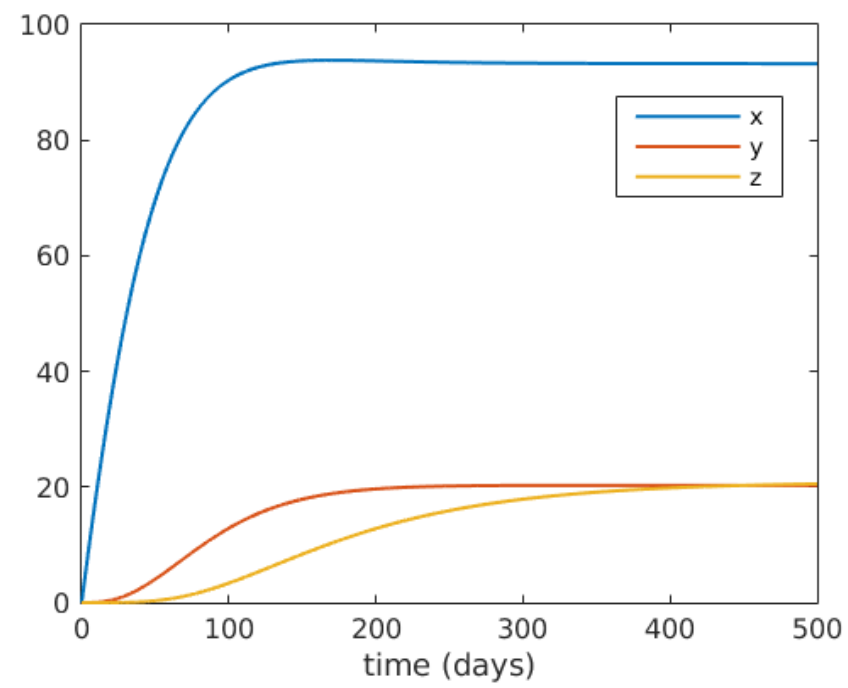

First, we describe the degradation taking into account just the local effect based on (10.7). This is just a test of the model and would refer to the abstract situation in which the initial malfunctioning of the neurons affects all the REVs in the same way (thus canceling every prion-like transmission). We set $\theta=10^{-3}$ and assume that at given time (taken as $t=0$ ), in each REV $a$ jumps to a value 0.02 and therefore $\lambda=2.548$. We take $T$ as 180 days, i.e. we update the value of $a$ twice a year. The results are displayed in Figures 75, 76 and 77. More precisely, Figure 75 represents the evolution of the degradation $a$; Figure 76 shows the normalised number of toxic oligomers in each REV; Figure 77 shows how the production rate of monomers in each REV evolves with time. In these graphs, $t$ is expressed in years after the onset of the disease. In the early stage of the disease the shape of the curve appearing in Figures 76 and 77 fits the clinical data presented in the literature (see (JACK et al., 2013) and also (BERTSCH et al., 2017)). On the other hand, the decrease in the concentration of $\mathrm{A} \beta$ is well known in clinical practice: quoting from (BALLARD et al., 2011), "meta-analyses suggest that AD can be differentiated from other dementias by the detection of lower concentration of $\mathrm{A} \beta_{1-42} \ldots$.". Moreover, low concentration of $\mathrm{A} \beta_{42}$ in CSF (Celebral Splinal Fluid) is listed among diagnostic criteria and differential diagnosis of AD from other dementias.

We point out that the stability of the model (more precisely of a similar model) is discussed in detail in (BERTSCH et al., 2020)).

\subsubsection{Including the non-local effect}

To simplify the numerical simulation and the visualisation of the results, from now on we consider a 2D model: a square grid of $20 \times 20$ REV's. The setting of the parameters, for uniformity, will be the same as before: $M_{i}=10^{-2}, k=10^{-4}$ and $k^{*}=5 \times 10^{-6}$. In addition to the local effect (10.7) (with $\theta=10^{-3}$ ), we will take into account the prion-like propagation 
Figure 75 - Progression of the average degradation $a$ of neurons in a REV (local effect) when the onset of the disease takes place at $t=0(a(0)=0.02)$.

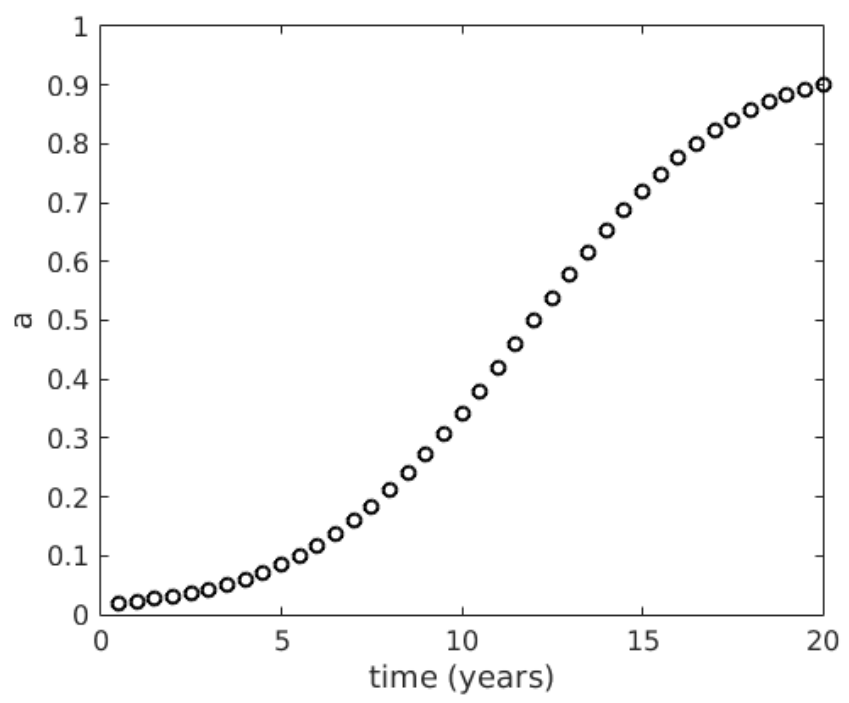

Figure 76 - Evolution of the (normalised) number of toxic oligomers in the REV during the progression of the disease.

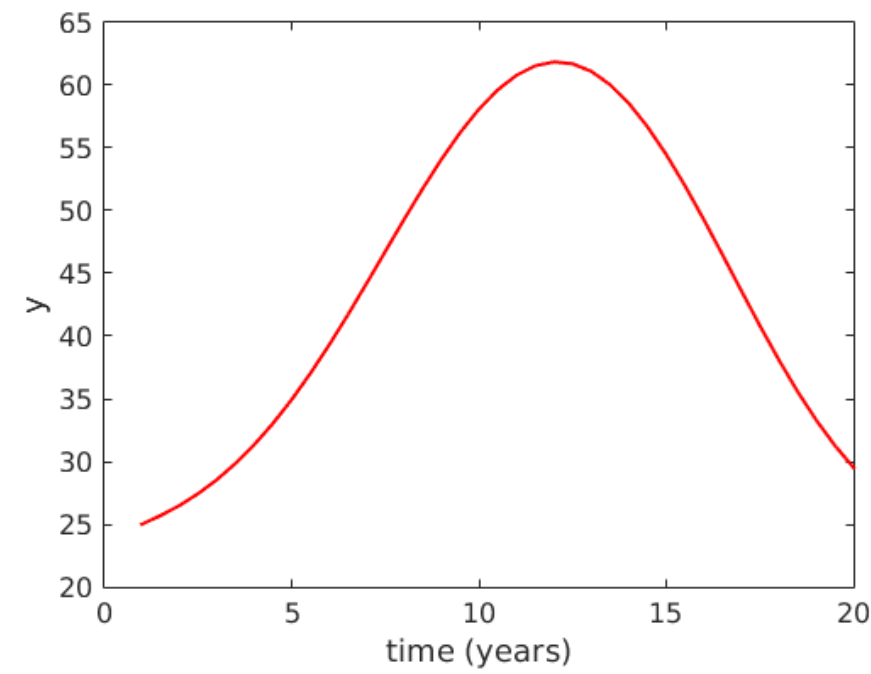

modelled by (10.8) starting with the case of isotropic propagation with $\sigma=0.05$. As initial condition, we assume for instance that $a=0$ in each REV with the exception of the square with coordinates $(6,6)$, where we put $a=0.02$. Figure 78 , which contains the screenshots at $10,12.5$, 15 and 17.5 years, shows the progressive degradation of the tissue.

To conclude this section we consider a case in which the propagation is anisotropic. In the following example case, we set the same initial condition of the previous example, i.e., $a=0.02$ in the region of coordinates $(6,6)$ and $a=0$ otherwise. But in this case, just to be specific, we assume that the propagation (corresponding to a value of $\sigma=0.2$ ) in the directions NE-SW and $\mathrm{E}-\mathrm{W}$ are 20 times larger than the propagation in the other directions, in the half-left-part and half-right-part of the brain respectively. In Figure 79 we can see the particular behaviour of the 
Figure 77 - Evolution of the average monomer production in the REV during the progression of the disease.

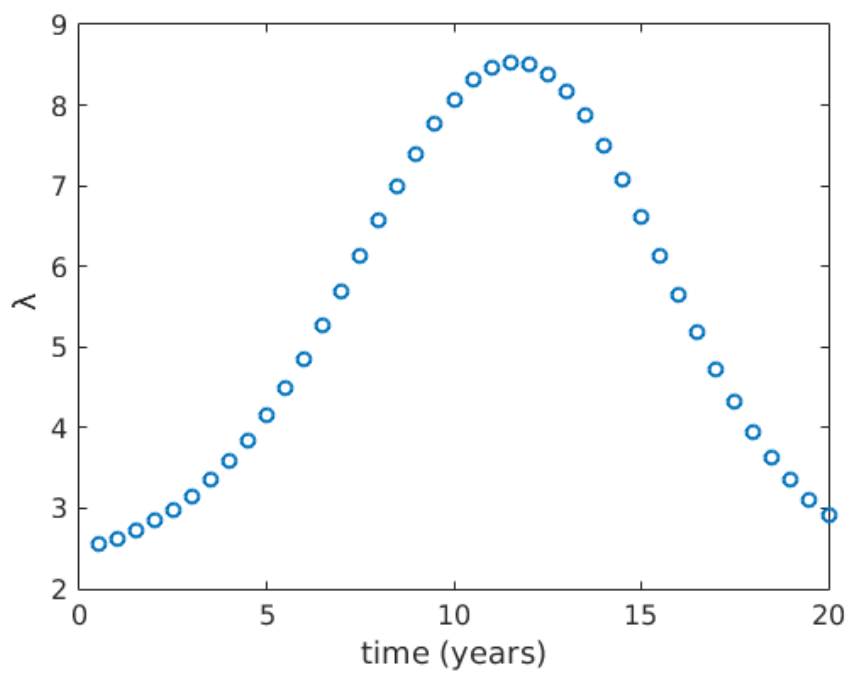

Figure 78 - Non-local effect in a 2D model. Screenshots at different times $(t=10, t=12.5, t=15, t=$ 17.5 years). The initial situation is such that only one REV (of coordinates $(6,6)$ ) has an initial malfunctioning index $a=0.02$.
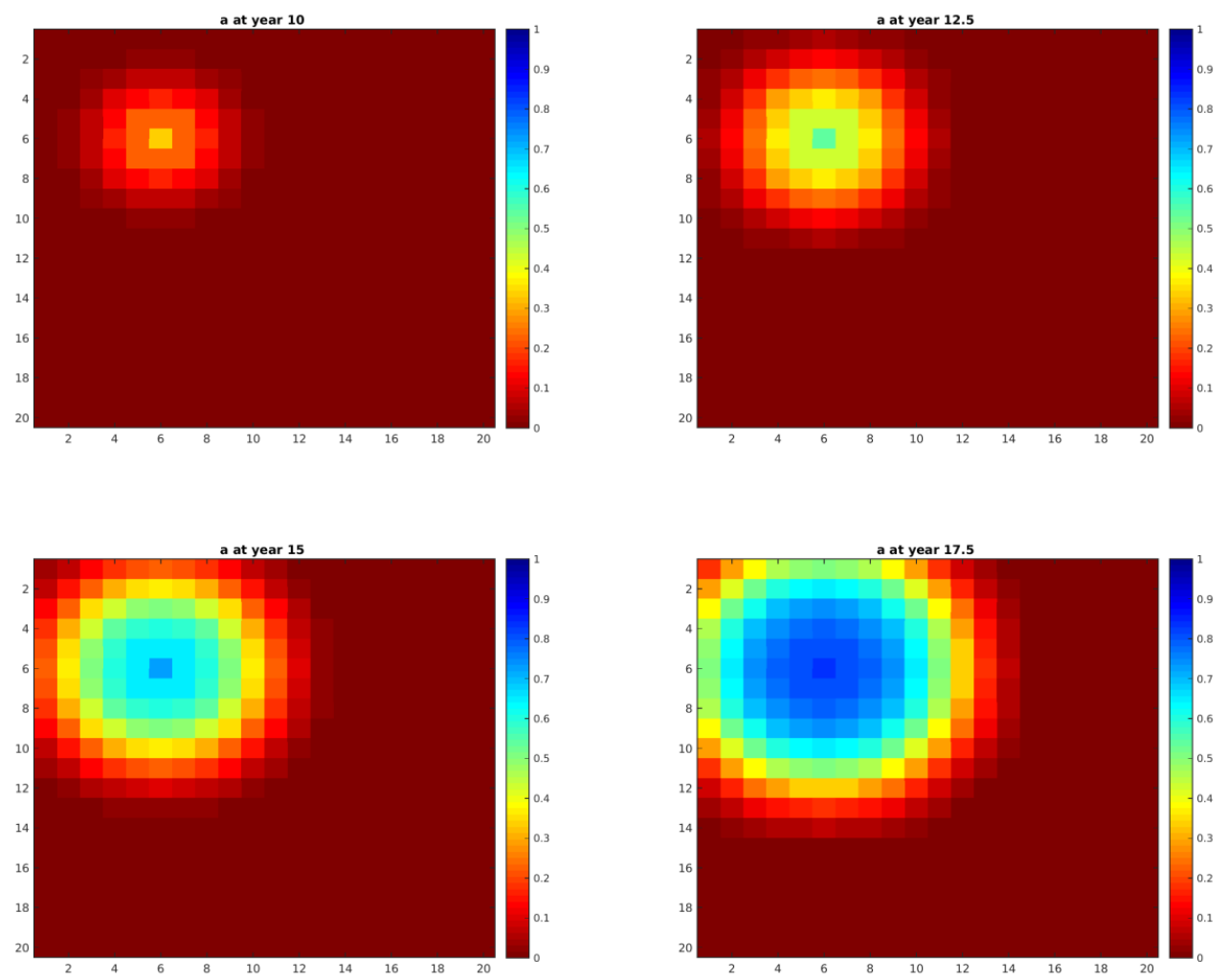

propagation of the disease as visualized through the screenshots at 10, 12.5, 15 and 17.5 years. 
Figure 79 - An example of non-isotropic propagation. Screenshots at different times $(t=10, t=12.5, t=$ $15, t=17.5$ years). The initial situation is such that only one REV (of coordinates $(6,6)$ ) has an initial malfunctioning index $a=0.02$.
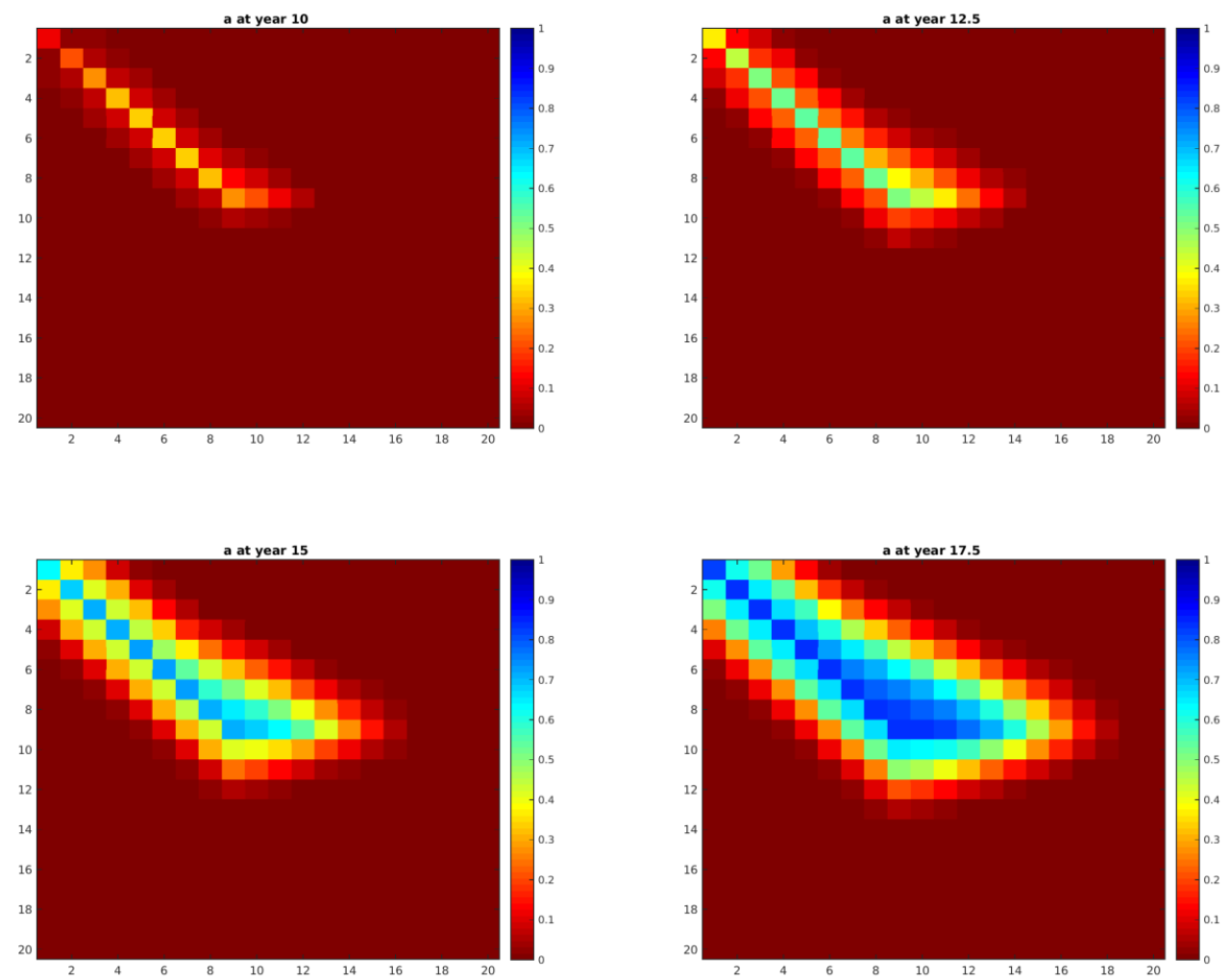

\subsubsection{Global existence of solutions of system (10.10)}

We consider the system (10.10) with positive initial data $\mathrm{X}(0), \mathrm{Y}(0), \mathrm{Z}(0)$. It is easy to show that the system has a global positive solution. Indeed, we show that $X, Y$ and $Z$ are bounded. For this purpose, we consider the system

$$
\left\{\begin{array}{l}
X^{\prime}=-k X^{2}-k X Y-k^{*} X Z-M_{1} X+\lambda, \\
Y^{\prime}=\frac{1}{2} k X^{2}-k X Y-k Y^{2}-k^{*} Y Z-M_{2} Y, \\
Z^{\prime}=\frac{1}{2} \nmid k Y^{2}+k X Y-M_{3} Z
\end{array}\right.
$$

with positive Cauchy data $X(0), Y(0), Z(0)$. Then $X(t)>0, Y(t)>0, Z(t)>0$ as long as the solution exists.

In addition

$$
\begin{gathered}
X^{\prime} \leq-k X^{2}+\lambda-k^{*} X Z \Rightarrow X(t) \leq \alpha:=\max \left\{X(0), \sqrt{\frac{\lambda}{k}}\right\}, \\
Y^{\prime} \leq k \alpha^{2}-k Y^{2}-k^{*} Y Z \Rightarrow Y(t) \leq \beta:=\max \{Y(0), \alpha\} \\
Z^{\prime} \leq \frac{1}{2} k \beta^{2}+k \alpha \beta-M_{3} Z
\end{gathered}
$$

so that also $Z$ is bounded. 


\subsubsection{Equilibrium solutions}

Let us consider the equilibrium solutions of system (10.10), i.e. the solutions of the algebraic system

$$
\left\{\begin{array}{l}
0=-k X^{2}-k X Y-k^{*} X Z-M_{1} X+\lambda \\
0=\frac{1}{2} k X^{2}-k X Y-k Y^{2}-k^{*} Y Z-M_{2} Y \\
0=\frac{1}{2} k Y^{2}+k X Y-M_{3} Z
\end{array}\right.
$$

We shall prove that system (10.10) has an equilibrium solution

$$
X \equiv X_{0}>0, \quad Y \equiv Y_{0}>0, \quad Z \equiv Z_{0}>0 .
$$

In addition, this solution is unique among all positive equilibrium solutions of (10.11).

Existence of equilibrium solutions: solutions of system (10.11) satisfy $Z=\frac{k}{2 M_{3}} Y^{2}+$ $\frac{k}{M_{3}} X Y$ and

$$
\left\{\begin{array}{l}
-k X^{2}-k X Y-k^{*} X\left(\frac{k}{2 M_{3}} Y^{2}+\frac{k}{M_{3}} X Y\right)-M_{1} X+\lambda=0, \\
\frac{1}{2} k X^{2}-k X Y-k Y^{2}-k^{*} Y\left(\frac{k}{2 M_{3}} Y^{2}+\frac{k}{M_{3}} X Y\right)-M_{2} Y=0
\end{array}\right.
$$

i.e. a system of 2 quadratic equations in $X$ :

$$
\left\{\begin{array}{l}
\left(1+\frac{k^{*}}{M_{3}} Y\right) X^{2}+\left(Y+\frac{k^{*}}{2 M_{3}} Y^{2}+\frac{M_{1}}{k}\right) X-\frac{\lambda}{k}=0, \\
X^{2}-2\left(Y+\frac{k^{*}}{M_{3}} Y^{2}\right) X-2 Y^{2}-\frac{k^{*}}{M_{3}} Y^{3}-\frac{2 M_{2}}{k} Y=0 .
\end{array}\right.
$$

The positive solutions of the quadratic equations are

$$
X=X_{1}(Y)=\frac{-\left(Y+\frac{k^{*}}{2 M_{3}} Y^{2}+\frac{M_{1}}{k}\right)+\sqrt{\left(Y+\frac{k^{*}}{2 M_{3}} Y^{2}+\frac{M_{1}}{k}\right)^{2}+\frac{4 \lambda}{k}\left(1+\frac{k^{*}}{M_{3}} Y\right)}}{2\left(1+\frac{k^{*}}{M_{3}} Y\right)}
$$

and

$$
X=X_{2}(Y)=Y+\frac{k^{*}}{M_{3}} Y^{2}+\sqrt{\left(Y+\frac{k^{*}}{M_{3}} Y^{2}\right)^{2}+2 Y^{2}+\frac{k^{*}}{M_{3}} Y^{3}+\frac{2 M_{2}}{k} Y .}
$$

Observe that $X_{2}(Y)$ is strictly increasing,

$$
X_{2}(0)=0, \quad X_{2}(\infty)=\infty, \quad X_{1}(0)=-\frac{M_{1}}{2 k}+\frac{1}{2} \sqrt{\left(\frac{M_{1}}{k}\right)^{2}+\frac{4 \lambda}{k}}>0
$$

and

$$
\begin{aligned}
X_{1}(Y) & =\frac{Y+\frac{k^{*}}{2 M_{3}} Y^{2}+\frac{M_{1}}{k}}{2\left(1+\frac{k^{*}}{M_{3}} Y\right)}\left(-1+\sqrt{\left.1+\frac{\frac{4 \lambda}{k}\left(1+\frac{k^{*}}{M_{3}} Y\right)}{\left(Y+\frac{k^{*}}{2 M_{3}} Y^{2}+\frac{M_{1}}{k}\right)^{2}}\right)}\right. \\
& =\frac{Y+\frac{k^{*}}{2 M_{3}} Y^{2}+\frac{M_{1}}{k}}{2\left(1+\frac{k^{*}}{M_{3}} Y\right)}\left(\frac{\frac{4 \lambda}{k}\left(1+\frac{k^{*}}{M_{3}} Y\right)}{2\left(Y+\frac{k^{*}}{2 M_{3}} Y^{2}+\frac{M_{1}}{k}\right)^{2}}\right)(1+o(1)) \rightarrow 0, \quad \text { as } Y \rightarrow \infty .
\end{aligned}
$$


Hence the curves $X=X_{1}(Y)$ and $X=X_{2}(Y)$ have at least one intersection point $\left(X_{0}, Y_{0}\right)$, which corresponds to an equilibrium solution $\left(X_{0}, Y_{0}, Z_{0}\right)$, where $Z_{0}=\frac{k}{2 M_{3}} Y_{0}^{2}+\frac{k}{M_{3}} X_{0} Y_{0}$.

Uniqueness of equilibrium solutions: let us notice preliminarily that a solution $\left(X_{0}, Y_{0}, Z_{0}\right)$ of system (10.11) such that $X_{0}>0, Y_{0}>0, Z_{0}>0$ satisfies (10.12).

The last equation gives

$$
M_{3} Z=\frac{1}{2} k Y^{2}+k X Y .
$$

Replacing in (10.11) we have

$$
\left\{\begin{array}{l}
0=-k X^{2}-k X Y-\frac{k^{*}}{M_{3}} X\left(\frac{1}{2} k Y^{2}+k X Y\right)-M_{1} X+\lambda \\
0=\frac{1}{2} k X^{2}-k X Y-k Y^{2}-\frac{k^{*}}{M_{3}} Y\left(\frac{1}{2} k Y^{2}+k X Y\right)-M_{2} Y \\
0=\frac{1}{2} k Y^{2}+k X Y-M_{3} Z
\end{array}\right.
$$

Multiplying the first equation by $-Y$ and the second by $X$ we obtain

$$
\left\{\begin{array}{l}
0=k X^{2} Y+k X Y^{2}+\frac{k^{*}}{M_{3}} X Y\left(\frac{1}{2} k Y^{2}+k X Y\right)+M_{1} X Y-\lambda Y \\
0=\frac{1}{2} k X^{3}-k X^{2} Y-k X Y^{2}-\frac{k^{*}}{M_{3}} X Y\left(\frac{1}{2} k Y^{2}+k X Y\right)-M_{2} Y X \\
0=\frac{1}{2} k Y^{2}+k X Y-M_{3} Z
\end{array}\right.
$$

Summing up the two first equations in (10.14) we get

$$
\begin{aligned}
\lambda Y= & k X^{2} Y+k X Y^{2}+\frac{k k^{*}}{2 M_{3}} X Y^{3}+\frac{k k^{*}}{M_{3}} X^{2} Y^{2}+M_{1} X Y \\
& +\frac{1}{2} k X^{3}-k X^{2} Y-k X Y^{2}-\frac{k k^{*}}{2 M_{3}} X Y^{3}-\frac{k k^{*}}{M_{3}} X^{2} Y^{2}-M_{2} Y X,
\end{aligned}
$$

and eventually

$$
Y\left(\lambda+\left(M_{2}-M_{1}\right) X\right)=\frac{k}{2} X^{3} .
$$

Since $X>0$, necessarily, $\lambda+\left(M_{2}-M_{1}\right) X>0$.

Consider now the function

$$
f(X)=\frac{\frac{k}{2} X^{3}}{\lambda+\left(M_{2}-M_{1}\right) X},
$$

defined on the interval

$$
I:=\left\{X>0, \lambda+\left(M_{2}-M_{1}\right) X>0\right\} .
$$

We notice now that $f^{\prime}(X)>0$ in $I$, since

$$
\begin{aligned}
& \frac{3 k}{2} X^{2}\left(\lambda+\left(M_{2}-M_{1}\right) X\right)-\frac{k}{2} X^{3}\left(M_{2}-M_{1}\right) \\
& \quad=k X^{3}\left(M_{2}-M_{1}\right)+\frac{3 k \lambda}{2} X^{2}=k X^{2}\left(X\left(M_{2}-M_{1}\right)+\frac{3 \lambda}{2}\right) \\
& \quad>\frac{\lambda}{2}>0 .
\end{aligned}
$$


Thus, by contradiction, if $\left(X_{0}, Y_{0}, Z_{0}\right)$ and $\left(X_{1}, Y_{1}, Z_{1}\right)$ are different solutions of (10.11) with $0<X_{0}<X_{1}$, we have $0<Y_{0}<Y_{1}$. Then the first equations of (10.13) gives

$$
\begin{aligned}
\lambda= & k X_{0}^{2}+k X_{0} Y_{0}+\frac{k^{*}}{M_{3}} X_{0}\left(\frac{1}{2} k Y_{0}^{2}+k X_{0} Y_{0}\right)+M_{1} X_{0} \\
& <k X_{1}^{2}+k X_{1} Y_{1}+\frac{k^{*}}{M_{3}} X_{1}\left(\frac{1}{2} k Y_{1}^{2}+k X_{1} Y_{1}\right)+M_{1}=\lambda,
\end{aligned}
$$

yielding a contradiction.

To be specific, we substitute the values $M_{i}=10^{-2}, k=10^{-4}$ and $k^{*}=5 \times 10^{-6}$, as in our simulations and we find that $X_{1}(Y)$ is decreasing and that the intersection of the two curves $X=X_{1}(Y)$ and $X=X_{2}(Y)$ corresponds to the values found in Figure ?? of Section 10.3, as it can be seen in Figure 80.

Figure 80 - Graphs of the curves $X=X_{1}(Y)$ and $X=X_{2}(Y)$ with $M_{i}=10^{-2}, k=10^{-4}$ and $k^{*}=5 \times 10^{-6}$.

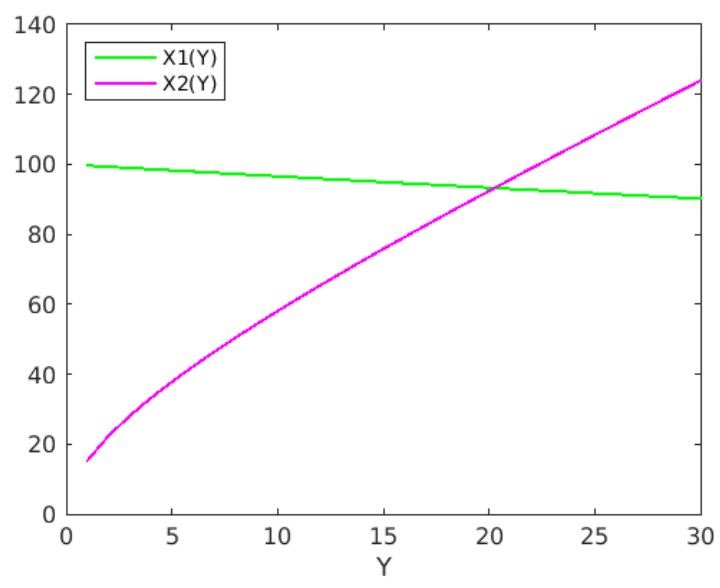

Stability of equilibrium solutions: for sake of simplicity, from now on we assume $M_{1}=M_{2}=M_{3}=: M$. In order to prove the asymptotic stability of the equilibrium solution, we have to prove that the eigenvalues of the Jacobian matrix

$$
\left(\begin{array}{ccc}
-2 k X-k Y-k^{*} Z-M & -k X & -k^{*} X \\
k X-k Y & -k X-2 k Y-k^{*} Z-M & -k^{*} Y \\
k Y & k X+k Y & -M
\end{array}\right)
$$

of the map defined by (10.11), evaluated at the critical point $(X, Y, Z)$, have negative real parts, keeping in mind that $X>0, Y>0, Z>0$. In other words, we have to prove that the characteristic polynomial of the Jacobian matrix of (10.11) is a so-called Hurwitz polynomial (see, e.g. (HAHN, 1967), Chapter II, Theorem 4.2). We write the characteristic polynomial in the form

$$
-\lambda^{3}+a \lambda^{2}+b \lambda+c
$$

An elementary computation shows that $c<0$, so that the polynomial (10.17) has a real negative solution. In order to prove that the remaining solutions have a negative real part, we apply to (10.17) the so-called Routh-Hurwitz criterion (see, e.g., (HAHN, 1967), Chapter II, 
Table 10 - Dependence of $\hat{Y}$ on the values chosen for $k$.

\begin{tabular}{cc}
\hline \hline$k$ & $\hat{Y}$ \\
\hline $10^{-4}$ & 20.26 \\
$0.5 \times 10^{-4}$ & 20.51 \\
$2 \times 10^{-4}$ & 18.00 \\
\hline \hline
\end{tabular}

Theorem 6.1). It is straightforward to see that $a<0, b<0$, so that we have but to show (see equation (6.1) in (HAHN, 1967)) that

$$
a b+c>0 .
$$

A cumbersome but elementary computation shows now that

$$
\begin{aligned}
a b+c & =k^{2}\left(9 k-k^{*}\right) X^{3}+21 k^{3} X^{2} Y+18 k^{3} X Y^{2}+6 k^{3} Y^{3}+3 k^{2} k^{*} X^{2} Y+ \\
& 15 k^{2} k^{*} X^{2} Z+6 k^{2} k^{*} X Y^{2}+26 k^{2} k^{*} X Y Z+ \\
& 2 k^{2} k^{*} Y^{3}+13 k^{2} k^{*} Y^{2} Z+24 k^{2} M X^{2}+44 k^{2} M X Y+22 k^{2} M Y^{2}+2 k k^{* 2} X Y Z+ \\
& 9 k k^{* 2} X Z^{2}+k k^{* 2} Y^{2} Z+9 k k^{* 2} Y Z^{2}+4 k k^{*} M X Y+ \\
& 30 k k^{*} M X Z+2 k k^{*} M Y^{2}+30 k k^{*} M Y Z+24 k M^{2} X+24 k M^{2} Y+ \\
& 2 k^{* 3} Z^{3}+10 k^{* 2} M Z^{2}+16 k^{*} M^{2} Z+8 M^{3}>0,
\end{aligned}
$$

provided $k \geq \frac{1}{9} k^{*}$

Thus the equilibrium solution of the system (10.11) with positive Cauchy data is asymptotically stable when $k \geq \frac{1}{9} k^{*}$. This condition is coherent with the choice of $k, k^{*}$ in Figure 80, i.e. $k^{*}=\frac{k}{20}$.

\subsubsection{Parameter dependence}

Here we sketch a numerical investigation on the dependence of the model on the values chosen for the parameters $k$ and $M_{i}$. In particular, we show how their variations influence the values of the asymptotic value of $\mathrm{Y}$ (that will be denoted by $\hat{Y}$ ) which is the key factor in the evolution of AD.

We have already noted that multiplying $k$ and $k^{*}$ by a factor $m$ and dividing $\lambda$ by the same factor, then $X, Y$, and $Z$ are also divided by $m$. Maintaining the same relationship between $k$ and $k^{*}$ as in our simulations $\left(k^{*}=k / 20\right)$ we see that doubling or dividing by 2 the value of $k$ does not induce a relevant variation in $\hat{Y}$ (see Table 10).

On the other hand, as evident from the data in the Table 11, the dependence on $M$ (which, as in our simulations is the common value of $M_{1}, M_{2}$ and $M_{3}$ ) is much more critical. 
Table 11 - Dependence of $\hat{Y}$ on the values chosen for $M$.

\begin{tabular}{cc}
\hline \hline$M$ & $\hat{Y}$ \\
\hline $10^{-2}$ & 20.26 \\
$0.5 \times 10^{-2}$ & 29.62 \\
$2 \times 10^{-2}$ & 9.06 \\
\hline \hline
\end{tabular}

\subsection{Achievements and future opportunities}

We have proposed a relatively simple model for the so-called amyloid cascade hypothesis for $\mathrm{AD}$ and its effect on the state of degradation of neurons. Dividing a portion of the brain in sufficiently small subregions and averaging the state of degradation in each subregion, we have formulated a system of ordinary differential equations for the number of monomeric and oligomeric beta-amyloid polymers and the insoluble amyloid plaques. The typical time scale of the amyloid peptides evolution is much faster than that of the evolution of the state of degradation of the neurons. The latter process can take into account a prion-like diffusion of the disease. We have also presented some numerical results in 2D to illustrate the behaviour of the solutions. A MATLAB implementation of the mathematical model to simulate the results presented in this work is available in GitHub at the URL: <https://github.com/LucaMeacci/ Alzheimer_MathModel_Ejam>.

The key point of the model is its high level of flexibility. Additional features could be implemented in the model. For instance:

- diffusion of soluble amyloid within the cerebral parenchima;

- toxic effect of phosphorylated $\tau$ protein inside neurons and its interaction with $\beta$-amyloid;

- more realistic anatomy of the brain;

- clearance of the amyloid due to the continuous production and removal of the cerebrospinal fluid.

The possibility of including different phenomena in the model is particularly important since the role of the various mechanisms in the development of AD is not yet well understood. In other words, numerical simulations can be used to test different modelling hypotheses. 
CHAPTER

11

\section{PANDEMIC FATIGUE IMPACT INTO COVID-19 SPREAD: A MATHEMATICAL MODELING ANSWER TO THE ITALIAN SCENARIO}

The year 2020 will not go into oblivion. The COVID-19 virus pandemic has spread unprecedented challenges around the world. Governments, institutions, the economical system, scientific research, and entire populations have been facing an anti-epidemic battle. Even very many families and individuals are been subjected to tremendous psychological pressure.

The World Health Organization (WHO) has declared the 2019-nCoV acute respiratory disease outbreak as a 'Public Health Emergency of International Concern' on 30 January 2020, specifically to enhance the level of preparedness of countries that need additional support (World Health Organization 2020b) (RABAJANTE, 2020)

The COVID-19 disease was named by the WHO on 11 February 2020 (ORGANIZATION et al., 2020b) and it is due to the virus "severe acute respiratory syndrome" coronavirus 2 (SARSCoV-2), which was detected in December 2019 in the city Wuhan in China (VELAVAN; MEYER, 2020). This novel virus belongs to the large coronavirus family that cause respiratory illness as the Middle East respiratory Disease (MERS) and the Severe Acute respiratory Syndrome (SARS). However, COVID-19 is characterized by a peculiar aggressiveness in terms of reproducibility (LIU et al., 2020a) and mortality (RUAN et al., 2020). Common symptoms are fever, dry cough, sore throat, tiredness, headache, myalgia and breathlessness. Some patients can suffer acute respiratory distress syndrome and multiorgan dysfunction (SINGHAL, 2020).

The COVID-19 contagion is due to human transmission through respiratory droplets or contact with infected surfaces by a sick person. For this reason, non-pharmaceutical interventions have been adopted and put in place with measures that vary, according to the severity of the 
Chapter 11. Pandemic fatigue impact into COVID-19 spread: a mathematical modeling answer to the 178 Italian scenario

situation, from the use of personal protections such as breathing masks and hand gloves, to social distancing and up to public health measures of lockdown of entire regions or countries (ORGANIZATION et al., 2020a; FLAXMAN et al., 2020). Such measures were essentially the primary means of preventing and mitigating the spread of the epidemic. Despite these interventions, on 31 December 2020, the WHO reported 81.475.053 confirmed cases and 1.798.050 deaths for COVID-19 in the world.

Since the first part of 2020, the WHO has declared COVID-19 epidemic a public heath emergency of international concern, then as a pandemic and has called on countries to take actions and evidence-based decisions (SOHRABI et al., 2020). This necessity represents an opportunity and at the same time a challenge for mathematical modelers to project the epidemic forward. Although in the presence of limited data and information on the virus, the mathematical modelling community did not fail to quickly produce efforts to assist the decision-making processes (SHAO; WU, 2020; CURRIE et al., 2020). Many significant works have been produced, ranging from statistical approaches (WU et al., 2020; PAIVA et al., 2020; SAHOO; SAPRA, 2020; CHAKRABORTY; GHOSH, 2020), to deterministic compartmental models (SARKAR; KHAJANCHI; NIETO, 2020; MARTELLONI; MARTELLONI, 2020; LEE; LI; KIM, 2020; KHAN; ATANGANA, 2020; MAHAJAN; SIVADAS; SOLANKI, 2020; ZHAO; CHEN, 2020) and including through stochastic procedures (HELLEWELL et al., 2020; KUCHARSKI et al., 2020). Some of them aim to estimate of the transmission risk (TANG et al., 2020), to estimate the number of unreported cases (LIU et al., 2020b), or to take into account both the age and social contact structure (SINGH; ADHIKARI, 2020). In general, one common question is to understand how major public policies restricting social movement, such as social distancing, "stay-at-home" measures, quarantine, and public closings, mitigate the epidemic spread. The idea is not only to predict the outbreak behavior, such as its final size and its peak time (ARINO; PORTET, 2020), but also to address the challenge to help in planning effective control strategies and public health interventions (MAMO, 2020). Specifically, mathematical models based on dynamical equations present the advantage to identify more clearly the mechanisms beyond the epidemic dynamics (COOPER; MONDAL; ANTONOPOULOS, 2020; NDAIROU et al., 2020; BRAUER, 2017). In the COVID-19 case, although its epidemiological characteristics are yet to be fully elucidated and the introduction of a certain degree of estimation error is unavoidable (POSTNIKOV, 2020), Ordinary Differential Equations (ODEs) models can provide an accurate description of the spreading on the level of a country-wide averaged statistics (FANELLI; PIAZZA, 2020). Such models kinds allow to rather naturally define physical-based parameters and, by the tuning of them, easier simulate political strategies and social-environmental features with respect to more complex models which may not necessarily more reliable (CACCAVO, 2020; RODA et al., 2020).

A general and impactful consequence of the COVID-19 pandemic is given by the psychological repercussions on the population. The emotional pressure throughout the outbreak period has raised several concerns regarding the psychological status and its mental health effect 
on patients (VINDEGAARD; BENROS, 2020), on quarantined persons (PENG et al., 2020; LEI et al., 2020; LUO et al., 2020), and on hospital and front-line staff (SAGHERIAN et al., 2020; TENG et al., 2020). The COVID-19 fear has caused a parallel epidemic of anxiety, stress, and depression in the population in general (ASMUNDSON; TAYLOR, 2020b; TAYLOR et al., 2020); a rather extensive and evident phenomenon in several individuals, including distressing emotions, thoughts and images of danger and avoidance, and other defensive behaviors (ÖZDIN; ÖZDIN, 2020). For this reason, such impact forms have been the subject of extensive studies in various contexts (ZHAO et al., 2020; FUSAR-POLI; BRAMBILLA; SOLMI, 2020; YANG et al., 2020) with remarkable implications ranging from uncertainty and worry to a sort of coronaphobia (ASMUNDSON; TAYLOR, 2020a).

Indeed, although the preventive measures, such as social distancing, home confinement and lockdown, contributed to slowing and containing the pandemic spread of the pandemic, they negatively affected people's way of life, with consequences for mental and psychological health and well-being of the general population. (MARROQUÍN; VINE; MORGAN, 2020; JIAO et al., 2020; MAJUMDAR; BISWAS; SAHU, 2020; SINGH et al., 2020; KAPASIA et $a l .$, 2020; MARGARITIS et al., 2020). To comply with precautionary and defense policies generated common disorders in terms of tiredness, loneliness, irritability, sleep disturbance, lack of motivation, and loss of interest in previously enjoyed activities (FIELD et al., 2020; BARTOSZEK et al., 2020).

The WHO used the expression "pandemic fatigue" to indicate the psychological response to the prolonged public health crisis and to sustained and unresolved adversities in people's lives. In the COVID-19 case, this kind of distress, also due to the implementation of invasive measures with unprecedented impacts on people's daily lives, has led to complacency, alienation and hopelessness (ORGANIZATION et al., 2020c). The pandemic fatigue, emerging gradually over time and affected by a number of emotions, experiences and perceptions, can express itself as emerging demotivation to engage in protection behaviours. Many countries reported an increase in collective fatigue, evident from the fact that "people are feeling demotivated about following recommended behaviours to protect themselves and others from the virus" (EUROPE, 2020). Although there are some sporadic conflicting ideas on the subject (HARVEY, 2020; MAHASE, 2020), there is no doubt that this social phenomenon can affect our behavior and therefore also in the consequent evolution of the pandemic. This means that pandemic fatigue not only represents a cause of the virus advent but at the same time becomes a cause of the COVID-19 spread. Steven Taylor in the book "The Psychology of Pandemics" states that "remarkably little attention has been devoted to the psychological factors that influence the spread of pandemic infection and the associated emotional distress and social disruption. Psychological factors [...] play a role in nonadherence to vaccination and hygiene programs, and play an important role in how people cope with the threat of infection" (TAYLOR, 2019). The tiredness of living with the discomforts induced by the health regulations imposed, including a heightened state of distrust towards these measures, can easily lead a considerable number of individuals to let their guard down and even 
Chapter 11. Pandemic fatigue impact into COVID-19 spread: a mathematical modeling answer to the 180

Italian scenario

to unjustifiably minimize the danger and vulnerability to the virus (CULLEN; GULATI; KELLY, 2020).

In this work, we consider and introduce the non-negligible role of the pandemic fatigue, being the human behaviour central to COVID-19 transmission (WEST et al., 2020). There are attempts to take into account the individual reaction but they are rare (LIN et al., 2020); the merit of our mathematical model compared to other existing works is to add and mimic the pandemic fatigue impact into COVID-19 spread. We opt for a simple mean-filed model, being suitable a general quantitative picture of the epidemic spreading, including restrictive public health interventions and the social/emotional population response to them. The mathematical model consists in a SIQRD (susceptible-infected-quarantined-recovered-dead) model performed by Ordinary Differential Equations (ODEs). We apply the model in the Italian context during 2020 (already the subject of other publications (PICCOLOMINI; ZAMA, 2020; CACCAVO, 2020; GIORDANO et al., 2020; PICCOLOMIINI; ZAMA, 2020)), to test the consistency of the hypotheses applied to the results obtainable with the model simulations and compared with the statistical data. This novel study aims to show a possible explanation of the pandemic behavior with respect to the people's relaxation/resistance to comply with public health restrictions and, therefore, albeit with a certain degree of approximation, to evaluate how much the pandemic fatigue impact affected the COVID-19 outbreak in Italy in 2020.

The paper contents are organized as follows. In Section 11.1 we describe the assumptions adopted, develop the mathematical model, and prove its mathematical well-posedness. Section 11.2 contains the numerical results of the simulations performed with our model, where the parameters calibration is acquired by the Italian preventive actions during 2020. Primarily, the comparison with the data collected by the competent authorities on the evolution of positive cases, quarantined people, and dead is also presented. Subsequently, the model is used predictively to evaluate the hypothetical results on the basis of different administrative choices and more or less virtuous behavior of the population. The estimated cost in terms of infected people and deaths due to the negative effects of pademic fatigue is reported. Finally, in the Section 11.3 we provide a brief discussion of the achievements of this work and the implications in terms of measures to contain or prevent the negative effects of pandemic fatigue. We include some comments on possible future developments and applications of the model.

\subsection{Model}

Let us propose to study the evolution dynamics of the COVID-19 outbreak with respect to time $t \geq t_{0}$, representing $t_{0}$ the initial time. We assume that the entire population is divided into five compartments. We denote with $S(t)$ the number of individuals susceptible to infection at time $t$ and $I(t)$ the number of infected people who, however, is still at time $t$ in an asymptomatic 
state. We apply the simplifying hypothesis ${ }^{1}$ that, after an average time of virus incubation, each infected person presents symptoms and consequently is placed in quarantine. With $Q(t)$ we therefore indicate the number of infected people at time $t$, who have symptoms and are automatically isolated, that is, from the point of view of the model, they are no longer able to infect a susceptible person. Finally, every sick person, after quarantine, can die or recover. $D(t)$ counts the total number of deaths and $R(t)$ recovered individuals at time $t$. We suppose that a recovered person does not go back to being susceptible to the virus. We assume also that the whole population is constant and equal to $N$. This approximation is justifiable because the pandemic outbreak covers a period of time in which the variation in the total individual number is negligible.

Figure 81 - Schematic flow diagram representation of the model.

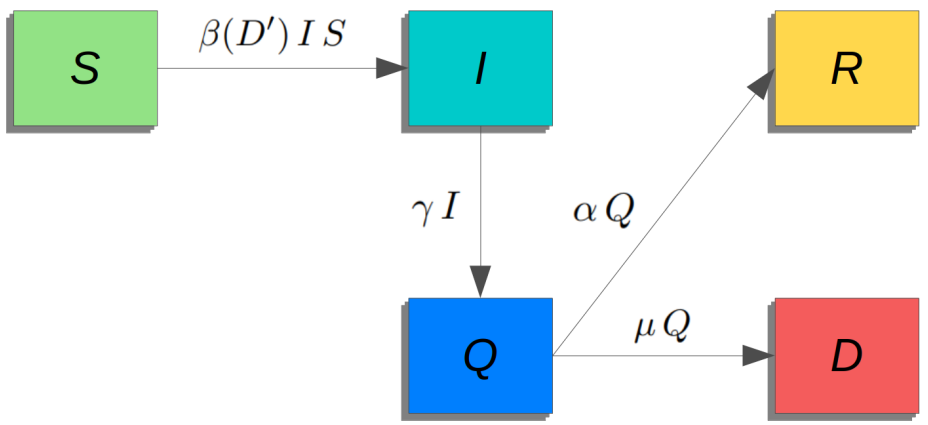

The dynamics between these compartments are illustrated in the flow diagram of Figure 81 and governed by some parameters. We assume to consider them piecewise constant and fixed, according to their meaning. Setting the time unit, we define $\gamma \geq 0$ the average period during which an infected individual becomes symptomatic, i.e. the average incubation time. Then, we use the parameters $\alpha \geq 0$ and $\mu \geq 0$ to rule the transition from the compartment of symptomatically ill and quarantined persons to the compartment of those who are healed or dead, respectively. We note that $\alpha$ and $\mu$ are given by the combination of the average time to exit the quarantined class and the probability of defeating or not the virus. Concerning the transition between the susceptible to the infected population, we introduce $\beta$ transmission rate obeying to the mass action law. The definition of $\beta$ deserves particular attention, because it must have the information content suitable for describing (i) the effect of the social isolation measures and, at the same time, (ii) the population response due to pandemic fatigue. We opt to introduce the transmission rate as a composite function as follows

$$
\beta\left(D^{\prime}(t), t\right)=\frac{\eta(t)}{\exp (\kappa(t) \mu(t) Q(t))} .
$$

The virus contagiousness is expressed by the fixed piecewise factor $\eta \geq 0$. A public health intervention that includes a measure such as "stay-at-home" or lockdown directly affects the

1 A possible generalization of the model can be easily constructed. 
decay of the value of this factor. It is also hypothesized that the state of fatigue in respecting the measures during the pandemic period is reflected in reducing the state of alert and consequently the suggested good preventive practices. This negative effect is inversely directed with respect to the feeling of vulnerability. It is reasonable to assume that the perception of danger is induced by deaths in the unit of time. This assumption leads to the definition of the denominator of the (11.1), where $\kappa \geq 0$ is a piecewise constant and a fixed parameter denoting the population sensitivity to the occurrence of deaths. The use of the exponential function with respect to the increase in deaths is a reasonable model choice compared to the severe reaction that this data reflects on people's behavior ${ }^{2}$.

Based on the above considerations, we obtain the mathematical model consisting of the following system of nonlinear ODEs,

$$
\left\{\begin{aligned}
S^{\prime}(t) & =-\frac{\eta}{\exp (\kappa \mu Q(t))} I(t) S(t), \\
I^{\prime}(t) & =\frac{\eta}{\exp (\kappa \mu Q(t))} I(t) S(t)-\gamma I(t), \\
Q^{\prime}(t) & =\gamma I(t)-(\alpha+\mu) Q(t), \\
R^{\prime}(t) & =\alpha Q(t), \\
D^{\prime}(t) & =\mu Q(t),
\end{aligned}\right.
$$

where, $t \geq t_{0}$. The system is supplemented by the initial conditions

$$
\begin{gathered}
S\left(t_{0}\right)=S_{0}>0, \quad I\left(t_{0}\right)=I_{0} \geq 0, \quad Q\left(t_{0}\right)=Q_{0} \geq 0, \\
R\left(t_{0}\right)=R_{0} \geq 0, \quad D\left(t_{0}\right)=D_{0} \geq 0 .
\end{gathered}
$$

We remark that it is easy to prove that entire population is constant, being that $S^{\prime}(t)+$ $I^{\prime}(t)+Q^{\prime}(t)+R^{\prime}(t)+D^{\prime}(t)=0$, and therefore iqual to $S_{0}+I_{0}+Q_{0}+R_{0}+D_{0}=N$. Furthermore, we recall the simple notion that the values $S(t), R(t)$, and $D(t)$ report the total number of individuals in the class at $t$. In the case of asymptomatic and symptomatic (quarantined) infected, the cumulative values at time $t$ are

$$
C I(t)=-\int_{t_{0}}^{t} S(t) d t, \quad C Q(t)=\gamma \int_{t_{0}}^{t} I(t) d t
$$

In Table 12 we summarize the parameters of the mathematical model and their descriptions.

In order to prove the well-posedness of the formulation, we proceed to simplify the model (11.2). We can rewrite the system considering the new normalized variables $s(t)=$

2 Although adaptive behavior is well documented in real life, in general mathematical epidemiology, individuals rarely endogenously adapt their contact behavior during epidemics, as this occurs in social distancing based on fear. To explore the effect of including such fear behavior in models of epidemic dynamics, we suggest to see (EPSTEIN et al., 2008) 
Table 12 - Parameters of the mathematical model.

\begin{tabular}{ccc}
\hline Symbol & Description & State \\
\hline$t_{0}$ & Beginning time of pandemic evolution & fixed \\
$S_{0}$ & Number of susceptible individuals at $t_{0}$ & fixed \\
$I_{0}$ & Number of asymptomatic infected people at $t_{0}$ & fixed \\
$Q_{0}$ & Number of symptomatic infected and quarantined people at $t_{0}$ & fixed \\
$R_{0}$ & Number of recovered individuals at $t_{0}$ & fixed \\
$D_{0}$ & Number of dead individuals at $t_{0}$ & fixed \\
$\eta$ & Transmission rate & piecewise non-negative \\
$\kappa$ & Population sensitivity of alert state & piecewise non-negative \\
$\gamma$ & Average virus incubation period & piecewise non-negative \\
$\alpha$ & Average rate/probability of healing & piecewise non-negative \\
$\mu$ & Average rate/probability of death & piecewise non-negative \\
\hline
\end{tabular}

$S(t) / N, i(t)=I(t) / N, q(t)=Q(t) / N, r(t)=R(t) / N$, and $d(t)=D(t) / N$. With a little abuse of notation, we redefine $\eta=\eta N$ and $\kappa=\kappa N$. Now, under the new normalized formulation, $s(t)+i(t)+q(t)+r(t)+d(t)=1, \forall t \geq t_{0}$; therefore we can remove the first equation of the system (11.2) and obtain the following new system of 4 ODEs,

$$
\left\{\begin{aligned}
i^{\prime}(t) & =\frac{\eta}{\exp (\kappa \mu q(t))}(1-i(t)-q(t)-r(t)-d(t)) i(t)-\gamma i(t), \\
q^{\prime}(t) & =\gamma i(t)-(\alpha+\mu) q(t), \\
r^{\prime}(t) & =\alpha q(t) \\
d^{\prime}(t) & =\mu q(t)
\end{aligned}\right.
$$

with the corresponding initial conditions

$$
i\left(t_{0}\right)=i_{0} \geq 0, \quad q\left(t_{0}\right)=q_{0} \geq 0, \quad r\left(t_{0}\right)=r_{0} \geq 0, \quad d\left(t_{0}\right)=d_{0} \geq 0 .
$$

Expressed in a compact form, the feasible domain of the system (11.5) is

$$
\Gamma=\left\{(i, q, r, d) \in \mathbb{R}_{+}^{4}: i+q+r+d \leq 1\right\} .
$$

An initial-value problem for a ODEs system is mathematically well-posed when a solution exists, the solution is unique, and it depends continuously on the data. We aim to prove that $\Gamma$ is positively invariant to show the well-posedness of the model (YORKE, 1967).

Theorem 11.1. The problem for the ODEs system (11.2) with initial conditions (11.3) is well-posed.

Proof. Let us denote $x(t)=(i(t), q(t), r(t), d(t))^{T}$. The system (11.2) is equivalent to the system (11.5), which can be seen as

$$
\frac{d x(t)}{d t}=f(x(t)), \quad t \geq t_{0},
$$

where

$$
\begin{aligned}
f(x(t))= & {\left[\frac{\eta}{\exp (\kappa \mu q(t))}(1-i(t)-q(t)-r(t)-d(t)) i(t)-\gamma i(t),\right.} \\
& \gamma i(t)-(\alpha+\mu) q(t), \alpha q(t), \mu q(t)]^{T} .
\end{aligned}
$$


To prove that $\Gamma$ is positively invariant, we observe that $\Gamma$ is a compact set. We need to prove that if $x(0) \in \Gamma$, then $x(t) \in \Gamma, \forall t \geq 0$. Let us consider the boundary $\delta \Gamma$ that consists of 5 plane segments, as follows

$$
\begin{gathered}
P_{1}=\left\{(i, q, r, 0):(i, q, r) \in \mathbb{R}_{+}^{3}, i+q+r \leq 1\right\}, \\
P_{2}=\left\{(i, q, 0, d):(i, q, d) \in \mathbb{R}_{+}^{3}, i+q+d \leq 1\right\}, \\
P_{3}=\left\{(i, 0, r, d):(i, r, d) \in \mathbb{R}_{+}^{3}, i+r+d \leq 1\right\}, \\
P_{4}=\left\{(0, q, r, d):(q, r, d) \in \mathbb{R}_{+}^{3}, q+r+d \leq 1\right\}, \\
P_{5}=\left\{(i, q, r, d) \in \mathbb{R}_{+}^{4}: i+q+r+d=1\right\} .
\end{gathered}
$$

In addition, let us consider the corresponding outer normal vector to these plane segments, i.e., $v_{1}=(0,0,0,-1), v_{2}=(0,0,-1,0), v_{3}=(0,-1,0,0), v_{4}=(-1,0,0,0)$, and $v_{5}=(1,1,1,1)$. We can prove that the scalar products of $f(x)$ and the normal vectors $\left(v_{1}, v_{2}, v_{3}, v_{4}, v_{5}\right)$ of the boundary planes are less or equal to zero in order to conclude that $x(0) \in \Gamma$, then $x(t) \in \Gamma \forall t \geq 0$. These are easily verifiable, as reported below,

$$
\begin{gathered}
\left\langle\left. f(x(t))\right|_{x \in P_{1}}, v_{1}\right\rangle=-\mu q(t) \leq 0, \\
\left\langle\left. f(x(t))\right|_{x \in P_{2}}, v_{2}\right\rangle=-\alpha q(t) \leq 0, \\
\left\langle\left. f(x(t))\right|_{x \in P_{3}}, v_{3}\right\rangle=-\gamma i(t) \leq 0, \\
\left\langle\left. f(x(t))\right|_{x \in P_{4}}, v_{4}\right\rangle=0, \\
\left\langle\left. f(x(t))\right|_{x \in P_{5}}, v_{5}\right\rangle=0
\end{gathered}
$$

Q.E.D.

\subsection{The case of Italy: numerical results}

During 2020, one of the most severely affected countries by the pandemic was Italy and can be considered as a case study for the reaction of public health authorities through containment measures and with respect to the psychological/behavioral response of the general population. On January 31, 2020, the Italian Council of Ministers declares a state of emergency as a result of the health risks associated with the spread of the epidemic. On 31 December 2020, Italy counts 2.107.166 positive cases since the emergency beginning and 74.159 victims. The data of surveillance for COVID-19 are managed and communicated by the Italian Civil Protection Department and they are publicly available. They are collected in a dataset published in the GitHub repository (CIVILE, 2020) and presented in (MORETTINI et al., 2020). We refer to these data to test our model in the Italian scenario during 2020. 
The Italian government has established various interventions and it is possible to consult their detailed list ${ }^{3}$. However, for modeling purposes, we resume the anti-contagion policy with the following main events:

- 9 March 2020: National lockdown. "Stay-at-home" requirements enacted and commercial businesses halted throughout the national territory;

- 23 March 2020: Tightening of lockdown rules. Closure of production, industries and commercial services considered non-essential. Reduction of the possibility of transport, even of a private nature;

- 3 June 2020: Reopening of the Country. Activities and mobility are allowed;

- 6 November 2020: Restrictive lockdown measures, on a national scale, for the second wave of contagion.

From the point of view of the model and system dynamics, these interventions are reflected in the value of the transmission rate $\eta$. The graph in Figure 82 visually represents how these events can be taken into account in the $\eta$ setting. For example, the two evident jumps to the downside correspond to the first national lockdowns on 9 and 23 March 2020, respectively. The restrictions imposed on work activities, on mobility and the order to stay at home directly decrease the possibility that an infected person meets with a susceptible individual. Referring to what is reported in the graph, this means that with the intervention of 9 March 2020, the probability of contagion was reduced by half and on on 23 March 2020 further to a quarter. The order of magnitude of the $\eta$ value takes into account the susceptible people basin, a priori equal to the entire number of the Italian population ${ }^{4}$.

The setting of other parameters to simulate the COVID-19 spreading dynamics into the Italian scenario using our model, can be imported by the collected statistics. We consider 24 February 2020 as the starting day because it is the first day with the data recording. The initial conditions (11.3) can be set, obtaining ${ }^{5}: S_{0}=6.0 \times 10^{8}, I_{0}=3.5 \times 10^{4}, Q_{0}=221, R_{0}=30$, and $D_{0}=7$. We establish the unit of time in 1 day, and therefore we can fix $\gamma=0.07$, meaning that the virus can have an incubation period up to over 2 weeks ${ }^{6}$.

3 An overview of Italian government initiatives to prevent and restrain the COVID-2019 pandemic is available here (DEPUTIES, 2021).

4 The choice to consider this setup is a simplification; a more articulated use of the parameter can be used, without compromising the considerations and objectives of this work.

5 In the case of Italy and of the study of populations with a number of inhabitants of several orders of magnitude higher than the other variables, a possibility consists in considering the number of susceptible people constant, resulting in a further simplification of the model.

6 The setting of $\gamma$ is opinionable; the incubation period is not so clear (LAUER et al., 2020; LEUNG, 2020). We remark that the parameters values are hypothetical and the result of averages and simplifying choices. For example, it seems that a COVID-19 infected person is not homogeneously contagious during the infectious period and yet begins to be able to transmit the virus after a few days. This effect in the model it taken into account by operating a slight shifting of the $\eta$ values of a few days. 
Figure 82 - Transmission rate $\eta$ with the main Italian Government COVID-19 measures.

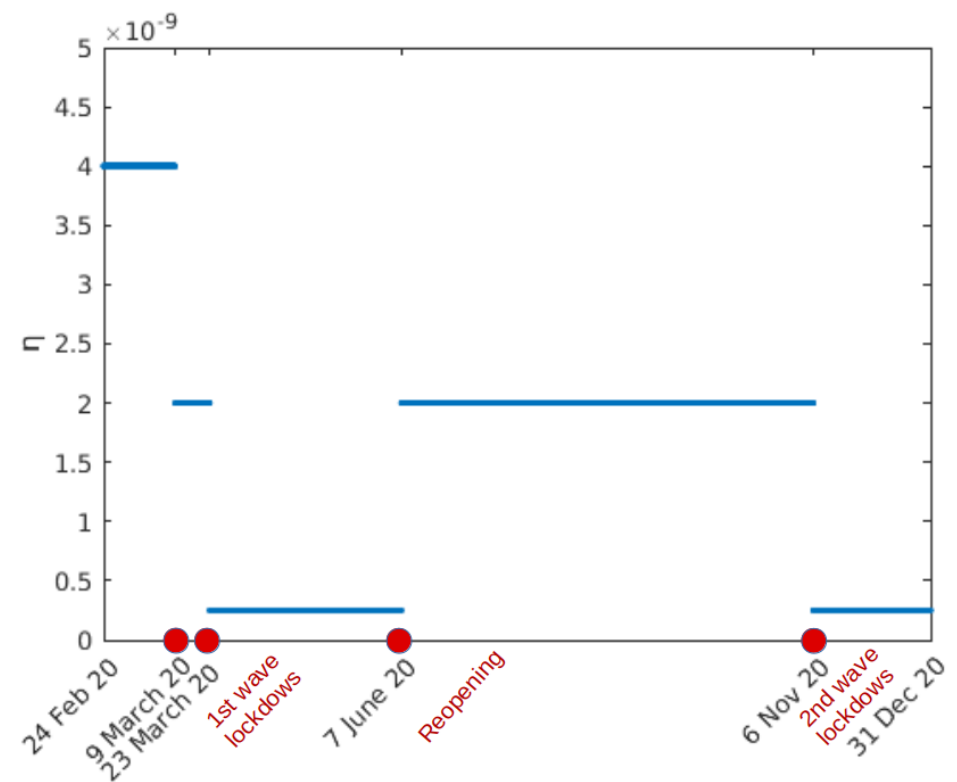

The trend of the pandemic during 2020 in Italy can be macroscopically divided into 2 phases, the first and the second wave, to indicate a significant increase in cases in two distinct moments. The first wave took place between the months of February and May. Let us primarily focus on this first phase, proceed to numerically solve the system (11.2), and denote these simulation as "Interventions-based Transmission Test". Three parameters are still to be fixed; we benefit from the fitting of the statistical data to establish that $\kappa=0.22$ and the proportions between the sick people number and the recovered or dead people number fix that $\alpha=0.015$ and $\mu=0.006$ until the intervention of the tightened lockdown of 23 March 2020 and subsequently $\alpha=a=0.023$ and $\mu=0.003$. The fact that it $\alpha$ has increased and $\mu$ has decreased reflects a better efficiency gained in healthfully managing the disease. In Figure 83, we display the graphs (using the continuous line) of the infected people, of the recovered and dead people, obtained by the numerical solution of our model. Comparing these solutions with the reported statistical data (using circles), we appreciate a similar behavior during this first left phase, which concerns the first wave of infections ${ }^{7}$.

Let us now turn our attention to the second wave phase. By maintaining the same parameters setup, the results of our model deviate significantly from the statistical data. We observe that this evidence occurs despite taking into account the various preventive measures, and therefore the different $\eta$ values, throughout the year. The parameters have also been updated with respect to the increased efficiency of medical assistance, imposing a further reduction of the $\mu=0.0015$ and $\mu=0.0005$ after July and November, respectively. Nonetheless, the deviation

7 We note a certain excess approximation of the variables results of the model with respect to the collected data. The possibility of the existence of non-reporting cases, especially in the first instances of the pandemic, is to be kept in mind for a correct assessment (HORTAÇSU; LIU; SCHWIEG, 2021; ZHAO et al., 2020). 
Figure 83 - Numerical solutions of the simulation of COVID-19 spread in Italy during 2020, performed by our model according the "Interventions-based Transmission Test". The statistical data are also reported.

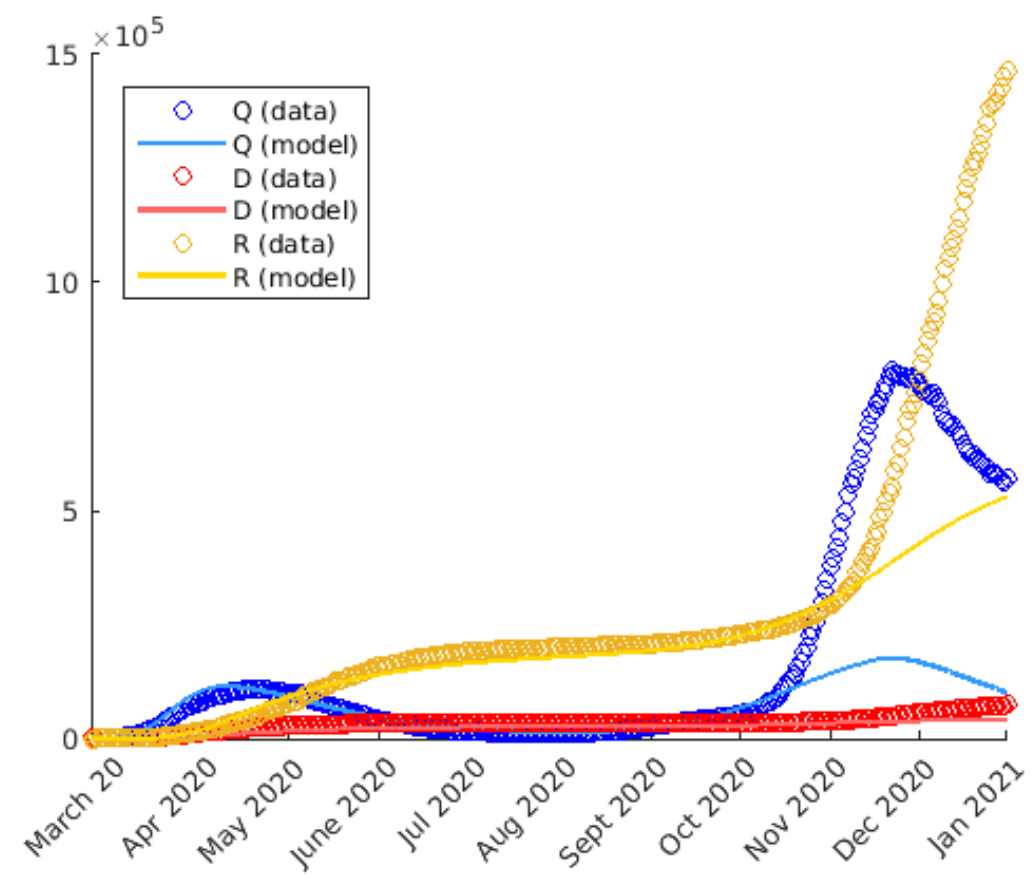

of the model results and the real data turns out to be satisfactory. The hypothesis proposed is that this significant difference is due to a different social response with respect to showy behavior and compliance with the rules of precaution imposed.

Let us therefore perform a second test; we call it "Pandemic Fatigue Impact Test". As argued above, there is evidence that prolonged exposure to a pandemic and to rules that affect daily life exerts a strain on the individual that can lead to a more or less gradual loss of diligence in complying with anti-contagion measures. We remark that, by construction, the model already takes into account the individual reaction with respect to the danger perception induced by an increase in deaths. According to (11.1), people spontaneously adapt their precautionary behavior based on the virus's heightened fatality. Nevertheless, to mimic the effect of pandemic fatigue, we have to introduce a change in people sensitivity. For how the model is developed, this feature can be easy implemented by varying the value of $\kappa$. In Figure 84, we display the numerical results of the simulation, where we simply adapt the people sensitivity setting $\kappa=0.0007$ after after mid-July 2020 and $\kappa=0.00018$ after mid-September 2020.

The graphs in Figure show that, although the results obtained with our model are not exactly identical to the data, they are nevertheless close enough to accept that the dynamics described by the system reflect what may occur during COVID-19 outbreak in real life. If the theory presented in this study is consistent, this means that the model could be use to hypothesize with a certain degree of confidence not only a possible justification for the size of the second wave but to provide an estimate of the impact of pandemic fatigue in terms of deaths. Concerning 
Figure 84 - Numerical solutions of the simulation of COVID-19 spread in Italy during 2020, performed by our model according the "Pandemic Fatigue Impact Test". The statistical data are also reported.

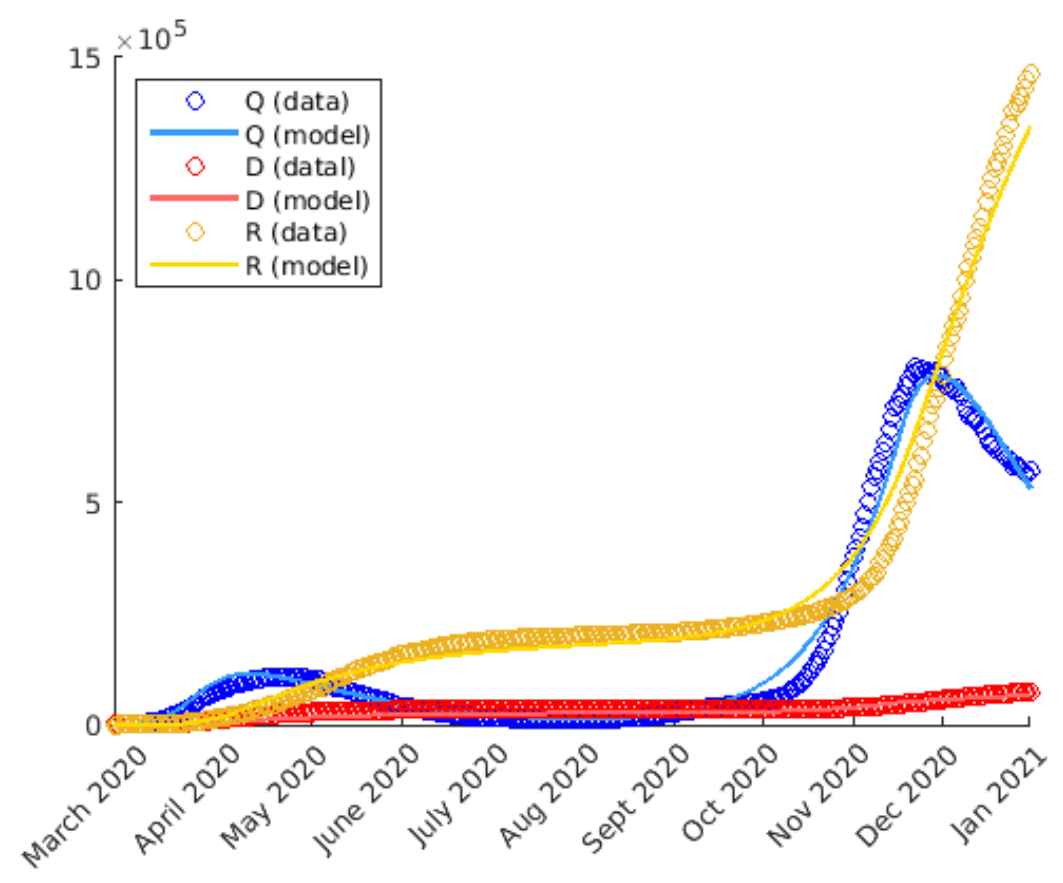

Italy, according to our results the insensitivity to the state of emergency may have cost 27,000 lives at the end of 2020 .

This model, albeit with simplifications and inevitable limitations, can be used not only for a posteriori analysis of Covid-19 or epidemic scenarios, but also preventively to make containment and precautionary decisions, giving a tool to measure possible future impacts due to pandemic fatigue.

\subsection{Achievements and future opportunities}

Nowadays, strategies to face COVID-19 pandemic must be identified. The fight against this novel virus has called for countless people from various fields of knowledge to propose studies and evidences. In this paper, we have presented a ODEs mathematical model to project the pandemic forward, with public health interventions and including individual behavioural responses due to pandemic fatigue.

Our model is relatively simple, however it has the merit to consider essential elements. It can easily simulate the dynamics resulting from the application of measures affecting the rate of transmission. Simple tests can highlight unexpected results, showing a high sensitivity to governmental actions, and forecast eventualities to escape. Such predictions emphasize the importance of interventions in controlling COVID-19 outbreak. The key feature of our model is to allow to incorporate a possible lowering of guard and negligence in observing precautionary 
practices for the emotional pressure of the emergency perpetration and the social discomforts resulting from a different and in some cases hostile daily life. According to the model, in Italy, a decreased sensitivity of the population to a state of alarm could have caused 27,000 more deaths by the end of 2020 .

Our study involves the (i) formulation and well-posedness prove of the mathematical model, (ii) a consistency test with publicly accessible data sources, and (iii) the availability of the numerical code. Our Matlab implementation is freely downloadable and utilizable from GitHub repository (MEACCI, 2020).

The model aims to be descriptive and predictive; "since all models are wrong" it should be considered as a baseline model for further improvement (BOX, 1976). A challenging opportunity consists in introducing the spatial dependence of variables and parameters. This development can lead to a considerable complication of the model, by adopting the theory of differential equations. Other modeling choices, such as agent-based model or cellular automata, could turn out to be a viable and simultaneously relevant options (FUENTES; KUPERMAN, 1999; WHITE; REY; SÁNCHEZ, 2007). Some study objectives may be represented by the identification of an optimal preventive strategy under territorial basis, the study of the behavioral mutual influences of different types of populations, and the effects on the restriction of internal and international mobility.

Our study underlines the importance to develop not only direct restrictive strategies. Such measures are vital and are strictly necessary. Nonetheless, positive impacting results can be achieved by working to promote indirect strategies, oriented to alleviate the fatigue sense of the population. For example, promoting the idea that physical distancing does not imply emotional detachment with others is useful. Social connections can potentially buffer against negative physical and mental health outcomes (NITSCHKE et al., 2020). Also advertising opportunities for reduced academic and work stress, increased time with family, or other advantages of the new situation can encourage positive attitudes (SERLACHIUS; BADAWY; THABREW, 2020; ORGANIZATION et al., 2020c). A more acute containment strategy coupled with an effort in improving the social responsibility and resilience can mitigate terrible consequences of COVID-19 pandemic. 

CHAPTER

12

\section{CONCLUSIONS}

In the presentation of the thesis, we preferred the presentation in editorial form of the results obtained over a dissertative illustration. This document consists of seven published articles and four papers in the submission or revision phase. For this reason each chapter can be considered an autonomous reading: although each of them is part of a research stream, it can be independently considered and understood.

These results are part of a project of the National Institute of Science and Technology in Medicine Assisted by Scientific Computing (INCT - MACC), within the Brazilian Program of National Institutes of Science and Technology, to consolidate its associated network as an international player among the few leading organizations in computational modeling in medicine and its applications in health-care.

\subsection{Main contribution}

The main contribution of this thesis is represented by the presentation of a new twocomponent model for the red blood cell membrane. The merit of this new formulation is to mimic the two main component of the red blood cell membrane, i.e., the cytoskeleton and the lipid bilayer, as the most sophisticated current particle-based models of Li et al. (2014), Tang et al. (2017), and Fu et al. (2017), but with a continuum approach for the lipid bilayer component. Continuous models provide consistent approximations of large scale molecular systems with less adjustable parameters than particle-based approaches, and allow to benefit from the basin of numerical methods for continuum mechanics. Specifically, we adopt the viscous liquid-shell model with Canham-Helfrich bending energy described by Arroyo, DeSimone and Heltai (2010) with the discretization proposed by Rodrigues et al. (2015a). We thus arrive at a hybrid model, with a discrete network for the cytoskeleton and a continuous surface fluid for the lipid bilayer. The two components are coupled by adhesion forces that mimic the attachment of the cytoskeleton nodes to the bilayer integral proteins. A fully-discrete numerical scheme is 
proposed and tested.

The delivery of the two-component model allowed to virtually explore and get physical measurements of particular inter-cellular behaviors, such as bilayer's reaction to exogenous bodies, or the cytoskeleton impact on the local bilayer curvature and into the resulting bending energy, the average surface tension and internal fluid pressure. In particular, we visualized and studied extreme events like the detachment of the cytoskeleton from the lipid bilayer and the opposite case of a folding phenomenon of the spectrins due to an overtension condition, leading to a complex evolution of the bilayer energy and a perturbation of its biconcave shape.

The novelty was accompanied by studies dedicated ad hoc to illustrate the innovations introduced and to evaluate their effects separately. At the same time, this decision was intended to cover a further exigency. In order to enable a deeper scientific understanding of red blood cell disease developments and their preventions, there is a need to continue to develop and implement the latest and most advanced single-cell mathematical/computational models to complement experimental findings. In this context, one challenge is to bridge the gap making the mathematical approach accessible to a wider interdisciplinary readership and allow its implementation.

We have provided a detailed explanation of the cytoskeletal component-based model and given some insights on its mechanical behaviour. In summary, some main points addressed are: (a) red blood cell (RBC) cytoskeleton is a complex structure with non-trivial dynamics, (b) adoption of worm-like-chain forces and principle of virtual work for RBC cytoskeleton modeling, (c) delivering of a cytoskeletal component-based mathematical model, (d) focus on single spectrin equilibrium, (e) cytoskeletal symmetry defects impact on mechanical system response, (f) coarse graining system techniques, (g) virtual sole cytoskeleton optical tweezers experiment, and (f) commented ready-to-use code.

At the same time, a particular effort has been made to introduce a method to simulate the adhesion between the membrane components through the trans-membrane proteins. Adopting the recent progress on the computational modeling of adhesive contact, the introduction of the effects of cytoskeleton-bilayer interaction has represented an original contribution to the entire red blood cell model. The publication of this method may open to further improvements in the simulation of this significant effect and its understanding

One special work was dedicated to a membrane application with the presentation of the first detailed mathematical modelling of the oleosome, a biologically and technologically relevant plant organelle. This pioneeristic approach we proposed in the study can be valuable in promoting interdisciplinary research between food, biological and mathematical sciences.

Thanks to these previous advances in building and refining models concerning various aspects of the red blood cell membrane, we were able to present our most generalized and sophisticated mathematical/computational formulation. Such model is multi-component; it takes into account the lipid bilayer behavior, the cytoskeleton dynamics, the interaction activity 
between them, and the internal cytoplasm flow. This method is among the most advanced in terms of simulating the mechanical behavior of a single red blood cell, both for the consideration of the four components and for the advantages of a continuous modeling of the cytosplam and the lipid bilayer. In summary, from the computational point of view, it concerns a discrete stabilized formulation for the bilayer with surface triangular P1-linear finite elements and surface area/volume controllers, a discrete network representation for the cytoskeleton, both interacting through appropriate adhesion forces, and a P1 tetrahedral finite element discretization of the bulk fluids, resolving a Stokes problem under Newtonian behavior.

This main achievement allows us to believe in the opening of numerous interdisciplinary studies with this new mathematical tool. In Section 12.3 we present a brief overview of some of them, providing specific research opportunities.

\subsection{Other contributions}

Our research work contributed also towards other directions; we aim to highlight the followings achievements.

Concerning the microfluidic control, a growing interest exists in in the steering of particles in microfluidic systems, especially in the biological and medical fields. In our work reported on Chapter 7, an interconnection of various mathematical problems, such as Stokes equations, Poisson equation for electrical potential, linear optimization, control problem, Bellman's principle and Hamilton-Jacobi equation was shown. Our method automatically identifies a safe timeoptimal trajectory. The simulations made by the numerical resolution of the model have allowed to point out some important remarks and to show how counter-intuitive the optimal trajectories can be in this kind of problem, therefore proving the utility of using a mathematical and computational approach for designing performing trajectories.

With respect to models for disease progression we addressed two illness forms: tumor and Alzheimer's disease.

The heterogeneity of tumour cells is now widely accepted and cancer stem cells (CSCs) have been identified in many cancers, as well as in sarcomas and leukemia. The CSCs relevance in the progression of these and other tumours appears to be evident and the investigation of their role has been the object of many experimental papers as well as of more theoretically-oriented articles. One of the features of tumours with CSC is the so-called tumour-growth-paradox that consists in the fact that a tumour with a larger death rate for ordinary (non-stem) cancer cells might grow faster than a tumour with a lower death rate for the same class of cells. Of course, since the death rate is influenced by medical treatment, understanding the reasons of this paradox can be relevant in the control of tumours. We presented, discussed and compared various models involving different mathematical ingredients and different approaches: agent-based models with the use of cellular automata, ordinary differential equations mean field approaches, integro- 
differential equations models, and reaction-diffusion models. We wrote these works with the aim of being accessible to a wide readership, maintaining an interdisciplinary character for bringing experts and researchers from different but complementary sectors in the comprehension of the phenomenon but at the same time with the necessary formalism of mathematics. Numerical simulations are displayed for each class of models, in order to not only contribute to elucidate fundamental principles of the elements that occur in this important issue and to develop a consistent mathematical model but also to make certain predictions experimentally verifiable as well to exhibit the tumour-growth paradox. We dedicated special attention to the impact of crowing and ageing of cell, obtaining conclusions about some main points: (a) crowding of cells during the growth of tumours with stem cells induces an accelerated growth in presence of cytotoxic treatment (tumour paradox); (b) cellular automata provide an useful tool to describe the process including senescence of cells and to evidentiate the paradoxical behaviour; (c) because of crowding, compartmental models describing the evolution of different lineages of differentiated cells result in systems of ODE's that are not hierarchical; and (d) to take age-structure of cells into account in a continuous model, a non-standard boundary value problem for a first order PDE is consistent.

From the Alzheimer perspective, the existence of soluble toxic oligomers of beta-amyloid seems to be the cause of the degradation of neurons. Our strategy was to mimic the two main phenomena involved in the following manner. First, we modeled the evolution from monomeric amyloid produced by neurons to senile plaques by a system of ordinary differential equations, in accordance with the Smoluchowski equation. Then, we considered the brain tissue divided into small representative regions. The latter process concerns the disease progression taking into account the interaction among adjacent regions. These two problems are interdependent, because the monomers production depends on the average degradation of the neurons and in turn, the degradation is caused by the number of toxic oligomers (generated by the monomers). The results obtained regarding the time of the Alzheimer's evolution are in line with the statistical data, indicating a physical consistency of the model joint with a mathematical well-posedness which in the work has been reported in detail.

Finally, an epidemiological contribution was presented for the the COVID-19 outbreak. The psychological effects and the lack of resilience to coexistence with the pandemic were found to be relevant for the evolution and spread of coronavirus itself. Our proposed model has the merit of considering this component of social reaction through a model with ordinary partial differential equations. The model is proved mathematically well-posed and has been tested in the text to offer a possible explanation of the acceleration of cases in the pandemic second wave in Italy during 2020. Our study highlights how vital is to apply restrictive strategies, but at the same time to promote complementary strategies, oriented to treat the pandemic fatigue of the population. 


\subsection{Ongoing and future works}

The future goals are directed to a necessary and constant effort to improve the models and cover the use of them. This goal underlines the need to increase collaborations and to concretize the conjunction of mathematical achievements with experimental results in laboratories and in the medical field. We believe this aspect is vital to the quality of this kind of science production and to the usability of the findings by the scientific community. Some of the future opportunities are listed in the last sections of each Chapter, according to the specific topic.

However, concerning the RBC membrane model, we believe that the following two points, on which we have already begun to work, deserve some mention.

In the first place, we are in the process of using the model to refine virtual experiments, for which there is a physical equivalent that can be conducted in the laboratory. Thanks to new technologies, it is increasingly possible to obtain measurements and data even at the microscopic level. The strategy of working in parallel with experiments in the laboratory can accelerate the results and the reliability of the deductions. Several of these types of techniques and experiments are shown in Figure 85. The Figure 86 shows our first attempt to apply our model to simulate the micropipette aspiration experiment (HERANT; MARGANSKI; DEMBO, 2003).

Figure 85 - Major techniques for cell mechanics study. (a) Atomic force spectroscopy; (b) magnetic twisting cytometry; (c) cytoindentation; (d) microplate stretcher; (e) micropipette aspiration; (f) laser/optical tweezers; (g) optical stretcher; (h) shear flow; (i) microfluidic assay; (j) microfabricated post array; (k) particle tracking microrheology; (l) magnetic nanoparticlebased stimuli. The imgae is taken from Unal et al. (2014)

(a)
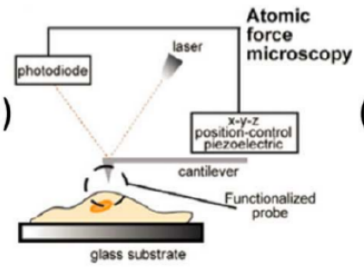

(e)

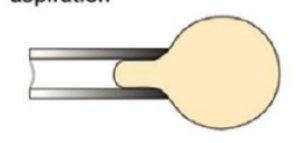

(i)

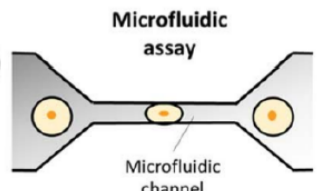

(b)

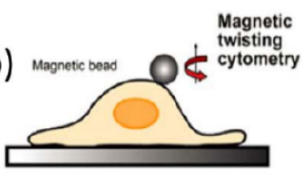

(f)

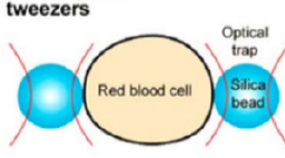

(c)
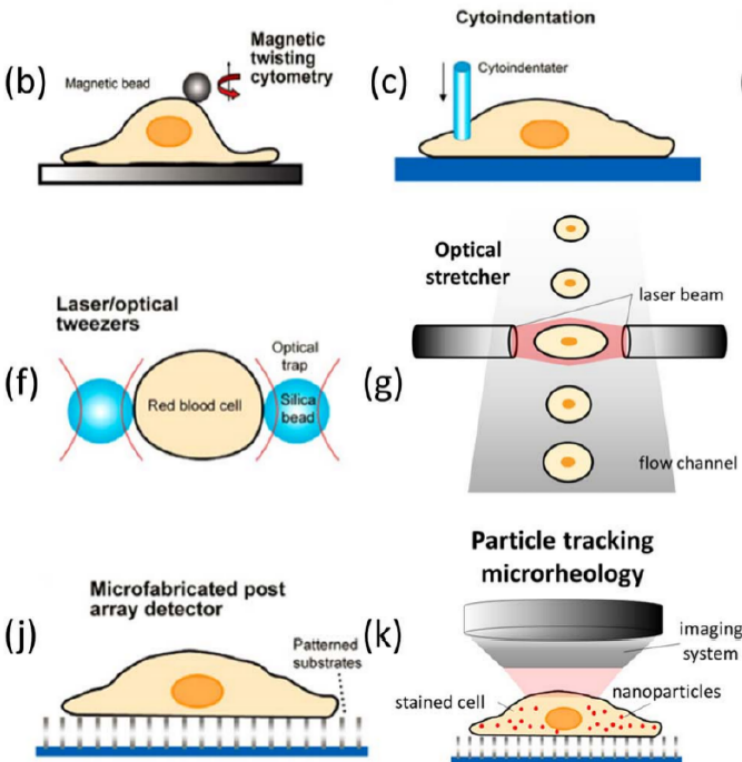

Particle tracking

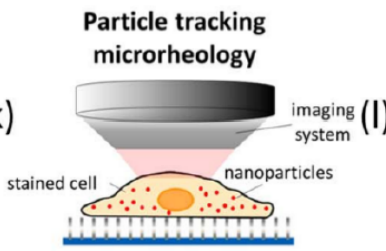

(d)
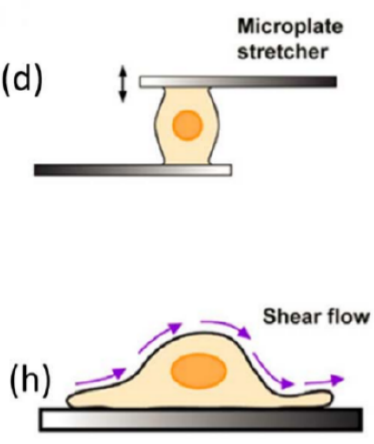

Magnetic nanoparticle stimuli

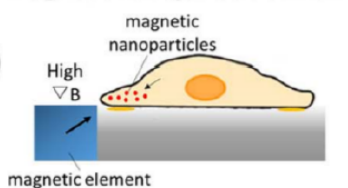

Another challenging perspective is represented by introducing stochastic fluctuations in our model. In particular, the treatment of fluctuations can be adopted by stochastic integration with implicit treatment of the stiffer deterministic terms. Methods of increasing temporal accuracy 
Figure 86 - Virtual aspiration of a RBC by two micropipettes at different times showing the cell deformation and the underlying triangular mesh used for discretization of the lipidic membrane.
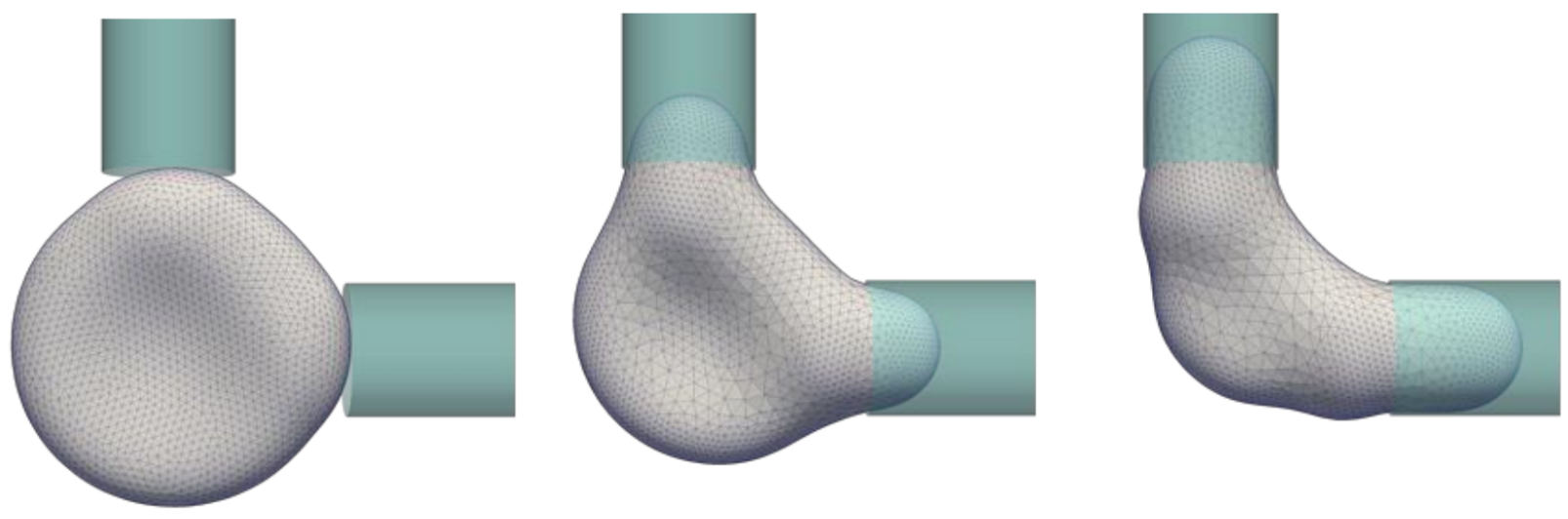

with less restrictive time steps can be studied by decomposition of operators (HOWARD; MILNER, 2011; MILSTEIN; TRETYAKOV, 2013). A first approach to induce the stomatocyte transition using stochastic force in shown in Figure 87, where we can observe the tendency of the biconcave membrane to tend towards diseased morphology, with respect to the increase in thermal fluctuations imposed.

Figure 87 - Attempt to induce the typical morphology of the RBC membrane under stomatocyte appearance, increasing the thermal fluctuations approach stomacyte transition simulations, including stochastic fluctuations in the model.

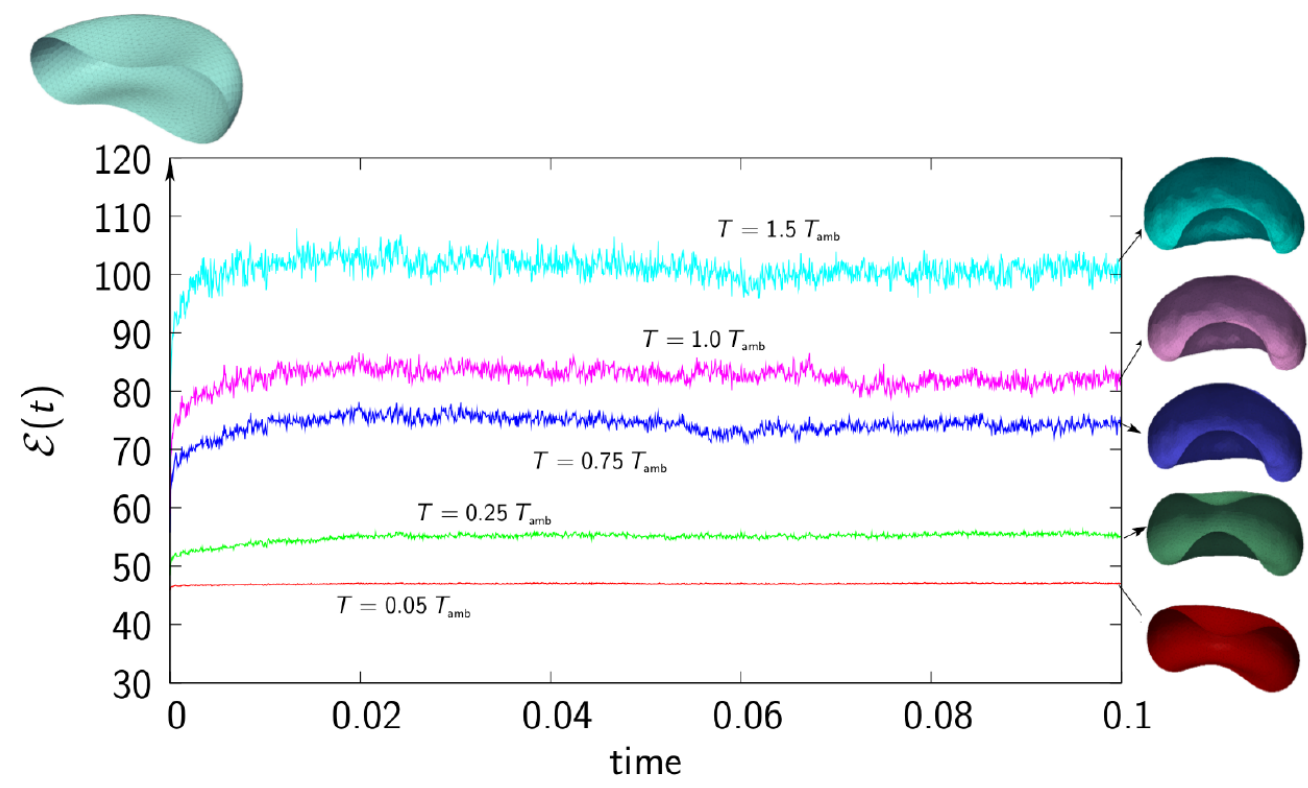


ACHDOU, Y.; FRANCHI, B.; MARCELLO, N.; TESI, M. C. A qualitative model for aggregation and diffusion of $\beta$-amyloid in Alzheimer's disease. J. Math. Biol., v. 67, n. 6-7, p. 1369-1392, 2013. ISSN 0303-6812. Citations on pages 159 and 162.

ADAMS, G. G.; IMRAN, S.; WANG, S.; MOHAMMAD, A.; KOK, M. S.; GRAY, D. A.; CHANNELL, G. A.; HARDING, S. E. Extraction, isolation and characterisation of oil bodies from pumpkin seeds for therapeutic use. Food chemistry, Elsevier, v. 134, n. 4, p. 1919-1925, 2012. Citation on page 74 .

AL-HAJJ, M.; WICHA, M. S.; BENITO-HERNANDEZ, A.; MORRISON, S. J.; CLARKE, M. F. Prospective identification of tumorigenic breast cancer cells. Proceedings of the National Academy of Sciences, National Acad Sciences, v. 100, n. 7, p. 3983-3988, 2003. Citations on pages 125 and 141 .

ANDERSON, A.; REJNIAK, K. Single-cell-based models in biology and medicine. [S.1.]: Springer Science \& Business Media, 2007. Citation on page 47.

ANDERSON, A. R.; WEAVER, A. M.; CUMMINGS, P. T.; QUARANTA, V. Tumor morphology and phenotypic evolution driven by selective pressure from the microenvironment. Cell, Elsevier, v. 127, n. 5, p. 905-915, 2006. Citation on page 142.

ANDERSON, E. C.; HESSMAN, C.; LEVIN, T. G.; MONROE, M. M.; WONG, M. H. The role of colorectal cancer stem cells in metastatic disease and therapeutic response. Cancers, Molecular Diversity Preservation International, v. 3, n. 1, p. 319-339, 2011. Citation on page 247.

ARINO, J.; PORTET, S. A simple model for covid-19. Infectious Disease Modelling, Elsevier, 2020. Citation on page 178.

ARMANI, M.; CHAUDHARY, S.; PROBST, R.; WALKER, S.; SHAPIRO, B. Control of microfluidic systems: two examples, results, and challenges. International Journal of Robust and Nonlinear Control, Wiley Online Library, v. 15, n. 16, p. 785-803, 2005. Citations on pages 111 and 112 .

ARMANI, M.; CUMMINS, Z.; GONG, J.; MATHAI, P.; PROBST, R.; ROPP, C.; WAKS, E.; WALKER, S.; SHAPIRO, B. Feedback control of microflows. In: Feedback Control of MEMS to Atoms. [S.1.]: Springer, 2012. p. 269-319. Citations on pages 111 and 112.

ARROYO, M.; DESIMONE, A. Relaxation dynamics of fluid membranes. Physical review E, APS, v. 79, n. 3, p. 031915, 2009. Citation on page 22.

ARROYO, M.; DESIMONE, A.; HELTAI, L. The role of membrane viscosity in the dynamics of fluid membranes. arXiv preprint arXiv:1007.4934, 2010. Citations on pages 30, 33, 49, and 191. 
ASMUNDSON, G. J.; TAYLOR, S. Coronaphobia: Fear and the 2019-ncov outbreak. Journal of anxiety disorders, Elsevier, v. 70, p. 102196, 2020. Citation on page 179.

How health anxiety influences responses to viral outbreaks like covid-19: What all decisionmakers, health authorities, and health care professionals need to know. Journal of anxiety disorders, Elsevier, v. 71, p. 102211, 2020. Citation on page 179.

AVSIEVICH, T.; ZHU, R.; POPOV, A.; BYKOV, A.; MEGLINSKI, I. The advancement of blood cell research by optical tweezers. Reviews in Physics, Elsevier, v. 5, p. 100043, 2020. Citation on page 106.

BACYINSKI, A.; XU, M.; WANG, W.; HU, J. The paravascular pathway for brain waste clearance: Current understanding, significance and controvers. Front Neuroanat., v. 101, p. 101, 2017. Citation on page 164 .

BALLARD, C.; GAUTHIER, S.; CORBETT, A.; BRAYNE, C.; AARSLAND, D.; JONES, E. Alzheimer's disease. The Lancet, v. 377, p. 1019-1031, 2011. Citation on page 168.

B ̈̈NSCH, E. Finite element discretization of the navier-stokes equations with a free capillary surface. Numerische Mathematik, Springer, v. 88, n. 2, p. 203-235, 2001. Citations on pages 79 and 97.

BAO, G. Mechanics of biomolecules. Journal of the Mechanics and Physics of Solids, Elsevier, v. 50, n. 11, p. 2237-2274, 2002. Citation on page 51.

BAO, G.; BAZILEVS, Y.; CHUNG, J.-H.; DECUZZI, P.; ESPINOSA, H. D.; FERRARI, M.; GAO, H.; HOSSAIN, S. S.; HUGHES, T. J.; KAMM, R. D. et al. Usnctam perspectives on mechanics in medicine. Journal of The Royal Society Interface, The Royal Society, v. 11, n. 97, p. 20140301, 2014. Citations on pages 19, 29, and 47.

BAO, G.; SURESH, S. Cell and molecular mechanics of biological materials. Nature materials, Nature Publishing Group, v. 2, n. 11, p. 715, 2003. Citations on pages 47 and 74.

BARRE, A.; SIMPLICIEN, M.; CASSAN, G.; BENOIST, H.; ROUGÉ, P. Oil bodies (oleosomes): Occurrence, structure, allergenicity. Revue Française d'Allergologie, Elsevier, 2018. Citation on page 83 .

BARRETT, J. W.; GARCKE, H.; NÜRNBERG, R. Numerical computations of the dynamics of fluidic membranes and vesicles. Physical review E, APS, v. 92, n. 5, p. 052704, 2015. Citation on page 22 .

A stable numerical method for the dynamics of fluidic membranes. Numerische Mathematik, Springer, v. 134, n. 4, p. 783-822, 2016. Citation on page 88.

BARTHES-BIESEL, D. Motion and deformation of elastic capsules and vesicles in flow. Annual Review of fluid mechanics, Annual Reviews, v. 48, p. 25-52, 2016. Citations on pages 21 and 49.

BARTOSZEK, A.; WALKOWIAK, D.; BARTOSZEK, A.; KARDAS, G. Mental well-being (depression, loneliness, insomnia, daily life fatigue) during covid-19 related home-confinement-a study from poland. International journal of environmental research and public health, Multidisciplinary Digital Publishing Institute, v. 17, n. 20, p. 7417, 2020. Citation on page 179. 
BATEMAN, R. J.; MUNSELL, L. Y.; MORRIS, J. C.; SWARM, R.; YARASHESKI, K. E.; HOLTZMAN, D. M. Quantifying cns protein production and clearance rates in humans using in vivo stable isotope labeling, immunoprecipitation, and tandem mass spectrometry. Nature medicine, NIH Public Access, v. 12, n. 7, p. 856, 2006. Citation on page 167.

BATLlE, E.; CLEVERS, H. Cancer stem cells revisited. Nature Medicine, Nature Publishing Group, v. 23, n. 10, p. 1124, 2017. Citation on page 141.

BAUMGART, T.; HESS, S. T.; WEBB, W. W. Imaging coexisting fluid domains in biomembrane models coupling curvature and line tension. Nature, Nature Publishing Group, v. 425, n. 6960, p. 821,2003 . Citation on page 40 .

BELLOMO, N.; DELITALA, M. From the mathematical kinetic, and stochastic game theory to modelling mutations, onset, progression and immune competition of cancer cells. Physics of Life Reviews, Elsevier, v. 5, n. 4, p. 183-206, 2008. Citations on pages 141 and 142.

BELLOMO, N.; LI, N.; MAINI, P. K. On the foundations of cancer modelling: selected topics, speculations, and perspectives. Mathematical Models and Methods in Applied Sciences, World Scientific, v. 18, n. 04, p. 593-646, 2008. Citation on page 141.

BENÍTEZ, L.; BARBERIS, L.; CONDAT, C. Modeling tumorspheres reveals cancer stem cell niche building and plasticity. Physica A: Statistical Mechanics and its Applications, Elsevier, v. 533, p. 121906, 2019. Citation on page 141.

BENNETT, V. The spectrin-actin junction of erythrocyte membrane skeletons. Biochimica et Biophysica Acta (BBA)-Reviews on Biomembranes, Elsevier, v. 988, n. 1, p. 107-121, 1989. Citation on page 58.

BENNETT, V.; BAINES, A. J. Spectrin and ankyrin-based pathways: metazoan inventions for integrating cells into tissues. Physiological reviews, Am Physiological Soc, v. 81, n. 3, p. 1353-1392, 2001. Citation on page 48.

BERETTA, E.; CAPASSO, V.; MOROZOVA, N. Mathematical modelling of cancer stem cells population behavior. Mathematical Modelling of Natural Phenomena, EDP Sciences, v. 7, n. 1, p. 279-305, 2012. Citation on page 247.

BERETTA, E.; MOROZOVA, N.; CAPASSO, V.; HAREL-BELLAN, A. Some results on the population behavior of cancer stem cells. In: New Challenges for Cancer Systems Biomedicine. [S.1.]: Springer, 2012. p. 145-172. Citation on page 247.

BERTSCH, M.; FRANCHI, B.; MARCELLO, N.; TESI, M. C.; TOSIN, A. Alzheimer's disease: a mathematical model for onset and progression. Math. Med. Biol., v. 34, n. 2, p. 193-214, 2017. ISSN 1477-8599. Available: <https://doi.org/10.1093/imammb/dqw003>. Citations on pages 159,162 , and 168 .

BERTSCH, M.; FRANCHI, B.; MESCHINI, V.; TESI, M.; TOSIN, A. A sensitivity analysis of a mathematical model for the synergistic interplay of Amyloid beta and tau on the dynamics of Alzheimer's disease. 2020. Preprint, ArXiv 2006.01749. Citation on page 168.

BERTSCH, M.; FRANCHI, B.; TESI, M.; TOSIN, A. Microscopic and macroscopic models for the onset and progression of Alzheimer's disease. J. Phys. A, v. 50, n. 41, p. 414003, 22, 2017. ISSN 1751-8113. Available: <https://doi.org/10.1088/1751-8121/aa83bd>. Citations on pages 159, 162, and 163. 
Well-posedness of a mathematical model for Alzheimer's disease. SIAM J. Math. Anal., v. 50, n. 3, p. 2362-2388, 2018. ISSN 0036-1410. Available: <https://doi.org/10.1137/ 17M1148517>. Citation on page 162.

BETTERIDGE, R.; OWEN, M. R.; BYRNE, H. M.; ALARCÓN, T.; MAINI, P. K. The impact of cell crowding and active cell movement on vascular tumour growth. Networks and heterogeneous media, v. 1, n. 4, p. 515-535, 2006. Citations on pages 125, 126, 127, 128, 142, and 248.

BETTINI, S.; VERGARA, D.; BONSEGNA, S.; GIOTTA, L.; TOTO, C.; CHIEPPA, M.; MAFFIA, M.; GIOVINAZZO, G.; VALLI, L.; SANTINO, A. Efficient stabilization of natural curcuminoids mediated by oil body encapsulation. RSC advances, Royal Society of Chemistry, v. 3 , n. 16 , p. 5422-5429, 2013. Citation on page 74 .

BETZ, T.; LENZ, M.; JOANNY, J.-F.; SYKES, C. Atp-dependent mechanics of red blood cells. Proceedings of the National Academy of Sciences, National Acad Sciences, v. 106, n. 36, p. 15320-15325, 2009. Citation on page 42.

BIRIA, A.; MALEKI, M.; FRIED, E. Continuum theory for the edge of an open lipid bilayer. Advances in Applied Mechanics, Elsevier, v. 46, p. 1-68, 2013. Citation on page 87.

BONITO, A.; DEMLOW, A.; NOCHETTO, R. H. Finite element methods for the laplacebeltrami operator. In: Handbook of Numerical Analysis. [S.1.]: Elsevier, 2020. v. 21, p. 1-103. Citation on page 93.

BONITO, A.; NOCHETTO, R. H.; PAULETTI, M. S. Parametric fem for geometric biomembranes. Journal of Computational Physics, Elsevier, v. 229, n. 9, p. 3171-3188, 2010. Citations on pages 34,77 , and 88 .

BOONDIREK, A.; TRIAMPO, W.; NUTTAVUT, N. A review of cellular automata models of tumor growth. In: CITESEER. International Mathematical Forum. [S.1.], 2010. v. 5, n. 61, p. 3023-3029. Citation on page 248.

BORGHI, N.; BROCHARD-WYART, F. Tether extrusion from red blood cells: integral proteins unbinding from cytoskeleton. Biophysical journal, Elsevier, v. 93, n. 4, p. 1369-1379, 2007. Citation on page 48.

BORSI, I.; FASANO, A.; PRIMICERIO, M.; HILLEN, T. Mathematical properties of a non-local integro-pde model for cancer stem cells. Mathematical Medicine and Biology, v. 34, p. 59-75, 2015. Citations on pages 142 and 248.

A non-local model for cancer stem cells and the tumour growth paradox. Mathematical Medicine and Biology, IMA, p. dqv037, 2015. Citations on pages 125, 126, 127, 131, 132, and 133.

BOURGEOIS, C.; GOMAA, A. I.; LEFÈVRE, T.; CANSELL, M.; SUBIRADE, M. Interaction of oil bodies proteins with phospholipid bilayers: A molecular level elucidation as revealed by infrared spectroscopy. International journal of biological macromolecules, Elsevier, 2018. Citation on page 73 .

BOUSSINESQ, M. Sur l'existance d'une viscosite superficielle, dans la mince couche de transition separant un liquide d'une autre fluide contigue. Ann. Chim. Phys, v. 29, p. 349-357, 1913. Citations on pages 77 and 87. 
BOX, G. E. Science and statistics. Journal of the American Statistical Association, Taylor \& Francis, v. 71, n. 356, p. 791-799, 1976. Citation on page 189.

BRAAK, H.; TREDICI, K. D. Alzheimer's pathogenesis: is there neuron-to-neuron propagation? Acta Neuropathol., Springer-Verlag, v. 121, n. 5, p. 589-595, 2011. ISSN 0001-6322. Citation on page 160 .

BRASAEMLE, D. L.; WOLINS, N. E. Packaging of fat: an evolving model of lipid droplet assembly and expansion. Journal of Biological Chemistry, ASBMB, v. 287, n. 4, p. 2273-2279, 2012. Citation on page 73.

BRAUER, F. Mathematical epidemiology: Past, present, and future. Infectious Disease Modelling, Elsevier, v. 2, n. 2, p. 113-127, 2017. Citation on page 178.

BUSCAGLIA, G. C.; AUSAS, R. F. Variational formulations for surface tension, capillarity and wetting. Computer Methods in Applied Mechanics and Engineering, Elsevier, v. 200, n. 45-46, p. 3011-3025, 2011. Citation on page 87.

CACACE, S.; CRISTIANI, E.; FALCONE, M. Can local single-pass methods solve any stationary hamilton-jacobi-bellman equation? SIAM Journal on Scientific Computing, SIAM, v. 36, n. 2, p. A570-A587, 2014. Citations on pages 115 and 116.

CACCAVO, D. Chinese and italian covid-19 outbreaks can be correctly described by a modified sird model. medRxiv, Cold Spring Harbor Laboratory Press, 2020. Citations on pages 178 and 180 .

Cancer Stem Cell News. Web site. https://www. cancerstemcellnews.com/reviews/. 2018. Citation on page 141.

CANHAM, P. B. The minimum energy of bending as a possible explanation of the biconcave shape of the human red blood cell. Journal of theoretical biology, Elsevier, v. 26, n. 1, p. 61IN777-76IN881, 1970. Citations on pages 33, 40, 76, and 88.

CAPASSO, V.; SERIO, G. A generalization of the kermack-mckendrick deterministic epidemic model. Mathematical Biosciences, Elsevier, v. 42, n. 1, p. 43-61, 1978. Citation on page 136.

CAPECCHI, D. History of virtual work laws: a history of mechanics prospective. [S.1.]: Springer Science \& Business Media, 2012. Citation on page 86.

CARBONELL, F.; ITURRIA-MEDINA, Y.; EVANS, A. Mathematical modeling of protein misfolding mechanisms in neurological diseases: A historical overview. Front. Neurol., v. 9:37, p. 1-16, 2018. Citations on pages 159, 162, and 163.

CARLO, D. D.; LEE, L. P. Dynamic single-cell analysis for quantitative biology. [S.1.]: ACS Publications, 2006. Citations on pages 30 and 47.

CHAKRABORTY, T.; GHOSH, I. Real-time forecasts and risk assessment of novel coronavirus (covid-19) cases: A data-driven analysis. Chaos, Solitons \& Fractals, Elsevier, p. 109850, 2020. Citation on page 178 .

CHAN, Y.; HAVERKAMP, R. G.; HILL, J. M. Force-extension formula for the worm-like chain model from a variational principle. Journal of theoretical biology, Elsevier, v. 262, n. 3, p. 498-504, 2010. Citation on page 51. 
CHANG, H.-Y.; LI, X.; KARNIADAKIS, G. E. Modeling of biomechanics and biorheology of red blood cells in type 2 diabetes mellitus. Biophysical journal, Elsevier, v. 113, n. 2, p. 481-490, 2017. Citations on pages 30, 49, and 67.

CHANG, H.-Y.; LI, X.; LI, H.; KARNIADAKIS, G. E. Md/dpd multiscale framework for predicting morphology and stresses of red blood cells in health and disease. PLoS computational biology, Public Library of Science, v. 12, n. 10, p. e1005173, 2016. Citations on pages 49, 67, 100, 101, 104, and 106.

CHAPMAN, K. D.; DYER, J. M.; MULLEN, R. T. Biogenesis and functions of lipid droplets in plants thematic review series: lipid droplet synthesis and metabolism: from yeast to man. Journal of lipid research, ASBMB, v. 53, n. 2, p. 215-226, 2012. Citation on page 73.

CHAUDHARY, S.; SHAPIRO, B. Arbitrary steering of multiple particles independently in an electro-osmotically driven microfluidic system. IEEE Transactions on Control Systems Technology, IEEE, v. 14, n. 4, p. 669-680, 2006. Citations on pages 111, 112, and 115.

CHEN, C. Y.; TSENG, Y. H.; WARD, J. P. A mathematical model demonstrating the role of interstitial fluid flow on the clearance and accumulation of amyloid $\beta$ in the brain. Math. Biosci., v. 317, p. 108258, 2019. ISSN 0025-5564. Available: <https://doi.org/10.1016/j.mbs. 2019.108258>. Citation on page 162.

CHEN, H.; MEISBURGER, S. P.; PABIT, S. A.; SUTTON, J. L.; WEBB, W. W.; POLLACK, L. Ionic strength-dependent persistence lengths of single-stranded rna and dna. Proceedings of the National Academy of Sciences, National Acad Sciences, v. 109, n. 3, p. 799-804, 2012. Citation on page 51 .

CHIMON, S.; SHAIBAT, M.; JONES, C.; CALERO, D.; B., A.; Y., I. Evidence of fibril-like $\beta$-sheet structures in a neurotoxic amyloid intermediate of alzheimerÕs $\beta$-amyloid. Nature Structural \& Molecular Biology, v. 14, n. 12, p. 1157-1164, 2007. Citation on page 160.

CHIRICO, S. D.; BARI, V. di; FOSTER, T.; GRAY, D. Enhancing the recovery of oilseed rape seed oil bodies (oleosomes) using bicarbonate-based soaking and grinding media. Food chemistry, Elsevier, v. 241, p. 419-426, 2018. Citation on page 74.

CHO, R. W.; CLARKE, M. F. Recent advances in cancer stem cells. Current opinion in genetics \& development, Elsevier, v. 18, n. 1, p. 48-53, 2008. Citation on page 247.

CHOPARD, B.; DROZ, M. Cellular automata modeling of physical systems. 1998. Cambridge UP, p. 122-137. Citation on page 127.

CIVILE, P. del Consiglio dei Ministri Dipartimento della P. Dati COVID-19 Italia. [S.1.]: GitHub, 2020. <https://github.com/pcm-dpc/COVID-19>. Citation on page 184.

CLARKE, M. F.; FULLER, M. Stem cells and cancer: two faces of eve. Cell, Elsevier, v. 124, n. 6, p. 1111-1115, 2006. Citation on page 247.

CLÉMENT, F; MARTIN, V. The lax-milgram theorem. a detailed proof to be formalized in coq. arXiv preprint arXiv:1607.03618, 2016. Citation on page 93.

CLEVERS, H. The cancer stem cell: premises, promises and challenges. Nature Medicine, Nature Publishing Group, v. 17, n. 3, p. 313, 2011. Citations on pages 141 and 247. 
CODINA, R.; BLASCO, J.; BUSCAGLIA, G. C.; HUERTA, A. Implementation of a stabilized finite element formulation for the incompressible navier-stokes equations based on a pressure gradient projection. International Journal for Numerical Methods in Fluids, Wiley Online Library, v. 37, n. 4, p. 419-444, 2001. Citation on page 96.

COHEN, A. D.; RABINOVICI, G. D.; MATHIS, C. A.; JAGUST, W. J.; KLUNK, W. E.; IKONOMOVIC, M. D. Using pittsburgh compound $\mathrm{b}$ for in vivo pet imaging of fibrillar amyloidbeta. In: MICHAELIS, E. K.; MICHAELIS, M. L. (Ed.). Current State of Alzheimer's Disease Research and Therapeutics. Academic Press, 2012, (Advances in Pharmacology, v. 64). p. 27 - 81. Available: <http://www.sciencedirect.com/science/article/pii/B9780123948168000027>. Citation on page 163 .

COHEN, J. E. Mathematics is biology's next microscope, only better; biology is mathematics' next physics, only better. Plos biol, Public Library of Science, v. 2, n. 12, p. e439, 2004. Citation on page 19.

COOPER, I.; MONDAL, A.; ANTONOPOULOS, C. G. A sir model assumption for the spread of covid-19 in different communities. Chaos, Solitons \& Fractals, Elsevier, v. 139, p. 110057, 2020. Citation on page 178 .

COVENEY, P. V. Computational biomedicine. Part 1: molecular medicine. [S.1.]: The Royal Society, 2020. Citation on page 19.

CRAFT, D.; WEIN, L.; SELKOE, D. A mathematical model of the impact of novel treatments on the a beta burden in the alzheimer's brain, csf and plasma. Bull. Math .Biol., v. 64, n. 5, p. 1011-1031, 2002. Citation on page 162.

CRISTIANI, E. Numerical methods for optimal control problems: part II: local single-pass methods for stationary HJ equations. Roma, 2013. P. 21. Class notes. Citation on page 120.

CRUZ, L.; URBANC, B.; BULDYREV, S. V.; CHRISTIE, R.; GóMEZ-ISLA, T.; HAVLIN, S.; MCNAMARA, M.; STANLEY, H. E.; HYMAN, B. T. Aggregation and disaggregation of senile plaques in Alzheimer disease. P. Natl. Acad. Sci. USA, v. 94, n. 14, p. 7612-7616, 1997. Citation on page 159 .

CULLEN, W.; GULATI, G.; KELLY, B. Mental health in the covid-19 pandemic. QJM: An International Journal of Medicine, Oxford University Press, v. 113, n. 5, p. 311-312, 2020. Citation on page 180 .

CURRIE, C. S.; FOWLER, J. W.; KOTIADIS, K.; MONKS, T.; ONGGO, B. S.; ROBERTSON, D. A.; TAKO, A. A. How simulation modelling can help reduce the impact of covid-19. Journal of Simulation, Taylor \& Francis, v. 14, n. 2, p. 83-97, 2020. Citation on page 178.

CURTIS, J. E.; KOSS, B. A.; GRIER, D. G. Dynamic holographic optical tweezers. Optics communications, Elsevier, v. 207, n. 1, p. 169-175, 2002. Citation on page 111.

DALE, D. C.; MACKEY, M. C. Understanding, treating and avoiding hematological disease: better medicine through mathematics? Bulletin of mathematical biology, Springer, v. 77, n. 5, p. 739-757, 2015. Citation on page 19.

DAO, M.; LIM, C. T.; SURESH, S. Mechanics of the human red blood cell deformed by optical tweezers. Journal of the Mechanics and Physics of Solids, Elsevier, v. 51, n. 11-12, p. 2259-2280, 2003. Citations on pages 61, 71, and 105. 
DEACONU, M.; TANRÉ, E. Smoluchowski's coagulation equation: probabilistic interpretation of solutions for constant, additive and multiplicative kernels. Ann. Scuola Norm. Sup. Pisa Cl. Sci. (4), v. 29, n. 3, p. 549-579, 2000. ISSN 0391-173X. Available: <http://www.numdam.org/ item?id=ASNSP_2000_4_29_3_549_0>. Citations on pages 162 and 163.

DEPUTIES, I. C. of. Overview of initiatives to prevent and restrain the new COVID2019 pandemic. 2021. <https://www.camera.it/temiap/documentazione/temi/pdf/1203754.pdf? _1613070762706>. Citation on page 185.

DINGLI, D.; MICHOR, F. Successful therapy must eradicate cancer stem cells. Stem cells, Wiley Online Library, v. 24, n. 12, p. 2603-2610, 2006. Citations on pages 125 and 142.

DINGLI, D.; TRAULSEN, A.; PACHECO, J. M. Compartmental architecture and dynamics of hematopoiesis. PloS one, Public Library of Science, v. 2, n. 4, p. e345, 2007. Citation on page 127.

DISCHER, D. E. New insights into erythrocyte membrane organization and microelasticity. Current opinion in hematology, LWW, v. 7, n. 2, p. 117-122, 2000. Citations on pages 48 and 52 .

DISCHER, D. E.; BOAL, D. H.; BOEY, S. K. Simulations of the erythrocyte cytoskeleton at large deformation. ii. micropipette aspiration. Biophysical Journal, Elsevier, v. 75, n. 3, p. 1584-1597, 1998. Citations on pages 49 and 51.

DITTMAR, T.; ZÄNKER, K. S. Role of cancer stem cells in cancer biology and therapy. [S.1.]: CRC Press, 2013. Citation on page 125.

DRAKE, R. A general mathematical survey of the coagulation equation. In: Topics in Current Aerosol Research (Part 2), International Reviews in Aerosol Physics and Chemistry. Oxford, UK: Pergamon Press, 1972. p. 203-376. Citation on page 162.

DUBOVSKII, P. B. Mathematical theory of coagulation. [S.1.]: Seoul National University, Research Institute of Mathematics, Global Analysis Research Center, Seoul, 1994. ii+169 p. (Lecture Notes Series, v. 23). Citation on page 162.

DUMITRU, A. C.; PONCIN, M. A.; CONRARD, L.; DUFRÊNE, Y. F.; TYTECA, D.; ALSTEENS, D. Nanoscale membrane architecture of healthy and pathological red blood cells. Nanoscale Horizons, Royal Society of Chemistry, v. 3, n. 3, p. 293-304, 2018. Citation on page 47.

DZIUK, G.; ELLIOTT, C. M. Finite elements on evolving surfaces. IMA journal of numerical analysis, Oxford University Press, v. 27, n. 2, p. 262-292, 2007. Citation on page 34.

. Finite element methods for surface pdes. Acta Numerica, Cambridge University Press, v. 22, p. 289-396, 2013. Citation on page 34.

EDELSTEIN-KESHET, L.; SPIROSS, A. Exploring the formation of Alzheimer's disease senile plaques in silico. J. Theor. Biol., v. 216, n. 3, p. 301-326, 2002. ISSN 0022-5193. Citations on pages 159,161 , and 166.

ENDERLING, H.; ANDERSON, A. R.; CHAPLAIN, M. A.; BEHESHTI, A.; HLATKY, L.; HAHNFELDT, P. Paradoxical dependencies of tumor dormancy and progression on basic cell kinetics. Cancer research, AACR, v. 69, n. 22, p. 8814-8821, 2009. Citations on pages 125, $126,127,128,142$, and 248. 
ENDERLING, H.; HAHNFELDT, P. Cancer stem cells in solid tumors: Is 'evading apoptosis'a hallmark of cancer? Progress in biophysics and molecular biology, Elsevier, v. 106, n. 2, p. 391-399, 2011. Citations on pages 247 and 248.

EPSTEIN, J. M.; PARKER, J.; CUMMINGS, D.; HAMMOND, R. A. Coupled contagion dynamics of fear and disease: mathematical and computational explorations. PLoS One, Public Library of Science, v. 3, n. 12, p. e3955, 2008. Citation on page 182.

EUROPE, W. H. O. How to counter pandemic fatigue and refresh public commitment to COVID-19 prevention measures. 2020. Available: <https://www.euro.who.int/en/health-topics/ health-determinants/behavioural-and-cultural-insights-for-health/news2/news/2020/10/ how-to-counter-pandemic-fatigue-and-refresh-public-commitment-to-covid-19-prevention-measures $>$. Citation on page 179.

EVANS, E. A. Mechanics and thermodynamics of biomembranes. [S.1.]: CRC press, 2018. Citation on page 56.

FAI, T. G.; LEO-MACIAS, A.; STOKES, D. L.; PESKIN, C. S. Image-based model of the spectrin cytoskeleton for red blood cell simulation. PLoS computational biology, Public Library of Science, v. 13, n. 10, p. e1005790, 2017. Citation on page 65.

FANELLI, D.; PIAZZA, F. Analysis and forecast of covid-19 spreading in china, italy and france. Chaos, Solitons \& Fractals, Elsevier, v. 134, p. 109761, 2020. Citation on page 178.

FASANO, A.; MANCINI, A.; PRIMICERIO, M. Tumours with cancer stem cells: A pde model. Mathematical biosciences, Elsevier, v. 272, p. 76-80, 2016. Citations on pages 125, 126, 127 , 137,142 , and 248 .

FASANO, A.; SEQUEIRA, A. Hemomath: The mathematics of blood. [S.1.]: Springer, 2017. Citation on page 29.

FEDOSOV, D.; CASWELL, B.; KARNIADAKIS, G. A multiscale red blood cell model with accurate mechanics, rheology and dynamics. Biophys. J., v. 98, p. 2215-2225, 2010. Citations on pages $22,34,40,52,53,63$, and 90 .

FEDOSOV, D. A.; CASWELL, B.; KARNIADAKIS, G. E. Coarse-grained red blood cell model with accurate mechanical properties, rheology and dynamics. In: IEEE. 2009 Annual International Conference of the IEEE Engineering in Medicine and Biology Society. [S.1.], 2009. p. 4266-4269. Citations on pages 34 and 89.

Systematic coarse-graining of spectrin-level red blood cell models. Computer Methods in Applied Mechanics and Engineering, Elsevier, v. 199, n. 29-32, p. 1937-1948, 2010. Citations on pages 30 and 56.

FEDOSOV, D. A.; LEI, H.; CASWELL, B.; SURESH, S.; KARNIADAKIS, G. E. Multiscale modeling of red blood cell mechanics and blood flow in malaria. PLoS computational biology, Public Library of Science, v. 7, n. 12, p. e1002270, 2011. Citations on pages 34 and 89.

FENG, F.; KLUG, W. S. Finite element modeling of lipid bilayer membranes. Journal of Computational Physics, Elsevier, v. 220, n. 1, p. 394-408, 2006. Citation on page 49.

FIELD, T.; MINES, S.; POLING, S.; DIEGO, M.; BENDELL, D.; VEAZEY, C. Young, alone, and young alone during a covid-19 lockdown. Journal of Mental Health \& Clinical Psychology, v. 4, n. 4, 2020. Citation on page 179. 
FISCHER, T. M. Shape memory of human red blood cells. Biophysical journal, Elsevier, v. 86, n. 5, p. 3304-3313, 2004. Citation on page 40.

FLAXMAN, S.; MISHRA, S.; GANDY, A.; UNWIN, H. J. T.; MELLAN, T. A.; COUPLAND, H.; WHITTAKER, C.; ZHU, H.; BERAH, T.; EATON, J. W. et al. Estimating the effects of non-pharmaceutical interventions on covid-19 in europe. Nature, Nature Publishing Group, p. 1-5, 2020. Citation on page 178.

FORD, J. Red blood cell morphology. International journal of laboratory hematology, Wiley Online Library, v. 35, n. 3, p. 351-357, 2013. Citation on page 99.

FORNARI, S.; SCHAEFER, A.; JUCKER, M.; GORIELY, A.; KUHL, E. Prion-like spreading of alzheimer's disease within the brain's connectome. J. R. Soc. Interface, v. 159, p. 20190356, 2019. Citation on page 163.

FORNARI, S.; SCHÃ €FER, A.; KUHL, E.; GORIELY, A. Spatially-extended nucleationaggregation-fragmentation models for the dynamics of prion-like neurodegenerative proteinspreading in the brain and its connectome. Journal of Theoretical Biology, v. 486, p. 110102, 2020. ISSN 0022-5193. Available: <http://www.sciencedirect.com/science/article/pii/ S0022519319304710>. Citation on page 163.

FOROUZANNIA, F.; ENDERLING, H.; KOHANDEL, M. Mathematical modeling of the effects of tumor heterogeneity on the efficiency of radiation treatment schedule. Bulletin of mathematical biology, Springer, v. 80, n. 2, p. 283-293, 2018. Citation on page 247.

FRANCHI, B.; HEIDA, M.; LORENZANI, S. A Mathematical model for Alzheimer's disease: An approach via stochastic homogenization of the Smoluchowski equation. arXiv e-prints, p. arXiv:1904.11015, Apr 2019. Citations on pages 162 and 163.

FRANCHI, B.; LORENZANI, S. From a microscopic to a macroscopic model for Alzheimer disease: two-scale homogenization of the Smoluchowski equation in perforated domains. J. Nonlinear Sci., v. 26, n. 3, p. 717-753, 2016. ISSN 0938-8974. Available: <https://doi.org/10. 1007/s00332-016-9288-7>. Citations on pages 162 and 163.

Smoluchowski equation with variable coefficients in perforated domains: homogenization and applications to mathematical models in medicine. In: Harmonic analysis, partial differential equations and applications. [S.1.]: Birkhäuser/Springer, Cham, 2017, (Appl. Numer. Harmon. Anal.). p. 49-67. Citations on pages 162 and 163.

FRANCHI, B.; TESI, M. A qualitative model for aggregation-fragmentation and diffusion of $\beta$-amyloid in Alzheimer's disease. Rend. Semin. Mat. Univ. Politec. Torino, v. 70, n. 1, p. 75-84, 2012. ISSN 0373-1243. Citation on page 162.

FRANDSEN, G. I.; MUNDY, J.; TZEN, J. T. Oil bodies and their associated proteins, oleosin and caleosin. Physiologia plantarum, Wiley Online Library, v. 112, n. 3, p. 301-307, 2001. Citation on page 83 .

FREUND, J. B. Numerical simulation of flowing blood cells. Annual review of fluid mechanics, Annual Reviews, v. 46, p. 67-95, 2014. Citation on page 21.

FREUND, L.; LIN, Y. The role of binder mobility in spontaneous adhesive contact and implications for cell adhesion. Journal of the Mechanics and Physics of Solids, Elsevier, v. 52, n. 11, p. 2455-2472, 2004. Citations on pages 35 and 90. 
FRIEDMAN, A.; HAO, W. Mathematical model on Alzheimer's disease. BMC Systems Biology, v. 108, n. 10 , p. $1-18,2016$. Citation on page 159.

FU, S.-P.; PENG, Z.; YUAN, H.; KFOURY, R.; YOUNG, Y.-N. Lennard-jones type pair-potential method for coarse-grained lipid bilayer membrane simulations in lammps. Computer Physics Communications, Elsevier, v. 210, p. 193-203, 2017. Citations on pages 22, 43, 47, and 191.

FUENTES, M.; KUPERMAN, M. Cellular automata and epidemiological models with spatial dependence. Physica A: Statistical Mechanics and its Applications, Elsevier, v. 267, n. 3-4, p. 471-486, 1999. Citation on page 189.

FUSAR-POLI, P.; BRAMBILLA, P.; SOLMI, M. Learning from covid-19 pandemic in northen italy: Impact on mental health and clinical care. Journal of affective disorders, Elsevier, v. 275, p. 78, 2020. Citation on page 179 .

GANGULY, R.; PURI, I. Mathematical model for the cancer stem cell hypothesis. Cell proliferation, Wiley Online Library, v. 39, n. 1, p. 3-14, 2006. Citations on pages 125, 126, 141, 142, and 247.

GEEKIYANAGE, N. M.; BALANANT, M. A.; SAURET, E.; SAHA, S.; FLOWER, R.; LIM, C. T.; GU, Y. A coarse-grained red blood cell membrane model to study stomatocyte-discocyteechinocyte morphologies. PloS one, Public Library of Science San Francisco, CA USA, v. 14, n. 4, p. e0215447, 2019. Citations on pages 97 and 98.

GENTRY, S. N.; JACKSON, T. L. A mathematical model of cancer stem cell driven tumor initiation: implications of niche size and loss of homeostatic regulatory mechanisms. PloS One, Public Library of Science, v. 8, n. 8, p. e71128, 2013. Citations on pages 141 and 142.

GILLAM, J.; MACPHEE, C. Modelling amyloid fibril formation kinetics: Mechanisms of nucleation and growth. Journal of Physics: Condensed Matter. IOPScience, v. 25, n. 37, p. 373101, 2013. Citation on page 167.

GIORDANO, G.; BLANCHINI, F.; BRUNO, R.; COLANERI, P.; FILIPPO, A. D.; MATTEO, A. D.; COLANERI, M. Modelling the covid-19 epidemic and implementation of population-wide interventions in italy. Nature Medicine, Nature Publishing Group, p. 1-6, 2020. Citation on page 180 .

GIUFFRIDA, M. L.; CARACI, F.; PIGNATARO, B.; CATALDO, S.; BONA, P. D.; V., B.; G., M.; G., P.; A., M.; A., P.; D., G.; F., N.; E., R.; A., C. $\beta$-amyloid monomers are neuroprotective. J. Neurosci., v. 29, p. 10582-10587, 2009. Citation on page 161.

GÓMEZ, F.; SILVA, L. S.; ARAÚJO, G. R. de S.; FRASES, S.; PINHEIRO, A. A. S.; AGERO, U.; PONTES, B.; VIANA, N. B. Effect of cell geometry in the evaluation of erythrocyte viscoelastic properties. Physical Review E, APS, v. 101, n. 6, p. 062403, 2020. Citation on page 49 .

GOOD, T. A.; MURPHY, R. M. Effect of $\beta$-amyloid block of the fast-inactivating $\mathrm{K}^{+}$channel on intracellular $\mathrm{Ca}^{2+}$ and excitability in a modeled neuron. P. Natl. Acad. Sci. USA, v. 93, p. 15130-15135, 1996. Citations on pages 159 and 160.

GOV, N.; SAFRAN, S. Red blood cell membrane fluctuations and shape controlled by atpinduced cytoskeletal defects. Biophysical journal, Elsevier, v. 88, n. 3, p. 1859-1874, 2005.

Citation on page 58. 
GOV, N.; ZILMAN, A.; SAFRAN, S. Cytoskeleton confinement and tension of red blood cell membranes. Physical review letters, APS, v. 90, n. 22, p. 228101, 2003. Citation on page 42.

GOV, N. S. Active elastic network: cytoskeleton of the red blood cell. Physical Review E, APS, v. 75, n. 1, p. 011921, 2007. Citation on page 58.

GRATZER, W. The red cell membrane and its cytoskeleton. Biochemical Journal, Portland Press Ltd, v. 198, n. 1, p. 1, 1981. Citations on pages 34, 48, and 89.

GREER, M.; PUJO-MENJOUET, L.; WEBB, G. A mathematical analysis of the dynamics of prion proliferation. J. Theoret. Biol., v. 242, n. 3, p. 598-606, 2006. ISSN 0022-5193. Available: <https://doi.org/10.1016/j.jtbi.2006.04.010>. Citation on page 163.

GRIFFIN, W.; SHENG, J.; ROYSTON, M.; GENTLEMAN, S.; MCKENZIE, J.; GRAHAM, D.; ROBERTS, G.; MRAK, R. Glial-neuronal interactions in Alzheimer's disease: the potential role of a cytokine cycle in disease progression. Brain Pathol., Blackwell Publishing Ltd, v. 8, n. 1, p. 65-72, 1998. ISSN 1750-3639. Citation on page 160.

GROSS, S.; REUSKEN, A. Numerical methods for two-phase incompressible flows. [S.1.]: Springer Science \& Business Media, 2011. Citations on pages 51, 77, and 87.

GUCKENBERGER, A.; GEKLE, S. Theory and algorithms to compute helfrich bending forces: A review. Journal of Physics: Condensed Matter, IOP Publishing, v. 29, n. 20, p. 203001, 2017. Citation on page 80 .

GUROVA, K. V.; GUDKOV, A. V. Paradoxical role of apoptosis in tumor progression. Journal of cellular biochemistry, Wiley Online Library, v. 88, n. 1, p. 128-137, 2003. Citations on pages 125,142 , and 248 .

HAASS, C.; SELKOE, D. J. Soluble protein oligomers in neurodegeneration: lessons from the Alzheimer's amyloid beta-peptide. Nat. Rev. Mol. Cell. Biol., v. 8, n. 2, p. 101-112, 2007. Citation on page 161 .

HAHN, W. Stability of motion. [S.1.]: Springer-Verlag New York, Inc., New York, 1967. xi+446 p. (Translated from the German manuscript by Arne P. Baartz. Die Grundlehren der mathematischen Wissenschaften, Band 138). Citations on pages 174 and 175.

HAINFELD, J. F.; STECK, T. L. The sub-membrane reticulum of the human erythrocyte: A scanning electron microscope study. Journal of Supramolecular Structure, Wiley Online Library, v. 6, n. 3, p. 301-311, 1977. Citation on page 48.

HANSEN, J.; SKALAK, R.; CHIEN, S.; HOGER, A. An elastic network model based on the structure of the red blood cell membrane skeleton. Biophysical journal, Elsevier, v. 70, n. 1, p. 146-166, 1996. Citations on pages 34, 52, and 89.

HARVEY, N. Behavioral fatigue: Real phenomenon, naïve construct, or policy contrivance? Frontiers in Psychology, Frontiers Media SA, v. 11, 2020. Citation on page 179.

HEINTZ, A. A numerical method for simulation dynamics of incompressible lipid membranes in viscous fluid. Journal of Computational and Applied Mathematics, Elsevier, v. 289, p. 87-100, 2015. Citation on page 22. 
HELAL, M.; HINGANT, E.; PUJO-MENJOUET, L.; WEBB, G. F. Alzheimer's disease: analysis of a mathematical model incorporating the role of prions. J. Math. Biol., Springer Berlin Heidelberg, v. 69, n. 5, p. 1-29, 2013. ISSN 0303-6812. Citations on pages 159 and 163.

HELFRICH, W. Elastic properties of lipid bilayers: theory and possible experiments. Zeitschrift für Naturforschung C, Verlag der Zeitschrift für Naturforschung, v. 28, n. 11-12, p. 693-703, 1973. Citations on pages 33, 76, and 88.

HELLEWELL, J.; ABBOTT, S.; GIMMA, A.; BOSSE, N. I.; JARVIS, C. I.; RUSSELL, T. W.; MUNDAY, J. D.; KUCHARSKI, A. J.; EDMUNDS, W. J.; SUN, F. et al. Feasibility of controlling covid-19 outbreaks by isolation of cases and contacts. The Lancet Global Health, Elsevier, 2020. Citation on page 178.

HENON, S.; LENORMAND, G.; RICHERT, A.; GALLET, F. A new determination of the shear modulus of the human erythrocyte membrane using optical tweezers. Biophysical journal, Elsevier, v. 76, n. 2, p. 1145-1151, 1999. Citation on page 61.

HERANT, M.; MARGANSKI, W. A.; DEMBO, M. The mechanics of neutrophils: synthetic modeling of three experiments. Biophysical Journal, Elsevier, v. 84, n. 5, p. 3389-3413, 2003. Citation on page 195.

HERRERO, M. A.; RODRIGO, M. A note on Smoluchowski's equations with diffusion. Appl. Math. Lett., v. 18, n. 9, p. 969-975, 2005. ISSN 0893-9659. Available: <https://doi.org/10.1016/ j.aml.2004.09.015>. Citation on page 162 .

HILLEN, T.; ENDERLING, H.; HAHNFELDT, P. The tumor growth paradox and immune system-mediated selection for cancer stem cells. Bulletin of mathematical biology, Springer, v. 75, n. 1, p. 161-184, 2013. Citations on pages 125, 126, 127, 128, 142, and 247.

HILLRINGHAUS, S.; DASANNA, A. K.; GOMPPER, G.; FEDOSOV, D. A. Importance of erythrocyte deformability for the alignment of malaria parasite upon invasion. Biophysical journal, Elsevier, v. 117, n. 7, p. 1202-1214, 2019. Citation on page 39.

HILLRINGHAUS, S.; DASANNA, A. K.; GOMPPER, G.; FEDOSOV, D. Stochastic bond dynamics facilitates alignment of malaria parasite at erythrocyte membrane upon invasion. bioRxiv, Cold Spring Harbor Laboratory, 2020. Citation on page 39.

HOCHMUTH, R. M.; WORTHY, P.; EVANS, E. A. Red cell extensional recovery and the determination of membrane viscosity. Biophysical journal, Elsevier, v. 26, n. 1, p. 101-114, 1979. Citations on pages 63 and 100.

HOPPENSTEADT, F. C.; PESKIN, C. S. Mathematics in medicine and the life sciences. [S.1.]: Springer Science \& Business Media, 2013. Citation on page 19.

HORTAÇSU, A.; LIU, J.; SCHWIEG, T. Estimating the fraction of unreported infections in epidemics with a known epicenter: an application to covid-19. Journal of econometrics, Elsevier, v. 220, n. 1, p. 106-129, 2021. Citation on page 186.

HOWARD, M. P.; MILNER, S. T. Numerical simulation methods for the rouse model in flow. Physical Review E, APS, v. 84, n. 5, p. 051804, 2011. Citation on page 196.

HUANG, A. Oleosins and oil bodies in seeds and other organs. Plant physiology, American Society of Plant Biologists, v. 110, n. 4, p. 1055, 1996. Citation on page 73. 
HUANG, A. H. Oil bodies and oleosins in seeds. Annual review of plant biology, Annual Reviews 4139 El Camino Way, PO Box 10139, Palo Alto, CA 94303-0139, USA, v. 43, n. 1, p. 177-200, 1992. Citation on page 73.

HUNTLY, B. J.; GILLILAND, D. G. Cancer biology: summing up cancer stem cells. Nature, Nature Publishing Group, v. 435, n. 7046, p. 1169, 2005. Citations on pages 141 and 247.

HURD, M. D.; MARTORELL, P.; DELAVANDE, A.; MULLEN, K. J.; LANGA, K. M. Monetary costs of dementia in the United States. New Engl. J. Med., v. 368, n. 14, p. 1326-1334, 2013. Citation on page 159.

ISRAELACHVILI, J. N. Intermolecular and surface forces. [S.1.]: Academic press, 2011. Citations on pages 35 and 90.

JACK, J. C. R.; KNOPMAN, D. S.; JAGUST, W. J.; SHAW, L. M.; AISEN, P. S.; WEINER, M. W.; PETERSEN, R. C.; TROJANOWSKI, J. Q. Tracking pathophysiological processes in Alzheimer's disease: an updated hypothetical model of dynamic biomarkers. Lancet Neurol., v. 12, n. 2, p. 207-216, 2013. ISSN 1474-4422. Citation on page 168.

JIAO, W. Y.; WANG, L. N.; LIU, J.; FANG, S. F.; JIAO, F. Y.; PETTOELLO-MANTOVANI, M.; SOMEKH, E. Behavioral and emotional disorders in children during the covid-19 epidemic. The journal of Pediatrics, Elsevier, v. 221, p. 264, 2020. Citation on page 179.

JU, M.; YE, S. S.; NAMGUNG, B.; CHO, S.; LOW, H. T.; LEO, H. L.; KIM, S. A review of numerical methods for red blood cell flow simulation. Computer methods in biomechanics and biomedical engineering, Taylor \& Francis, v. 18, n. 2, p. 130-140, 2015. Citations on pages 21,29 , and 52 .

KAKARALA, M.; WICHA, M. S. Implications of the cancer stem-cell hypothesis for breast cancer prevention and therapy. Journal of Clinical Oncology, American Society of Clinical Oncology, v. 26, n. 17, p. 2813-2820, 2008. Citations on pages 125 and 141.

KAMETANI, F.; HASEGAWA, M. Reconsideration of amyloid hypothesis and tau hypothesis in alzheimer's disease. New Engl. J. Med., v. 368, n. 14, p. 1326-1334, 2013. Citation on page 163.

KAPASIA, N.; PAUL, P.; ROY, A.; SAHA, J.; ZAVERI, A.; MALLICK, R.; BARMAN, B.; DAS, P.; CHOUHAN, P. Impact of lockdown on learning status of undergraduate and postgraduate students during covid-19 pandemic in west bengal, india. Children and Youth Services Review, Elsevier, v. 116, p. 105194, 2020. Citation on page 179.

KARI, J. Theory of cellular automata: A survey. Theoretical computer science, Elsevier, v. 334, n. 1, p. 3-33, 2005. Citation on page 127.

KARRAN, E.; MERCKEN, M.; STROOPER, B. D. The amyloid cascade hypothesis for Alzheimer's disease: an appraisal for the development of therapeutics. Nat Rev. Drug Discov., v. 10, n. 9, p. 698-712, 2011. Citations on pages 160 and 167.

KASHIWABARA, T.; COLCIAGO, C. M.; DEDÈ, L.; QUARTERONI, A. Well-posedness, regularity, and convergence analysis of the finite element approximation of a generalized robin boundary value problem. SIAM Journal on Numerical Analysis, SIAM, v. 53, n. 1, p. 105-126, 2015. Citation on page 93. 
KAVEH, K.; KOHANDEL, M.; SIVALOGANATHAN, S. Replicator dynamics of cancer stem cell: Selection in the presence of differentiation and plasticity. Mathematical Biosciences, Elsevier, v. 272, p. 64-75, 2016. Citations on pages 141 and 142.

KEY, B. Diseases of Red Blood Cells. 2016. Urlhttps://basicmedicalkey.com/diseases-of-redblood-cells/. Citation on page 100.

KHAN, M. A.; ATANGANA, A. Modeling the dynamics of novel coronavirus (2019-ncov) with fractional derivative. Alexandria Engineering Journal, Elsevier, 2020. Citation on page 178.

KIM, J.; LEE, H.; SHIN, S. Advances in the measurement of red blood cell deformability: A brief review. Journal of Cellular Biotechnology, IOS Press, v. 1, n. 1, p. 63-79, 2015. Citation on page 67.

KIRBY, B. J. Micro-and nanoscale fluid mechanics: transport in microfluidic devices. [S.1.]: Cambridge university press, 2010. Citation on page 117.

KUCHARSKI, A. J.; RUSSELL, T. W.; DIAMOND, C.; LIU, Y.; EDMUNDS, J.; FUNK, S.; EGGO, R. M.; SUN, F.; JIT, M.; MUNDAY, J. D. et al. Early dynamics of transmission and control of covid-19: a mathematical modelling study. The lancet infectious diseases, Elsevier, 2020. Citation on page 178.

KUUSELA, E.; ALT, W. Continuum model of cell adhesion and migration. Journal of mathematical biology, Springer, v. 58, n. 1-2, p. 135, 2009. Citations on pages 35 and 90.

LANCZOS, C. The variational principles of mechanics, 4th edn. [S.1.]: Toronto University Press, Toronto, 1970. Citations on pages 32, 54, and 76.

LAPIDOT, T.; SIRARD, C.; VORMOOR, J.; MURDOCH, B.; HOANG, T.; CACERESCORTES, J.; MINDEN, M.; PATERSON, B.; CALIGIURI, M. A.; DICK, J. E. A cell initiating human acute myeloid leukaemia after transplantation into scid mice. Nature, Nature Publishing Group, v. 367, n. 6464, p. 645, 1994. Citation on page 141.

LATHIA, J. D.; MACK, S. C.; MULKEARNS-HUBERT, E. E.; VALENTIM, C. L.; RICH, J. N. Cancer stem cells in glioblastoma. Genes \& development, Cold Spring Harbor Lab, v. 29, n. 12, p. 1203-1217, 2015. Citations on pages 125 and 141.

LAUER, S. A.; GRANTZ, K. H.; BI, Q.; JONES, F. K.; ZHENG, Q.; MEREDITH, H. R.; AZMAN, A. S.; REICH, N. G.; LESSLER, J. The incubation period of coronavirus disease 2019 (covid-19) from publicly reported confirmed cases: estimation and application. Annals of internal medicine, American College of Physicians, v. 172, n. 9, p. 577-582, 2020. Citation on page 185 .

LAURENÇOT, P.; MISCHLER, S. Global existence for the discrete diffusive coagulationfragmentation equations in $L^{1}$. Rev. Mat. Iberoamericana, v. 18, n. 3, p. 731-745, 2002. ISSN 0213-2230. Available: <https://doi.org/10.4171/RMI/334>. Citations on pages 162 and 163.

LEE, C.; LI, Y.; KIM, J. The susceptible-unidentified infected-confirmed (suc) epidemic model for estimating unidentified infected population for covid-19. Chaos, Solitons \& Fractals, Elsevier, v. 139, p. 110090, 2020. Citation on page 178. 
LEI, L.; HUANG, X.; ZHANG, S.; YANG, J.; YANG, L.; XU, M. Comparison of prevalence and associated factors of anxiety and depression among people affected by versus people unaffected by quarantine during the covid-19 epidemic in southwestern china. Medical science monitor: international medical journal of experimental and clinical research, International Scientific Information, Inc., v. 26, p. e924609-1, 2020. Citation on page 179.

LEUNG, C. The difference in the incubation period of 2019 novel coronavirus (sars-cov-2) infection between travelers to hubei and nontravelers: The need for a longer quarantine period. Infection Control \& Hospital Epidemiology, Cambridge University Press, v. 41, n. 5, p. 594596, 2020. Citation on page 185.

LI, C.; LIU, K. Nanomechanical characterization of red blood cells using optical tweezers. Journal of Materials Science: Materials in Medicine, Springer, v. 19, n. 4, p. 1529-1535, 2008. Citations on pages 63 and 104.

LI-GUO, J.; HENG-AN, W.; XIAO-ZHOU, Z.; XIU-XI, W. Coarse-grained molecular dynamics simulation of a red blood cell. Chinese Physics Letters, IOP Publishing, v. 27, n. 2, p. 028704 , 2010. Citation on page 29.

LI, H.; CHANG, H.; YANG, J.; LU, L.; TANG, Y.; LYKOTRAFITIS, G. Modeling biomembranes and red blood cells by coarse-grained particle methods. Applied Mathematics and Mechanics, Springer, v. 39, n. 1, p. 3-20, 2018. Citation on page 22.

LI, H.; LYKOTRAFITIS, G. Two-component coarse-grained molecular-dynamics model for the human erythrocyte membrane. Biophysical journal, Elsevier, v. 102, n. 1, p. 75-84, 2012. Citations on pages 22 and 49.

Erythrocyte membrane model with explicit description of the lipid bilayer and the spectrin network. Biophysical journal, Elsevier, v. 107, n. 3, p. 642-653, 2014. Citations on pages 22 and 68 .

LI, H.; YANG, J.; CHU, T. T.; NAIDU, R.; LU, L.; CHANDRAMOHANADAS, R.; DAO, M.; KARNIADAKIS, G. E. Cytoskeleton remodeling induces membrane stiffness and stability changes of maturing reticulocytes. Biophysical journal, Elsevier, v. 114, n. 8, p. 2014-2023, 2018. Citation on page 106.

LI, J.; DAO, M.; LIM, C.; SURESH, S. Spectrin-level modeling of the cytoskeleton and optical tweezers stretching of the erythrocyte. Biophysical journal, Elsevier, v. 88, n. 5, p. 3707-3719, 2005. Citation on page 97.

LI, J.; LYKOTRAFITIS, G.; DAO, M.; SURESH, S. Cytoskeletal dynamics of human erythrocyte. Proceedings of the National Academy of Sciences, National Acad Sciences, v. 104, n. 12, p. 4937-4942, 2007. Citations on pages 47 and 58.

LI, X.; LU, H.; PENG, Z. Continuum-and particle-based modeling of human red blood cells. Handbook of Materials Modeling: Applications: Current and Emerging Materials, Springer, p. 2593-2609, 2020. Citations on pages 21 and 50.

LI, X.; PENG, Z.; LEI, H.; DAO, M.; KARNIADAKIS, G. E. Probing red blood cell mechanics, rheology and dynamics with a two-component multi-scale model. Philosophical Transactions of the Royal Society A: Mathematical, Physical and Engineering Sciences, The Royal Society Publishing, v. 372, n. 2021, p. 20130389, 2014. Citations on pages 30, 49, 67, and 191. 
LI, X.; VLAHOVSKA, P. M.; KARNIADAKIS, G. E. Continuum-and particle-based modeling of shapes and dynamics of red blood cells in health and disease. Soft Matter, Royal Society of Chemistry, v. 9, n. 1, p. 28-37, 2013. Citation on page 47.

LIM, C.; ZHOU, E.; QUEK, S. Mechanical models for living cells-a review. Journal of biomechanics, Elsevier, v. 39, n. 2, p. 195-216, 2006. Citations on pages 47 and 74.

LIN, Q.; ZHAO, S.; GAO, D.; LOU, Y.; YANG, S.; MUSA, S. S.; WANG, M. H.; CAI, Y.; WANG, W.; YANG, L. et al. A conceptual model for the outbreak of coronavirus disease 2019 (covid-19) in wuhan, china with individual reaction and governmental action. International journal of infectious diseases, Elsevier, 2020. Citation on page 180.

LIU, S.-C.; DERICK, L. H.; PALEK, J. Visualization of the hexagonal lattice in the erythrocyte membrane skeleton. The Journal of cell biology, v. 104, n. 3, p. 527-536, 1987. Citations on pages 48 and 60 .

LIU, S.-C.; DERICK, L. H.; ZHAI, S.; PALEK, J. Uncoupling of the spectrin-based skeleton from the lipid bilayer in sickled red cells. Science, American Association for the Advancement of Science, v. 252, n. 5005, p. 574-576, 1991. Citation on page 68.

LIU, Y.; GAYLE, A. A.; WILDER-SMITH, A.; ROCKLÖV, J. The reproductive number of covid-19 is higher compared to sars coronavirus. Journal of travel medicine, 2020. Citation on page 177.

LIU, Z.; MAGAL, P.; SEYDI, O.; WEBB, G. Understanding unreported cases in the covid-19 epidemic outbreak in wuhan, china, and the importance of major public health interventions. Biology, Multidisciplinary Digital Publishing Institute, v. 9, n. 3, p. 50, 2020. Citation on page 178.

LÖHNER, R. Regridding surface triangulations. Journal of Computational Physics, Elsevier, v. 126, n. 1, p. 1-10, 1996. Citation on page 34.

LOPEZ, B. J.; VALENTINE, M. T. Molecular control of stress transmission in the microtubule cytoskeleton. Biochimica et Biophysica Acta (BBA)-Molecular Cell Research, Elsevier, v. 1853, n. 11, p. 3015-3024, 2015. Citation on page 64.

LUO, X.; ESTILL, J.; WANG, Q.; LV, M.; LIU, Y.; LIU, E.; CHEN, Y. The psychological impact of quarantine on coronavirus disease 2019 (covid-19). Psychiatry research, v. 291, p. 113193, 2020. Citation on page 179.

LUX, S. E. Dissecting the red cell membrane skeleton. Nature, Nature Publishing Group, v. 281, n. 5731, p. 426-428, 1979. Citation on page 48.

Anatomy of the red cell membrane skeleton: unanswered questions. Blood, American Society of Hematology, v. 127, n. 2, p. 187-199, 2016. Citations on pages 21 and 48.

MADDALENA, L. Analysis of an integro-differential system modeling tumor growth. Applied Mathematics and Computation, Elsevier, v. 245, p. 152-157, 2014. Citations on pages 125, 126, and 133.

MAHAJAN, A.; SIVADAS, N. A.; SOLANKI, R. An epidemic model sipherd and its application for prediction of the spread of covid-19 infection in india. Chaos, Solitons \& Fractals, Elsevier, v. 140, p. 110156, 2020. Citation on page 178. 
MAHASE, E. Covid-19: Was the decision to delay the UK's lockdown over fears of "behavioural fatigue" based on evidence? [S.1.]: British Medical Journal Publishing Group, 2020. Citation on page 179 .

MAJUMDAR, P.; BISWAS, A.; SAHU, S. Covid-19 pandemic and lockdown: cause of sleep disruption, depression, somatic pain, and increased screen exposure of office workers and students of india. Chronobiology International, Taylor \& Francis, v. 37, n. 8, p. 1191-1200, 2020. Citation on page 179.

MAMO, D. K. Model the transmission dynamics of covid-19 propagation with public health intervention. Results in Applied Mathematics, v. 7, p. 100123, 2020. ISSN 2590-0374. Available: <http://www.sciencedirect.com/science/article/pii/S2590037420300339>. Citation on page 178 .

MARCINIAK-CZOCHRA, A.; STIEHL, T.; WAGNER, W. Modeling of replicative senescence in hematopoietic development. Aging (Albany NY), Impact Journals, LLC, v. 1, n. 8, p. 723, 2009. Citations on pages 141 and 142.

MARGARITIS, I.; HOUDART, S.; OUADRHIRI, Y. E.; BIGARD, X.; VUILLEMIN, A.; DUCHÉ, P. How to deal with covid-19 epidemic-related lockdown physical inactivity and sedentary increase in youth? adaptation of anses' benchmarks. Archives of Public Health, Springer, v. 78, p. 1-6, 2020. Citation on page 179.

MARKO, J. F.; SIGGIA, E. D. Stretching dna. Macromolecules, ACS Publications, v. 28, n. 26, p. $8759-8770,1995$. Citation on page 51.

MARROQUÍN, B.; VINE, V.; MORGAN, R. Mental health during the covid-19 pandemic: Effects of stay-at-home policies, social distancing behavior, and social resources. Psychiatry research, Elsevier, v. 293, p. 113419, 2020. Citation on page 179.

MARTELLONI, G.; MARTELLONI, G. Modelling the downhill of the sars-cov-2 in italy and a universal forecast of the epidemic in the world. Chaos, Solitons \& Fractals, Elsevier, v. 139, p. 110064, 2020. Citation on page 178.

MARZAGALLI, M.; FONTANA, F.; RAIMONDI, M.; LIMONTA, P. Cancer stem cells-key players in tumor relapse. Cancers, Multidisciplinary Digital Publishing Institute, v. 13, n. 3, p. 376, 2021. Citation on page 247.

MATHAI, P. P.; BERGLUND, A. J.; LIDDLE, J. A.; SHAPIRO, B. A. Simultaneous positioning and orientation of a single nano-object by flow control: theory and simulations. New Journal of Physics, IOP Publishing, v. 13, n. 1, p. 013027, 2011. Citation on page 115.

MATTHÄUS, F. Diffusion versus network models as descriptions for the spread of prion diseases in the brain. Journal of Theoretical Biology, v. 240, n. 1, p. $104-113,2006$. ISSN 0022-5193. Available: <http://www.sciencedirect.com/science/article/pii/S0022519305003711>. Citation on page 163 .

MCHEDLISHVILI, G.; MAEDA, N. Blood flow structure related to red cell flow: determinant of blood fluidity in narrow microvessels. The Japanese journal of physiology, Physiological Society of Japan, v. 51, n. 1, p. 19-30, 2001. Citation on page 29. 
MCWHIRTER, J. L.; NOGUCHI, H.; GOMPPER, G. Flow-induced clustering and alignment of vesicles and red blood cells in microcapillaries. Proceedings of the National Academy of Sciences, National Academy of Sciences, v. 106, n. 15, p. 6039-6043, 2009. ISSN 0027-8424. Available: <https://www.pnas.org/content/106/15/6039>. Citation on page 22.

MEACCI, L. How and how much to invest for fighting cheaters: from an ode to a cellular automata model. Mathematical Sciences, Springer, v. 9, n. 3, p. 137-143, 2015. Citation on page 127.

Covid-19 spread model with pandemic fatigue impact. Numerical implementation. [S.1.]: GitHub, 2020. <https://github.com/LucaMeacci/ Covid-19-math-model-pandemic-fatigue $>$. Citation on page 189.

MEACCI, L.; AUSAS, R. F.; MUT, F.; BARI, V. di; BUSCAGLIA, G. C. Modeling a red blood cell cytoskeleton: insights, tips, and code. To be published, 2021. Citation on page 89.

MEACCI, L.; BUSCAGLIA, G. C.; AUSAS, F. R.; MUT, F. Modelando um citoesqueleto de glóbulo vermelho com forças do tipo worm-like chain. In: Caderno de trabalhos completos e resumos do ERMAC 2019. Unesp, Bauru/SP - Brazil: [s.n.], 2019. p. 248 - 254. Citations on pages 50 and 144 .

MEACCI, L.; BUSCAGLIA, G. C.; AUSAS, R. F.; MUT, F. A red blood cell cyto-bilayer interaction model. Proceeding Series of the Brazilian Society of Computational and Applied Mathematics, v. 7, n. 1, 2020. Citations on pages 36, 65, and 91.

MEACCI, L.; BUSCAGLIA, G. C.; MUT, F.; AUSAS, R. F.; PRIMICERIO, M. A new twocomponent approach in modeling red blood cells. Communications in Applied and Industrial Mathematics, Sciendo, v. 11, n. 1, p. 55-71, 2020. Citations on pages 91 and 98.

MEACCI, L.; MEDEIROS, D. de O.; BUSCAGLIA, G.; PRIMICERIO, M. O paradoxo do crescimento tumoral atraves de um modelo $3 \mathrm{~d}$ de automatos celulares com celulastronco cancer1genas. CQD-Revista Eletronica Paulista de Matematica, v. 14, p. 132-146, 2019. Citation on page 248 .

MEACCI, L.; NUÑO, J. C. S.; PRIMICERIO, M. Fighting tax evasion: a cellular automata approach. Advances in Mathematical Sciences and Applications, Gakko Tosho, v. 22, n. 2, p. 597-610, 2012. Citation on page 127.

MEACCI, L.; PRIMICERIO, M. Mathematical models for tumours with cancer stem cells. Computational and Applied Mathematics, Springer, p. 6544-6559, 2018. Citations on pages 142 and 248.

MEACCI, L.; PRIMICERIO, M.; BUSCAGLIA, G. C. Growth of tumours with stem cells: The effect of crowding and ageing of cells. Physica A: Statistical Mechanics and its Applications, Elsevier, v. 570, p. 125841, 2021. Citation on page 248.

MEACCI, L.; ROCHA, F. F.; SILVA, A. A.; PRAMIU, P. V.; BUSCAGLIA, C. G. Planejamento de trajetória para a manipulação de partículas em microfluídica. CQD-Revista Eletrônica Paulista de Matemática, C.Q.D., v. 10, p. 19-37, 2017. Citations on pages 25 and 117.

MEYER-LUEHMANN, M.; SPIRES-JONES, T.; PRADA, C.; GARCIA-ALLOZA, M.; CALIGNON, A. D.; ROZKALNE, A.; KOENIGSKNECHT-TALBOO, J.; HOLTZMAN, D. M.; BACSKAI, B. J.; HYMAN, B. T. Rapid appearance and local toxicity of amyloid- $\beta$ plaques in a 
mouse model of Alzheimer's disease. Nature, v. 451, n. 7179, p. 720-724, 2008. Citations on pages 161 and 162 .

MICHOR, F. Mathematical models of cancer stem cells. Journal of Clinical Oncology, American Society of Clinical Oncology, v. 26, n. 17, p. 2854-2861, 2008. Citations on pages 125, 141 , and 247.

MICHOR, F.; HUGHES, T. P.; IWASA, Y.; BRANFORD, S.; SHAH, N. P.; SAWYERS, C. L.; NOWAK, M. A. Dynamics of chronic myeloid leukaemia. Nature, Nature Publishing Group, v. 435, n. 7046, p. 1267-1270, 2005. Citations on pages 127, 141, and 142.

MILLS, J.; QIE, L.; DAO, M.; LIM, C.; SURESH, S. Nonlinear elastic and viscoelastic deformation of the human red blood cell with optical tweezers. Molecular \& Cellular Biomechanics, Tech Science Press, v. 1, n. 3, p. 169, 2004. Citation on page 61.

MILSTEIN, G. N.; TRETYAKOV, M. V. Stochastic numerics for mathematical physics. [S.l.]: Springer Science \& Business Media, 2013. Citation on page 196.

MOHANDAS, N.; GALLAGHER, P. G. Red cell membrane: past, present, and future. Blood, Am Soc Hematology, v. 112, n. 10, p. 3939-3948, 2008. Citations on pages 48 and 89.

MOLINA-PEÑA, R.; ÁLVAREZ, M. M. A simple mathematical model based on the cancer stem cell hypothesis suggests kinetic commonalities in solid tumor growth. PloS one, Public Library of Science, v. 7, n. 2, p. e26233, 2012. Citation on page 247.

MOLINA-PEÑA, R.; TUDON-MARTINEZ, J. C.; AQUINES-GUTIÉRREZ, O. A mathematical model of average dynamics in a stem cell hierarchy suggests the combinatorial targeting of cancer stem cells and progenitor cells as a potential strategy against tumor growth. Cancers, Multidisciplinary Digital Publishing Institute, v. 12, n. 9, p. 2590, 2020. Citation on page 247.

MONTEAGUDO, Á.; SANTOS, J. Studying the capability of different cancer hallmarks to initiate tumor growth using a cellular automaton simulation. application in a cancer stem cell context. Biosystems, Elsevier, v. 115, p. 46-58, 2014. Citations on pages 142 and 248.

Treatment analysis in a cancer stem cell context using a tumor growth model based on cellular automata. PloS One, Public Library of Science, v. 10, n. 7, p. e0132306, 2015. Citations on pages 142 and 248 .

MORETTINI, M.; SBROLLINI, A.; MARCANTONI, I.; BURATTINI, L. Covid-19 in italy: Dataset of the italian civil protection department. Data in brief, Elsevier, v. 30, p. 105526, 2020. Citation on page 184.

MOSCONI, L.; BERTI, V.; GLODZIK, L.; PUPI, A.; SANTI, S. D.; LEON, M. de. Preclinical detection of Alzheimer's disease using FDG-PET, with or without amyloid imaging. J. Alzheimer's Dis., v. 20(3), p. 843-854, 2010. Citation on page 163.

MOSES, W. Fundamental limits of spatial resolution in pet. Nucl Instrum Methods Phys Res A., v. 648, n. Supplement 1, p. S236-S240, 2011. Citation on page 165.

MURPHY, D. J.; VANCE, J. Mechanisms of lipid-body formation. Trends in biochemical sciences, Elsevier, v. 24, n. 3, p. 109-115, 1999. Citation on page 73. 
MURPHY, R. M.; PALLITTO, M. M. Probing the kinetics of $\beta$-amyloid self-association. J. Struct. Biol., v. 130, n. 2-3, p. 109-122, 2000. ISSN 1047-8477. Citations on pages 159, 162, and 167.

NAG, S.; SARKAR, B.; BANDYOPADHYAY, A.; SAHOO, B.; SREENIVASAN, V.; KOMBRABAIL, M.; MURALIDHARAN, C.; MAITI, S. Nature of the amyloid- $\beta$ monomer and the monomer-oligomer equilibrium. J. Biol. Chem., v. 286, n. 16, p. 13827-13833, 2011. Citation on page 166.

NANS, A.; MOHANDAS, N.; STOKES, D. L. Native ultrastructure of the red cell cytoskeleton by cryo-electron tomography. Biophysical journal, Elsevier, v. 101, n. 10, p. 2341-2350, 2011. Citations on pages 48 and 49.

NAPIER, J. A.; STOBART, A. K.; SHEWRY, P. R. The structure and biogenesis of plant oil bodies: the role of the er membrane and the oleosin class of proteins. Plant molecular biology, Springer, v. 31, n. 5, p. 945-956, 1996. Citation on page 73.

NDAIROU, F.; AREA, I.; NIETO, J. J.; TORRES, D. F. Mathematical modeling of covid-19 transmission dynamics with a case study of wuhan. Chaos, Solitons \& Fractals, Elsevier, p. 109846, 2020. Citation on page 178.

NIKIFORIDIS, C. V. Structure and functions of oleosomes (oil bodies). Advances in colloid and interface science, Elsevier, p. 102039, 2019. Citation on page 73.

NIKIFORIDIS, C. V.; MATSAKIDOU, A.; KIOSSEOGLOU, V. Composition, properties and potential food applications of natural emulsions and cream materials based on oil bodies. RSC Advances, Royal Society of Chemistry, v. 4, n. 48, p. 25067-25078, 2014. Citation on page 74.

NITSCHKE, J. P.; FORBES, P. A.; ALI, N.; CUTLER, J.; APPS, M. A.; LOCKWOOD, P. L.; LAMM, C. Resilience during uncertainty? greater social connectedness during covid-19 lockdown is associated with reduced distress and fatigue. British Journal of Health Psychology, Wiley Online Library, 2020. Citation on page 189.

NOGUCHI, H. Membrane simulation models from nanometer to micrometer scale. Journal of the Physical Society of Japan, The Physical Society of Japan, v. 78, n. 4, p. 041007-041007, 2009. Citations on pages 56 and 58.

NOGUCHI, H.; GOMPPER, G. Shape transitions of fluid vesicles and red blood cells in capillary flows. Proceedings of the National Academy of Sciences, National Acad Sciences, v. 102, n. 40, p. 14159-14164, 2005. Citation on page 22.

NUNES, T.; HAMDAN, D.; LEBOEUF, C.; BOUCHTAOUI, M. E.; GAPIHAN, G.; NGUYEN, T. T.; MELES, S.; ANGELI, E.; RATAJCZAK, P.; LU, H. et al. Targeting cancer stem cells to overcome chemoresistance. International journal of molecular sciences, Multidisciplinary Digital Publishing Institute, v. 19, n. 12, p. 4036, 2018. Citation on page 247.

O'BRIEN, R.; WONG, P. Amyloid precursor protein processing and Alzheimer's disease. Annual Review of Neuroscience, Annual Reviews Inc., v. 34, p. 185-204, 7 2011. ISSN 0147006X. Citation on page 160.

ONO, K.; CONDRON, M. M.; TEPLOW, D. B. Structure-neurotoxicity relationships of amyloid $\beta$-protein oligomers. P. Natl. Acad. Sci. USA, v. 106, n. 35, p. 14745-14750, 2009. Citation on page 160 . 
ORGANIZATION, W. H. et al. Coronavirus disease 2019 (covid-19): situation report, 72. World Health Organization, 2020. Citation on page 178.

Naming the coronavirus disease (COVID-19) and the virus that causes it. 2020. Citation on page 177.

Pandemic fatigue: reinvigorating the public to prevent COVID-19: policy framework for supporting pandemic prevention and management: revised version November 2020. [S.1.], 2020. Citations on pages 179 and 189.

ÖZDIN, S.; ÖZDIN, Ş. B. Levels and predictors of anxiety, depression and health anxiety during covid-19 pandemic in turkish society: The importance of gender. International Journal of Social Psychiatry, SAGE Publications Sage UK: London, England, v. 66, n. 5, p. 504-511, 2020. Citation on page 179.

PAIVA, H. M.; AFONSO, R. J. M.; OLIVEIRA, I. L. de; GARCIA, G. F. A data-driven model to describe and forecast the dynamics of covid-19 transmission. PloS one, Public Library of Science San Francisco, CA USA, v. 15, n. 7, p. e0236386, 2020. Citation on page 178.

PAJIC-LIJAKOVIC, I.; MILIVOJEVIC, M. Modeling analysis of the lipid bilayer-cytoskeleton coupling in erythrocyte membrane. Biomechanics and modeling in mechanobiology, Springer, v. 13, n. 5, p. 1097-1104, 2014. Citations on pages 35 and 90.

PALLITTO, M. M.; MURPHY, R. M. Mathematical model of the kinetics of beta-amyloid fibril growth from the denatured state. Biophys. J., v. 81, n. 3, p. 109-122, 2001. ISSN 1805-1822. Citations on pages 162 and 167.

PAN, W.; CASWELL, B.; KARNIADAKIS, G. E. A low-dimensional model for the red blood cell. Soft matter, Royal Society of Chemistry, v. 6, n. 18, p. 4366-4376, 2010. Citation on page 52 .

PARESCHI, L.; TOSCANI, G. Interacting Multiagent Systems: Kinetic equations and Monte Carlo methods. [S.1.]: Oxford University Press, 2013. Citation on page 163.

PARK, Y.; BEST, C. A.; BADIZADEGAN, K.; DASARI, R. R.; FELD, M. S.; KURIABOVA, T.; HENLE, M. L.; LEVINE, A. J.; POPESCU, G. Measurement of red blood cell mechanics during morphological changes. Proceedings of the National Academy of Sciences, National Acad Sciences, v. 107, n. 15, p. 6731-6736, 2010. Citation on page 99.

PENG, C.-C.; TZEN, J. T. Analysis of the three essential constituents of oil bodies in developing sesame seeds. Plant and cell physiology, Oxford University Press, v. 39, n. 1, p. 35-42, 1998. Citations on pages 81 and 83 .

PENG, M.; MO, B.; LIU, Y.; XU, M.; SONG, X.; LIU, L.; FANG, Y.; GUO, T.; YE, J.; YU, Z. et al. Prevalence, risk factors and clinical correlates of depression in quarantined population during the covid-19 outbreak. Journal of affective disorders, Elsevier, v. 275, p. 119-124, 2020. Citation on page 179 .

PENG, Z.; ASARO, R. J.; ZHU, Q. Multiscale simulation of erythrocyte membranes. Physical Review E, APS, v. 81, n. 3, p. 031904, 2010. Citations on pages 22, 49, 97, and 99.

Multiscale modelling of erythrocytes in stokes flow. Journal of Fluid Mechanics, Cambridge University Press, v. 686, p. 299, 2011. Citation on page 22. 
PENG, Z.; LI, X.; PIVKIN, I. V.; DAO, M.; KARNIADAKIS, G. E.; SURESH, S. Lipid bilayer and cytoskeletal interactions in a red blood cell. Proceedings of the National Academy of Sciences, National Acad Sciences, v. 110, n. 33, p. 13356-13361, 2013. Citations on pages 22, $30,35,49,68$, and 90 .

PICAS, L.; RICO, F.; DEFORET, M.; SCHEURING, S. Structural and mechanical heterogeneity of the erythrocyte membrane reveals hallmarks of membrane stability. ACS nano, ACS Publications, v. 7, n. 2, p. 1054-1063, 2013. Citation on page 48.

PICCOLOMIINI, E. L.; ZAMA, F. Monitoring italian covid-19 spread by an adaptive seird model. medRxiv, Cold Spring Harbor Laboratory Press, 2020. Citation on page 180.

PICCOLOMINI, E. L.; ZAMA, F. Monitoring italian covid-19 spread by a forced seird model. PloS one, Public Library of Science San Francisco, CA USA, v. 15, n. 8, p. e0237417, 2020. Citation on page 180.

PIVKIN, I. V.; KARNIADAKIS, G. E. Accurate coarse-grained modeling of red blood cells. Physical review letters, APS, v. 101, n. 11, p. 118105, 2008. Citations on pages 22, 40, 56, 61, and 89.

PLOG, B.; NEDERGAARD, M. The glymphatic system in central nervous system health and disease: Past, present, and future. Annual Review of Pathology: Mechanisms of Disease, v. 13, p. 379-394, 02 2018. Citation on page 164.

POLESZCZUK, J.; ENDERLING, H. A high-performance cellular automaton model of tumor growth with dynamically growing domains. Applied Mathematics, NIH Public Access, v. 5, n. 1, p. 144, 2014. Citations on pages 142 and 248.

POLESZCZUK, J.; HAHNFELDT, P.; ENDERLING, H. Evolution and phenotypic selection of cancer stem cells. PLoS Computational Biology, Public Library of Science, v. 11, n. 3, p. e1004025, 2015. Citation on page 142 .

PONTES, B.; VIANA, N.; SALGADO, L.; FARINA, M.; NETO, V. M.; NUSSENZVEIG, H. Cell cytoskeleton and tether extraction. Biophysical journal, Elsevier, v. 101, n. 1, p. 43-52, 2011. Citation on page 106.

POPEL, A. S.; JOHNSON, P. C. Microcirculation and hemorheology. Annu. Rev. Fluid Mech., Annual Reviews, v. 37, p. 43-69, 2005. Citation on page 29.

POSTNIKOV, E. B. Estimation of covid-19 dynamics "on a back-of-envelope": Does the simplest sir model provide quantitative parameters and predictions? Chaos, Solitons \& Fractals, Elsevier, v. 135, p. 109841, 2020. Citation on page 178.

POZRIKIDIS, C. Modeling and simulation of capsules and biological cells. [S.1.]: CRC Press, 2003. Citation on page 63.

Numerical simulation of the flow-induced deformation of red blood cells. Annals of biomedical engineering, Springer, v. 31, n. 10, p. 1194-1205, 2003. Citation on page 104.

PRIETO-VILA, M.; TAKAHASHI, R.-u.; USUBA, W.; KOHAMA, I.; OCHIYA, T. Drug resistance driven by cancer stem cells and their niche. International journal of molecular sciences, Multidisciplinary Digital Publishing Institute, v. 18, n. 12, p. 2574, 2017. Citation on page 247. 
PROBST, R.; SHAPIRO, B. Three-dimensional electrokinetic tweezing: device design, modeling, and control algorithms. Journal of Micromechanics and Microengineering, IOP Publishing, v. 21, n. 2, p. 027004, 2011. Citations on pages 111 and 112.

PRüSS, J.; PUJO-MENJOUET, L.; WEBB, G. F.; ZACHER, R. Analysis of a model for the dynamics of prions. Discrete Contin. Dyn. Syst. Ser. B, v. 6, n. 1, p. 225-235, 2006. ISSN 1531-3492. Available: <https://doi.org/10.3934/dcdsb.2006.6.225>. Citation on page 163.

RABAJANTE, J. F. Insights from early mathematical models of 2019-ncov acute respiratory disease (covid-19) dynamics. arXiv preprint arXiv:2002.05296, 2020. Citation on page 177.

RAJ, A.; KUCEYESKI, A.; WEINER, M. A network diffusion model of disease progression in dementia. Neuron, v. 73, n. 6, p. 1204-1215, 2012. ISSN 0896-6273. Citations on pages 160 and 163.

RAJ, A.; LOCASTRO, E.; KUCEYESKI, A.; TOSUN, D.; RELKIN, N.; WEINER, M. Network diffusion model of progression predicts longitudinal patterns of atrophy and metabolism in alzheimerâs disease. Cell Reports, v. 10, n. 3, p. 359 - 369, 2015. ISSN 2211-1247. Available: $<$ http://www.sciencedirect.com/science/article/pii/S2211124714010638>. Citation on page 163.

RAJAGOPAL, V.; HOLMES, W. R.; LEE, P. V. S. Computational modeling of single-cell mechanics and cytoskeletal mechanobiology. Wiley Interdisciplinary Reviews: Systems Biology and Medicine, Wiley Online Library, v. 10, n. 2, p. e1407, 2018. Citations on pages 65 and 74.

RAMANUJAN, S.; POZRIKIDIS, C. Deformation of liquid capsules enclosed by elastic membranes in simple shear flow: large deformations and the effect of fluid viscosities. Journal of Fluid Mechanics, Cambridge University Press, v. 361, p. 117-143, 1998. Citation on page 21.

RAUCHER, D. Application of laser tweezers to studies of membrane-cytoskeleton adhesion. Methods in cell biology, Elsevier, v. 89, p. 451-466, 2008. Citation on page 105.

REYA, T.; MORRISON, S. J.; CLARKE, M. F.; WEISSMAN, I. L. Stem cells, cancer, and cancer stem cells. Nature, Nature Publishing Group, v. 414, n. 6859, p. 105, 2001. Citations on pages 141 and 247.

RIGLAR, D. T.; RICHARD, D.; WILSON, D. W.; BOYLE, M. J.; DEKIWADIA, C.; TURNBULL, L.; ANGRISANO, F.; MARAPANA, D. S.; ROGERS, K. L.; WHITCHURCH, C. B. et al. Super-resolution dissection of coordinated events during malaria parasite invasion of the human erythrocyte. Cell host \& microbe, Elsevier, v. 9, n. 1, p. 9-20, 2011. Citation on page 39.

ROCHA, H.; ALMEIDA, R.; LIMA, E.; RESENDE, A.; ODEN, J.; YANKEELOV, T. A hybrid three-scale model of tumor growth. Mathematical Models and Methods in Applied Sciences, World Scientific, v. 28, n. 01, p. 61-93, 2018. Citation on page 142.

RODA, W. C.; VARUGHESE, M. B.; HAN, D.; LI, M. Y. Why is it difficult to accurately predict the covid-19 epidemic? Infectious Disease Modelling, Elsevier, 2020. Citation on page 178.

RODRIGUES, D. S.; AUSAS, R. F.; MUT, F.; BUSCAGLIA, G. C. A semi-implicit finite element method for viscous lipid membranes. Journal of Computational Physics, Elsevier, v. 298, p. 565-584, 2015. Citations on pages 22, 30, 37, 38, 49, 68, 79, 88, 93, 97, and 191. 
RODRIGUES, R. O.; PINHO, D.; FAUSTINO, V.; LIMA, R. A simple microfluidic device for the deformability assessment of blood cells in a continuous flow. Biomedical microdevices, Springer, v. 17, n. 6, p. 108, 2015. Citation on page 34.

RODRIGUEZ-BRENES, I. A.; KOMAROVA, N. L.; WODARZ, D. Evolutionary dynamics of feedback escape and the development of stem-cell-driven cancers. Proceedings of the National Academy of Sciences, National Acad Sciences, v. 108, n. 47, p. 18983-18988, 2011. Citation on page 142 .

RODRÍGUEZ-GARCÍA, R.; LÓPEZ-MONTERO, I.; MELL, M.; EGEA, G.; GOV, N. S.; MONROY, F. Direct cytoskeleton forces cause membrane softening in red blood cells. Biophysical journal, Elsevier, v. 108, n. 12, p. 2794-2806, 2015. Citations on pages 30 and 106.

RUAN, Q.; YANG, K.; WANG, W.; JIANG, L.; SONG, J. Clinical predictors of mortality due to covid-19 based on an analysis of data of 150 patients from wuhan, china. Intensive care medicine, Springer, v. 46, n. 5, p. 846-848, 2020. Citation on page 177.

RUEF, P.; LINDERKAMP, O. Deformability and geometry of neonatal erythrocytes with irregular shapes. Pediatric research, Nature Publishing Group, v. 45, n. 1, p. 114-119, 1999. Citation on page 99.

RUSU, R. E. An algorithm for the elastic flow of surfaces. Interfaces and Free Boundaries, v. 7, n. 3, p. 229-239, 2005. Citation on page 34.

SAFEUKUI, I.; BUFFET, P. A.; DEPLAINE, G.; PERROT, S.; BROUSSE, V.; NDOUR, A.; NGUYEN, M.; MERCEREAU-PUIJALON, O.; DAVID, P. H.; MILON, G. et al. Quantitative assessment of sensing and sequestration of spherocytic erythrocytes by the human spleen. Blood, The Journal of the American Society of Hematology, American Society of Hematology Washington, DC, v. 120, n. 2, p. 424-430, 2012. Citation on page 68.

SAGHERIAN, K.; STEEGE, L. M.; COBB, S. J.; CHO, H. Insomnia, fatigue and psychosocial well-being during covid-19 pandemic: A cross-sectional survey of hospital nursing staff in the united states. Journal of clinical nursing, Wiley Online Library, 2020. Citation on page 179.

SAHOO, B. K.; SAPRA, B. K. A data driven epidemic model to analyse the lockdown effect and predict the course of covid-19 progress in india. Chaos, Solitons \& Fractals, Elsevier, v. 139, p. 110034, 2020. Citation on page 178.

SARKAR, K.; KHAJANCHI, S.; NIETO, J. J. Modeling and forecasting the covid-19 pandemic in india. Chaos, Solitons \& Fractals, Elsevier, v. 139, p. 110049, 2020. Citation on page 178.

SAUER, R. A. Computational contact formulations for soft body adhesion. Advances in Soft Matter Mechanics, Springer, p. 55, 2012. Citations on pages 30, 35, 62, 69, and 90.

SAUER, R. A.; LI, S. A contact mechanics model for quasi-continua. International journal for numerical methods in engineering, Wiley Online Library, v. 71, n. 8, p. 931-962, 2007. Citation on page 241.

SCHÄFER, A.; WEICKENMEIER, J.; KUHL, E. The interplay of biochemical and biomechanical degeneration in alzheimerâs disease. Computer Methods in Applied Mechanics and Engineering, v. 352, p. 369 - 388, 2019. ISSN 0045-7825. Available: <http://www.sciencedirect. com/science/article/pii/S0045782519302312>. Citation on page 163. 
SCRIVEN, L. Dynamics of a fluid interface equation of motion for newtonian surface fluids. Chemical Engineering Science, Elsevier, v. 12, n. 2, p. 98-108, 1960. Citations on pages 33, 77 , and 87.

SEGUIN, B.; FRIED, E. Microphysical derivation of the canham-helfrich free-energy density. Journal of mathematical biology, Springer, v. 68, n. 3, p. 647-665, 2014. Citation on page 33.

SEIFERT, U. Configurations of fluid membranes and vesicles. Advances in physics, Taylor \& Francis, v. 46, n. 1, p. 13-137, 1997. Citation on page 40.

SEIFERT, U.; BERNDL, K.; LIPOWSKY, R. Shape transformations of vesicles: Phase diagram for spontaneous-curvature and bilayer-coupling models. Physical review A, APS, v. 44, n. 2 , p. 1182, 1991. Citation on page 97.

SERLACHIUS, A.; BADAWY, S. M.; THABREW, H. Psychosocial challenges and opportunities for youth with chronic health conditions during the covid-19 pandemic. JMIR Pediatrics and Parenting, JMIR Publications Inc., Toronto, Canada, v. 3, n. 2, p. e23057, 2020. Citation on page 189 .

SETHIAN, J. A.; VLADIMIRSKY, A. Ordered upwind methods for static hamilton-jacobi equations. Proceedings of the National Academy of Sciences, National Acad Sciences, v. 98, n. 20, p. 11069-11074, 2001. Citation on page 116.

SHANKAR, G. M.; LI, S.; MEHTA, T. H.; GARCIA-MUNOZ, A.; SHEPARDSON, N. E.; SMITH, I.; BRETT, F. M.; FARRELL, M. A.; ROWAN, M. J.; LEMERE, C. A.; REGAN, C. M.; WALSH, D. M.; SABATINI, B. L.; SELKOE, D. J. Amyloid-beta protein dimers isolated directly from alzheimer's brains impair synaptic plasticity and memory. Nat. Med., v. 14, p. 837-842, 2008. Citation on page 161.

SHAO, Y.; WU, J. Idm editorial statement on the 2019-ncov. Infectious Disease Modelling, v. 5, p. $233-234,2020$. ISSN 2468-0427. Available: <http://www.sciencedirect.com/science/ article/pii/S2468042720300038>. Citation on page 178.

SHEETZ, M. P.; SABLE, J. E.; DÖBEREINER, H.-G. Continuous membrane-cytoskeleton adhesion requires continuous accommodation to lipid and cytoskeleton dynamics. Annu. Rev. Biophys. Biomol. Struct., Annual Reviews, v. 35, p. 417-434, 2006. Citation on page 106.

SIGÜENZA, J.; MENDEZ, S.; NICOUD, F. How should the optical tweezers experiment be used to characterize the red blood cell membrane mechanics? Biomechanics and modeling in mechanobiology, Springer, v. 16, n. 5, p. 1645-1657, 2017. Citation on page 61.

SINGH, R.; ADHIKARI, R. Age-structured impact of social distancing on the covid-19 epidemic in india. arXiv preprint arXiv:2003.12055, 2020. Citation on page 178.

SINGH, S.; ROY, M. D.; SINHA, C. P. T. M. K.; PARVEEN, C. P. T. M. S.; SHARMA, C. P. T. G.; JOSHI, C. P. T. G. Impact of covid-19 and lockdown on mental health of children and adolescents: A narrative review with recommendations. Psychiatry research, Elsevier, p. 113429, 2020. Citation on page 179.

SINGHAL, T. A review of coronavirus disease-2019 (covid-19). The Indian Journal of Pediatrics, Springer, p. 1-6, 2020. Citation on page 177.

SMOLUCHOWSKI, M. Versuch einer mathematischen theorie der koagulationskinetik kolloider lsungen. IZ. Phys. Chem., v. 92, p. 129Đ168, 1917. Citation on page 161. 
SOHRABI, C.; ALSAFI, Z.; O’NEILL, N.; KHAN, M.; KERWAN, A.; AL-JABIR, A.; IOSIFIDIS, C.; AGHA, R. World health organization declares global emergency: A review of the 2019 novel coronavirus (covid-19). International Journal of Surgery, Elsevier, 2020. Citation on page 178 .

SOLÉ, R. V.; RODRÍGUEZ-CASO, C.; DEISBOECK, T. S.; SALDANA, J. Cancer stem cells as the engine of unstable tumor progression. Journal of theoretical biology, Elsevier, v. 253, n. 4, p. 629-637, 2008. Citation on page 125.

SPANGLER, E. J.; HARVEY, C. W.; REVALEE, J. D.; KUMAR, P. S.; LARADJI, M. Computer simulation of cytoskeleton-induced blebbing in lipid membranes. Physical Review E, APS, v. 84 , n. 5, p. 051906, 2011. Citation on page 106.

STEIGMANN, D. J. The Role of Mechanics in the Study of Lipid Bilayers. [S.1.]: Springer, 2017. Citation on page 74.

STEWART, I. The mathematics of life. [S.1.]: Basic Books, 2011. Citation on page 19.

STIEHL, T.; MARCINIAK-CZOCHRA, A. Mathematical modeling of leukemogenesis and cancer stem cell dynamics. Mathematical Modelling of Natural Phenomena, EDP Sciences, v. 7, n. 1, p. 166-202, 2012. Citation on page 126.

SUN, Z.; KOMAROVA, N. L. Stochastic control of proliferation and differentiation in stem cell dynamics. Journal of mathematical biology, Springer, v. 71, n. 4, p. 883-901, 2015. Citation on page 126.

SURESH, S. Mechanical response of human red blood cells in health and disease: some structureproperty-function relationships. Journal of materials research, Cambridge University Press, v. 21, n. 8, p. 1871-1877, 2006. Citations on pages 49, 67, and 68.

SURESH, S.; SPATZ, J.; MILLS, J. P.; MICOULET, A.; DAO, M.; LIM, C.; BEIL, M.; SEUFFERLEIN, T. Connections between single-cell biomechanics and human disease states: gastrointestinal cancer and malaria. Acta biomaterialia, Elsevier, v. 1, n. 1, p. 15-30, 2005. Citation on page 104.

SVAHN, H. A.; BERG, A. van den. Single cells or large populations? Lab on a Chip, Royal Society of Chemistry, v. 7, n. 5, p. 544-546, 2007. Citation on page 29.

SVETINA, S.; KUZMAN, D.; WAUGH, R. E.; ZIHERL, P.; ŽEKŠ, B. The cooperative role of membrane skeleton and bilayer in the mechanical behaviour of red blood cells. Bioelectrochemistry, Elsevier, v. 62, n. 2, p. 107-113, 2004. Citation on page 49.

SWIHART, A.; MIKRUT, J.; KETTERSON, J.; MACDONALD, R. Atomic force microscopy of the erythrocyte membrane skeleton. Journal of microscopy, Wiley Online Library, v. 204, n. 3, p. 212-225, 2001. Citations on pages 56 and 58.

TAKEUCHI, M.; MIYAMOTO, H.; SAKO, Y.; KOMIZU, H.; KUSUMI, A. Structure of the erythrocyte membrane skeleton as observed by atomic force microscopy. Biophysical Journal, Elsevier, v. 74, n. 5, p. 2171-2183, 1998. Citation on page 58.

TAKEUCHI, Y.; IWASA, Y.; SATO, K. Mathematics for life science and medicine. [S.1.]: Springer Science \& Business Media, 2007. Citation on page 19. 
TAN, S.; XU, M. Numerical studies of shape recovery of a red blood cell using dissipative particle dynamics. International Journal of Computational Methods, World Scientific, p. 1950032, 2019. Citation on page 63.

TANG, B.; WANG, X.; LI, Q.; BRAGAZZI, N. L.; TANG, S.; XIAO, Y.; WU, J. Estimation of the transmission risk of the 2019-ncov and its implication for public health interventions. Journal of clinical medicine, Multidisciplinary Digital Publishing Institute, v. 9, n. 2, p. 462, 2020. Citations on pages 100 and 178.

TANG, Y.-H.; LU, L.; LI, H.; EVANGELINOS, C.; GRINBERG, L.; SACHDEVA, V.; KARNIADAKIS, G. E. Openrbc: a fast simulator of red blood cells at protein resolution. Biophysical journal, Elsevier, v. 112, n. 10, p. 2030-2037, 2017. Citations on pages 22, 43, 47, and 191.

TASSO, I. V.; BUSCAGLIA, G. C. A finite element method for viscous membranes. Computer Methods in Applied Mechanics and Engineering, Elsevier, v. 255, p. 226-237, 2013. Citation on page 75 .

TATARNIKOVA, O. G.; ORLOV, M. A.; N.V, B. Beta-amyloid and tau protein: Structure, interaction and prion-like properties. Biochemistry (Moscow), v. 80, n. 13, p. 1800-1819, 2015. Citation on page 163.

TAYLOR, S. The psychology of pandemics: Preparing for the next global outbreak of infectious disease. [S.1.]: Cambridge Scholars Publishing, 2019. Citation on page 179.

TAYLOR, S.; LANDRY, C. A.; PALUSZEK, M. M.; FERGUS, T. A.; MCKAY, D.; ASMUNDSON, G. J. Development and initial validation of the covid stress scales. Journal of Anxiety Disorders, Elsevier, v. 72, p. 102232, 2020. Citation on page 179.

TENG, Z.; WEI, Z.; QIU, Y.; TAN, Y.; CHEN, J.; TANG, H.; WU, H.; WU, R.; HUANG, J. Psychological status and fatigue of frontline staff two months after the covid-19 pandemic outbreak in china: A cross-sectional study. Journal of affective disorders, Elsevier, 2020. Citation on page 179 .

TOMASETTI, C.; LI, L.; VOGELSTEIN, B. Stem cell divisions, somatic mutations, cancer etiology, and cancer prevention. Science, American Association for the Advancement of Science, v. 355, n. 6331, p. 1330-1334, 2017. Citations on pages 141 and 247.

TOMASETTI, C.; VOGELSTEIN, B.; PARMIGIANI, G. Half or more of the somatic mutations in cancers of self-renewing tissues originate prior to tumor initiation. Proceedings of the National Academy of Sciences, National Acad Sciences, v. 110, n. 6, p. 1999-2004, 2013. Citations on pages 141 and 247.

TUVAL, I.; MEZIĆ, I.; BOTTAUSCI, F.; ZHANG, Y. T.; MACDONALD, N. C.; PIRO, O. Control of particles in microelectrode devices. Physical review letters, APS, v. 95, n. 23, p. 1-4, 2005. Citation on page 111.

TZEN, J. Seed oil bodies of sesame and their surface proteins, oleosin, caleosin, and steroleosin. Sesame, the Genus Sesamum, CRC Press London, UK, v. 48, p. 187-200, 2011. Citation on page 73 .

TZEN, J.; HUANG, A. Surface structure and properties of plant seed oil bodies. The Journal of cell biology, Rockefeller University Press, v. 117, n. 2, p. 327-335, 1992. Citation on page 73. 
TZEN, J. T. Integral proteins in plant oil bodies. ISRN Botany, Hindawi Publishing Corporation, v. 2012, 2012. Citations on pages 73 and 83 .

ULKER, P.; SATI, L.; CELIK-OZENCI, C.; MEISELMAN, H.; BASKURT, O. Mechanical stimulation of nitric oxide synthesizing mechanisms in erythrocytes. Biorheology, IOS Press, v. 46, n. 2, p. 121-132, 2009. Citation on page 29.

UNAL, M.; ALAPAN, Y.; JIA, H.; VARGA, A. G.; ANGELINO, K.; ASLAN, M.; SAYIN, I.; HAN, C.; JIANG, Y.; ZHANG, Z. et al. Micro and nano-scale technologies for cell mechanics. Nanobiomedicine, SAGE Publications Sage UK: London, England, v. 1, p. 5, 2014. Citations on pages 47 and 195 .

URBANC, B.; CRUZ, L.; BULDYREV, S. V.; HAVLIN, S.; IRIZARRY, M. C.; STANLEY, H. E.; HYMAN, B. T. Dynamics of plaque formation in Alzheimer's disease. Biophys. J., v. 76, n. 3, p. 1330-1334, 1999. ISSN 0006-3495. Citation on page 159.

VASQUEZ, P. A.; HULT, C.; ADALSTEINSSON, D.; LAWRIMORE, J.; FOREST, M. G.; BLOOM, K. Entropy gives rise to topologically associating domains. Nucleic acids research, Oxford University Press, v. 44, n. 12, p. 5540-5549, 2016. Citation on page 51.

VÁZQUEZ, J. L. The importance of mathematics in the development of science and technology. Boletín de la Sociedad Española de Matemática Aplicada, v. 19, p. 69-112, 2001. Citation on page 19.

VEERAPANENI, S. K.; RAHIMIAN, A.; BIROS, G.; ZORIN, D. A fast algorithm for simulating vesicle flows in three dimensions. Journal of Computational Physics, Elsevier, v. 230, n. 14, p. 5610-5634, 2011. Citation on page 21.

VEERAPANENI, S. K.; RAJ, R.; BIROS, G.; PUROHIT, P. K. Analytical and numerical solutions for shapes of quiescent two-dimensional vesicles. International Journal of NonLinear Mechanics, Elsevier, v. 44, n. 3, p. 257-262, 2009. Citation on page 42.

VELAVAN, T. P.; MEYER, C. G. The covid-19 epidemic. Tropical medicine \& international health, Wiley-Blackwell, v. 25, n. 3, p. 278, 2020. Citation on page 177.

VINDEGAARD, N.; BENROS, M. E. Covid-19 pandemic and mental health consequences: Systematic review of the current evidence. Brain, behavior, and immunity, Elsevier, v. 89, p. 531-542, 2020. Citation on page 179.

VISVADER, J. E.; LINDEMAN, G. J. Cancer stem cells in solid tumours: accumulating evidence and unresolved questions. Nature Reviews Cancer, Nature Publishing Group, v. 8, n. 10, p. 755, 2008. Citations on pages 141 and 247.

WALSH, D. M.; SELKOE, D. J. A $\beta$ oligomers: a decade of discovery. J. Neurochem., Blackwell Publishing Ltd, v. 101, n. 5, p. 1172-1184, 2007. ISSN 1471-4159. Citation on page 160.

WANNER, G.; FORMANEK, H.; THEIMER, R. The ontogeny of lipid bodies (spherosomes) in plant cells. Planta, Springer, v. 151, n. 2, p. 109-123, 1981. Citation on page 73.

WEEKES, S. L.; BARKER, B.; BOBER, S.; CISNEROS, K.; CLINE, J.; THOMPSON, A.; HLATKY, L.; HAHNFELDT, P.; ENDERLING, H. A multicompartment mathematical model of cancer stem cell-driven tumor growth dynamics. Bulletin of Mathematical Biology, Springer, v. 76, n. 7, p. 1762-1782, 2014. Citations on pages 141, 142, and 247. 
WEICKENMEIER, J.; JUCKER, M.; GORIELY, A.; KUHL, E. A physics-based model explains the prion-like features of neurodegeneration in alzheimer's disease, parkinson's disease, and amyotrophic lateral sclerosis. J. of the Mechanics and Physics of Solids, v. 124, p. 264-281, 2019. Citation on page 163.

WEISS, L. D.; DRIESSCHE, P. van den; LOWENGRUB, J. S.; WODARZ, D.; KOMAROVA, N. L. Effect of feedback regulation on stem cell fractions in tissues and tumors: Understanding chemoresistance in cancer. Journal of Theoretical Biology, Elsevier, v. 509, p. 110499, 2021. Citation on page 248 .

WEISS, L. D.; KOMAROVA, N. L.; RODRIGUEZ-BRENES, I. A. Mathematical modeling of normal and cancer stem cells. Current Stem Cell Reports, Springer, v. 3, n. 3, p. 232-239, 2017. Citations on pages 142 and 247.

WERNER, B.; DINGLI, D.; LENAERTS, T.; PACHECO, J. M.; TRAULSEN, A. Dynamics of mutant cells in hierarchical organized tissues. PLoS computational biology, Public Library of Science, v. 7, n. 12, p. e1002290, 2011. Citations on pages 127, 141, and 142.

WERNER, B.; SCOTT, J. G.; SOTTORIVA, A.; ANDERSON, A. R.; TRAULSEN, A.; ALTROCK, P. M. The cancer stem cell fraction in hierarchically organized tumors can be estimated using mathematical modeling and patient-specific treatment trajectories. Cancer research, AACR, v. 76, n. 7, p. 1705-1713, 2016. Citations on pages 127, 141, and 142.

WEST, B. J. A mathematics for medicine: the network effect. Frontiers in physiology, Frontiers, v. 5 , p. 456, 2014. Citation on page 19.

WEST, R.; MICHIE, S.; RUBIN, G. J.; AMLÔT, R. Applying principles of behaviour change to reduce sars-cov-2 transmission. Nature Human Behaviour, Nature Publishing Group, v. 4, n. 5, p. 451-459, 2020. Citation on page 180.

WHITE, S. H. Small phospholipid vesicles: internal pressure, surface tension, and surface free energy. Proceedings of the National Academy of Sciences, National Acad Sciences, v. 77, n. 7, p. 4048-4050, 1980. Citation on page 79.

WHITE, S. H.; REY, A. M. D.; SÁNCHEZ, G. R. Modeling epidemics using cellular automata. Applied mathematics and computation, Elsevier, v. 186, n. 1, p. 193-202, 2007. Citation on page 189.

WODARZ, D.; KOMAROVA, N. Can loss of apoptosis protect against cancer? Trends in Genetics, Elsevier, v. 23, n. 5, p. 232-237, 2007. Citations on pages 125, 142, and 248.

WRZOSEK, D. Existence of solutions for the discrete coagulation-fragmentation model with diffusion. Topol. Methods Nonlinear Anal., v. 9, n. 2, p. 279-296, 1997. ISSN 1230-3429. Available: <https://doi.org/10.12775/TMNA.1997.014>. Citations on pages 162 and 163.

WU, J. T.; LEUNG, K.; BUSHMAN, M.; KISHORE, N.; NIEHUS, R.; SALAZAR, P. M. de; COWLING, B. J.; LIPSITCH, M.; LEUNG, G. M. Estimating clinical severity of covid-19 from the transmission dynamics in wuhan, china. Nature Medicine, Nature Publishing Group, v. 26, n. 4, p. 506-510, 2020. Citation on page 178.

XIAO, L.; LIU, Y.; CHEN, S.; FU, B. Simulation of deformation and aggregation of two red blood cells in a stenosed microvessel by dissipative particle dynamics. Cell biochemistry and biophysics, Springer, v. 74, n. 4, p. 513-525, 2016. Citation on page 53. 
YANG, Y.; ZHU, J.-f.; YANG, S.-y.; LIN, H.-J.; CHEN, Y.; ZHAO, Q.; FU, C.-w. Prevalence and associated factors of poor sleep quality among chinese returning workers during the covid-19 pandemic. Sleep medicine, Elsevier, v. 73, p. 47-52, 2020. Citation on page 179.

YAZDANI, A. Z. K.; BAGCHI, P. Phase diagram and breathing dynamics of a single red blood cell and a biconcave capsule in dilute shear flow. Phys. Rev. E, American Physical Society, v. 84, p. 026314, Aug 2011. Available: <https://link.aps.org/doi/10.1103/PhysRevE.84.026314>. Citation on page 22.

YORKE, J. A. Invariance for ordinary differential equations. Mathematical systems theory, Springer, v. 1, n. 4, p. 353-372, 1967. Citation on page 183.

YUN, Z.; XIANG, C.; WANG, L. Effect of a high-frequency vibration boundary on rbc. Journal of Mechanics in Medicine and Biology, World Scientific, v. 18, n. 08, p. 1840032, 2018. Citation on page 53.

ZHANG, P.; BARI, V. D.; BRIARS, R.; TAHER, Z. M.; YUAN, J.; LIU, G.; GRAY, D. Influence of pecan nut pretreatment on the physical quality of oil bodies. Journal of Food Quality, Hindawi, v. 2017, 2017. Citation on page 74.

ZHANG, R.; BROWN, F. L. Cytoskeleton mediated effective elastic properties of model red blood cell membranes. The Journal of chemical physics, American Institute of Physics, v. 129, n. 6, p. 08B603, 2008. Citation on page 58.

ZHAO, H.; HE, X.; FAN, G.; LI, L.; HUANG, Q.; QIU, Q.; KANG, Z.; DU, T.; HAN, L.; DING, L. et al. Covid-19 infection outbreak increases anxiety level of general public in china: involved mechanisms and influencing factors. Journal of affective disorders, Elsevier, v. 276, p. 446-452, 2020. Citation on page 179.

ZHAO, S.; CHEN, H. Modeling the epidemic dynamics and control of covid-19 outbreak in china. Quantitative Biology, Springer, p. 1-9, 2020. Citation on page 178.

ZHAO, S.; MUSA, S. S.; LIN, Q.; RAN, J.; YANG, G.; WANG, W.; LOU, Y.; YANG, L.; GAO, D.; HE, D. et al. Estimating the unreported number of novel coronavirus (2019-ncov) cases in china in the first half of january 2020: a data-driven modelling analysis of the early outbreak. Journal of clinical medicine, Multidisciplinary Digital Publishing Institute, v. 9, n. 2, p. 388, 2020. Citation on page 186 .

ZHOU, L.; ZHANG, Y.-Q.; DENG, X.-L.; LIU, M. Iccm2015 (paper id: 1148): Dpd simulation of the movement and deformation of bioconcave cells. International Journal of Computational Methods, World Scientific, v. 13, n. 04, p. 1641003, 2016. Citation on page 53.

ZHOU, Y.; WANG, Y.; LIN, Q. A microfluidic device for continuous-flow magnetically controlled capture and isolation of microparticles. Journal of Microelectromechanical Systems, IEEE, v. 19, n. 4, p. 743-751, 2010. Citation on page 111.

ZOU, K.; GONG, J. S.; YANAGISAWA K.AND MICHIKAWA, M. A novel function of monomeric amyloid-protein serving as an antioxidant molecule against metal-induced oxidative damage. J. Neurosci., v. 22, p. 4833-4841, 2002. Citation on page 161.

ZWEYTICK, D.; ATHENSTAEDT, K.; DAUM, G. Intracellular lipid particles of eukaryotic cells. Biochimica et Biophysica Acta (BBA)-Reviews on Biomembranes, Elsevier, v. 1469, n. 2, p. 101-120, 2000. Citation on page 73. 

APPENDIX

\section{A}

\section{CODE FOR MODELING A RED BLOOD CELL \\ CYTOSKELETON}

In this section, we present the computational implementation of the mathematical model described in Chapter 3. In particular, we numerically solve the system (3.6) with the NewtonRaphson method (3.6), arising from the principle of virtual work and adopting a friction model for the dissipation energy, as previously described. To promote the execution of the code and mathematical model, we provide a code in Octave, a free software and language. This code can be useful in any case to identify a possible way of numerically solving the model and with some simple modifications it can be adapted to other commonly used coding languages such as Matlab or C.

Below, we report the main routine of the program, which we can name with Cyto_main.m. The routine recalls some functions that we will propose and explain below. The main steps of Cyto_main.m are commented with reference to the lines of code following the same routine.

1: global $\mathrm{kp}=5 ; \%$ Spring POW constant

2: global $\mathrm{p}=0.0375 ; \%$ Persistence length

3 : betav $=1 ; \%$ Friction param .

4:

5: \%\% PRE-PROCESSING

6:

7:\% Read mesh data

8: coor=load ('coordinates.txt') ; coor=coor',

9: edges=load (' edges.txt') ; edges=edges ',

10:

11: \% Extract edge-node connectivity

12: mesh = struct ('p', coor, 'e', edges, ' $\mathrm{x} 0$ ', 0$)$;

13: 
14: nnodes $=\operatorname{size}(\operatorname{mesh} \cdot \mathrm{p}, 2)$;

15: nedges $=$ size $($ mesh.e, 2$)$;

16:

17: $\mathrm{Nv}=$ nnodes;

18: $\mathrm{tNv}=3 * \mathrm{Nv}$;

19:

20: \% Initial cytoskeleton spring lengths

21: mesh $\cdot \mathrm{x} 0=$ ComputeEdgeLengths $($ mesh $)$;

22:

23: \% Initial cytoskelenton energy

24: energy $=$ ComputeUs ( mesh $)$;

25:

26: \% Initial Cyto-force (- derivative of energy)

27: force $=$ ComputeAnalFs (mesh);

28:

29: \% Non-linear system

30:

31: iden $=$ speye $(\mathrm{tNv}) ; \%$ Sparse identity matrix

32:

33: $\mathrm{dt}=1 \mathrm{e}-2 ; \%$ Time step

34: tini $=0.0 ; \%$ Initial time

35: tend $=0.1 ; \%$ Final time

36: freqout $=1$;

37:

38: TOLr $=1 \mathrm{e}-7 ; \%$ Newton-Raphson tolerance

39: MAXIT $=10 ; \%$ Newton-Raphson $\max$ iterations

40:

41: Fext $=$ zeros $(\mathrm{tNv}, 1) ; \%$ External forcing ( Set by user)

42:

43: $X=\operatorname{mesh} \cdot \mathrm{p}(:)$;

44: $\mathrm{Zn}=[\mathrm{X} ; \operatorname{zeros}(\mathrm{tNv}, 1)]$;

45: $\mathrm{Z}=\mathrm{Zn}$;

46: $\mathrm{tt}=\mathrm{tini}$

47:

48: fid = fopen ("evol.txt", "w");

49: fprintf (fid, "\%1.10f \%1.10f \n", tt, energy); fflush(fid);

50 :

51: fprintf(stdout, "\n | INITIALIZATION COMPLETE $\backslash \mathrm{n} ")$;

52: 
53: fprintf( stdout, "\n । BEGIN LOOP OVER TIME STEPS $\backslash n ")$; fflush ( stdout );

54: $\mathrm{n}=1$;

55: countf $=1$;

56:

57: \% Initialization post-processing

58: Z_pos=Z;

59: $\mathrm{t}_{-}$pos $=\mathrm{tt}$;

60:

61: \%\% CORE-PROCESSING

62:

63: while $(\mathrm{tt}<$ tend $) \%$ Loop over time steps

64:

65: fprintf(stdout, $" \backslash \mathrm{n} \quad \mathrm{I}$ Time: \%g, $\mathrm{dt}=\% \mathrm{~g} \backslash \mathrm{n} ", \mathrm{tt}, \mathrm{dt}) ; \mathrm{fflush}($ stdout );

66:

67: iter $=1$; normRES $=10 *$ TOLr;

68: while (normRES > TOLr \&\& iter $<=$ MAXIT) \% Newton-Raphson loop 69:

70: $\quad$ flag $\mathrm{J}=1$;

$71:$

72: $[$ Rv Rf jacRf fWLC fPOW] = ComputeSystemMembers $($ mesh, Zn, Z, Fext, betav, dt, flagJ ) ;

73:

74: $\quad$ JAC $=$ [

75: iden $\quad-\mathrm{dt} *$ iden $; \ldots$

76: $\quad$ sparse $(j a c R f) \quad-d i a g(b e t a v) * i d e n$

77: ] ;

$78:$

79: $\quad$ RES $=[$

80: $\quad$ Rv $; \ldots$

81: $\quad \mathrm{Rf}$

82: ] ;

83:

84: $\quad \mathrm{dZ}=\mathrm{JAC} \backslash$ RES ;

$85:$

86: $\quad Z=Z-d Z$;

87: $\quad$ mesh. $\mathrm{p}=\operatorname{reshape}(\mathrm{Z}(1: \mathrm{tNv}), 3, \mathrm{Nv})$;

88: 
89: $\quad$ normRES $=$ norm $($ RES $)$;

90: fprintf(stdout, " $\quad::>\quad \mathrm{N}-\mathrm{R}$ iter \%d । normRES $=\% \mathrm{e} \backslash \mathrm{n} "$, iter, normRES);

91: $\quad$ fflush ( stdout);

92:

93: $\quad$ iter $=$ iter +1 ;

94:

95: end \% End Newton-Raphson loop

96:

97: $\quad \mathrm{Zn}=\mathrm{Z}$;

98:

99: $\quad \mathrm{tt}=\mathrm{tt}+\mathrm{dt}$;

100: $\mathrm{n}=\mathrm{n}+1$;

101:

102: $\quad$ video $=1$;

103: \% Plotting 3D

104: if video $==1$

105: $\quad$ te $r=3 * N v$;

106: $\quad x p p=Z(1: 3:$ ter, 1$)$;

107: $\quad \operatorname{ypp}=Z(2: 3:$ ter, 1$)$;

108: $\quad z p p=Z(3: 3:$ ter, 1$)$;

109: plot3 (xpp, ypp, zpp, ' o ', 'MarkerSize ', 10 , 'MarkerFaceColor ', ' r ') ;

110: $\quad$ axis $\left(\left[\begin{array}{llllll}-2 & 2 & -2 & 2 & -2 & 2\end{array}\right]\right)$;

111: $\quad x$ label ("x");

112: ylabel ("y");

113: zlabel (" z");

114: hold on

115: for $1 \mathrm{c}=1:$ nedges

116: $\quad \operatorname{xee}=[\operatorname{xpp}(\operatorname{edges}(1,1 \mathrm{c}), 1) \operatorname{xpp}(\operatorname{edges}(2,1 \mathrm{c}), 1)]$;

117: $\quad \operatorname{yee}=[\operatorname{ypp}(\operatorname{edges}(1,1 \mathrm{c}), 1) \quad \operatorname{ypp}(\operatorname{edges}(2,1 \mathrm{c}), 1)]$;

118: $\quad$ plot (xee, yee, ' b')

119: end

120: hold off

121: filenamev=sprintf(',output/\%05d.png', tt );

122: print (filenamev) ;

123: end

124:

125: energy $(\mathrm{n})=$ ComputeUs (mesh);

126: fprintf (fid, "\%1.10f \% $1.10 \mathrm{f} \backslash \mathrm{n} ", \mathrm{tt}$, energy (n)); fflush(fid); 
127:

128: \% Collecting data

129: Z_pos=[Z_pos Z];

130: $\mathrm{t}_{-}$pos $=\left[\mathrm{t}_{-}\right.$pos $\left.\mathrm{tt}\right]$;

131:

132: end \% End loop over time steps

133:

134: fclose (fid);

135: fprintf( stdout, " $\backslash \mathrm{n}$ । NORMAL COMPLETION $\backslash \mathrm{n} "$ );

In the Cyto_main.m routine, we remark that the following lines refer to:

- Lines 1-2. Definition of global variables. These kind of variables can be used also inside the functions called;

- Line 3. Definition of the parameter $\beta_{v}$ of friction that will be applied in the NewtonRaphson method;

- Lines 8-18. Reading and loading of input archives related to the geometry of the problem and construction of the mesh, describing the system as a structure array. The file coordinates.txt contains the spatial coordinates of the nodes while the file edges.txt lists all the connection between the nodes (see the Appendix);

- Lines 21-27. Calculation of the length of the spectrin edges, energy and forces of the initial configuration of the system through the functions ComputeEdgeLengths .m, ComputeUs.m and ComputeAnalFs.m respectively, shown below and commented in this section.

- Lines 31-39. Initialization of data for the resolution of the non-linear system deriving from the Newton-Raphson method;

- Line 41. Setting of any external forces at the nodes. Note that the vectors with dimension $(\mathrm{tNv}, 1)$ correspond to consider the spatial coordinates of the nodes listed in one only column for all the nodes (see line 18);

- Lines 44-45. Initialization of the unknowns $\mathrm{Z}$ and $\mathrm{Zn}$ at the current and previous time respectively. They contain the coordinates of the nodes and the respective velocities in a single column;

- Lines 48-49. Opening of the output file evol. txt reporting the energy of the system for each simulation time (see the Appendix);

- Lines 51-53. Setting messages to appear on screen communicating the start of the coreprocessing loop. Similar messages are sometime clearly reported in the code below; 
- Lines 51-53. Setting messages to appear on screen communicating the start of the coreprocessing loop. Similar messages are sometime clearly reported in the code below;

- Line 63. Initialization of the core-processing while loop over time steps;

- Line 68. Initialization of the Newton-Raphson while loop;

- Line 70. Flag to activate the calculation of Jacobian of force residual;

- Line 72. Calculation of the members necessary for resolution with the Newton-Raphson method through the function ComputeSystemMembers.m, described below in this section;

- Lines 74-77. Assembly of the Jacobian matrix;

- Lines 79-82. Assembly of the the residue vector;

- Lines 84-93. System resolution and data update;

- Line 95. End of NewtonRaphson loop;

- Lines 102-123. 3-dimensional plotting of system nodes and saving of screens in the output folder;

- Lines 125-126. Calculation of the new energy value with the ComputeUs .m function and saving the value in the evol. txt file;

- Lines 129-130. Collecting the nodes positions and the times;

- Line 132. End of the loop over time steps;

- Line 134. Closure of the output file evol . txt.

We report below the functions used in the previous routine. The function ComputeEdgeLengths .m calculates the lengths of all the edges of the system. The function has as input the mesh structure vector mesh and calculates the Euclidean distance returning to the output the row vector res of dimension of the edges number.

1: function res = ComputeEdgeLengths $($ mesh $)$

2:

3: $\quad$ res $=$ sqrt $\left((\operatorname{mesh} \cdot \mathrm{p}(1, \operatorname{mesh} \cdot \mathrm{e}(1,:))-\operatorname{mesh} \cdot \mathrm{p}(1, \operatorname{mesh} \cdot \mathrm{e}(2,:))) \cdot{ }^{\wedge} 2\right.$ $+\ldots$

4: $\quad(\operatorname{mesh} \cdot \mathrm{p}(2, \operatorname{mesh} \cdot \mathrm{e}(1,:))-\operatorname{mesh} \cdot \mathrm{p}(2, \operatorname{mesh} \cdot \mathrm{e}(2,:))) \cdot{ }^{\wedge} 2+\ldots$

5: $\quad(\operatorname{mesh} \cdot \mathrm{p}(3, \operatorname{mesh} . \mathrm{e}(1,:))-\operatorname{mesh} \cdot \mathrm{p}(3, \operatorname{mesh} \cdot \mathrm{e}(2,:))) \cdot \wedge 2)$;

6: end

The following ComputeUs.m function evaluates the energy of the system according to the equation ?? and considering as input the mesh. 
1: function energy $=$ ComputeUs (mesh)

2:

3: global kp

4: global p

5:

6: $\quad \operatorname{lm}=2.2 *$ mesh.$x 0 ; \%$ Maximum extension of each edge

7: $\quad$ kBT_lm $=1 \mathrm{~m}$;

$8:$

9: $\quad$ nedges $=$ size $($ mesh.e, 2$)$;

10:

11: $\quad$ lcur $=$ ComputeEdgeLengths (mesh) ;

12:

13: $\quad \mathrm{xx}=1 \mathrm{cur} . / \mathrm{lm}$;

14:

15: energy $=\operatorname{sum}\left(\mathrm{kBT} \_l \mathrm{~lm} . *\left(3 * \mathrm{xx} \cdot{ }^{\wedge} 2-2 * \mathrm{xx} \cdot{ }^{\wedge} 3\right) . /(4 * \mathrm{p} *(1-\mathrm{xx}))+\mathrm{kp}\right.$ . / lcur) ;

16:

17: end

In particular, we observe that the following command lines of the ComputeUs .m function are attributed to:

- Lines 3-4. Calling of global variables;

- Line 6-7. Calculation of the maximum extension $\ell_{m}$ of the spectrines and of the product of the parameters $k_{B} T \ell_{m}$ (being in this case the temperature $T$ and the parameter $k_{B}$ chosen arbitrarily equal to 1$)$;

- Line 9. Counting the number of edges;

- Line 11. Calculation of the current length of the edges through the function ComputeEdgeLengths .m;

- Line 13. Calculation of the the dimensionless ratio between the current and the maximum lengths of the edges;

- Line 15. Calculation of the global energy of the system according to the equation (??).

The ComputeAnalFs.m function computes the WLC-POW forces (for each node), considering as input the mesh and calculating the analytical derivative of energy. In particular, such derivative is calculated passing trough the derivative of the energy respect to the edges (being the energy defined over the relative extensions of spectrins). For more details over the function, we list some remarks below the code. 
1: function force $=$ ComputeAnalFs (mesh)

2:

3: global kp; \% Spring POW constant

4: $\quad$ global p; \% Persistence length

5: $\quad \operatorname{lm}=2.2 *$ mesh. $x 0 ; \%$ Maximum extension of each edge

6: $\quad$ lcur = ComputeEdgeLengths (mesh);

7:

8: $\quad \mathrm{tNv}=3 * \operatorname{size}(\operatorname{mesh} \cdot \mathrm{p}, 2)$;

9: $\quad$ force $=$ zeros $(\mathrm{tNv}, 1)$;

10:

11: nedges $=\operatorname{size}(\operatorname{mesh} . \mathrm{e}, 2)$;

12: $\quad$ listedges $=[1:$ nedges $]$;

13: $\quad \mathrm{xx}=1 \mathrm{cur}(1$ istedges $) . / \operatorname{lm}(1$ istedges $)$;

14: $\quad$ kBT_lm $=1 \mathrm{~m}($ listedges $)$;

15: $\quad \mathrm{fed}=\mathrm{kBT} \_l \mathrm{~lm} /(4 * \mathrm{p}) . *\left(6 *\left(\mathrm{xx}-\mathrm{xx} \cdot{ }^{\wedge} 2\right) *(1-\mathrm{xx})+\left(3 * \mathrm{xx} \cdot{ }^{\wedge} 2-2 *\right.\right.$

$$
\left.\left.\mathrm{xx} \cdot{ }^{\wedge} 3\right)\right) . /(1-\mathrm{xx}) .^{\wedge} 2-\mathrm{kp} . /\left(\operatorname{lm}(\operatorname{listedges}) \cdot * \mathrm{xx} \cdot{ }^{\wedge} 2\right) \text {; }
$$

16:

17: $\operatorname{tn}=[1: 3]$

18: for $\mathrm{j}=1:$ nedges

19: $\quad \mathrm{j} 1=\operatorname{mesh} . \mathrm{e}(1, \mathrm{j}) ; \mathrm{j} 2=\operatorname{mesh} . \mathrm{e}(2, \mathrm{j})$;

20: $\quad \mathrm{tj} 1=3 *(\mathrm{j} 1-1) ; \mathrm{tj} 2=3 *(\mathrm{j} 2-1)$;

21: $\operatorname{drdx}(1: 3)=[(\operatorname{mesh} \cdot \mathrm{p}(:, \mathrm{j} 1)-\operatorname{mesh} \cdot \mathrm{p}(:, \mathrm{j} 2))] /(\operatorname{lcur}(\mathrm{j}) * \operatorname{lm}(\mathrm{j}$ )) ;

22:

23: $\quad$ aux $=[\operatorname{fed}(j)$ fed $(j)$ fed $(j)$ fed $(j)$ fed $(j)$ fed $(j)]$;

24: $\quad \operatorname{dfdx}=\operatorname{aux} * *[\mathrm{drdx},-\mathrm{drdx}]$;

25:

26: $\quad$ inds $\left.=\left[\begin{array}{lll}\mathrm{tj} 1 \mathrm{tj} 1 \mathrm{tj} 1\end{array}\right]+\mathrm{tn},[\mathrm{tj} 2 \mathrm{tj} 2 \mathrm{tj} 2]+\mathrm{tn}\right]$;

27: $\quad$ force $($ inds $)=$ force $($ inds $)-\operatorname{dfdx}$;

28: end

29: force $=$ full( $\operatorname{reshape}($ force $, 3, \operatorname{size}(\operatorname{mesh} \cdot \mathrm{p}, 2)))$;

30:

31: end

To understand the various commands of the ComputeAnalFs.m, we refer the reader to the notes of the ComputeUs.m function. Nevertheless, we remark that the following lines are relevant:

- Line 15. Calculation of the derivative of the energy of equation (??) with respect to the 
relative extensions $\mathrm{xx}$ of the spectrines;

- Lines 18-28. With the cycle for $j=1$ :nedges, the code evaluates the derivative of the relative extensions of the edges with respect to the nodes and computes the forces for each node.

The next ComputeSystemMembers.m function has the goal to compute the residuals Rv and Rf, and the Jacobian matrix jacRf for the resolution of the system of the Newton-Raphson method. Indeed, the function evaluates the pure WLC and POW forces. The input arguments are clearly identified as previously described in this section.

1: function [Rv Rf jacRf fWLC fPOW] = ComputeSystemMembers (mesh, $\mathrm{Zn}, \mathrm{Z}$, Fext, betav, dt, flagJ )

2: $\quad \mathrm{global} \mathrm{kp}$

3: global p

4:

5: $\quad$ thetaint $=1.0$;

6: $\quad \mathrm{Nv}=\operatorname{size}(\operatorname{mesh} \cdot \mathrm{p}, 2)$;

7: $\quad \mathrm{tNv}=3 * \mathrm{Nv}$;

8: $\quad$ nedges $=$ size $($ mesh .e, 2$)$;

9:

10: $\quad \mathrm{Rv}=\operatorname{zeros}(\mathrm{tNv}, 1) ; \%$ Velocity residual

11: $\operatorname{Rf}=\operatorname{zeros}(\mathrm{tNv}, 1) ; \%$ Force residual

12:

13: \%-_- Velocity residual

14: $\quad \mathrm{Rv}=\mathrm{Z}(1: \mathrm{tNv})-\mathrm{Zn}(1: \mathrm{tNv})-\mathrm{Z}((\mathrm{tNv}+1):(2 * \mathrm{tNv})) * \mathrm{dt}$;

15:

16: \%--- Force residual

17: $\operatorname{meshn}=$ mesh;

18: $\quad$ meshn $\cdot \mathrm{p}=\operatorname{reshape}(\mathrm{Zn}(1: \mathrm{tNv}), 3, \mathrm{Nv})$;

19:

20: Fintn $=$ ComputeAnalFs $($ meshn $)$;

21: Fintp $=$ ComputeAnalFs (mesh);

22: Fint $=$ thetaint $*$ Fintp $+(1-$ thetaint $) *$ Fintn;

23:

24: $\quad$ Fric $=$ zeros $(\mathrm{tNv}, 1)$;

25: $\quad$ Fric $(1: \mathrm{tNv})=-$ betav $* \mathrm{Z}((\mathrm{tNv}+1):(2 * \mathrm{tNv}))$;

26: $\operatorname{Rf}=$ Fint $(:)+$ Fric $(:)-$ Fext $(:)$;

27:

28: \%-- Jacobian of force residual

29: $\quad$ if ( fl ag J )

30: 
31: $\quad$ jacRf $=$ zeros $(\mathrm{tNv}, \mathrm{tNv})$;

32:

33: $\quad \operatorname{lm}=2.2 *$ mesh.$x 0 ; \%$ Maximum extension of each edge

34: $\quad$ lcur = ComputeEdgeLengths (mesh);

35 :

36: $\quad$ listedges $=[1:$ nedges $]$;

37: $\quad \mathrm{xx}=1 \mathrm{cur}(1$ istedges $) . / 1 \mathrm{~m}($ listedges $)$;

38: $\quad$ kBT_lm $=\operatorname{lm}($ listedges $)$;

39: $\quad$ fWLC $=\mathrm{kBT} \_l \mathrm{~lm} /(4 * \mathrm{p}) . *\left(6 *\left(\mathrm{xx}-\mathrm{xx} .^{\wedge} 2\right) * *(1-\mathrm{xx})+\left(3 * \mathrm{xx} \cdot{ }^{\wedge} 2-\right.\right.$ $2 * x x \cdot \wedge 3) \quad$. / $(1-x x) \wedge^{\wedge} 2 ; \%$ attractive wormlike chain force

40: $\quad$ fPOW $=-\mathrm{kp} \cdot /\left(\operatorname{lm} . * \mathrm{xx} \cdot{ }^{\wedge} 2\right) ; \%$ repulsive power force

41: $\mathrm{fed}=\mathrm{fWLC}+\mathrm{fPOW}$;

42: $\quad \mathrm{fed} 2=\mathrm{kBT} \_\mathrm{lm} /(4 * \mathrm{p}) . *\left((12 * \mathrm{xx} \cdot \wedge 2-18 * \mathrm{xx}+6) . *(1-\mathrm{xx}) .^{\wedge} 2+\right.$ $2 *(4 * \mathrm{xx} \cdot \wedge 3-9 * \mathrm{xx} \cdot \wedge 2+6 * \mathrm{xx}) \cdot *(1-\mathrm{xx})) . /(1-\mathrm{xx}) \cdot \wedge 4+2 * \mathrm{kp}$ .$/(\operatorname{lm} . * x x \cdot \wedge 3)$;

43: $\quad 1 \mathrm{curlm}=1 \mathrm{cur} . * 1 \mathrm{~m}$;

44:

45: $\quad \mathrm{j} 1=\operatorname{mesh} . \mathrm{e}(1,:) ; \mathrm{j} 2=\operatorname{mesh} . \mathrm{e}(2,:)$;

46: $\quad \mathrm{tj} 1=3 *(\mathrm{j} 1-1) ; \mathrm{tj} 2=3 *(\mathrm{j} 2-1)$;

47: $\quad \operatorname{tn}=[1: 3]$;

48: $\quad$ iden $=$ eye $(3)$;

49: for $\mathrm{j}=1$ : nedges

50:

51: $\quad \operatorname{drdx}(1: 3)=[(\operatorname{mesh} \cdot \mathrm{p}(:, \mathrm{j} 1(\mathrm{j}))-\operatorname{mesh} \cdot \mathrm{p}(:, \mathrm{j} 2(\mathrm{j})))] /$ $\operatorname{lcurlm}(\mathrm{j})$;

52: $\quad \operatorname{drdx} 2=\mathrm{drdx} * \mathrm{drdx}$;

53: $\quad$ Ae2 $=$ [

54: $\quad \operatorname{drdx} 2,-\operatorname{drdx} 2$;

55: $\quad-\mathrm{drdx} 2, \mathrm{drdx} 2$

56: ] $\quad]$

57: $\quad$ aux1 $=$ iden/lcurlm $(j)$;

58: $\quad$ Ae1 $=[$

59: $\quad$ aux $1,-\operatorname{aux} 1$;

60: $\quad-$ aux 1, aux 1

61: $\quad]-\operatorname{lm}(\mathrm{j}) * \operatorname{Ae} 2 / \operatorname{lcur}(\mathrm{j})$;

62:

63:

64: $\quad$ inds $=[[\operatorname{tj} 1(\mathrm{j}) \mathrm{tj} 1(\mathrm{j}) \mathrm{tj} 1(\mathrm{j})]+\mathrm{tn},[\mathrm{tj} 2(\mathrm{j}) \operatorname{tj} 2(\mathrm{j}) \operatorname{tj} 2(\mathrm{j}$ ) $+\operatorname{tn}]$; 
65: jacRf (inds, inds $)=$ jacRf $($ inds, inds $)-$ thetaint $*$ fed $(j) * \operatorname{Ael}$ - thetaint $*$ fed $2(\mathrm{j}) * \operatorname{Ae} 2$;

66:

67: end

68: end

69: end

In the case of the ComputeSystemMembers.m function, we add the following final remarks, specifying the instructions:

- Lines 10-11 Initialization of Rv and Rf, the velocity and force residuals, respectively;

- Line 14. Calculation of the velocity residual $\mathrm{Rv}$, using the current state $\mathrm{Z}$ and the previous one $\mathrm{Zn}$ of the unknown;

- Lines 17-26. Calculation of the force residual Rf, considering the WLC-POW force Fint, the friction Fric and the external force Fext. In the case of the evaluation of the WLC-POW force Fint the code consider the possibility to use a $\theta$-method in order to balance the contribution the the current Fintp and the previous Fintn state of the force;

- Line 29. With the control if(flagJ), the code decides whether to mount the Jacobian matrix jacRf;

- Lines 39-40. Calculation of the attractive WLC force fWLC and the repulsive POW force fPOW;

- Lines 49-67. Within the cycle for $\mathrm{j}=1$ :nedges, the code computes the first and second derivative of the relative extensions of the edges with respect to the nodes in order to update the components of the Jacobian matrix;

- Line 65. Final assembly of the Jacobian matrix jacRf. We note that the term thetaint establishes also in this phase the balance of the temporal contribution according to a $\theta$-method.

For a set of archives to test the code, we addres the reader to GitHub at the URL: $<$ https://github.com/LucaMeacci/RBC_cytoskeleton_mathmodel>. 

APPENDIX

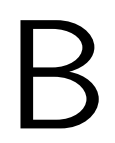

(2)

\section{EXPLORING A LENNARD-JONES POTENTIAL APPLICATION FOR MODELING THE RED BLOOD CELL CYTOMEMBRANE ADHESION: A WOLFRAM MATHEMATICA IMPLEMENTATION}

To model the interaction between the lipid bilayer and the cytoskeleton of a red blood cell means important applications in medicine. We hypothesize the possibility to apply the LennardJones potential in order to provide a mathematical formulation of a soft-body adhesion model between the above-mentioned components of an erythrocyte. The Mathematica use is effective to simulate and quickly visualize the interaction energy and force by changing parameters.

This study is aimed to provide a mathematical formulation of the forces that model the interactions between the cytoskeleton, composed of a discrete set of nodes connected by WLC-type forces and the bi-lipid membrane modeled as a continuous surface that behaves as a two-dimensional fluid. According to the approach of Sauer and Li (2007), we can assume that two atoms (or molecules) located at interact with each other via an interatomic potential $\phi(r)$, where $r$ is the distance between them. We refer the reader to the Chapter 4 for definitions and calculations.

\section{B.1 A little study with Mathematica}

$\mathrm{LJ}\left[\mathrm{r}_{-}\right.$, eps_, $\left.\mathrm{r}_{-}\right]:=\mathrm{eps}(\mathrm{r} 0 / r)^{\wedge} 6-2 \mathrm{eps} *(\mathrm{r} 0 / r)^{\wedge} 3$

$\operatorname{Plot}[\mathrm{LJ}[r, 0.1,0.15],\{r, 0.1,0.8\}]$ 
Manipulate[Plot[LJ $[r$, eps, r0], $\{r, 0.1,0.5\}$, PlotRange $\rightarrow\{-0.6,0.1\}],\{$ eps, $0.1,0.5\},\{\mathrm{r} 0,0.15,0.25\}]$ (Figure 88)

Figure 88 - Dynamical visualization of Lennard Jones potential graph.

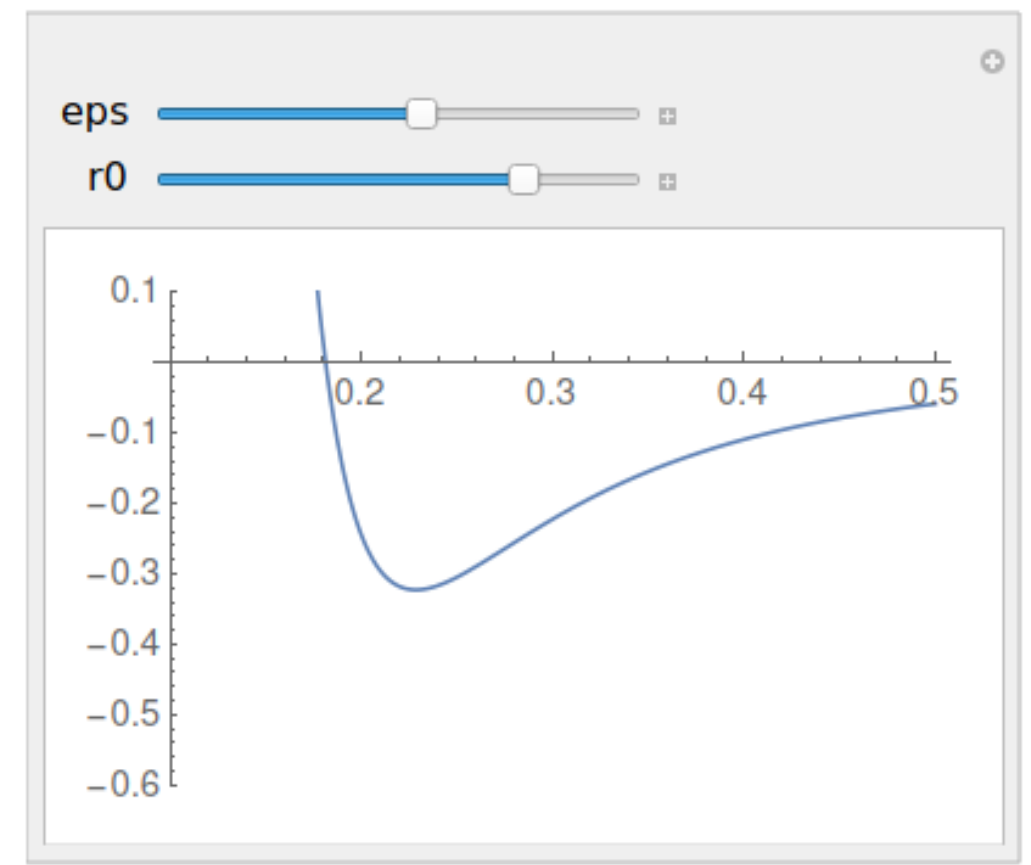

$D[\mathrm{LJ}[r, \mathrm{eps}, \mathrm{r} \mathrm{r}], r]$

$\mathrm{DLJ}\left[\mathrm{r}_{-}, \mathrm{eps}, \mathrm{rO} \mathrm{O}_{-}\right]:=\frac{6 \mathrm{epsr} 0^{3}}{\mathrm{r}^{4}}-\frac{6 \mathrm{epsi0^{6 }}}{r^{7}}$

Manipulate[Plot[DLJ $r$, eps, r0], $\{r, 0.1,0.5\}$, PlotRange $\rightarrow\{-0.6,5\}],\{$ eps $, 0.1,0.5\},\{r 0,0.15,0.25\}]$

(Figure 88)

Solve $[\mathrm{DLJ}[r, \mathrm{eps}, \mathrm{r} 0]==0, r]$

Out put : $\left\{\{r \rightarrow \mathrm{r} 0\},\left\{r \rightarrow-(-1)^{1 / 3} \mathrm{r} 0\right\},\left\{r \rightarrow(-1)^{2 / 3} \mathrm{r} 0\right\}\right\}$

\section{$\mathrm{LJ}[\mathrm{r} 0, \mathrm{eps}, \mathrm{r} \mathbf{0}]$}

Output : -eps

To compute the global contact energy we have to perform integrals on $\Gamma$ (surface of membrane), taking advantage of our hypothesis that the adhesive surface $\Upsilon$ of the cytoskeleton consists of rigid spheres (see Chapter 4). These calculations lead (under these assumptions) to an explicit expression for the total interaction energy.

betagamma $=10$;

betacyto $=1$;

eps $=1$;

$R=0.2$

$\mathrm{r} 0=0.05$;

$\mathrm{Wq}=100$; 
Figure 89 - Dynamical visualization of Lennard Jones potential derivative graph.

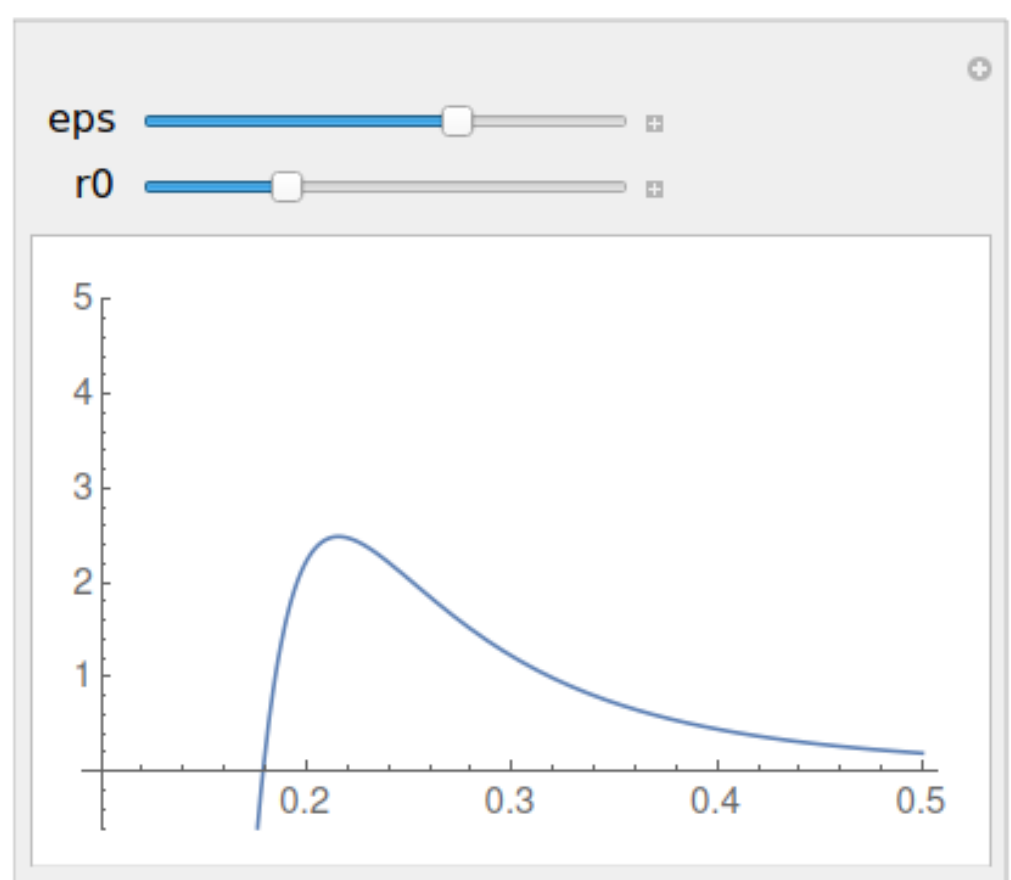

Econt[d_]:=betagammabetacyto $(2 \mathrm{Pieps}) /(1+d / R)\left(\left(2\left(\mathrm{r}^{\wedge} 3\right) /(d+2 R)-\ldots\right.\right.$ $\left.\left.\ldots-1 / 4\left(\mathrm{r0}^{\wedge} 6\right) /\left((d+2 R)^{\wedge} 4\right)\right)-\left(2\left(\mathrm{r}^{\wedge} 3\right) /(d)-1 / 4\left(\mathrm{r0}^{\wedge} 6\right) /\left(d^{\wedge} 4\right)\right)\right)$;

Manipulate[Econt $[\boldsymbol{d}],\{\boldsymbol{d}, \mathbf{0 . 0 1}, \mathbf{0 . 1}\}]$ (Figure 90)

Figure 90 - Dynamical evaluation of the global contact energy.

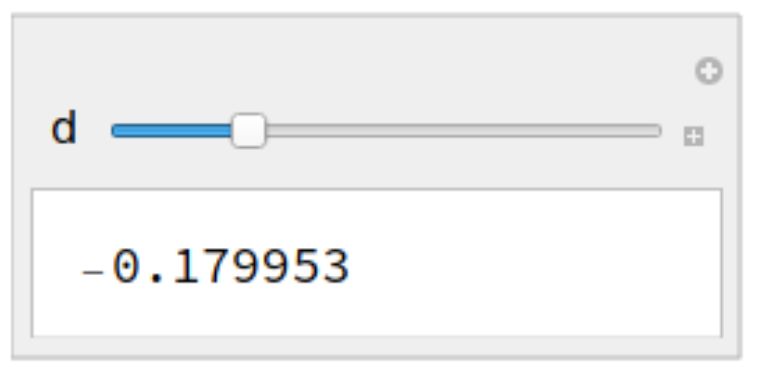

Manipulate[

\section{GraphicsColumn $[\{$}

Graphics3D $[\{$ ColorData[ [ “Rainbow", "Reverse” $\}][$ Econt $[d]]$, Sphere $[\{0,0,1.2+d\}, .2]$, Red, Polygon $[\{\{10,-10,1\},\{-10,-10,1\},\{-10,10,1\},\{10,10,1\}\}]\}$,

ViewPoint $\rightarrow$ Left, PlotRange $\rightarrow\{\{0.4,-0.4\},\{-0.5,0.5\}$, All $\}$, ImageMargins $\rightarrow 0, \ldots$

... ImagePadding $\rightarrow$ None, Boxed $\rightarrow$ False],

Show $[$ Plot $[$ Econt $[d],\{d$, dmin, dmax $\}$, PlotRange $\rightarrow\{\{-0.3,1.2\}\}$,

ColorFunction $\rightarrow$ ColorData[\{ "Rainbow", "Reverse" $\}]$,

PlotStyle $\rightarrow$ Normal, ImageMargins $\rightarrow$ 0, ImagePadding $\rightarrow$ None, BaseStyle- $>$ \{FontFamily $\rightarrow$ “Arial”, $\ldots$ ...FontSize $\rightarrow$ 10\},

Axes $\rightarrow$ True], 
Graphics $[\{$ Black, Disk $[\{d$, Econt $[d]\},\{.001, .020\}]\}]$

]

\}, Alignment $\rightarrow\{\{$ Center,Center $\},\{$ Center, Top $\}\}$, Spacings $\rightarrow 0.1$, ImageSize $\rightarrow\{400,300\}$

]

,$\{\{d, d m i n$, "distance (d)" $\}$, dmin, dmax $\}$, Initialization $: \rightarrow($

betagamma $=10$;

betacyto $=1$;

eps $=1$;

$R=0.2$;

$\mathrm{r} 0=0.05$

$\mathrm{Wq}=100$;

Econt[d_]:=betagammabetacyto(2Pieps) $/(1+d / R)\left(\left(2\left(\mathrm{r}^{\wedge} 3\right) /(d+2 R)-\ldots\right.\right.$

$\left.\left.\ldots-1 / 4\left(\mathrm{r}^{\wedge} 6\right) /\left((d+2 R)^{\wedge} 4\right)\right)-\left(2\left(\mathrm{r}^{\wedge} 3\right) /(d)-1 / 4\left(\mathrm{r} 0^{\wedge} 6\right) /\left(d^{\wedge} 4\right)\right)\right)$;

$\operatorname{dmax}=0.1$;

$\mathrm{dmin}=\mathbf{0 . 0 1 5}$;

$\operatorname{ymin}=-0.3$;

$\operatorname{ymax}=1.2 ;$ ), SaveDefinitions $\rightarrow$ True]

Figure 91 - Dynamic visualization of the interaction between a plane and a sphere through the Manipulate function of Wolfram Mathematica.

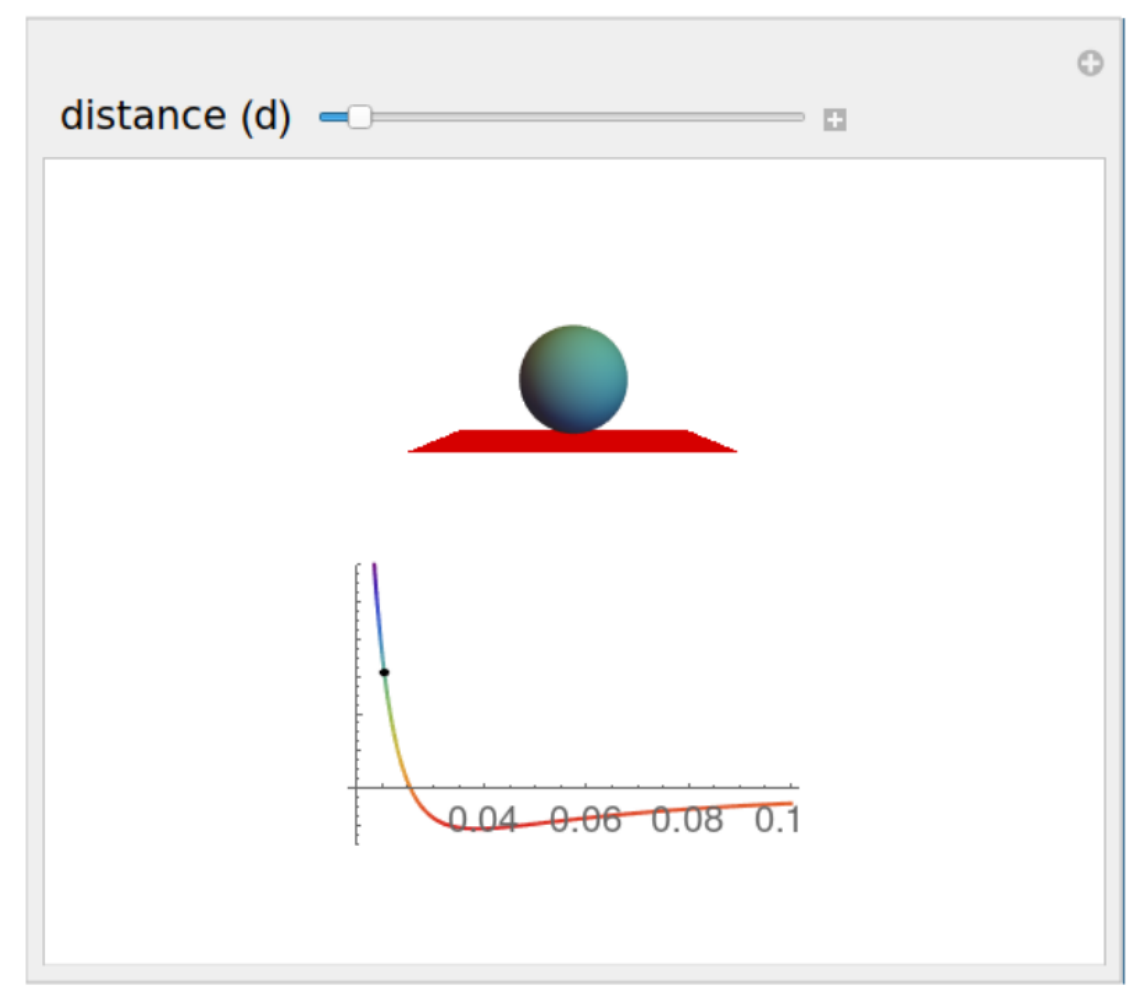




\section{B.1.1 A hybrid potential}

The numerical implementation of the formulation for the cyto-bilayer interaction model can be used for simulating the mechanical behaviour of a RBC. The mechanical behavior of a red blood cell subjected to forces of deformation and violent stretching. This requires the application of numerical methods such as the finite elements for the continuous membrane and Newton Raphson iteration procedure to solve the resulting linear systems. During these tests, the potential of Lennard-Jones has not always proved effective due to its divergence in the origin. We therefore propose a hybrid potential, derived from the Lennard Jones one.

$\mathrm{r} 0=0.20$

alpha $=0.8$

dstar $=$ alpha $*$ r0;

eps $=0.5$;

Plot[If $[d<$ dstar, DLJ [dstar, eps, r0] $d+\mathrm{LJ}[\mathrm{dstar}, \mathrm{eps}, \mathrm{rO}]-\ldots$

$\ldots-\mathrm{DLJ}[\mathrm{dstar}, \mathrm{eps}, \mathrm{r} 0] \mathrm{dstar}, \mathrm{LJ}[\boldsymbol{d}, \mathrm{eps}, \mathrm{r} 0]],\{d, 0.01,0.5\}]$ (Figure 92)

Figure 92 - Graph of the new hybrid Lennard Jones Potential

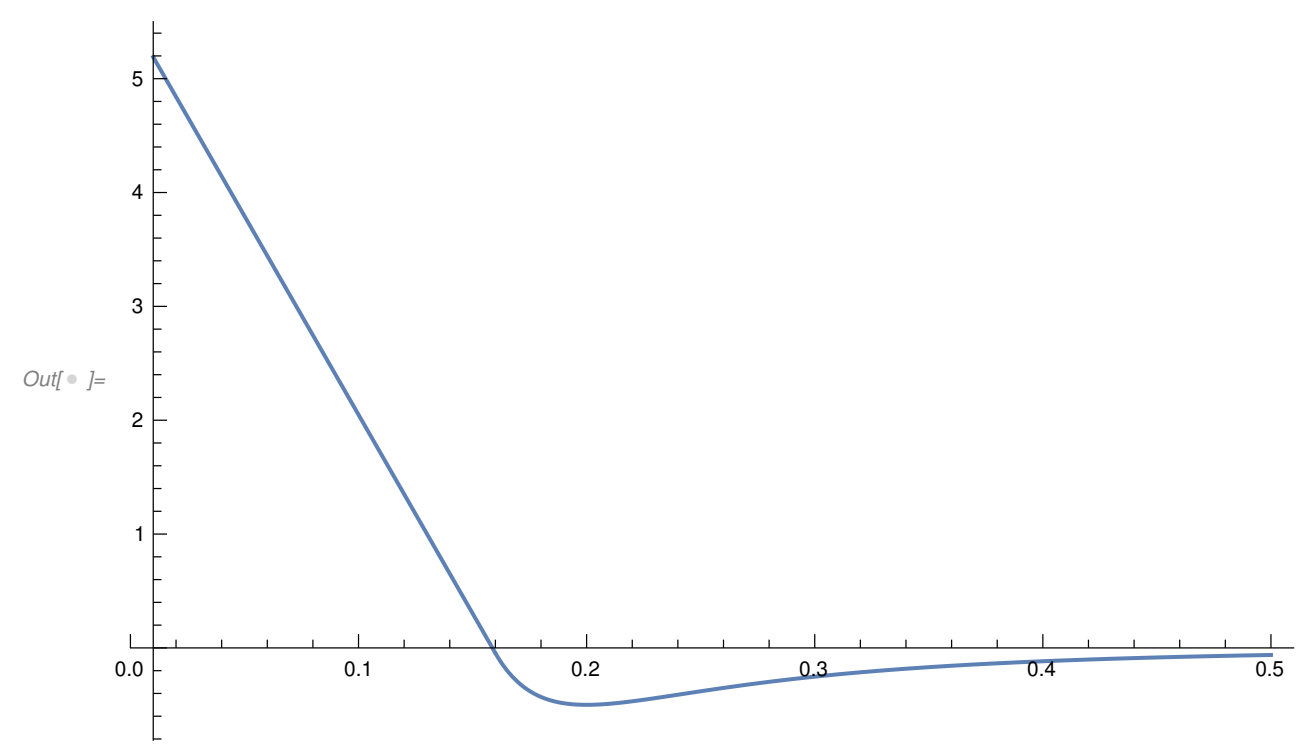



APPENDIX

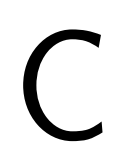

(1)

\section{INTERACTION BETWEEN CROWDING AND GROWTH IN TUMOURS WITH STEM CELLS: CONCEPTUAL MATHEMATICAL MODELLING}

Growth of tumors in the presence of stem cells is the object of a steadily increasing number of papers dealing both with theoretical and experimental research (see, e.g., (HUNTLY; GILLILAND, 2005; MICHOR, 2008; CLEVERS, 2011; VISVADER; LINDEMAN, 2008; REYA et al., 2001; CHO; CLARKE, 2008; CLARKE; FULLER, 2006; ANDERSON et al., 2011; PRIETO-VILA et al., 2017; NUNES et al., 2018; MARZAGALLI et al., 2021)).

Mathematical modeling of this phenomenon frequently uses methods of population dynamics. The basic idea consists in studying the evolution and mutual interactions of two subpopulations of malignant cells: cancer stem cells (CSCs) and differentiated cancer cells (CCs). CSCs are assumed to be immortal and capable of proliferating indefinitely; their mitosis can be symmetric (i.e., producing two new CSCs) or asymmetric (i.e. producing one CSC and one $\mathrm{CC}$ ). The differentiated cells CCs may belong to different lineages and undergo the usual process of maturity, replication, and apoptosis. There is an extensive bibliography in this area ( (TOMASETTI; LI; VOGELSTEIN, 2017; TOMASETTI; VOGELSTEIN; PARMIGIANI, 2013; BERETTA; CAPASSO; MOROZOVA, 2012; BERETTA et al., 2012; FOROUZANNIA; ENDERLING; KOHANDEL, 2018; ENDERLING; HAHNFELDT, 2011; GANGULY; PURI, 2006; WEEKES et al., 2014; WEISS; KOMAROVA; RODRIGUEZ-BRENES, 2017; MOLINAPEÑA; ÁLVAREZ, 2012; MOLINA-PEÑA; TUDON-MARTINEZ; AQUINES-GUTIÉRREZ, 2020) and the references quoted therein).

In (HILLEN; ENDERLING; HAHNFELDT, 2013) it has been suggested that an important role in the replication of cells could be played by the local "crowding" of cells. From a 
APPENDIX C. Interaction between crowding and growth in tumours with stem cells: conceptual mathematical modelling

mathematical point of view, this corresponds to assuming that the replication potential of each cell may depend on its position, in the sense that it can be hindered by the presence of other cells in its vicinity. This fact has been incorporated in different classes of models: (i) models that can be classified "agent-based" i.e., considering the evolution of each cell, using computer simulations and/or cellular automata methods ((MONTEAGUDO; SANTOS, 2014; MONTEAGUDO; SANTOS, 2015; POLESZCZUK; ENDERLING, 2014; MEACCI et al., 2019), and the review (BOONDIREK; TRIAMPO; NUTTAVUT, 2010)), (ii) models that introduce partial differential equations and integral terms to take into account the space-dependence of the cellular dynamics (BORSI et al., 2015a; FASANO; MANCINI; PRIMICERIO, 2016), (iii) models that are "mean field approximations" of the dynamics of individual cells, taking as unknown the averaged density of each sub-population and modifying the compartmental schemes of the population dynamics multiplying the replication potential by a monotonic function of the density of cells, decreasing to zero when all available space is filed by other cells (BETTERIDGE et al., 2006; MEACCI; PRIMICERIO, 2018; MEACCI; PRIMICERIO; BUSCAGLIA, 2021).

Introducing the effect of "crowding" allows us to describe the tumor growth paradox that consists of an accelerated tumor growth that can be found with an increased death rate of CC that can be the consequence of cytotoxic treatment (ENDERLING et al., 2009; WODARZ; KOMAROVA, 2007; GUROVA; GUDKOV, 2003; WEISS et al., 2021).

In Section C.1, we will introduce and discuss a 3D model with cellular automata (CA) whose evolution is governed by stochastic rules. Several lineages of CC's can be present and each cell is characterized by a different degree of maturity (newborn, juvenile, adult, and senescent) and thus by different probabilities for ageing, replication and apoptosis. Crowding effect is also taken into account. In a generalized version of the model all the probabilities can be supposed to be space-dependent and variable in time; also the case of a proliferation capacity decreasing at each cycle (see (ENDERLING; HAHNFELDT, 2011)) could be taken into account. In Subsection C.1.1 we display some simulations. The model is conceptual, in the sense that the values of the parameters are chosen in a speculative way as the role of this kind of conceptual model consists not in giving exact numerical results but rather a correct qualitative insight in this complex phenomenon. In particular, the results we obtain show the evidence of the tumor paradox and thus the possible counterintuitive effect of cytotoxic treatment.

In Section C. 2 we present a deterministic model that describes the growth of the tumor in terms of the total number of the cells of each type and lineage in the domain under consideration. The age distribution of the CCs is assumed to be continuous and consequently its evolution is governed by a first-order partial differential equation (PDE). The crowding effect is no longer considered as a local effect but is averaged over the whole region in the typical spirit of mean field approximations. The mathematical well-posedness of the model is proved in the Section C.3. In Subsection C.2.1 we display some numerical results of the model after reducing it to a system of ordinary differential equations (ODEs), as it is usual in compartmental models. We 
obtain global information on the growth of the tumor that confirm the evidence of the tumour growth paradox.

Finally, we report some concluding comments in Section C.4.

\section{C.1 Cellular automata modelling}

To illustrate the model and simplify the notation we consider the case in which there exists just one lineage of CCs. Of course, the same procedure applies (with different coefficients) to the case of an arbitrary number of lineages. For instance, in the simulations of Subsection C.1.1, we will consider three different lineages.

We imagine the cells live in a cubic lattice of $50 \times 50 \times 50$ size. At each time step, each site in the lattice can be either vacant (white) or occupied by a CSC (black), or by a CC. In the lattice case, the $\mathrm{CC}$ might be newborn, juvenile, adult or senescent, and we denote them with colors blue, green, yellow, and red, respectively.

Starting from a given time $t_{k}$ we consider to apply the following rules in order to update the system:

1. Each CC may undergo apoptosis in the following time interval with probability $\mu_{(1)}, \mu_{(2)}$, $\mu_{(3)}, \mu_{(4)}$ (according to its age class). In this case the corresponding site becomes white;

2. Each cell may undergo dormancy with probability $\delta$ in case of CSC, or $\delta_{1}, \delta_{2}, \delta_{3}, \delta_{4}$ for the CCs (according to its age class). In this case the cell remains in the same state ( $j$-th colour) and does not replicate in the time step $\left(t_{k}, t_{k+1}\right)$;

3. Each surviving and not dormant cell is potentially replicant with probability $\rho, \rho_{1}, \rho_{2}, \rho_{3}$, $\rho_{4}$. But we assume that mitosis is possible only if there are vacant sites in a neighbourhood of the would-be mother cell (crowding effect) (see Remark C.1.). In this case, the replicative potential $\rho$ is multiplied by the fraction of the vacant sites in the neighbourhood. In the case of the mitosis occurrence of a $\mathrm{CC}$, the site of the mother cell becomes pale blue as well as one of the vacant sites of the neighbourhood randomly chosen. If the mother cell is a CSC, them a daughter CSC appears in its neighbourhood with probability $(1-d)$. Otherwise the new cell is a pale blue CC;

4. Each surviving and non dormant $\mathrm{CC}$ that did not replicate may change its state (from newborn to juvenile, form juvenile to adult, from adult to senescent) with probabilities $p_{(1)}, p_{(2)}, p_{(3)}$.

Remark C.1. In the simplest case, the neighborhood of the would-be mother cell in which the newborn cell can be located is a cubic shell of $3 \times 3 \times 3$ lattice sites centered in the site of the replicating cell. A possible generalization could consist in considering a sequence of 
APPENDIX C. Interaction between crowding and growth in tumours with stem cells: conceptual 250 mathematical modelling

cubic shells of $3 \times 3 \times 3,5 \times 5 \times 5$, and so on that when no vacant sites are present in the first shell, the second shell can be investigated, and so on. But in this case, in each step the replication potential will be reduced. In the sequel we will use the $3 \times 3 \times 3$ shell.

Remark C.2. In the definition of the crowding effect, instead of multiplying the replication potential $\rho$ by the fraction $\phi$ of the vacant sites, we could multiply it by a chosen function $F(\phi)$ belonging to $[0,1]$, with $F((0)=0$ and $F(1)=1$.

Figure 93 - Flowchart of cellular automata algorithm concerning the model for the tumour progression with cancer stem cells.

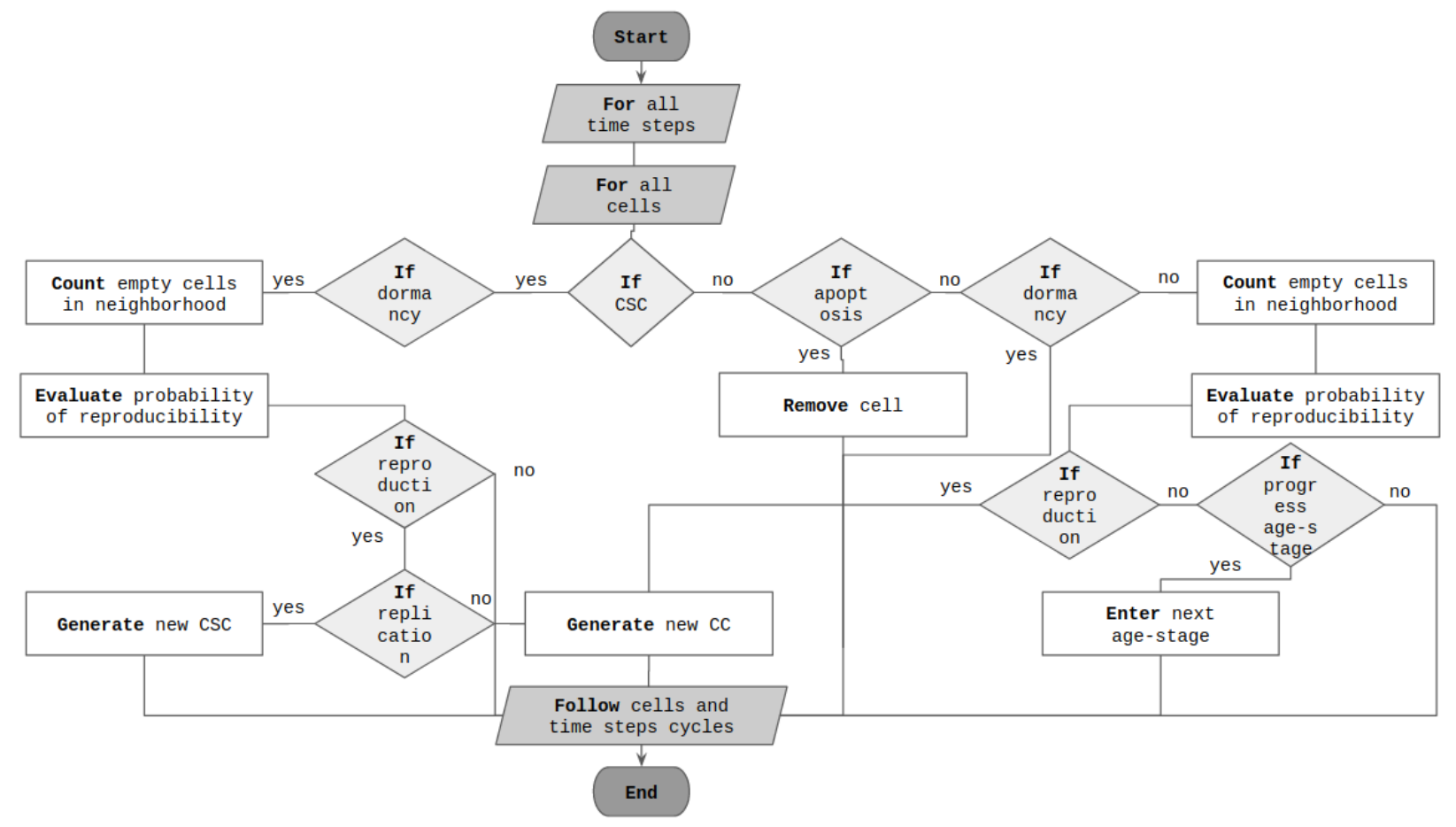

Starting from a specific initial condition, the cellular automata algorithm runs through all the positions of the lattice for each unit of time and, based on the presence or absence of a certain type of cell and its state with respect to the age progression, it decides how to update the system. In Figure 93 we report a flowchart that implements the hypotheses described above. We note that the algorithm makes decisions that are not deterministic but respond to probabilities and therefore have a certain degree of randomness. Nevertheless, the execution of a sufficient number of simulations shows that on average the overall behavior tends to a specific situation with respect to a certain parameter setup. The usefulness of an approach based on cellular automata is given by the possibility of establishing local development rules (on single cells) and studying over time the collective behavior of the system, in our case, the tumor progression.

By following the previous instructions, the model can predict and visualize the threedimensional evolution of tumor growth, retaining the detail of the characterization of each single cell, with respect to type, age and lineage. For example, let us consider 3 cell lineages. The panels of Figure 94 show how starting from a condition of a small central nucleus of 
$3 \times 3 \times 3$ CSCs (see panel (a)), it is possible to study the evolution of the tumoral tissue for a certain time in the future. Panel (b) shows the visualization by age class, while panel (c) displays the lineages of the tumor cells. A version implemented in Matlab code of the computational model is freely accessible in the GitHub repository <https://github.com/LucaMeacci/ ConceptualCSCmathmodellingForCancers $>$.

Figure 94 - Progression of the tumor tissue using the computational model of cellular automata. The panel (a) shows the initial condition of a $3 \times 3 \times 3$ CSCs nucleus in the center. The panels (b) and (c) display the prediction of tumor progression after a certain time by displaying the respective age cell classes and cell lineages, respectively.

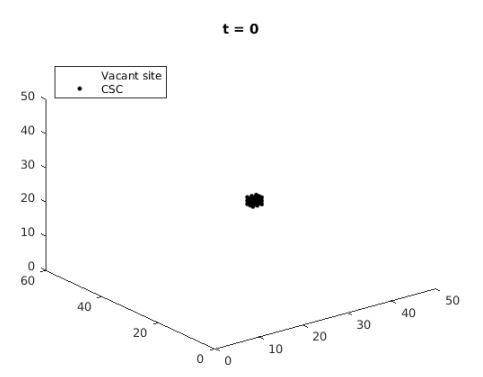

(a)

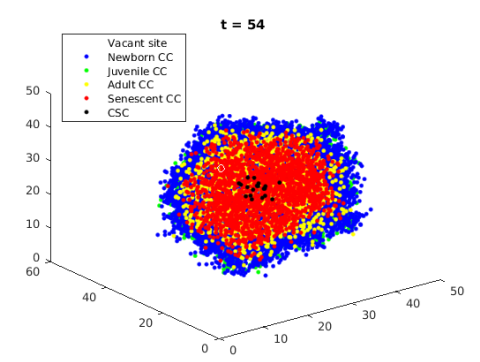

(b)

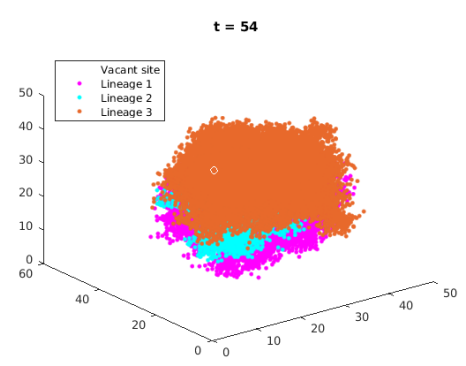

(c)

\section{C.1.1 Cellular automata simulations}

We aim to use this level of detail of the model to study and evaluate the impact of some phenomena. We have performed the following simulations with a Processor Intel Xeon(R) CPU E3-1270 v6 3.80GHz x 8 (32 GiB RAM).

First of all, we intend to evaluate the tumor response against a hypothetical medical treatment. To this purpose, We suppose to mimic the treatment effect progressively increasing the cell mortality according to the parameter $\xi$. The new mortality for each cell age class $j$ results to be $\xi \mu_{(j)}$.

We also mention some premises. The time unit can be arbitrary chosen as long as the parameters are adjusted. In particular, the various values of $\mu, \rho$ and $p$ are time dependent parameters. We have assumed the time unit of time equal to the time needed by a CSC to reproduce it in a new cell. Furthermore, the dormancy parameters $\delta$ result in a slowing down of the process. For this reason, without losing generality, we can assume to consider them null in the simulations. In accordance with these hypotheses and premises, we list the setup of parameters in Table 13.

We set as initial condition 27 CSCs arranged in a cube in the lattice center. Under this configuration, we study the evolution of the system with respect to the mortality variation. In Figure 95 we display the graphs of the fraction of tumor cells (CSCs and all classes of CCs) in the lattice, tuning the mortality trough the parameter $\xi$. 
APPENDIX C. Interaction between crowding and growth in tumours with stem cells: conceptual

Table 13 - Setup of parameters for the cellular automata simulations.

\begin{tabular}{ccccc}
\hline CSC & Newborn CC & Juvenile CC & Adult CC & Senescent CC \\
\hline$\rho=1$ & $\rho_{1}=0.5$ & $\rho_{2}=0.7$ & $\rho_{3}=0.8$ & $\rho_{4}=0.3$ \\
- & $\mu_{1}=0.1$ & $\mu_{2}=0.1$ & $\mu_{3}=0.1$ & $\mu_{4}=0.15$ \\
- & $p_{1}=1$ & $p_{2}=1$ & $p_{3}=1$ & - \\
$\delta=0$ & $\delta_{1}=0$ & $\delta_{2}=0$ & $\delta_{3}=0$ & $\delta_{4}=0$ \\
$d=0.8$ & - & - & - & - \\
\hline
\end{tabular}

Figure 95 - Quantitative evolution of the tumoral cells (CSCs and CCS) with respect to the mortality variation, using the cellular automata model.

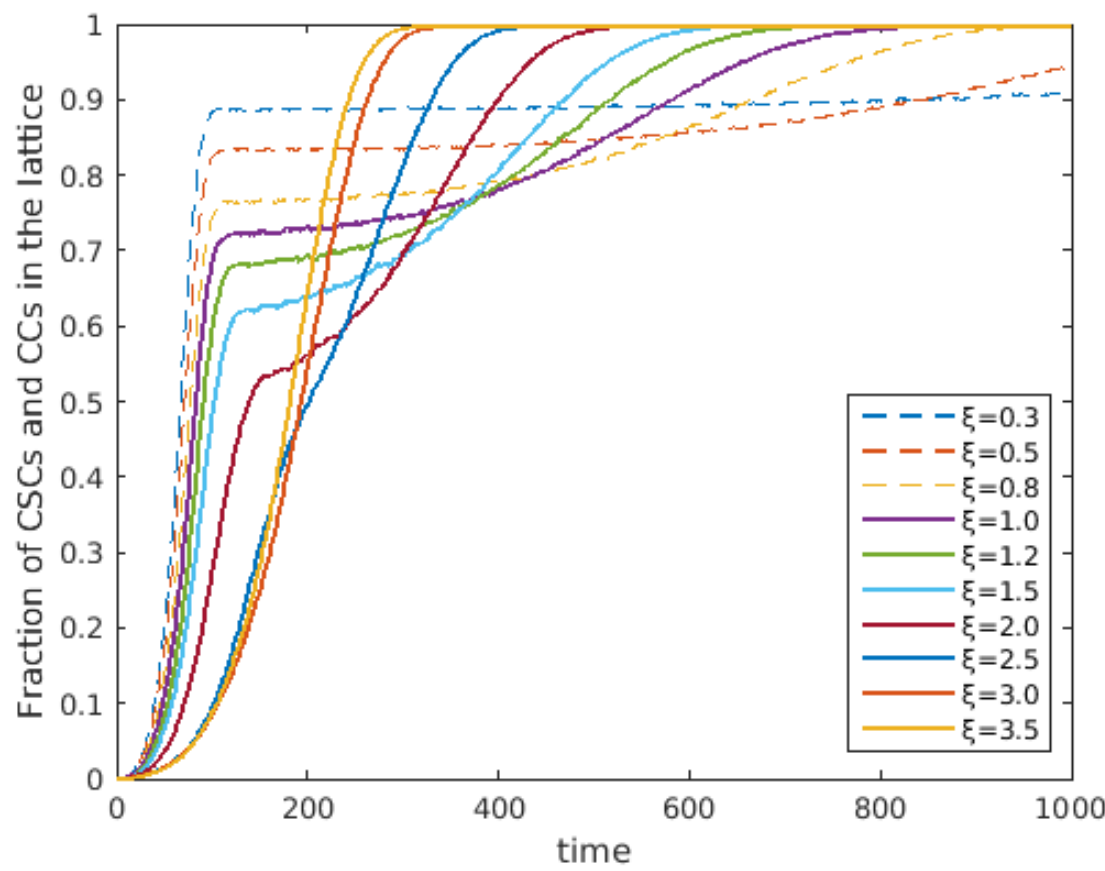

The results appear to be counter-intuitive at first glance. After a certain time, the tumor volume turns out to be greater as mortality increases. This occurrence would mean that a more aggressive treatment would not benefit the sick patient. This occurrence is called the "tumor growth paradox" because an increased mortality of the CCs may produce a faster growth of the tumour. Nevertheless, the model helps to understand why this situation can be expected under our assumptions. Let us consider the two cases with $\xi=1$ and $\xi=2$; let us focus on the evolution of CSCs and CCs for the age groups. In Figure 96 we report the corresponding graphs.

CSCs are not affected by the increase in mortality. On the contrary, in (b) case the CCs are significantly reduced. From the point of view of the tumour progression, in this second case, therefore the CSCs (more aggressive and able to reproduce) have more free space and freedom of growth than in (a) case. In a certain sense, the greater presence of the CCs because of a lower mortality operated as a confinement for an CSCs accelerated evolution.

Having reached these conclusions, we can proceed with a further step forward. Although 
Figure 96 - Quantitative evolution of the lattice fraction of CSCs and CCs divided into age classes (where newborn stands for 1 and senescent stands for 4). The case (a) corresponds to a mortality value induced by $\xi=1$, while the case (b) results from $\xi=2$.

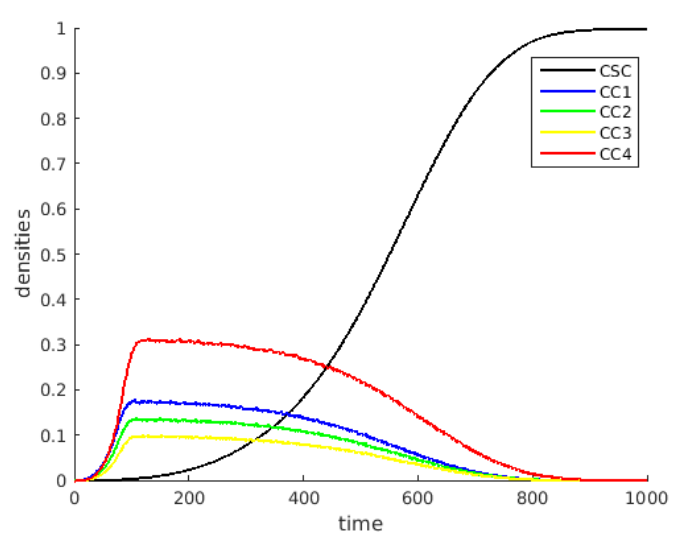

(a)

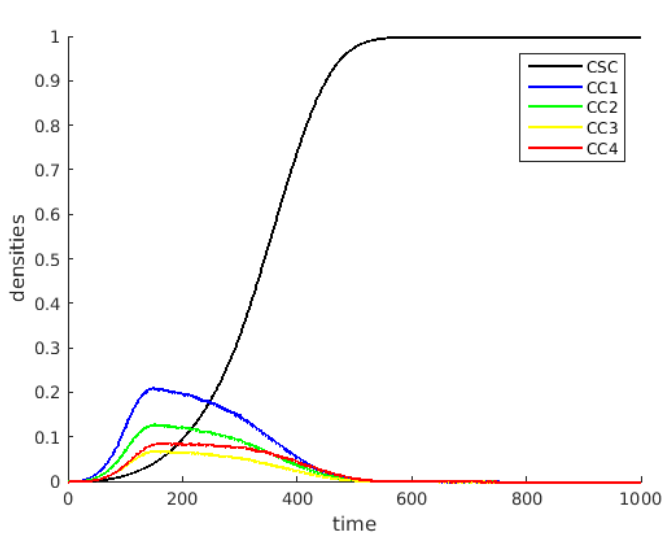

(b)

common characteristics and properties can be differentiated and clustered into types of cancerous cells (ordinary or stem cells) or with respect to the phase of the cellular life cycle (newborn, juvenile, adult or senescent), there exists a certain heterogeneity with respect to the cell lineages that can play a significant role in the evolution of the tumor. If we assume to consider 3 lineages, each of them may differ to some extent with respect to certain characteristics. From the model point of view, this eventuality can be translated with a parameters setup, further declined on the basis of the lineage. We suppose to consider the previous configuration of the parameters with $\xi=3.5$ but, moreover, to differentiate by lineage some of them as in the Table 14 .

Table 14 - Update of parameters setup for the cellular automata simulations, according to the cell lineages. In variables, the subscript stands for age and the superscript stands for lineage.

\begin{tabular}{ccc}
\hline Lineage 1 & Lineage 2 & Lineage 3 \\
\hline$\rho_{1}^{1}=0.8$ & $\rho_{1}^{2}=0.5$ & $\rho_{1}^{3}=0.5$ \\
$\rho_{2}^{1}=1.0$ & $\rho_{2}^{2}=0.7$ & $\rho_{2}^{3}=0.7$ \\
$\rho_{3}^{1}=1.1$ & $\rho_{3}^{2}=0.8$ & $\rho_{3}^{3}=0.8$ \\
$\rho_{4}^{1}=0.6$ & $\rho_{4}^{2}=0.3$ & $\rho_{4}^{3}=0.3$ \\
$\mu_{1}^{1}=0.09$ & $\mu_{1}^{2}=0.10$ & $\mu_{1}^{3}=0.10$ \\
$\mu_{2}^{1}=0.09$ & $\mu_{2}^{2}=0.10$ & $\mu_{2}^{3}=0.10$ \\
$\mu_{3}^{1}=0.09$ & $\mu_{3}^{2}=0.10$ & $\mu_{3}^{3}=0.10$ \\
$\mu_{4}^{1}=0.14$ & $\mu_{4}^{2}=0.15$ & $\mu_{4}^{3}=0.15$ \\
\hline
\end{tabular}

The level of detail of the model allows us to intervene with specificity in order to simulate the strategies to combat the disease. Analogously to the previous study, we can hypothesize to model medical treatment to reduce the cancer cells. The further assumption is to choose an intervention time aimed at eliminating the CCs of one specific lineage, the number 1 in the following simulations. The algorithm is instructed to completely remove the CCs of the first lineage at the time $t=100$. In Figure 97, we can see the time evolution of the total fraction of 
APPENDIX C. Interaction between crowding and growth in tumours with stem cells: conceptual mathematical modelling

tumoral cells (CSCs and CCs) without this kind of intervention and opting for the first lineage removal.

Figure 97 - Quantitative evolution of the tumoral cells, without intervention and opting for removing the CCs of lineage 1 at time $t=100$.

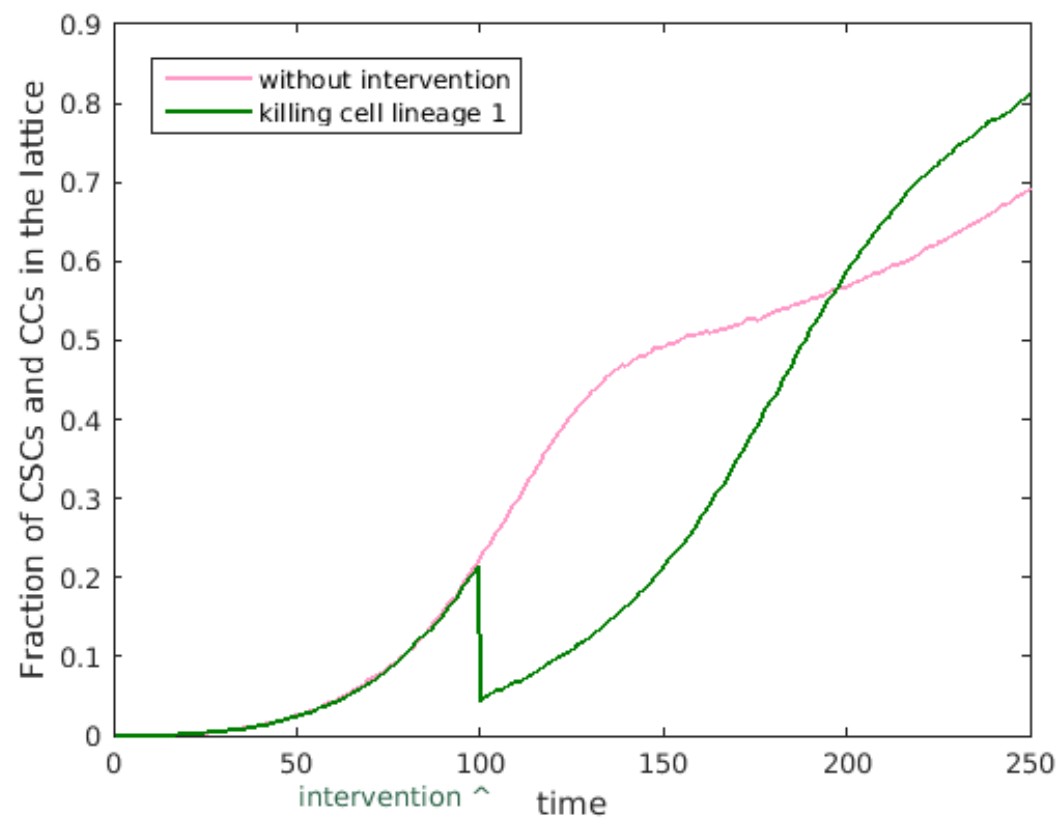

The sudden decrease in tumor volume is evident in the proximity of the intervention. Nonetheless, once again, a non-intuitive trend appears. It seems in fact that, in this case, the action of removing a greater number of CCs (of lineage 1) is unfavorable with respect to the progression of the disease.

We can analyze what happened at the level of age classes and lineages in the two cases. The respective behaviors are plotted in Figure 98.

Once again, a non-intuitive conclusion may be justified. Due to the particular evolutionary characteristics of lineage 1 , at the intervention time, the CCs of the first lineage turn out to be in the majority. Its removal facilitates the proliferation of CSCs which, finding more space available, induce an acceleration in the global growth of the tumor volume.

\section{C.2 Modelling based on differential equations}

In this Section we describe the growth of the tumor in terms of the total number of cells in the region under consideration. Thus, we will not take into account the space dependence of the densities of the CSCs and of the CCs, but we average such densities over the entire region. Of course, we will lose the local and stochastic character of the approach with CA but the "global" information that will be obtained is in accordance with the model presented in the previous model, by averaging in a proper way the "crowding" effect. 


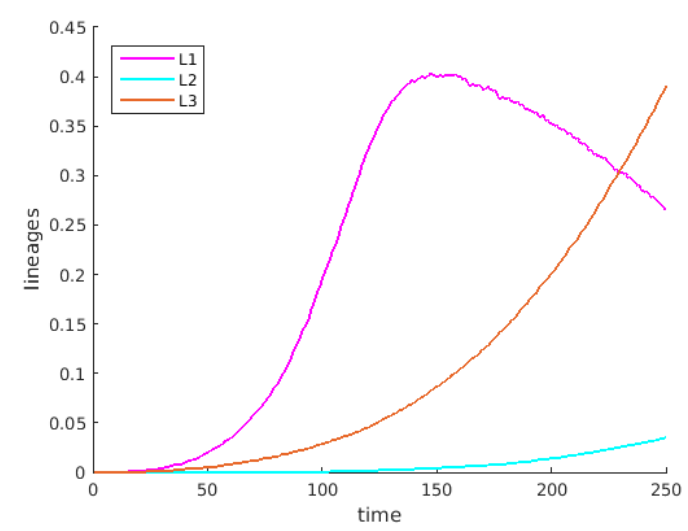

(a)

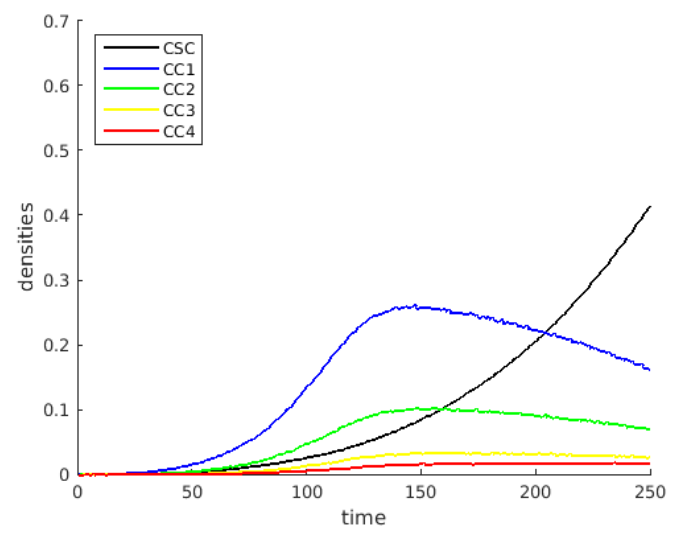

(c)

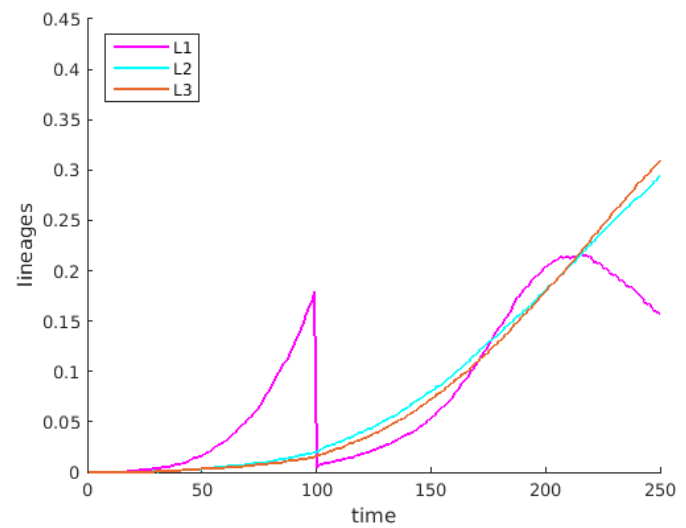

(b)

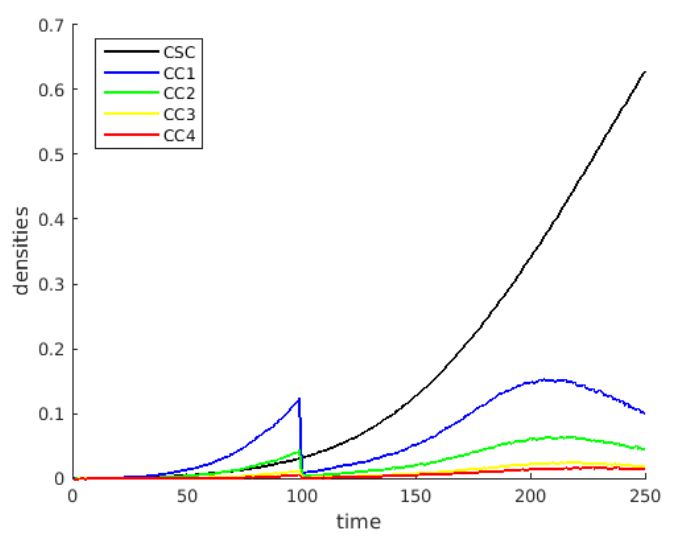

(d)

Figure 98 - Quantitative progression of the tumour displayed according to the lineages (panel (a) without intervention and panel (b) considering $t=100$ as removal time) and divided into age classes (panels (c) and (d) without and with the treatment).

To be specific, we define by $U(t), V^{1}(t), V^{2}(t), \ldots, V^{M}(t)$ the densities of the CSCs and of the $M$ lineages of CCs in the region and we assume that there exist numbers $d^{i}(i=1,2, \ldots, M)$ such that, if $d=\sum_{i} d^{i}<1$, then $1-d$ represents the fraction of symmetric replication of CSC (i.e. mitosis originating $2 \mathrm{CSCs}$ ) while each $d^{i}$ is the fraction of the mitosis of CSC that originates a CC of the $i$-th lineage. Therefore, the time evolution of the number of CSCs in the region (to save notation, assume the volume is 1 so that the numbers coincide with densities) is governed by the following equation:

$$
\dot{U}(t)=\rho(1-d) U(t) F(P(t)), \quad V(t)=\sum_{i} V^{i}(t)
$$

where

$$
P(t)=U(t)+V(t), \quad U(0)=U_{0},
$$

$\rho$ is the replication potential of CSC and $F$ is a monotonic decreasing function such that $F(0)=1$ and $F(1)=0$.

To take into account the ageing and apoptosis of the CCs we introduce the age distribution functions $v^{i}(a, t)$ where $a$ denotes the "age" of the cell and for any $t>0$ and $0<a_{1}<a_{2}$, 
APPENDIX C. Interaction between crowding and growth in tumours with stem cells: conceptual

$\int_{a_{1}}^{a_{2}} v^{i}(a, t) d a$ is the number of cells of the $i$-th lineage that, at time $t$, have age between $a_{1}$ and $a_{2}$. This means that $V^{i}(t)=\int_{0}^{\infty} v^{i}(a, t) d a$.

Assuming that biological age coincides with chronological age, the evolution of the age distribution function of the $i$-th lineage of $\mathrm{CC}$ is governed by the following equations

$$
\frac{\partial v^{i}(a, t)}{\partial t}+\frac{\partial v^{i}(a, t)}{\partial a}=-\mu^{i}(a) v^{i}(a, t)-\rho^{i}(a) v^{i}(a, t) F(P(t)), \quad i=1,2, \ldots, M,
$$

where $\mu^{i}(a)$ and $\rho^{i}(a)$ represent the mortality and the replication potential of the cells of the $i$-th lineage in function of their age.

Equations (C.3) are supplemented by the following initial-boundary conditions

$$
\begin{gathered}
v^{i}(a, 0)=v^{i, 0}(a)>0, \quad \sum_{i} v^{i, 0}(a)>0, \quad i=1,2, \ldots, M, \\
v^{i}(0, t)=\rho d^{i} F(P(t)) U(t)+2 \int_{0}^{\infty} \rho^{i}(a) v^{i}(a, t) F(P(t)) d a, \quad i=1,2, \ldots, M,
\end{gathered}
$$

The well-posedness of problem (C.1) - (C.5) is later in the Section C.3.

To simplify numerical calculations and compare this model with the results of simulations based on space-dependent mechanisms and stochastic rules, we consider a special case in which the functions $\rho^{1}(a)$ and $\mu^{i}(a)$ are piecewise constant. To be specific, we assume that there exist 4 age intervals $\left(0, a_{1}\right),\left(a_{1}, a_{2}\right),\left(a_{2}, a_{3}\right),\left(a_{3}, A\right)$ (newborn, juveniles, adult and senescent cells) and that $A$ is the maximum age of the cells. We denote by $\rho_{(1)}, \rho_{(2)}, \rho_{(3)}, \rho_{(4)}$ and by $\mu_{(1)}, \mu_{(2)}$, $\mu_{(3)}, \mu_{(4)}$ the values of $\rho$ and $\mu$ in these intervals. Moreover, we indicate with $V_{(1)}^{i}, V_{(2)}^{i}, V_{(3)}^{i}$, $V_{(4)}^{i}$ the numbers of CC's of the age classes of the $i$-th lineage.

Then, we integrate the equation C.3 in each of the age intervals and we take into account the equation C.5, thus obtaining

$$
\left\{\begin{aligned}
\dot{V}_{(1)}^{i}(t)= & \rho d F(P(t)) U(t)+\left(\rho_{(1)} V_{(1)}^{i}(t)+2 \rho_{(2)} V_{(2)}^{i}(t)+2 \rho_{(3)} V_{(3)}^{i}(t)+2 \rho_{(4)} V_{(4)}^{i}(t)\right) F(P(t))- \\
& -v^{i}\left(a_{1}, t\right)-\mu_{(1)} V_{(1)}^{i}(t), \\
\dot{V}_{(2)}^{i}(t)= & v^{i}\left(a_{1}, t\right)-v^{i}\left(a_{2}, t\right)-\rho_{(2)} V_{(2)}(t) F(P(t))-\mu_{(2)} V_{(2)}^{i}(t) \\
\dot{V}_{(3)}^{i}(t)= & v^{i}\left(a_{2}, t\right)-v^{i}\left(a_{3}, t\right)-\rho_{(3)} V_{(3)}(t) F(P(t))-\mu_{(3)} V_{(3)}^{i}(t), \\
\dot{V}_{(4)}^{i}(t)= & v^{i}\left(a_{3}, t\right)-\rho_{(4)} V_{(4)}(t) F(P(t))-\mu_{(4)} V_{(4)}^{i}(t) .
\end{aligned}\right.
$$

The total number of CCs is thus given by

$$
\dot{V}(t)=\rho d F(P(t)) U(t) \sum_{i=1}^{M} \sum_{j=1}^{4} \rho_{(j)} V_{(j)}^{i} F(P(t))-\sum_{j=1}^{4} \mu_{(j)} V_{(j)}^{i} .
$$

As it is usual in compartmental models we can consider the case in which the age distribution within each class is negligible and we write

$$
\psi_{(j)} v^{i}\left(a_{j}, t\right)=V_{(j)}^{i}(t),
$$


where $\psi_{(j)}$ measures the width of the $j$-th age class (or the time spent by the cell in the $j$-th class).

Hence, omitting the lineages for further simplification of the notation and combining the equations (C.1), (C.6) and (C.8), we have the following complete system

$$
\left\{\begin{aligned}
\dot{U}(t)= & \rho(1-d) U(t) F(P(t)) \\
\dot{V}_{(1)}(t) & =\rho d F(P(t)) U(t)-\left(\gamma_{(1)}+\mu_{(1)}\right) V_{(1)}(t)+ \\
& +\left(\rho_{(1)} V_{(1)}(t)+2 \rho_{(2)} V(2)(t)+2 \rho_{(3)} V_{(3)}(t)+2 \rho_{(4)} V_{(4)}(t)\right) F(P(t)) \\
\dot{V}_{(2)}(t)= & \gamma_{(1)} V_{(1)}(t)-\left(\gamma_{(2)}+\mu_{(2)}\right) V_{(2)}(t)-\rho_{(2)} V_{(2)}(t) F(P(t)) \\
\dot{V}_{(3)}(t)= & \gamma_{(2)} V_{(2)}(t)-\left(\gamma_{(3)}+\mu_{(3)}\right) V_{(3)}(t)-\rho_{(3)} V_{(3)}(t) F(P(t)) \\
\dot{V}_{(4)}(t)= & \gamma_{(3)} V_{(3)}(t)-\mu_{(4)} V_{(4)}(t)-\rho_{(4)} V_{(4)}(t) F(P(t))
\end{aligned}\right.
$$

with $\gamma_{(j)}=1 / \psi_{(j)}$, for $j=1,2,3$.

An oversimplified case is the one in which $\rho$ and $\mu$ are constant and hence the integration of the equation (C.3) yields

$$
\dot{V}^{i}(t)=\rho d^{i} F(P(t)) U(t)+\rho V^{i} F(P(t))-\mu V^{i}, \quad i=1,2, \ldots, M
$$

and summing over $i$ hence we have the following system of ordinary differential equations (ODEs)

$$
\left\{\begin{array}{l}
\dot{V}(t)=\rho d F(P(t)) U(t)+\rho V(t) F(P(t))-\mu V(t) \\
\dot{U}(t)=\rho(1-d) F(P(t)) U(t)
\end{array}\right.
$$

\section{C.2.1 Numerical simulations}

We numerically solve the problem (C.9), adopting to consider 3 cell lineages. We use ode45 Matlab solver based on explicit Runge-Kutta method. Since the crowding effect is averaged over the entire available space, it is natural to consider a duration of the phenomenon such that a large part of the entire domain can be occupied by the cells. At this point, it is in order to note that, if $U(0)>0$, then system (C.9) has just one equilibrium state, i.e., $U=1$ and $V^{i}=0$. The initial conditions are analogous to the simulations performed with CA, i.e., $U(0)=27 / 50^{3}$ and $V_{(j)}^{i}(0)=0$, for all $i$ and $j$. The other parameters are listed in Table 15 .

Similarly to the case with cellular automata, we study the behavior of the system with respect to the variation in mortality, updating this last parameter values with the multiplicative factor $\xi$. Figure 99 contains the graphs of the total (CSCs and CCs of all ages and lineages) volume fraction of the tumour. Again, the tumour growth paradox occurs. Let us see into detail, considering once again, what happens at the level of age classes and lineages, choosing the mortality values arising from setting $\xi$ equals to 0.6 and 1.5 . 
APPENDIX C. Interaction between crowding and growth in tumours with stem cells: conceptual 258 mathematical modelling

Table 15 - Setup of parameters for the differential equations numerical simulations. In variables, the subscript stands for age and the superscript stands for lineage. When the superscript is not reported all the lineages have the same configuration.

\begin{tabular}{|c|c|c|c|c|c|c|}
\hline CSC & \multicolumn{3}{|c|}{ Newborn CC } & \multicolumn{3}{|c|}{ Juvenile CC } \\
\hline \multirow[b]{2}{*}{$\begin{array}{c}\rho=1 \\
d=0.8\end{array}$} & Lineage 1 & Lineage 2 & Lineage 3 & Lineage 1 & Lineage 2 & Lineage 3 \\
\hline & $\begin{array}{c}\rho_{(1)}^{1}=0.6 \\
\mu_{(1)}^{1}=0.09\end{array}$ & $\begin{array}{c}\rho_{(1)}^{2}=0.5 \\
\mu_{(1)}^{2}=0.10\end{array}$ & $\begin{array}{c}\rho_{(1)}^{3}=0.4 \\
\mu_{(1)}^{3}=0.11\end{array}$ & $\begin{array}{c}\rho_{(2)}^{1}=0.8 \\
\mu_{(2)}^{1}=0.09\end{array}$ & $\begin{array}{c}\rho_{(2)}^{2}=0.7 \\
\mu_{(2)}^{2}=0.10\end{array}$ & $\begin{array}{c}\rho_{(2)}^{3}=0.6 \\
\mu_{(2)}^{3}=0.11\end{array}$ \\
\hline- & \multicolumn{3}{|c|}{$\gamma_{(1)}=1$} & \multicolumn{3}{|c|}{$\gamma_{(2)}=1$} \\
\hline
\end{tabular}

\begin{tabular}{|c|c|c|c|c|c|}
\hline \multicolumn{3}{|c|}{ Adult CC } & \multicolumn{3}{|c|}{ Senescent CC } \\
\hline Lineage 1 & Lineage 2 & Lineage 3 & Lineage 1 & Lineage 2 & Lineage 3 \\
\hline $\begin{array}{c}\rho_{(3)}^{1}=0.9 \\
\mu_{(3)}^{1}=0.09\end{array}$ & $\begin{array}{c}\rho_{(3)}^{2}=0.8 \\
\mu_{(3)}^{2}=0.10\end{array}$ & $\begin{array}{c}\rho_{(3)}^{3}=0.7 \\
\mu_{(3)}^{3}=0.11\end{array}$ & $\begin{array}{c}\rho_{(4)}^{1}=0.4 \\
\mu_{(4)}^{1}=0.14\end{array}$ & $\begin{array}{c}\rho_{(4)}^{2}=0.3 \\
\mu_{(4)}^{2}=0.15\end{array}$ & $\begin{array}{c}\rho_{(4)}^{3}=0.2 \\
\mu_{(4)}^{3}=0.16\end{array}$ \\
\hline \multicolumn{3}{|c|}{$\gamma_{(3)}=1$} & \multicolumn{3}{|c|}{-} \\
\hline
\end{tabular}

Figure 99 - Quantitative evolution of the tumoral cells with respect to the mortality variation, adopting the differential equations model.

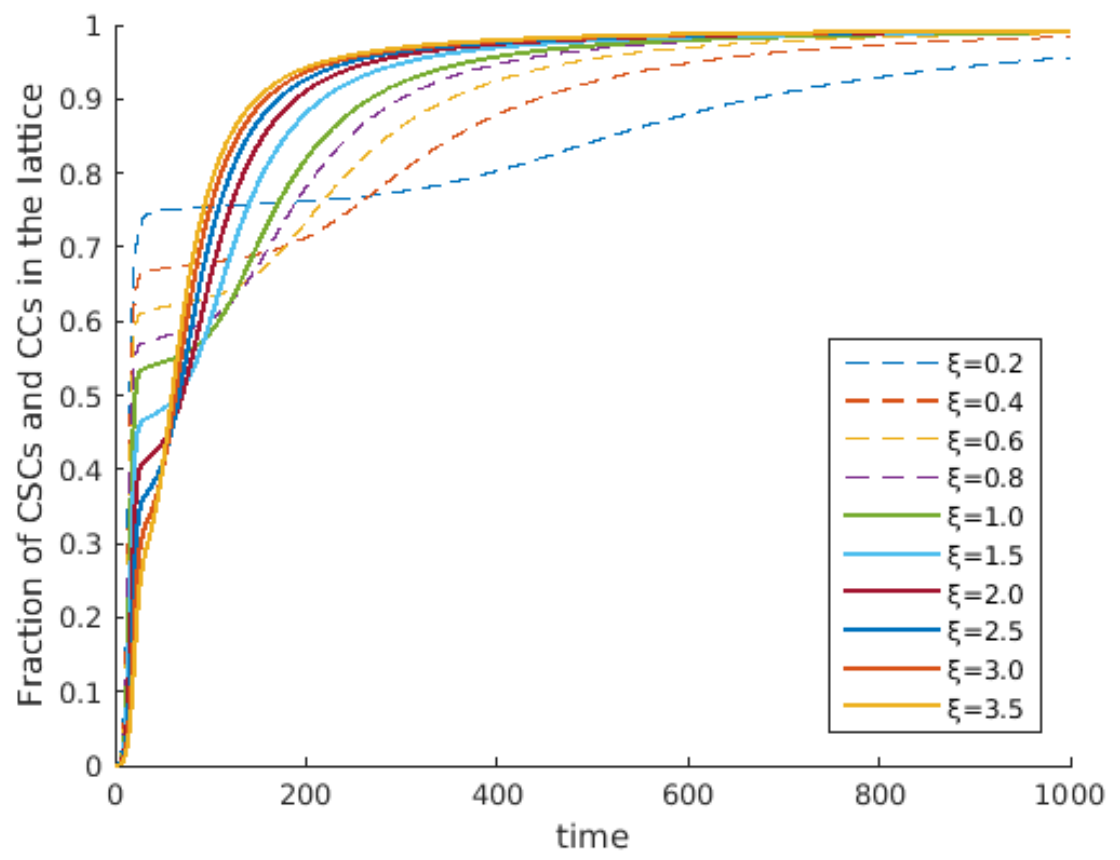

In the case of lower induced mortality, the initial phase of tumor evolution proposes a greater crowding of CCs (panels (a), (b), and (c)), in particular with respect to the lineage 1 (panel (a)). In the case with a higher mortality (which could be lowered with medical treatment) initially the progression of the disease seems to benefit from a significant lowering of the CCs (panels (d), (e), and (f)). However, this same event also results to be not positive over time. One more time, the differential equations model suggest that the presence of CCs contributes to 
Figure 100 - Quantitative cancer progression according to age class and lineage. The panels (a), (b) and (c) show the results for a mortality value resulting from $\xi=0.6$, while the panels for $(\mathbf{d}),(\mathbf{e})$ and (f) concern the simulations with $\xi=1.5$. In the legends L1, L2 and L3 stand for first, second and third lineage, respectively.

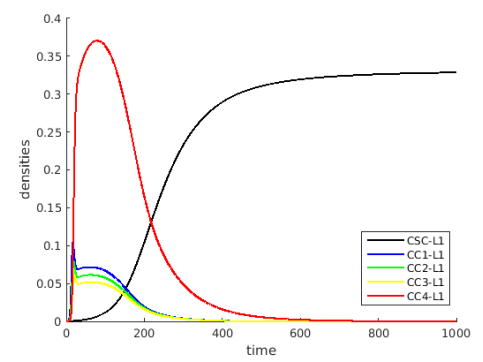

(a)

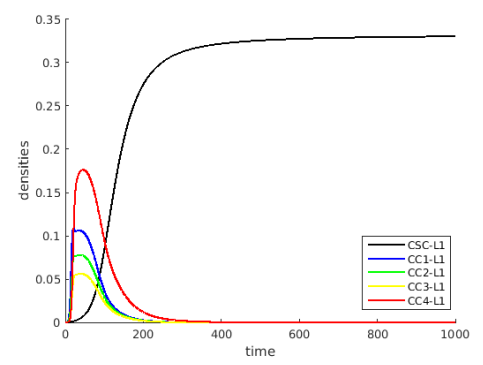

(d)

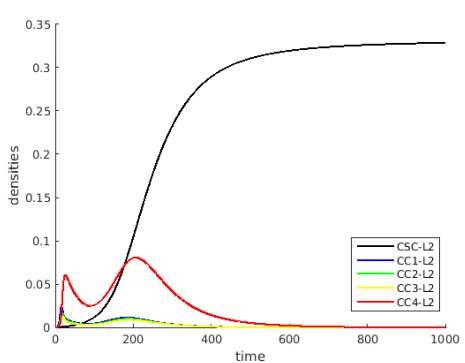

(b)

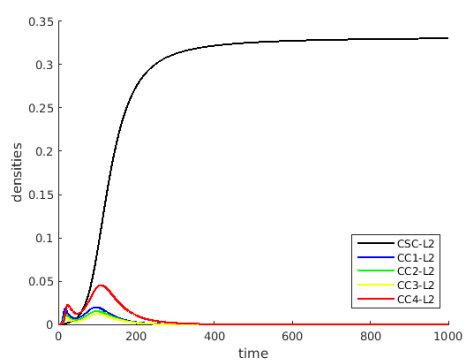

(e)

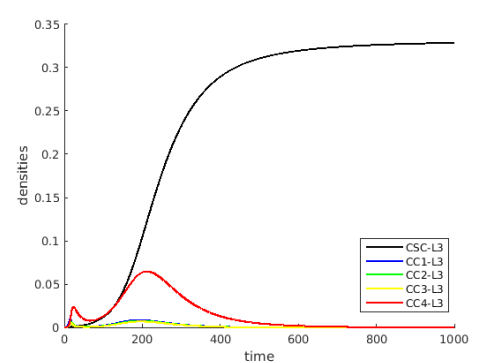

(c)

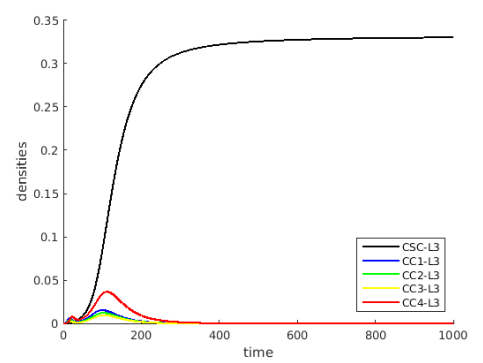

(f)

limiting an accelerated tumor growth induced by CSCs.

\section{C.3 Well-posedness of the differential equations based model}

We aim to prove the well-posedness (existence and uniqueness of the solution whose value depends continuously on the data) of problem (C.1) - (C.5) in a time interval $(O, T)$. With no lack of generality and in order to simplify notation, we will treat the case of just one lineage of CCs. Let us proceed in 2 steps: first, we will introduce an auxiliary problem and prove that it has a unique solution, then we will use this result to solve the main problem.

Step 1. For any prescribed function $\Psi(t), 0 \leq \Psi(t) \leq 1$, with Lipschitz constant $K$ in $[0, T]$, we solve the following auxiliary problem

$$
\left\{\begin{array}{llrl}
v_{a}+v_{t}=h(a, t) v, & & a \in(0, A), t \in(0, T), \\
v(a, 0)=v_{0}(a), & & a \in(0, A), \\
v(0, t)=\Phi(t)+2 \Psi(t) \int_{0}^{A} \rho(a) v(a, t) d a, & & t \in(0, T),
\end{array}\right.
$$

where, we have defined

$$
h(a, t)=-\mu(a) v-\rho(a), \quad a \in(0, A), t \in(0, T),
$$




$$
\Phi(t)=\rho_{0} d \Psi(t) U_{0} \exp \left[\rho_{0}(1-d) \int_{0}^{t} \Psi(t)(\tau) d \tau\right], \quad t \in(0, T) .
$$

Concerning the initial data, we assume that:

H1 $U_{0}>0^{1}$,

$\mathrm{H} 2 v_{0}(a)$ is a continuous non-negative function with support in $(0, \alpha)$ and such that $U_{0}+$ $\int_{0}^{\alpha} v_{0}(a) d a \leq 1$

Finally, in (C.12) we take $A=\alpha+T$.

Proposition C.3. The auxiliary problem (C.12)-(C.14), under the hypotheses (H1)-(H2) is well-posed, having a unique solution.

Proof of Proposition C.3. To solve problem (C.12)-(C.14), we use a fixed point argument. We assign a non-negative function $X(t) \in C^{1}(0, T)$ such that

$$
0 \leq X(t) \leq K_{1}, \quad|\dot{X}(t)| \leq K_{2}
$$

(where $K_{1}$ and $K_{2}$ are constants to be chosen) and we solve the linear problem

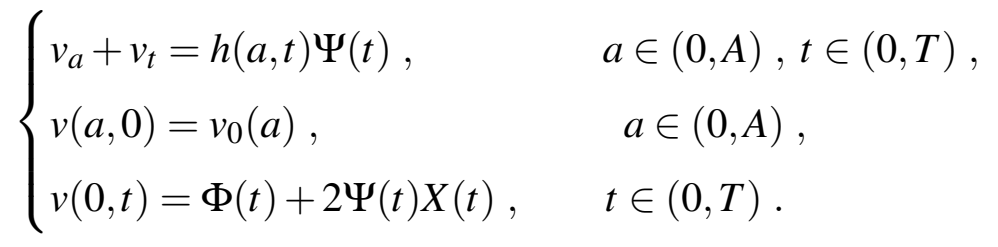

Since the data are non-negative and bounded and $h(a, t)$ in non-positive, we have that $v(a, t)$ is non-negative and bounded in $(0, A) \times(0, T)$. If we define

$$
\widetilde{X}(t)=\int_{0}^{A} \rho(a) v(a, t) d a, \quad t \in(0, T)
$$

where $v(a, t)$ is the solution of the problem (C.16), we have defined a mapping $\mathscr{T}$

$$
\widetilde{X}(t)=\mathscr{T} X(t), \quad t \in(0, T)
$$

Since $v(a, t)$ vanishes for $+t<a<A, 0<t<T$, we have

$$
\widetilde{X}(t)=\int_{0}^{t} \rho(a) v(a, t) d a+\int_{t}^{\alpha+t} \rho(a) v(a, t) d a .
$$

Clearly, $\widetilde{X}(t)$ in non-negative. Moreover, the second term is bounded by a constant $B=A \max \rho$ while the first term (the only one depending on $X(t)$ ) is $O(t)$. Therefore, choosing e.g. $K_{1}=2 B$, $T$ can be determined so that

$$
0 \leq \widetilde{X}(t) \leq K_{1}
$$

\footnotetext{
1 The case $U_{0}=0$ corresponds to the trivial case in which no CSCs are present.
} 
In addition, it is

$$
|\dot{\widetilde{X}}(t)| \leq \max \rho\left[\int_{0}^{A}\left|\frac{\partial v}{\partial t}\right| d a\right]=\max \rho\left[\int_{0}^{t}\left|\frac{\partial v}{\partial t}\right| d a+\int_{t}^{\alpha+T}\left|\frac{\partial v}{\partial t}\right| d a\right] .
$$

The second term in square brackets is bounded independently of $K_{2}$ (it does not contain $X$ nor $\dot{X}$ ) while the first term is $O(t)$. Hence, with this choice of the constants $K_{1}$ and $K_{2}, \mathscr{T}$ maps the set defined by (C.21) into itself.

Next, we have to evaluate the uniform norm of $\widetilde{X}_{1}-\widetilde{X}_{2}$ where, for $i=1,2$ it is $\widetilde{X}_{i}=$ $\rho(a) v_{i}(a, t) d a$ and $v_{i}(a, t)$ is the solution of the problem (C.16) with boundary datum

$$
v_{i}(0, t)=\Phi(t)+2 \Psi(t) X_{i}(t), \quad t \in(0, T) .
$$

We obtain that

$$
\left|\widetilde{X}_{1}(t)-\widetilde{X}_{2}(t)\right| \leq \max \rho \int_{0}^{t}\left|v_{1}(a, t)-v_{2}(a, t)\right| d a .
$$

In turn, the difference $\left|v_{1}(a, t)-v_{2}(a, t)\right|$ can be estimated in terms of the difference of the boundary data. Consequently, reducing $T$ if necessary we have that the transformation is a contraction and the Banach fixed point theorem ensures that $\mathscr{T}$ has a unique fixed point. Hence problem (C.12)-(C.14) has a unique solution. Q.E.D

Step 2. We use the previous result to prove the following theorem.

Theorem C.4. The problem (C.1) - (C.5) is well-posed in a suitable domain $(0, A) \times$ $(0, T)$.

Proof of Theorem C.4. As the previous case of Proposition C.3., we will opt for a fixed point technique. For a given function $p(t) \in C^{1}(0, T)$, such that

$$
0<P_{1} \leq p(t) \leq P_{2}, \quad|\dot{p}(t)|<P_{3},
$$

we define the function $\Psi(t)=F(p(t))$. Then, with this choice of $\Psi(t)$ we solve the problem (C.12)-(C.14), as did in Step 1, obtaining a function $v(a, t)$. We define

$$
\widetilde{p}(t)=U_{0} \exp \left[\rho(1-d) \int_{0}^{t} F(p(\tau)) d \tau\right]+\int_{0}^{A} v(a, t) d a .
$$

Then, choosing $P_{1}$ and $P_{2}$ appropriately, we find that $p(t)$ satisfies the first inequalities in (C.22). Moreover, it is

$$
\dot{\widetilde{p}}(t)=U_{0} \exp \left[\rho(1-d) \int_{0}^{t} F(p(\tau)) d \tau\right] F(p(t))+\int_{0}^{A} \frac{\partial v}{\partial t}(a, t) d a,
$$

and thus $\dot{\tilde{p}}(t)$ is bounded independently of $\widetilde{p}(t)$. Also the third inequality in (C.22) holds for $\widetilde{p}(t)$. This means that the transformation $\mathscr{P}$ defined by (C.24) maps the set (C.22) into itself. We prove that $T$ can be chosen so that $\mathscr{P}$ is a contraction. Indeed

$$
\left|\widetilde{p}_{1}(t)-\widetilde{p}_{2}(t)\right|=U_{0}\left|\exp \left[\rho(1-d) \int_{0}^{t} F\left(p_{1}(\tau)\right) d \tau\right]-\exp \left[\rho(1-d) \int_{0}^{t} F\left(p_{2}(\tau)\right) d \tau\right]\right|+
$$


APPENDIX C. Interaction between crowding and growth in tumours with stem cells: conceptual

$$
+\int_{0}^{A}\left|v_{1}(a, t)-v_{2}(a, t)\right| d a .
$$

The first term is estimated in term of $\left|p_{1}-p_{2}\right| O(t)$ because of the Lipschitz continuity of $F$. The integral in the second term can be splitted

$$
\int_{0}^{A}\left|v_{1}(a, t)-v_{2}(a, t)\right| d a=\int_{0}^{t}\left|v_{1}(a, t)-v_{2}(a, t)\right| d a+\int_{t}^{A}\left|v_{1}(a, t)-v_{2}(a, t)\right| d a .
$$

In the second term the initial data of $v_{1}$ and $v_{2}$ are equal and the difference is estimated in terms of the integral of $\left|h_{1}-h_{2}\right|$ and thus of $\left|F\left(p_{1}(\tau)\right)-F\left(p_{2}(\tau)\right]\right| O(t)$. The integrand of the first term is estimated in terms of the difference in the boundary data plus the integral of $\left|h_{1}-h_{2}\right|$. Thus the first term is $\left|p_{1}-p_{2}\right| O(t)$. Consequently $T$ can be chosen so that $\left|\widetilde{p}_{1}-\widetilde{p}_{2}\right|<\beta\left|p_{1}-p_{2}\right|$ with $\beta<1$. Using again the Banach fixed point theorem, we finally conclude the proof of the well-posedness of the problem (C.1) - (C.5). Q.E.D

\section{C.4 Concluding comments}

We have presented and discussed a conceptual model for the growth of tumors, in presence of CSCs and of several lineages of ordinary CCs. It is assumed that mitosis can be hindered by crowding of cells. Aging and apoptosis of CCs was also taken into account.

The model has been implemented in the framework of 3D cellular automata where the rules governing the evolution of $\mathrm{CA}$ are expressed in terms of stochastic dynamics. We have displayed simulations that show the development of the tumor and the fractions of the different lineages and age classes of CCs. Moreover, we have shown that the model exhibits the so-called tumor paradox, i.e., the fact that the speed of tumor growth can be greater when the mortality of the CCs increases. Consequently, we have provided examples in which the effect of cytotoxic intervention has a counterproductive effect.

We have also shown that similar results are obtained taking as unknown the fractions of families of cells over the total volume and using the same assumptions for replication, aging, and apoptosis. This is remarkable, since in this mathematical formulation based on differential equations, the crowding is no longer a local effect but is averaged over the total volume. Moreover, in these simulations the tumor paradox is shown to occur. 
ANNEX
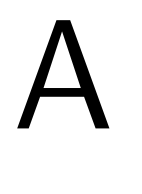

\section{LIST OF PUBLICATIONS AND CONFERENCE PARTICIPATIONS}

A list of publications and conference participations during last four years is provided:

\section{Publications in journals}

MEACCI, L.; DI BARI, V.; AUSAS, R. F.; MUT, F.; GRAY, D. A.; BUSCAGLIA, G. C. A mathematical model of a single seed oleosome. Results in Applied Mathematics, v. 9, p. 100128, 2021.

DOI: https://doi.org/10.1016/j.rinam.2020.100128

MEACCI, L.; PRIMICERIO, M.; BUSCAGLIA, G. C. Growth of tumours with stem cells: The effect of crowding and ageing of cells. Physica A: Statistical Mechanics and its Applications, 2021, p. 125841, (Elsevier), 2021.

DOI: https://doi.org/10.1016/j.physa.2021.125841

BERTSCH, M. ; FRANCHI, B. ; MEACCI, L. ; PRIMICERIO, M. ; TESI, M.C. The amyloid cascade hypothesis and Alzheimer's disease: A mathematical model. EUROPEAN JOURNAL OF APPLIED MATHEMATICS, v. 01, p. 1-20, 2020.

DOI: https://doi.org/10.1017/S0956792520000339

MEACCI, L.; BUSCAGLIA, G. C.; MUT, F.; AUSAS, R. F.; PRIMICERIO, M. A new twocomponent approach in modeling red blood cells. COMMUNICATIONS IN APPLIED AND INDUSTRIAL MATHEMATICS, v. 11, p. 55-71, 2020.

DOI: https://doi.org/10.2478/caim-2020-0004

REDDY, G. M. M.; SEITENFUSS, A. B.; DE OLIVEIRA MEDEIROS, D. ; MEACCI, L.; ASSUNÇÃO, M. ; VYNNYCKY, M. A Compact FEM Implementation for Parabolic Integro-Differential Equations in 2D. Algorithms, v. 13, p. 242, 2020.

DOI: https://doi.org/10.3390/a13100242 
MEACCI, L. Modeling a Geothermal Field: A Not-Trivial Starter Kit. Open Access Library Journal, vol. 7, no 10, p. 1, 2020.

DOI: https://doi.org/10.4236/oalib.1106836.

MEACCI, L.; DE OLIVEIRA MEDEIROS, D.; BUSCAGLIA, G. c.; PRIMICERIO, M.. O paradoxo do crescimento tumoral através de um modelo 3D de autômatos celulares com células-tronco cancerígenas. REVISTA ELETRÔNICA PAULISTA DE MATEMÁTICA, v. 14, p. 132-146, 2019.

DOI: http://dx.doi.org/10.21167/cqdvol14ermac201923169664lmdomgcbmp132146

MEACCI, L.; FARINA, A.; PRIMICERIO, M. Fluid Mechanics - A free boundary model for the evolution of a geothermal system. Rendiconti Lincei-Matematica e Applicazioni, vol. 30, no 1, p. 125-137, 2019.

DOI: http://dx.doi.org/10.4171/RLM/838

MEACCI, L.; PRIMICERIO, M. Mathematical models for tumours with cancer stem cells. Computational and Applied Mathematics, vol. 37, no 5, p. 6544-6559, 2018.

DOI: https://doi.org/10.1007/s40314-018-0707-2

MEACCI, L.; FRACALOSSI ROCHA, F.; SILVA ALVES, A.; PRAMIU, P. V.; BUSCAGLIA, G. C. . A Trajectory Planning Model for the Manipulation of Particles in Microfluidics. TEMA. Tendências em Matemática Aplicada e Computacional, v. 19, p. 509, 2018. DOI: http://dx.doi.org/10.5540/tema.2018.019.03.509

MEACCI, L.; FRACALOSSI ROCHA, F.; ALVES SILVA, A.; PRAMIU, V. P.; BUSCAGLIA, C. G . Planejamento de trajetória para a manipulação de partículas em microfluídica. REVISTA ELETRÔNICA PAULISTA DE MATEMÁTICA, v. 10, p. 19-37, 2017.

DOI: http://dx.doi.org/10.21167/cqdvol10ermac201723169664lmffraaspvpgcb1937

\section{Conference Participations}

MEACCI, L.; BUSCAGLIA, G. C. AUSAS; MUT, F.A Red Blood Cell Cyto-Bilayer Interaction Model. XXXIX Congresso Nacional de Matemática Aplicada e Computacional - CNMAC 2019, Uberlândia (MG), September 16-20th, 2019. Proceeding Series of the Brazilian Society of Computational and Applied Mathematics, 7(1), 2020.

DOI: https://doi.org/10.5540/03.2020.007.01.0344

MEACCI, L.; BUSCAGLIA, G. C. AUSAS; MUT, F. A red blood cell multi-component mathematical model for slow transient virtual experiments. ICMC Summer Meeting on Differential Equations - 2020 Chapter, Special Session on Integral and Functional Differential Equations, São Carlos/SP, Brazil, February 3-5th, 2020.

RIBEIRO, M. P.; AUSAS, R. F.; MEACCI, L.; BUSCAGLIA, G. C. Modelagem computacional de flutuaçoes termicas em globulos vermelhos. XXXIX Congresso Nacional 
de Matemática Aplicada e Computacional - CNMAC 2019, Uberlândia (MG), September 16-20th, 2019. Proceeding Series of the Brazilian Society of Computational and Applied Mathematics, 7(1), 2020

MEACCI, L.; BUSCAGLIA, G. C. AUSAS; MUT, F. Modelando um citoesqueleto de glóbulo vermelho com forças do tipo worm-like chain. In: VI ENCONTRO REGIONAL DE MATEMÁTICA APLICADA E COMPUTACIONAL, 2019, Bauru. Caderno de trabalhos completos e resumos. Bauru: Unesp, Faculdade de Ciências, p. 248 254, 2019.

SITE: https://www.fc.unesp.br/!/departamentos/matematica/eventos2341/ermac-2019/cadernode-trabalhos-e-resumos/

MEACCI, L. Exploring a Lennard-Jones Potential Application for Modeling the Red Blood Cell Cytomembrane Adhesion. Brazilian Wolfram Technology Conference 2019, São Paulo (SP), November 23th, 2019

MEACCI, L.; BUSCAGLIA, G. C. AUSAS; MUT, F. A two-component discrete-continuum red blood cell mathematical model, 9th International Congress on Industrial and Applied Mathematics - ICIAM 2019, Valencia (Spain), July 15-19th, 2019

MEACCI, L. A new two-component red blood cell discrete-continuum model. Escola Latino Americana de Matemática, Unión Matemática de América Latina y el Caribe, CMCC - Centro de Matemática, Computação e Cognição UFABC, 27 de Agosto - 06 de Setembro de 2018, Santo André (SP).

MEACCI, L. A two-component red blood cell model. Researcher Links Workshop: BioSoft-Matter Agrifood Challenges, 19 - 23 March 2018, Guaruja, São Paulo, Brazil.

BUSCAGLIA, G. C.; MEACCI L.; AUSAS, R. F. A Two-Component Fluid-Solid Finite Element Model of the Red Blood Cell, XXIII Congreso sobre Métodos Numéricos y sus Aplicaciones. Universidad Nacional de La Plata, AR. November 7, 2017. 


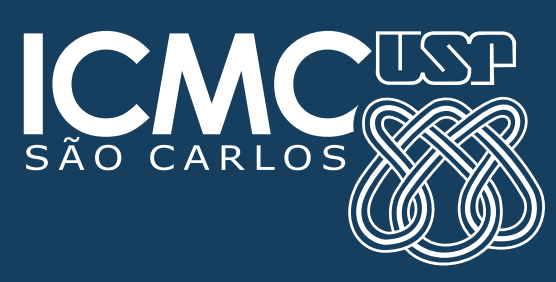

